\title{
Mathematical modeling of the structure and function of inner hair cell ribbon synapses
}

\author{
Dissertation \\ (Cumulative Dissertation) \\ for the award of the degree \\ "Doctor rerum naturalium" \\ of the Georg-August-Universität Göttingen \\ within the doctoral program Sensory and Motor Neuroscience \\ of the Georg-August University School of Science (GAUSS)
}

Submitted by

Mantas Gabrielaitis

from Lithuania

Göttingen 2015 


\section{Examination committee}

\section{Prof. Dr. Fred Wolf}

Department of Nonlinear Dynamics

Max Planck Institute for Dynamics and Self-Organization, Göttingen

Reviewer, Member of the Thesis Committee

\section{Prof. Dr. Tobias Moser}

Institute for Auditory Neuroscience and InnerEarLab

University Medical Center Göttingen

Reviewer, Member of the Thesis Committee

Prof. Dr. Erwin Neher

Department of Membrane Biophysics

Max Planck Institute for Biophysical Chemistry, Göttingen

Member of the Thesis Committee

\section{Prof. Dr. Tim Gollisch}

Research Group Sensory Processing in the Retina

Department of Ophthalmology

University Medical Center Göttingen

\section{Prof. Dr. Martin Göpfert}

Department of Cellular Neurobiology

University of Göttingen

\section{Dr. Robert Gütig}

Max Planck Research Group Theoretical Neuroscience

Max Planck Institute for Experimental Medicine, Göttingen 


\section{Statement of originality}

I hereby ensure that this dissertation entitled "Mathematical modeling of the structure and function of inner hair cell ribbon synapses" has been written independently and with no other sources and aids than quoted.

Mantas Gabrielaitis

Göttingen, October 2015 


\section{Papers submitted for this thesis}

A.B. Wong*, M.A. Rutherford*, M. Gabrielaitis*, T. Pangršič, F. Göttfert, T. Frank, S. Michanski, S. Hell, F. Wolf, C. Wichmann, and T. Moser. Developmental refinement of hair cell synapses tightens the coupling of $\mathbf{C a}^{\mathbf{2}+}$ influx to exocytosis. EMBO J 33 (3): 247 - 264. (c) 2014 The authors.

T. Pangršič*, M. Gabrielaitis*, S. Michanski, B. Schwaller, F. Wolf, N. Strenzke, and T. Moser. EF-hand protein $\mathrm{Ca}^{2+}$ buffers regulate $\mathrm{Ca}^{2+}$ influx and exocytosis in sensory hair cells. Proc Natl Acad Sci USA 112 (9): E1028 - E1037. (C) 2015 The authors. 


\section{Acknowledgments}

It is my pleasure to thank the people who accompanied me during this journey to a doctorate.

First of all, I sincerely thank my advisors Fred Wolf and Tobias Moser for providing the opportunity to work on exciting problems in their excellent groups. I feel deeply indebted for all their advice, guidance, trust, and support during these years. I would also like to express my warmest gratitude to Erwin Neher, the third member of my thesis committee, for highly valuable discussions and comments on my work.

As it can be seen from the contents of this dissertation, I had a privilege to work closely with many wonderful scientists during my stay here in Göttingen. For our collaboration, I would like to acknowledge Nicola Strenzke, Beat Schwaller, Susann Michanski, Mark Rutherford, Tina Pangršič, Aaron Wong, and Carolin Wichmann. I would like to thank Nikolai Chapochnikov for his advice and inspiring discussions during the early stage of my studies. Many thanks to all the members of the InnerEarLab and the department of Nonlinear Dynamics, especially, José Casadiego Bastidas, Tzu-Lun Wang, Tūreiti Keith, Christian Vogl, Juan Daniel Flórez-Weidinger, Chao-Hua Huang, Wenqi Wu, Iliana Panou, Markus Helmer, Rituparna Chakrabarti, Agostina Palmigiano, Andreas Neef, and Jakob Metzger, for the exchange of ideas and other academic interactions.

Crucial to my work was access to the high performance computing facilities, and it is hard to imagine better support than that provided by Yorck-Fabian Beensen, Denny Fliegner, and Hecke Schrobsdorff. Thank you.

I am very grateful to Nicola Strenzke and Peter Heil for sharing their data of the auditory nerve fiber responses.

A big thank-you to Ayse Bolik, Viktoria Novak, Regina Wunderlich, Barbara Guichemer, and the GGNB team for their excellent assistance with administrative aspects of my work and studies.

Finally, I would like to thank my family, Madina, and friends. I am immensely grateful to you for your love, support, and all the moments we have spent together. Thank you! 


\section{Contents}

$\begin{array}{ll}\text { List of Abbreviations } & 1\end{array}$

1 General Introduction $\quad 2$

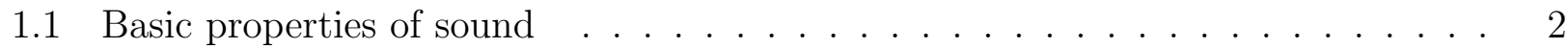

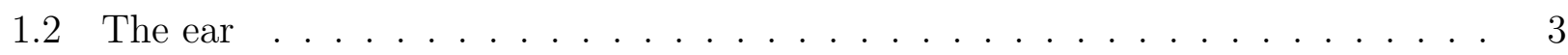

1.3 Inner hair cell ribbon synapses _. . . . . . . . . . . . . . . . . . 9

$1.4 \mathrm{Ca}^{2+}$ dynamics at presynaptic active zones $\ldots \ldots \ldots \ldots$. . . . . . . . 14

1.5 Active zone topography . . . . . . . . . . . . . . . . . . . 18

1.6 Spiral ganglion neurons . . . . . . . . . . . . . . . . . . . 21

2 Developmental Refinement of Hair Cell Synapses Tightens the Coupling of $\mathrm{Ca}^{2+}$ Influx to Exocytosis $\quad 26$

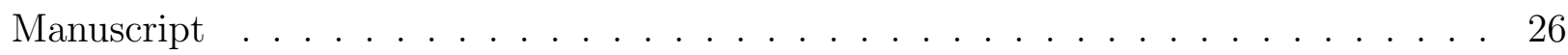

Supplementary material . . . . . . . . . . . . . . . . . 46

Additional results . . . . . . . . . . . . . . . . . . . . . . . . 74

3 EF-hand Protein $\mathrm{Ca}^{2+}$ Buffers Regulate $\mathrm{Ca}^{2+}$ Influx and Exocytosis in Sen$\begin{array}{ll}\text { sory Hair Cells } & 82\end{array}$

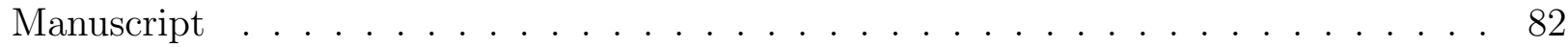

Supplementary material . . . . . . . . . . . . . . . . . . . . 94

4 Presynaptic Mechanisms of Heterogeneous Information Transfer to the Au$\begin{array}{ll}\text { ditory Nerve } & 124\end{array}$

List of abbreviations . . . . . . . . . . . . . . . . . . . . 125

4.1 Introduction . . . . . . . . . . . . . . . . . . . . . 128

4.2 Formulation of the model . . . . . . . . . . . . . . . . . . . . . . . . 129

4.3 Analytical treatment of the model . . . . . . . . . . . . . . . . . . . . 139

4.4 Results . . . . . . . . . . . . . . . . . . . . . . . . . . 148

4.5 Methods . . . . . . . . . . . . . . . . . . . . . . 184

Supplementary material $1 \ldots \ldots$. . . . . . . . . . . . . . . . . 189

Supplementary material $2 \ldots \ldots \ldots 1$

Supplementary material $3 \ldots \ldots \ldots \ldots$. . . . . . . . . . . . . . . . . . . . . . . . . . 
Supplementary material $4 \ldots \ldots \ldots \ldots \ldots$

Supplementary material $5 \ldots \ldots \ldots$. . . . . . . . . . . . . . . 224

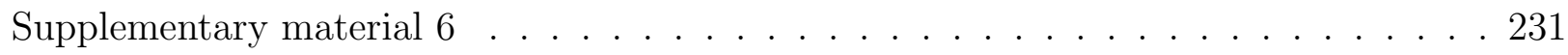

Supplementary material $7 \ldots \ldots \ldots \ldots \ldots$

5 General Discussion $\quad 244$

5.1 Presynaptic mobile $\mathrm{Ca}^{2+}$ buffers . . . . . . . . . . . . . . . . 244

5.1 .1 Summary . . . . . . . . . . . . . . . . . . . . . 244

5.1 .2 Overview of the main experimental findings . . . . . . . . . . . 245

5.1 .3 Overview of the main modeling results . . . . . . . . . . . . . . . 245

5.1 .4 Concentrations . . . . . . . . . . . . . . . . . . 246

5.1 .5 Functional role . . . . . . . . . . . . . . . . . . . 246

5.2 Active zone topography and coupling of $\mathrm{Ca}^{2+}$ influx to exocytosis . . . . . . . 247

5.2 .1 Summary . . . . . . . . . . . . . . . . . . . 247

5.2 .2 Overview of the main experimental findings . . . . . . . . . . . . 248

5.2 .3 Overview of the main modeling results . . . . . . . . . . . . . . 248

5.2 .4 A note on heterogeneity . . . . . . . . . . . . . . . . . . 249

5.2.5 Properties of $\mathrm{Ca}^{2+}$ dynamics within the active zones . . . . . . . . . . 249

5.2 .6 Developmental changes . . . . . . . . . . . . . . . . . . . 250

5.2 .7 Functional implications . . . . . . . . . . . . . . . . . . 251

5.3 Analytically tractable biophysical model of IHC ribbon synapses . . . . . . . . . 252

5.4 Presynaptic mechanisms of information decomposition in the auditory nerve . . 252

5.4 .1 Motivation . . . . . . . . . . . . . . . . . 252

5.4 .2 Approach . . . . . . . . . . . . . . . . . . 253

5.4 .3 Heterogeneity of rate-level functions . . . . . . . . . . . . . . . 253

5.4 .4 Heterogeneity of spike train statistics . . . . . . . . . . . . . . . 258

Bibliography $\quad 262$ 


\section{List of Abbreviations}

$p_{o}$

open probability of presynaptic $\mathrm{Ca}^{2+}$ channels

$R_{c}$ effective coupling distance between presynaptic $\mathrm{Ca}^{2+}$ channels and $\mathrm{Ca}^{2+}$ sensors of exocytosis

$S P L$

sound pressure level

$V_{m}$

(presynaptic) membrane potential

$V_{\text {rest }}$

(presynaptic) resting membrane potential

AZ

(presynaptic) active zone

CB-D28k

calbindin-D28k

CF

characteristic frequency

CR

calretinin

IEI

inter-release time interval

$\mathrm{IHC}$

inner hair cell

ISI

inter-spike time interval

$\mathrm{OHC}$

outer hair cell

PMCA

plasma membrane $\mathrm{Ca}^{2+}$ ATPase

$\mathrm{PV}-\alpha$

parvalbumin- $\alpha$

RLF

rate-level function

RRP

readily releasable pool of vesicles

SGN

spiral ganglion neuron

SR

spontaneous spike rate 


\section{Chapter 1}

\section{General Introduction}

In this chapter, we introduce the context of the problems addressed in this dissertation. We also provide a general overview of the structure and function of the peripheral auditory system, inner hair cells and their ribbon synapses, and spiral ganglion neurons.

\subsection{Basic properties of sound}

In physical terms, sound is a propagating wave of a variation of the pressure and mass density of the medium in which the wave propagates from their equilibrium levels. Given that the auditory system decomposes sounds into their spectral components in an early stage (see section 1.2), a harmonic function is a natural choice as the basic unit used to characterize any sound wave. The equation of a harmonic sound wave, also called a pure tone, propagating in air along axis $x$ can be written in terms of the pressure as

$$
P(t, x)=A_{p} \cdot \sin (2 \cdot \pi \cdot f \cdot(t-x / v)+\phi)
$$

Here, $P(t, x)$ is the deviation of air pressure from its mean level at moment $t$ and position $x$, $A_{p}$ is the peak pressure above this level, $f$ is the sound frequency, $v$ is the speed of propagation of the wave $\left(343 \mathrm{~m} \cdot \mathrm{s}^{-1}\right.$ in the air at $\left.20^{\circ} \mathrm{C}\right), \phi$ is the phase of the wave. In fact, after the decomposition of a sound wave into its spectral components in the auditory system, only the temporal component remains, and thus, Eq. (1.1) simplifies to $P(t)=A_{p} \cdot \sin (2 \cdot \pi \cdot f \cdot t+\phi)$. The sound intensity is usually quantified in a form of a logarithmic relative measure called sound pressure level $(S P L)$

$$
S P L=20 \cdot \log _{10}\left(A_{p} / A_{0}\right),
$$

where, $A_{0}$ is a reference sound pressure, which is usually set to $20 \mu \mathrm{Pa}$. $S P L$ is measured in decibels $(\mathrm{dB})$. The range of sound levels which can be perceived by an animal is usually wide. For example, in humans it is between 0 and $120 \mathrm{~dB}$, which corresponds to the difference in $A_{p}$ of six orders of magnitude (Viemeister, 1988). The range of audible frequencies varies from one species to another. For humans, this range is from $20 \mathrm{~Hz}$ to $20 \mathrm{kHz}$ (Purves et al., 2004). 
For cats and mice, two important laboratory animals used in investigations of the auditory system, it is $50 \mathrm{~Hz}-85 \mathrm{kHz}$ and $1 \mathrm{kHz}-80 \mathrm{kHz}$, respectively (Heffner \& Heffner, 1985; Heffner \& Heffner, 2001).

\subsection{The ear}

Detection and processing of acoustic signals in animals are performed by the auditory system. This system contains special sensory endorgans which gather and convert sound into electrochemical signals. In the case of mammals, this is performed by the tympanic ears (Fay \& Popper, 1994; Popper \& Fay, 1995; Whitlow et al., 2000; Pickles, 2008). The information about the auditory stimulus is then transmitted through the auditory nerve to the CNS for further processing. In this section, we review the key stages and structures of sound processing in the ear of terrestrial mammals.

\section{The outer ear}

In terrestrial mammals, the ear is divided into three parts: the outer, the middle, and the inner ears (Fig. 1.1). The outer ear consists of the pinna, which includes a resonant hollow called the concha, and the external auditory meatus (the ear canal). The main function of the external ear is to collect airborne sound waves and transmit them efficiently to the middle ear. Moreover, the geometrical features of the pinna and concha enable mammals to distinguish between sounds coming from below or above, and front or behind (Pickles, 2008).

\section{The middle ear}

At the end of the ear canal, sound waves hit the tympanic membrane (eardrum) separating the outer and middle ears (Fig. 1.1). The tympanic membrane is rigidly connected to the flexible membrane of the oval window in the cochlea (which belongs to the inner ear) by three small middle-ear bones: the malleus, the incus and the stapes. The main function of the middle ear is to match the sound impedances of air in the ear canal and the cochlear fluids of the inner ear (Pickles, 2008). Much of the sound energy would be reflected away without the middle ear mechanism because the sound impedance is much lower in gas than in liquid. The impedance matching is based on the twenty-fold larger area of the tympanic membrane than the oval window (see the upper right panel of Fig. 1.1). 


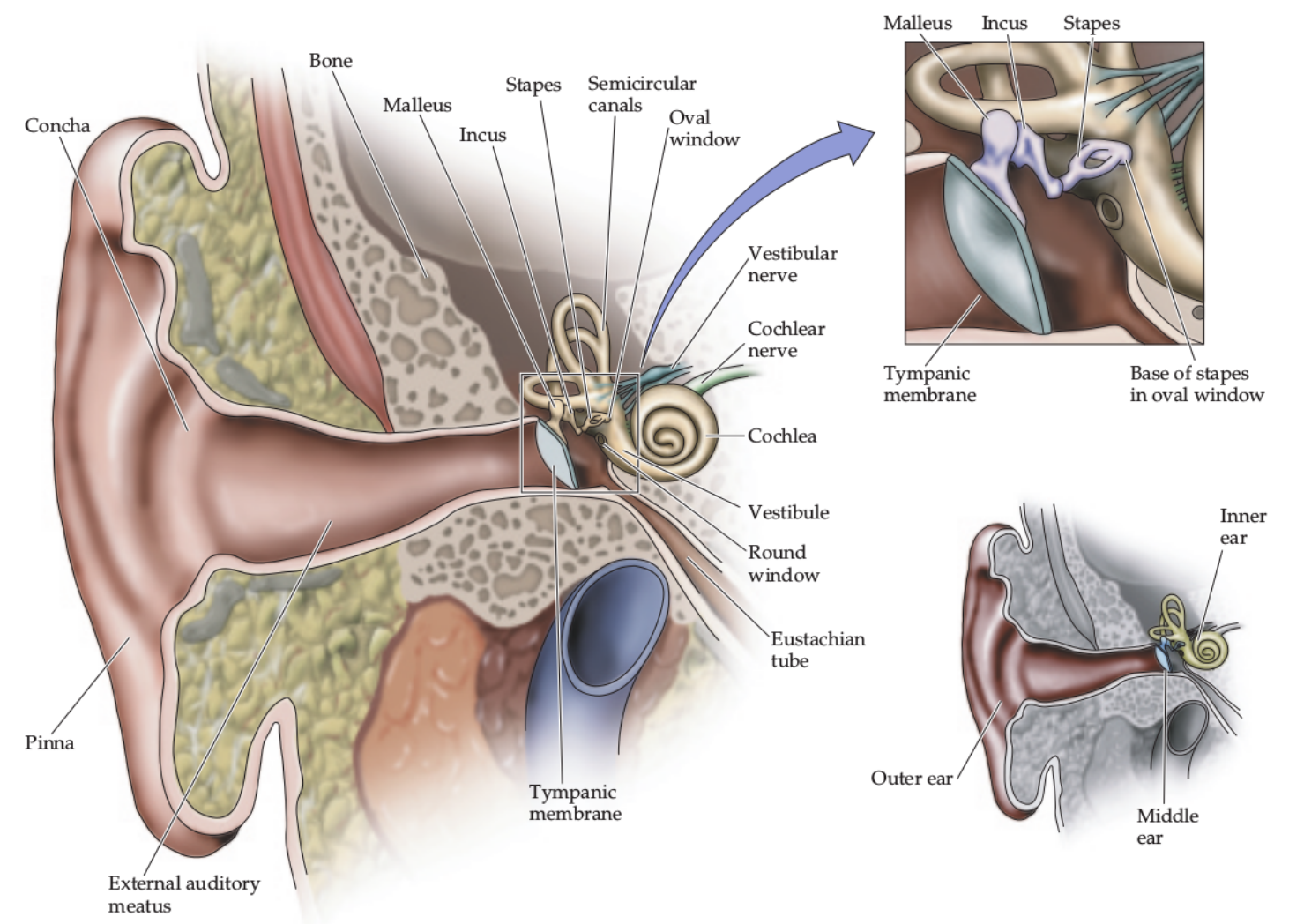

Figure 1.1: The main elements of a human ear. The lower right panel defines the three main compartments of the ear. An enlarged view of the assembly of the components of the middle ear is shown in the upper right panel. Source: (Purves et al., 2004).

\section{The inner ear: the cochlea}

The cochlea is the auditory part of the inner ear, which also carries the vestibular apparatus (Fig. 1.2). The cochlea is a coiled hollow duct of bone with three parallel chambers filled with fluids. In humans, it is $10 \mathrm{~mm}$ wide and $5 \mathrm{~mm}$ high. From base to apex, the coil makes $\sim 2.5$ turns (Pickles, 2008). A cross-section of the cochlear duct is pictured in the upper right panel of Fig. 1.2. Two of the cochlear chambers, the scala vestibuli and the scala tympani are filled with perilymph (similar in ionic composition to normal extracellular fluid) and connect at the apex of the cochlea. They are separated by a third chamber, the scala media, which is filled with endolymph (rich in $\mathrm{K}^{+}$and low in $\mathrm{Na}^{+}$, like a typical intracellular solution). The scala media is separated from the scala vestibuli by the Reissner's membrane, and from the scala tympani by the basilar membrane, a fibrous structure (Fig. 1.2).

The scala media harbors the auditory transducer called the organ of Corti, which sits on the basilar membrane and extends along the cochlear canal from the base (where the oval window is located) to the apex (Fig. 1.2). The organ of Corti consists of hair cells, which are the mechanosensory receptors, and a multitude of supporting cells providing structural and metabolic support. The hair cells are of two kinds. The inner hair cells (IHCs) are distributed 


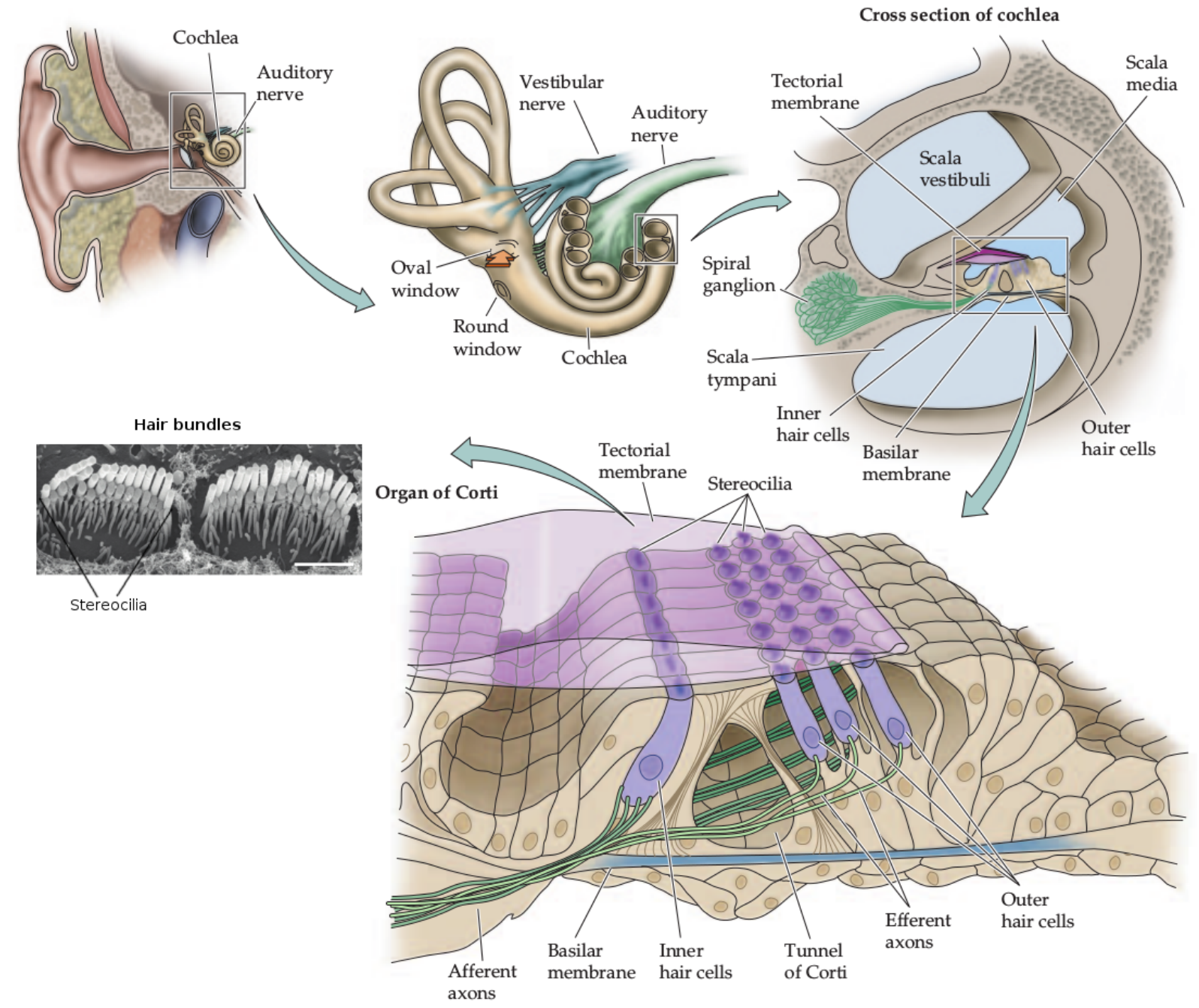

Figure 1.2: Organization of the cochlea and the organ of Corti. An enlarged view of the cochlea is provided by the top middle panel. Subsequent two panels show cross sections of the cochlear duct and the organ of Corti. The lower left panel is an electron micrograph of the surface of the organ of Corti with hair bundles of two inner hair cells exposed (the scale bar, $2 \mu \mathrm{M}$ ). Source: the lower left panel taken from (Kazmierczak \& Müller, 2012), the rest adapted from (Purves et al., 2004). 
in one row along the organ of Corti closer to the modiolus (the cochlear central axis). The outer hair cells (OHCs) are aligned in three to five rows, further away from the modiolus (Fig. 1.2). The scala media also contains the stria vascularis (Fig. 1.2), a tissue which is responsible for maintaining the ionic composition and high positive potential of the endolymph (Marcus et al., 2002; Wangemann, 2006), crucial for the electromechanical conversion of sounds by the organ of Corti.

The vibrations of sound at the oval window, which opens onto the scala vestibuli, induce displacements of the cochlear fluids coupled to the transversal motion of the basilar membrane and all structures fixed to it. This commences a mechanical wave traveling towards the apex of the cochlea. The motion of the incompressible fluids is allowed by a second membranous patch, the so-called round window, which opens onto the scala tympani (Fig. 1.2). The transversal movement of the basilar membrane induces a shearing motion of the fluid between it and the so-called tectorial membrane, a gelatinous sheet which extends above the organ of Corti in the scala media (Fig. 1.2). The shearing motion deflects the hair bundles of IHCs and OHCs periodically triggering their electrical depolarization (as described in the next section).

From the perspective of a propagating sound wave, the cochlea is very different from a homogeneous and isotropic medium, like air. The cochlea acts as a narrow-bandwidth filter with the resonance frequency gradually decreasing from very high at the base to very low at the apex. Thus, the sound wave is decomposed into its harmonic components in space as it propagates along the cochlear duct. This gives rise to the tonotopic organization of the cochlea: a certain spectral component is predominantly encoded by the transversal motion of only a narrow, unique segment of the basilar membrane, and leads to excitement of relatively few hair cells. However, it should be noted that the spectral resolution is worsened when the sound amplitude is increased. The basis for frequency tuning of the cochlea lies in the bulk linear mechanical properties of the basilar membrane and the cochlear fluids (Bekesy, 1960; Ruggero \& Robles, 2001; Pickles, 2008). Additional sharpening of the tuning is provided by the active mechanical amplification of the basilar membrane deflection (Nobili et al., 1998; Pickles, 2008). This amplification is most likely produced by OHCs, which, upon mechanical activation, feed additional energy back into the traveling wave. Two candidate mechanisms of the active amplification are currently being considered. The first of them is the active motion of the hair bundle of OHCs (Crawford \& Fettiplace 1985; Howard \& Hudspeth, 1987; Manley et al., 2001; Fettiplace, 2006). The second mechanism is based on contractions of OHC bodies which are induced upon depolarization and mediated by piezoelectric protein prestin expressed in the plasma membrane of these cells (Brownell et al., 1986; Zheng et al., 2000; Dallos et al., 2006).

\section{Inner hair cells}

Information about the spatially decomposed sound waves is thought to be passed to the brain by the type I spiral ganglion neurons excited by IHCs (Kiang et al., 1982; Weisz et al., 2009). 
IHCs are flask-shaped cells (Fig. 1.3A) with typical length and diameter of $35 \mu \mathrm{M}$ and $10 \mu \mathrm{M}$, respectively (Pickles, 2008). There are $~ 3500$ IHCs per cochlea in humans (Moller, 2000). The name "hair" arises from the apically positioned hair bundles which bear the mechanotransduction apparatus (see lower left panel of Fig. 1.2). The hair bundles of IHCs consist of $40-80$ hairs (also called sterecocilia) aligned in three rows in increasing height in a nearly linear fashion (Fig. 1.2; Garfinkle \& Saunders, 1983; Lim, 1986). Each stereocilium contains an ordered cytoskeleton made of actin filaments and is relatively stiff (reviewed by Peng et al., 2011). They are anchored in the cuticular plate at the apical surface of IHCs and bend only at the anchoring points upon mechanical stimulation. Stereocilia within the bundle are cross-linked by thin proteinaceous fibers (Pickles et al., 1984). This linking together with the hydrodynamic coupling and the mechanical constraints at the insertion points are thought to explain why the stereocilia move coherently as a unit upon mechanical stimulation (Kozlov et al., 2007).

A deflection of the hair bundle from the resting position towards the row of the tallest stereocilia increases the open probability of the mechanotransducer channels which carry an inward current of positively charged ions. It was suggested based on $\mathrm{Ca}^{2+}$ imaging and electrophysiological recordings that one to two of these channels are located at the tips of stereocilia, 50 - 100 channels per bundle (Denk et al., 1995; Beurg et al., 2006; Beurg et al., 2009). The mechanical tension necessary to open their pores is believed to be mediated by the so-called tip links, which connect the tips of the shorter stereocilia with the neighboring longer stereocilia (Pickles, et al., 1984; Assad et al., 1991; Kazmierczak \& Mueller, 2012). The activation kinetics of the mechanotransducer channel is very fast: the current estimates of several tens of $\mu$ s only reflect the upper limit of the temporal resolution of the measuring devices (Corey \& Hudspeth, 1979; Ricci et al., 2005). The mechanotransducer channels are nonselective, high conductance $(150-300 \mathrm{pS})$ cation channels which, in physiological conditions, mostly mediate inward $\mathrm{K}^{+}$ and $\mathrm{Ca}^{2+}$ currents (Lumpkin et al., 1997; Ricci et al., 2003; Beurg et al., 2006). It has to be noted that a small fraction of the mechanotransducer channels are open even in the absence of mechanical stimulation, and contribute to the resting membrane potential $\left(V_{\text {rest }}\right)$, (Hudspeth \& Corey, 1977). Molecular identity of these channels is not known yet, though the transmembrane channel-like proteins were recently suggested as potential candidates (Pan et al., 2013).

Mature IHCs respond to mechanical stimuli by graded changes of the membrane potential $\left(V_{m}\right)$. Fig. 1.3B shows the dynamics of $V_{m}$ in response to pure-tone sound stimuli of fixed $S P L$ (Palmer \& Russell, 1986). Low frequency tones induce periodic oscillations of $V_{m}$ with vanishing DC component. When the sound frequency is increased, the DC component increases while the $\mathrm{AC}$ component decreases, becoming vanishingly small above $3-5 \mathrm{kHz}$. This is thought to mainly reflect the low pass filtering properties of IHC plasma membrane, which has a time constant of $\sim 1 \mathrm{~ms}$ at rest (Sellick \& Russell, 1980; Russell \& Sellick, 1986; Raybould et al., 2001). Besides the mechanotransducer channels in the hair bundle, the resting and receptor potentials of mature IHCs are known to be considerably influenced by several different voltagegated $\mathrm{K}^{+}$channels expressed basolaterally. There is a slowly activating delayed rectifier $\mathrm{K}^{+}$ 
current mediated by 4-aminopyridine sensitive channels. KCNQ4 channels underlie the inwardly rectifying current (Oliver et al., 2003). The fast $\mathrm{K}^{+}$current is mediated by large-conductance $\mathrm{Ca}^{2+}$-dependent BK channels (Kros et al., 1998). These channels are expressed within the first few weeks after birth of an animal and considerably reduce the membrane constant, endowing mature IHCs with higher frequency response (Kros et al., 1998). Also, the fast $\mathrm{K}^{+}$current prevents initiation of $\mathrm{Ca}^{2+}$ mediated spikes, which are observed in immature IHCs. It has to be noted that the basolateral part of the IHC is exposed to perilymph, thus, these $\mathrm{K}^{+}$currents are outward.

A

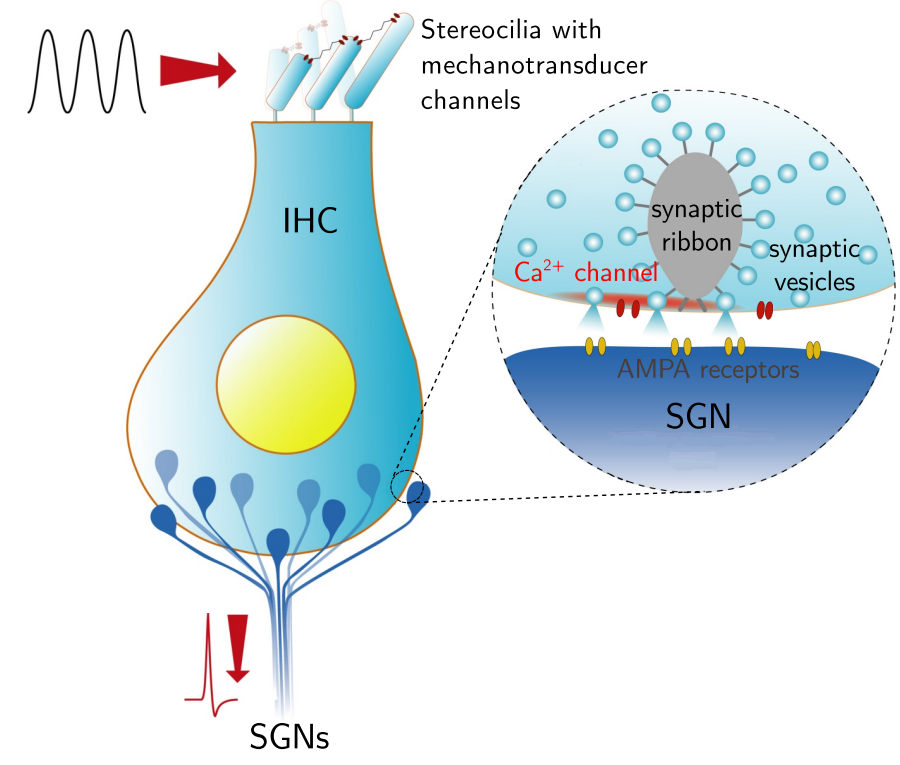

B
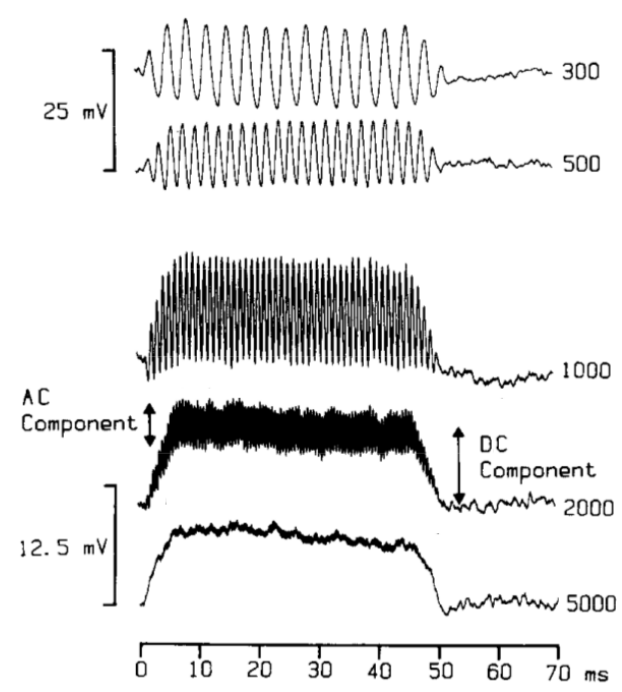

Figure 1.3: The main components and electric responses of inner hair cells. (A) The key structural and functional elements of IHC and its ribbon synapses involved in sound encoding. (B) Responses of IHC membrane potential to $80 \mathrm{~dB}$ SPL sound bursts of different frequencies. The numbers on the right of each trace indicate the frequencies of sound stimuli. The upper scale bar is for the two top traces, the lower scale bar is for the remaining three traces. Source: panel B adapted from (Palmer \& Russell, 1986).

The information about auditory stimuli encoded in changes of $V_{m}$ of IHCs is transferred to the CNS by $\mathrm{Ca}^{2+}$-dependent neurotransmitter release at excitatory ribbon synapses made between IHCs and spiral ganglion neurons (SGNs) (see Fig. 1.3A). Around a dozen synapses are formed at the base of each IHC, with the exact number varying dependent on the species and tonotopic position along the cochlea (Meyer et al., 2009; Meyer \& Moser, 2010). Each of these synapses drive separate SGNs and provide the sole afferent inputs received by these neurons (Liberman, 1980). The structure and function of the presynaptic sites of these synapses are reviewed in the next section. 


\subsection{Inner hair cell ribbon synapses}

\section{Synaptic ribbons and active zones}

The distinguishing feature of all ribbon synapses is a structural specialization, the so-called synaptic ribbon, seen as a dense body at the presynaptic active zones (AZs) in electronmicrographs (Fig. 1.4A; Matthews \& Fuchs, 2010). The name of this structure stems from its characteristic morphology, an elongated, electron-dense sphere in parallel with the plasma membrane, which resembles a ribbon. The synaptic ribbons are surrounded by a halo of synaptic vesicles that are connected to it via filamentous tethers. The main structural constituent of the synaptic ribbons is the protein RIBEYE (Schmitz et al., 2000; Khimich et al., 2005), which consists of two key domains. The so-called A domain is thought to participate in oligomerization of different RIBEYE molecules to form the scaffold of the presynaptic body (Magupalli et al., 2008; Schmitz, 2009). The so-called B domain was suggested to be involved in the formation as well as tethering of vesicles (Schmitz et al., 2000; Schmitz, 2009). A stripe-shaped electron-dense area, the so-called presynaptic density, is located below the ribbon (Fig. 1.4A, B).

Within this area, $\mathrm{Ca}^{2+}$ channels, the protein components of the vesicular fusion machinery, and cytomatrix proteins, which spatially arrange all molecular components involved in exocytosis, are clustered (reviewed in Rutherford \& Pangršič, 2012; Wichmann \& Moser, 2015). Bassoon, one of the cytomatrix proteins, is crucial for anchoring the ribbons to the presynaptic densities via interaction of the B domain of RIBEYE (Dieck et al., 2005; Khimich 2005; Frank et al., 2010; Regus-Leidig et al., 2014).

\section{Voltage-gated $\mathrm{Ca}^{2+}$ channels}

Depolarization of an IHC leads to opening of voltage-gated $\mathrm{Ca}^{2+}$ channels and subsequent influx of $\mathrm{Ca}^{2+}$ at the presynaptic AZs. About $90 \%$ of the $\mathrm{Ca}^{2+}$-influx is mediated by L-type $\mathrm{Ca}_{\mathrm{V}} 1.3$ channels, genetic elimination of which reduces the presynaptic exocytosis to marginal levels and leads to deafness (Platzer et al., 2000; Brandt et al., 2003). Inactivation of Cav1.3 channels is weak (Cui, et al., 2007; Neef et al., 2009), which makes them well suited for encoding prolonged stimuli. In hearing animals, STED immunolocalization experiments indicate that these $\mathrm{Ca}^{2+}$ channels are predominantly clustered in the plasma membrane just below the presynaptic densities (Fig. 1.4C; Frank et al., 2010). Here, bassoon and Rab3-interacting molecules (RIM2 $\alpha$ and RIM $2 \beta$ ) were shown to be involved in clustering of Cav1.3 channels for efficient exocytosis at IHC ribbon synapses (Frank et al., 2010; Jung et al., 2015). Based on nonstationary fluctuation analysis of $\mathrm{Ca}^{2+}$-tail currents, the estimated number of $\mathrm{Ca}_{\mathrm{V}} 1.3$ channels per IHC in the apical region of the cochlea is $\sim 1800$ (Brandt et al., 2005). Assuming thirteen synapses per IHC (Meyer et al., 2009), this would amount to $\sim 140$ channels per synapse. Which part of these channels corresponds to the presynaptic density is, however, not known. 
A

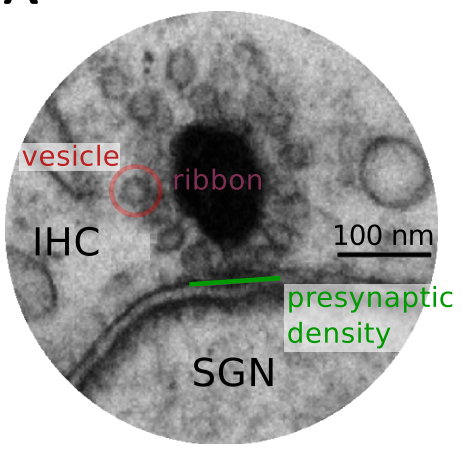

C

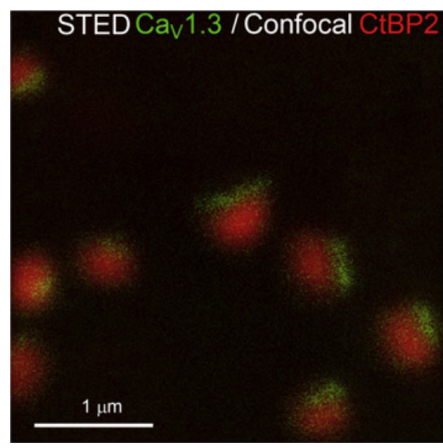

E

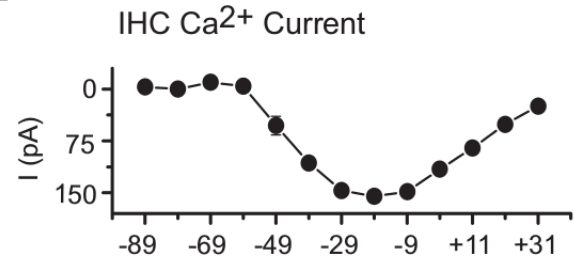

AF Average Response

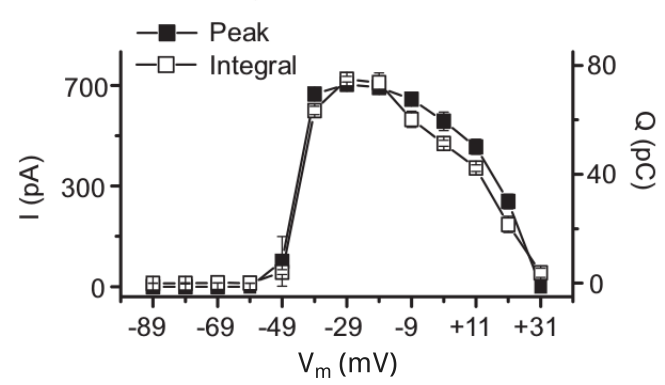

B

D
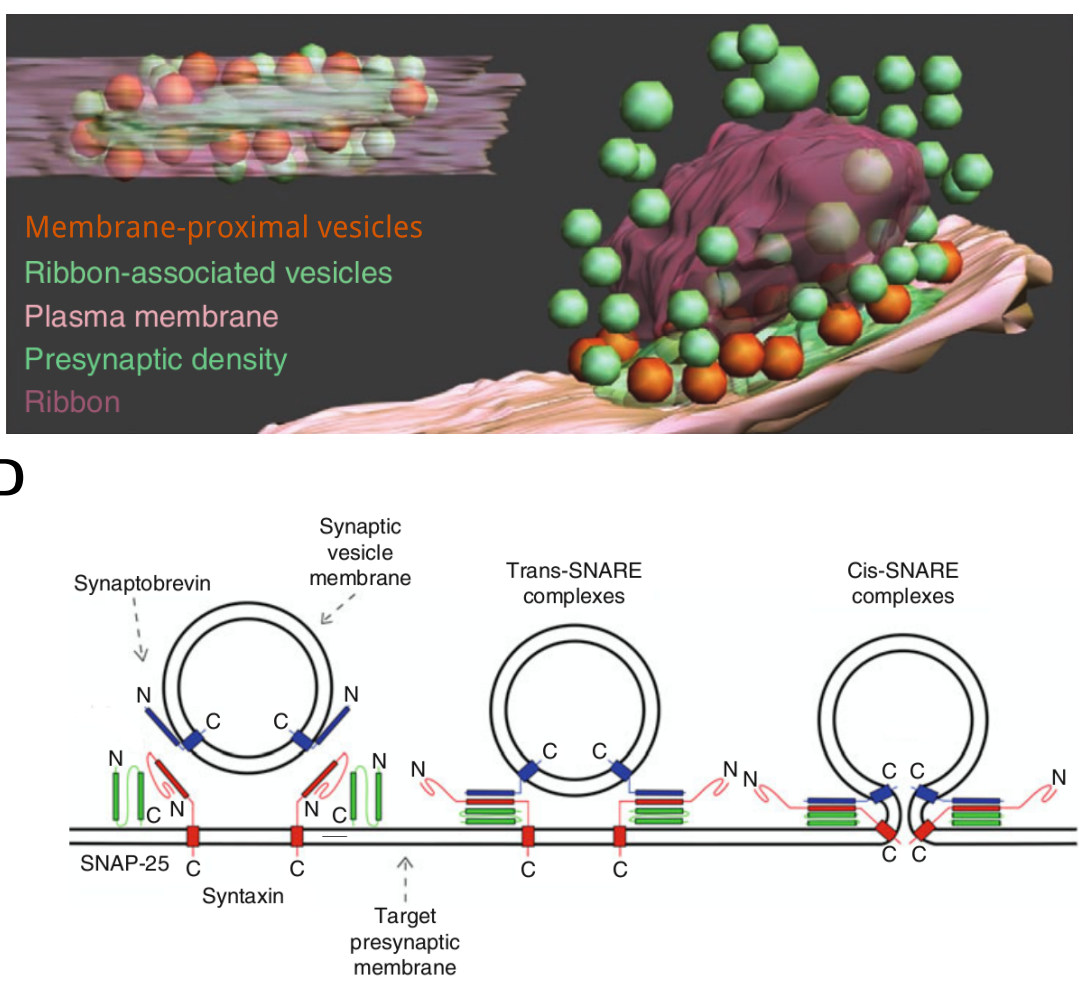

$\mathbf{F}$

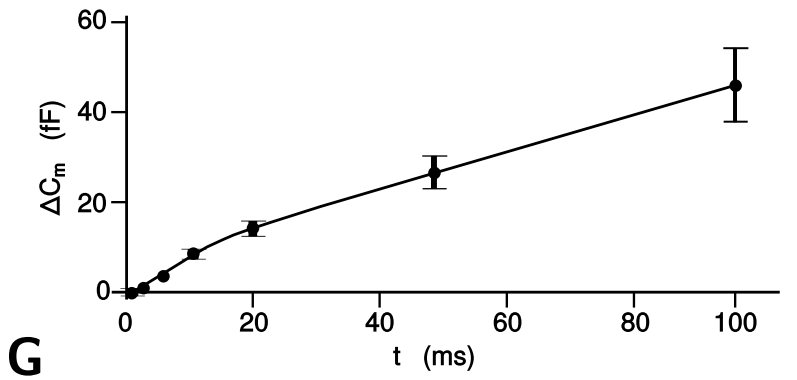

G
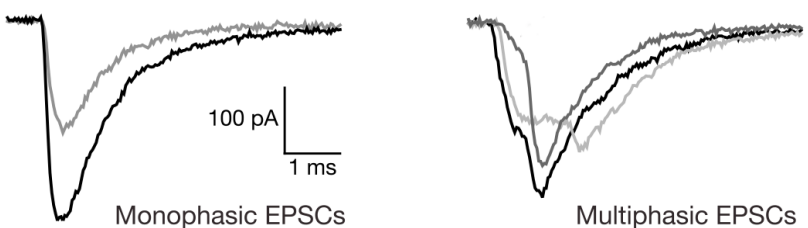

Figure 1.4: Structure and function of inner hair cell ribbon synapses. (A) An electron micrograph of a ribbon synapse (source: Frank et al., 2010). (B) 3D tomographic reconstruction of a ribbon synapse (source: Pangršič et al., 2010). (C) Single optical sections of ribeye (confocal) and Cav1.3 (STED) immunofluorescence (source: Frank et al., 2010). (D) The molecular mechanism of vesicle fusion at conventional synapses (source: Cohen, 2013). (E) $V_{m}$-dependencies of the presynaptic $\mathrm{Ca}^{2+}$ current (upper subpanel) and extent of exocytosis (lower subpanel). The extent of exocytosis was quantified either as a peak postsynaptic current or as an integral of the postsynaptic current over $200 \mathrm{~ms}$ from 6 IHCs (source: Goutman \& Glowatzki, 2007). (F) Exocytic presynaptic membrane capacitance increment $\left(\Delta \mathrm{C}_{\mathrm{m}}\right)$ as a function of the depolarizing step duration (source: Pangršič et al., 2010). (G) Examples of monophasic and mutiphasic EPSCs (source: Chapochnikov et al., 2014). 


\section{Readily releasable pool of synaptic vesicles}

$\mathrm{Ca}^{2+}$ ions which enter the presynaptic sites upon opening of the voltage-gated channels bind to the $\mathrm{Ca}^{2+}$-sensors of exocytosis and initiate neurotransmitter release. Here, fusion-competent vesicles tethered to the plasma membrane at the circumference of the presynaptic density release glutamate into the synaptic cleft upon stimulation (Fig. 1.4B). These vesicles are commonly referred to as the readily releasable pool of synaptic vesicles (RRP) (Lenzi et al., 2002; Frank et al., 2010; Pangršič et al., 2010). Results from electron tomography of the presynaptic sites and electrophysiological measurements of the vesicle fusion kinetics suggested that the RRP of IHC ribbon synapses consists of approximately a dozen of vesicles, on average (Pangršič et al., 2010). These vesicles are tethered either to the presynaptic membrane or synaptic ribbon (Wichmann \& Moser, 2015). Together with the presynaptic density protein bassoon, the synaptic ribbons are crucial for maintaining large and functional RRPs (Khimich 2005; Frank et al., 2010; Jing et al., 2013).

\section{Vesicle fusion machinery}

The molecular machinery which mediates fusion of synaptic vesicles with the plasma membrane at IHC synapses is poorly understood (reviewed by Rutherford \& Pangršič, 2012). At CNS synapses, this task is performed by the SNARE proteins synaptobrevin, syntaxin 1, and SNAP25 (reviewed in Jahn \& Fasshauer, 2012). Once brought in contact, the four snare motifs of these proteins assemble into a stable bundle of interlaced $\alpha$-helices to form the trans-SNARE complex (Fig. 1.4D). An inward force which pulls both membranes towards each other is then induced. The full merging of the membranes is believed to be prevented by a clamp formed upon binding of complexins, small cytosolic proteins. This clamp is removed and the irreversible fusion is induced by synaptotagmin, the major $\mathrm{Ca}^{2+}$-sensor of exocytosis, upon binding of $\mathrm{Ca}^{2+}$-ions. However, IHC ribbon synapses appear to operate without neuronal isoforms of these proteins. Indeed, none of SNAP-25, syntaxin 1, and synaptobrevin $1-3$ could be found by immunohistochemistry in mice IHCs (Nouvian et al., 2011). In agreement, IHC exocytosis was insensitive to the application of neurotoxins targeted to, and genetic deletion of, those proteins. Complexins $1-4$ were not detected in IHCs either and were shown to be dispensable for normal hearing in mice (Strenzke et al., 2009; Uthaiah \& Hudspeth, 2010). The role of synaptotagmins in IHC exocytosis is controversial and requires further investigation; however, Syt1 and Syt2, which are the main synaptotagmin isoforms at conventional synapses, are not expressed in adult IHCs (reviewed in Rutherford \& Pangršič, 2012). In this regard, the accumulating evidence points towards otoferlin, a multi-C2 domain, $\mathrm{Ca}^{2+}$-binding protein with structural homology to synaptotagmin and Munc13-like priming factors, as the major $\mathrm{Ca}^{2+}$-sensor of exocytosis at IHC ribbon synapses (reviewed in Pangršič et al., 2012). In the absence of otoferlin, no depolarization-induced RRP fusion at IHCs ribbon synapses was observed. Moreover, a role of otoferlin in vesicular priming and replenishment has been postulated (Roux et al., 2006; 
Pangršič et al., 2010). In accordance with these findings, mutations or deletion of the otoferlin gene lead to deafness (Yasunaga et al., 1999; Roux et al., 2006).

\section{Kinetics and $\mathrm{Ca}^{2+}$ dependence of presynaptic vesicle fusion}

Based on electrophysiological estimates of the exocytosis vs $\mathrm{Ca}^{2+}$ influx relations, it was suggested that fusion of RRP vesicles at IHC synapses happens upon sequential binding of $4-5$ $\mathrm{Ca}^{2+}$ ions to the $\mathrm{Ca}^{2+}$ sensor of exocytosis (Beutner et al., 2001; Goutman \& Glowatzki, 2007). This is in line with the findings from CNS synapses (Pang \& Südhof, 2010). The upper subpanel of Fig. 1.4E shows the dependence of the whole-cell $\mathrm{Ca}^{2+}$ current $\left(I_{C a}\right)$ on $V_{m}$. $I_{C a}$ activates around $-60 \mathrm{mV}$ and reaches the peak level at $-20 \mathrm{mV}$ due to increased open probability of presynaptic Cav1.3 channels. Further depolarization of the cell leads to only negligible elevations of the open probability and the amplitude of $I_{C a}$ starts decreasing, as $V_{m}$ approaches the reversal potential of the current $(\sim+40 \mathrm{mV})$. Exocytosis reflects the $V_{m}$ vs $I_{C a}$ relation (Fig. $\left.1.4 \mathrm{E}\right)$.

In the physiological $V_{m}$ range, auditory hair cell ribbon synapses feature a pronounced shortterm depression and no facilitation (Moser \& Beutner, 2000; Goutman \& Glowatzki., 2011; Cho et al., 2011). A transient depolarization of IHC from the resting potential to another fixed level leads to an initial fast component of vesicular release, which slows down with ongoing stimulation and reaches a steady state within $20 \mathrm{~ms}$ (Fig. 1.4F). This decrease in exocytosis rate during ongoing stimulation was proposed to be solely due to a partial depletion of the RRP during ongoing stimulation (Moser \& Beutner, 2000; Pangršič et al., 2010). Paired-pulse facilitation, but only mild, is observed only if the holding membrane potential is decreased to -90 mV or less (Cho et al., 2011; Goutman \& Glowatzki, 2011).

Neurotransmitter release at IHC ribbon synapses induces large EPSCs, with peak amplitudes of up to $800 \mathrm{pA}$ (average $\sim 300 \mathrm{pA}$ ), at the postsynaptic boutons of afferent spiral ganglion neurons (Glowatzki \& Fuchs, 2002; Grant et al., 2010; Chapochnikov et al., 2014). The majority of these EPSCs are single peaked (Fig. 1.4G), however, the remaining have a pronounced multiphasic character. Based on these findings (size and shape), it was suggested that single EPSCs are induced by the fusion of multiple quanta (Glowatzki \& Fuchs, 2002; Neef et al., 2007). Here, two main scenarios were proposed: (1) synchronized fusion of multiple synaptic vesicles simultaneously, or (2) $\mathrm{Ca}^{2+}$-dependent homotypic fusion of multiple vesicles followed by fusion of the formed compound vesicles. In the first scenario, the synchronized vesicle fusion is supposed to happen without a synchronizing effect of action potentials. In a recent publication, both of these hypotheses were challenged by combined results from electrophysiology, light and electron microscopy, as well as computational modeling (Chapochnikov et al., 2014). In particular inconsistent with the previously proposed models is the fact that the EPSC shape properties do not depend on the stimulation intensity, and thus, on the the vesicle release rate (Glowatzki \& Fuchs, 2002; Goutman \& Glowatzki, 2007; Chapochnikov et al., 2014), and that variable EPSCs are observed even in the absence of $\mathrm{Ca}^{2+}$ (Chapochnikov et al., 2014). Moreover, the total charge of all EPSCs is similar, independent of their shape (Grant et al., 
2010; Chapochnikov et al., 2014). Hence, it was proposed that each EPSC is rather induced by fusion of a single vesicle at IHC ribbon synapses (Chapochnikov et al., 2014). As shown by biophysical modeling, the multiphasic shapes of EPSCs can, in fact, be explained by dynamic flickering of the fusion pore between the vesicle and the plasma membrane.

\section{Vesicle replenishment and endocytosis}

One of the distinguishing functional features of IHC ribbon synapses is their ability to release vesicles at very high rates over prolonged periods of time. Indeed, the postsynaptic SGNs are capable of spiking at rates above $100 \mathrm{~Hz}$ during continuous stimulation over 10 min (Kiang et al., 1965). Taking into account the phasic nature of SGN responses (see below), the underlying vesicle fusion rate at the presynaptic $\mathrm{AZ}$ cannot be smaller. The necessary conditions for this are (i) high capacity refilling of the RRP, (ii) fast clearance of the presynaptic membrane from fused vesicle components and (iii) an efficient endocytosis machinery to replenish vesicles during ongoing stimulation. To date, only a few fragments of the whole picture of the molecular mechanisms underlying endocytosis and replenishment of vesicles at these synapses have been exposed.

The RRP refilling at IHC as well as other ribbon synapses is believed to happen directly from the reserve pool of vesicles tethered to the synaptic ribbons (Lenzi et al., 2002; Schmitz, 2009; Frank et al., 2010; Snellman et al., 2011). Here, active as well as passive (i.e., diffusion), transport mechanisms of the ribbon-attached vesicles towards the AZ were hypothesized (Bunt, 1971; Lenzi \& von Gersdorff, 2001; Graydon et al., 2014). However, little is known about the kinetics. It was shown though that application of $2.5 \mathrm{mM}$ of the slow mobile $\mathrm{Ca}^{2+}$-binding buffer EGTA specifically slows vesicular replenishment to the presynaptic AZs of IHCs, without affecting the RRP kinetics (Moser \& Beutner, 2000). This finding suggests the dependence of replenishment on $\mathrm{Ca}^{2+}$ hundreds of nm away from the clusters of presynaptic $\mathrm{Ca}^{2+}$ channels. Adaptor protein 2, one of the key players in clathrin-dependent endocytosis at CNS synapses (reviewed in Jahn \& Boyken, 2013) and interaction partner of otoferlin in IHCs has recently been shown to be important for fast vesicle replenishment at IHC ribbon synapses (Pangršič et al., 2010; Duncker et al., 2013; Jung et al., 2015). The mechanisms of priming of the replenished vesicles in this system remain elusive. Lately, it was demonstrated that IHC ribbon synapses function in the absence of Munc13-like proteins (Vogl et al., 2015), which are employed to establish fusion competence of vesicles at CNS synapses (Südhof, 2012). Otoferlin as well as the ribbon itself were proposed to be involved in vesicle priming (Pangršič et al., 2010; Snellman et al., 2011).

The ribbon-associated pool of vesicles is thought to be directly replenished by endocytic processes. Current findings support the presence of both, clathrin mediated, and bulk endocytosis at IHC ribbon synapses. In this context, clathrin-coated vesicles, as well as large invaginations of the plasma membrane were found in direct vicinity of IHC AZs (Frank et al., 2010; Neef et al., 2014; Kamin et al., 2014). Recently, these findings were further substanti- 
ated by live-cell imaging experiments using the newly developed plasma membrane trafficking marker mCling in combination with super-resolution fluorescence microscopy, which revealed that depolarization-induced recycling of IHC membrane happens around AZs at the base of IHCs (Revelo et al., 2014). In particular, bulk exocytosis was evident from these measurements as large membranous cisterns were converted into small, synaptic-like organelles within a few minutes after stimulation. Moreover, recent electrophysiological measurements of the kinetics of the plasma membrane turnover showed that the clathrin-mediated endocytosis poses the predominant membrane retrieval mechanism in response to mild depolarizations and happens at constant rate (Neef et al., 2014). This component of endocytosis could specifically be blocked with clathrin inhibitors and pharmacological or genetic disruption of dynamin 1. Upon prolonged depolarizations, corresponding to exocytosis of three or more equivalents of RRP, an additional, faster mode of exocytosis was initiated (Neef et al., 2014). It was proposed to reflect bulk endocytosis. To date, the exact mechanism of synaptic vesicle genesis from endocytic cisterns remains to be clarified, but recent evidence suggests a role of presynaptic ribbons in this process (Schmitz, 2009; Kantardzhieva et al., 2013).

\section{$1.4 \mathrm{Ca}^{2+}$ dynamics at presynaptic active zones}

\section{Factors shaping $\left[\mathrm{Ca}^{2+}\right]$ at presynaptic active zones}

An open presynaptic $\mathrm{Ca}^{2+}$ channel can be treated as a point source of $\mathrm{Ca}^{2+}$ located on the reflective surface of the plasma membrane to a very good approximation. Assuming no reactions of $\mathrm{Ca}^{2+}$ ions with other molecules or reflections from other surfaces, the spatial profile of an increment of $\left[\mathrm{Ca}^{2+}\right]$ above the background level due to a steadily open channel is given by

$$
\delta\left[\mathrm{Ca}^{2+}\right](\mathrm{r})=\frac{i_{C a}}{4 \cdot \pi \cdot F \cdot D_{C a} \cdot r},
$$

where $i_{C a}$ is the absolute value of the single $\mathrm{Ca}^{2+}$ channel current, $D_{C a}$ is the diffusion constant of $\mathrm{Ca}^{2+}$ in the cytoplasm, $F$ is Faraday's constant, and $r$ is the distance from the mouth of the channel (Naraghi \& Neher, 1997). Thus, $\delta\left[\mathrm{Ca}^{2+}\right](\mathrm{r})$ is inversely proportional to $r$. Fig. 1.5A shows a $\delta\left[\mathrm{Ca}^{2+}\right]$ vs $r$ relation with $i_{C a}=0.3 \mathrm{pA}$, a physiological estimate of $\mathrm{Ca}^{2+}$ influx through Cav1.3 channels of IHC ribbon synapses (Zampini et al., 2013).

Once $\mathrm{Ca}^{2+}$ ions enter the presynaptic site, they not only passively diffuse but also bind to other molecules which either freely move in the cytosol or are fixed in space, called mobile and immobile $\mathrm{Ca}^{2+}$ buffers, respectively (Schwaller, 2010; Matthews \& Dietrich, 2015). In the most simple case, when the buffer molecules have single $\mathrm{Ca}^{2+}$ binding sites, the $\delta\left[\mathrm{Ca}^{2+}\right]$ is described by

$$
\delta\left[\mathrm{Ca}^{2+}\right](\mathrm{r})=\frac{i_{C a}}{4 \cdot \pi \cdot F \cdot\left(\kappa_{B} \cdot D_{B}+D_{C a}\right) \cdot r} \cdot\left(1+\frac{\kappa_{B} \cdot D_{B}}{D_{C a}} \cdot e^{-r / \lambda_{B}}\right),
$$




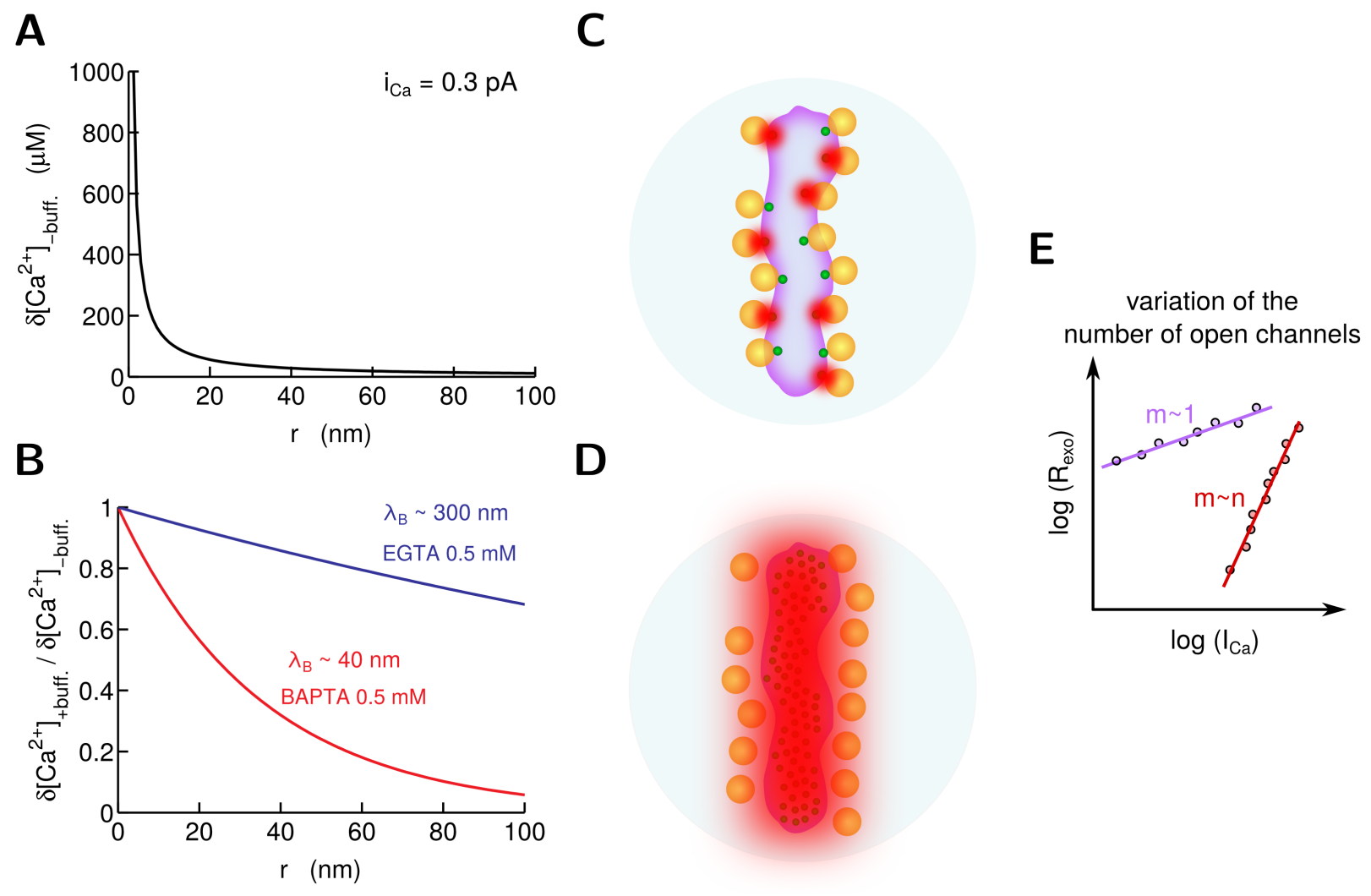

Figure 1.5: Basic properties of presynaptic $\left[\mathrm{Ca}^{2+}\right]$ domains and active zone topographies. (A) An increment of intracellular $\left[\mathrm{Ca}^{2+}\right]$ due to a permanently open $\mathrm{Ca}^{2+}$ channel with $i_{C a}=0.3 \mathrm{pA}$ at different distances from the channel mouth in the absence of $\mathrm{Ca}^{2+}$ buffers. (B) Ratios of intracellular $\left[\mathrm{Ca}^{2+}\right]$ increments due to a permanently open channel with $i_{C a}=0.3$ in the presence and absence of a single mobile $\mathrm{Ca}^{2+}$ buffer with one $\mathrm{Ca}^{2+}$-binding site at different distances from the channel mouth. Two commonly used exogenous $\mathrm{Ca}^{2+}$ buffers EGTA and BAPTA were considered, both with the total concentration of $0.5 \mathrm{mM}$. (C) An example of an extreme case of $\mathrm{Ca}^{2+}$-nanodomain coupling scenario. Magenta - presynaptic density area, orange - RRP vesicles, green - presynaptic $\mathrm{Ca}^{2+}$ channels, red $\mathrm{Ca}^{2+}$ domains around open $\mathrm{Ca}^{2+}$ channels. (D) An example of an extreme case of $\mathrm{Ca}^{2+}$-microdomain coupling scenario. (E) Effects of changed $\mathrm{Ca}^{2+}$ influx $\left(I_{C a}\right)$ on the exocytosis rate $\left(R_{\text {exo }}\right)$ upon manipulation of the number of open $\mathrm{Ca}^{2+}$ channels in the $\mathrm{Ca}^{2+}$-nanodomain and $\mathrm{Ca}^{2+}$-microdomain coupling scenarios. 
where $D_{B}$ is the diffusion constant of the buffer molecules, $\lambda_{B}$ is the buffering length constant, and $\kappa_{B}$ is the buffering capacity (Naraghi \& Neher, 1997). In the case of immobile $\mathrm{Ca}^{2+}$ buffers, $D_{B}=0$. Three important facts follow from Eq. (1.4). First, immobile buffers do not affect $\delta\left[\mathrm{Ca}^{2+}\right](\mathrm{r})$ (they only affect the time course of the build-up of the spatial profile). Second, mobile buffers do not influence $\delta\left[\mathrm{Ca}^{2+}\right](\mathrm{r})$ at $r=0$ but make the decay of $\delta\left[\mathrm{Ca}^{2+}\right](\mathrm{r})$ exponentially faster at $r>0$. This point is illustrated in Fig. $1.5 \mathrm{~B}$, where ratios of $\delta\left[\mathrm{Ca}^{2+}\right](\mathrm{r})$ are plotted for two exemplary mobile $\mathrm{Ca}^{2+}$ buffers. Finally, the buffer scales down the level of $\delta\left[\mathrm{Ca}^{2+}\right](\mathrm{r})$ by $\left(D_{C a}+\kappa_{B} \cdot D_{B}\right) / D_{C a}$ at $r \gg \lambda_{B}$. In neurons, $\lambda_{B}$ is usually in the range of tens or hundreds of nanometers, while $\kappa$ is in the range of tens or hundreds (Matthews \& Dietrich, 2015). Thus, endogenous mobile buffers can strongly localize $\mathrm{Ca}^{2+}$ signals to the vicinity of the presynaptic $\mathrm{Ca}^{2+}$ channel clusters and greatly reduce the bulk $\left[\mathrm{Ca}^{2+}\right]$ outside them.

The spatial profile of $\left[\mathrm{Ca}^{2+}\right]$ within the presynaptic AZ is determined by the overlap of $\delta\left[\mathrm{Ca}^{2+}\right](\mathrm{r})$ of individual voltage-gated $\mathrm{Ca}^{2+}$ channels. However, these contributions do not necessarily add linearly because $\mathrm{Ca}^{2+}$ reacts with the buffer molecules. This may have consequences for the properties of $\mathrm{Ca}^{2+}$ signaling and exocytosis (Neher, 1998). Not less important to note is that, upon application of depolarizing stimuli, $\mathrm{Ca}^{2+}$ channels fluctuate between the open and closed states, resulting in an ongoing build-up and collapse of $\mathrm{Ca}^{2+}$ domains around the channels. The kinetics of equilibration of $\delta\left[\mathrm{Ca}^{2+}\right]$ can greatly affect the instantaneous values of $\left[\mathrm{Ca}^{2+}\right]$ at the AZs, especially at locations further away from the channel mouths (Naraghi $\&$ Neher, 1997). Finally, the $\delta\left[\mathrm{Ca}^{2+}\right](\mathrm{r})$ may be influenced by reflections of $\mathrm{Ca}^{2+}$ from the presynaptic vesicles if they are located in a close proximity to the $\mathrm{Ca}^{2+}$ channels (Shahrezaei $\&$ Delaney, 2004). Some authors even suggested the presynaptic ribbons as potential reflectors of $\mathrm{Ca}^{2+}$, which could boost $\left[\mathrm{Ca}^{2+}\right]$ at presynaptic AZs (Roberts, 1994; Graydon et al., 2011). The overlap of the single channel $\mathrm{Ca}^{2+}$ domains, the kinetics of their build-up and collapse, and the effect of $\mathrm{Ca}^{2+}$ impermeable organelles on $\left[\mathrm{Ca}^{2+}\right]$ at $\mathrm{AZs}$ of IHC ribbon synapses were among the questions considered in this dissertation (chapters $2-4$ ).

\section{Mechanisms of $\mathrm{Ca}^{2+}$ extrusion}

Influx of $\mathrm{Ca}^{2+}$ through voltage-gated $\mathrm{Ca}^{2+}$ channels elevates $\left[\mathrm{Ca}^{2+}\right]$ at presynaptic AZs dramatically. The resting bulk $\left[\mathrm{Ca}^{2+}\right]$ in IHCs is around $50 \mathrm{nM}$ or even lower (Moser \& Beutner, 2001). In contrast, three orders of magnitude larger time-averaged $\left[\mathrm{Ca}^{2+}\right]$ was estimated at the vesicular release sites in response to IHC depolarizations in a physiological range (Beutner et al., 2001). Such contrasting levels of $\left[\mathrm{Ca}^{2+}\right]$ at presynaptic AZs are crucial for temporally precise encoding of $V_{m}$ changes into neurotransmitter release by IHCs. An efficient removal of $\mathrm{Ca}^{2+}$ from the AZs, and ultimately from the cells, is required for maintenance of these gradients.

As it follows from the previous discussion, it is the passive diffusion and $\mathrm{Ca}^{2+}$ buffering which take away $\mathrm{Ca}^{2+}$ from presynaptic AZs into the bulk cytosol. IHCs have particularly high capacity for this clearance due to exceptionally large presynaptic volume (whole IHC) and high concentrations of $\mathrm{Ca}^{2+}$ buffers (see below). The accumulating bulk $\mathrm{Ca}^{2+}$ is further removed 
by the standard machinery: plasmalemmal $\mathrm{Ca}^{2+}$ pumps (PMCAs) and $\mathrm{Na}^{+} / \mathrm{Ca}^{2+}$ antiporters (reviewed in Berridge et al., 2003). In addition, mitochondria and internal $\mathrm{Ca}^{2+}$ stores of endoplasmic reticulum can act as temporary absorbers of intracellular $\mathrm{Ca}^{2+}$ (reviewed in Berridge et al., 2003). Contribution of these components of the $\mathrm{Ca}^{2+}$-signaling toolkit was demonstrated to noticeably affect bulk $\mathrm{Ca}^{2+}$ transients, and in turn, exocytosis at some presynaptic sites in the CNS (Billups \& Forsythe, 2002; Kim et al., 2005; Lee et al., 2007; Scullin \& Partridge, 2010). The situation is apparently quite different in hair cells. Large scale $\mathrm{Ca}^{2+}$ modeling of $\mathrm{Ca}^{2+}$ imaging data of vestibular hair cells indicated that the effect of PMCA and SERCA (endoplasmic reticulum) pumps on global $\mathrm{Ca}^{2+}$ becomes noticeable only some seconds after the onset of an ongoing stimulation which evokes maximal $\mathrm{Ca}^{2+}$ influx (Bortolozzi et al., 2008). The blockage of $\mathrm{Ca}^{2+}$-induced $\mathrm{Ca}^{2+}$ release from endoplasmic reticulum by ryanodine also caused a noticeable change in exocytosis only after more than 10 min (Lelli et al., 2003). Further, there was no significant contribution of mitochondria to $\mathrm{Ca}^{2+}$ microdomains around the presynaptic AZs initiated by peak $\mathrm{Ca}^{2+}$ influx upon $150 \mathrm{~ms}$ long depolarization at mature IHC ribbon synapses (Frank et al., 2009). Finally, SGN responses suggest that, differently from some of the CNS synapses, the elevations of bulk $\mathrm{Ca}^{2+}$ do not contribute to exocytosis considerably during ongoing activity for physiologically relevant time periods at IHC ribbon synapses. Indeed, SGNs are capable of maintaining rates around the same level during ongoing strong stimulation over $10 \mathrm{~min}$ (Kiang et al., 1965). Moreover, no decline in phase locking of SGN spiking in response to ongoing stimulation by low frequency sounds is seen over periods of at least tens of seconds (Kiang et al., 1965; Rose et al., 1967).

\section{$\mathrm{Ca}^{2+}$ buffers of inner hair cells}

Due to the involvement of $\mathrm{Ca}^{2+}$ in a plethora of cellular signaling processes, cells, including presynaptic sites, contain many different protein species capable of binding $\mathrm{Ca}^{2+}$ (Berridge et al., 2003; Schwaller, 2012). Most of these proteins act as $\mathrm{Ca}^{2+}$ sensors in $\mathrm{Ca}^{2+}$ induced chains of intracellular signaling and are quite often present at low concentrations (see, e.g., Yang et al., 2014). When talking about $\mathrm{Ca}^{2+}$-binding proteins, the term $\mathrm{Ca}^{2+}$-buffer is usually assigned to a small group of them: parvalbumins $\alpha$ and $\beta(\mathrm{PV}-\alpha / \beta)$, Calbindin-D9k (CB-D9k), calbindinD28k (CB-D28k) and calretinin (CR) (Schwaller, 2010). These proteins contain distinctive $\mathrm{Ca}^{2+}$ binding domains of amino acid residues, the so-called EF-hands, and are members of a large family of the EF-hand proteins (Nakayama et al., 2000). PVs, CBs, and CR are found in micro- to millimolar concentrations and are thought to be primarily used for capturing $\mathrm{Ca}^{2+}$ in cells (Schwaller, 2010).

CB-D28k, CR, and PV- $\alpha$ of the aforementioned EF-hand proteins are expressed in IHCs (Hackney et al., 2005). The estimates of concentrations of CB-D28k, CR, and PV $\alpha$ based on immunogold labeling were respectively $60 \mu \mathrm{M}, 20 \mu \mathrm{M}$, and $90 \mu \mathrm{M}$ in apical IHC of 16 day postnatal rats (Hackney et al., 2005). Noteworthy, CB-D28k, CR, and PV- $\alpha$ have, respectively, four, five, and two $\mathrm{Ca}^{2+}$ binding sites, with some of them being highly cooperative in the case 
of CR (Schwaller, 2010). Similar concentrations were found in the basal IHCs. However, given the limitations of immunohistochemistry, the real concentrations may be higher than the above estimates.

Another important endogenous $\mathrm{Ca}^{2+}$ buffer in IHCs is ATP. Its total concentration in IHCs is not known. However, reduction of its level below $2 \mathrm{mM}$ leads to a fast run-down of the $\mathrm{Ca}^{2+}$ influx and, in turn, abortion of exocytosis, due to the arrest of PMCA activity (Weiler et al., 2014). It is approximately this concentration to which IHCs are usually clamped during whole-cell patch clamp recordings (see e.g., Moser \& Beutner, 2000; Goutman \& Glowatzki, 2007). In some neurons, immobile buffers were characterized kinetically based on their effect on transient $\left[\mathrm{Ca}^{2+}\right]$ in (Matthews \& Dietrich, 2015). However, neither the origin, nor $\mathrm{Ca}^{2+}$-binding properties and concentrations of immobile buffers in IHCs are known.

How the endogenous mobile $\mathrm{Ca}^{2+}$ buffers shape the presynaptic $\left[\mathrm{Ca}^{2+}\right]$ and exocytosis at IHC ribbon synapses is not well understood and is one of the main questions considered in chapter 3 of this dissertation.

\subsection{Active zone topography}

\section{Background}

Due to sharp localization of $\mathrm{Ca}^{2+}$ domains around the voltage-gated $\mathrm{Ca}^{2+}$ channels, the spatial profile of $\left[\mathrm{Ca}^{2+}\right]$ at presynaptic AZs is largely affected by the exact positions of the channels. From the perspective of exocytosis, the location of RRP vesicles are also important. The particular spatial positioning of $\mathrm{Ca}^{2+}$ channels and vesicles at an $\mathrm{AZ}$ is termed "active zone topography" (Meinrenken et al., 2002; Shahrezaei \& Delaney, 2005). Interchangeable, but more loosely defined, is a term "coupling between $\mathrm{Ca}^{2+}$ channels and sensors of exocytosis" (Eggermann et al., 2012). Two different limiting scenarios of the presynaptic AZ topography have been recognized during ongoing research (reviewed in Augustine et al., 2003; Eggermann et al., 2012). In the so-called $\mathrm{Ca}^{2+}$-nanodomain coupling scenario, fusion of a given synaptic vesicle is induced by one or a few $\mathrm{Ca}^{2+}$ channels located in a close proximity to the $\mathrm{Ca}^{2+}$ sensor of exocytosis of that vesicle. The "nanodomain" refers to a highly localized $\mathrm{Ca}^{2+}$ domain of a size in the range of nanometers contributed mainly by one or a few closest channels. In the so-called $\mathrm{Ca}^{2+}$-microdomain coupling scenario, exocytosis is initiated by many channels located further away from the fusion machinery of the RRP vesicles. The "microdomain" refers to a cloud of $\mathrm{Ca}^{2+}$ ions which spreads over the entire presynaptic AZ upon depolarization of the presynaptic site.

Strict quantitative definitions of the $\mathrm{Ca}^{2+}$-nanodomain and $\mathrm{Ca}^{2+}$-microdomain scenarios have not been presented. However, the $\mathrm{Ca}^{2+}$-nanodomain coupling scenario is usually assigned to a synapse if the average distance traveled by $\mathrm{Ca}^{2+}$ ions from the $\mathrm{Ca}^{2+}$ channels to the sensors of exocytosis is up to several tens of nm (Eggermann et al., 2012). If this distance is 
above $100 \mathrm{~nm}$, the coupling between the $\mathrm{Ca}^{2+}$ channels and sensors of exocytosis is considered to be $\mathrm{Ca}^{2+}$-microdomain like. Extreme cases of the $\mathrm{Ca}^{2+}$-nanodomain and $\mathrm{Ca}^{2+}$-microdomain scenarios are illustrated in Fig. 1.5C, D. In the first scenario, each vesicular release site contains one very tightly coupled channel. Due to sharp localization of $\mathrm{Ca}^{2+}$ domains around separate channels (see above), $\left[\mathrm{Ca}^{2+}\right]$ at any of the vesicles is contributed mostly by the nearest channel. In the second scenario, the AZ contains considerably more channels than vesicular release sites. Moreover, vesicles are located at a larger distance from any of the channels and there are many channels with similar proximity to any of the vesicles. Thus, $\left[\mathrm{Ca}^{2+}\right]$ at any of the $\mathrm{Ca}^{2+}$ sensors of exocytosis is not dominated by contribution of a particular channel.

\section{Functional significance}

Different AZ topography scenarios endow synapses with contrasting information processing features, such that the suitability of each design may depend on the task as well as metabolic and energetic resources of the cells (reviewed in Eggermann et al., 2012). For example, considerably higher $\left[\mathrm{Ca}^{2+}\right]$ is created at the $\mathrm{Ca}^{2+}$ sensors of exocytosis in the $\mathrm{Ca}^{2+}$-nanodomain rather than $\mathrm{Ca}^{2+}$-microdomain coupling regime due to the sharp decay of $\left[\mathrm{Ca}^{2+}\right]$ with distance from the channels. Thus, synapses with the former coupling scenario are considerably more resistant to accumulating bulk $\left[\mathrm{Ca}^{2+}\right]$ during prolonged depolarizations. Moreover, the build-up and collapse of $\left[\mathrm{Ca}^{2+}\right]$ at vesicular release sites are faster, due to tighter coupling between the channels and vesicles. These features may be advantageous for encoding at synapses at the starting stages of sensory pathways, when a fast reaction to changes of stimuli is necessary, but may be inadequate for synapses involved in memory formation, when stimulation history effects play a positive role. The $\mathrm{Ca}^{2+}$-nanodomain topographies are more favorable energetically as fewer $\mathrm{Ca}^{2+}$ channels mean smaller metabolic expense for their maintenance as well as smaller energetic costs of $\mathrm{Ca}^{2+}$ extrusion from the cytoplasm by PMCA pumps. On the other hand, encoding properties of synapses may be degraded by the stochastic opening of the $\mathrm{Ca}^{2+}$ channels in the case of the $\mathrm{Ca}^{2+}$-nanodomain coupling scenario. The intensity of this type of noise is considerably reduced in the case of the $\mathrm{Ca}^{2+}$-microdomain coupling topographies due to averaging over many channels which contribute to $\left[\mathrm{Ca}^{2+}\right]$ at the vesicular release sites.

\section{Determining AZ topography: Methodologies}

Two approaches based on intracellular electrophysiology are commonly used to probe the coupling regime between presynaptic $\mathrm{Ca}^{2+}$ channels and sensors of exocytosis (Augustine et al., 1991; Eggermann et al., 2012). One of those approaches relies on estimating the dependence between $\mathrm{Ca}^{2+}$ influx and the rate of exocytosis of the readily releasable pool upon manipulation of the number of open $\mathrm{Ca}^{2+}$ channels. In the case of the $\mathrm{Ca}^{2+}$ microdomain scenario, a change in the number of open channels proportionally scales $\left[\mathrm{Ca}^{2+}\right]$ at the presynaptic $\mathrm{AZ}$ (assuming the linear relation between the $\mathrm{Ca}^{2+}$ influx and the $\left[\mathrm{Ca}^{2+}\right]$ ). As mentioned in section 
1.3, $\mathrm{Ca}^{2+}$-dependent exocytosis is highly cooperative in many synapses (happens upon binding of $4-5 \mathrm{Ca}^{2+}$ ions) and, thus, the dependence of its rate on $\left[\mathrm{Ca}^{2+}\right]$ and the $\mathrm{Ca}^{2+}$ influx is supralinear. The corresponding relation is usually well described by a power function with an exponent (which we denote by $m$ ) similar to the degree of the intrinsic $\mathrm{Ca}^{2+}$ cooperativity of exocytosis (denoted by n, Fig. 1.5F). The situation is, however, different in the case of the $\mathrm{Ca}^{2+}$ nanodomain scenario. Then, reducing the number of active channels leads to a proportional elimination of operational vesicular release sites. As a consequence, the exocytosis rate scales proportionally to the $\mathrm{Ca}^{2+}$ influx, i.e., $m=1$. The real synapses work neither in the ideal nanodomain nor in the ideal microdomain coupling scenarios, thus, $m$ is never exactly equal to 1 or $n$. A detailed mathematical modeling is necessary for interpreting the intermediate values of $m$ in terms of the number of channels coupled to vesicular release sites (Meinrenken et al., 2002; Shahrezaei et al., 2006).

The second approach used to probe the AZ topography is based on measuring presynaptic exocytosis rates in different intracellular $\mathrm{Ca}^{2+}$ buffering conditions, which are changed by dialyzing the presynaptic sites with solutions containing different exogenous $\mathrm{Ca}^{2+}$ buffers. For a given AZ topography, $\mathrm{Ca}^{2+}$ buffers affect $\left[\mathrm{Ca}^{2+}\right]$ at presynaptic vesicular release sites depending on the parameters $\lambda_{B}$ and $\kappa_{B}$, which are intrinsic properties of the buffers. Given that the rate of exocytosis is proportional to a power $n$ of $\left[\mathrm{Ca}^{2+}\right]$, we can write $\left(R_{e x o, 1} / R_{e x o, 2}\right)=\left(\left[\mathrm{Ca}^{2+}\right]_{1} /\left[\mathrm{Ca}^{2+}\right]_{2}\right)^{n}$, where $R_{\text {exo }}$ is the exocytosis rate, and subscript indexes 1 and 2 stand for two different $\mathrm{Ca}^{2+}$ buffering conditions. If the AZ contained only one channel and one vesicular release site, we could combine this expression with Eq. (1.4) (section 1.4) and then express the distance $r$ as a function of $R_{e x o, 1} / R_{e x o, 2}$. The same procedure has been applied for real AZs with multiple $\mathrm{Ca}^{2+}$ channels and vesicular release sites in a range of studies (see e.g., Neher, 1998; Bucurenciu et al., 2008; Baur et al., 2015). The estimated distance is then called the coupling distance between the $\mathrm{Ca}^{2+}$ channels and sensors of exocytosis (Eggermann et al., 2012). We denoted this quantity by $R_{c}$ in this dissertation. Intuitively, $R_{c}$ reflects the proximity between the $\mathrm{Ca}^{2+}$ channels and the vesicular release sites within the AZ. However, as it was shown in chapter 3 of this dissertation, the exact value of $R_{c}$ depends also on such properties as $\mathrm{Ca}^{2+}$ cooperativity of exocytosis and, especially, $\mathrm{Ca}^{2+}$-binding kinetics of intracellular mobile $\mathrm{Ca}^{2+}$ buffers. Thus, a detailed computational modeling is necessary for interpreting $R_{c}$ in terms of spatial positioning of the $\mathrm{Ca}^{2+}$ channels.

Despite the fact that values of the exponent $m$ and the coupling distance carry a lot of information, they are not sufficient to fully constrain the topographical arrangement of the presynaptic $\mathrm{Ca}^{2+}$ channels and vesicular release sites. In some highly accessible synapses, like frog neuromuscular junction or the calyx of Held, electron microscopy provided a great deal of additional information (see, e.g., Harlow et al., 2001; Nakamura et al., 2015). Recently developed super-resolution fluorescence microscopy methods, especially the localization methods like STORM (see, e.g., Sauer, 2013), are promising tools to advance the understanding about the spatial organization of presynaptic AZ further. 


\section{Current findings}

To this date, the coupling between presynaptic $\mathrm{Ca}^{2+}$ channels and sensors of exocytosis have been studied with less or more scrutiny in different synapses. Probably the best understood are the squid giant synapses and the frog neuromuscular junctions, where the nanodomain coupling scenario was reported (Adler et al., 1991; Augustine et al., 1991; Harlow et al., 2001; Shahrezaei et al., 2006; Mariney \& Dittrich, 2013). The same coupling regime was also reported in other systems, like ciliary ganglion calyx synapses (Stanley, 1993), synapses between basket and granule cells in hippocampus (Bucurenciu et al., 2008), cerebellar synapses between parallel fibers and Purkinje cells (Schmidt et al., 2013). Microdomain coupling between $\mathrm{Ca}^{2+}$ channels and sensors of exocytosis was, for example, documented in the immature calyx of Held (Meinrenken et al., 2002) and synapses between mossy fibers and CA3 pyramidal neurons in hippocampus (Vyleta \& Jonas, 2014). Interestingly, tightening of the coupling was found upon maturation in some synapses, including the calyces of Held (Fedchyshyn \& Wang, 2005; Wang et al., 2009; Nakamura et al., 2015) and synapses between cerebellar cortical parallel fibers and Purkinje cells (Baur et al., 2015).

At mature IHC ribbon synapses, tight coupling between the $\mathrm{Ca}^{2+}$ channels and $\mathrm{Ca}^{2+}$ sensors of exocytosis was suggested. It was shown that $5 \mathrm{mM}$ BAPTA $\left(\lambda_{B} \sim 10 \mathrm{~nm}\right)$ but not $5 \mathrm{mM}$ $\operatorname{EGTA}\left(\lambda_{B} \sim 100 \mathrm{~nm}\right)$ decreases exocytosis rate significantly (Moser \& Beutner, 2000). The estimate of the exponent $m$ of the exocytosis vs $\mathrm{Ca}^{2+}$ influx relation based on a change of the number of active channels was $\sim 1.4$, relatively close to one (Brandt et al., 2005). In agreement to these findings, it was shown that, given the same total $\mathrm{Ca}^{2+}$ influx, more exocytosis is evoked at stronger depolarizations (more open channels, smaller single channel current) than at weaker depolarizations (fewer open channels, larger single channel current) (Brandt et al., 2005).

Despite the aforementioned findings, a more quantitative and detailed view of the topographic arrangement of the $\mathrm{Ca}^{2+}$ channels and $\mathrm{RRP}$ vesicles at $\mathrm{AZs}$ of IHC ribbon synapses is still missing. Functional significance of the potential regime of $\mathrm{Ca}^{2+}$ nanodomain control of exocytosis at these synapses is not well understood either. These questions were approached in chapters $2-4$ of this dissertation by exploiting mathematical modeling combined with experimental approaches. A developmental aspect of the coupling regime between the $\mathrm{Ca}^{2+}$ channels and sensors of exocytosis was considered in chapter 2 as well, but to a lesser extent.

\subsection{Spiral ganglion neurons}

\section{Basic properties}

Neurotransmitter release at IHC ribbon synapses drives spiral ganglion neurons of type one (herein simply denoted by SGNs). SGNs are bipolar, with cell bodies located in the spiral ganglion. The peripheral axon innervates one IHC by making a single synaptic contact. The centrally-projecting axons of SGNs innervate the cochlear nucleus, located in the brainstem 
(already a part of the CNS). These axons represent $\sim 90-95 \%$ of fibers found in the auditory nerve (Liberman et al., 1990). The remaining afferent axons comprising the auditory nerve belong to the spiral ganglion neurons of type two, which innervate OHCs.

SGNs are strongly myelinated. However, the initial part of the peripheral axon which begins at the IHC as a compact postsynaptic bouton is unmyelinated. The site for initial spike generation in SGNs has been proposed to be located there (Hossain et al., 2005; Rutherford et al., 2012). The action potentials are initiated by excitatory inputs in the form of EPSCs following vesicle fusion at a single IHC ribbon synapse (see section 1.3). The EPSCs are mediated by large ring-shaped postsynaptic AMPA receptor clusters (Meyer et al., 2009; Chapochnikov et al., 2014). In vitro recordings revealed that SGNs show (Hodgkin) type III excitability and respond to the excitatory inputs very phasically (Rutherford et al., 2012). Similarly, in vivo and in vitro recordings of SGN spontaneous responses showed that each EPSC evokes a single spike, unless falling shortly after the previous spike, in which case spike failures are observed due to SGN refractoriness (Siegel, 1992; Rutherford et al., 2012). Altogether, the current knowledge strongly suggests that SGNs act as reliable transmitters of information encoded in the form of presynaptic vesicle fusion event times to the CNS.

Besides the synaptic contacts with IHCs, the peripheral axons of SGNs are also innervated by efferent fibers of the lateral olivocochlear bundle, which descends from the superior olivary complex in the brainstem (reviewed in Robertson, 2009). It was shown that activation of the olivocochlear bundle can modulate SGN activity by increasing as well as decreasing their spike rates (Groff \& Liberman, 2003). This is compatible with the presence of both, excitatory and inhibitory synaptic transmission systems involved in functioning of the efferent innervation (Eybalin, 1993; Ruel et al., 2001). However, the exact mechanisms of action of these systems are not well understood.

\section{Responses to sound stimuli}

SGNs receive inputs from single IHCs in the cochlea and preserve the tonotopic organization of the cochlea as they innervate the brainstem. For weak sounds, each SGN responds with considerable changes in its spike rate only to sound stimuli from a narrow range of frequencies. The frequency to which a particular SGN is the most sensitive is called its characteristic frequency (CF). Interestingly, SGNs generate spikes at lower rates in the absence of sounds too, most likely, due to spontaneous vesicle fusion at the presynaptic sites (Siegel \& Relkin, 1987; Robertson \& Paki, 2002). The spike trains triggered in response to pure tone stimuli depend on the sound frequencies. For frequencies below $1 \mathrm{kHz}$, the SGN spiking is phase-locked to the sound wave (Fig. 1.6A; Kiang et al., 1965; Rose et al., 1967), however the spike times are essentially independent on the sound phase for frequencies above $2-4 \mathrm{kHz}$ (Fig. 1.6B; Johnson, 1980; Palmer \& Russell, 1986). The deterioration of phase locking with increased CF is largerly explained by the decay of the AC component of the IHC receptor potential (Fig. 1.3B; Palmer \& Russell, 1986). However, additional stages of presynaptic signal processing, like $\mathrm{Ca}^{2+}$ dynamics 
at AZs or exocytosis, might contribute due to their finite speed.

In response to a tone burst of fixed amplitude, SGNs respond with adapting firing rates (Kiang et al., 1965). This can be seen best from the post-stimulus time histograms of high-CF neurons (Fig. 1.6C). The onset spike rate, which can be as high as $1000 \mathrm{~s}^{-1}$, quickly decays over the first few tens of milliseconds and reaches a steady state level, which is typically in the range of $100-300 \mathrm{~s}^{-1}$. In fact, the adaptation of spike rate continues further if stimulation is prolonged but is slow and mild (Kiang et al., 1965). One of the key reasons behind the spike rate depression is thought to be the partial depletion of the RRP of the presynaptic vesicles during ongoing stimulation (Furukawa et al., 1978; Moser \& Beutner, 2000; Goutman \& Glowatzki, 2007). The steady state spike trains of high-CF SGNs in response to sound bursts at a given SPL are irregular and often approximated by a homogeneous Poisson process convolved with refractoriness of the spike generator (Kiang et al., 1965; Li \& Young, 1993). The irregularity of spike intervals primarily arises from the stochasticity of presynaptic vesicle fusion times (Glowatzki \& Fuchs, 2002; Grant et al., 2010). It should be noted that experimental data deviates from the above model considerably in some cases (Li \& Young, 1993; Prijs et al., 1993). Moreover, it was shown that small but significant correlations between interspike intervals during spontaneous activity of SGNs exist, which are not taken into account by the Poisson model (Prijs et al., 1993; Peterson et al., 2014).

SGNs encode sound amplitudes with varying spike rates, but only in a limited range of sound levels. A typical sound-level tuning curve of these neurons, the so-called rate-level function (RLF), has a sigmoid shape (Fig. 1.6D, E; Sachs \& Abbas, 1974; Winter et al., 1990). These tuning curves are usually constructed by using spike rates estimated in the adapted part of the response (Sachs \& Abbas, 1974; Winter et al., 1990; Heil et al., 2011). RLFs are characterized by four parameters: the spontaneous spike rate (SR), the maximum spike rate, the threshold $S P L$, and the dynamic range. The SR equals to the spike rate in the absence of sound stimuli. The threshold is the SPL at which the spike rate of the neuron exceeds the SR by a chosen amount, usually by $10 \%$ of the difference between the maximum and spontaneous spike rates (Sachs \& Abbas, 1974). The dynamic range is defined as the difference between $S P L$ levels which trigger spike rates slightly smaller than the maximum rate and slightly larger than the SR. Usually, "slightly" means $10 \%$ of the difference between the maximum and spontaneous spike rates (Taberner et al., 2005) but see also (Nizami, 2002).

\section{Heterogeneity of responses to pure tones}

One of the remarkable features of RLFs is that they differ considerably even between SGNs which innervate IHCs with virtually the same cochlear tonotopic positions and, most likely, even the same IHCs (Fig. 1.6D, E; Liberman, 1980; Liberman, 1982). For example, SRs vary from considerably smaller than $1 \mathrm{~s}^{-1}$ to $150 \mathrm{~s}^{-1}$ (Liberman, 1978; Winter et al., 1990; Taberner \& Liberman, 2005). The thresholds may differ by up to $50 \mathrm{~dB}$ (Liberman, 1978; Winter et al., 1990; Taberner \& Liberman, 2005) and the dynamic ranges in some SGNs can be five or even 

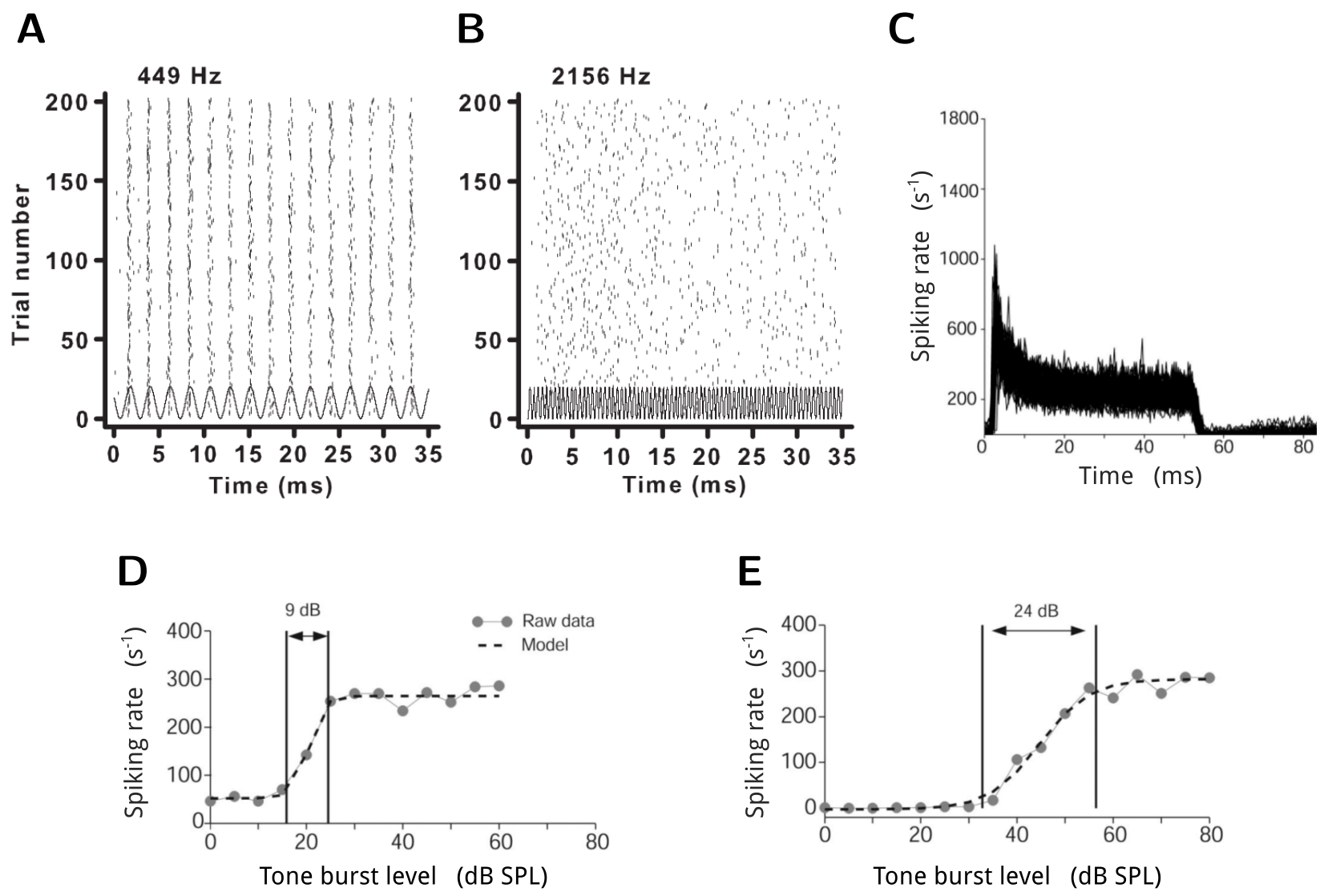

Figure 1.6: Basic properties of spiral ganglion neuron responses to sound stimuli. (A) Raster plots of single SGN responses to low frequency $(449 \mathrm{~Hz})$ tone bursts at CF (source: Avissar et al., 2007). (B) The same as in (A) but with sound frequency (and CF) equal to $2156 \mathrm{~Hz}$ (source: Avissar et al., 2007). (C) Superimposed post-stimulus-time histograms from different high-CF SGNs in response to pure tone bursts at $\mathrm{CF}$ and amplitudes $30 \mathrm{~dB}$ above the spiking threshold (source: Taberner \& Liberman, 2005). (D) The rate-level function of a high spontaneous rate SGN (source: Taberner \& Liberman, 2005). (E) The same as (D) but for a low spontaneous rate SGN. Note the higher spiking threshold and wider dynamic range than in the previous example (source: Taberner \& Liberman, 2005). 
more times larger than in others (Yates et al., 1990; Taberner \& Liberman, 2005). Moreover, certain correlations between different parameters of the same RLF were identified. For instance, low SR SGNs usually have higher thresholds and wider dynamic ranges than high SR SGNs (Fig. 1.6D, E). The interspike interval statistics were shown to be heterogeneous as well (Li \& Young, 1993; Prijs et al., 1993; Peterson et al., 2014).

The RLF heterogeneity is thought to be crucial for the wide range of $S P L \mathrm{~s}$ that humans and other mammals are able to discriminate (Viemeister 1988; Colburn et al., 2003). However, physiological mechanisms of this important aspect of the auditory system functioning are still not well understood. Three mutually non-exclusive hypotheses were proposed: (1) variation of the intrinsic biophysical properties of SGNs (Liberman, 1982; Liberman et al., 2011; Liu et al., 2014), (2) variation of the efferent innervation of SGNs by the lateral olivocochlear system (Liberman 1990; Groff \& Liberman, 2003) (3) variation of the presynaptic properties of IHCs (Merchan-Perez \& Liberman, 1996; Robertson \& Paki, 2002; Frank et al., 2009). The hypothesis of the presynaptic mechanisms was addressed in chapter 4 of this dissertation by using extensive mathematical modeling constrained by in vivo recordings from SGNs. 


\section{Chapter 2}

\section{Developmental Refinement of Hair Cell Synapses Tightens the Coupling of $\mathrm{Ca}^{2+}$ Influx to Exocytosis}

\section{Citation}

A.B. Wong*, M.A. Rutherford*, M. Gabrielaitis*, T. Pangršič, F. Göttfert, T. Frank, S. Michanski, S. Hell, F. Wolf, C. Wichmann, and T. Moser. Developmental refinement of hair cell synapses tightens the coupling of $\mathbf{C a}^{2+}$ influx to exocytosis. EMBO J 33 (3): $247-264$. (c) 2014 The authors. $\quad *$ - equal contribution.

\section{Detailed contributions of M. Gabrielaitis}

(1) Designed the mathematical model of the presynaptic AZ of an IHC ribbon synapse, including $\mathrm{Ca}^{2+}$ dynamics and presynaptic vesicle fusion, based on the experimental findings of the coauthors of the article. (2) Performed the model simulations and analyzed the simulation results to constrain the active zone topographies of mature and immature IHC synapses based on the experimentally estimated exocytosis vs $\mathrm{Ca}^{2+}$ influx relations. (3) Prepared figures 8, S9, S10, S11, S12, S13, S14. (4) Together with the coauthors, wrote the main part of the paper. Prepared Supplementary Material 9.

\section{Supplementary material}

Nine sections of supplementary material, which is a part of the original publication, follow the main body of the article. Important experimental data (sections $1-8$ ), information on the mathematical model, and modeling results (section 9), which could not be included in the main text due to the space limitations, are provided there.

\section{Additional results}

Important additional modeling results, which extend the material presented in the original 
publication, are provided here. This section was prepared independently by the author of the present dissertation. 


\title{
Developmental refinement of hair cell synapses tightens the coupling of $\mathrm{Ca}^{2+}$ influx to exocytosis
}

\author{
Aaron B Wong ${ }^{1,2,3, \dagger}$, Mark A Rutherford ${ }^{1,3,4, \dagger}$, Mantas Gabrielaitis ${ }^{1,5,6, \dagger}$, Tina Pangrši $\check{c}^{1,3, \$}$, Fabian \\ Göttfert ${ }^{7}$, Thomas Frank ${ }^{1, \S}$, Susann Michanski ${ }^{1,8}$, Stefan Hell7,9, Fred Wolf ${ }^{3,5,6,{ }^{* \star *}}$, Carolin Wichmann ${ }^{3,8, * *}$ \\ \& Tobias Moser ${ }^{1,2,3,6,9,{ }^{*}}$
}

\begin{abstract}
Cochlear inner hair cells (IHCS) develop from pre-sensory pacemaker to sound transducer. Here, we report that this involves changes in structure and function of the ribbon synapses between $\mathrm{IHCs}$ and spiral ganglion neurons (SGNs) around hearing onset in mice. As synapses matured they changed from holding several small presynaptic active zones (AZs) and apposed postsynaptic densities (PSDs) to one large AZ/PSD complex per SGN bouton. After the onset of hearing (i) IHCS had fewer and larger ribbons; (ii) Cav1.3 channels formed stripe-like clusters rather than the smaller and round clusters at immature AZs; (iii) extrasynaptic Cav1.3channels were selectively reduced, (iv) the intrinsic $\mathrm{Ca}^{2+}$ dependence of fast exocytosis probed by $\mathrm{Ca}^{2+}$ uncaging remained unchanged but (v) the apparent $\mathrm{Ca}^{2+}$ dependence of exocytosis linearized, when assessed by progressive dihydropyridine block of $\mathrm{Ca}^{2+}$ influx. Biophysical modeling of exocytosis at mature and immature $\mathrm{AZ}$ topographies suggests that $\mathrm{Ca}^{2+}$ influx through an individual channel dominates the $\left[\mathrm{Ca}^{2+}\right]$ driving exocytosis at each mature release site. We conclude that IHC synapses undergo major developmental refinements, resulting in tighter spatial coupling between $\mathrm{Ca}^{2+}$ influx and exocytosis.
\end{abstract}

Keywords active zone; $\mathrm{Ca}^{2+}$ channel; $\mathrm{Ca}^{2+}$ dependence; exocytosis; vesicle Subject Categories Neuroscience

DOI 10.1002/embj.201387110 | Received 9 October 2013| Revised 24

November 2013 | Accepted 28 November 2013

EMBO Journal (2014) 33, 247-264

\section{Introduction}

Inner hair cells (IHC) ribbon synapses are molecularly and morphologically specialized to transmit acoustic information at hundreds of $\mathrm{Hz}$ with sub-millisecond precision over long periods of time (recently reviewed in Matthews \& Fuchs, 2010; Rutherford \& Pangršič, 2012; Pangršič et al, 2012; Safieddine et al, 2012). The IHC receptor potential is coupled to glutamate exocytosis by $\mathrm{Ca}^{2+}$ influx through $\mathrm{Ca}_{v} 1.3 \mathrm{Ca}^{2+}$ channels (Platzer et al, 2000; Brandt et al, 2003; Dou et al, 2004). Tens of these channels are thought to cluster within the presynaptic density (Brandt et al, 2005; Frank et al, 2010; Zampini et al, 2013). One to two dozen synaptic vesicles are tethered at the plasma membrane within nanometer distance of the $\mathrm{Ca}^{2+}$ channel cluster and are thought to represent the putative readily releasable pool (RRP) of vesicles for exocytosis (Moser \& Beutner, 2000; Zenisek et al, 2000; Lenzi et al, 2002; Rutherford \& Roberts, 2006; Frank et al, 2010). Precisely how $\mathrm{Ca}^{2+}$ channels couple to exocytosis and which mechanism of exocytosis governs transmitter release is not well understood.

Exocytosis at individual IHC AZs has been suggested to be multivesicular (Glowatzki \& Fuchs, 2002), whereby $\mathrm{Ca}^{2+}$ is thought to regulate the rate of release events without affecting the number of vesicles released per event. $\mathrm{Ca}^{2+}$-nanodomain coupling, in which exocytosis is controlled by very few nearby $\mathrm{Ca}^{2+}$ channels (Augustine et al, 1991; Stanley, 1993; Mintz et al, 1995; Wang et al, 2008; reviewed in Augustine et al, 2003; Moser et al, 2006; Neher \& Sakaba, 2008; Eggermann et al, 2012), has been put forward to explain the physiological coupling of $\mathrm{Ca}^{2+}$ influx to RRP exocytosis

\footnotetext{
InnerEarLab, Department of Otolaryngology, University Medical Center Cöttingen, Göttingen, Germany

IMPRS Neuroscience, Göttingen Graduate School for Neuroscience and Molecular Biosciences, Göttingen, Germany

Collaborative Research Center 889 , University of Göttingen, Göttingen, Germany

Department of Otolaryngology, Washington University School of Medicine, St. Louis, MO, USA

Theoretical Neurophysics Group, Max Planck Institute for Dynamics and Self-Organization, Cöttingen, Germany

Bernstein Center for Computational Neuroscience, University of Göttingen, Göttingen, Germany

Department of Nanobiophotonics, Max Planck Institute for Biophysical Chemistry, Göttingen, Germany

Molecular Architecture of Synapses Group, InnerEarLab, Department of Otolaryngology, University Medical Center Cöttingen, Göttingen, Germany

9 Center for Nanoscale Microscopy and Molecular Physiology of the Brain, University of Cöttingen, Göttingen, Germany

*Corresponding author. Tel: +49 55139 8968, -22803, -22837; Fax: +49 551 3912950; E-mail: tmoser@gwdg.de

**Corresponding author. Tel: +49551 39 22604; Fax: +49551 3912950; E-mail: cwichma@gwdg.de

***Corresponding author. Tel: +49 551 5176423; Fax: +49 551 5176409; E-mail: fred@nld.ds.mpg.de

${ }^{\dagger}$ These authors contributed equally to this work.

ॠCurrent address: Synaptic Physiology of Mammalian Vestibular Hair Cells Junior Research Group, InnerEarLab, Department of Otolaryngology, University Medical Center

Göttingen, Göttingen, Germany

${ }^{\S}$ Current address: Friedrich Miescher Institute for Biomedical Research, Basel, Switzerland
} 
in mature IHCs (Brandt et al, 2005; Goutman \& Glowatzki, 2007; Zampini et al, 2013). A Ca ${ }^{2+}$ nanodomain may synchronize multivesicular release (Jarsky et al, 2010; Graydon et al, 2011). Alternatively, $\mathrm{Ca}^{2+}$ microdomain coupling (Heil \& Neubauer, 2010), in which exocytosis is controlled by many channels cooperatively, and a linear intrinsic $\mathrm{Ca}^{2+}$ dependence of fusion (Johnson et al, 2010) have been suggested to explain the coupling of $\mathrm{Ca}^{2+}$ influx to RRP exocytosis in mature IHCs and these concepts, too, may coexist with multivesicular release.

Before the onset of hearing, exocytosis is evoked by $\mathrm{Ca}^{2+}$ action potentials (Beutner \& Moser, 2001; Glowatzki \& Fuchs, 2002; Johnson et al, 2005) with low " $\mathrm{Ca}^{2+}$ efficiency" (Beutner \& Moser, 2001; Johnson et al, 2005). Non-mutually exclusive hypotheses for the increase in $\mathrm{Ca}^{2+}$ efficiency of exocytosis over development include (i) progressive confinement of $\mathrm{Ca}^{2+}$ influx to the $\mathrm{AZ}$, (ii) establishment of nanodomain coupling between $\mathrm{Ca}_{\mathrm{V}} 1.3$ channels and release sites, and (iii) a change in intrinsic $\mathrm{Ca}^{2+}$ dependence of the molecular exocytosis machinery. Upregulation of synaptotagmin IV during postnatal development has been proposed to "linearize” the intrinsic $\mathrm{Ca}^{2+}$ dependence of IHC exocytosis around the onset of hearing (Johnson et al, 2010), supporting hypothesis iii. However, this contrasts with $\mathrm{Ca}^{2+}$ uncaging experiments that found a supralinear intrinsic $\mathrm{Ca}^{2+}$ dependence of exocytosis in mature IHCs (Beutner et al, 2001). Moreover, in immature IHCs, a thorough biophysical analysis of the intrinsic $\mathrm{Ca}^{2+}$ dependence of exocytosis or of the spatial coupling between $\mathrm{Ca}^{2+}$ influx and exocytosis was lacking until now.

Here, we combined morphological approaches: transmission electron microscopy (TEM), immunohistochemistry, confocal and stimulated emission depletion (STED) microscopy; with physiological techniques: electrophysiology, confocal $\mathrm{Ca}^{2+}$ imaging, $\mathrm{Ca}^{2+}$ uncaging; and biophysical modeling of $\mathrm{AZ}$ function to study the IHC afferent synapse during postnatal maturation. We find the intrinsic $\mathrm{Ca}^{2+}$ dependence of fast exocytosis to be similar in immature and mature IHCs, arguing against hypothesis iii. On the other hand, we find a diminution of extrasynaptic $\mathrm{Ca}^{2+}$ channels (supporting hypothesis i) and indicate a developmental switch from $\mathrm{Ca}^{2+}$ microdomain to $\mathrm{Ca}^{2+}$-nanodomain control of vesicle fusion around hearing onset (supporting hypothesis ii).

\section{Results}

\section{Postnatal maturation of AZ ultrastructure in IHC afferent synapses}

Afferent synaptic contacts between IHCs and type I SGNs are present in mice already at birth (Shnerson et al, 1981) and increase in number during the first postnatal week (Shnerson et al, 1981; Sobkowicz et al, 1982; recently reviewed in Bulankina \& Moser, 2012). Around the onset of hearing (p12, Mikaelian et al, 1965), synaptic contacts mature (Sobkowicz et al, 1982) and the number of presynaptic ribbons and postsynaptic AMPA receptor clusters decreases (Huang et al, 2007, 2012; Sendin et al, 2007). By p21, IHC-afferent synapses are considered predominantly mature morphologically (Bulankina \& Moser, 2012; Safieddine et al, 2012) and functionally (Khimich et al, 2005; Grant et al, 2010; Wong et al, 2013). We first investigated the molecular and structural changes within individual IHC-SGN contacts during the 2-week period around hearing onset.

We triple-labeled afferent IHC synapses in whole-mounts of the organ of Corti for (i) RIBEYE/CtBP2 (the major component of the synaptic ribbon (Schmitz et al, 2000; Khimich et al, 2005)), (ii) GluA2/3 (AMPA receptor subunits of the SGN PSD (Matsubara et al, 1996; Khimich et al, 2005)), and (iiia) bassoon (AZ scaffold contributing to ribbon anchorage (tom Dieck et al, 1998; Khimich et al, 2005)), or (iiib) the $\mathrm{Na}^{+} / \mathrm{K}^{+}$-ATPase $\alpha 3$ subunit marking the SGN membrane (McLean et al, 2009). Immature synapses displayed several small appositions of AZs and PSDs (p6, Fig 1A). These AZ/PSD complexes often appeared to form circular structures $>1 \mu \mathrm{m}$ in diameter (Fig 1B), larger than the ring-like GluA clusters of mature synapses (p20, Fig 1C; up to approximately $700 \mathrm{~nm}$, Meyer et al, 2009). $\mathrm{Na}^{+} / \mathrm{K}^{+}$-ATPase labeling of SGN indicated that these circular structures localized to the periphery of the contact between each individual immature postsynaptic bouton and an IHC (Fig 1C,D, p6). Some of the AZ/PSD complexes lacked detectable RIBEYE immunofluorescence and likely represented ribbonless AZs (quantified in Supplementary Fig S2). After the onset of hearing each IHCSGN synapse displayed a single AZ/PSD complex (p20, Fig 1).

TEM confirmed the presence of extended immature synaptic contacts that included ribbon-occupied and ribbonless AZ/PSD appositions (Fig 2A, p6 \& p9). An exemplary synapse reconstructed from serial ultrathin sections (Fig 2D, p6) illustrates three ribbonoccupied AZs and one ribbonless AZ (black arrowhead). Compared with more mature ribbons (Fig 2B, p15 \& p20), immature ribbons assumed more spherical or ovular shapes (Fig 2C), were smaller (Fig 2E), associated with fewer vesicles (Fig 2E and tomogram in Supplementary Fig S1), and were juxtaposed to shorter PSDs (Fig 2E). After hearing onset, droplet- or wedge-shaped ribbons became prevalent (Fig 2C). Different ribbon shapes were observed in the same IHCs but we did not analyze their subcellular distribution. A typical p20 ribbon spanned four 70-nm-thin sections, compared to 1.5 sections on average for p6 ribbons. Floating ribbons tethering vesicles were observed near membrane-anchored ribbons at p6 (Fig 2A, green arrowhead). All preparations for TEM were fixed and processed at around noon time, minimizing possible effects of circadian changes (Hull et al, 2006).

Comparisons of confocal RIBEYE immunofluorescence between p6 and p20 revealed an approximate halving of ribbon number per IHC and an approximate doubling of the integrated pixel intensities per ribbon (Fig 2F,G and Supplementary Fig S2). An increase in fullwidth-at-half-maximum (FWHM, Fig 2F) of these RIBEYE spots is consistent with the increase in ribbon size observed in TEM. For GluA2/3, both the reduction in puncta number and the increase in integrated intensity per punctum were larger than for ribbons (Supplementary Fig S2C,E). We note that confocal measurements provide only a semi-quantitative comparison because immunolabeling may not be linear or equally efficient amongst puncta and because some ribbons or GluA2/3 puncta can be smaller than the resolution limit of confocal microscopy, especially in immature IHCs. Nevertheless, the confocal images indicate an increase in the ratio of ribbon and GluA2/3 puncta number from approximately 0.6 at p6 to nearly 1 for p20 IHCs (Supplementary Fig S2D), consistent with our observations of ribbonless AZs in immature IHCs and with the idea of a 1:1 correspondence between ribbons and receptor clusters per SGN bouton upon maturation (shown in Fig 1C,D, p20). 

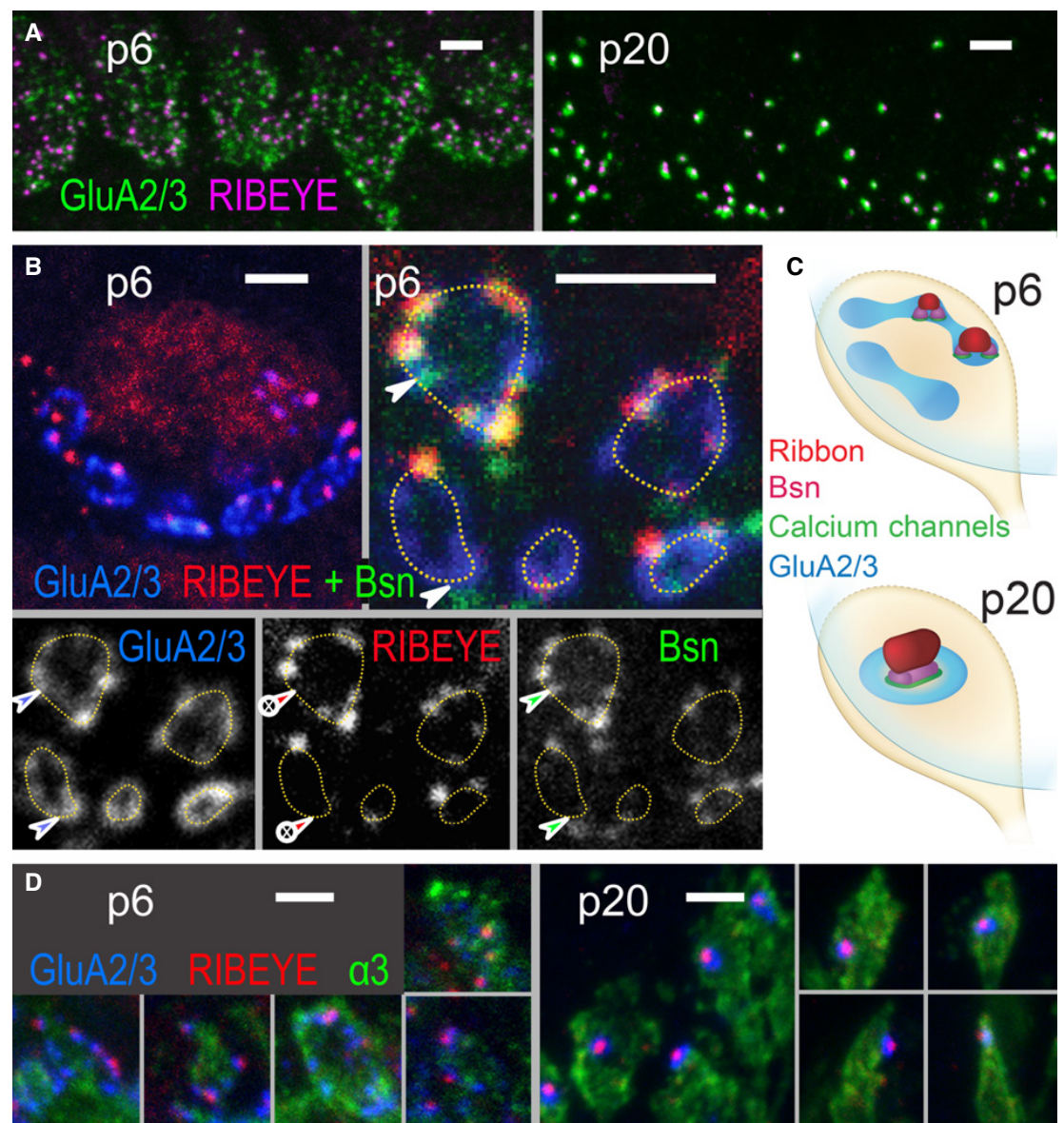

Figure 1. Developmental changes in morphology of hair cell afferent synapses.

A Projection of confocal images showing GluA2/3 (green), RIBEYE (magenta) across the basal region of a row of IHCS at p6 (left) and p20 (right). Scale bar, $2 \mu \mathrm{m}$.

B Single confocal sections. Upper left: At p6, the basal pole of each IHC was contacted by several patches of postsynaptic glutamate receptors (GluA2/3, blue) forming circular structures (highlighted by dashed lines in upper right and lower left). Upper right: Each GluA2/3 spot was juxtaposed to an AZ spot, marked by protein bassoon (green) and in some cases by a ribbon (RIBEYE, red). Two ribbonless AZs marked by colocalizing bassoon and GluA2/3 puncta were highlighted by arrowheads. Individual fluorescence channels are presented separately in gray scale below. Scale bars, $2 \mu \mathrm{m}$.

C Schematized distribution of ribbon, bassoon, $\mathrm{Ca}^{2+}$ channels and GluA2/3-containing receptors on immature (p6, upper) and mature (p20, lower) IHC-SCN contacts. Immature IHC-SGN contacts hold multiple AZ/PSD complexes. Mature IHC-SGN contacts show a single and larger AZ/PSD complex.

D Projection of confocal images showing GluA2/3 (blue), RIBEYE (red), and $\mathrm{Na}^{+} / \mathrm{K}^{+}$-ATPase $\alpha 3$ subunit (green) at p6 (left) and p20 (right). The latter antibody labels the membranes of SGNs, each of which terminates onto the IHC with a bouton-type contact. In p6 preparations, the large circular structures formed by GluA spots partially encompass the perimeter of the bouton contacts. At p20, each postsynaptic bouton contains only a single condensed glutamate receptor cluster that is juxtaposed to a single presynaptic ribbon. Scale bars, $2 \mu \mathrm{m}$.

Immature ribbons were anchored to the $\mathrm{AZ}$ membrane via up to two rootlets (presynaptic densities, Figs $2 \mathrm{~A}$ and $3 \mathrm{~A}, \mathrm{~B})$. In contrast, a single continuous density attached the entire base of the mature ribbon to the AZ (Fig 3C,D). Using anti-bassoon immuno-EM (Fig 3F) we indicate that bassoon localizes to the presynaptic density underneath the ribbon, where the majority of immuno-gold was found (on average $1.4 \pm 0.3$ particles at the base and $0.3 \pm 0.1$ elsewhere at the ribbon, $P<0.001, n=21$ labeled synapses). This is consistent with a previous immuno-EM study on photoreceptor ribbon synapses (Dick et al, 2001) and also supported by present STED microscopy of hair cell synapses (see Fig $3 \mathrm{G}$ and below). The notion of ribbon-anchor consolidation was supported by the observation of fewer electron-dense connections between membrane and ribbon in random sections of ribbon-occupied AZs from p6 to p20 (Supplementary Fig S3).

Next, we studied the topography of bassoon and $\mathrm{Ca}_{\mathrm{V}} 1.3$ at the mature AZ, employing dual-color STED microscopy of immunolabeled IHCs in whole-mounts. Bassoon and $\mathrm{Ca}_{\mathrm{V}} 1.3$ immunofluorescence often formed elongated stripes that aligned intimately with each other (Fig 3G). Most p19 synapses had one $\mathrm{Ca}^{2+}$-channel stripe, some had more than one stripe in parallel, and others had round or more complex configurations (Fig 4B). Before the onset of hearing we observed only spot-like $\mathrm{Ca}^{2+}$-channel clusters (Fig 4A). These spots are reminiscent of the small presynaptic densities seen 
in immature AZs (Fig 2A), suggesting that $\mathrm{Ca}^{2+}$ channels cluster within the presynaptic density of immature IHCs, as they do in the stripe-like densities of mature IHCs (Frank et al, 2010). As depicted in Fig 3E, TEM analysis of membrane-proximal vesicles and presynaptic densities, and STED imaging of $\mathrm{Ca}_{\mathrm{V}} 1.3$ channels suggest that synapse maturation leads to a more ordered topology of $\mathrm{Ca}^{2+}$ channels and membrane-proximal vesicles. In summary IHC-SGN synapses underwent major structural changes around the onset of hearing characterized by larger ribbons, continuously anchored to a single elongated presynaptic density, juxtaposed to a single postsynaptic receptor cluster.

\section{Progressive confinement of $\mathrm{Ca}_{\mathrm{v}} \mathrm{1.3}$ and $\mathrm{Ca}^{2+}$ influx to AZs over development}

$\mathrm{Ca}_{\mathrm{V}} 1.3$ channels conduct $>90 \%$ of the voltage-gated $\mathrm{Ca}^{2+}$ influx in IHCs before and after the onset of hearing (Platzer et al, 2000; Brandt et al, 2003). Over development, we tracked changes in abundance and location of $\mathrm{Ca}_{\mathrm{V}} 1.3$ with immunofluorescence and confocal $\mathrm{Ca}^{2+}$ imaging (Fig 5), in experiments run in parallel under identical conditions for the different age groups. Using $\mathrm{Ca}_{V} 1.3$ knockout mice as a negative control we observed non-specific immunoreactivity only near the cuticular plate at the apical pole of p9 IHCs (Supplementary Fig S4), as previously shown for IHCs of hearing mice (Brandt et al, 2005).

$\mathrm{Ca}_{\mathrm{V}} 1.3$ immunofluorescence near ribbons was detectable already at p6 (arrowheads in Fig 5A) and increased until p14 (mean intensity around ribbons at p6: $6494 \pm 366$ a.u. versus p14: $7721 \pm 265$ a.u., $P=0.007)$. Thereafter, we did not find significant changes of synaptic $\mathrm{Ca}_{\mathrm{V}} 1.3$ immunofluorescent spots except for a subtle increase in the long axis (p14: $0.336 \pm 0.004$ versus p20: $0.350 \pm 0.003 \mu \mathrm{m}$, $P=0.005$ ). Consistent with a previous report (Zampini et al, 2010) we also noted $\mathrm{Ca}_{\mathrm{V}} 1.3$ immunofluorescence away from ribbons, appearing spot-like or diffuse along the entire basolateral plasma membrane of immature IHCs. Upon the onset of hearing, $\mathrm{Ca}_{\mathrm{V}} 1.3$ immunoreactivity became largely confined to ribbon-occupied AZs (Fig 5A, lower panels). We quantified the density of $\mathrm{Ca}_{\mathrm{V}} 1.3$ immunofluorescence immediately around ribbons (synaptic) versus distant from ribbons (extrasynaptic, see Materials and Methods). At p6, the density of extrasynaptic fluorescence was around $43 \%$ of that in synaptic regions, while by p20, it had decreased to only $9 \%$.

Next, we studied changes in intracellular $\mathrm{Ca}^{2+}$ concentration $\left(\left[\mathrm{Ca}^{2+}\right]_{\mathrm{i}}\right)$ elicited by step depolarizations (to $-7 \mathrm{mV}$ for 200 or $254 \mathrm{~ms}$ ) in IHCs using fast confocal $\mathrm{Ca}^{2+}$ imaging before and after the onset of hearing (Fig 5B). To preferentially visualize sites of $\mathrm{Ca}^{2+}$ influx, we used the low-affinity $\mathrm{Ca}^{2+}$ indicator Fluo-5N (0.4 mM) in conjunction with the slow $\mathrm{Ca}^{2+}$ chelator EGTA (2 mM; Frank et al, 2009), and a fluorescently tagged RIBEYE-binding peptide (Francis et al, 2011) for identification of ribbon-occupied AZs. Different from the synapse-confined $\mathrm{Ca}^{2+}$ signals of mature IHCs (Fig 5B, p16), in immature IHCs we found a spatially extended rise of $\left[\mathrm{Ca}^{2+}\right]_{i}$ (Fig 5B, p10) consistent with substantial extrasynaptic $\mathrm{Ca}^{2+}$ influx and with immunohistochemistry (Fig 5A), and also compatible with potential $\mathrm{Ca}^{2+}$-induced $\mathrm{Ca}^{2+}$ release (Kennedy \& Meech, 2002). Occasionally (two out of seven IHCs) we found wide-spread "global" $\mathrm{Ca}^{2+}$ signals (two uppermost rows in Fig 5B, and Supplementary Fig S5). In the other five immature IHCs the peak $\mathrm{Ca}^{2+}$ signal was "spot-like" around ribbons (third row in Fig 5B, and Supplementary Fig S5), further suggesting that $\mathrm{Ca}_{\mathrm{V}} 1.3$ clusters at AZs already before the onset of hearing. We approximated the strength of the extrasynaptic $\mathrm{Ca}^{2+}$ signal as the ratio of $\Delta \mathrm{F}$ away from the ribbon over $\Delta \mathrm{F}$ at the ribbon, which was greatest in immature IHCs with "global" signals, smaller in immature IHCs with "spot-like" signals, and even smaller in IHCs after the onset of hearing (Supplementary Fig S5).

The intense, broadly distributed $\mathrm{Ca}_{\mathrm{V}} 1.3$ immunofluorescence in immature IHCs suggests that the reduction of the whole-cell $\mathrm{Ca}^{2+}$ current around the onset of hearing reflects a reduction in the number of $\mathrm{Ca}^{2+}$ channels, primarily in the extrasynaptic membrane. We studied the IHC $\mathrm{Ca}^{2+}$ current before and after the onset of hearing using whole-cell patch-clamp recordings. We did not detect significant differences in the voltage dependence of activation (Supplementary Fig S6A,B) or in the extent or kinetics of inactivation (Supplementary Fig S6C) between p10 and p14 IHCs. Nonstationary fluctuation analysis of $\mathrm{Ca}^{2+}$-tail currents (Roberts et al, 1990; Frank et al, 2010) indicated that the smaller number of $\mathrm{Ca}^{2+}$ channels almost completely accounted for the reduction of the whole-cell current $(P<0.001$, ANOVA, Supplementary Fig S6D,E). The single channel current $\left(i_{\mathrm{Ca}}\right)$ estimate did not change significantly $(P=0.1$, ANOVA, Supplementary Fig S6F) and the estimated maximal open probability $\left(P_{\text {open,max }}\right)$ decreased slightly $(P<0.001$, ANOVA; Supplementary Fig S6F), whereas single channel recordings indicated a reduction in $i_{\mathrm{Ca}}$ and an increase in $P_{\text {open }}$ over development (Zampini et al, 2010, 2013). While both approaches agree on the notion of much higher total $\mathrm{Ca}^{2+}$ channel numbers in immature cells, they differ in their estimates of $i_{\mathrm{Ca}}$ and $P_{\text {open }}$ and also report opposite trends over development likely for technical reasons (see text accompanying Supplementary Fig S6 for discussion). In summary, immature IHCs contain thousands of synaptic and extrasynaptic $\mathrm{Ca}_{\mathrm{V}} 1.3$ channels that together support presensory $\mathrm{Ca}^{2+}$ action potentials. Upon maturation extrasynaptic $\mathrm{Ca}_{\mathrm{V}} 1.3 \mathrm{Ca}^{2+}$ channels are downregulated and $\mathrm{Ca}^{2+}$ influx becomes confined to AZs, supporting hypothesis 1 in introduction.

\section{Maturation of the coupling between $\mathrm{Ca}^{2+}$ influx and exocytosis}

The developmental confinement of $\mathrm{Ca}^{2+}$ influx to AZs together with the changes in ultrastructure and molecular nanoanatomy of the AZ prompted us to test for parallel changes in the coupling of $\mathrm{Ca}^{2+}$ influx to exocytosis. In particular, we tested hypothesis ii: tighter functional coupling between $\mathrm{Ca}^{2+}$ channels and the RRP, and hypothesis iii: a change in the intrinsic (biochemical) $\mathrm{Ca}^{2+}$ dependence of the molecular exocytosis machinery.

\section{No change in the intrinsic $\mathrm{Ca}^{2+}$ dependence of fast exocytosis}

First, we addressed the intrinsic $\mathrm{Ca}^{2+}$ dependence of IHC exocytosis before the onset of hearing, which had not been done previously. UV-laser photolysis of caged $\mathrm{Ca}^{2+}$ was used to elevate $\left[\mathrm{Ca}^{2+}\right]$ homogenously throughout the cytosol in p6-8 IHCs and in p14-18 IHCs for comparison. $\left[\mathrm{Ca}^{2+}\right]$ was monitored with ratiometric imaging and related to the resulting exocytic increase of membrane capacitance $\left(\Delta C_{m}\right)$. Approximately half (22 out of 40 ) of p6-8 IHCs and nearly all (32 out of 33) of p14-18 IHCs responded with a $C_{m}$ rise that comprised two kinetic components with time constants of a few milliseconds and tens of millisecond, respectively (Fig 6A,C). The total 


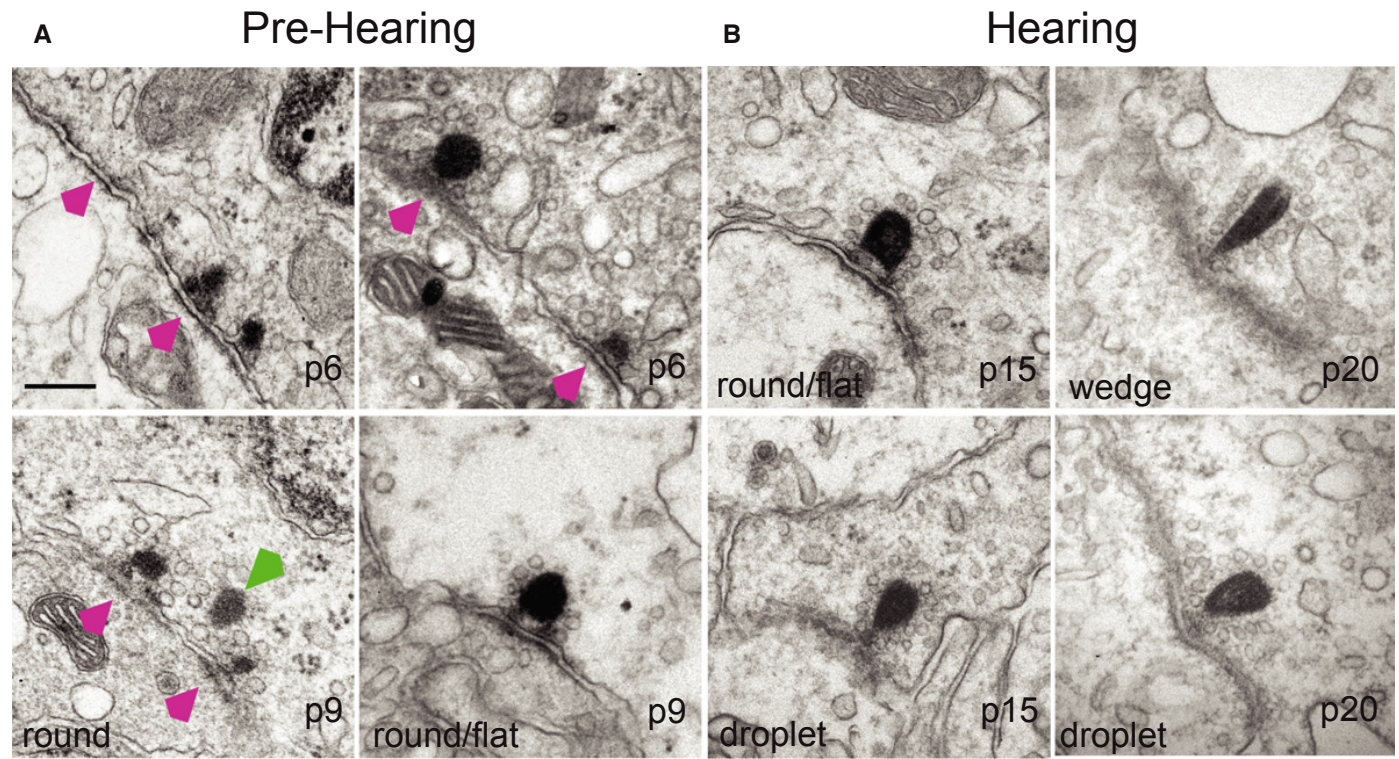

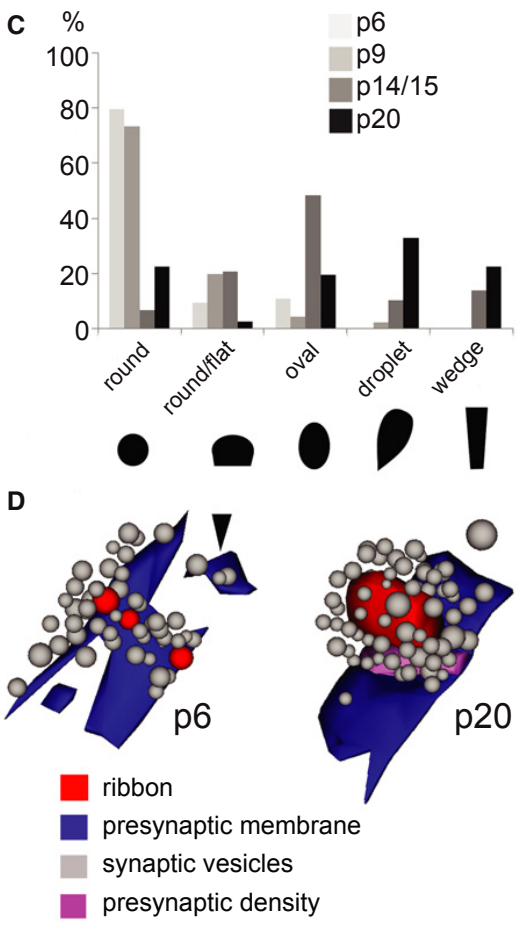

Electron microscopy
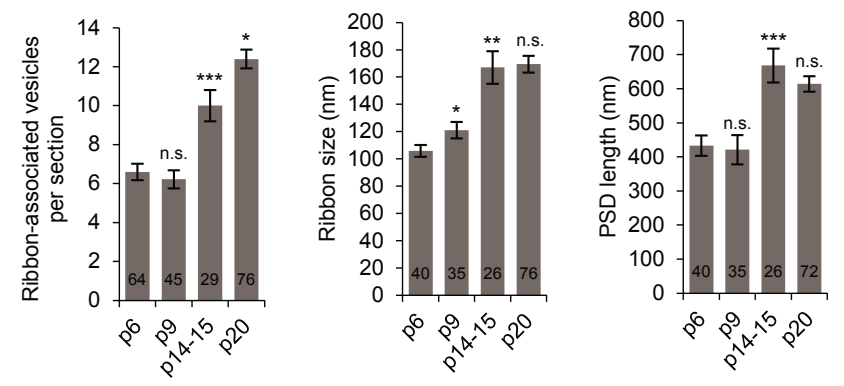

F
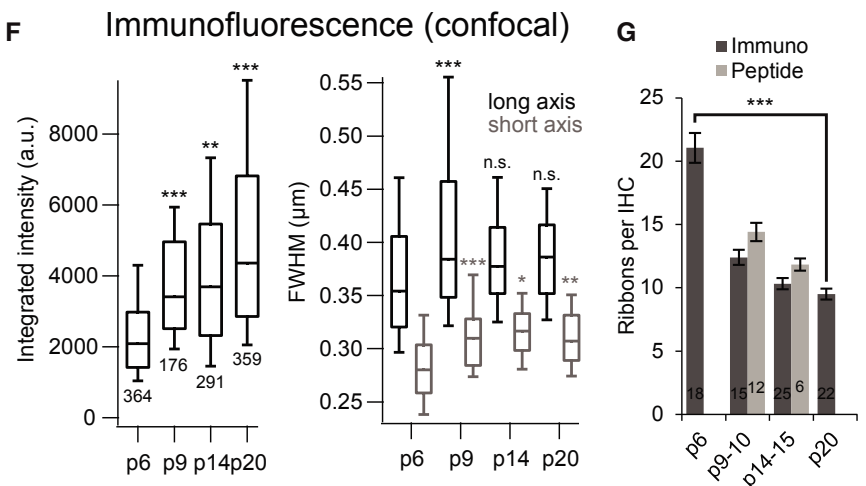

amplitude of the $C_{m}$ rise was largely independent of $\left[\mathrm{Ca}^{2+}\right]$ at both developmental stages (Fig 6D), as previously reported for p14-25 IHCs (Beutner et al, 2001). However, it was approximately 3.6 times smaller in p6-8 IHCs than in p14-18 IHCs $(324 \pm 24 \mathrm{fF} ; n=40$ versus $1161 \pm 104 \mathrm{fF} ; n=31 ; P=1.5 \times 10^{-13}$, Wilcoxon Rank Test), potentially relating to the lower number of synaptic vesicles found at the AZs of p6-7 IHCs (Fig 2E). Moreover, when both kinetic components were present in p6-8 IHCs we found a greater contribution of the slow one $(69 \%)$, while the two components contributed equally in p14-18 IHCs (Supplementary Fig S7). In the other p6-8 IHCs the $C_{m}$ rise was best approximated by a single exponential function with relatively slow time constants within the range of those of the slow component of the $C_{m}$ rise in IHCs with bi-exponential responses (Fig 6C). Focusing on the fast component of exocytosis, we probed the intrinsic $\mathrm{Ca}^{2+}$ dependence of exocytosis by measuring the delay and rate constant of the $C_{m}$ rise for a range of $\left[\mathrm{Ca}^{2+}\right]_{\mathrm{i}}$ (Fig 6C,E). We found a similar supralinear intrinsic $\mathrm{Ca}^{2+}$ dependence in p6-8 and p14-18 IHCs, indicating that the $\mathrm{Ca}^{2+}$. 
Figure 2. Maturation of ribbon synapse ultrastructure.

A, B Representative electron micrographs of IHC ribbon synapses in pre-hearing (A) and hearing (B) mice. Before the onset of hearing (A), at p6 and p9, multiple appositions of small discontinuous pre- and postsynaptic densities were found (magenta arrowheads) typically accompanied by one or more round-shaped ribbon(s) anchored close to the presynaptic membrane via two rootlets. In some cases ribbons were floating in the cytoplasm at a greater distance from the membrane (green arrowhead). After the onset of hearing (B), at p15 and p20, typically one ribbon occupied the presynaptic cytoplasm at the AZ. In comparison to before the onset of hearing, the mature pre- and postsynaptic densities were relatively extended and continuous. The sections were cut parallel to the ribbons short axes, the perspective from which ribbon-shape was measured. Scale bar of $200 \mathrm{~nm}$ in (A) applies also to (B).

C Through development the ribbon-shape changed from predominantly round to oval, droplet-like, or wedge-like shapes, as shown in (A) and (B).

D 3D reconstructions from serial sections before (left, p6) and after (right, p20) the onset of hearing. Presynaptic densities connect the ribbon to the plasma membrane.

E Quantification of synaptic parameters from random EM sections. Number of ribbon-associated vesicles, size of synaptic ribbon as well as the extent of postsynaptic density (PSD) increase with age (student's t-test). Only sections parallel to the ribbons' short axes with sharply delineated PSD were analyzed. Ribbon size was measured as the length of the longest extent across the synaptic ribbon.

$\mathrm{F}$ Increase of ribbon size/content during development was corroborated by the increase in integrated puncta intensity from quantification of RIBEYE immunofluorescence. A developmental increase in full-width-of-half-maximum (FWHM) of Gaussian fits to puncta was also observed, Wilcoxon rank test.

G Decrease of number of synaptic ribbon during development estimated by immunohistochemistry (immuno) and live imaging (peptide), student's $t$-test.

Data information: ${ }^{*} P<0.05 ;{ }^{* *} P<0.01 ;{ }^{* * *} P<0.001$. n.s., no significant difference.

binding properties of the molecule(s) mediating fast exocytosis do not change upon the onset of hearing. Because of the steep $\mathrm{Ca}^{2+}$ dependence we applied statistical comparison among p6-8 and p14-17 IHCs for the rate constants of the fast component and the exocytic delays within a narrow $\left[\mathrm{Ca}^{2+}\right]$ range $(15-25 \mu \mathrm{M}$, for which we found the best comparable representation of $\left[\mathrm{Ca}^{2+}\right]$ changes). Neither the rate constants $(P=0.7$, Student's $t$-test $)$ nor the delays $(P=0.86$, Student's $t$-test) were significantly different. The rate constants were larger and the delays shorter for the fast component in the present study (Fig 6C,E) than those observed previously at comparable $\left[\mathrm{Ca}^{2+}\right]$, which we attribute to the use of shorter stimuli (100 $\mu$ s laser stimulation versus $1 \mathrm{~ms}$ long arc lamp flashes) that achieved a faster rise of $\left[\mathrm{Ca}^{2+}\right]$.

\section{Linearization of the apparent $\mathrm{Ca}^{2+}$ dependence of exocytosis is limited to changes in the number of open $\mathrm{Ca}^{2+}$ channels}

Next, we addressed the apparent $\mathrm{Ca}^{2+}$ dependence of exocytosis (Matveev et al, 2011) by studying the coupling between $\mathrm{Ca}^{2+}$ influx and RRP exocytosis in p6, p9-10, and p14-17 IHCs. We used strong $(-17 \mathrm{mV})$ and brief $(20 \mathrm{~ms})$ depolarizations to evoke exocytosis of the RRP (Moser \& Beutner, 2000; Beutner \& Moser, 2001) in perforated-patch recordings. Following the framework of our previous analysis (Brandt et al, 2005) we studied the relationship between RRP exocytosis $\left(\Delta C_{m, 20 \mathrm{~ms}}\right)$ and the integrated $\mathrm{Ca}^{2+}$ influx $\left(Q_{\mathrm{Ca}}\right)$ while either varying the single $\mathrm{Ca}^{2+}$ channel current $\left(i_{\mathrm{Ca}}\right)$ or changing the number of open $\mathrm{Ca}_{\mathrm{V}} 1.3$ channels $\left(N_{\mathrm{Ca}} \times P_{\text {open }}\right)$. The apparent $\mathrm{Ca}^{2+}$ cooperativity $m$ was obtained by fitting the exocytosis- $Q_{\mathrm{Ca}}$ relationship for each cell with a power function: $\Delta C_{m}=A\left(Q_{\mathrm{Ca}}\right)^{m}$ (Augustine et al, 1991), limited to the $Q_{\mathrm{Ca}}$ range in which no obvious saturation of the exocytosis was observed. For $\mathrm{Ca}^{2+}$-microdomain control (see Introduction), $m$ should be similar to the intrinsic $\mathrm{Ca}^{2+}$ cooperativity (4-5, Beutner et al, 2001), regardless of how the $\mathrm{Ca}^{2+}$ influx was manipulated. For $\mathrm{Ca}^{2+}$-nanodomain control (see Introduction), the $m$ should differ between the two manipulations, because there, the number of released RRP vesicles is linearly determined by $N_{\text {Ca }} \times P_{\text {open }}(m \sim 1$, Augustine et al, 1991, see our biophysical model below).

To vary $i_{\text {Ca }}$, we slowly changed the extracellular $\left[\mathrm{Ca}^{2+}\right]$ (Fig 7A,C) in both directions while supplementing divalent ions $\left(\mathrm{Mg}^{2+}\right)$ at low $\mathrm{Ca}^{2+}$ concentration $\left(<1.3 \mathrm{mM}\left[\mathrm{Ca}^{2+}\right]_{\mathrm{e}}\right)$. We observed a supralinear exocytosis- $Q_{\mathrm{Ca}}$ relationship in the range of low $Q_{\mathrm{Ca}}$ (Fig 7C, average of the maximal $Q_{\mathrm{Ca}}$ used for fitting indicated by dashed vertical lines) before and after the onset of hearing (supralinear apparent $\mathrm{Ca}^{2+}$ dependence, p6-8: $m=3.10 \pm 0.39, n=6 \mathrm{IHCs} ; \mathrm{p} 14-17$ : $m=2.91 \pm 0.38, n=7$ IHCs). Next, to gradually reduce $N_{\mathrm{Ca}} \times P_{\text {open }}$ (Fig 7D) while maintaining $i_{\mathrm{Ca}}$ (Hess et al, 1984), we slowly bathperfused preparations with the antagonistic dihydropyridine isradipine $(10 \mu \mathrm{M})$. In immature IHCs, we found a supralinear apparent $\mathrm{Ca}^{2+}$ dependence $(m=2.35 \pm 0.18, n=7 \mathrm{IHCs})$ consistent with control of any given release site by several channels (" $\mathrm{Ca}^{2+}$ microdomainlike" control of exocytosis). The exponent $m$ declined with maturation to a quasi-linear apparent $\mathrm{Ca}^{2+}$ dependence after the onset of hearing (p14-p17: $1.42 \pm 0.13 ; n=7$ IHCs, see also Supplementary Fig S8 for corroborating data), suggesting a change towards " $\mathrm{Ca}^{2+}$ nanodomain-like” control of exocytosis.

\section{Linearization of the apparent $\mathrm{Ca}^{2+}$ dependence of exocytosis for $N_{\text {Ca }} \times P_{\text {open }}$ changes indicates tightening of $\mathrm{Ca}^{2+}$ influx- exocytosis coupling: insights from modeling}

How precisely $\mathrm{Ca}^{2+}$ influx through nearby $\mathrm{Ca}^{2+}$ channels regulates the exocytosis of a given RRP vesicle depends on several parameters, including the number, distance and open probability of $\mathrm{Ca}^{2+}$ channels, the concentration and binding kinetics of $\mathrm{Ca}^{2+}$ buffers and the $\mathrm{Ca}^{2+}$-binding properties of the $\mathrm{Ca}^{2+}$ sensor of exocytosis (Moser et al, 2006; Matveev et al, 2011). Given this complexity we turned to biophysical modeling in order to reconcile the topography of membrane-proximal vesicles (here assumed to represent the RRP) and $\mathrm{Ca}^{2+}$ channels at the $\mathrm{AZ}$ with the apparent $\mathrm{Ca}^{2+}$ dependence of exocytosis observed in IHCs during changes of the number of open channels $\left(N_{\mathrm{Ca}} \times P_{\text {open }}\right)$ or $i_{\mathrm{Ca}}$ before and after the onset of hearing. We based the models on morphological and biophysical information (e.g. data from EM, STED, $\mathrm{Ca}^{2+}$ channel analysis, $\mathrm{Ca}^{2+}$ uncaging of this study and published work: for parameters and computational methods see Supplementary Material S9). We then studied paradigmatic topographies of $\mathrm{Ca}^{2+}$ channels and $\mathrm{Ca}^{2+}$ sensors for mature AZ (scenarios "M1-3", Fig 8A and Supplementary Material S9) and immature AZs (scenarios "IM", Fig 8B and Supplementary Material S9).

For the mature AZ, 14 vesicles (40-nm-diameter red disks in Fig $8 \mathrm{~A}, \mathrm{~B})$ were randomly distributed at the longer edges of an $80 \times 420 \mathrm{~nm}$ presynaptic density (seven vesicles per one side), with 

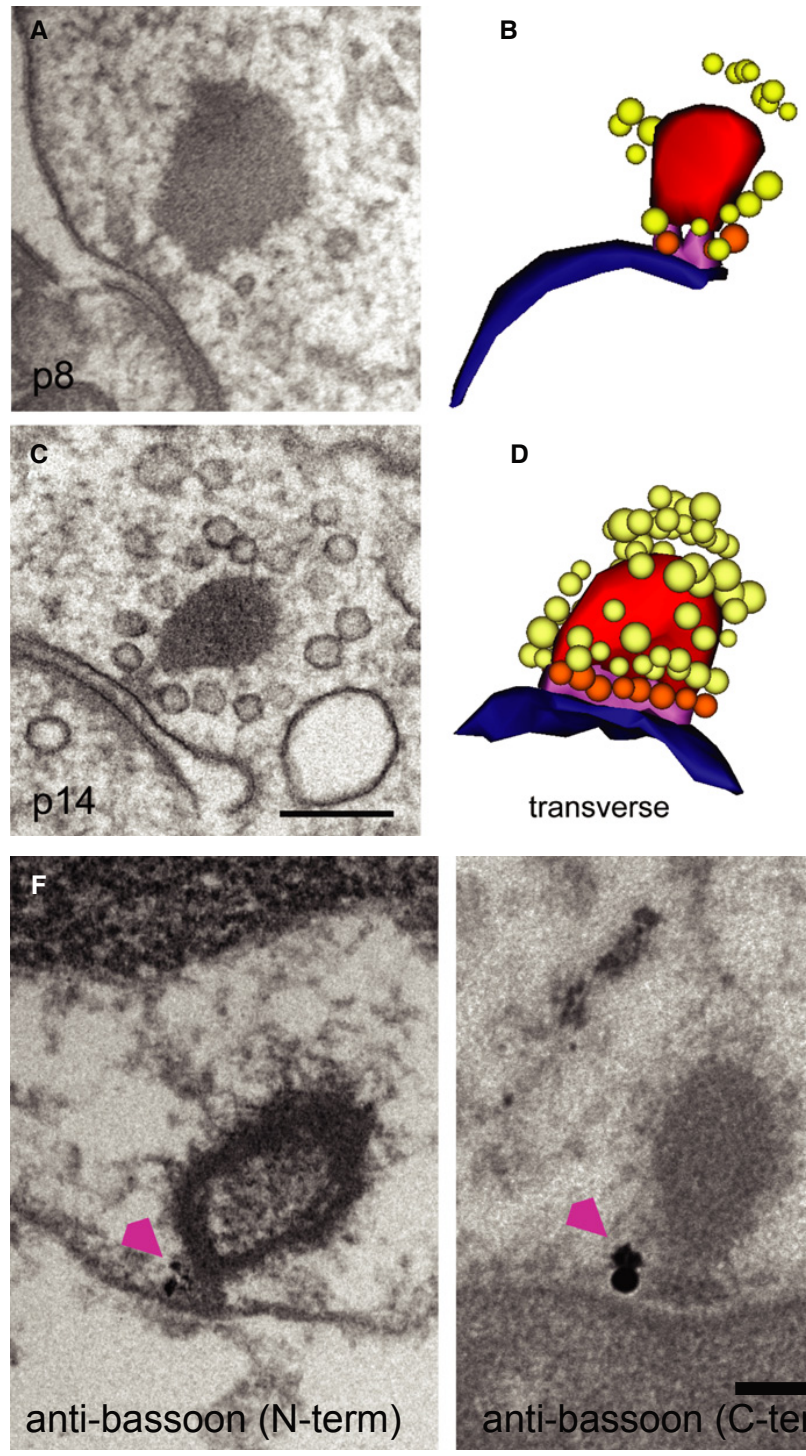

D

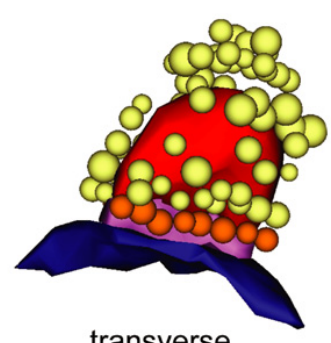

transverse

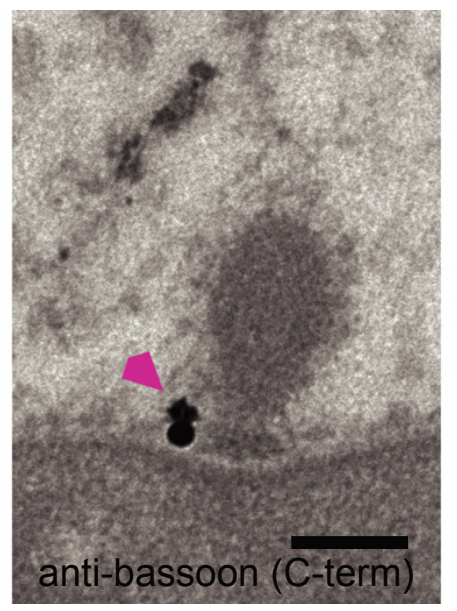

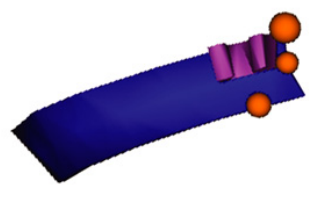

E

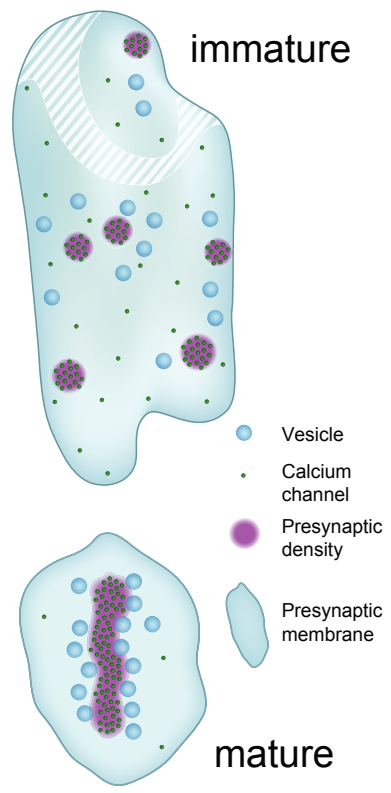

top view

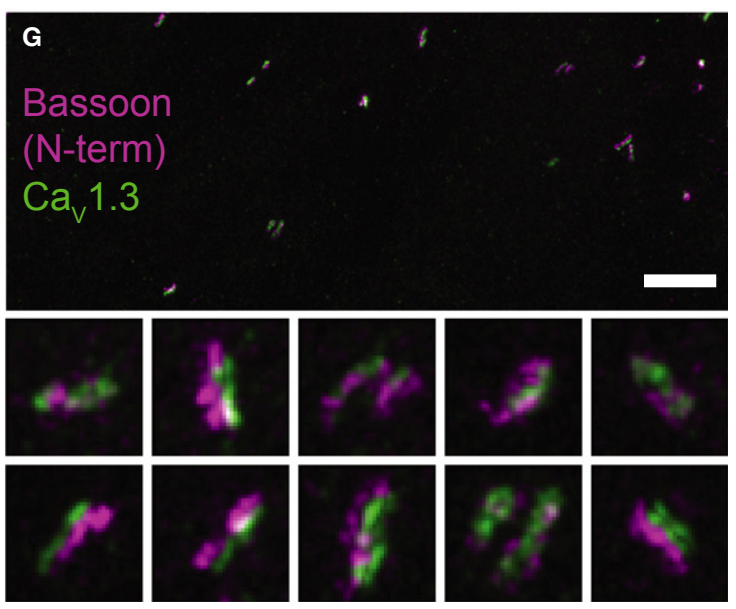

Figure 3. Nanoanatomy of IHC AZs.

A TEM of an immature IHC ribbon synapse using high-pressure freezing and freeze substitution (HPF/FS).

B Corresponding serial 3D reconstruction of (A), showing the immature ribbon connected via two rootlets to the presynaptic membrane. For color code see (D)

C Representative TEM of a mature ribbon, connected with one continuous presynaptic density to the membrane, as demonstrated in the 3D reconstruction in (D).

D 3D reconstruction: ribbon: red; AZ membrane: blue; rootlet (immature)/presynaptic density (mature): magenta; membrane-proximal SVs: orange; ribbon-associated SVs: yellow.

E Schematics of immature (top) and mature (bottom) AZs inspired by exemplary 3D reconstructions and STED imaging of Cav 1.3 immunofluorescence. Ca ${ }^{2+}$ channels formed small clusters and vesicles were less organized at the immature AZ. Stripe-like $\mathrm{Ca}^{2+}$-channel cluster with vesicles positioned regularly along each side at the mature $A Z$.

F Pre-embedding immunogold labeling for bassoon (arrowhead) was detected at the ribbon-anchoring presynaptic density of mature ribbons. Two primary antibodies specific to the $\mathrm{N}$-terminal or C-terminal of bassoon, respectively, were used (see Materials and Methods). Scale bar, $200 \mathrm{~nm}$.

G STED image of immunolabeled bassoon (magenta) and Cav1.3 channel clusters (green) in mature IHCs: stripe-like morphology and closely aligned immunofluorescence of bassoon and Cav1.3 reminiscent of the presynaptic density reconstructed from TEM (D). The image is a maximum intensity projection after smoothing with a Gaussian ( $\sigma=1$ pixel) in Imagej. The color lookup was chosen to optimize contrast and visibility of dimmer structures. Pixel size, $20 \mathrm{~nm}$. Scale bar, $2 \mu \mathrm{m}$. Insets, $700 \times 700 \mathrm{~nm}$. 


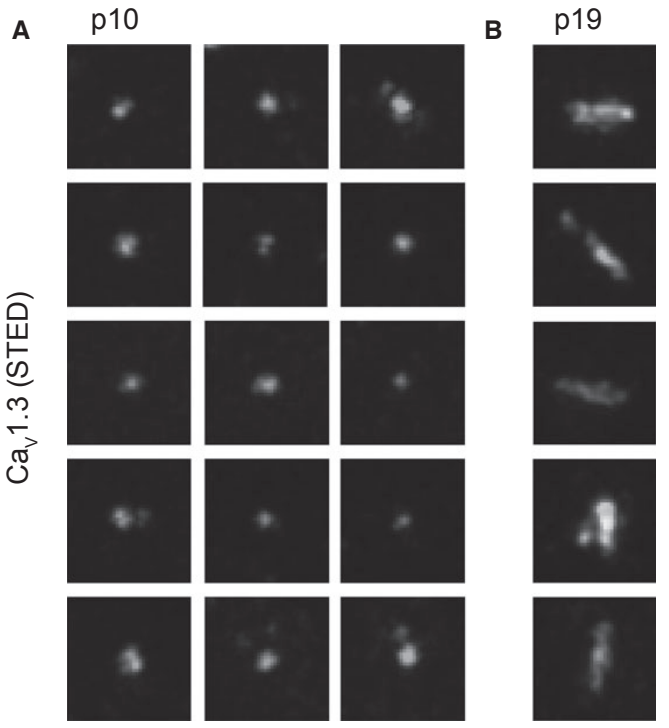

Figure 4. Development of Cav1.3 cluster morphology.

A Projections of STED sections of immunolabeled Cav1.3 clusters in the organ of Corti whole-mount at p10 (0.6 $\mu \mathrm{m}$ step size, total z covered: $4 \mu \mathrm{m})$. Cav1.3 clusters in pre-hearing IHCs appeared spot-like (A), in contrast to the stripe-like appearance in mature IHCS (B). Images were processed as in Fig 3G. Pixel size, $20 \mathrm{~nm}, 700 \times 700 \mathrm{~nm}$ regions-ofinterest centered at individual Cav1.3 clusters.

B Projections of STED sections of immunolabeled CaV1.3 clusters in the organ of Corti whole-mount at p19 $(0.6 \mu \mathrm{m}$ step size, total z covered: $4 \mu \mathrm{m})$. Cav1.3 clusters in pre-hearing IHCs appeared spot-like (A), in contrast to the stripe-like appearance in mature IHCS (B). Images were processed as in Fig 3G. Pixel size, $20 \mathrm{~nm}, 700 \times 700 \mathrm{~nm}$ regions-of interest centered at individual CaV1.3 clusters.

the vesicular $\mathrm{Ca}^{2+}$ sensor (black spots in Fig 8A,B) in contact with the density in the plane of the plasma membrane. The size of the presynaptic density (grey area in Fig 8A,B) was chosen based on exemplary synapses reconstructed from serial EM sections (e.g. Fig 3D), and is consistent with $\mathrm{Ca}_{\mathrm{V}} 1.3$ immunofluorescence in STED microscopy (Figs 4G and 5B). We placed 14-90 channels within the presynaptic density, representing different scenarios of channelvesicle coupling. In scenario M1 (Fig 8A, upper panel), 36 channels were distributed randomly in the presynaptic density area. In scenario M2 (Fig 8A, middle panel), in addition to the 36 randomly distributed ones, 14 channels were placed in physical contact with the vesicle $\mathrm{Ca}^{2+}$ sensors, simulating molecular coupling (review in Eggermann et al, 2012) and nicknamed hereafter as "private channel" to highlight its dominance on the $\mathrm{Ca}^{2+}$ signal of a given vesicle. In scenario M3, only those 14 channels in physical contact with the vesicles were implemented (Fig 8A, lower panel). For each scenario, 100 different realizations of random channel and vesicle positions were considered. Analogous to experimental analysis, we compared the slope $m$ of vesicle release against $\mathrm{Ca}^{2+}$ influx in double-logarithmic plots among scenarios of different degree of coupling. Scenarios M1-3 resulted in different exponents $m$ for $N_{\mathrm{Ca}} \times P_{\text {open }}$ change, all below 2: 1.8 for M1, 1.2 for M2 and 1.1 for M3 (Fig 8E), while $m$ estimates for $i_{\text {Ca }}$ change were all approximately 4 (Fig 8F). As another measure of $\mathrm{Ca}^{2+}$ influx to exocytosis coupling, we studied the effective number of contributing channels $n_{c h}$ (Fig $8 \mathrm{D}$ for scenario M2), which is the ratio of the total mean steady state $\left[\mathrm{Ca}^{2+}\right]$ (Fig $8 \mathrm{C}$ ) to the mean steady state $\left[\mathrm{Ca}^{2+}\right]$ elicited by the channel that contributed the most. With increasing dominance of the nearest channel, the effective number $n_{c h}$ of channels contributing to $\left[\mathrm{Ca}^{2+}\right]$ at the $\mathrm{Ca}^{2+}$ sensor went down from 4.4 for M1 to 2.1 for M2 and 1.2 for M3. Specifically, $n_{c h}=2.3$ (1.2) in scenario M2 (M3) indicates that each "private channel" on average contributes $44 \%$ $(83 \%)$ of $\left[\mathrm{Ca}^{2+}\right]$ at the corresponding $\mathrm{Ca}^{2+}$ sensor, whereas $n_{c h}=4.4$ in scenario M1 implies a contribution of only $23 \%$ by the nearest channel. Figure 8D illustrates for the case of scenario M2 how $n_{c h}$ depends on the spatial position of channels and $\mathrm{Ca}^{2+}$ sensors in the plane of the plasma membrane. Simply moving the sensor $20 \mathrm{~nm}$ away from the "private channel" towards the vesicle center in M2 (scenario M2d, Supplementary Figs S9 and S14), increased $n_{c h}$ to 5.8 and $m$ to 2.5 for $N_{\mathrm{Ca}} \times P_{\text {open }}$ changes, highlighting the strong effect of spatial proximity for the relevance of the nearest channel in controlling release of a given vesicle. In conclusion, modeling confirmed that the experimentally tractable $\mathrm{Ca}^{2+}$ cooperativity $m$ during $N_{\mathrm{Ca}} \times P_{\text {open }}$ changes is diagnostic for the relevance of the nearest channel in controlling fusion and support the notion of a $\mathrm{Ca}^{2+}$-nanodomain control of exocytosis in mature IHCs.

Despite the fact that implementing molecular coupling (M2-3) was sufficient to reproduce $m$ near unity during $N_{\mathrm{Ca}} \times P_{\text {open }}$ changes, this may not be the case if the channel density at the $\mathrm{AZ}$ is very high. Indeed, when we placed 40 additional randomly distributed $\mathrm{Ca}^{2+}$ channels in scenario M2 (a total of 90 channels, scenario M2b in Supplementary Fig S9), $m$ increased from 1.2 to 2.0 and $n_{c h}$ from 2.3 to 3.5 . One possibility to preserve the dominance of the "private channels" is to implement an exclusion zone around them where no other channels can be located. Scenario M2c (Supplementary Figs S9 and S14, same density of channels outside the exclusion zone as in $\mathrm{M} 2 \mathrm{~b}$ ) assumed circular exclusion zones with the width of a channel diameter and resulted in estimates of $m=1.2$ and $n_{c h}=2.1$ (very similar to those of scenario M2). On the other hand, if the density of the channels in the presynaptic density was low, $m$ near unity could be reproduced even with two "private channels". For example, addition of one more "private channel" per vesicle in M3 (scenario M3b in Supplementary Figs S9 and S14) led to $m=1.2\left(N_{\mathrm{Ca}} \times P_{\text {open }}\right.$ change $)$ and $n_{c h}=2.7$, close to the values obtained for scenario M2. Placing additional $\mathrm{Ca}^{2+}$ channels outside the presynaptic density $\left(10 / \mu \mathrm{m}^{2}\right.$, likely a strong overestimate of the average extrasynaptic density, see Brandt et al, 2005; Zampini et al, 2013) had negligible impact. In all above-mentioned scenarios $m$ stayed high, approximately 4 , when manipulating $i_{\text {Ca. }}$.

Modelling the apparent $\mathrm{Ca}^{2+}$ dependence of immature IHCs based on AZ topography is more challenging because (i) of the large fraction of extrasynaptic $\mathrm{Ca}^{2+}$ channels (possibly driving extrasynaptic exocytosis) and (ii) the fact that half of the IHCs lacked the fast component of exocytosis elicited by $\mathrm{Ca}^{2+}$ uncaging (which was used for modelling). However, higher $m$ predictions for $N_{\mathrm{Ca}} \times P_{\text {open }}$ changes than found for the mature scenarios M2-3 were readily obtained with models based on realistic AZ topography (IM1), such as that depicted in Fig 8B. There, a presynaptic density area similar to that of M1-3 was distributed within $1 \mu \mathrm{m}$ diameter disk in the form of separate patches corresponding to either pairs of smaller or single bigger densities (consistent with EM reconstructions), wherein $60 \mathrm{Ca}^{2+}$ channels were distributed with similar average 

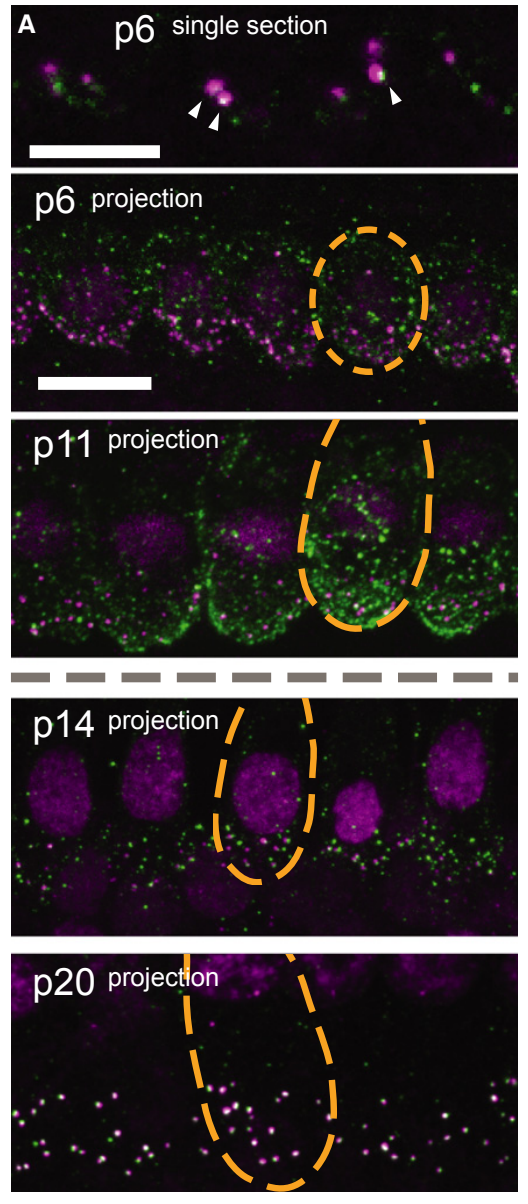
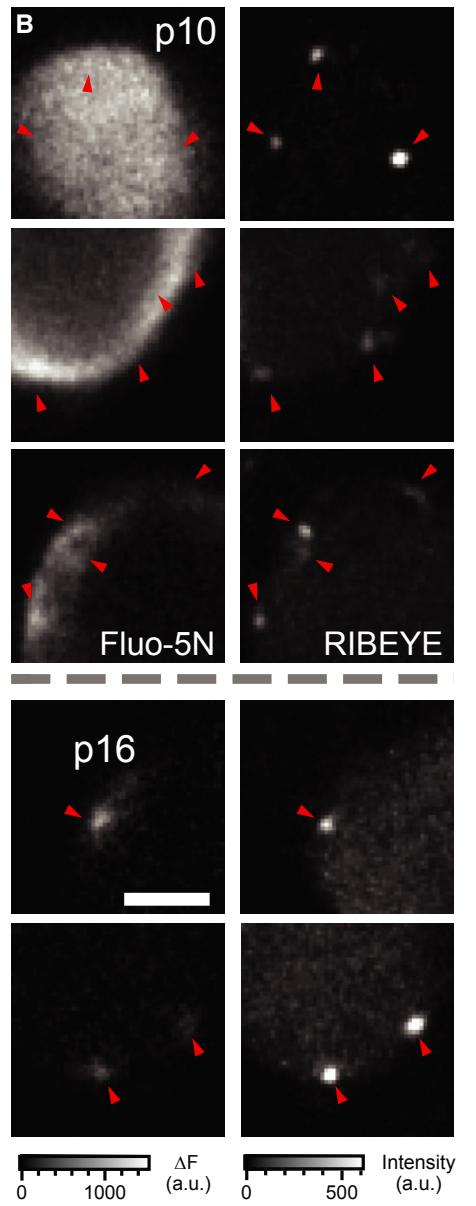

Figure 5. Maturation of hair cell $\mathrm{Ca}^{2+}$ signaling.

A Organs of Corti immunolabeled for Cav1.3 (green) and RIBEYE/CtBP2 (magenta). At p6 (top), a single section shows both ribbon-occupied (arrowheads) and ribbonless Cav1.3 spots as well as diffuse membrane staining. At p11 (middle, projection) puncta of Cav1.3 were observed throughout the basolateral membrane, especially the basal pole. By p14 the clusters of Cav1.3 were concentrated in the basal poles near synaptic ribbons, while the entire inner hair cell membrane remained diffusely labeled for Cav1.3. At p20 there was very little extrasynaptic labeling and the cell membrane was no longer diffusely immunoreactive for Cav1.3. Each synaptic ribbon was accompanied by a cluster of Cav1.3 and only few Cav1.3 clusters remained ribbonless. Scale bars, $10 \mu \mathrm{m}$.

B Examples of XY scans across the basolateral portion of IHCs before (upper, p10) and after (lower, p14) the onset of hearing. Left and right panels show the increase in Fluo-5N fluorescence during a step depolarization to $-7 \mathrm{mV}$ for $254 \mathrm{~ms}\left(2 \mathrm{mM}[\mathrm{EGTA}]_{\mathrm{i}}\right.$ ) and peptide-labeled synaptic ribbons (position marked with arrowheads). Note the relatively widespread submembraneous $\mathrm{Ca}^{2+}$ signal in immature IHCs as compared to the local $\mathrm{Ca}^{2+}$ rise in mature IHCs. Still, the highest $\left[\mathrm{Ca}^{2+}\right]$ was usually observed at the ribbons-occupied AZs. Scale bar, $3 \mu \mathrm{m}$.

density as in M2. Scenario IM1 did not force physical contact of $\mathrm{Ca}^{2+}$ channel and vesicular $\mathrm{Ca}^{2+}$ sensor, which was placed in shortest distance to the nearest presynaptic density. In addition, 60 channels were distributed randomly outside the presynaptic density patches. The predicted $m\left(N_{\mathrm{Ca}} \times P_{\text {open }}\right.$ change $)$ was 2.1 , which is similar to the experimental observation (2.3) and considerably higher than the $m\left(N_{\mathrm{Ca}} \times P_{\text {open }}\right.$ change $)$ estimates of M2 or M3 (Fig $8 \mathrm{E}, \mathrm{F})$. On the other hand, $m$ ( $i_{\mathrm{Ca}}$ change) was 4.1 , which is very similar to those of M1-3: $m=3.9-4.1$ (Fig 8F). However, $m$ considerably decreased for $N_{\mathrm{Ca}} \times P_{\text {open }}\left(m=1.1\right.$ ) but not for $i_{\mathrm{Ca}}$ changes $(m=4.0)$ when implementing "private channels" as in scenarios M2 and M3 (scenario IM1b, Supplementary Material S9, Supplementary Figs S9 and S14).
In our model, we realized that RRP depletion (fusion exceeding vesicle replenishment, see Supplementary Material S9) could cause exocytosis to saturate with increasing $\mathrm{Ca}^{2+}$ influx depending on the model parameters (Figs 8D,E and Supplementary Fig S10). Saturation would lead to underestimation of $m$ if data of this $Q_{\mathrm{Ca}}$ range was included into the fit. Release leveled-off more in the case of $i_{\mathrm{Ca}}$ variation, where, different from $N_{\mathrm{Ca}} \times P_{\text {open }}$ changes (Fig 7D, see also Supplementary Fig S8), saturation was also observed experimentally and fitting was restricted to the non-saturating range (Fig 7C). Our treatment of this "release saturation" is described in Supplementary Material S9. Using simulations, we also explored the $Q_{\text {Ca }}$ range in which the number of open channels per cluster is very low, which is difficult to achieve experimentally because of the 

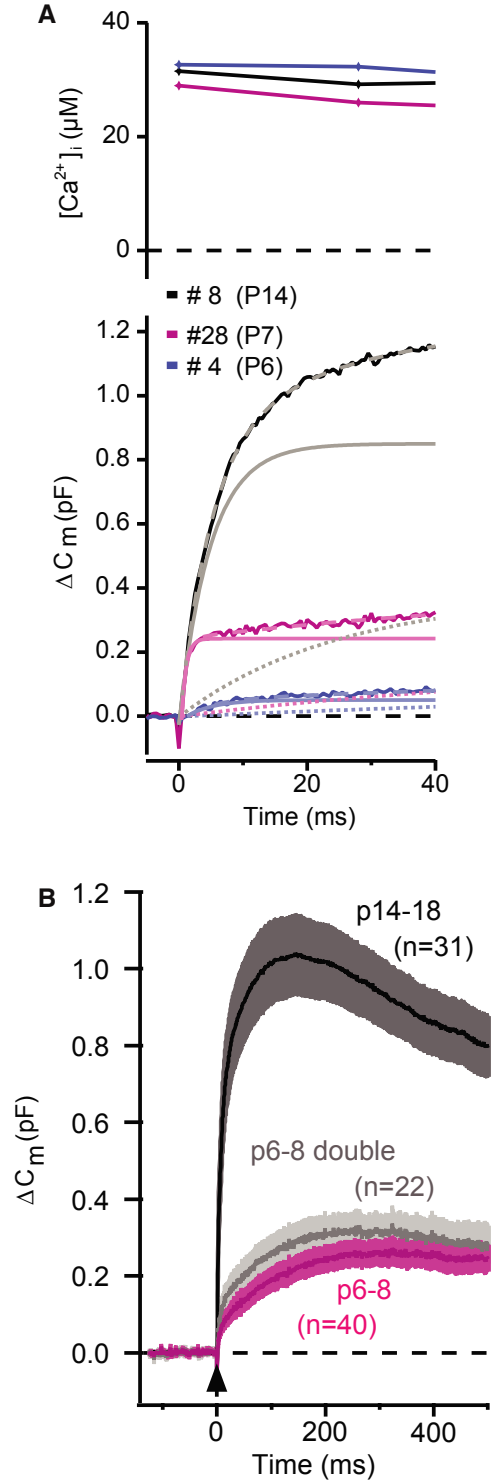

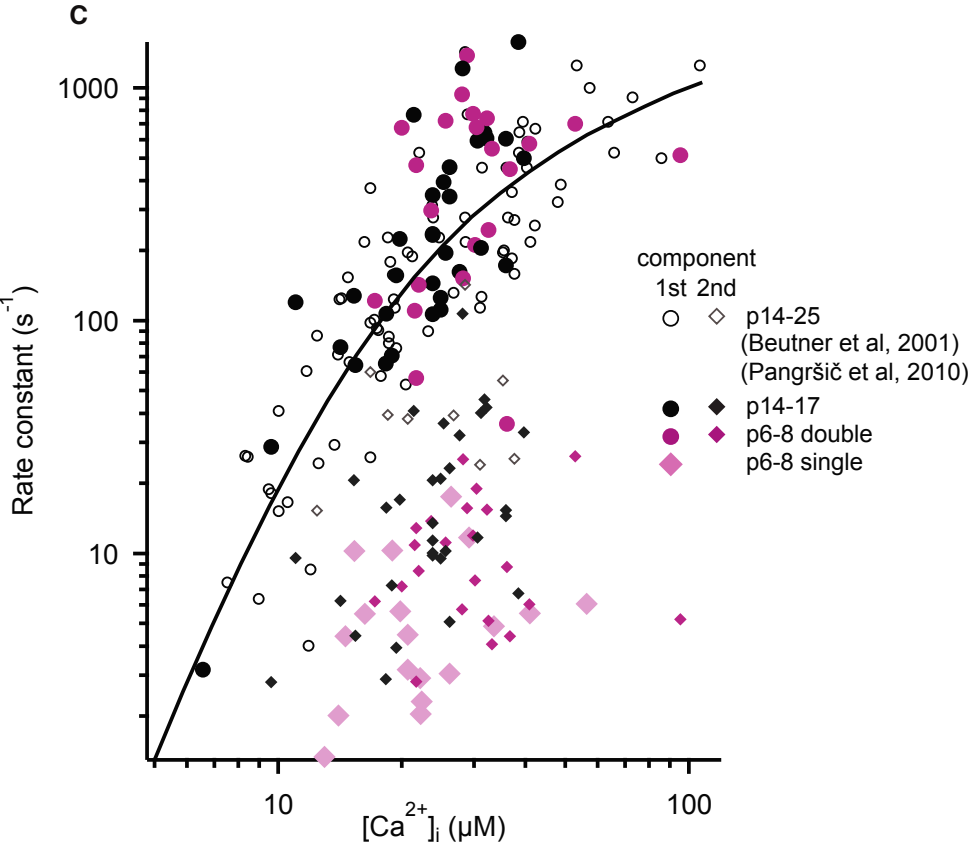

D

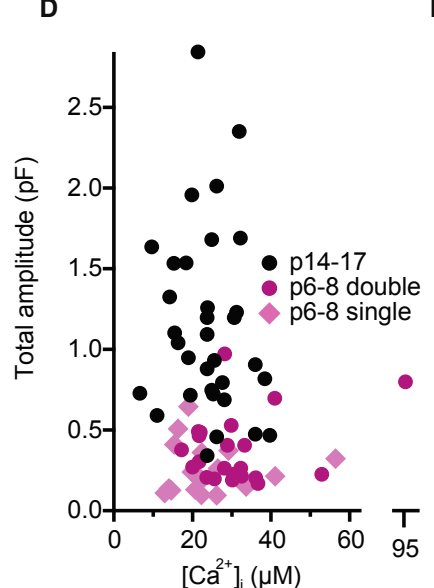

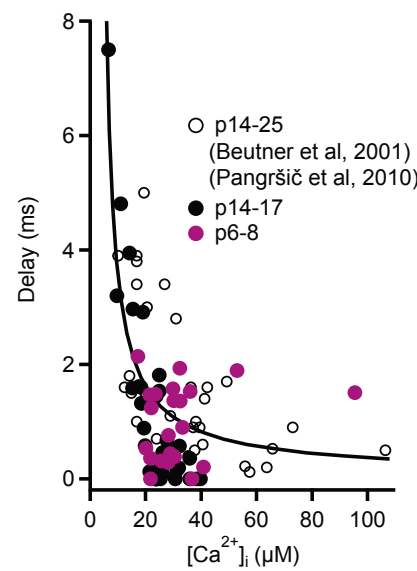

Figure 6. Intrinsic $\mathrm{Ca}^{2+}$ dependence of exocytosis in pre-hearing IHCs.

A Representative examples of UV-laser evoked capacitance rise $\left(\Delta C_{m}\right)$ of IHCs from mice of the specified ages. Upper: Intracellular [Ca $\left.{ }^{2+}\right]$ of a p14 (black), a $p 7$ (magenta) and a p6 (blue) IHCS after UV-flash, measured ratiometrically with mag-fura-2. Lower: $\Delta C_{m}$ of the corresponding IHCs (solid lines). Lighter dashed lines represent bi-exponential fits to the data and lighter solid and dotted lines represent the fast and slow components respectively.

B The average ( \pm SEM) $\Delta C_{m}$ of p14-18 (black) and p6-8 IHCs (magenta: all cells; grey: only cells with bi-exponential $\Delta C_{m}$ ).

C Rate constants from exponential fits to the $\Delta C_{m}$ upon flash photolysis plotted against post-flash $\left[\mathrm{Ca}^{2+}\right]_{i}$. Circles mark the rate constants for first (fast) component and rhombi mark those for the second (slow) component of the bi-exponential fits. Rate constants of responses in p6-8 IHCS, which were best fitted with a single exponential are also marked with rhombi. Note the overlap between pre-hearing (p6-8) and hearing (p14+) age groups. Black (p14-18) and magenta (p6-8) symbols are newly acquired data, while open circles represent previously published data on mature IHCs (Beutner et al, 2001; Pangršič et al, 2010).

D Total response amplitudes (sum of first and second component, in case of double exponential fits) were largely independent of post-flash [Ca $\left.{ }^{2+}\right]_{\mathrm{i}}$.

E Delay of responses plotted against post-flash $\left[\mathrm{Ca}^{2+}\right]_{\mathrm{i}}$.

Data information: Solid lines in (C) and (E) are model fits from a previous publication (Beutner et al, 2001).

approximately $20 \%$ residual current with isradipine block. As expected, $m$ approached unity regardless of the topography, because release becomes dependent on $\mathrm{Ca}^{2+}$ influx through single openings of the few available channels. In contrast, for $i_{\mathrm{Ca}}$ changes $m$ values remained near constant even at considerably lowered $Q_{\mathrm{Ca}}$ outside the saturation region. In conclusion, biophysical modeling supports 


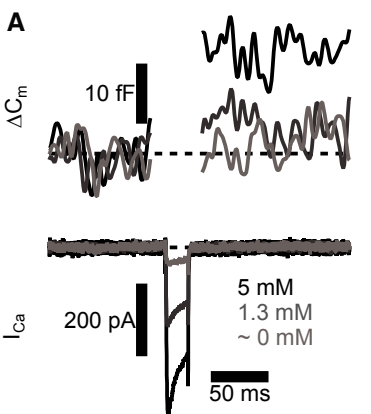

C
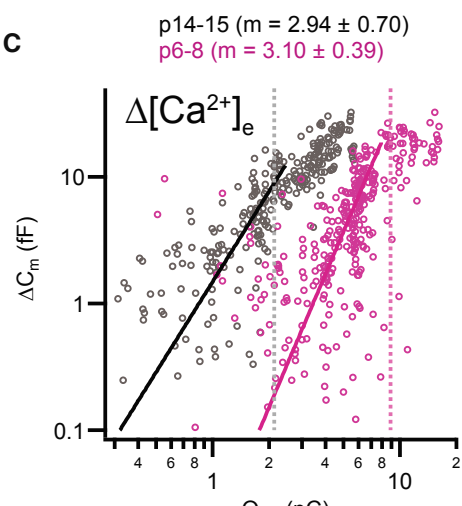

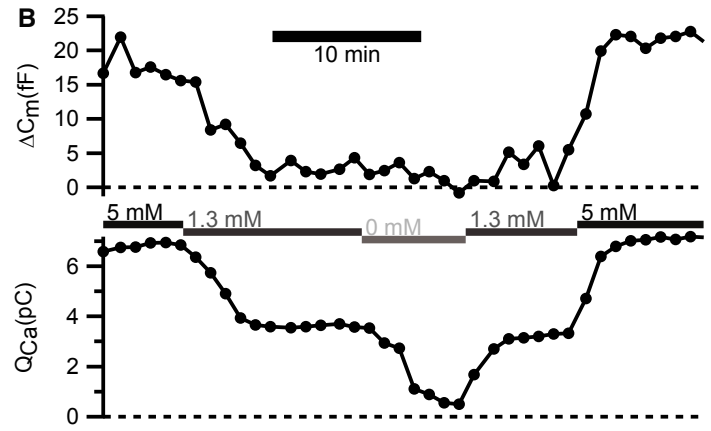

D $\quad \mathrm{p} 15-16(\mathrm{~m}=1.42 \pm 0.13)$

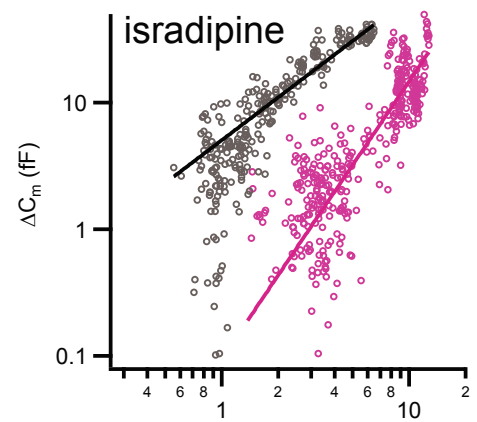

$\mathrm{Q}_{\mathrm{Ca}}(\mathrm{pC})$

Figure 7. Maturation of $\mathrm{IHCs} \mathrm{Ca}^{2+}$ influx-exocytosis coupling: experimental data.

A Depolarizations (20 ms to $-17 \mathrm{mV}$ ) elicited inward $\mathrm{Ca}^{2}+$ current $\left(I_{\mathrm{Ca}}\right.$, lower panel) and triggered $\Delta \mathrm{C}_{m}$ (upper panel) in a representative pre-hearing ( $\left.\mathrm{p} 7\right)$ IHC. Increasing $\left[\mathrm{Ca}^{2}+\right]_{\mathrm{e}}$ enlarged both $I_{\mathrm{Ca}}$ and $\Delta \mathrm{C}_{m}$.

B Time course for $\Delta C_{m}$ and integrated $\mathrm{Ca}^{2+}$ influx $\left(Q_{\mathrm{Ca}}\right)$ of the same IHC in (A) for repetitive depolarizations (20 ms to $-17 \mathrm{mV}$ ) at $60 \mathrm{~s}$ interval. Experiment started at $5 \mathrm{mM}\left[\mathrm{Ca}^{2+}\right]_{\mathrm{e}}$ and periods where bath solution was slowly perfused with a different $\left[\mathrm{Ca}^{2+}\right]_{\mathrm{e}}$ are marked by horizontal bars.

C Plotting $\Delta C_{m}, 20 \mathrm{~ms}$ against $Q_{\mathrm{Ca}}$ reveals a supralinear apparent $\mathrm{Ca}^{2+}$ dependence of exocytosis for low $Q_{\mathrm{Ca}}$. Solid lines represent best fit power functions $\left(\Delta C_{m}=A\right.$ $\left.\left(Q_{c a}\right)^{m}\right)$ to the whole data set in this range. Each small symbol represents an individual response to depolarization ( $p 15-16$, grey, $n=7 \mathrm{IHCs} ; \mathrm{p} 6, \mathrm{magenta}, n=7$ IHCS). Dashed vertical lines indicate the average maximal $Q_{\mathrm{ca}}$ limiting the $Q_{\mathrm{ca}}$ range used for fitting.

D Decreasing apparent $\mathrm{Ca}^{2+}$ cooperativity of exocytosis through blocking L-type $\mathrm{Ca}^{2+}$ channels with isradipine. Each small symbol represents an individual response to depolarization (p14-15, grey, $n=6 \mathrm{IHCS}$; p6-8, magenta, $n=7 \mathrm{IHCS}$ )

Data information: In (C) and (D), solid lines show best fit power functions to the whole data set. Average values of the exponent $m$ are indicated on top.

the experimentally derived notion of a maturational tightening of $\mathrm{Ca}^{2+}$ channel-exocytosis coupling.

\section{Discussion}

Here we studied the maturation of afferent IHC synapses around the onset of hearing. We found a transformation from an immature synaptic contact containing multiple small and peripheral spot-like AZ/PSD complexes into a consolidated contact with a single large AZ/PSD complex per SGN bouton. $\mathrm{Ca}^{2+}$ channels and $\mathrm{Ca}^{2+}$ influx became progressively confined to synaptic AZs over development. $\mathrm{Ca}_{V} 1.3$ channels first formed spot-like clusters whereas, following AZ remodeling, they formed an extended stripe alongside bassoon protein within the presynaptic density. Synaptic ribbons were larger in size and more flat in shape after the onset of hearing. $\mathrm{Ca}^{2+}$ uncaging triggered a fast component of exocytosis in only half of the immature IHCs, where it was smaller than in mature IHCs but had a similar intrinsic $\mathrm{Ca}^{2+}$ dependence. A "linearization" of the apparent $\mathrm{Ca}^{2+}$ dependence of exocytosis observed for $N_{\mathrm{Ca}} \times P_{\text {open }}$ changes, together with computational modeling of immature and mature $\mathrm{AZ}$ topographies, indicated a developmental switch from more $\mathrm{Ca}^{2+}$ microdomain-like to more $\mathrm{Ca}^{2+}$ nanodomain-like coupling between $\mathrm{Ca}_{\mathrm{V}} 1.3$ channels and vesicular release sites.

\section{Maturation of molecular nanoanatomy of IHC afferent synapses}

High-resolution immunofluorescence and electron microscopy jointly demonstrated major structural changes of IHC synapses around the onset of hearing, schematized in Figs $1 \mathrm{C}$ and 3E. We propose that this change to a more ordered stripe-like AZ topography, together with the dramatic reduction of extrasynaptic $\mathrm{Ca}_{\mathrm{V}} 1.3$ channels, enhances the $\mathrm{Ca}^{2+}$ efficiency of exocytosis and tightens the spatial coupling between $\mathrm{Ca}_{\mathrm{V}} 1.3$ channels and vesicular release sites (Figs 7 and 8). We consider two not mutually exclusive candidate mechanisms for the structural refinement of the synapse over 

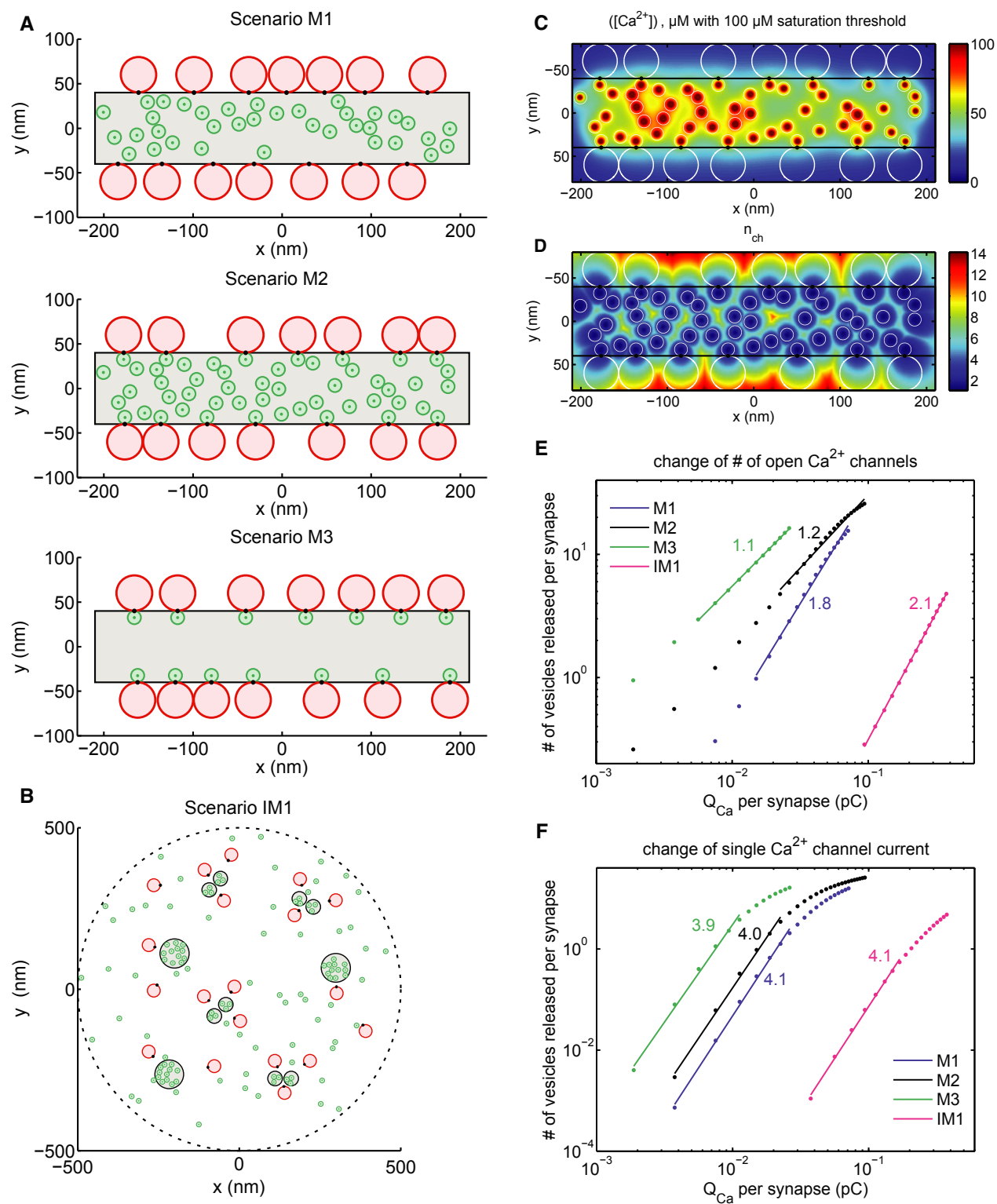

Figure 8. Maturation of IHCs $\mathrm{Ca}^{2+}$ influx-exocytosis coupling: modeling.

A Scenarios M1-3 of the mature AZ topography. Grey area: presynaptic density, red discs: RRP vesicles, green discs: $\mathrm{Ca}^{2+}$ channels, black spots: Ca ${ }^{2+}$ sensors of exocytosis. Scenario M1: random positioning of channels within the presynaptic density, $\mathrm{Ca}^{2+}$ sensor pointing towards the channel cluster. Scenario $\mathrm{M2}$ : introduction of private channels simulating molecular coupling of release site and channel. Scenario M3: only private channels, unrealistically low number of channels. Plots correspond to one out of a hundred realizations considered in the simulations.

B Scenario IM1 of the immature AZ topography. Legend as in (A). Note the more extended synaptic contact with several $\mathrm{Ca}^{2+}$ channel clusters, which were placed at the sites of presynaptic densities.

C Total mean steady state $\left[\mathrm{Ca}^{2+}\right]$ profile at the AZ membrane for a particular realization of scenario $\mathrm{M} 2$.

D Effective number of channels contributing to total mean steady state $\left[\mathrm{Ca}^{2+}\right]$ shown in (C).

E Average overall release (number of vesicles released) versus $\mathrm{Ca}^{2+}$ charge influx per synapse after the first 20 ms after the stimulus onset when $N_{\text {Ca }} \times P_{\text {open }}$ was changed by blocking channels (modeling the isradipine experiment). Dots correspond to the values averaged over different synapses and realizations as described in Supplementary Material S9. Straight lines are linear fits in log-log scale within the interval of fivefold decrease in $Q_{\mathrm{ca}}$. Numbers correspond to the slope of fits, representing the apparent $\mathrm{Ca}^{2+}$ cooperativity of release, $m$. Blue —-mature scenario $\mathrm{M} 1$, black—-mature scenario $\mathrm{M} 2$, green-mature scenario $\mathrm{M} 3$, magentaimmature scenario IMI.

$F \quad$ The same as (E) but $Q_{\mathrm{Ca}}$ was varied by manipulating $i_{\mathrm{Ca}}$ instead of $N_{\mathrm{Ca}} \times P_{\mathrm{open}}$. 
development: (i) merging of the individual synaptic specializations within a contact and (ii) pruning of all but one AZ/PSD complex per contact.

Merging may be mediated by interactions of pre- and/or post-synaptic scaffold molecules and could involve trans-synaptic regulation. Such cluster-cluster interactions may join multiple small ribbons, $\mathrm{Ca}^{2+}$-channel clusters, or GluA clusters into single large structures. Several lines of evidence indicate an important role of bassoon in this process. First, the involvement of cytomatrix of the AZ proteins in synaptogenesis was demonstrated for other synapses (Zhai et al, 2000, 2001; Shapira et al, 2003) whereby bassoon also recruits other proteins to the AZ (Shapira et al, 2003; Takao-Rikitsu et al, 2004; Maas et al, 2012). Second, bassoon resides in the stripelike presynaptic density of the mature IHC synapse of wild-type mice (Fig 3) and also in the immature presynaptic density (Fig 1). Third, while synapses are still formed in IHCs lacking functional bassoon (Khimich et al, 2005), the presynaptic density and $\mathrm{Ca}^{2+}$ channel clusters remain spot-like (Frank et al, 2010). Notably, around hearing onset bassoon mRNA is upregulated in the mouse organ of Corti under control of thyroid hormone (Sendin et al, 2007). Perhaps bassoon facilitates AZ merging via interactions with CAST (Takao-Rikitsu et al, 2004), RIBEYE (tom Dieck et al, 2005), piccolo (Wang et al, 2009), and other proteins. The fusion of neighboring AZ-anchored ribbons is plausible given the strong RIBEYE-RIBEYE interactions (Magupalli et al, 2008), reports of ribbon-ribbon fusion during retinal synaptogenesis (Regus-Leidig et al, 2009), their stimulus-dependent structural plasticity (SpiwoksBecker et al, 2004), and our observation of floating ribbons near anchored ones at immature IHC AZs (Fig 2).

Alternatively, or in parallel with merging, all but one of the AZs may be degraded. Pruning is a well-established mechanism for synaptic refinement in general (Goda \& Davis, 2003). In the organ of Corti, pruning is thought to refine SGN afferent connectivity at the level of fibers and contacts (Sobkowicz et al, 1982; Huang et al, 2007, 2012; Sendin et al, 2007) but its role in development within a synaptic contact is not clear. Molecularly, the pruning of AZs might involve protein degradation, which appears to be counteracted by bassoon and piccolo via negative regulation of ubiquitin-ligase activity (Waites et al, 2013), such that their greater abundance might protect the largest of the initially formed AZs. In addition, a mutual regulation of ribbon size and $\mathrm{Ca}^{2+}$ channel clusters by $\mathrm{Ca}^{2+}$ influx and RIBEYE expression levels, respectively, was indicated in zebrafish lateral line hair cells during early development (Sheets et al, 2011, 2012). Super-resolution live-imaging of fluorescently-tagged ribbons and other AZ/PSD components in organotypic organ of Corti cultures will be required to directly evaluate the hypotheses of merging and pruning in future studies.

\section{Maturation of $\mathrm{Ca}^{2+}$ influx-exocytosis coupling in IHCs}

So far, neither the intrinsic $\mathrm{Ca}^{2+}$ dependence of exocytosis nor the $\mathrm{Ca}^{2+}$ influx-exocytosis coupling in immature IHCs was well understood. We characterized the intrinsic $\mathrm{Ca}^{2+}$ dependence of exocytosis and the spatial coupling between $\mathrm{Ca}^{2+}$ influx and exocytosis in IHCs before and after the onset of hearing. We note that the amplitude of the fast $C_{m}$ rise evoked by $\mathrm{Ca}^{2+}$ uncaging, as used for studying the intrinsic $\mathrm{Ca}^{2+}$ dependence of exocytosis, was about 50 times larger than that reflecting the exocytosis of the RRP, analyzed for apparent $\mathrm{Ca}^{2+}$ dependence. Moreover, the mediating vesicle population(s) remain to be elucidated and might involve parallel extrasynaptic fusion of vesicles or vesicle priming and subsequent fusion (Fuchs et $a l, 2003)$. We assume that fast $C_{m}$ rise reports synaptic vesicle fusion and the $\mathrm{Ca}^{2+}$ dependence of transmitter release, based on the observations of (i) partial cross-depletion of exocytosis elicited by $\mathrm{Ca}^{2+}$ influx and uncaging (Beutner et al, 2001) and (ii) the abolition of fast exocytosis in the absence of the putative priming factor and $\mathrm{Ca}^{2+}$ sensor of hair cell exocytosis otoferlin (Roux et al, 2006; Pangršič et al, 2010). The $\mathrm{Ca}^{2+}$ dependent kinetics of the fast component were, when present, comparable between IHCs before and after the onset of hearing, suggesting a common $\mathrm{Ca}^{2+}$ sensor of exocytosis, most likely otoferlin. It will be interesting to study the molecular changes underlying the emergence of the fast component in immature IHCs in future experiments.

What then accounts for the major developmental increase in efficiency of whole-cell $\mathrm{Ca}^{2+}$ influx to evoke exocytosis? In agreement with a recent single-channel study (Zampini et al, 2010) we find that immature IHCs express several thousands of $\mathrm{Ca}_{V} 1.3$ channels. Upon maturation and the cessation of $\mathrm{Ca}^{2+}$ action potential firing (Kros et al, 1998; Brandt et al, 2007), $\mathrm{Ca}^{2+}$ influx was largely restricted to AZs (Fig 5B). This reduction of extrasynaptic $\mathrm{Ca}^{2+}$ channels likely accounts for much of the dramatic developmental increase in the efficiency of $\mathrm{Ca}^{2+}$ influx to drive exocytosis (Beutner \& Moser, 2001; Johnson et al, 2005) and lowers the metabolic expenditure for clearing $\mathrm{Ca}^{2+}$. Within synapses, $\mathrm{Ca}^{2+}$ channels appear to be retained and consolidated and, likely due to stabilizing interactions with scaffolding proteins.

Biophysical experiments and modeling indicated tighter spatial coupling between $\mathrm{Ca}^{2+}$ influx and RRP exocytosis after the onset of hearing. In agreement with our own and other published work, $m$ was near unity for manipulation of $N_{\mathrm{Ca}} \times P_{\text {open }}$ in mature IHCs (Fig 7D). This means that release scaled linearly with the number of open channels, suggesting either a $\mathrm{Ca}^{2+}$-nanodomain-like coupling or a linear intrinsic $\mathrm{Ca}^{2+}$-dependence of exocytosis. However, we found a larger $m$ when manipulating $i_{\mathrm{Ca}}$, consistent with a nonlinear $\mathrm{Ca}^{2+}$-dependence also seen with $\mathrm{Ca}^{2+}$ uncaging. On the other hand, in immature IHCs we found a supralinear apparent $\mathrm{Ca}^{2+}$ dependence of exocytosis when manipulating $i_{\mathrm{Ca}}$ or $N_{\mathrm{Ca}} \times P_{\text {open }}$, consistent with previous reports (Johnson et al, 2005) and indicative of a more $\mathrm{Ca}^{2+}$-microdomain-like coupling with a cooperative $\mathrm{Ca}^{2+}$ sensor. Together, these findings argue against the hypothesis that the $\mathrm{Ca}^{2+}$ dependence of IHC exocytosis is "linearized" upon maturation as a result of a change in the $\mathrm{Ca}^{2+}$ sensor of fusion (Johnson et al, 2010). Moreover, the "linearization" of $\mathrm{Ca}^{2+}$ dependence of exocytosis observed in whole-cell $C_{m}$ recordings is unlikely to result from summation of heterogeneously supralinear AZs (Heil \& Neubauer, 2010) because in such a scenario the apparent $\mathrm{Ca}^{2+}$ dependence should be linear no matter how it was manipulated.

Does the experimentally observed "linearization" of apparent $\mathrm{Ca}^{2+}$ dependence during manipulation of $N_{\mathrm{Ca}} \times P_{\text {open }}$ indeed reflect the establishment of a $\mathrm{Ca}^{2+}$-nanodomain control of exocytosis in mature IHCs? After all, these protocols are thought to apply only to initial release at stimulus onset when pool depletion has not commenced (Augustine et al, 1991; Stanley, 1993; Mintz et al, 1995). Here, however, we had to accept considerable release of the RRP 
when probing exocytosis with $20 \mathrm{~ms}$ depolarizations in elevated $\left[\mathrm{Ca}^{2+}\right]_{\mathrm{e}}$ to obtain a good signal to noise ratio. Therefore, we performed biophysical modeling to critically assess the consistency of our conclusions and also to gain further insights into the mechanism of IHC stimulus-secretion coupling. Combining data from a variety of different experimental approaches, we carefully investigated the effects of release saturation, resulting primarily from RRP depletion, on the predicted apparent $\mathrm{Ca}^{2+}$ dependence of exocytosis. Saturation was most pronounced when increasing $i_{\mathrm{Ca}}$ and less prevalent during manipulation of $N_{\mathrm{Ca}} \times P_{\text {open }}$. Importantly, RRP depletion is counteracted by fast vesicle replenishment at the IHC AZ (Pangršič et al, 2010; Goutman 2012), and for experimental $m$ estimation during $i_{\mathrm{Ca}}$ manipulation we restricted the $Q_{\mathrm{Ca}}$ range outside the saturating range. The $m$ estimates derived from full and reduced $Q_{\mathrm{Ca}}$ range did not differ much for $N_{\mathrm{Ca}} \times P_{\text {open }}$ (Supplementary Fig S8). Because $m$ was much lower for manipulation of $N_{\mathrm{Ca}} \times P_{\text {open }}$ than for $i_{\mathrm{Ca}}$, opposite of the expected result if saturation impacted our analysis, we trust that it indicates $\mathrm{Ca}^{2+}$-nanodomain control of exocytosis at mature AZs. The $m$ estimate of approximately 1.4 placed strong constrains on the topography of the AZ models. Within our modeling framework and explored parameter space this was reproduced best when favoring control of exocytosis by "private channels”, which may indeed reflect molecular coupling (e.g. Liu et al, 2011).

The notion of a developmental tightening of the spatial $\mathrm{Ca}^{2+}$ channel release site coupling is further supported by a decrease of the EGTA-sensitivity of RRP exocytosis $\left(6 \% \Delta C_{m}\right.$ reduction with $5 \mathrm{mM}[\mathrm{EGTA}]_{\mathrm{i}}$ compared with perforated-patch in p14-25 IHCs versus $37 \%$ in p6 IHCs for $10 \mathrm{~ms}$ depolarization, Beutner \& Moser, 2001). A similar conclusion was reached at the calyx of Held synapse (Wang et al, 2008), another auditory synapse with high rates of synaptic transmission and high fidelity. There, developmental downregulation of the AZ component septin 5 seems critically involved in the transition from $\mathrm{Ca}^{2+}$-microdomain control before to $\mathrm{Ca}^{2+}$-nanodomain control after the onset of hearing (Yang et al, 2010). The precise molecular mechanism governing the maturation of coupling between $\mathrm{Ca}^{2+}$ channels and release sites at the IHC AZ remains to be elucidated in future studies.

\section{Materials and Methods}

\section{Animals}

C57Bl/6 mice (aged 6-30 days) mice were used for experiments. All experiments complied with national animal care guidelines and were approved by the University of Göttingen Board for animal welfare and the animal welfare office of the state of Lower Saxony.

Patch-clamp and confocal $\mathrm{Ca}^{2+}$ imaging

IHCs from apical coils of freshly dissected organs of Corti were patch-clamped as described (Moser \& Beutner, 2000). The standard pipette solution contained (in mM): $115 \mathrm{Cs}$-glutamate, 13 TEA-Cl, $20 \mathrm{CsOH}$-HEPES, $1 \mathrm{MgCl}_{2}, 2 \mathrm{MgATP}, 0.3 \mathrm{NaGTP}, 10$ EGTA, 0.4 Fluo-5N (Penta- ${ }^{+}$salt, Invitrogen), and carboxytetramethyl-rhodamine(TAMRA)-conjugated RIBEYE-binding dimer peptide $(2 \mu \mathrm{M}$, Francis et al, 2011) for $\mathrm{Ca}^{2+}$ imaging. The pipette solution for perforated patch experiments contained (in $\mathrm{mM}$ ): 135 Cs-gluconate, 10 TEA-Cl, 10 4-aminopyridine, $10 \mathrm{CsOH}$-HEPES, $1 \mathrm{MgCl}_{2}$, and $250 \mu \mathrm{g} / \mathrm{ml}$ amphotericin. The extracellular solution contained (in $\mathrm{mM}$ ): $104 \mathrm{NaCl}, 35 \mathrm{TEA}-\mathrm{Cl}, 2.8 \mathrm{KCl}, 5 \mathrm{CaCl}_{2}, 1 \mathrm{MgCl}_{2}, 10 \mathrm{NaOH}$-HEPES, 10 D-glucose, $\mathrm{pH}$ 7.3. Extracellular $\left[\mathrm{Ca}^{2+}\right]$ was varied between nominally $\mathrm{Ca}^{2+}$-free to $10 \mathrm{mM}$ for experiments manipulating $i_{\mathrm{Ca}}$. In all cases, $\mathrm{NaCl}$ concentration was adjusted for osmolarity of solutions with different $\mathrm{CaCl}_{2}$ concentrations. Currents were low-pass filtered at $2.9 \mathrm{kHz}$ (8.5 kHz for fluctuation analysis) and sampled at $50 \mathrm{kHz}$ (100 kHz for fluctuation analysis). Cells with holding current greater than -50 pA were discarded. An EPC-9 amplifier or an EPC10 amplifier and "Patchmaster" software (HEKA Elektronik, Lambrecht, Germany) was used for measurements. Whole-cell capacitance measurement was performed in perforated-patch configuration as previously described (Moser \& Beutner, 2000), except that $\Delta C_{m}$ was estimated as the difference of the mean $C_{m}$ over $100 \mathrm{~ms}$ after the end of the depolarization (the initial $40 \mathrm{~ms}$ were skipped). All voltages were corrected for liquid-junction potentials and voltage-drops across series resistance. $\mathrm{Ca}^{2+}$ currents were further isolated from background current using a $P / n$ protocol. Confocal $\mathrm{Ca}^{2+}$ imaging was performed as described (Frank et al, 2009). In brief, presynaptic $\mathrm{Ca}^{2+}$ signal of IHCs were observed as changes of $\mathrm{Ca}^{2+}$ indicator fluorescence in XY scans using long (200-254 ms) step depolarizations to $-7 \mathrm{mV}$.

\section{UV-laser photolysis of caged calcium}

UV-laser photolysis of caged calcium was performed as previously described (Nouvian et al, 2011). Briefly, to obtain step-wise increases in intracellular calcium, $100 \mu$ s of pulsed laser light from a DPSL-355/1000 UV laser (Rapp OptoElectronic, Hamburg, Germany) were applied after achieving the whole-cell configuration. $\left[\mathrm{Ca}^{2+}\right]_{\mathrm{i}}$ was measured by ratiometric imaging using the calcium indicator dye mag-fura-2 (Invitrogen, Darmstadt, Germany). The dye was excited by a monochromatic light source alternating between 340 and $380 \mathrm{~nm}$, and imaged using a CCD camera (TILL Photonics, Gräfelfing, Germany). $\left[\mathrm{Ca}^{2+}\right]_{\mathrm{i}}$ was determined as previously described (Beutner et al, 2001). The pipette solution for flash-photolysis contained (in mM): 83 Cs-gluconate, 16 TEA-Cl, 18 Cs-HEPES (pH 7.2), 0.3 mag-fura-2, 10 DM-nitrophen (gift of A. Leonov and C. Griesinger, Göttingen; or Calbiochem, Darmstadt, Germany), 5 1,3diaminopropan-2-ol-tetraacetic acid and $10 \mathrm{CaCl}_{2}$. The extracellular solution for flash-photolysis contained (in mM): $97 \mathrm{NaCl}, 35$ TEA-Cl, $2.8 \mathrm{KCl}, 10 \mathrm{CaCl}_{2}, 1 \mathrm{MgCl}_{2}, 10 \mathrm{Na}$-HEPES, $1 \mathrm{CsCl}, 11.1 \mathrm{D}$-glucose (pH 7.2). A double exponential function with variable delay was used to fit the change in $C_{m}$, from which rate constants and response delay were extracted. In cases where the rate constants of the two components differed by less than a factor of four, the traces were re-fitted with a single exponential function. IHCs in which no $C_{m}$ responses could be elicited were excluded from analysis.

\section{Immunohistochemistry and immunofluorescence microscopy}

Apical cochlear turns were fixed for $25 \mathrm{~min}$ in $99 \%$ methanol at $-20^{\circ} \mathrm{C}$. Primary antibodies: mouse anti-CtBP2 (1:200; BD Biosciences), rabbit anti-GluA2/3 (1:200; Chemicon), rabbit anti-Ca 1.3 (1:50; Alomone Labs), goat anti-CtBP2 (1:150; Santa Cruz Biotech), mouse anti-GluA2 (1:75 Chemicon), mouse anti-Sap7f407 to 
bassoon $\left(1: 1,000\right.$; Abcam), mouse anti- $\mathrm{Na}^{+} / \mathrm{K}^{+}$ATPase $\alpha 3$ subunit (1:200; Thermo Scientific). Secondary antibodies: AlexaFluor488, 594, and 647 (1:200; Molecular Probes). Images were acquired with an SP5 confocal microscope (Leica) with a $100 \times$ oil-immersion objective $(\mathrm{NA}=1.4)$. Experiments were repeated until all antigens were stained with relative uniformity in at least one preparation from each age group processed in batch. Each preparation yielded several images. Those with readily-apparent bleaching were discarded. Each image had 5-7 IHCs. Stacks from two preparations per age, stained and acquired in parallel across age groups, were analysed with Igor Pro 6 (Wavemetrics). Intensity per synapse was calculated in the optical section with the peak intensity. A region-ofinterest (ROI) was determined by fitting a 2D Gaussian function on a $1 \mu \mathrm{m}^{2}$ region surrounding each $\mathrm{CtBP} 2$ or $\mathrm{Ca}_{\mathrm{V}} 1.3$ immunofluorescent spot (Frank et al, 2010). Comparison of plasma membrane versus ribbon-associated $\mathrm{Ca}_{\mathrm{V}} 1.3$ immunofluorescence was performed by first connecting all ribbons within $3 \mu \mathrm{m}$ of each other in $3 \mathrm{D}$, and then using the connecting lines to define the axes of cylinders of $800 \mathrm{~nm}$ diameter to demarcate a volume of basal plasma membrane. The average intensity of this basal plasma membrane was compared with that of the ribbon-proximal region, defined as $3 \mathrm{D}$ spheres of $800 \mathrm{~nm}$ diameter centered at all connected ribbons. Three IHCs per age group were analyzed like this. Average voxel intensity of the stack was subtracted as background.

Two-color STED images were acquired on a custom setup with a stage-scanner (Mad City Labs) using Atto590- and Atto647Nconjugated secondary antibodies (Atto-Tec) excited with pulsed diode lasers (PicoQuant) at 595 and $640 \mathrm{~nm}$, temporally interleaved at $50 \mathrm{~ns}$ intervals. After excitation, the fluorescence of dyes in the periphery of the excited spot was suppressed through stimulated emission by the STED laser, emitting $1.2 \mathrm{~ns}$ pulses of $775 \mathrm{~nm}$ light at $20 \mathrm{MHz}$ (IPG Photonics). The STED beam was guided through a polymeric phase plate (vortex pattern; RPC Photonics) and circularly-polarized before coupling into the objective. Because emitted fluorescence is confined to the zero intensity center of the STED beam, using one STED-beam for both dyes ensured that a small misalignment of the excitation beams would not show in the resulting images. The fluorescence was detected at 600-640 nm (Atto590) and 650-690 nm (Atto 647N) with SPCM-AQRH13 fiber-coupled photon-counting modules (Perkin Elmer). The fiber core acted as a confocal pinhole of 1.1 Airy disks.

\section{Transmission electron microscopy}

Cochleae were explanted around noon and perfusion-fixed on ice with $4 \%$ PFA and $0.5 \%$ glutaraldehyde in $1 \times$ PBS, pH 7.2. After $1 \mathrm{~h}$ of incubation, the apical cochlear turns were explanted in $1 \times$ PBS and fixed overnight on ice with secondary fixative comprising $2 \%$ glutaraldehyde in 0.1 M sodium cacodylate buffer, $\mathrm{pH}$ 7.2. The samples were washed in sodium cacodylate buffer and postfixed on ice for $1 \mathrm{~h}$ with $1 \%$ osmium tetroxide $((\mathrm{v} / \mathrm{v})$ in $0.1 \mathrm{M}$ sodium cacodylate buffer), followed by a $1 \mathrm{~h}$ washing step in sodium cacodylate buffer and three brief washing steps in distilled water. The samples were stained en bloc with $1 \%(\mathrm{v} / \mathrm{v})$ uranyl acetate in distilled water for $1 \mathrm{~h}$ on ice. After a brief wash with distilled water, samples were dehydrated at room temperature in increasing ethanol concentrations, infiltrated in Epon resin (100\% EtOH/Epon 1:1 (v/v), 30 and $90 \mathrm{~min}$; $100 \%$ Epon, overnight), and embedded for $48 \mathrm{~h}$ at $70^{\circ} \mathrm{C}$. Following conventional embedding $65-75 \mathrm{~nm}$ sections were obtained approaching from the anterior edge using an Ultracut $\mathrm{E}$ (Reichert-Jung). Slices were postfixed and -stained with uranyl acetate/lead citrate following standard protocols. Micrographs were taken with a JEOL electron microscope (JEM 1011) equipped with a Gatan Orius 1200A camera using the Digital Micrograph software package at an 8000-fold magnification.

Quantitative image analysis was performed as follows: For the size of ribbons, the longest axis of each ribbon in a section, excluding the membrane-bound rootlet region, was measured. For ribbonassociated synaptic vesicles the first row of vesicles around the ribbon were counted per section. For analysis, Student's t-test was used, if not otherwise indicated. Three-dimensional reconstruction was performed on 4-7 serial $70 \mathrm{~nm}$ sections with the free software Reconstruct (Fiala, 2005).

For electron-tomography, $250 \mathrm{~nm}$ conventionally embedded sections were applied to Formvar-coated 100 copper mesh grids and stained with $4 \%$ uranyl acetate and Reynold's lead citrate, subsequently $10 \mathrm{~nm}$ gold particles were applied to the grid. A single tilt series was acquired at a JEOL JEM 2100 electron microscope at $200 \mathrm{kV}$ from -48 to $+60^{\circ}$ with $1^{\circ}$ increment using Serial-EM software. The tomogram was generated using the IMOD package etomo and model was rendered using $3 \mathrm{dmod}$ (bio3d.colorado.edu/imod/).

\section{High-pressure freezing}

For high-pressure freezing organs of Corti were explanted as described above and placed in aluminium specimen carrier of $200 \mu \mathrm{m}$ (type A) depth filled with inhibiting solution (Pangršič et al, 2010). The aluminium lid (type B) has been dipped in hexadecen (Sigma-Aldrich) before placing onto the sample. Samples were frozen immediately using the HPM010 (Bal-tec) and rapidly transfered into liquid nitrogen for storage. Freeze substitution was performed in an EM AFS2 (Leica) according to (Rostaing et al, 2006; Siksou et al, 2007). Briefly, samples were incubated for 4 days in $0.1 \%$ tannic acid in acetone at $-90^{\circ} \mathrm{C}$. Before raising the temperature to $-20^{\circ} \mathrm{C}\left(5^{\circ} \mathrm{C} / \mathrm{h}\right)$ and three washing steps with aceton $2 \%$ osmium tetroxide in acetone has been added and incubated for $16 \mathrm{~h}$. Finally temperature has been raised to $4^{\circ} \mathrm{C}\left(10^{\circ} \mathrm{C} / \mathrm{h}\right)$ and samples were washed in acetone and warmed to RT. Finally samples were infiltrated and embedded in Epon resin.

\section{Immunogold pre-embedding}

Immunogold pre-embedding labeling on p14 wild-type mice was performed according to (Nieratschker et al, 2009), using the guinea pig anti-bassoon antibody (anti-bassoon (Cterm) in Fig 3F; Synaptic Systems; 1:500), mouse anti-Sap7f407 antibody (anti-bassoon (Nterm) in Fig 3F, 1:400; Abcam) and goat anti-guinea pig or antimouse nanogold IgG from Nanoprobes. For silver enhancement the Nanoprobes HQ Silver ${ }^{\mathrm{TM}}$ enhancement kit (Nanoprobes) was used.

\section{Data analysis and statistical tests}

Data are presented as mean \pm SEM, unless otherwise specified. Normality was assessed with the Jarque-Bera test. F-test was used to assess equality of variance in normally distributed data sets. Unpaired, two-tailed Wilcoxon rank test (also known as Mann-Whitney $U$-test) 
was used to compare data of non-normal distribution or when variances of experimental groups were unequal. In case of normally-distributed equal-variance data, Student's unpaired two-tailed $t$-test was used to compare two samples (* indicates $P<0.05$ ). Comparison of dispersion was performed with a modified Levene's test (Brown-Forsythe test, Brown \& Forsythe, 1974), using median instead of mean for improved robustness under non-normality. One-way ANOVA followed by Tukey's test was used to detect differences in multiple comparisons for FA.

\section{Computational model}

Supplementary Material S9 describes the model implementation in detail. In brief, the spatial positioning of RRP vesicles and $\mathrm{Ca}^{2+}$ channels (AZ topography) at mature and immature synapses was based on functional (fluctuation analysis, $\mathrm{Ca}^{2+}$ imaging, published single channel recording data) and morphological (EM and STED) estimates. $\mathrm{Ca}^{2+}$ channel gating was modelled as a three state Markov chain with gating rates based on the experimental data by (Neef et al, 2009). The spatial $\left[\mathrm{Ca}^{2+}\right]$ profile was assumed to equilibrate instantaneously upon channel opening and closing. $\left[\mathrm{Ca}^{2+}\right]$ levels were estimated by treating open channels as hemispherical $\mathrm{Ca}^{2+}$ sources and by using the linearized buffer approximation (Naraghi \& Neher, 1997). $\mathrm{Ca}^{2+}$ diffusion was constrained by the reflective plasma membrane but considered unaffected by synaptic vesicles or the synaptic ribbon. $\mathrm{Ca}^{2+}$ profile was assumed to be shaped by the following mobile $\mathrm{Ca}^{2+}$ buffers: calretinin, calbindin, parvalbumin, and ATP. The concentrations of endogeneous $\mathrm{Ca}^{2+}$ buffers were taken from (Hackney et al, 2005) and [ATP] was set to $2 \mathrm{mM}$. $\mathrm{Ca}^{2+}$ triggered fusion of the readily releasable pool vesicles followed the seven state Markov chain model proposed by (Beutner et al, 2001) for both mature and immature synapses. The refilling of the vesicle docking sites was treated as a single step process with a fixed rate. The value of the refilling rate was set based on the experimental data by (Pangršič et al, 2010). Channel gating and vesicle fusion-replenishment dynamics were propagated by using Gillespie's algorithm (Gillespie, 1977) and its extended version for time dependent reaction rates. All calculations were performed in MATLAB. To decrease the computation time to convenient level, $100 \mathrm{CPU}$ cores were used for simulating a chosen synapse scenario.

Supplementary information for this article is available online: http://emboj.embopress.org

\section{Acknowledgments}

We thank G. Hoch for developing image analysis routines and S. Gerke, C. Senger-Freitag for expert technical assistance. We thank Nikolai M. Chapochnikov for inspiring discussions and contributions to the modelling approach used in this study and Drs A. Bulankina, T. Dresbach, and E. Neher for critical reading of a previous version of the MS. A.B.W. was supported by a Lichtenberg fellowship of the state of Lower Saxony (through the "Neurosenses" PhD program). This work was supported by grants of the German Research Foundation through the collaborative research center 889 (projects A2 to T.M., A7 to C.W. and C6 to F.W.) and the Center for Nanoscopy and Molecular Physiology of the Brain (to T.M. and S.H.) and the German Federal Ministry of Education and Research (through the Bernstein Center grant 01GQ0433 to T.M. and 01GQ0811 to F.W.).

\section{Author contributions}

ABW, MAR, CW and TM designed the study. ABW performed electrophysiology, $\mathrm{Ca}^{2+}$ imaging and analysis of immunohistochemical data. ABW and TP performed calcium uncaging experiments. MAR performed immunohistochemistry, confocal and STED microscopy. MG and FW constructed the biophysical model with input from ABW, MAR, CW and TM. All numerical simulations were performed and analyzed by MG. FG built the two-channel STED microscope. TF performed non-stationary fluctuation analysis. CW performed electron microscopy, SM did part of the immuno-EM. ABW, MG, MAR, CW and TM prepared the manuscript.

\section{Conflict of interest}

The authors declare that they have no conflict of interest.

\section{References}

Augustine GJ, Adler EM, Charlton MP (1991) The calcium signal for transmitter secretion from presynaptic nerve terminals. Ann N Y Acad Sci 635: 365-381 Augustine GJ, Santamaria F, Tanaka K (2003) Local calcium signaling in neurons. Neuron 40: $331-346$

Beutner D, Moser T (2001) The presynaptic function of mouse cochlear inner hair cells during development of hearing. J Neurosci 21: 4593

Beutner D, Voets T, Neher E, Moser T (2001) Calcium dependence of exocytosis and endocytosis at the cochlear inner hair cell afferent synapse. Neuron 29: 681-690

Brandt A, Khimich D, Moser T (2005) Few CaV1. 3 channels regulate the exocytosis of a synaptic vesicle at the hair cell ribbon synapse. J Neurosci 25: 11577

Brandt A, Striessnig J, Moser T (2003) CaV1. 3 channels are essential for development and presynaptic activity of cochlear inner hair cells. J Neurosci 23: 10832-10840

Brandt N, Kuhn S, Münkner S, Braig C, Winter H, Blin N, Vonthein R, Knipper M, Engel J (2007) Thyroid hormone deficiency affects postnatal spiking activity and expression of $\mathrm{Ca}^{2+}$ and $\mathrm{K}^{+}$channels in rodent inner hair cells. J Neurosci 27: $3174-3186$

Brown MB, Forsythe AB (1974) Robust tests for the equality of variances. J Am Stat Assoc 69: 364-367

Bulankina AV, Moser T (2012) Neural circuit development in the mammalian cochlea. Physiology (Bethesda) 27: 100-112

Dick O, Hack I, Altrock WD, Garner CC, Gundelfinger ED, Brandstätter JH (2001) Localization of the presynaptic cytomatrix protein Piccolo at ribbon and conventional synapses in the rat retina: comparison with Bassoon. J Comp Neurol 439: 224-234

tom Dieck S, Altrock WD, Kessels MM, Qualmann B, Regus H, Brauner D, Fejtová A, Bracko O, Gundelfinger ED, Brandstätter JH (2005) Molecular dissection of the photoreceptor ribbon synapse: physical interaction of Bassoon and RIBEYE is essential for the assembly of the ribbon complex. J Cell Biol 168: 825-836

tom Dieck S, Sanmartí-Vila L, Langnaese K, Richter K, Kindler S, Soyke A, Wex H, Smalla KH, Kämpf U, Fränzer JT, Stumm M, Garner CC, Gundelfinger ED (1998) Bassoon, a novel zinc-finger CAG/glutamine-repeat protein selectively localized at the active zone of presynaptic nerve terminals. J Cell Biol 142: 499-509

Dou H, Vazquez AE, Namkung Y, Chu H, Cardell EL, Nie L, Parson S, Shin H-S, Yamoah EN (2004) Null mutation of alpha1D Ca ${ }^{2+}$ channel gene results in deafness but no vestibular defect in mice. J Assoc Res Otolaryngol 5: 215-226 
Eggermann E, Bucurenciu I, Goswami SP, Jonas P (2012) Nanodomain coupling between $\mathrm{Ca}^{2+}$ channels and sensors of exocytosis at fast mammalian synapses. Nat Reu Neurosci 13: 7-21

Fiala JC (2005) Reconstruct: a free editor for serial section microscopy. J Microsc 218: $52-61$

Francis AA, Mehta B, Zenisek D (2011) Development of new peptide-based tools for studying synaptic ribbon function. J Neurophysiol 106: $1028-1037$

Frank T, Khimich D, Neef A, Moser T (2009) Mechanisms contributing to synaptic $\mathrm{Ca}^{2+}$ signals and their heterogeneity in hair cells. Proc Natl Acad Sci USA 106: 4483

Frank T, Rutherford MA, Strenzke N, Neef A, Pangršič T, Khimich D, Fejtova A, Gundelfinger ED, Liberman MC, Harke B, Bryan KE, Lee A, Egner A (2010) Bassoon and the synaptic ribbon organize $\mathrm{Ca}^{2+}$ channels and vesicles to add release sites and promote refilling. Neuron 68: 724-738

Fuchs PA, Glowatzki E, Moser T (2003) The afferent synapse of cochlear hair cells. Curr Opin Neurobiol 13: $452-458$

Gillespie DT (1977) Exact stochastic simulation of coupled chemical reactions. J Phys Chem 81: 2340-2361

Glowatzki E, Fuchs PA (2002) Transmitter release at the hair cell ribbon synapse. Nat Neurosci 5: 147-154

Goda Y, Davis CW (2003) Mechanisms of synapse assembly and disassembly. Neuron 40: 243-264

Goutman JD (2012) Transmitter release from cochlear hair cells is phase locked to cyclic stimuli of different intensities and frequencies. J Neurosci 32: $17025-17036$

Goutman JD, Glowatzki E (2007) Time course and calcium dependence of transmitter release at a single ribbon synapse. Proc Natl Acad Sci USA 104: $16341-16346$

Grant L, Yi E, Glowatzki E (2010) Two modes of release shape the postsynaptic response at the inner hair cell ribbon synapse. J Neurosci 30 $4210-4220$

Graydon CW, Cho S, Li G-L, Kachar B, von Gersdorff H (2011) Sharp Ca ${ }^{2+}$ nanodomains beneath the ribbon promote highly synchronous multivesicular release at hair cell synapses. J Neurosci 31: 16637-16650

Hackney CM, Mahendrasingam S, Penn A, Fettiplace R (2005) The concentrations of calcium buffering proteins in mammalian cochlear hair cells. J Neurosci 25: 7867

Heil P, Neubauer H (2010) Summing across different active zones can explain the quasi-linear $\mathrm{Ca}^{2+}$-dependencies of exocytosis by receptor cells. Front Synaptic Neurosci 2: 148

Hess P, Lansman JB, Tsien RW (1984) Different modes of Ca channel gating behaviour favoured by dihydropyridine $\mathrm{Ca}$ agonists and antagonists. Nature 311: $538-544$

Huang L-C, Barclay M, Lee K, Peter S, Housley GD, Thorne PR, Montgomery JM (2012) Synaptic profiles during neurite extension, refinement and retraction in the developing cochlea. Neural Deu 7: 1-17

Huang L-C, Thorne PR, Housley GD, Montgomery JM (2007) Spatiotemporal definition of neurite outgrowth, refinement and retraction in the developing mouse cochlea. Development 134: 2925-2933

Hull C, Studholme K, Yazulla S, von Gersdorff H (2006) Diurnal changes in exocytosis and the number of synaptic ribbons at active zones of an ON-type bipolar cell terminal. J Neurophysiol 96: 2025-2033

Jarsky T, Tian M, Singer JH (2010) Nanodomain control of exocytosis is responsible for the signaling capability of a retinal ribbon synapse. J Neurosci 30: 11885-11895

Johnson SL, Franz C, Kuhn S, Furness DN, Ruttiger L, Munkner S, Rivolta MN, Seward EP, Herschman HR, Engel J, Knipper M, Marcotti W (2010)
Synaptotagmin IV determines the linear $\mathrm{Ca}^{2+}$ dependence of vesicle fusion at auditory ribbon synapses. Nat Neurosci 13: 45-52

Johnson SL, Marcotti W, Kros CJ (2005) Increase in efficiency and reduction in $\mathrm{Ca}^{2+}$ dependence of exocytosis during development of mouse inner hair cells. J Physiol 563: 177-191

Kennedy HJ, Meech RW (2002) Fast $\mathrm{Ca}^{2+}$ signals at mouse inner hair cell synapse: a role for $\mathrm{Ca}^{2+}$-induced $\mathrm{Ca}^{2+}$ release. J Physiol 539: 15-23 Khimich D, Nouvian R, Pujol R, tom Dieck S, Egner A, Gundelfinger ED, Moser $T$ (2005) Hair cell synaptic ribbons are essential for synchronous auditory signalling. Nature 434: 889-894

Kros CJ, Ruppersberg JP, Rüsch A (1998) Expression of a potassium current in inner hair cells during development of hearing in mice. Nature 394: $281-284$

Lenzi D, Crum J, Ellisman MH, Roberts WM (2002) Depolarization redistributes synaptic membrane and creates a gradient of vesicles on the synaptic body at a ribbon synapse. Neuron 36: 649-659

Liu KS, Siebert M, Mertel S, Knoche E, Wegener S, Wichmann C, Matkovic T, Muhammad K, Depner H, Mettke C, Bückers J, Hell SW, Müller M, Davis GW, Schmitz D, Sigrist SJ (2011) RIM-binding protein, a central part of the active zone, is essential for neurotransmitter release. Science 334: $1565-1569$

Maas C, Torres VI, Altrock WD, Leal-Ortiz S, Wagh D, Terry-Lorenzo RT, Fejtova A, Gundelfinger ED, Ziv NE, Garner CC (2012) Formation of Golgi-derived active zone precursor vesicles. J Neurosci 32: 11095-11108

Magupalli VG, Schwarz K, Alpadi K, Natarajan S, Seigel GM, Schmitz F (2008) Multiple RIBEYE-RIBEYE interactions create a dynamic scaffold for the formation of synaptic ribbons. J Neurosci 28: $7954-7967$

Matsubara A, Laake JH, Davanger S, Usami S, Ottersen OP (1996) Organization of AMPA receptor subunits at a glutamate synapse: a quantitative immunogold analysis of hair cell synapses in the rat organ of Corti. J Neurosci 16: 4457

Matthews G, Fuchs P (2010) The diverse roles of ribbon synapses in sensory neurotransmission. Nat Reu Neurosci 11: 812-822

Matveev V, Bertram R, Sherman A (2011) Calcium cooperativity of exocytosis as a measure of $\mathrm{Ca}^{2+}$ channel domain overlap. Brain Res 1398: $126-138$

McLean WJ, Smith KA, Glowatzki E, Pyott SJ (2009) Distribution of the Na, K-ATPase $\alpha$ subunit in the rat spiral ganglion and organ of corti. J Assoc Res Otolaryngol 10: $37-49$

Meyer AC, Frank T, Khimich D, Hoch G, Riedel D, Chapochnikov NM, Yarin YM, Harke B, Hell SW, Egner A, Moser T (2009) Tuning of synapse number, structure and function in the cochlea. Nat Neurosci 12: 444-453

Mikaelian D, Alford BR, Ruben RJ (1965) Cochlear potentials and 8 nerve action potentials in normal and genetically deaf mice. Ann Otol Rhinol Laryngol 74: 146-157

Mintz IM, Sabatini BL, Regehr WG (1995) Calcium control of transmitter release at a cerebellar synapse. Neuron 15: 675-688

Moser T, Beutner D (2000) Kinetics of exocytosis and endocytosis at the cochlear inner hair cell afferent synapse of the mouse. Proc Natl Acad Sci USA 97: $883-888$

Moser T, Neef A, Khimich D (2006) Mechanisms underlying the temporal precision of sound coding at the inner hair cell ribbon synapse. J Physiol 576: $55-62$

Naraghi M, Neher E (1997) Linearized buffered $\mathrm{Ca}^{2+}$ diffusion in microdomains and its implications for calculation of $\left[\mathrm{Ca}^{2+}\right]$ at the mouth of a calcium channel. J Neurosci 17: 6961

Neef J, Gehrt A, Bulankina AV, Meyer AC, Riedel D, Gregg RG, Strenzke N, Moser $\mathrm{T}$ (2009) The $\mathrm{Ca}^{2+}$ channel subunit beta2 regulates $\mathrm{Ca} 2+$ channel 
abundance and function in inner hair cells and is required for hearing J Neurosci 29: $10730-10740$

Neher E, Sakaba T (2008) Multiple roles of calcium ions in the regulation of neurotransmitter release. Neuron 59: $861-872$

Nieratschker V, Schubert A, Jauch M, Bock N, Bucher D, Dippacher S, Krohne G, Asan E, Buchner S, Buchner E (2009) Bruchpilot in ribbon-like axonal agglomerates, behavioral defects, and early death in SRPK79D kinase mutants of Drosophila. PLoS Genet 5: e1000700

Nouvian R, Neef J, Bulankina AV, Reisinger E, Pangršič T, Frank T, Sikorra S, Brose N, Binz T, Moser T (2011) Exocytosis at the hair cell ribbon synapse apparently operates without neuronal SNARE proteins. Nat Neurosci 14: $411-413$

Pangršič T, Lasarow L, Reuter K, Takago H, Schwander M, Riedel D, Frank T, Tarantino LM, Bailey JS, Strenzke N, Brose N, Müller U, Reisinger E, Moser $T$ (2010) Hearing requires otoferlin-dependent efficient replenishment of synaptic vesicles in hair cells. Nat Neurosci 13: 869-876

Pangršič T, Reisinger E, Moser T (2012) Otoferlin: a multi-C2 domain protein essential for hearing. Trends Neurosci 35: 671-680

Platzer J, Engel J, Schrott-Fischer A, Stephan K, Bova S, Chen H, Zheng H, Striessnig J (2000) Congenital deafness and sinoatrial node dysfunction in mice lacking class D L-type $\mathrm{Ca}^{2+}$ channels. Cell 102: 89-97

Regus-Leidig H, tom Dieck S, Specht D, Meyer L, Brandstätter JH (2009) Early steps in the assembly of photoreceptor ribbon synapses in the mouse retina: the involvement of precursor spheres. J Comp Neurol 512: 814-824

Roberts WM, Jacobs RA, Hudspeth AJ (1990) Colocalization of ion channels involved in frequency selectivity and synaptic transmission at presynaptic active zones of hair cells. J Neurosci 10: $3664-3684$

Rostaing P, Real E, Siksou L, Lechaire J-P, Boudier T, Boeckers TM, Gertler F, Gundelfinger ED, Triller A, Marty S (2006) Analysis of synaptic ultrastructure without fixative using high-pressure freezing and tomography. Eur J Neurosci 24: 3463-3474

Roux I, Safieddine S, Nouvian R, Grati M, Simmler M-C, Bahloul A, Perfettini I, Le Gall M, Rostaing P, Hamard G, Triller A, Avan P, Moser T, Petit C (2006) Otoferlin, defective in a human deafness form, is essential for exocytosis at the auditory ribbon synapse. Cell 127: 277-289

Rutherford MA, Pangršič T (2012) Molecular anatomy and physiology of exocytosis in sensory hair cells. Cell Calcium 52: 327-337

Rutherford MA, Roberts WM (2006) Frequency selectivity of synaptic exocytosis in frog saccular hair cells. Proc Natl Acad Sci USA 103: 2898

Safieddine S, El-Amraoui A, Petit C (2012) The auditory hair cell ribbon synapse: from assembly to function. Annu Reu Neurosci 35: 509-528

Schmitz F, Königstorfer A, Südhof TC (2000) RIBEYE, a component of synaptic ribbons: a proteins journey through evolution provides insight into synaptic ribbon function. Neuron 28: 857-872

Sendin G, Bulankina AV, Riedel D, Moser T (2007) Maturation of ribbon synapses in hair cells is driven by thyroid hormone. J Neurosci 27 : $3163-3173$

Shapira M, Zhai RG, Dresbach T, Bresler T, Torres VI, Gundelfinger ED, Ziv NE, Garner CC (2003) Unitary assembly of presynaptic active zones from Piccolo-Bassoon transport vesicles. Neuron 38: 237-252

Sheets L, Kindt KS, Nicolson T (2012) Presynaptic CaV1.3 channels regulate synaptic ribbon size and are required for synaptic maintenance in sensory hair cells. J Neurosci 32: 17273-17286

Sheets L, Trapani JG, Mo W, Obholzer N, Nicolson T (2011) Ribeye is required for presynaptic CaV1.3a channel localization and afferent innervation of sensory hair cells. Development 138: 1309-1319
Shnerson A, Devigne C, Pujol R (1981) Age-related changes in the C57BL/6] mouse cochlea. II. Ultrastructural findings. Brain Res 254: 77-88

Siksou L, Rostaing P, Lechaire J-P, Boudier T, Ohtsuka T, Fejtová A, Kao H-T, Greengard P, Gundelfinger ED, Triller A, Marty S (2007) Three-dimensional architecture of presynaptic terminal cytomatrix. J Neurosci 27: 6868-6877

Sobkowicz HM, Rose JE, Scott GE, Slapnick SM (1982) Ribbon synapses in the developing intact and cultured organ of Corti in the mouse. J Neurosci 2: 942

Spiwoks-Becker I, Glas M, Lasarzik I, Vollrath L (2004) Mouse photoreceptor synaptic ribbons lose and regain material in response to illumination changes. Eur J Neurosci 19: 1559-1571

Stanley EF (1993) Single calcium channels and acetylcholine release at a presynaptic nerve terminal. Neuron 11: 1007-1011

Takao-Rikitsu E, Mochida S, Inoue E, Deguchi-Tawarada M, Inoue M, Ohtsuka T, Takai Y (2004) Physical and functional interaction of the active zone proteins, CAST, RIM1, and Bassoon, in neurotransmitter release. J Cell Biol 164: $301-311$

Waites CL, Leal-Ortiz SA, Okerlund N, Dalke H, Fejtova A, Altrock WD, Gundelfinger ED, Garner CC (2013) Bassoon and Piccolo maintain synapse integrity by regulating protein ubiquitination and degradation. EMBO J 32: 954-969

Wang L-Y, Neher E, Taschenberger $H$ (2008) Synaptic vesicles in mature calyx of Held synapses sense higher nanodomain calcium concentrations during action potential-evoked glutamate release. J Neurosci 28 : $14450-14458$

Wang X, Hu B, Zieba A, Neumann NG, Kasper-Sonnenberg M, Honsbein A, Hultqvist G, Conze T, Witt W, Limbach C, Geitmann M, Danielson H, Kolarow R, Niemann G, Lessmann V, Kilimann MW (2009) A protein interaction node at the neurotransmitter release site: domains of Aczonin/Piccolo, Bassoon, CAST, and rim converge on the N-terminal domain of Munc13-1. J Neurosci 29: 12584-12596

Wong AB, Jing Z, Rutherford MA, Frank T, Strenzke N, Moser T (2013) Concurrent maturation of inner hair cell synaptic $\mathrm{Ca}^{2+}$ influx and auditory nerve spontaneous activity around hearing onset in mice. J Neurosci 33: $10661-10666$

Yang Y-M, Fedchyshyn MJ, Grande G, Aitoubah J, Tsang CW, Xie H, Ackerley CA, Trimble WS, Wang L-Y (2010) Septins regulate developmental switching from microdomain to nanodomain coupling of $\mathrm{Ca}^{2+}$ influx to neurotransmitter release at a central synapse. Neuron 67: 100-115

Zampini V, Johnson SL, Franz C, Knipper M, Holley MC, Magistretti J, Masetto S, Marcotti W (2013) Burst activity and ultrafast activation kinetics of CaV1.3 $\mathrm{Ca}^{2+}$ channels support presynaptic activity in adult gerbil hair cell ribbon synapses. J Physiol 591: 3811-3820

Zampini V, Johnson SL, Franz C, Lawrence ND, Münkner S, Engel J, Knipper M, Magistretti J, Masetto S, Marcotti W (2010) Elementary properties of CaV1.3 $\mathrm{Ca}(2+)$ channels expressed in mouse cochlear inner hair cells. J Physiol 588: 187-199

Zenisek D, Steyer JA, Almers W (2000) Transport, capture and exocytosis of single synaptic vesicles at active zones. Nature 406: 849-854

Zhai R, Olias G, Chung WJ, Lester RAJ, tom Dieck S, Langnaese K, Kreutz MR, Kindler S, Gundelfinger ED, Garner CC (2000) Temporal appearance of the presynaptic cytomatrix protein bassoon during synaptogenesis. Mol Cell Neurosci 15: 417-428

Zhai R, Vardinon-Friedman H, Cases-Langhoff C, Becker B, Gundelfinger ED, Ziv NE, Garner CC (2001) Assembling the presynaptic active zone: a characterization of an active one precursor vesicle. Neuron 29: 131-143 
Supplementary Material 1 Electron tomography of ribbon synapse of a prehearing IHC
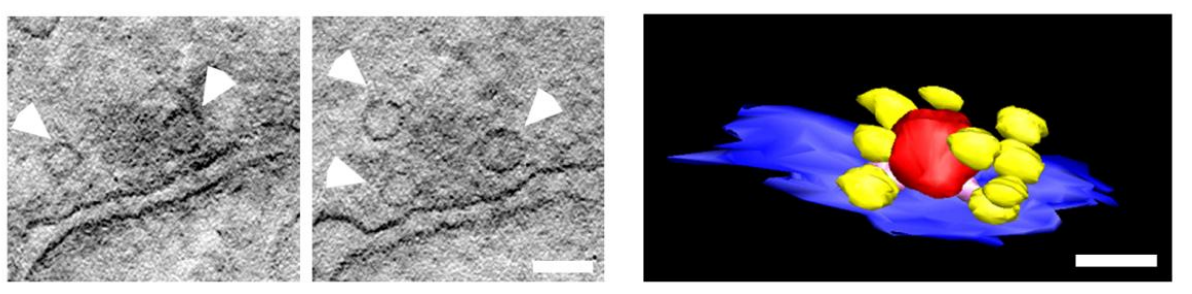

Figure S1. Electron tomography of ribbon synapse of a prehearing IHC.

Two example virtual sections of a tomogram (left) and corresponding model (right) of a pre-hearing p6 AZ. The small spherical ribbon (red) is attached via two rootlets (magenta) to the AZ membrane (blue). Nine synaptic vesicles (yellow, arrowheads) are found attached to the ribbon. Scale bars: $50 \mathrm{~nm}$ 
Supplementary Material 2: Quantification of CtBP2 and GluA2/3 puncta number and intensity
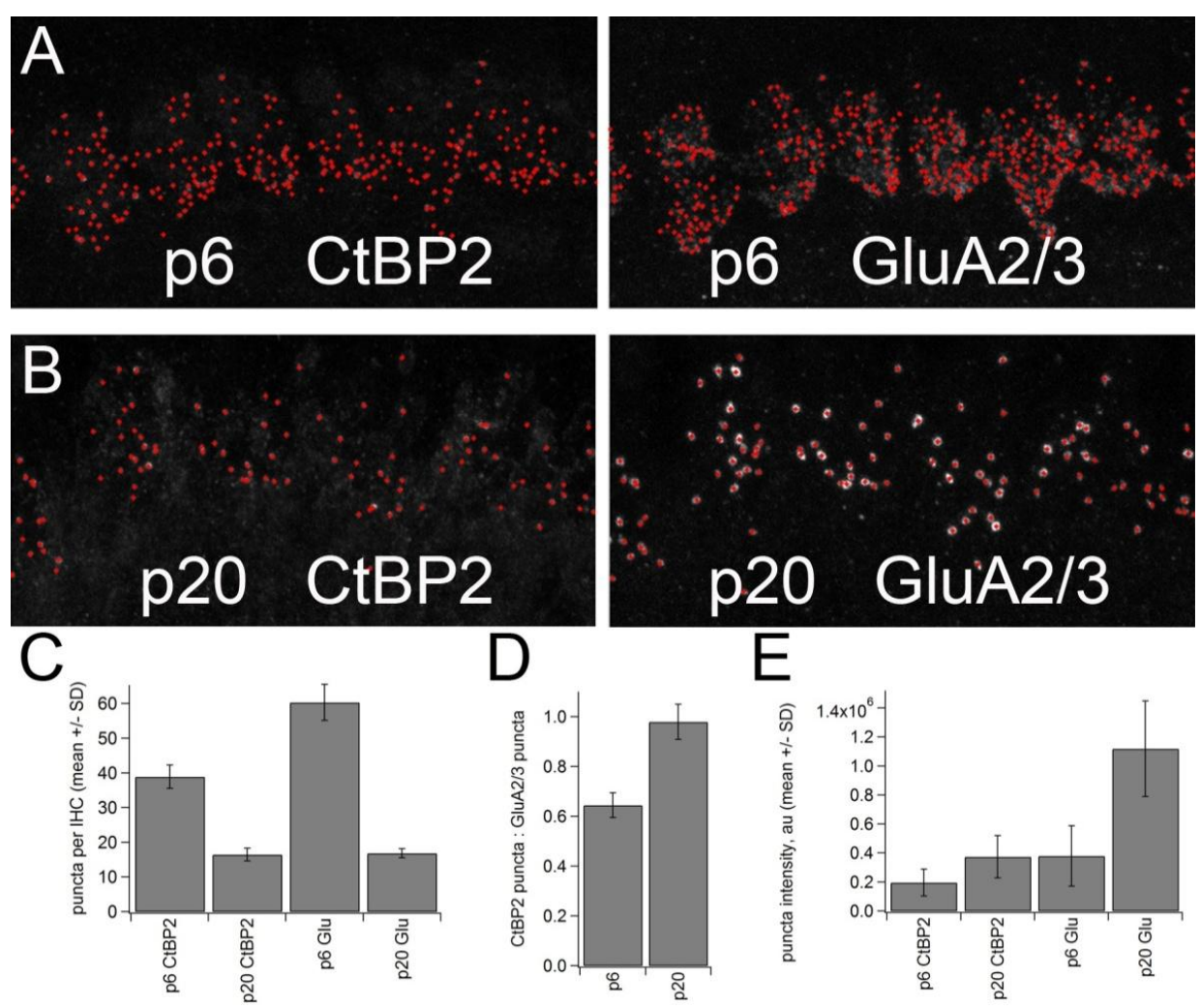

Figure S2. Quantification of CtBP 2 and GluA2/3 puncta number and intensity

(A-B) Projection of confocal images across approximately 6 IHCs in the organ or Corti wholemount at p6 (A) and p20 (B). The same images are presented in merged color in Figure 1 of the main text. Images are single-channel gray scale immunofluorescence for RIBEYE/CtBP2 (left) and GluA2/3 (right). With custom procedures in Matlab software, the locations of synapses in confocal image stacks were defined as the centers of mass of fluorescent spots after thresholding by a subjective intensity criterion for each channel. The background (defined as the average voxel intensity in the entire synaptic region excluding voxels that exceeded the threshold value) was subtracted. Gaussian functions were fitted in all three dimensions to determine the center of mass of each cluster. Each red symbol marks the center of mass, in 3D, of a ribbon or a punctum of 
glutamate receptors. Immunofluorescence intensity was measured as the integral of the voxel values within a defined region of interest $(13 \times 13 \times 5$ voxels in the $X, Y$, and $Z$ direction, which has the volume of $\sim 0.7 \times 0.7 \times 2.0 \mu \mathrm{m}$ ) centered on the voxel where the center of mass of each punctum was located.

(C) Puncta per IHC (mean \pm s.d.) for 24 hair cells from 4 different image stacks. From p6 to p20 ribbons are reduced by $\sim 50 \%$ and GluA2 $/ 3$ puncta are reduced to $\sim 30 \%$ in number.

(D) Ratio of ribbons to glutamate receptor puncta per IHC (mean \pm s.d.).

(E) Summed voxel intensity in the defined region around each center of mass. Per puncta intensity approximately doubled for ribbons and approximately tripled for GluA2/3. 
Supplementary Material 3 Number of Electron-dense connection of vesicles to ribbon.

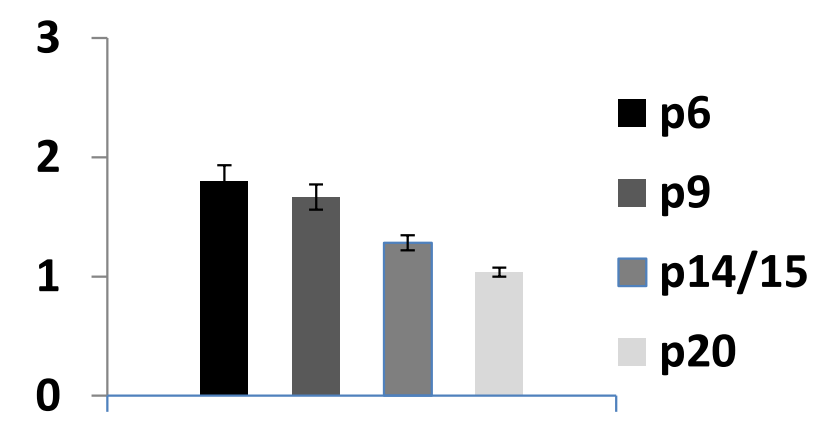

Figure S3. Quantification of the number of electron-dense connections.

Number of electron-dense connections from the membrane of the active zone to the synaptic ribbon observed in random synaptic sections along development. 
Supplementary Material 4 Evaluation of specificity of $\mathrm{Ca}_{\mathrm{V}} 1.3$ immunolabeling

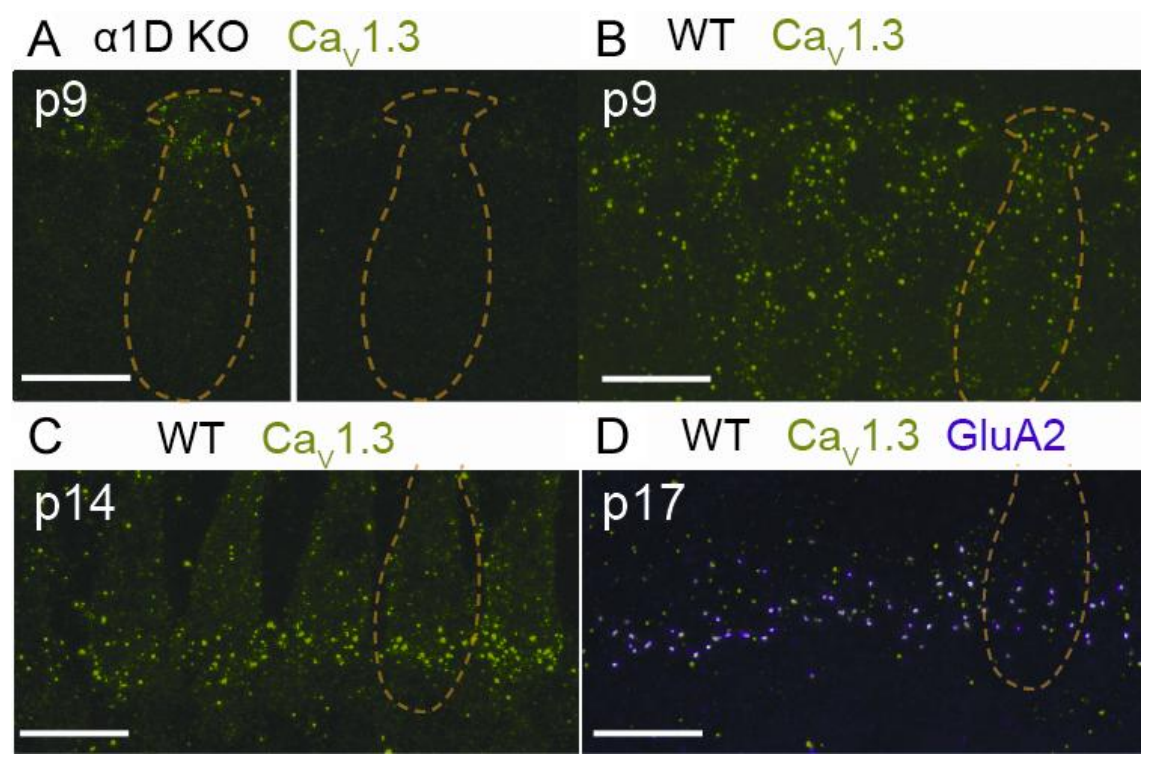

Figure S4. Evaluation of specificity of Cav1.3 immunolabeling

(A) Control experiments probed for Cav1.3 immunoreactivity at $\mathrm{p} 9$ in the $\alpha 1 \mathrm{D}$ knockout mouse ${ }^{1}$, which lacks the channel's pore-forming $\alpha$-subunit recognized by the antibody. The non-specific staining pattern was somewhat selective for the hair cell apical plate and neck region. However, there were no presynaptic Cav1.3 clusters and the basolateral membrane was not immuno-positive relative to surrounding tissue. (B) Same as in panel (A) but with wild-type mice at p9. (C) Cav1.3 in wild-type IHCs at p14. In addition to synaptic clusters, extrasynaptic Cav1.3 labeling highlights the IHC plasma membrane against the surrounding tissue in (B) and (C). (D) By p17 the IHC membrane is no longer discernible by diffuse Cav1.3 stain, however, some extrasynaptic Cav1.3 puncta persist. Each postsynaptic patch of glutamate receptors (GluA2, magenta) is juxtaposed to a presynaptic punctum of Cav1.3 that appears colocalized (white). All scale bars: $10 \mu \mathrm{m}$. 
Supplementary Material 5 Quantitative comparison of the spread of $\mathrm{Ca}^{2+}$ signal in IHCs during development
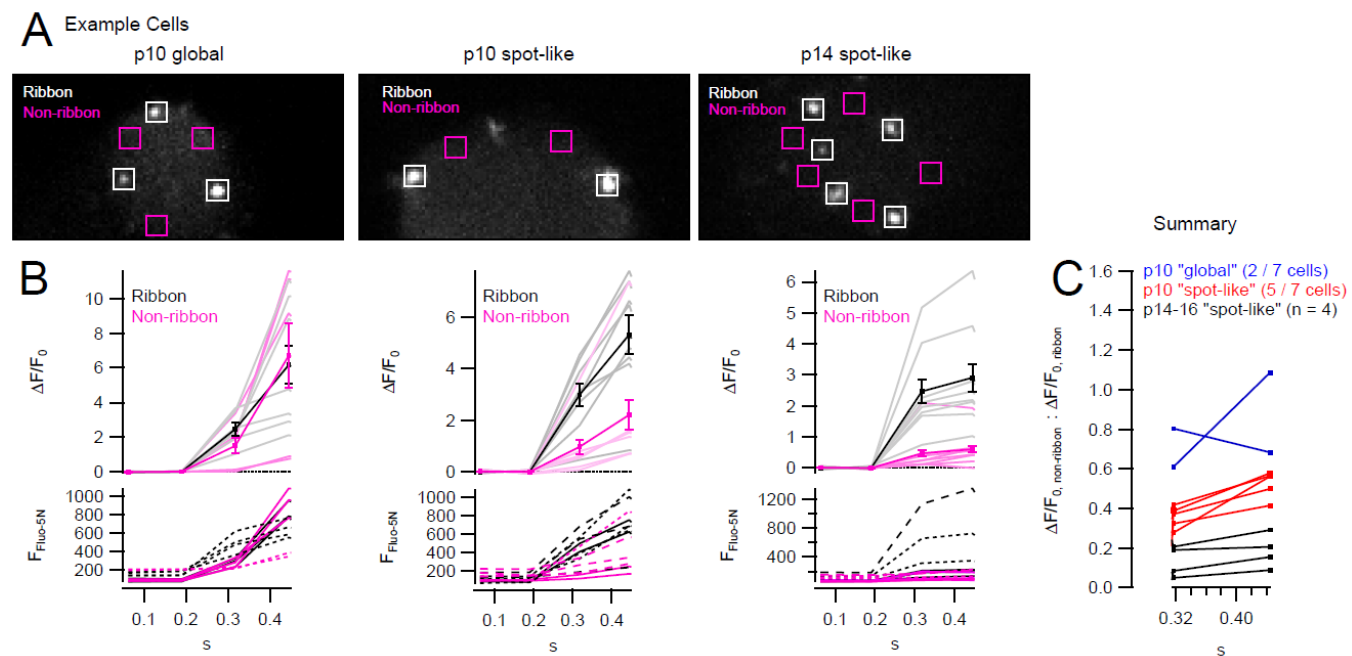

Figure S5. Quantitative comparison of the spread of $\mathrm{Ca}^{2+}$ signal in IHCs.

(A) Regions-of-Interest (ROIs) centered at ribbons (white boxes) and at non-ribbon (magenta boxes) areas, defined from presence or absence of Ribeye-binding peptide fluorescence. (B) Upper panels: Normalized fluorescence change $\left(\Delta \mathrm{F} / \mathrm{F}_{0}\right)$ of $\mathrm{Ca}^{2+}$ indicator Fluo-5N (400 $\left.\mu \mathrm{M}\right)$, upon a $254 \mathrm{~ms}$ depolarization to $-7 \mathrm{mV}(0.254-0.508 \mathrm{~s}$, last two data points). Black and grey traces are average and individual traces from ribbon-centered ROIs; Magenta and pink traces are average and individual traces from non-ribbon ROIs. Lower Panels: raw fluorescence at these ROIs, broken line and dotted lines are obtained from different confocal sections. (C) Ratio of average $\Delta \mathrm{F} / \mathrm{F}_{0}$ at nonribbon ROIs over ribbon-centered ROIs during depolarization, analyzed in a cell-by-cell basis and representing ratios of several pairs of neighboring ROIs per IHC. 
Supplementary Material 6 Characterization of the biophysical properties of $\mathrm{Ca}^{2+}$ channels in IHCs during postnatal development

A

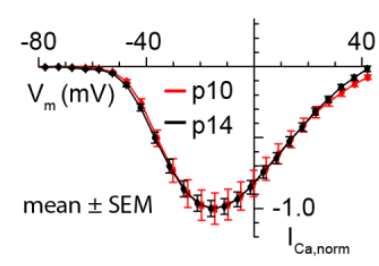

D

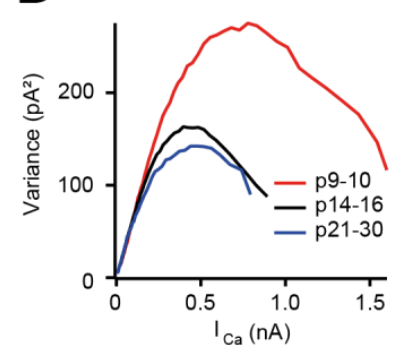

B

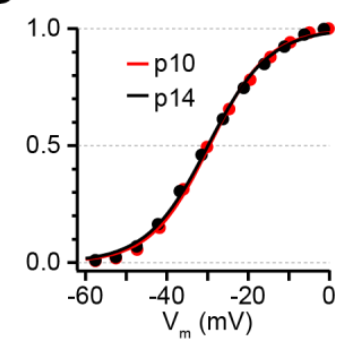

E

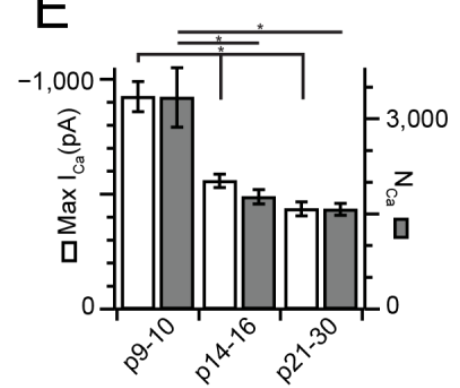

C

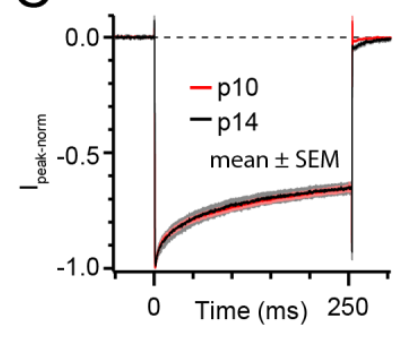

$\mathrm{F}$

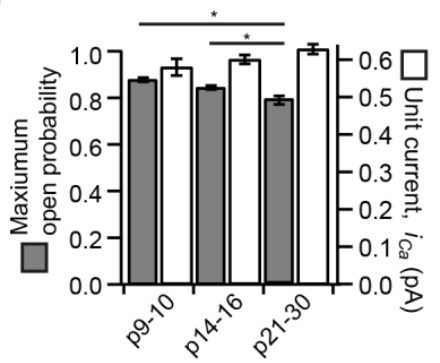

Figure S6. Biophysical properties of voltage-dependent whole-cell $\mathrm{Ca}^{2+}$ current $\left(\mathrm{I}_{\mathrm{Ca}}\right)$ (A) Average steady-state $\mathrm{I}_{\mathrm{Ca}}-\mathrm{V}$ for p10 (red) and p14-16 (black) IHCs in $5 \mathrm{mM}\left[\mathrm{Ca}^{2+}\right]_{\mathrm{e}}$, normalized to peak $\mathrm{I}_{\mathrm{Ca}}$ (for panels A and B: $\mathrm{p} 10, \mathrm{n}=23$ IHCs; $\mathrm{p} 14-16, \mathrm{n}=31 \mathrm{IHCs}$ ). (b) Activation curve for $\mathrm{I}_{\mathrm{Ca}}$, obtained by dividing the $\mathrm{I}_{\mathrm{Ca}}-\mathrm{V}$ curve in (a) by a line-fit to the linear portion $(-5$ to $+20 \mathrm{mV})$. Solid lines represent fitting of a Boltzmann function in the form of $1 /\left(1+\exp \left(\left(\mathrm{V}_{\text {half }} \mathrm{V}\right) / \mathrm{slope}\right)\right.$. No significant difference was found in the half-activation potential ( $V_{\text {half; }}$ p10: $-29.74 \pm 0.35 \mathrm{mV}$; p1416: $-29.73 \pm 0.36 \mathrm{mV}$; Student's t-Test, $\mathrm{p}=0.97$ ), despite a subtle difference in their slope (p10: $7.10 \pm 0.09 \mathrm{mV}$; p14-16: $7.39 \pm 0.06 \mathrm{mV}$; Wilcoxon Rank Test, $\mathrm{p}=0.0049) . \quad(\mathrm{C})$ Average inactivation of $\mathrm{I}_{\mathrm{Ca}}$ during a $254 \mathrm{~ms}$ depolarization in $5 \mathrm{mM}[\mathrm{Ca}]_{\mathrm{e}}$ and highly buffered $(10 \mathrm{mM}$ $\left.[E G T A]_{i}\right)$ condition. No obvious difference was observed between the inactivation kinetics of the two age groups (6 repetitions per cell; p10, $\mathrm{n}=7 \mathrm{IHCs} ; \mathrm{p} 14-16, \mathrm{n}=7 \mathrm{IHCs}$ ). (D) Grand averages of variance vs. mean relationships ( $\mathrm{p} 9-10: \mathrm{n}=13 \mathrm{IHCs}, \mathrm{p} 14-16: \mathrm{n}=31 \mathrm{IHCs}, \mathrm{p} 21-30: \mathrm{n}=27 \mathrm{IHCs}$ ) in nonstationary fluctuation analysis of $\mathrm{Ca}^{2+}$ tail-currents. $\left[\mathrm{Ca}^{2+}\right]_{\mathrm{e}}=10 \mathrm{mM}$, $[\text { BayK8644 }]_{\mathrm{e}}=5 \mathrm{\mu M}$. $(\mathrm{E})$ 
The decrease in whole cell $\mathrm{Ca}^{2+}$ current $\left(\mathrm{I}_{\mathrm{Ca}}\right.$, empty bars; $\mathrm{p} 9-10, \mathrm{n}=13$ IHCs; $\mathrm{p} 14-16$, $\mathrm{n}=31$ IHCs; $\mathrm{p} 21-30, \mathrm{n}=27 \mathrm{IHCs}$ ) was largely matched by the reduction in number of $\mathrm{Ca}^{2+}$ channel estimated by fluctuation analysis $\left(\mathrm{N}_{\mathrm{Ca}}\right.$, solid bars; $\mathrm{p} 9-10, \mathrm{n}=16$ IHCs; $\mathrm{p} 14-16$, $\mathrm{n}=33$ IHCs; $\mathrm{p} 21-30, \mathrm{n}=26 \mathrm{IHCs}$ ) during postnatal development (F) There was no significant change in the estimated single channel current (holding potential: $-68 \mathrm{mV}$; $\mathrm{p} 9-10: 0.58 \pm 0.02 \mathrm{pA}, \mathrm{n}$ $=13$ IHCs; p14-16: $0.60 \pm 0.01 \mathrm{pA}, \mathrm{n}=31$ IHCs; p21-30: $0.63 \pm 0.01 \mathrm{pA}, \mathrm{n}=27$ IHCs; ANOVA, $\mathrm{p}$ $=0.103)$ and only a small reduction in the estimated maximal open probability measured ( $\mathrm{p} 9-10$ : $0.88 \pm 0.01 ; \mathrm{p} 14-16: 0.84 \pm 0.01 ; \mathrm{p} 21-30: 0.79 \pm 0.02 ;$ Student's t-test, p9-10 vs p14-16, p = 0.27; p9-10 vs p20-30, p < 0.001; p14-16 vs p20-30, p = 0.006).

Comparing the estimation of microscopic channel properties by non-stationary fluctuation analysis and direct cell-attached single channel recordings

The main point of doing the fluctuation analysis was to seek confirmation of the hypothesis that the number of $\mathrm{Ca}^{2+}$ channels $\left(\mathrm{N}_{\mathrm{Ca}}\right)$ declines around the onset of hearing. This was, indeed, observed and also agrees with previously published work based on single channel recordings ${ }^{2}$. However, we note that both approaches differed in their estimates of single channel current $i_{\mathrm{Ca}}$ and open probability $\left(\mathrm{P}_{\mathrm{open}}\right)$, which we believe reflects technical differences that are discussed below.

Generally, fluctuation analysis tends to underestimate the $i_{\mathrm{Ca}}$ due to the limited bandwidth of recording $(8.5 \mathrm{kHz})$, and this seems to be the case here, too. Our estimates of approximately $-0.6 \mathrm{pA}$ at $-68 \mathrm{mV}$ compare to approximately $-1.0 \mathrm{pA}$ measured in cell-attached at $-68 \mathrm{mV}$ after hearing onset $^{3}$. For this reason we relied on the single channel recording estimates for $i_{\mathrm{Ca}}$ for our biophysical model.

We believe that likely methodological differences gave rise to the differences between the $\mathrm{P}_{\text {open }}$ estimates from single channel experiments $\left(0.15\right.$ at $-20 \mathrm{mV}$ for immature $\mathrm{IHCs}^{2}$ and 0.21 for mature $\mathrm{IHCs}^{3}$ of the gerbil) and fluctuation analysis (around 0.8 at approximately $+60 \mathrm{mV}$ in estimates from mature IHCs [present study and refs. 4,5] and slightly higher (0.88) for immature IHCs). Using BayK8644 and strong depolarization we aim to maximize the open probability (greater than 0.5) as required for faithful estimation of the channel properties by binomial fitting. As stated in the main manuscript we aim to estimate the total number of $\mathrm{Ca}^{2+}$ channels and identify our open probability estimates as "maximal open probability" to make these points. While the single channel work by ref. 
2,3 also used BayK8644, they used lower depolarizations and longer pulses (mostly $500 \mathrm{~ms}$ ). We consider it likely that the lower depolarization level (via less activation) and potentially also $\mathrm{Ca}^{2+}$ dependent inactivation led to lower estimates of open probability. Since $\mathrm{Ca}^{2+}$ dependent inactivation is greater in immature than in mature $\mathrm{IHCs}^{6}$ one would then expect lower open probability estimates in immature IHCs, which seems to be the case (see above). Further observations of ref. 2,3 fitting this hypothesis are that the bursts (mode 2 of gating) were preferentially observed at the beginning of the sweep and that inactivation is evident in the ensemble currents. For this reason in our biophysical model we relied on the $\mathrm{P}_{\text {open }}$ estimate of the fluctuation analysis. We used $\mathrm{P}_{\text {open }}$ of 0.4 , which we derived from the measured 0.8 and divided it by the factor of 2 by which BayK8644 enhances the whole cell $\mathrm{Ca}^{2+}$ current at the peak $\mathrm{Ca}^{2+}$ current potential $^{5,7}$. We note that this likely reflects an upper boundary, which would in the model work against a dominance of the nearest channel for controlling $\mathrm{Ca}^{2+}$ at a given vesicle (i.e. against $\mathrm{Ca}^{2+}$ nanodomain control, that we favor for the mature $\mathrm{AZ}$ ). 
Supplementary Material 7 Contribution of different kinetic components to the $\Delta \mathrm{C}_{\mathrm{m}}$ response upon $\mathrm{Ca}^{2+}$-uncaging
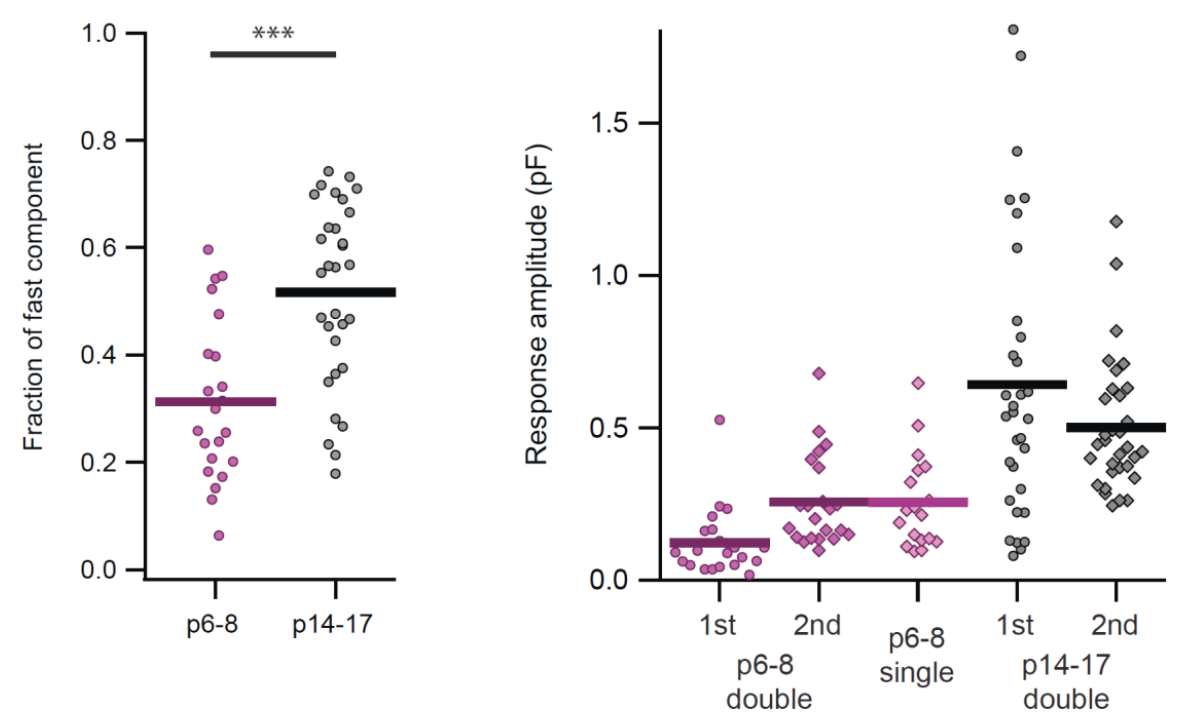

Figure S7. Contribution of different kinetic components to the $\Delta \mathrm{C}_{\mathrm{m}}$ response upon $\mathrm{Ca}^{2+}$-uncaging.

Quantification of the contributions of the kinetic components to the $\Delta \mathrm{C}_{\mathrm{m}}$ (left) and the amplitudes of kinetic components of the $\Delta \mathrm{C}_{\mathrm{m}}$ (right) triggered by UV-laser $\mathrm{Ca}^{2+}$ uncaging, as estimated by fitting double or single exponential functions to the $\Delta \mathrm{C}_{\mathrm{m}}$ traces. 
Supplementary Material 8 Supplementary figure for apparent $\mathrm{Ca}^{2+}$ cooperativity of exocytosis measurement

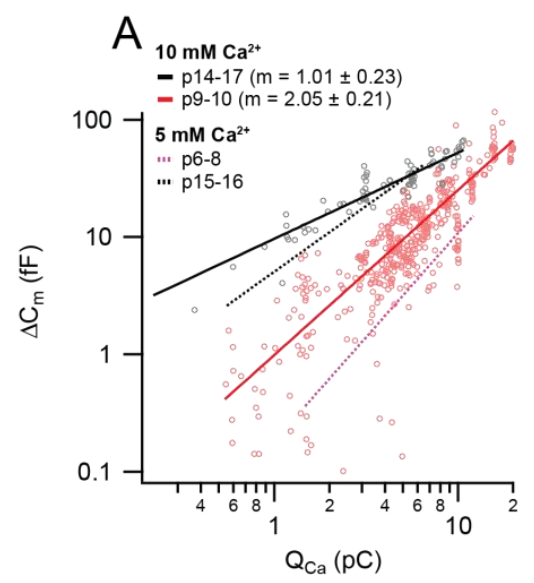

B
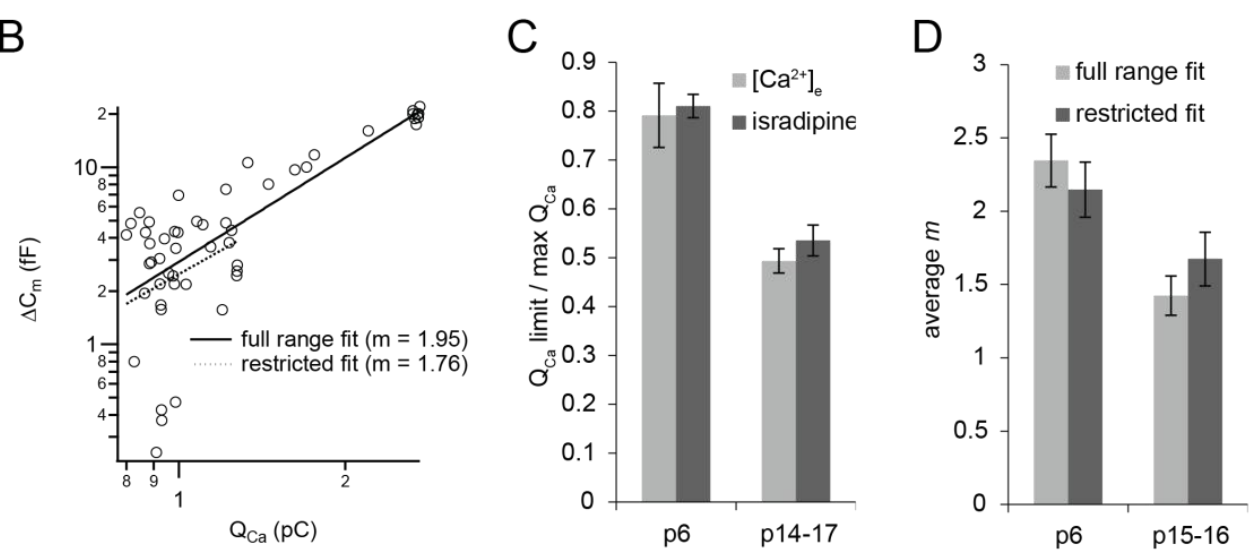

Figure S8. Additional data for apparent $\mathrm{Ca}^{2+}$-cooperativity of exocytosis measurement. (A) Apparent $\mathrm{Ca}^{2+}$-cooperativity of exocytosis in developing IHCs probed at $10 \mathrm{mM}$ extracellular calcium. Open circles are raw data points obtained from p14-17 (grey, n $=4$ IHCs) and p10 (pink, $\mathrm{n}=5 \mathrm{IHCs}$ ) with slow perfusion of $10 \mu \mathrm{M}$ isradipine, performed in the presence of $10 \mathrm{mM}\left[\mathrm{Ca}^{2+}\right]_{\mathrm{e}}$. Solid lines are power function fits to the combined datasets. Average exponents from fitting individual cells are: $1.01 \pm 0.23$ (p14-17) and $2.05 \pm 0.21$ (p9-10). For comparison, dotted lines show power fits to p15-16 (black) and p6-8 (magenta) data performed in $5 \mathrm{mM}\left[\mathrm{Ca}^{2+}\right]_{\mathrm{e}}$, as displayed in Figure 7 in the main figure. (B-D) Probing saturation with a restricted $\mathrm{Q}_{\mathrm{Ca}}$ fitting range. (B) Data from an example p15 IHC in isradipine experiments $\left(5 \mathrm{mM}\left[\mathrm{Ca}^{2+}\right]_{\mathrm{e}}\right)$, where datapoints were either fitted with the full (solid line) or restricted (dotted line) $\mathrm{Q}_{\mathrm{Ca}}$ range, as a fraction of the 
maximum $Q_{\text {Ca }}$ per cell (see panel C). (C) Average $Q_{\text {Ca }}$ range where limited saturation was found in $\left[\mathrm{Ca}^{2+}\right]_{\mathrm{e}}$ experiments, and the average actual $\mathrm{Q}_{\mathrm{Ca}}$ range used for restricted fitting for isradipine experiments, both expressed as a ratio to maximum $\mathrm{Q}_{\mathrm{Ca}}$. (D) Average power, $m$, from fitting the full and restricted $\mathrm{Q}_{\mathrm{Ca}}$ range in isradipine experiments. 


\section{Supplementary Material 9: Computational Modeling}

\subsection{Description of the model for studying the coupling between $\mathrm{Ca}^{2+}$ influx and exocytosis}

\subsubsection{AZ topography: positioning of RRP vesicles and presynaptic $\mathrm{Ca}^{2+}$ channels}

We considered three main scenarios M1-3 of the mature synapse AZ topography (Fig. 8A in the Results section). There, presynaptic $\mathrm{Ca}^{2+}$ channels were treated as $15 \mathrm{~nm}$ diameter ${ }^{8}$ disks and positioned in the plane of the plasma membrane within the area of the presynaptic density - assumed as a $420 \times 80 \mathrm{~nm}$ rectangular stripe. This choice of the presynaptic density area was based on an exemplary reconstruction of the synapse from serial EM sections and is consistent with the STED imaging of Cav 1.3 immunofluorescence (e.g. Fig 3D,G in the Results section). In scenario M1, 36 channels were distributed completely randomly within the stripe. In scenarios $\mathrm{M} 2$ and $\mathrm{M} 3,14 \mathrm{Ca}^{2+}$ channels were placed in contact with the vesicular $\mathrm{Ca}^{2+}$ sensors ("private channels"), simulating molecular coupling. In scenario M2, additional 36 channels were distributed randomly within the area of the presynaptic density. We also considered several modifications of M2-3: M2b-d and M3b (Fig. S9). M2b differed from M2 in that there were 76 instead of 36 channels distributed randomly within the presynaptic density, in addition to the 14 "private channels". M2c differed from M2 in that the "private channels" were surrounded by exclusion zones of a channel's diameter wide. M2d differed from M2 in that the $\mathrm{Ca}^{2+}$ sensors were shifted by $20 \mathrm{~nm}$ towards the center of the vesicles in the plane of the plasma membrane. M3b was identical to M3 except that each vesicle was assigned two "private channels".

In all aforementioned cases, 14 vesicles (40 nm diameter disks) were randomly distributed at the longer sides of the presynaptic density stripe ( 7 vesicles per side), approximating the number of membraneproximal, ribbon-associated vesicles, which we assume to represent the readily releasable pool (RRP). In the top view projection of the active zone, vesicles were in contact with the presynaptic density stripe and their $\mathrm{Ca}^{2+}$ sensors, located at the level of the plasma membrane, coincided with those contact points. Positions of the channels and the vesicular release sites were fixed throughout a particular simulation. 100 realizations with different positions of the vesicles and randomly placed channels of all those scenarios were used.

For the immature synapse, two scenarios of the AZ, IM1 (Fig. 8) and IM1b (Fig. S9), were considered. In these cases, a presynaptic density area similar to that of M1-3 was distributed within a $1 \mu$ m diameter circular area in the form of separate patches, wherein $60 \mathrm{Ca}^{2+}$ channels were distributed with similar average density as in M2. 4 pairs of smaller (45 nm diameter) and 3 single larger (90 nm diameter) density patches were assumed, based on an exemplary reconstruction from serial EM sections. In addition, 60 channels were distributed outside the presynaptic density patches within the $1 \mu \mathrm{m}$ circular area. In the case of IM1, all additional channels were distributed randomly outside the presynaptic density while in the case of IM1b, 19 of the additional channels were placed in physical contact with the vesicular $\mathrm{Ca}^{2+}$ sensors. In both, IM1 and IM1b, vesicles were distributed around the presynaptic density patches and over the remaining $\mathrm{AZ}$ as suggested by the EM data. The $\mathrm{Ca}^{2+}$ sensors were located at the edges of the vesicles 
in such a way that distances from them to the center of mass of the closest presynaptic densities were minimal. 100 realizations with different randomly placed channel positions were considered. Positions of the vesicles and the presynaptic density patches were identical over those 100 realizations.
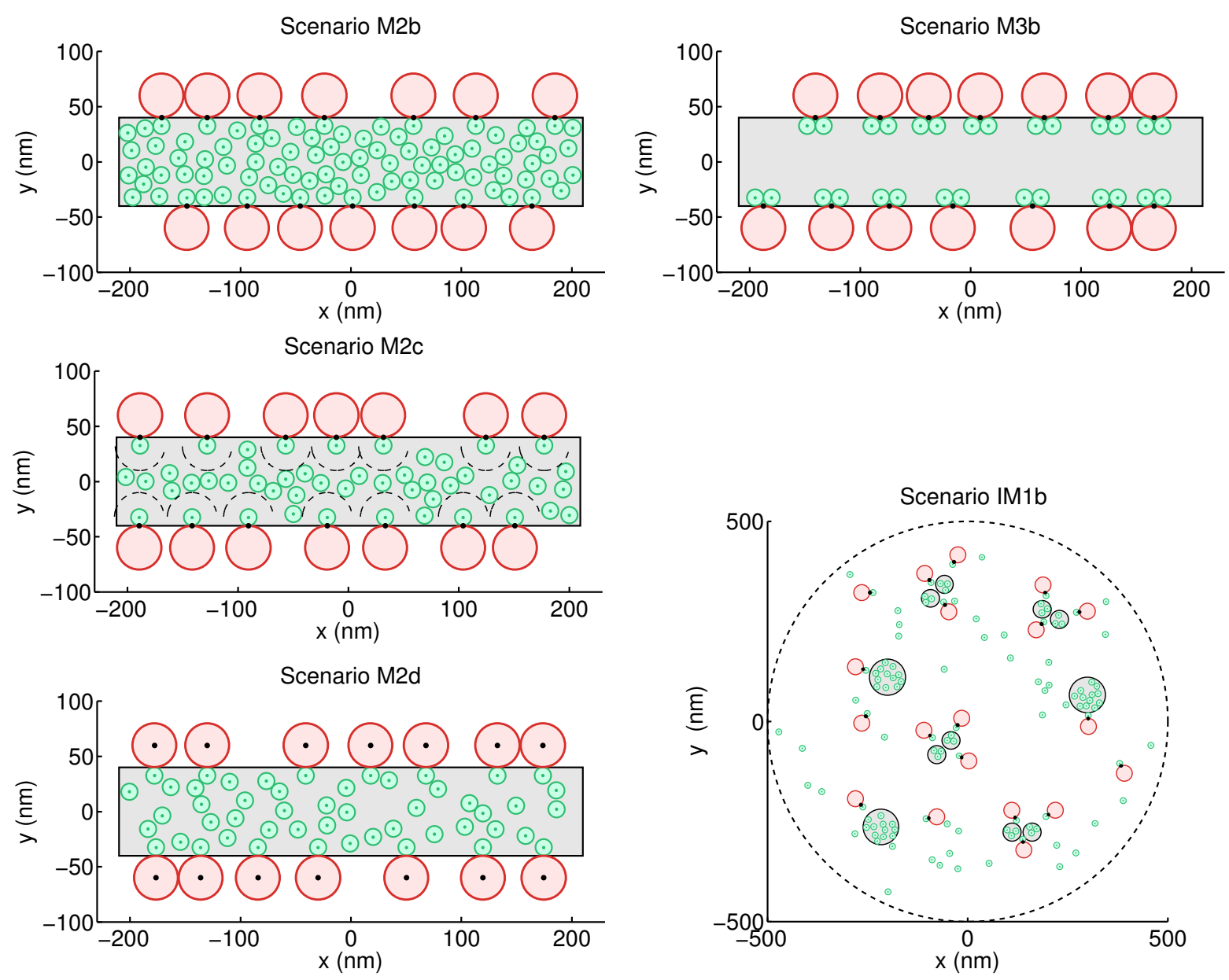

Figure S9: Scenarios M2b, M2c, M2d, M3b of the mature AZ topography and scenario IM1b of the immature AZ topography. Grey area: presynaptic density, red discs: RRP vesicles, green discs: $\mathrm{Ca}^{2+}$ channels, black spots: $\mathrm{Ca}^{2+}$ sensors of exocytosis.

\subsection{2 $\quad \mathrm{Ca}^{2+}$ channel gating dynamics}

A three state Markov chain model $C_{1} \underset{1 \cdot k_{-1}}{\stackrel{2 \cdot k_{+1}}{\rightleftarrows}} C_{2} \underset{2 \cdot k_{-1}}{\stackrel{1 \cdot k_{+1}}{\rightleftarrows}} O$ was used to describe the gating dynamics of the presynaptic $\mathrm{Ca}^{2+}$ channels: $C_{1,2}$ - closed channel states, $O$ - open channel state. Values of the gating rates $k_{+1}$ and $k_{-1}$ were calculated from the activation kinetics reported by ref.9. In particular, it follows from Fig. 1F, G in ref.9 that $\tau=\left(k_{+1}+k_{-1}\right)^{-1}=0.45 \mathrm{~ms}$ and $P_{o} / P_{o, \max }=\left(k_{+1} /\left(k_{+1}+k_{-1}\right)\right)^{2} / P_{o, \max }=0.8$ at membrane potential $V_{m}=-17 \mathrm{mV}$, which is the potential of the depolarizing pulse in our experiments. 
We assumed $P_{o, \max }=0.8$, as estimated from from the fluctuation analysis of $\mathrm{Ca}^{2+}$-tail currents. These values result in $k_{+1}=1.78 \mathrm{~ms}^{-1}$ and $k_{-1}=0.44 \mathrm{~ms}^{-1}$. It should be noted, however, that BayK8644, which increases the channel open probability ${ }^{10}$, was used by ref.9 while our aim was to model the channel gating in our current experiments in the absence of BayK8644. Given that the most pronounced effect of BayK8644 is to prolong channel residence times in the open state ${ }^{10}$, we increased $k_{-1}$ from $0.44 \mathrm{~ms}^{-1}$ to $1.37 \mathrm{~ms}^{-1}$ such that the open probability was halved. This is consistent with approximately twofold difference of the peak $\mathrm{Ca}^{2+}$ current with and without BayK86447.

\subsubsection{Intracellular $\left[\mathrm{Ca}^{2+}\right]$ dynamics}

$\left[\mathrm{Ca}^{2+}\right]$ levels in our model were estimated by treating open presynaptic $\mathrm{Ca}^{2+}$ channels as $\mathrm{Ca}^{2+}$ point sources and by using the linearized buffer approximation ${ }^{11}$. The total flux of $\mathrm{Ca}^{2+}$ through an open $\mathrm{Ca}^{2+}$ channel at the imposed membrane potential $\left(\mathrm{V}_{\mathrm{m}}=-17 \mathrm{mV}\right)$ was set to $i_{C a}=0.3 \mathrm{pA}$ for the mature synapse (Fig. 2B, ref.3) and $i_{C a}=0.5 \mathrm{pA}$ for the immature synapse (Fig. 3D, ref.2). We preferred to use directly measured single channel current values rather than their estimate based on the fluctuation analysis of $\mathrm{Ca}^{2+}$-tail currents. 3D $\mathrm{Ca}^{2+}$ diffusion was constrained by the reflective plasma membrane but considered unaffected by the synaptic vesicles or the synaptic ribbon. The spatial $\left[\mathrm{Ca}^{2+}\right]$ profile was assumed to reach steady state instantaneously upon opening and closing of the channels. Overall $\left[\mathrm{Ca}^{2+}\right]$ at any point of interest was obtained by summing the contributions of separate presynaptic $\mathrm{Ca}^{2+}$ channels as is valid in the linearized buffer approximation.

In our model, we considered the following endogeneous $\mathrm{Ca}^{2+}$ buffers: calretinin $(\mathrm{CR})$, calbindin $(\mathrm{CB})$, parvalbumin $(\mathrm{PV})$ and ATP. $\mathrm{Ca}^{2+}$ and mobile $\mathrm{Ca}^{2+}$ buffer concentrations, their binding and unbinding rates, and diffusion coefficients are given in Table S9. Diffusion coefficients of $\mathrm{Ca}^{2+}$ bound buffer molecules were assumed to be the same as the diffusion coefficients of the corresponding $\mathrm{Ca}^{2+}$-free buffer molecules. We assumed cooperative $\mathrm{Ca}^{2+}$ binding to $\mathrm{CR}$ and noncooperative binding to the other three buffers considered. Based on ref.12, we assumed that CR consists of one single binding site (CR1) and two identical, independent pairs of cooperative binding sites (CR2). In Table S9, CR2 $2_{\mathrm{T}}$ stands for the $\mathrm{Ca}^{2+}$ free $\mathrm{CR} 2$ while $\mathrm{CR} 2 \mathrm{R}$ stands for $\mathrm{CR} 2$ with one $\mathrm{Ca}^{2+}$ ion already bound.

Calculation of the steady state levels of $\mathrm{Ca}^{2+}$ and $\mathrm{Ca}^{2+}$ bound buffer species in our case followed exactly the same path as in ref.11 except that the Jacobian matrix A (Eq. 8 in ref.11) which corresponds to the equations of the $\mathrm{Ca}^{2+}$-buffer reaction dynamics had a different form due to the cooperative $\mathrm{Ca}^{2+}$ binding of CR. In our particular case, matrix A was defined as follows:

$$
A=\left(\begin{array}{ccccccc}
-a_{1} & b_{1} & 0 & 0 & 0 & 0 & c_{1} \\
b_{2} & -a_{2} & 0 & 0 & 0 & 0 & c_{2} \\
0 & 0 & -a_{3} & 0 & 0 & 0 & c_{3} \\
0 & 0 & 0 & -a_{4} & 0 & 0 & c_{4} \\
0 & 0 & 0 & 0 & -a_{5} & 0 & c_{5} \\
0 & 0 & 0 & 0 & 0 & -a_{6} & c_{6} \\
a_{1}-b_{2} & a_{2}-b_{1} & a_{3} & a_{4} & a_{5} & a_{6} & -\sum_{i=1}^{6} c_{i}
\end{array}\right) .
$$


Here, index 1 corresponds to $\mathrm{CR} 2_{\mathrm{T}}$, index 2 corresponds to $\mathrm{CR} 2_{\mathrm{R}}$, and $a_{1-2}, b_{1-2}, c_{1-2}$ are defined as follows:

$$
\begin{aligned}
& a_{1}=\left(k_{o f f, C R 2_{T}}+2 k_{o n, C R 2_{T}}\left[\mathrm{Ca}^{2+}\right]_{r e s t}\right), \\
& a_{2}=2 k_{o f f, C R 2_{R}}, \\
& b_{1}=-2 k_{\text {on }, C R 2_{T}}\left[\mathrm{Ca}^{2+}\right]_{\text {rest }} \text {, } \\
& b_{2}=k_{\text {on }, C R 2_{R}}\left[\mathrm{Ca}^{2+}\right]_{\text {rest }} \text {, } \\
& c_{1}=\frac{2 k_{o n, C R 2_{T}}[\mathrm{CR} 2]}{1+2 k_{o n, C R 2_{T}} / k_{o f f, C R 2_{T}}\left[\mathrm{Ca}^{2+}\right]_{\text {rest }}+\left(k_{o n, C R 2_{T}} k_{o n, C R 2_{R}}\right) /\left(k_{o f f, C R 2_{T}} k_{o f f, C R 2_{R}}\right)\left[\mathrm{Ca}^{2+}\right]_{r e s t}^{2}}, \\
& c_{2}=\frac{2\left(k_{o n, C R 2_{T}} k_{o n, C R 2_{R}}\right) / k_{o f f, C R 2_{T}}[\mathrm{CR} 2]\left[\mathrm{Ca}^{2+}\right]_{\text {rest }}}{1+2 k_{o n, C R 2_{T}} / k_{o f f, C R 2_{T}}\left[\mathrm{Ca}^{2+}\right]_{\text {rest }}+\left(k_{o n, C R 2_{T}} k_{o n, C R 2_{R}}\right) /\left(k_{o f f, C R 2_{T}} k_{o f f, C R 2_{R}}\right)\left[\mathrm{Ca}^{2+}\right]_{\text {rest }}^{2}} .
\end{aligned}
$$

Indexes 3-6 correspond to CR1, CB, PV, and ATP. $a_{3-6}, c_{3-6}$ correspond to $1 / \tau_{i}$ and $\kappa_{i} / \tau_{i}$ defined in ref.11:

$$
\begin{aligned}
& a_{i}=1 / \tau_{i}=\left(k_{o f f, i}+k_{o n, i}\left[\mathrm{Ca}^{2+}\right]_{\text {rest }}\right), \\
& c_{i}=\kappa_{i} / \tau_{i}=\frac{k_{o n, i} k_{\text {off }, i}[\mathrm{~B}]}{k_{o f f, i}+k_{o n, i}\left[\mathrm{Ca}^{2+}\right]_{\text {rest }}} .
\end{aligned}
$$

Calculation of $\left[\mathrm{Ca}^{2+}\right]$ at any point of interest was based on the diagonalization of the matrix $(\mathbf{D} \cdot \mathbf{A})$, where $\mathbf{D}$ is a diagonal matrix with the diffusion coefficients of the corresponding molecules as in $\mathbf{A}$ put on the diagonal $\mathbf{D}=\operatorname{diag}\left[D_{C R}, D_{C R}, D_{C R}, D_{C B}, D_{P V}, D_{A T P}, D_{\mathrm{Ca}}\right]$. In particular:

$$
\left[\mathrm{Ca}^{2+}\right](t)=\sum_{j=1}^{N_{C a}} s_{j}(t) / r_{j} \sum_{i=1}^{7} k_{i} u_{7, i} e^{-\sqrt{\mu_{i}} r_{j}} .
$$

Here, $r_{j}$ is the distance from the point of interest to the center of the $\mathrm{j}$-th channel and $s_{j}(t)$ stands for the state of the $\mathrm{j}$-th channel at moment $t: s_{j}=0$ if the channel is closed and $s_{j}=1$ if the channel is open. $u_{7, i}$ is the seventh component of the i-th eigenvector of $(\mathbf{D} \cdot \mathbf{A})$ and $\mu_{i}$ is the corresponding eigenvalue of $(\mathbf{D} \cdot \mathbf{A})$, while $\left\{k_{i}\right\}_{i=1}^{7}$ is the solution of $\left\{\sum_{i=1}^{7} u_{j, i} k_{i}=i_{C a} /\left(4 \pi D_{\mathrm{Ca}^{2+}}\right) \delta_{j, 7}\right\}_{j=1}^{7}$.

The free ATP concentration was calculated by assuming equilibration of $\mathrm{Mg}^{2+}$ to ATP binding with free cellular concentration of $\mathrm{Mg}^{2+}$ equal to $0.5 \mathrm{mM}^{19}$. The overall ATP concentration was set to $2 \mathrm{mM}$, and $K_{d, A T P}=45 \mu \mathrm{M}^{16}$. We did not explicitly take the competition between $\mathrm{Mg}^{2+}$ and $\mathrm{Ca}^{2+}$ for ATP into account, because $\left[\mathrm{Mg}^{2+}\right]=0.5 \mathrm{mM}$ is much higher than $\left[\mathrm{Ca}^{2+}\right]$ at the release sites, which leads to only negligible fluctuations of free ATP level around its mean value upon $\mathrm{Ca}^{2+}$ channel opening and closing. 


\begin{tabular}{|c||c|c|c|c|}
\hline & $C, \mathrm{mM}$ & $k_{o n},(\mathrm{mM} \cdot \mathrm{ms})^{-1}$ & $k_{o f f}, \mathrm{~ms}^{-1}$ & $D, \mu \mathrm{m}^{2} \cdot \mathrm{ms}^{-1}$ \\
\hline \hline CR2 & \multirow{2}{*}{$3.6 \cdot 10^{-2}\left(4.0 \cdot 10^{-2}\right)$, ref.14 } & 1.8, ref.12 & $5.3 \cdot 10^{-2}$, ref.12 & \multirow{2}{*}{$2.0 \cdot 10^{-1}$, ref.12 } \\
\cline { 3 - 5 } CR2 $\mathrm{R}$ & $3.1 \cdot 10^{2}$, ref.12 & $2.0 \cdot 10^{-2}$, ref.12 & \\
\hline CR1 & $1.8 \cdot 10^{-2}\left(2.0 \cdot 10^{-2}\right)$, ref.14 & 7.3, ref.12 & $2.52 \cdot 10^{-1}$, ref.12 & $2.0 \cdot 10^{-1}$, ref.12 \\
\hline CB & $2.32 \cdot 10^{-1}(1.7)$, ref.14 & $7.5 \cdot 10^{1}$, ref.13 & $2.95 \cdot 10^{-2}$, ref.13 & $2.0 \cdot 10^{-1}$, ref.17 \\
\hline PV & $1.88 \cdot 10^{-1}\left(1.38 \cdot 10^{-1}\right)$, ref.14 & $1.08 \cdot 10^{2}$, ref.15 & $9.8 \cdot 10^{-4}$, ref.15 & $4.3 \cdot 10^{-1}$, ref.18 \\
\hline ATP (free) & $1.65 \cdot 10^{-1}$ & $1.0 \cdot 10^{3}$, ref.16 & $9.0 \cdot 10^{1}$, ref.16 & 2.0, ref.11 \\
\hline Ca $^{2+}$ (rest) & $5.0 \cdot 10^{-5}$ & - & - & 2.0, ref.11 \\
\hline
\end{tabular}

Table S9 $\mathrm{Ca}^{2+}$ and mobile $\mathrm{Ca}^{2+}$ buffer binding site concentrations $(C)$, their binding $\left(k_{\text {on }}\right)$ and unbinding $\left(k_{o f f}\right)$ rates, and diffusion coefficients $(D)$ used in the model. Concentration values in the round brackets correspond to immature IHCs, if they differ from the mature IHCs. All concentration values were taken as reported by ref.14 for apical IHCs, which (in mice) were also used for our experiments. Note that the concentration entry for $\mathrm{CR} 2_{\mathrm{T}}$ and $\mathrm{CR} 2_{\mathrm{R}}$ refers to the concentration of $\mathrm{CR} 2$ binding site pairs.

\subsubsection{Fusion and replenishment dynamics of RRP vesicles}

In our simulations, $\mathrm{Ca}^{2+}$ triggered fusion of the $\mathrm{RRP}$ vesicles following the kinetic model proposed by ref.20:

$B(C a)_{0} \underset{b^{0} k_{\text {off }}}{\stackrel{5 k_{o n}\left[\mathrm{Ca}^{2+}\right]}{\longrightarrow}} B(\mathrm{Ca})_{1} \underset{b^{1} k_{\text {off }}}{\stackrel{4 k_{o n}\left[\mathrm{Ca}^{2+}\right]}{\longrightarrow}} B(\mathrm{Ca})_{2} \underset{b^{2} k_{\text {off }}}{\stackrel{3 k_{o n}\left[\mathrm{Ca}^{2+}\right]}{\longrightarrow}} B(\mathrm{Ca})_{3} \underset{b^{3} k_{\text {off }}}{\stackrel{2 k_{o n}\left[\mathrm{Ca}^{2+}\right]}{\longrightarrow}} B(\mathrm{Ca})_{4} \underset{b^{4} k_{\text {off }}}{\stackrel{1 k_{o n}\left[\mathrm{Ca}^{2+}\right]}{\longrightarrow}} B(\mathrm{Ca})_{5} \stackrel{\gamma}{\rightarrow}$ fused,

where $B(C a)_{n}$ denotes the state of an occupied vesicle release site and $n \mathrm{Ca}^{2+}$ ions bound to the $\mathrm{Ca}^{2+}$ sensor, while fused denotes an empty vesicle release site after a vesicle fusion. Values of the kinetic parameters were taken as reported by ref.20: $k_{o n}=27.6 \mathrm{mM}^{-1} \mathrm{~ms}^{-1}, k_{\text {off }}=2.150 \mathrm{~ms}^{-1}, b=0.4$, $\gamma=1.695 \mathrm{~ms}^{-1}$, except that, for the model realizations shown in the Fig. 8 of the Results section, we used two times smaller $k_{\text {on }}$ due to reasons discussed below (section 9.2).

Refilling of the empty vesicle release sites during the ongoing stimulation was considered as a single step process with a fixed rate $k_{r e p}$. Mathematically, this was implemented by adding an additional step fused $\stackrel{k_{r e p}}{\longrightarrow} B(C a)_{0}$ to the kinetic scheme (3). We set $k_{\text {rep }}=0.13 \mathrm{~ms}^{-1}$. This choice was based on the sustained release rate reported in ref.21. According to that study (Table 1, ref.21), each active zone releases 679 vesicles per second in the sustained phase, which is equivalent to 57 vesicles per second per release site (assuming 12 release sites per active zone). This value is the lower limit of the replenishment rate, which cannot be lower than the release rate. Moreover, it is likely that the replenishment rate is higher in a time window before the steady state is reached because number of the RRP surrounding vesicles, which are used to replenish the RRP, is likely to be higher at initial time moments. To take these effects into account, we arbitrarily set $k_{\text {rep }}$ two times larger than the vesicle release rate in steady state reported in ref.21. Increase or decrease of $k_{\text {rep }}$ by two times from that value did not have significant influence on the main aspects of the modelling results considered in this study. 


\subsubsection{Propagation of the dynamics of the system across time}

Initially, all the channels were set to be in the $C_{1}$ closed state and all the vesicle release sites were filled with vesicles, each in state $B(C a)_{0}$. This corresponds to the experimental conditions in which cells were kept at $V_{m}=-87 \mathrm{mV}$, with negligible $\left[\mathrm{Ca}^{2+}\right]$ levels at the active zone. After changing $V_{m}$ to $-17 \mathrm{mV}$ at $t=0$, gating dynamics of each $\mathrm{Ca}^{2+}$ channel was propagated across time by using Gillespie's method ${ }^{22}$. Vesicle fusion-replenishment dynamics was propagated by using an extended version of the Gillespie's method, which takes into account the time dependence of the transition rates. Briefly, at iteration $i$, the amount of time for a vesicle at a particular release site to stay in its current state $a$ was estimated by generating a uniformly distributed random variable $u \in(0,1)$ and solving the following equation for $t_{i+1}$

$$
\int_{t_{i}}^{t_{i+1}}\left(\sum_{b=1}^{N_{a \rightarrow}} k_{a \rightarrow b}(t)\right) d t=-\ln (u),
$$

where $k_{a \rightarrow b}(t)$ stands for the transition rate from state $a$ to state $b$ at time $t$ and $N_{a \rightarrow}$ is the number of possible transitions from state $a . t_{i}$ and $t_{i+1}$ are the times when the vesicle entered and left state $a$. It follows from (2) and (3) that the transition rates $k_{a \rightarrow b}(t)$ are piecewise constant functions of time. (4) was solved numerically exactly. To decide to which one of the possible final states the system moves at $t_{i+1}$, the unit interval $[0,1]$ was divided into $N_{a \rightarrow}$ subintervals, each corresponding to one of the possible

final states, with lengths $k_{a \rightarrow b}\left(t_{i+1}\right) / \sum_{b=1}^{N} k_{a \rightarrow b}\left(t_{i+1}\right)$. The final state was then chosen by generating a uniformly distributed random variable $u \in[0,1]$ which pointed to one of those subintervals. The vesicle fusion time was defined as the moment when $B(C a)_{5}$ transited to fused. The spatial position of the vesicle release sites remained constant over time, so that the sensor of a newly replenished vesicle was taking the same position as the sensor of the previously released vesicle.

\subsubsection{Estimation of the release vs. $\mathrm{Q}_{\mathrm{Ca}}$ dependencies}

To model the outcome of the channel blocking experiment, we randomly blocked a certain number of channels (in the range from 0 to $\mathrm{N}_{\mathrm{Ca}}-1$ ) for each scenario studied. For each number of blocked channels, we went through 100 different combinations of blocked channels. Simulations with synapse responses for each of those 100 blocked channel combinations were repeated 10 times. The same set of calculations was performed with 100 different realizations of each synapse topography scenarios generated as discussed above (section 9.1.1). Integrated $\mathrm{Ca}^{2+}$ influx $\left(\mathrm{Q}_{\mathrm{Ca}}\right)$ and number of released vesicles in response to $20 \mathrm{~ms}$ depolarization were calculated and averaged over (\# of the synapse realizations) · (\# of the blocked channel combinations) $\cdot \#$ of repetitions $)=10^{2} \cdot 10^{2} \cdot 10=10^{5}$ simulations for each number of blocked channels.

To simulate the $\mathrm{i}_{\mathrm{Ca}}$ manipulation experiment, we scaled down the single channel current $(0.3 \mathrm{pA}$ for mature synapses and $0.5 \mathrm{pA}$ for immature synapses) by a factor of $f_{s} \in\left[1, \mathrm{~N}_{\mathrm{Ca}}\right]$. Q $\mathrm{Q}_{\mathrm{Ca}}$ and number of released vesicles in response to $20 \mathrm{~ms}$ voltage steps were calculated and averaged over (\# of the synapse realizations $) \cdot(\#$ of repetitions $)=10^{2} \cdot 10^{3}=10^{5}$ simulations for each value of the single channel current scaling factor.

The estimates of the exponent $m$ of the release vs. $\mathrm{Q}_{\mathrm{Ca}}$ relationship under $\mathrm{N}_{\mathrm{Ca}} \times \mathrm{P}_{\text {open }}$ manipulation were determined by fitting the relationship in a double logarithmic plot with a line from the maximum 
$\mathrm{Q}_{\mathrm{Ca}}$ down to one-fifth of its value, similar to the that achieved experimentally (20\% residual current with $10 \mu \mathrm{M}$ isradipine). For $\mathrm{i}_{\mathrm{Ca}}$ manipulation, the exponent $m$ was determined in the following way. The line fitting started by using the first 5 points corresponding to released vesicle number bigger than $10^{-4}$. Then, additional points for fitting were included until the estimated value of the exponent $m$ decreased more than $5 \%$ compared to the initial one (based on the first five points). This limitation was required to avoid underestimations of $m$ due to the saturation of the release vs. $Q_{\mathrm{Ca}}$ relationship (see section 9.2).

\subsubsection{Considerations on the simplifications used in the model}

The linearized buffer approximation in general underestimates $\left[\mathrm{Ca}^{2+}\right]$ as it effectively corresponds to constant free buffer levels independent of $\mathrm{Ca}^{2+}$ coming from the sources. The overall mobile buffer concentration is indeed high $(0.7 \mathrm{mM}$ to $2 \mathrm{mM}$ binding sites) in our case, which enables substantial concentrations of $\mathrm{Ca}^{2+}$ free buffer at distances greater than the $\mathrm{Ca}^{2+}$ channel radius. Assuming instantaneous equilibration of $\left[\mathrm{Ca}^{2+}\right]$ upon the channel opening and closing, on the other hand, leads to an overestimation of the $\left[\mathrm{Ca}^{2+}\right]$. However, this overestimation is relatively substantial only for those $\mathrm{Ca}^{2+}$ sources that are located far from the point of interest (see ref.11). The absolute contribution of these faraway channels to the $\left[\mathrm{Ca}^{2+}\right]$ at the release sites is altogether small for the AZ topographies considered in our study. Thus, $\left[\mathrm{Ca}^{2+}\right]$ overestimation due to the assumption of instantaneous $\left[\mathrm{Ca}^{2+}\right]$ equilibration upon the channel opening and closing can be ignored.

\subsection{Further analysis of the release vs. $\mathrm{Ca}^{2+}$ influx dependencies}

\subsubsection{The saturation effect and its origins}

A pronounced saturation in the release vs. $\mathrm{Q}_{\mathrm{Ca}}$ dependencies was observed for some of the mature synapse scenarios considered in our study when strictly adhering to the model parameters as provided in the cited references. The saturation effect was particularly evident for the $\mathrm{i}_{\mathrm{Ca}}$ manipulation when the $k_{\text {on }}$ value reported by ref.20 was used. For example, in the case of scenario M2c, the relationship becomes linear in the a double log plot only when $\mathrm{Q}_{\mathrm{Ca}}$ drops fivefold below $\max \left[\mathrm{Q}_{\mathrm{Ca}}\right]$ based on $\mathrm{i}_{\mathrm{Ca}}$ manipulation (red curve Fig. S10, right panel). For $\mathrm{Q}_{\mathrm{Ca}}$ change based on $\mathrm{N}_{\mathrm{Ca}} \times \mathrm{P}_{\text {open }}$ manipulation, the saturation was smaller but still influential. For example, in the case of scenario M2c, the release vs. Q $\mathrm{Ca}_{\text {relationship }}$ shows saturation for the higher $\mathrm{Q}_{\mathrm{Ca}}$ and deviates considerably from a linear relationship in a double $\log$ plot in the range from $\max \left[\mathrm{Q}_{\mathrm{Ca}}\right] / 5$ to $\max \left[\mathrm{Q}_{\mathrm{Ca}}\right]$ (red curve Fig. S10, left panel). This leads to an underestimation of the exponent $m$ which reflects the apparent $\mathrm{Ca}^{2+}$ cooperativity.

The origin of the pronounced saturation in the release vs. $\mathrm{Q}_{\mathrm{Ca}}$ dependencies is fast initial RRP depletion (in respect to the width of the $20 \mathrm{~ms}$ observation time window) due to much higher vesicle fusion rate compared to the replenishment rate. Indeed, as shown by the red curve in Fig. S11, which represents scenario M2c, the exocytosis rate is so high that most of the initial RRP vesicles fuse within $6 \mathrm{~ms}$ after the stimulus onset. Then, the system enters steady state, during which exocytosis rate $\approx 0.1$ vesicle/ms per release site is limited by the replenishment step. During the next $14 \mathrm{~ms}$, around 1.4 vesicles fuse per release site. In other words, around half of all fusion events observed in the $20 \mathrm{~ms}$ 

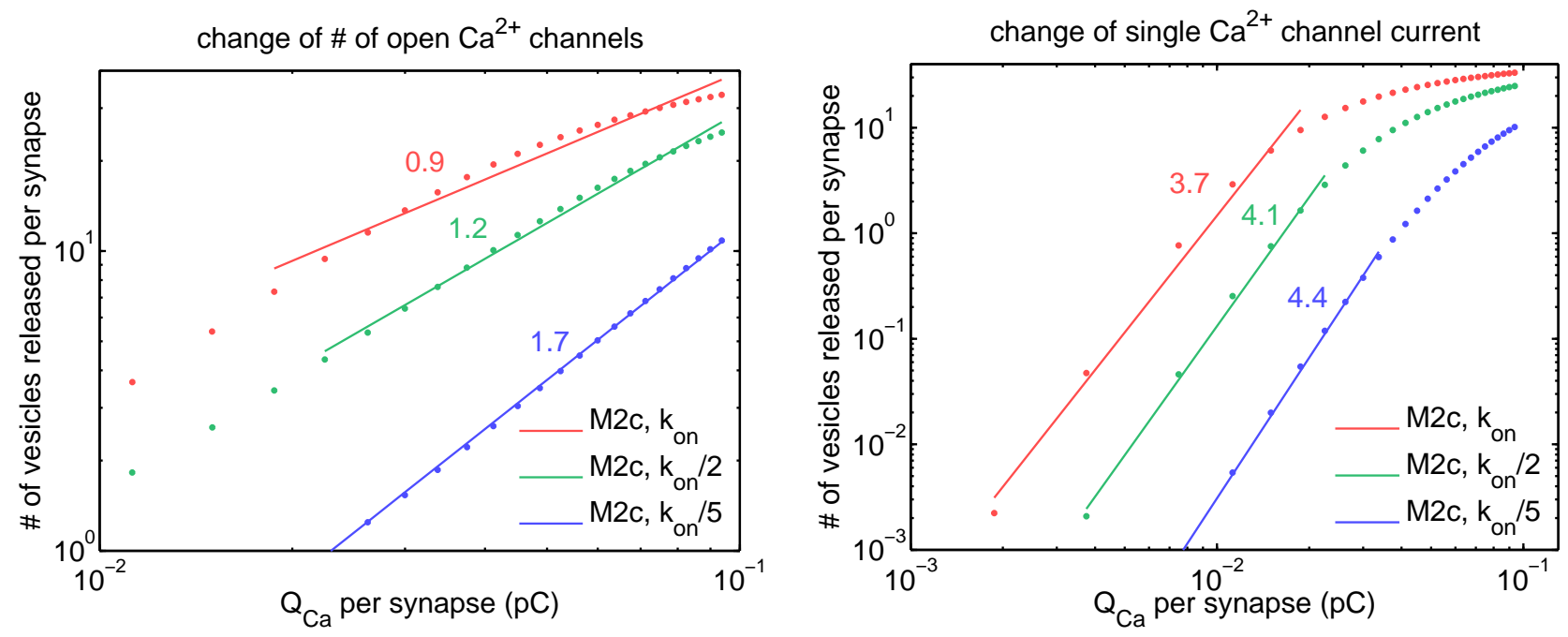

Figure S10: Release vs. $\mathrm{Q}_{\mathrm{Ca}} 20 \mathrm{~ms}$ after the stimulus onset for scenario M2c with different scaling of $k_{o n}$ values. Left $-\mathrm{N} \times \mathrm{P}_{\text {open }}$ manipulation, right $-\mathrm{i}_{\mathrm{Ca}}$ manipulation.

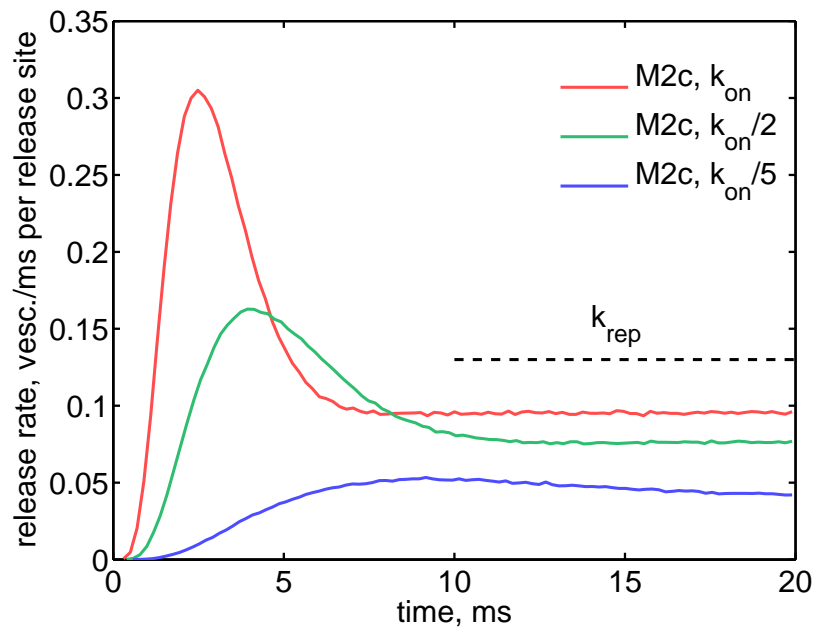

Figure S11: Vesicle release rate evolution after the stimulus application for scenario M2c with different scaling of $k_{o n}$ values. Dashed black line indicates to the replenishment rate $k_{\text {rep }}$ value on the ordinate axis. 
window happen within the first $6 \mathrm{~ms}$. This asymmetry in the exocytosis rate results in a relatively low decrease in overall release upon the reduction of the $\mathrm{Q}_{\mathrm{Ca}}$. For example, when $\mathrm{Q}_{\mathrm{Ca}}$ is reduced two times by reducing $\mathrm{i}_{\mathrm{Ca}}$ (which is equivalent to reducing $k_{o n}$ two times, green curve in Fig. S11), the RRP is depleted in $10 \mathrm{~ms}$ on average. During the next $10 \mathrm{~ms}, 0.8$ vesicles fuse per release site on average. Thus, despite the fact that $\mathrm{Ca}^{2+}$ is reduced two times, the overall release $20 \mathrm{~ms}$ after the stimulus application decreases only $2.4 / 1.8 \approx 1.3$ times. Note that, in all cases studied here, vesicle fusion rate was not limited by $\gamma$, the transition rate from $B(C a)_{5}$ to fused. Another, only a partial, source of the saturation is based on the fact that vesicle replenishment is not only the rate limiting step, (compare the steady release rates corresponding to the red curve with that showed by the black dashed line) but is also assumed to be $\mathrm{Ca}^{2+}$ independent.

When $\mathrm{Q}_{\mathrm{Ca}}$ is reduced sufficiently, the saturation becomes negligible. Then, the sensitivity of the release to change of $\mathrm{Ca}^{2+}$ reflects the real $\mathrm{Ca}^{2+}$ influx to exocytosis coupling (Fig. S10). Note, however, that $m$ values based on $\mathrm{N}_{\mathrm{Ca}} \times \mathrm{P}_{\text {open }}$ change approach 1 by definition when only a few channels remain unblocked. Thus, data for the lowest $\mathrm{Q}_{\mathrm{Ca}}$ have to be avoided. In contrast, $m$ values based on $\mathrm{i}_{\mathrm{Ca}}$ manipulation remain unchanged even at strongly reduced $\mathrm{Q}_{\mathrm{Ca}}$, assuming that there is no noticeable saturation in the region of interest. That is because the relative $\left[\mathrm{Ca}^{2+}\right]$ contribution to the vesicle sensors of a particular $\mathrm{Ca}^{2+}$ channel in respect to the rest of the $\mathrm{Ca}^{2+}$ channels remains unchanged during this manipulation and, thus, the sensitivity reflects the intrinsic $\mathrm{Ca}^{2+}$ dependence of exocytosis.

There are two ways to reduce the saturation effect in the release vs. $Q_{\text {Ca }}$ plots without changing the AZ topography or the observation time window. The first way is to increase the replenishment rate so that it becomes much higher than the fusion rate and the vesicle pool stays nearly full throughout the depolarizations. This, however, would strongly deviate from the physiological situation. The replenishment rate should be increased by an order of magnitude or more to achieve a considerable improvement. The second way to reduce the saturation effect is to decrease the fusion rate. This could be achieved by decreasing $k_{o n}$ or, equivalently, by decreasing the $\left[\mathrm{Ca}^{2+}\right]$ levels, which could be, for example, due to a stronger buffering or smaller single channel currents. The effect of the reduction of $k_{o n}$ is shown in Fig. $\mathrm{S} 10$. We see that the release vs. $\mathrm{Q}_{\mathrm{Ca}}$ relationship in a double log plot linearises in the range $\max \left[\mathrm{Q}_{\mathrm{Ca}}\right] / 5$ to $\max \left[\mathrm{Q}_{\mathrm{Ca}}\right]$ in the case of $\mathrm{N}_{\mathrm{Ca}} \times \mathrm{P}_{\text {open }}$ manipulation when $k_{\text {on }}$ is decreased (compare the red, green, and blue plots). This leads to a considerable increase in the exponent $m$ estimate. In the case of $\mathrm{i}_{\mathrm{Ca}}$ manipulation, the range of $\mathrm{Q}_{\mathrm{Ca}}$ with a constant slope of the release vs. $\mathrm{Q}_{\mathrm{Ca}}$ relationship in a double log plot increases considerably when $k_{\text {on }}$ decreases. Nevertheless, the exponent $m$ estimates based on the fitting to the linear part of the relationship is $\sim 4$ in all the cases considered. This is how it should be, because release vs. $\mathrm{Q}_{\mathrm{Ca}}$ relationships based on $\mathrm{i}_{\mathrm{Ca}}$ manipulation reflect the intrinsic $\mathrm{Ca}^{2+}$ dependence of exocytosis, not the $\mathrm{Ca}^{2+}$ influx to exocytosis coupling.

It is clearly seen by comparing plots in the left and the right panels in Fig. S10 that the saturation effect is more pronounced in the case of $\mathrm{i}_{\mathrm{Ca}}$ manipulation than $\mathrm{N}_{\mathrm{Ca}} \times \mathrm{P}_{\text {open }}$ manipulation. The explanation of this phenomena is based on disproportionate contributions of different $\mathrm{AZ} \mathrm{Ca}^{2+}$ channels to vesicle exocytosis rates at different release sites. This is seen the best by considering scenario M3. If we block half of the channels in that case, we effectively make the release nearly absent at half of the sites. 
On the contrary, reduction of the single channel current $\mathrm{i}_{\mathrm{Ca}}$ by two times only decreases the fusion rate, which is otherwise very high, at all sites. This reduction delays the pool depletion only by a few ms which is little compared to $20 \mathrm{~ms}$ width observation window. Moreover, the exocytosis rate of the subsequent replenished vesicles is limited by the replenishment, and is considerably lower than the depletion rate of the initial RRP. In this way, the delay of the pool depletion by a few ms due to the reduced $i_{\text {Ca }}$ has a smaller effect than the absence of vesicle release at a part of the release sites due to the equivalent $\mathrm{N}_{\mathrm{Ca}} \times \mathrm{P}_{\text {open }}$ reduction.

For most of the active zone scenarios considered in our simulations, we chose a two times reduced $k_{o n}$ value $\left(k_{o n} / 2\right)$ compared to that reported by ref.20. This helped to avoid a very pronounced saturation effect, which would result in unrealistic sublinear release vs. $\mathrm{Q}_{\mathrm{Ca}}$ relationships (described above). This choice is rather arbitrary and does not necessarily reflect the degree of the saturation observed in the experiments. It is important to note that the $\log \left(\mathrm{Q}_{\mathrm{Ca}}\right)$ range available for $m$ estimates based on $\mathrm{i}_{\mathrm{Ca}}$ change was wide in the case of the model. This guaranteed that these $m$ estimates were not noticeably affected by the saturation. If we limited the analysis of the modeling results to the same width of $\log \left(\mathrm{Q}_{\mathrm{Ca}}\right)$ range as in the case of experimental data, the modeling $m$ estimates based on $\mathrm{i}_{\mathrm{Ca}}$ change $(20 \mathrm{~ms}$ time window, $k_{o n} / 2$ ) would have been even smaller than the experimental ones. This potentially means that the effect of the saturation was more pronounced in the model (20 ms time window, $\left.k_{o n} / 2\right)$ than the experiment. Note that our modeling results $\left(20 \mathrm{~ms}\right.$ time window, $\left.k_{o n} / 2\right)$ showed that, among all the scenarios considered, experimental estimate $m=1.4$ based on $\mathrm{N}_{\mathrm{Ca}} \times \mathrm{P}_{\text {open }}$ change could be reproduced only in the case of the nanodomain coupling between the $\mathrm{Ca}^{2+}$ influx and exocytosis. If this is true with the considered level of saturation in the model, this would be true with a lower level of the saturation too. Below, we provide a more detailed analysis of the effect of the saturation on the exponent $m$ estimates for the considered AZ scenarios to constrain possible interpretations of experimental $m$ estimates in terms of the exocytosis to $\mathrm{Q}_{\mathrm{Ca}}$ influx coupling.

\subsubsection{Assessing the influence of the saturation effect}

A parameter-independent way to assess the influence of the saturation effect on the exponent $m$ estimates and reveal the real $\mathrm{Ca}^{2+}$ influx to exocytosis coupling is to use very short observation windows. This is not possible to achieve in the experiments due to low signal to noise ratio, but can be conveniently done in the modeling approach. In Fig. S12, release vs. $\mathrm{Q}_{\mathrm{Ca}}$ dependencies based on 3 ms observation windows are shown for cases $k_{\text {on }}$ and $k_{\text {on }} / 2$ for scenario M2c. The relationships based on $\mathrm{N}_{\mathrm{Ca}} \times \mathrm{P}_{\text {open }}$ change are, to a good approximation, linear in double $\log$ plots in the range $\max \left[\mathrm{Q}_{\mathrm{Ca}}\right] / 5$ to $\max \left[\mathrm{Q}_{\mathrm{Ca}}\right]$ (left panel). The saturation is rather mild for the dependencies based on $\mathrm{i}_{\mathrm{Ca}}$ change (right panel). Moreover, the estimates of the $m$ exponents are quite similar to those in the case of strongly reduced exocytosis rate $\left(k_{o n} / 5\right.$ in Fig. S10), as expected.

It should be noted that one of the possible concerns with using short time windows is that the estimate of the exponent $m$ can be influenced by the gating kinetics of the $\mathrm{Ca}^{2+}$ channels. However, in our case, the characteristic equilibration time of the channel gating is $0.45 \mathrm{~ms}$, which is much shorter than the 3 ms length observation window. 

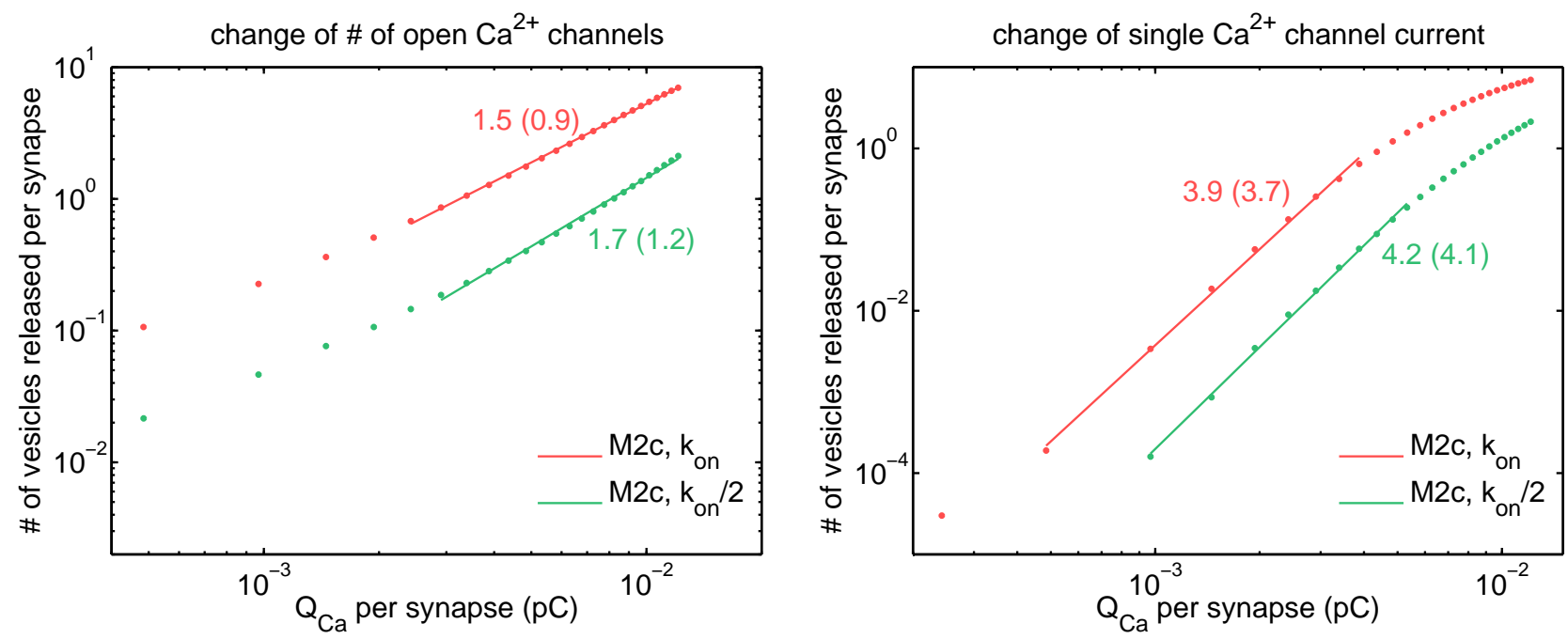

Figure S12: Release vs. $\mathrm{Q}_{\mathrm{Ca}} 3 \mathrm{~ms}$ after the stimulus application for scenario M2c with different scaling of $k_{\text {on }}$ values. Left $-\mathrm{N} \times \mathrm{P}_{\text {open }}$ manipulation, right $-\mathrm{i}_{\mathrm{Ca}}$ manipulation. Values in the round brackets correspond to the exponent $m$ estimated from the responses $20 \mathrm{~ms}$ (Fig. S11), rather than $3 \mathrm{~ms}$, after the stimulus onset.
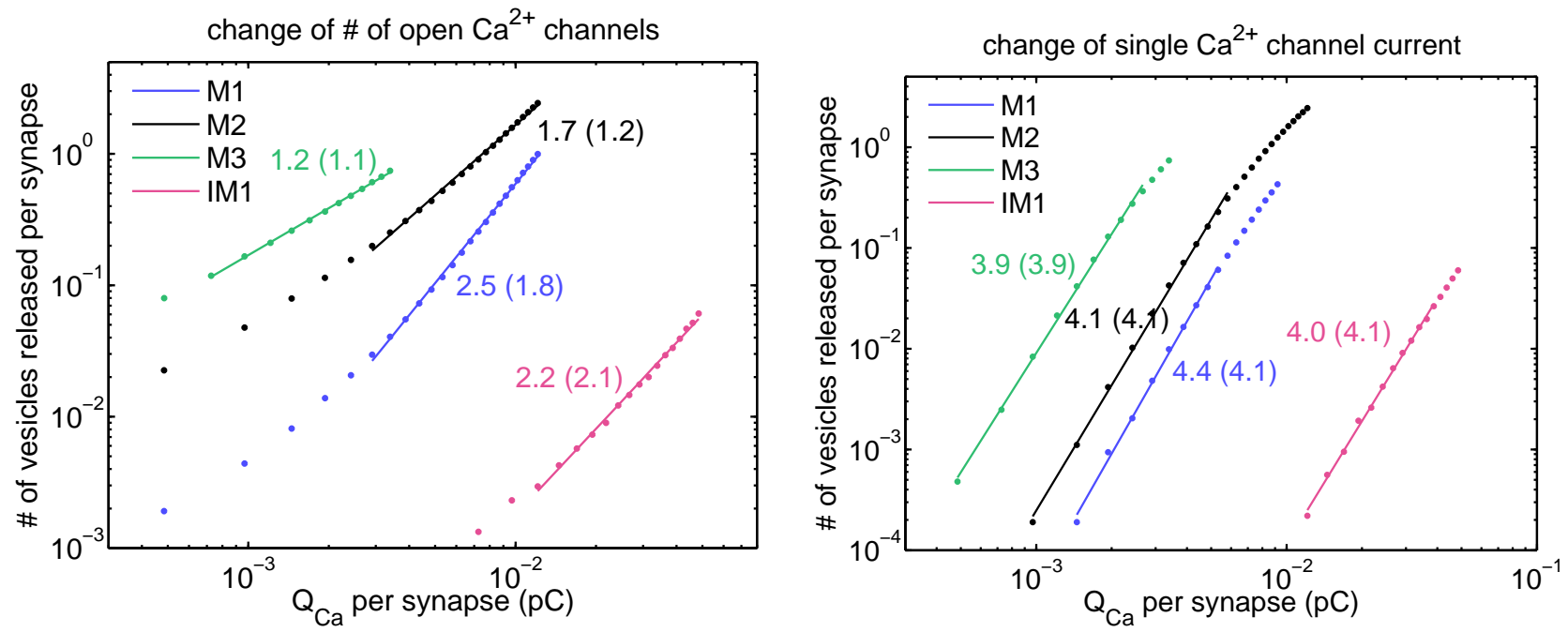

Figure S13: Release vs. $\mathrm{Q}_{\mathrm{Ca}} 3 \mathrm{~ms}$ after the stimulus application for scenarios M1-3 and IM1. Left $-\mathrm{N} \times \mathrm{P}_{\text {open }}$ manipulation, right $-\mathrm{i}_{\mathrm{Ca}}$ manipulation. Values in the round brackets correspond to the exponent $m$ estimated from the responses $20 \mathrm{~ms}$ (Fig. $8 \mathrm{E}, \mathrm{F}$ ), rather than $3 \mathrm{~ms}$, after the stimulus onset. $k_{o n} / 2$ was assumed. 

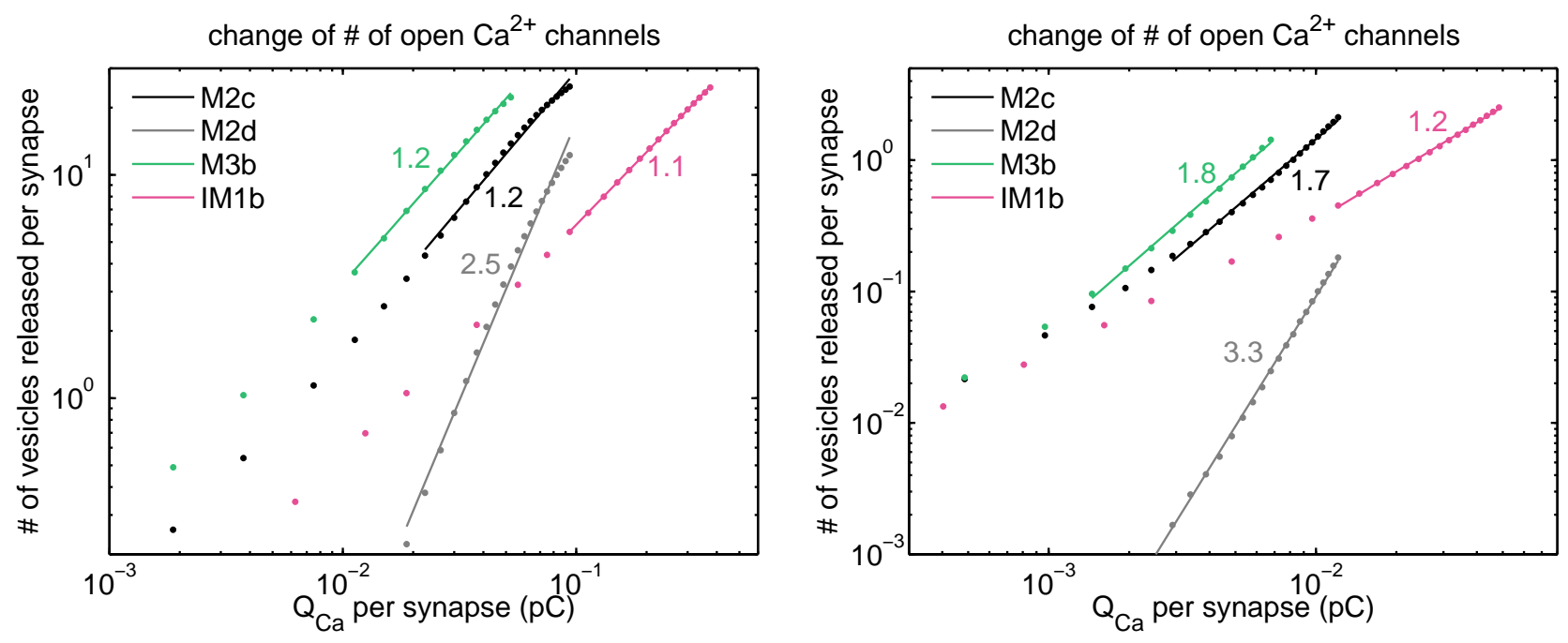

Figure S14: Release vs. $\mathrm{Q}_{\mathrm{Ca}}$ for scenarios M2c-d, M3b, and IM1b based on $\mathrm{N}_{\mathrm{Ca}} \times \mathrm{P}_{\text {open }}$ manipulation. Left $-20 \mathrm{~ms}$ after the stimulus onset, right $-3 \mathrm{~ms}$ after the stimulus onset. $k_{o n} / 2$ was assumed.

In Fig. S13, the release vs. $\mathrm{Q}_{\mathrm{Ca}}$ relationships and exponent $m$ estimates based on $3 \mathrm{~ms}$ observation windows for scenarios M1-3 and IM1 are shown and compared with the corresponding estimates based on 20 ms observation windows (values in round brackets) which were discussed in Results section. As can be expected from the previous discussion, $m$ values stayed $\sim 4$ for the $\mathrm{i}_{\mathrm{Ca}}$ manipulation for all considered scenarios, though the relationships became linear over a broader range of $\mathrm{Q}_{\mathrm{Ca}}$ values in the double log plots. In the case of $\mathrm{N}_{\mathrm{Ca}} \times \mathrm{P}_{\text {open }}$ manipulation, $m$ values stayed similar for scenarios $\mathrm{M} 3$ and IM1. In the case of scenario M3, that happened because this scenario corresponds to almost a pure $\mathrm{Ca}^{2+}$ nanodomain controlled vesicle release, when each release site has only one channel contributing to vesicle release at that site. Thus, blocking the channels results in a proportional decrease in the release. In the case of scenario IM1, the $m$ value did not change considerably, because the fusion rate was relatively moderate (due to relatively small $\left[\mathrm{Ca}^{2+}\right]$ levels) and, thus, the saturation effect was negligible even for the $20 \mathrm{~ms}$ observation window. The $m$ value increased from 1.8 to 2.3 for scenario M1 and from 1.2 to 1.7 for scenario M2. These examples show that, despite the fact that $m$ values based on $\mathrm{N}_{\mathrm{Ca}} \times \mathrm{P}_{\text {open }}$ change could be underestimated due to the saturation effect, their differences remain considerable among scenarios of different $\mathrm{Ca}^{2+}$ influx to exocytosis coupling.

Fig. S14 presents $m$ exponent estimates based on $\mathrm{N}_{\mathrm{Ca}} \times \mathrm{P}_{\text {open }}$ change with $20 \mathrm{~ms}$ (left) and $3 \mathrm{~ms}$ (right) depolarizations for scenarios M2c-d, M3b, and IM1b (Fig. S9). In the case of scenario IM1b, vesicle release at each release site is dominated by a single channel and, thus, $m$ is rather invariant for different time windows as in the case of scenario M3. For scenarios M2c and M3b, the $m$ value increases from 1.2 to 1.7-1.8, similar to what is observed for scenario M2 (Fig. S12). In contrast, in the case of scenario M2d, $m$ increases from 2.5 to 3.3. The main reason why the shift of the sensor by only 20 $\mathrm{nm}$ has a substantial effect on the $\mathrm{Ca}^{2+}$ to exocytosis coupling (M2c vs. M2d) is that a contribution to $\left[\mathrm{Ca}^{2+}\right]$ by a particular channel decreases with an increased distance faster at smaller than at larger 
distances. Thus, when the sensor is moved away from a "private channel" (Fig. S9), the contribution of this channel decreases more than the contributions of the other channels. 


\section{References}

1. Platzer, J. et al. Congenital deafness and sinoatrial node dysfunction in mice lacking class D L-type Ca2+ channels. Cell 102, 89-97 (2000).

2. Zampini, V. et al. Elementary properties of $\mathrm{CaV} 1.3 \mathrm{Ca}(2+)$ channels expressed in mouse cochlear inner hair cells. J Physiol 588, 187-199 (2010).

3. Zampini, V. et al. Burst activity and ultrafast activation kinetics of CaV1.3 Ca2+ channels support presynaptic activity in adult gerbil hair cell ribbon synapses. $J$ Physiol 591, 38113820 (2013).

4. Meyer, A. C. et al. Tuning of synapse number, structure and function in the cochlea. Nat Neurosci 12, 444-453 (2009).

5. Frank, T. et al. Bassoon and the synaptic ribbon organize $\mathrm{Ca}^{2}+$ channels and vesicles to add release sites and promote refilling. Neuron 68, 724-738 (2010).

6. Grant, L. \& Fuchs, P. Calcium- and Calmodulin-Dependent Inactivation of Calcium Channels in Inner Hair Cells of the Rat Cochlea. J Neurophysiol 99, 2183-2193 (2008).

7. Brandt, A., Khimich, D. \& Moser, T. Few CaV1. 3 channels regulate the exocytosis of a synaptic vesicle at the hair cell ribbon synapse. J Neurosci 25, 11577 (2005).

8. Wolf, M., Eberhart, A., Glossmann, H., Striessnig, J. \& Grigorieff, N. Visualization of the domain structure of an L-type Ca2+ channel using electron cryo-microscopy. J. Mol. Biol. 332, 171-182 (2003).

9. Neef, J. et al. The Ca2+ Channel Subunit beta2 Regulates Ca2+ Channel Abundance and Function in Inner Hair Cells and Is Required for Hearing. J Neurosci 29, 10730 (2009).

10. Hess, P., Lansman, J. B. \& Tsien, R. W. Different modes of Ca channel gating behaviour favoured by dihydropyridine Ca agonists and antagonists. Nature 311, 538-544 (1984).

11. Naraghi, M. \& Neher, E. Linearized buffered Ca2+ diffusion in microdomains and its implications for calculation of $[\mathrm{Ca} 2+]$ at the mouth of a calcium channel. J Neurosci 17, 6961 (1997).

12. Faas, G. C., Schwaller, B., Vergara, J. L. \& Mody, I. Resolving the fast kinetics of cooperative binding: Ca2+ buffering by calretinin. PLoS Biol. 5, e311 (2007).

13. Faas, G. C., Raghavachari, S., Lisman, J. E. \& Mody, I. Calmodulin as a direct detector of Ca2+ signals. Nat. Neurosci. 14, 301-304 (2011).

14. Hackney, C. M., Mahendrasingam, S., Penn, A. \& Fettiplace, R. The concentrations of calcium buffering proteins in mammalian cochlear hair cells. J Neurosci 25, 7867 (2005).

15. Lee, S.-H., Schwaller, B. \& Neher, E. Kinetics of Ca2+ binding to parvalbumin in bovine chromaffin cells: implications for $[\mathrm{Ca} 2+]$ transients of neuronal dendrites. $J$ Physiol 525, 419432 (2000).

16. Bollmann, J. H. \& Sakmann, B. Control of synaptic strength and timing by the release-site Ca2+ signal. Nat Neurosci 8, 426-434 (2005). 
17. Schmidt, H., Schwaller, B. \& Eilers, J. Calbindin D28k targets myo-inositol monophosphatase in spines and dendrites of cerebellar Purkinje neurons. Proc. Nat1. Acad. Sci. U.S.A 102, $5850-5855$ (2005).

18. Schmidt, H., Brown, E. B., Schwaller, B. \& Eilers, J. Diffusional Mobility of Parvalbumin in Spiny Dendrites of Cerebellar Purkinje Neurons Quantified by Fluorescence Recovery after Photobleaching. Biophysical Journal 84, 2599-2608 (2003).

19. Sinha, S. R., Wu, L.-G. \& Saggau, P. Presynaptic Calcium Dynamics and Transmitter Release Evoked by Single Action Potentials at Mammalian Central Synapses. Biophys J 72, 637-651 (1997).

20. Beutner, D., Voets, T., Neher, E. \& Moser, T. Calcium dependence of exocytosis and endocytosis at the cochlear inner hair cell afferent synapse. Neuron 29, 681-690 (2001).

21. Pangršič, T. et al. Hearing requires otoferlin-dependent efficient replenishment of synaptic vesicles in hair cells. Nat Neurosci 13, 869-876 (2010).

22. Gillespie, D. T. Exact stochastic simulation of coupled chemical reactions. J Phys Chem 81, 2340-2361 (1977). 


\section{Additional results}

In this section, we provide additional modeling results which resolve several potential issues related to the interpretation of exponent $m$ values and further constrain the AZ topography of mature IHC ribbon synapses. The effect of synaptic vesicles and ribbons as potential diffusion barriers on presynaptic $\left[\mathrm{Ca}^{2+}\right]$ is considered as well ${ }^{1}$.

\section{Updated concentrations of the endogenous $\mathrm{Ca}^{2+}$ buffers}

In the original publication of this chapter, mathematical modeling was based on the values of concentrations of three endogenous buffers, parvalbumin- $\alpha$, calbindin-D28k, and calretinin, which were taken from (Hackney et al., 2005). Our later results (presented in chapter 3) suggested, however, that concentrations of these buffers are considerably higher in mature IHCs. This prompted us to reevaluate exponents $m$ of the exocytosis vs $\mathrm{Ca}^{2+}$-influx relations in our model.

As follows from findings presented in chapter 3 , the effect of intracellular $\mathrm{Ca}^{2+}$ buffers on exocytosis in response to short $(20 \mathrm{~ms})$ depolarizations is well approximated by $0.5 \mathrm{mM}$ of BAPTA (total concentration). This is what we assumed in our new simulations. All other aspects of the model were implemented in the same way as described in the original publication of the present chapter.

We found no considerable differences between the new and the old estimates of exponents $m$ for AZ topographies tested, as summarized in panels A and B of Fig. 2.1 (new values in bold, old values in round brackets). This result is most likely explained by the compact arrangement of $\mathrm{Ca}^{2+}$ channels and RRP vesicles at the preynaptic densities which reduces the influence of mobile $\mathrm{Ca}^{2+}$ buffers on $\left[\mathrm{Ca}^{2+}\right]$ at the sensors of exocytosis (see chapter 3 for more details). We conclude that estimates of exponents $m$ from our previous modeling are compatible with the new estimates of the buffer concentrations.

\section{4 vs $5 \mathrm{Ca}^{2+}$ binding sites of the sensor of exocytosis}

Experimental estimates of the exponent $m$ based on the change of the single channel current reported in the original article of this chapter were $\sim 3$ (average value) in both, mature and immature, preparations. On the other hand, $m$ values obtained by computational modeling were $\sim 4$ and even slightly higher when assuming the updated mobile $\mathrm{Ca}^{2+}$ buffer concentrations (Fig. 2.1 A, B). This fact naturally rises the question of how mentioned deviation affects the interpretation of the exponent $m$ obtained based on the change of the number of open $\mathrm{Ca}^{2+}$ channels.

\footnotetext{
${ }^{1}$ The validity of the approximations of instantaneous build-up and collapse of $\mathrm{Ca}^{2+}$ domains upon gating of $\mathrm{Ca}^{2+}$ channels, and linearized $\left[\mathrm{Ca}^{2+}\right]$ dynamics, which were assumed in the original article, is analyzed in chapter 4 .
} 
A

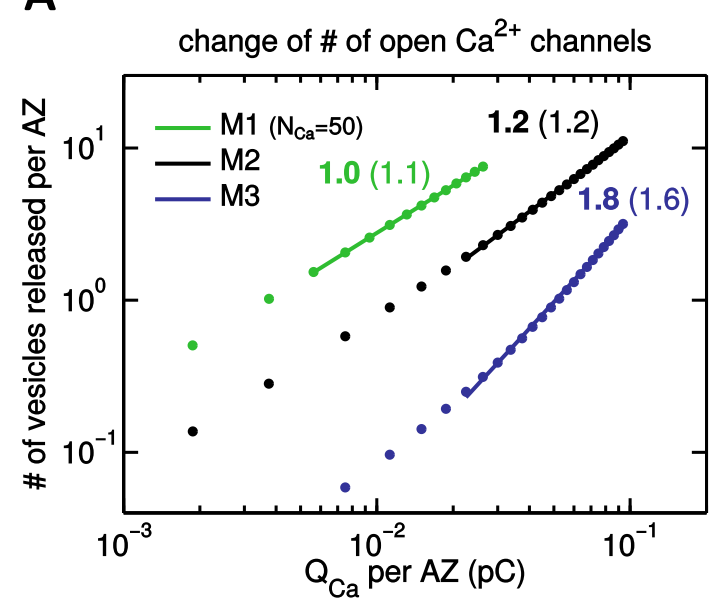

C

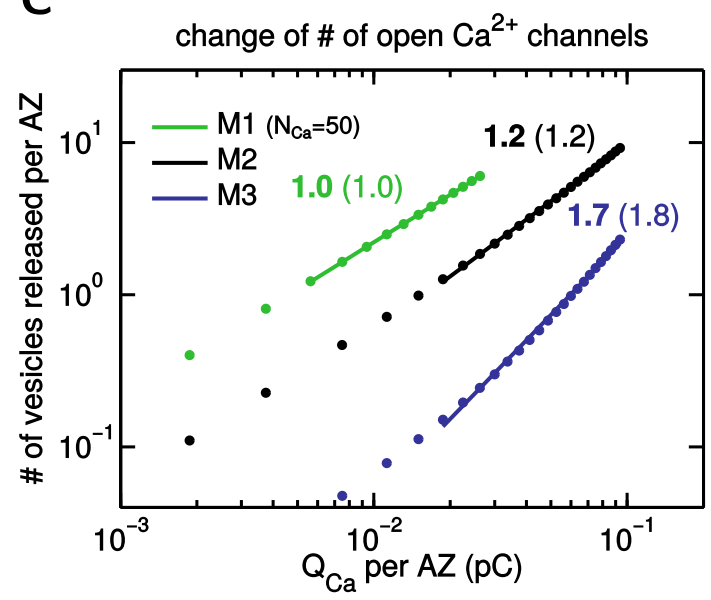

B

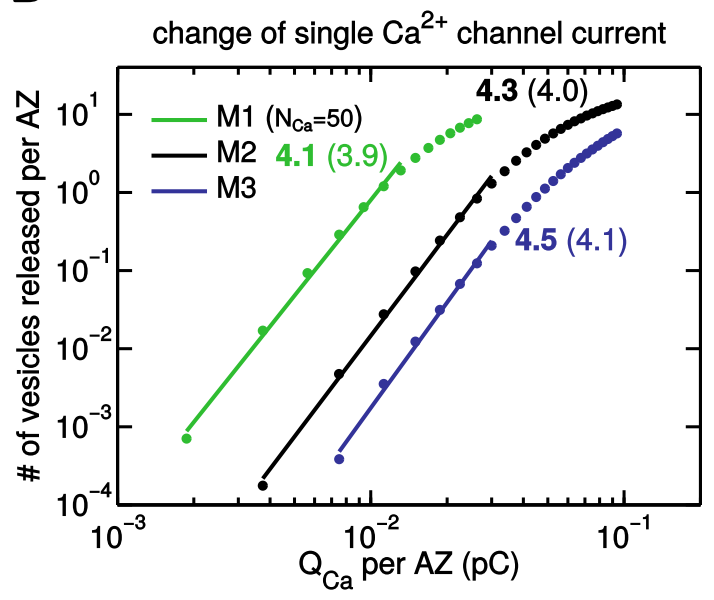

D

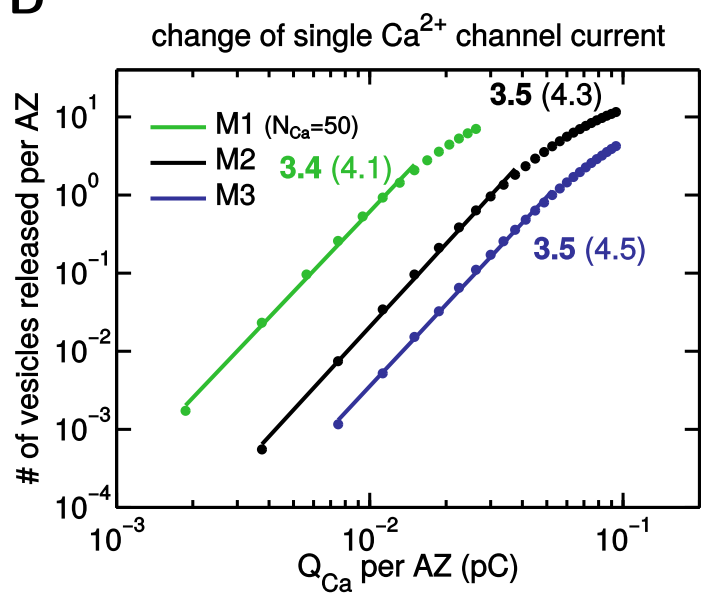

Figure 2.1: Reevaluation of the exponents $m$ of exocytosis vs $\mathrm{Ca}^{2+}$ influx relations. (A) Exponents $m$ estimated for AZ topography scenarios M1-3 by varying the number of open presynaptic $\mathrm{Ca}^{2+}$ channels. Here, the effect of intracellular $\mathrm{Ca}^{2+}$-buffers was approximated by $0.5 \mathrm{mM}$ BAPTA (the total concentration). Values of $m$ are displayed in bold. Numbers in round brackets show the corresponding estimates of $m$ obtained before. (B) The same as (A), but with exocytosis vs $\mathrm{Ca}^{2+}$ influx relations obtained by changing the single channel current. (C) The same as (A), but assuming only $4 \mathrm{Ca}^{2+}$ binding sites for the sensor of exocytosis. The new estimates of $m$ are shown in bold. Values of $m$ obtained by using the five binding site model earlier are put in round brackets. (D) The same as (C), but with exocytosis vs $\mathrm{Ca}^{2+}$ influx relations obtained by changing the single channel current. 
One possible explanation of the deviation of $m$ values between the model and the experiment is that the number of $\mathrm{Ca}^{2+}$ binding sites of the sensor of exocytosis is lower than five. Indeed, in the original study (Beutner et al., 2001), where the kinetics $\mathrm{Ca}^{2+}$ binding to the sensor of exocytosis was considered, it was noted that the model with four $\mathrm{Ca}^{2+}$-binding sites could reproduce the data nearly as good as the model with five binding sites. We, thus, reestimated the exponent $m$ based on the change of either the number of open channels or single channel current and by assuming only four $\mathrm{Ca}^{2+}$ bidning sites. As it is shown in Fig. 2.1C, D, $m$ values based on the change of the single channel current decreased to $\sim 3.5$ (panel D), which is within the uncertainty range of our experimental estimate, but stayed nearly the same for the change of the number of open $\mathrm{Ca}^{2+}$ channels (panel $\mathrm{C}$ ). This finding can be explained by the fact that few $\mathrm{Ca}^{2+}$ channels induce vesicle release in the considered scenarios, even in scenario M1 $(m=1.8)$, when all the channels are randomly distributed within the presynaptic density.

\section{Further refinement of the active zone topography}

In the original article of this chapter, topographies of presynaptic AZs were constrained by using only estimates of the exponents $m$ of exocytosis vs $\mathrm{Ca}^{2+}$ influx relations. In our next publication (chapter 3), we also obtained the effective coupling distance between the presynaptic $\mathrm{Ca}^{2+}$ channels and sensors of exocytosis (denoted by $R_{c}$ ). The average value of $R_{c}$ was $17 \mathrm{~nm}$ and the $5-95$ percentile was of $8-30 \mathrm{~nm}$. When taken together, $m$ and $R_{c}$ put stronger constraints on the AZ topographies. Here, we present the results of our additional efforts to refine the AZ topography of IHC ribbon synapses in hearing animals. All additional simulations were done as described in the original article of this chapter but with intracellular $\mathrm{Ca}^{2+}$ buffers approximated by $0.5 \mathrm{mM}$ BAPTA, as explained two pages above. The procedure of estimating $R_{c}$ is described in chapter 3 .

At first, we considered AZ topography scenario $\mathrm{M} 1\left(\mathrm{Ca}^{2+}\right.$ channels distributed randomly within the presynaptic density area) with different numbers of $\mathrm{Ca}^{2+}$ channels (denoted by $N_{C a}$ ) as shown in Fig. 2.2A. $m$ decreased from $\sim 1.8$ at $N_{C a}=75$ to $\sim 1.3$ at $N_{C a}=15 . m \sim 1.4$ could be reproduced with $N_{C a} \sim 20$ (Fig. 2.3A). However, the absolute release rate was unrealistically small then (less than 1 vesicle per $20 \mathrm{~ms}$ ). Moreover, the estimates of $R_{c}$ were $\sim 3$ times larger than our experimentally obtained value of $17 \mathrm{~nm}$ (Fig. $2.2 \mathrm{~A}$ ). In fact, even at $N_{C a}=75$, which corresponds to the presynaptic density quite tightly packed with $\mathrm{Ca}^{2+}$ channels, the effective coupling distance was $\sim 40 \mathrm{~nm}$. These findings suggested that some of the $\mathrm{Ca}^{2+}$ channels are preferentially close to the $\mathrm{Ca}^{2+}$ sensors of exocytosis. 
A
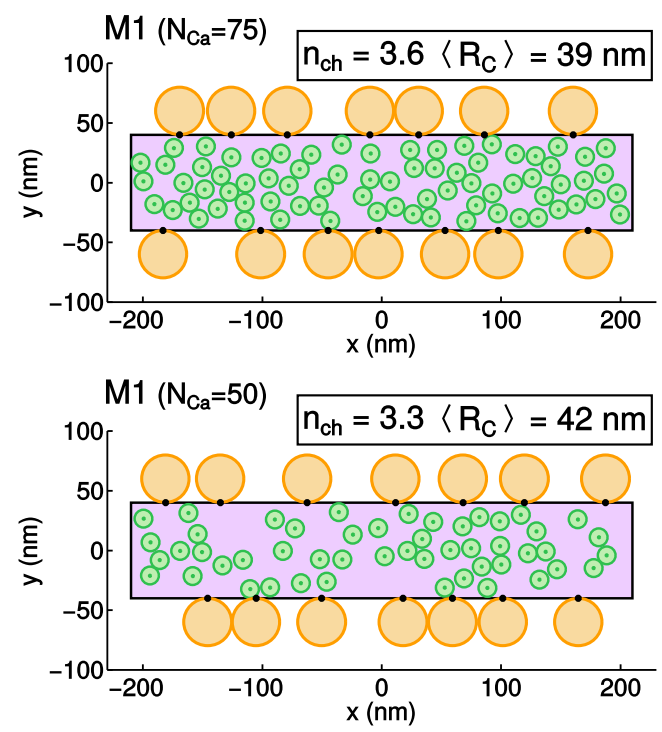

B
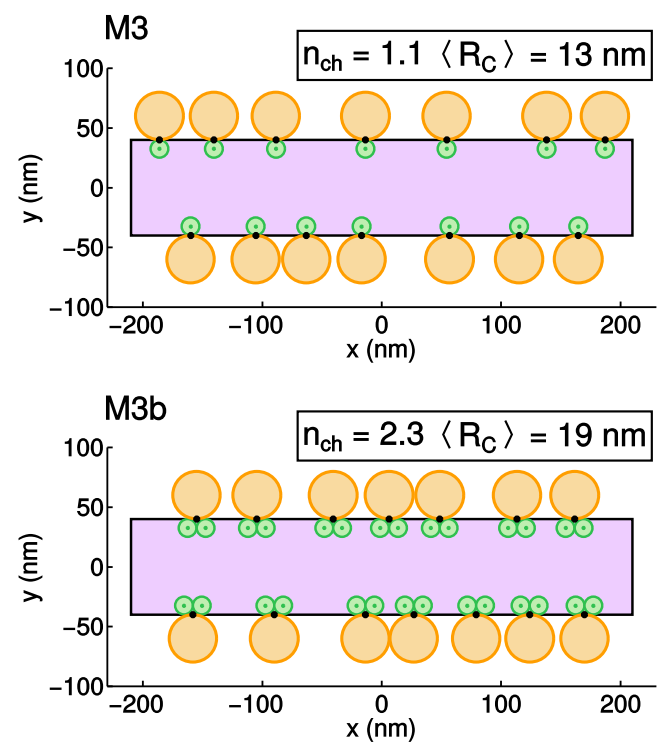
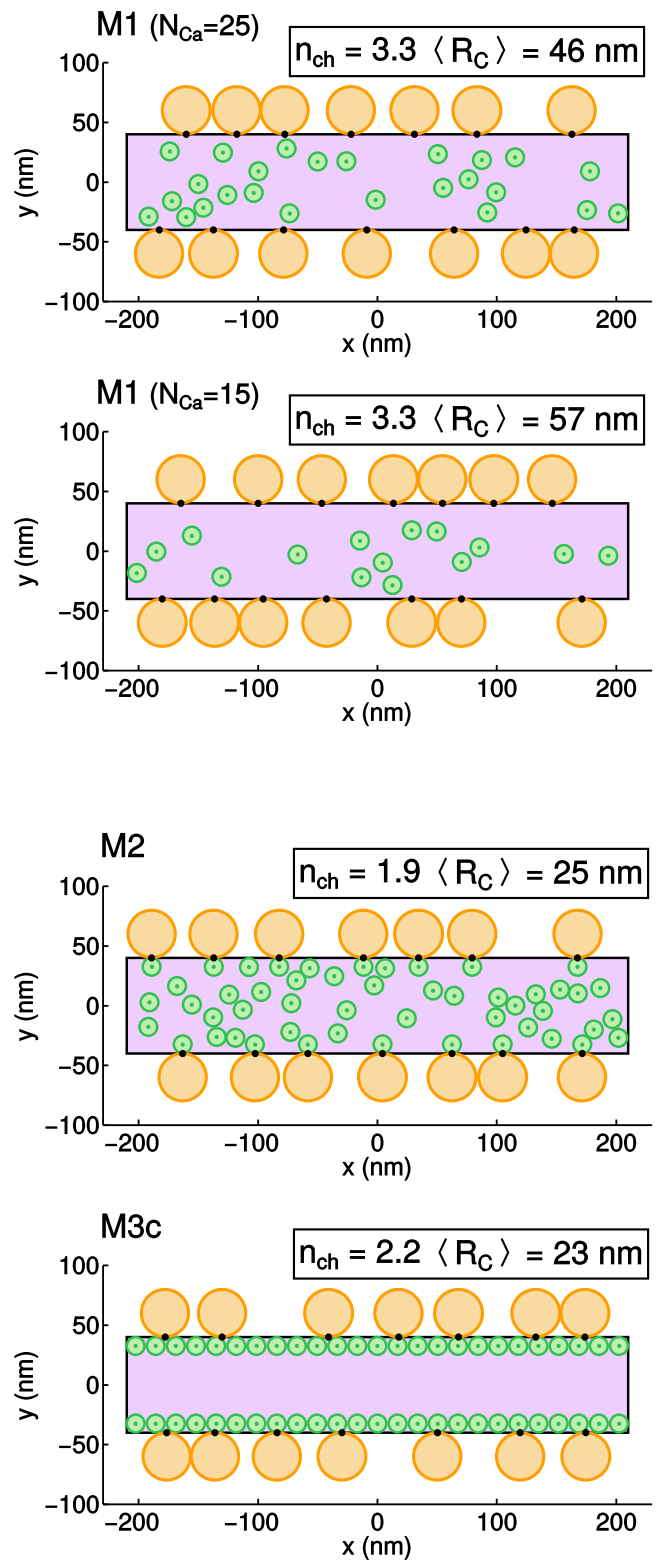

Figure 2.2: Refinement of the presynaptic AZ scenarios: part 1. (A) Different realizations of AZ scenario M1: $\mathrm{Ca}^{2+}$ channels distributed randomly within the presynaptic density. Different panels correspond to different number of channels. Estimates of the effective number of contributing channels and effective coupling distance are provided in insets of each panel. (B) AZ scenarios M3, M2, M3b, and M3c, which feature preferentially tight coupling of some of the $\mathrm{Ca}^{2+}$ channels to the RRP vesicles.

Guided by the above results, we next tried AZ topographies shown in Fig. 2.2B. Placement of only single nearby $\mathrm{Ca}^{2+}$ channel per RRP vesicle (scenario M3), reproduced $R_{c}=13 \mathrm{~nm}$ on average which is compatible with the experimental estimate $(17 \mathrm{~nm})$. However, the model estimate of exponent $m$ was $1.0\left(n_{c h}=1.1\right)$, considerably lower than 1.4 (Fig. 2.3B). We then found that $m$ of approximately 1.4 could be reproduced with such a placement of $\mathrm{Ca}^{2+}$ channels which results in the effective number of contributing channels, $n_{c h} \sim 2$ (see the original article 
of this paper for definition of $n_{c h}$ ). To preserve tight coupling, this could be achieved by either placing one more tightly coupled $\mathrm{Ca}^{2+}$ channel per vesicular release site (scenario M3b) or by adding more $\mathrm{Ca}^{2+}$ channels within the presynaptic density, which may be randomly distributed (scenario M2), or preferentially close to the edges of the presynaptic density (scenario M3c). Here, it is important to note that $n_{c h} \sim 2$ does not necessarily mean that there are exactly two physical channels close to each $\mathrm{Ca}^{2+}$ sensor of exocytosis. For example, in the case of scenario $\mathrm{M} 3 \mathrm{c}$, there are 3 to 4 channels that are rather close to the vesicle. However, due to the fact that $\left[\mathrm{Ca}^{2+}\right]$ decreases sharply with the distance from channel's mouth (Naraghi \& Neher, 1997), the contribution of the closest one or two channels is disproportionately large. It is interesting to note that $n_{c h}$ was approximately equal to 2 even for scenario M1 $\left(N_{C a} \sim 20\right)$, which could reproduce $m \sim 1.4$ (but not the right $R_{c}$ !). This signifies a tight relation between the variables $m$ and $n_{c h}$.

A

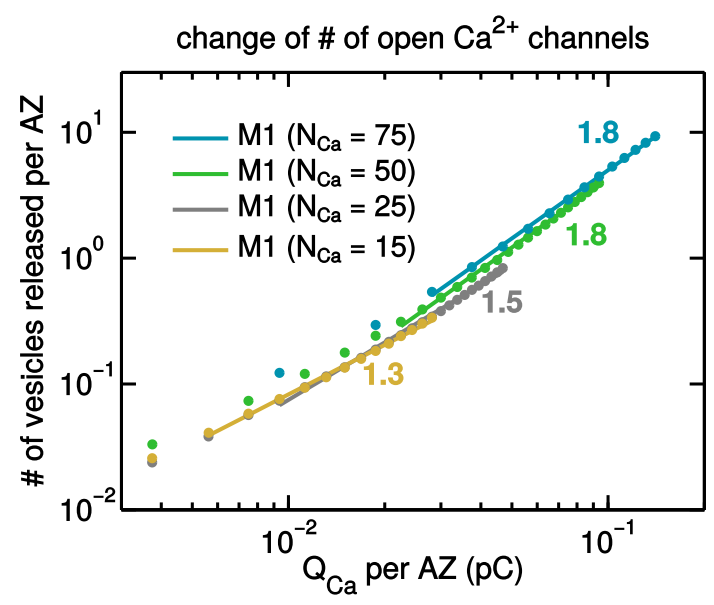

B

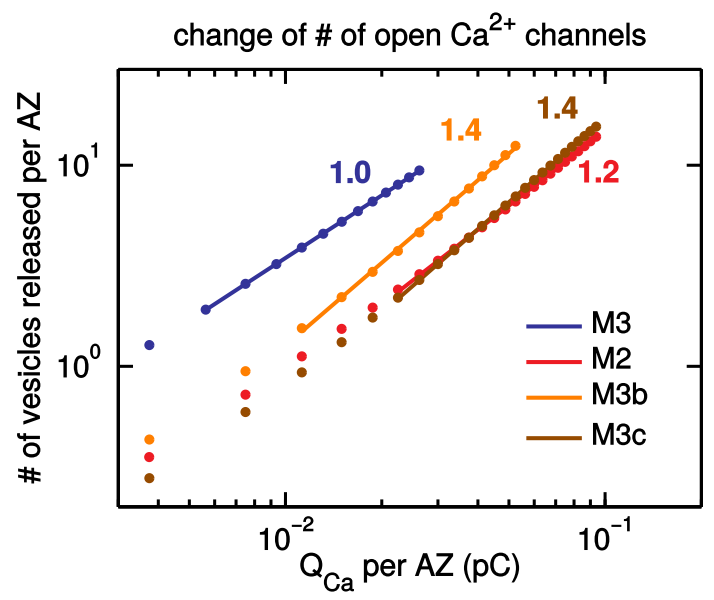

Figure 2.3: Refinement of the presynaptic AZ scenarios: part 2. (A) Exponents $m$ estimated for different realizations of AZ topography scenario M1 by varying the number of open presynaptic $\mathrm{Ca}^{2+}$ channels. The effect of intracellular $\mathrm{Ca}^{2+}$-buffers was approximated by $0.5 \mathrm{mM}$ BAPTA (the total concentration). (B) The same as (A), but for AZ scenarios M2, M3, M3b, and M3c.

Altogether, our findings suggest that vesicle fusion at IHC ribbon synapses is induced by two effective $\mathrm{Ca}^{2+}$ channels. One to two real channels are tightly coupled $(\lesssim 10 \mathrm{~nm})$ to each vesicle.

\section{Effect of diffusion barriers on presynaptic $\left[\mathrm{Ca}^{2+}\right]$}

In our simulations presented in the original article of this chapter, we did not take into account reflections of $\mathrm{Ca}^{2+}$ ions and buffer molecules from presynaptic vesicles. This is partially justified by the previous simulation results (Shahrezaei \& Delaney, 2004) which showed that $\left[\mathrm{Ca}^{2+}\right]$ is considerably increased due to a presynaptic vesicle acting as a diffusion barrier only if the vesicle 
is immediately (a few $\mathrm{nm}$ ) above the $\mathrm{Ca}^{2+}$ channel mouth. Thus, the reflections of $\mathrm{Ca}^{2+}$ ions could, at most, boost the contributions of those channels which are located just below the vesicles. Measures $m$ and $R_{c}$ do not allow to constrain the AZ topographies to such a certainty that the mentioned effect could be determined. Some researchers proposed that the synaptic ribbons could act as diffusion barriers as well (Roberts, 1994; Graydon et al., 2011). No evidence exists to support this idea so far. However, simulations showed that $\left[\mathrm{Ca}^{2+}\right]$ at the sensor of exocytosis could be elevated severalfold at hair cells from bullfrog sacculus (Roberts, 1994) or amphibian papilla (Graydon et al., 2011), if the ribbons were indeed reflective to $\mathrm{Ca}^{2+}$. It has to be noted, though, that synaptic ribbons of the aforementioned hair cells are much larger (wider) than the majority of ribbons observed in IHCs from mature mice (compare Fig. 1 from (Lenzi et al., 1999) and Fig. 2 of the original article of this chapter). Thus, our expectation was that ribbons at afferent IHC synapses should not influence $\left[\mathrm{Ca}^{2+}\right]$ at presynaptic AZs substantially even if they are reflective to $\mathrm{Ca}^{2+}$ ions and/or $\mathrm{Ca}^{2+}$ buffer molecules. In particular, we expect that the reflections from the ribbon do not affect the exponent $m$ values. Here, we provide additional results to evaluate the validity of this assumption.

To examine the effect of RRP vesicles and synaptic ribbon as diffusion barriers, we performed additional numerical simulations. Presynaptic AZ topography scenarios M1, M2, and M3 were considered as representatives of (relatively) loose and tight coupling between the $\mathrm{Ca}^{2+}$ channels and sensors of exocytosis. The spatiotemporal dynamics of $\mathrm{Ca}^{2+}$ was simulated by using CalC (version 7.7.4), a finite-difference solver of reaction-diffusion equations (Matveev et al., 2002). Our simulations featured a uniform 3D rectangular grid with $5 \mathrm{~nm}$ spatial resolution. Concentrations of $\mathrm{Ca}^{2+}$ at intermediate points in space were estimated by using the cubic spline interpolation. The simulation volume was defined by a rectangular box with the dimensions of $480 \mathrm{~nm} \times 820 \mathrm{~nm} \times 200 \mathrm{~nm}$. The AZ was positioned in the middle of the lower face of the box (dimensions $480 \mathrm{~nm} \times 820 \mathrm{~nm}$ ), so that the corresponding edges of both rectangles were in parallel. The lower face of the box was assumed to be reflective to $\mathrm{Ca}^{2+}$ ions and buffer molecules. Dirichlet boundary conditions were set on the remaining faces of the simulation volume, with concentrations of $\mathrm{Ca}^{2+}$ and buffer molecules fixed to their background levels $\left(\left[\mathrm{Ca}^{2+}\right]_{0}=50 \mathrm{~nm}\right.$, $\left.[\mathrm{BAPTA}]_{\mathrm{T}}=0.5 \mathrm{mM}\right)$. RRP vesicles, when treating them as diffusion barriers, were approximated by $30 \mathrm{~nm} \times 30 \mathrm{~nm} \times 30 \mathrm{~nm}$ cubes, placed in parallel with the plasma membrane, $5 \mathrm{~nm}$ above it. The ribbon was approximated by a rectangular box which was positioned with one of its faces in parallel with presynaptic density, $40 \mathrm{~nm}$ above the plasma membrane, as shown in Fig. 2.4A, B. The height of the ribbon was unlimited, i.e., was as high as allowed by the simulation volume. We considered two options for the ribbon width, $40 \mathrm{~nm}$ and $80 \mathrm{~nm}$, which correspond to half and full width of the presynaptic density respectively (Fig. 2.4A, B). While the first of them is supposed to account for "wedge" and "droplet" like ribbons (see Fig. 2 in the original publication of the present chapter), the second one represents the the "oval" ribbons. It has to be noted that, regarding its effect on $\mathrm{AZ}\left[\mathrm{Ca}^{2+}\right]$, the width of the ribbon is important only up to $100 \mathrm{~nm}$ or so above the plasma membrane. 


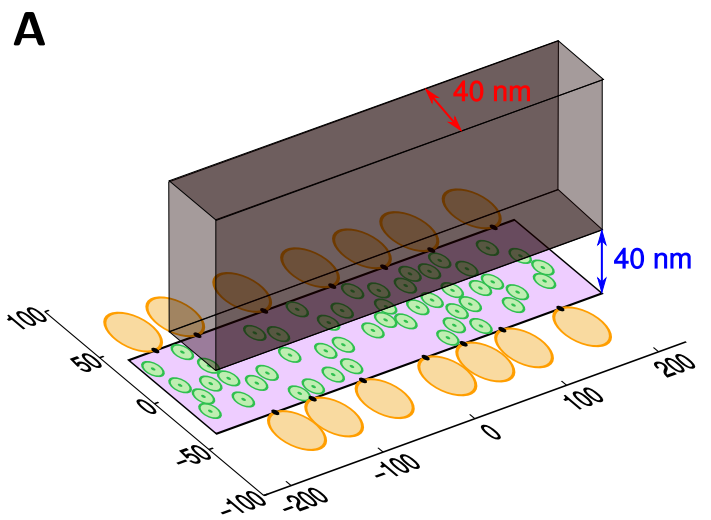

C

\begin{tabular}{|c|c|c|}
\hline M1 & $\begin{array}{l}\text { - vesicles } \\
\text { - ribbon }\end{array}$ & $\begin{array}{c}\text { + vesicles } \\
\text { + ribbon }\end{array}$ \\
\hline$\left\langle\left[\mathrm{Ca}^{2+}\right]_{{ }^{t}=+\infty}(\mu \mathrm{M})\right.$ & 54 & 66 \\
\hline $\mathrm{n}_{\mathrm{ch}}$ & 3.3 & 3.4 \\
\hline
\end{tabular}

\begin{tabular}{|c|c|c|}
\hline M2 & $\begin{array}{c}\text { - vesicles } \\
\text { - ribbon }\end{array}$ & $\begin{array}{c}\text { + vesicles } \\
\text { + ribbon }\end{array}$ \\
\hline$\left\langle\left[\mathrm{Ca}^{2+}\right]_{\bullet}\right\rangle_{t=+\infty}(\mu \mathrm{M})$ & 69 & 82 \\
\hline $\mathrm{n}_{\mathrm{ch}}$ & 1.9 & 2.0 \\
\hline
\end{tabular}

\begin{tabular}{|c|cc|}
\hline M3 & $\begin{array}{l}\text { - vesicles } \\
\text { - ribbon }\end{array}$ & $\begin{array}{c}\text { + vesicles } \\
\text { + ribbon }\end{array}$ \\
\hline$\left\langle\left[\mathrm{Ca}^{2+}\right]_{\bullet}\right\rangle_{\mathrm{t}=+\infty}(\mu \mathrm{M})$ & 37 & 40 \\
\hline $\mathrm{n}_{\mathrm{ch}}$ & 1.1 & 1.1 \\
\hline
\end{tabular}

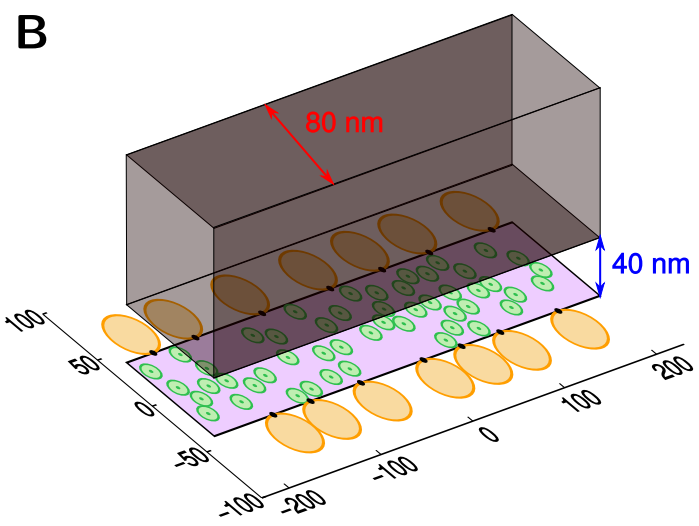

D

\begin{tabular}{|c|c|c|}
\hline M1 & $\begin{array}{l}\text { - vesicles } \\
\text { - ribbon }\end{array}$ & $\begin{array}{c}\text { + vesicles } \\
+ \text { ribbon }\end{array}$ \\
\hline$\left\langle\left[\mathrm{Ca}^{2+}\right]_{\bullet}\right\rangle_{t=+\infty}(\mu \mathrm{M})$ & 54 & 80 \\
\hline $\mathrm{n}_{\mathrm{ch}}$ & 3.3 & 3.6 \\
\hline
\end{tabular}

\begin{tabular}{|c|c|c|}
\hline M2 & $\begin{array}{c}\text { - vesicles } \\
\text { - ribbon }\end{array}$ & $\begin{array}{c}\text { + vesicles } \\
\text { + ribbon }\end{array}$ \\
\hline$\left\langle\left[\mathrm{Ca}^{2+}\right]_{\bullet}\right\rangle_{t=+\infty}(\mu \mathrm{M})$ & 69 & 96 \\
\hline $\mathrm{n}_{\mathrm{ch}}$ & 1.9 & 2.1 \\
\hline
\end{tabular}

\begin{tabular}{|c|c|c|}
\hline M3 & $\begin{array}{c}\text { - vesicles } \\
\text { - ribbon }\end{array}$ & $\begin{array}{c}\text { + vesicles } \\
\text { + ribbon }\end{array}$ \\
\hline$\left\langle\left[\mathrm{Ca}^{2+}\right]_{\bullet_{\mathrm{t}=+\infty}}(\mu \mathrm{M})\right.$ & 37 & 43 \\
\hline $\mathrm{n}_{\mathrm{ch}}$ & 1.1 & 1.2 \\
\hline
\end{tabular}

Figure 2.4: Effect of potential diffusion barriers on presynaptic $\left[\mathrm{Ca}^{2+}\right]$. (A) The geometry and placement of the synaptic ribbon with respect to the presynaptic density in the simulations. Width of the ribbon was set to $40 \mathrm{~nm}$ in this case. (B) The same as (A), but with the ribbon width set to $80 \mathrm{~nm}$. (C) Simulation results for AZ topography scenarios M1-3 corresponding to the ribbon geometry shown in (A). Entries of the columns "-vesicles -ribbon" correspond to the estimates of $n_{c h}$ and the time-averaged steady state $\left[\mathrm{Ca}^{2+}\right]$ by ignoring reflections of $\mathrm{Ca}^{2+}$ ions and buffer molecules from the ribbon and RRP vesicles. Whereas entries of the columns "+vesicles +ribbon" contain estimates of the same variables obtained with the aforementioned reflections taken into account. (D) the same as (C), but assuming the ribbon geometry shown in (B). 
As in the original article of the present chapter, we considered responses to depolarizations to $-17 \mathrm{mV}$, which evoke maximal $\mathrm{Ca}^{2+}$ influx. In order to calculate the averaged steady state $\left[\mathrm{Ca}^{2+}\right]$, we fixed all $\mathrm{Ca}^{2+}$ channels in their open states and set the current flowing through each of these channels to a product of a nominal single channel current $(0.3 \mathrm{pA})$ multiplied by the open probability of the channel (0.32). The steady state $\left[\mathrm{Ca}^{2+}\right]$ at the sensors of exocytosis was then calculated $2 \mathrm{~ms}$ after the stimulus onset, when initial equilibration of $\left[\mathrm{Ca}^{2+}\right]$ was reached. 20 random realizations of each of the three AZ topography scenarios M1-3 were considered and the results presented here represent average values over those 20 realizations. All the remaining parameters of the model were set to the same values as in the original article of this chapter. To decrease the computation time to a convenient level (order of a few hours), each realization was run on separate CPU cores in parallel (60 cores in total).

The simulation results are summarized in Fig. 2.4 C, D. When the ribbon width was set to $40 \mathrm{~nm}$, The elevations of $\left[\mathrm{Ca}^{2+}\right]$ at the sensor of exocytosis were $22 \%, 19 \%$, and $8 \%$ for scenarios M1, M2, and M3 respectively. These estimates increased to, respectively, 48\%, 39\%, and $16 \%$ when the ribbon width of $80 \mathrm{~nm}$ was assumed. In both cases, changes of the effective number of contributing channels, $n_{c h}$, were negligible, suggesting that the diffusion barriers proportionally scale up the contribution of all channels to $\left[\mathrm{Ca}^{2+}\right]$ at the sensors of exocytosis to a certain degree. Taking into account the strong correlation between $n_{c h}$ and exponent $m$ based on the change of the number of open channels (see previous section), these results suggest no considerable effect of the ribbon on the $m$ estimates for AZ topography scenarios considered in our work. 


\section{Chapter 3}

\section{EF-hand Protein $\mathrm{Ca}^{2+}$ Buffers Regulate $\mathrm{Ca}^{2+}$ Influx and Exocytosis in Sensory Hair Cells}

\section{Citation}

T. Pangršič*, M. Gabrielaitis*, S. Michanski, B. Schwaller, F. Wolf, N. Strenzke, and T. Moser. EF-hand protein $\mathrm{Ca}^{2+}$ buffers regulate $\mathrm{Ca}^{2+}$ influx and exocytosis in sensory hair cells. Proc Natl Acad Sci USA 112 (9): E1028 - E103\%. (c) 2015 The authors.

* - equal contribution.

\section{Detailed contributions of M. Gabrielaitis}

(1) Designed and performed mathematical modeling for estimating concentrations of the endogenous $\mathrm{Ca}^{2+}$ buffers and the effective coupling distance between presynaptic $\mathrm{Ca}^{2+}$ channels and sensors of exocytosis based on the experimental data. (2) Performed mathematical modeling for interpreting the physical meaning of the effective coupling distance. (3) Designed and performed mathematical modeling for predicting the sustained SGN spiking rates from the experimental data of exocytic capacitance increments, and evaluating the influence of the refractory periods of SGNs on them. (4) Analyzed all results of the mathematical modeling. (5) Analyzed some of the experimental data: 1) estimated the refractory periods (see Fig. S5), 2) fitted the data of the temporal profiles of exocytic plasma membrane capacitance increments as shown in Fig. S2A, 3) fitted the data of the $\Delta \mathrm{C}_{\mathrm{m}}$ vs $\mathrm{Q}_{\mathrm{Ca}}$ dependencies in wt and tko IHCs as shown in Fig. S6. (6) Prepared figures 8, 9, S2, S5, S6, S7, S8, S9, S10, S11, S12. (7) Together with the coauthors, wrote the main part of the article. Prepared the supplementary material with contributions by the coauthors.

\section{Supplementary material}

Eight sections of supplementary material, which is a part of the original publication, follow the 
main body of the article. Important experimental data (sections $1-6$ ), information on the mathematical model, and modeling results (sections $7-8$ ), which could not be included in the main text due to the space limitations, are provided there. 


\title{
EF-hand protein $\mathrm{Ca}^{2+}$ buffers regulate $\mathrm{Ca}^{2+}$ influx and exocytosis in sensory hair cells
}

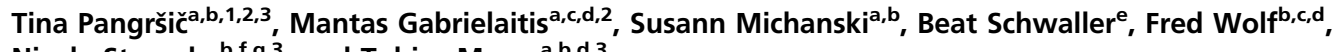 \\ Nicola Strenzke $e^{b, f, g, 3}$, and Tobias Moser ${ }^{a, b, d, 3}$
}

${ }^{a}$ Institute for Auditory Neuroscience and InnerEarLab, University Medical Center Göttingen, 37099 Göttingen, Germany; ${ }^{b}$ Collaborative Research Center 889, University of Göttingen, 37099 Göttingen, Germany; ${ }^{\circ}$ Theoretical Neurophysics Group, Max Planck Institute for Dynamics and Self-Organization, 37077 Göttingen, Germany; ${ }^{\mathrm{d} B e r n s t e i n}$ Center for Computational Neuroscience, Göttingen University, 37077 Göttingen, Germany; ${ }^{\mathrm{e}}$ Unit of Anatomy, Department of Medicine, University of Fribourg, 1700 Fribourg, Switzerland; ${ }^{f}$ Auditory Systems Physiology Group, InnerEarLab, Department of Otolaryngology, University Medical Center Göttingen, 37099 Göttingen, Germany; and ${ }^{9}$ Eaton Peabody Laboratory, Massachusetts Eye and Ear Infirmary, Harvard University, Boston, MA 02114

Edited* by A. J. Hudspeth, The Rockefeller University, New York, NY, and approved January 26, 2015 (received for review August 25, 2014)

EF-hand $\mathrm{Ca}^{2+}$-binding proteins are thought to shape the spatiotemporal properties of cellular $\mathrm{Ca}^{2+}$ signaling and are prominently expressed in sensory hair cells in the ear. Here, we combined genetic disruption of parvalbumin- $\alpha$, calbindin-D28k, and calretinin in mice with patch-clamp recording, in vivo physiology, and mathematical modeling to study their role in $\mathrm{Ca}^{2+}$ signaling, exocytosis, and sound encoding at the synapses of inner hair cells (IHCs). IHCs lacking all three proteins showed excessive exocytosis during prolonged depolarizations, despite enhanced $\mathrm{Ca}^{2+}$-dependent inactivation of their $\mathrm{Ca}^{2+}$ current. Exocytosis of readily releasable vesicles remained unchanged, in accordance with the estimated tight spatial coupling of $\mathrm{Ca}^{2+}$ channels and release sites (effective "coupling distance" of $17 \mathrm{~nm}$ ). Substitution experiments with synthetic $\mathrm{Ca}^{2+}$ chelators indicated the presence of endogenous $\mathrm{Ca}^{2+}$ buffers equivalent to $1 \mathrm{mM}$ synthetic $\mathrm{Ca}^{2+}$-binding sites, approximately half of them with kinetics as fast as 1,2-Bis(2-aminophenoxy)ethane$\mathrm{N}, \mathrm{N}, \mathrm{N}^{\prime}, \mathrm{N}^{\prime}$-tetraacetic acid (BAPTA). Synaptic sound encoding was largely unaltered, suggesting that excess exocytosis occurs extrasynaptically. We conclude that EF-hand $\mathrm{Ca}^{2+}$ buffers regulate presynaptic IHC function for metabolically efficient sound coding.

calcium buffers | exocytosis | calcium current | synapse | hair cell

ntracellular $\mathrm{Ca}^{2+}$ signaling regulates a multitude of cellular processes. In sensory hair cells, $\mathrm{Ca}^{2+}$ is crucial for electrical frequency tuning, afferent synaptic transmission, and efferent modulation (reviewed in ref. 1). To separate these signaling pathways and maintain high temporal fidelity of neurotransmission, $\mathrm{Ca}^{2+}$ signals must be temporally limited and spatially confined to the site of action. Cells typically achieve this by localizing $\mathrm{Ca}^{2+}$ entry and by rapidly removing free $\mathrm{Ca}^{2+}$ ions via binding to cytosolic "buffers" and finally $\mathrm{Ca}^{2+}$ extrusion (2-4). Of the various EF-hand $\mathrm{Ca}^{2+}$-binding proteins, some seem to function primarily as $\mathrm{Ca}^{2+}$-dependent signaling proteins (e.g., calmodulin and $\mathrm{Ca}^{2+}$ binding proteins $1-8, \mathrm{CaBP} 1-8)$, whereas others [parvalbumin- $\alpha$ $(\mathrm{PV} \alpha)$, calbindin-D28k $(\mathrm{CB})$, and calretinin $(\mathrm{CR})]$ are thought to mainly serve as mobile $\mathrm{Ca}^{2+}$ buffers.

Hair cells of various species strongly express the $\mathrm{Ca}^{2+}$-binding proteins $\mathrm{PV}, \mathrm{CB}$, and, in some cases, CR. This possibly reflects the need for buffers with different biophysical properties to functionally isolate different $\mathrm{Ca}^{2+}$ signaling mechanisms, which are spatially not well separated in these compact epithelial cells. $\mathrm{Ca}^{2+}$-binding proteins are particularly abundant in frog and chicken hair cells, which contain millimolar concentrations of parvalbumin-3 (5) as well as of CR $(6,7)$. An immune-EM study in rats indicated hundreds of micromolar of proteinaceous $\mathrm{Ca}^{2+}$-binding sites in inner hair cells (IHCs) (8). A patch-clamp study in gerbil IHCs reported endogenous buffers equivalent to approximately $0.4 \mathrm{mM}$ 1,2-Bis(2aminophenoxy)ethane-N,N, $\mathbf{N}^{\prime}, \mathbf{N}^{\prime}$-tetraacetic acid (BAPTA) (9). Genetic deletion of the EF-hand $\mathrm{Ca}^{2+}$-binding proteins $\mathrm{PV} \alpha, \mathrm{CB}$, and $\mathrm{CR}$ in mice has facilitated the analysis of their function (10-13; reviewed in ref. 14), but the combined deletion of these proteins remains to be studied. IHCs provide an experimentally wellaccessible presynaptic preparation that uses all three. Here, we studied IHC function and hearing in mice lacking the three buffers [triple buffer KO (TKO); $\mathrm{Pv}^{-/-} \mathrm{Cb}^{-/-} \mathrm{Cr}^{-/-}$]. By using perforated and ruptured-patch recordings, we analyzed voltage-gated $\mathrm{Ca}^{2+}$ currents and exocytosis of $\mathrm{Pv}^{-/-} \mathrm{Cb}^{-/-} \mathrm{Cr}^{--}$IHCs, in which we also substituted the deleted endogenous buffers with the synthetic $\mathrm{Ca}^{2+}$ chelators EGTA or BAPTA. Auditory systems function was probed by measuring otoacoustic emissions and auditory brainstem responses (ABRs) as well as by recordings from single spiral ganglion neurons (SGNs). We performed mathematical modeling to estimate concentrations of the endogenous mobile $\mathrm{Ca}^{2+}$ buffers and to better understand how these proteins control exocytosis at IHC synapses. We conclude that the endogenous buffer capacity of IHCs is well approximated by $1 \mathrm{mM}$ synthetic $\mathrm{Ca}^{2+}{ }^{+}$-binding sites with different kinetics. A tight spatial coupling between $\mathrm{Ca}^{2+}$ channels and sensors of exocytosis $\left(\mathrm{Ca}^{2+}\right.$ channel-exocytosis coupling) precludes interference of PV $\alpha, \mathrm{CB}$, and CR with fusion of the readily releasable pool of vesicles (RRP). Instead, we suggest that these buffers jointly regulate IHC presynaptic function by restricting neurotransmitter release to active zones (AZs).

Significance
$\mathrm{Ca}^{2+}$ ions serve as a key cellular signal and are tightly con-
trolled. One mechanism to limit free $\mathrm{Ca}^{2+}$ ions is buffering by
$\mathrm{Ca}^{2+}$-binding proteins, which are strongly expressed in sensory
hair cells of the ear. Here we studied how genetic disruption of
the $\mathrm{Ca}^{2+}$-binding proteins parvalbumin- $\alpha$, calbindin-D28k, and
calretinin affects exocytosis and sound encoding at the syn-
apses of mouse inner hair cells (IHCs) and spiral ganglion
neurons (SGNs). Mutant IHCs showed increased exocytosis, but
the sound-evoked spiking activity in SGNs was unaltered. To-
gether with mathematical modeling, this finding indicates that
a large fraction of exocytosis in mutant IHCs occurred outside
synapses. We conclude that Ca ${ }^{2+}$-binding proteins shape pre-
synaptic Ca ${ }^{2+}$ signals to restrict exocytosis to active zones, thus
enabling metabolically efficient sound encoding.

Author contributions: T.P., M.G., F.W., N.S., and T.M. designed research; T.P., S.M., and N.S. performed experiments; M.G. performed mathematical modeling; T.P., M.G., S.M., and N.S. analyzed data; T.P., M.G., B.S., F.W., N.S., and T.M. wrote the paper; and B.S. generated and characterized mouse knockouts.

The authors declare no conflict of interest.

*This Direct Submission article had a prearranged editor

'Present address: Synaptic Physiology of Mammalian Vestibular Hair Cells Junior Research Group, Institute for Auditory Neuroscience and InnerEarLab, University Medical Center Göttingen, 37099 Göttingen, Germany.

${ }^{2}$ T.P. and M.G. contributed equally to this work.

${ }^{3}$ To whom correspondence may be addressed. Email: tpangrs@gwdg.de, nstrenzke@med. uni-goettingen.de, or tmoser@gwdg.de.

This article contains supporting information online at www.pnas.org/lookup/suppl/doi:10. 1073/pnas.1416424112/-/DCSupplemental. 


\section{Results}

IHCs of Hearing Mice Express PV $\boldsymbol{\alpha}, \mathbf{C B}$, and CR. Hair cells in various species show specific expression patterns of the EF-hand $\mathrm{Ca}^{2+}$. binding proteins $\mathrm{PV} \alpha$, parvalbumin- $\beta$ (PV $\beta$ ) (oncomodulin), $\mathrm{CB}$, and $\mathrm{CR}(5,7,8,15)$, hereafter termed "mobile $\mathrm{Ca}^{2+}$ buffers" so as not to ignore the presence of other $\mathrm{Ca}^{2+}$ buffers such as ATP. We performed immunohistochemistry on apical organs of Corti of hearing C57BL/6 mice [postnatal day (P) 14-26] to characterize the expression of mobile $\mathrm{Ca}^{2+}$ buffers in mouse cochlear hair cells (Fig. 1). In agreement with results obtained in rats (8), we found mature mouse IHCs to express $\mathrm{PV} \alpha, \mathrm{CB}$, and CR (Fig. $1 A-C)$, but not PV $\beta$, which was strongly expressed in outer hair cells (OHCs) (Fig. 1D). Parallel immunostaining of organs of Corti from age-matched $\mathrm{Pv}^{-/-} \mathrm{Cb}^{-/-} \mathrm{Cr}^{-/-}$mice confirmed the absence of these three mobile $\mathrm{Ca}^{2+}$ buffers from all tissues (Fig. $1 E-G$ ) but the persistent expression of PV $\beta$ in OHCs (Fig. $1 H$ ).

Disruption of $\mathrm{PV} \alpha, C B$, and $\mathrm{CR}$ Increases the Amplitude and Inactivation of $\mathrm{Ca}^{2+}$ Current and Enhances Exocytosis in IHCs. Next, we studied the presynaptic function of $\mathrm{Pv}^{-/-} \mathrm{Cb}^{-/-} \mathrm{Cr}^{-/}$ IHCs by patch-clamp recordings of $\mathrm{Ca}^{2+}$ currents and exocytosis. We first examined the amplitude, voltage dependence, activation, and inactivation of the $\mathrm{IHC} \mathrm{Ca}{ }^{2+}$ current that is largely mediated by Cav1.3 channels (16-18). We used perforated-patch recordings to not interfere with the endogenous $\mathrm{Ca}^{2+}$ buffering. We found a $20 \%$ increase of the initial $\mathrm{Ca}^{2+}$ current, whereas its voltagedependence was unchanged (Fig. $2 A$ ). $\mathrm{Ca}^{2+}$-current kinetics were analyzed at the potential eliciting the peak $\mathrm{Ca}^{2+}$ current (on average at $-17 \mathrm{mV}$ ). The activation time constants were comparable between $\mathrm{Pv}^{-/-} \mathrm{Cb}^{-/-} \mathrm{Cr}^{-/-}$and WT $\left(\mathrm{Pv}^{+/+} \mathrm{Cb}^{+/+} \mathrm{Cr}^{+/+}\right)$IHCs (Fig. $2 B$ ). However, we observed a significant increase of $\mathrm{Ca}^{2+}$-current inactivation (nonnormalized currents; Fig. $2 C$ ). Inactivation was first assessed by analyzing the ratio of the current amplitude at the end of a 100-ms-long depolarization and the initial current amplitude. This ratio was smaller in $\mathrm{Pv}^{-/-} \mathrm{Cb}^{-/-} \mathrm{Cr}^{-/-}$than in $\mathrm{Pv}^{+/+}$ $\mathrm{Cb}^{+/+} \mathrm{Cr}^{+/+}$IHCs (mean \pm SEM, $0.71 \pm 0.02$ vs. $0.79 \pm 0.01 ; P=$

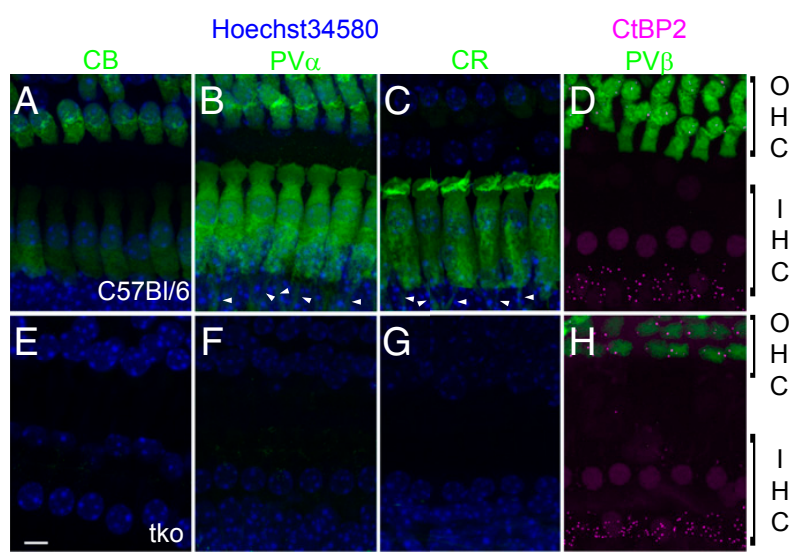

Fig. 1. IHCS of hearing mice express the EF-hand $\mathrm{Ca}^{2+}$-binding proteins $\mathrm{PV} \alpha_{\text {, }}$ $C B$, and $C R$. $(A-H)$ Maximum projections of confocal stacks from wholemount explants of the apical coils of P14-P26 organs of Corti of WT (Top) and $\mathrm{PV}^{-1-} \mathrm{Cb}^{-1-} \mathrm{Cr}^{-1-}$ (TKO; Bottom) mice immunolabeled for calbindin-D28k (CB; $A$ and $E)$, parvalbumin- $\alpha(P V \alpha ; B$ and $F)$, calretinin (CR; $C$ and $G)$, and parvalbumin- $\beta$ ( $P V \beta ; D$ and $H$, all in green). An antibody against $C$ tBP2 was used as a marker of presynaptic ribbons and cell nuclei (magenta, $D$ and $H$ ). In all other panels, cell nuclei were stained with Hoechst 34580 (blue). IHC bodies of WT but not TKO organs of Corti are homogeneously stained for three $\mathrm{Ca}^{2+}$ buffers, $C B, P V \alpha$, and $C R$. OHCs additionally contain PV $\beta$, not present in IHCs and not targeted for deletion in the TKO mice. PV $\alpha$ and CR show immunoreactivity also in the afferent fibers (arrowheads). (Scale bar: $5 \mu \mathrm{m}$.)
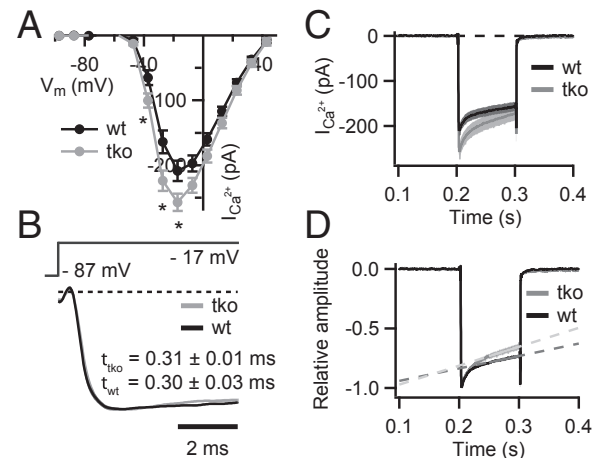

Fig. 2. Disruption of $P V_{\alpha}, C B$, and $C R$ enhances $\mathrm{Ca}^{2+}$-current amplitude and inactivation. $(A)$ Current-voltage relationship of the voltage-gated $\mathrm{Ca}^{2+}$ current in TKO (gray, $n=20$ ) and WT (black, $n=23$ ) IHCs from 2-3-wk-old mice. Note the slightly larger $\mathrm{Ca}^{2+}$ currents in the TKO IHCs $\left({ }^{*} P=0.01-0.03\right.$, Student $t$ test). (B) Normalized average $\mathrm{Ca}^{2+}$ currents in response to $10-100-\mathrm{ms}$ depolarizations to the peak $\mathrm{Ca}^{2+}$-current potential on an expanded time scale demonstrate that the kinetics of the activation were not different among TKO and WT IHCS $(P=0.13$, Wilcoxon rank-sum test). Data were fitted with $I(t)=I_{0}+I_{\max } \times\left(1-e^{-t / \tau}\right)^{p}$, whereby the power $(p)$ was fixed to 2 in most cases. ( $C$ and $D$ ) Absolute $(C)$ and normalized $(D) \mathrm{Ca}^{2+}$ currents in response to $100-\mathrm{ms}$ depolarizations to the peak $\mathrm{Ca}^{2+}$ current potential. A stronger $\mathrm{Ca}^{2+}$-current inactivation was observed in the TKO IHCs. Slopes of the linear fits $(1 / \mathrm{s})$ are $1.0 \pm 0.1$ and $1.6 \pm 0.2$ for the $\mathrm{Ca}^{2+}$ currents in WT $(n=$ 16) and TKO $(n=14) \mathrm{IHCS}$, respectively $(P=0.02$, Student $t$ test).

0.01, Wilcoxon rank-sum test). As a second measure of the $\mathrm{Ca}^{2+}$ current inactivation, we fitted a linear function to the last $50 \mathrm{~ms}$ of the normalized $\mathrm{Ca}^{2+}$ currents. Its steeper slope for $\mathrm{Pv}^{-1} \mathrm{Cb}^{-/-} \mathrm{Cr}^{-/-}$ IHCs corroborated the notion of a more pronounced inactivation (Fig. 2D).

To address potential mechanisms underlying the increased $\mathrm{Ca}^{2+}$-current amplitude, we evaluated the number and morphological appearance of the ribbon synapses in IHCs after hearing onset (P15-P18). The presynaptic ribbon protein Ribeye/CtBP2 and the postsynaptic glutamate receptor GluA $2 / 3$ were visualized by confocal microscopy of immunolabeled organs of Corti (19). We found comparable numbers of ribbons $(12.5 \pm 0.9$ and $13.9 \pm$ 0.5; SI Appendix, Fig. S1), glutamate receptor clusters (12.5 \pm 0.8 and $13.4 \pm 1.1)$, as well as ribbon synapses $(12.0 \pm 0.9$ and $12.6 \pm$ 0.9 in six WT and five TKO organs of Corti, respectively). This argues against a potential up-regulation of synapse number as a cause for the increased $\mathrm{Ca}^{2+}$ current. It also rules out an excitotoxic degradation of synapses that one might have expected from potentially enhanced glutamate release in the absence of the three $\mathrm{Ca}^{2+}$ buffers.

We then studied depolarization-induced exocytosis by using membrane capacitance $\left(C_{\mathrm{m}}\right)$ measurements, first in perforatedpatch experiments and second in ruptured-patch recordings from $\mathrm{Pv}^{-/} \mathrm{Cb}^{-/-} \mathrm{Cr}^{-/-}$IHCs with varying concentrations of BAPTA or EGTA in the pipette solution. To probe for potential effects of $\mathrm{Ca}^{2+}$-buffer deficiency on exocytosis, cells were depolarized for different durations and $C_{\mathrm{m}}$ was measured before and after depolarization (Fig. 3). Interestingly, exocytosis upon short stimuli was not significantly altered (Fig. $3 C$ and $D$ ), a finding that can, according to our mathematical model, best be explained by tight $\mathrm{Ca}^{2+}$ channel-exocytosis coupling (as detailed later). However, we found more exocytosis in the $\mathrm{Pv}^{-/-} \mathrm{Cb}^{-/-} \mathrm{Cr}^{-/-}$IHCs for longer stimuli $\left(P<0.05\right.$ for $\Delta C_{\mathrm{m}, 100 \mathrm{~ms}}$; Fig. $3 C$; figure legends provide $P$ values of other $\Delta C_{\mathrm{m}}$ measurements) despite comparable $\mathrm{Ca}^{2+}$-charge transfer. The $\Delta C_{\mathrm{m}}$ amplitude was more variable for 200-ms depolarizations, but the higher efficiency of $\mathrm{Ca}^{2+}$ influx to drive sustained exocytosis in $\mathrm{P}^{-1-} \mathrm{Cb}^{-/-} \mathrm{Cr}^{-1-}$ IHCs can readily be appreciated from the ratio of exocytic increments over 
$A$

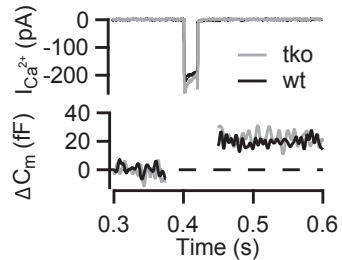

C

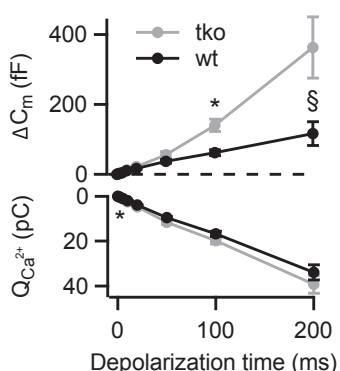

E

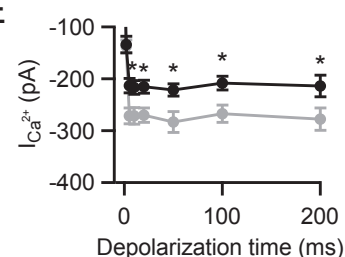

G

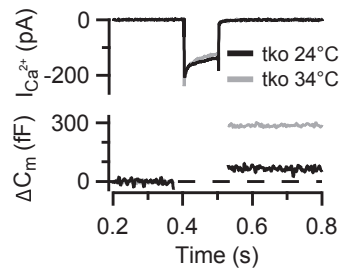

$\mathrm{B}$

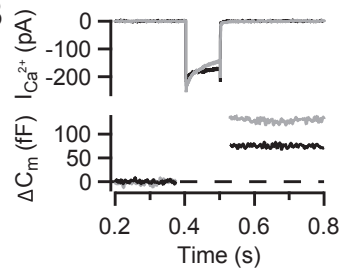

D

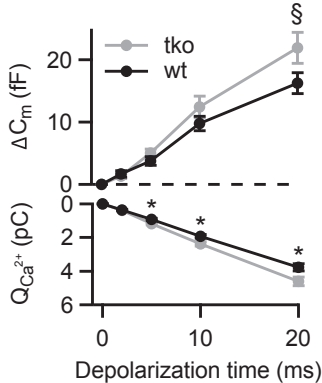

$\mathrm{F}$

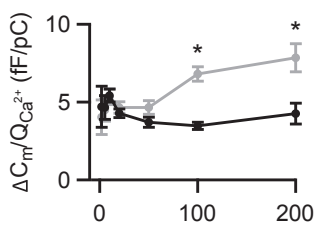

Depolarization time (ms)

$\mathrm{H}$

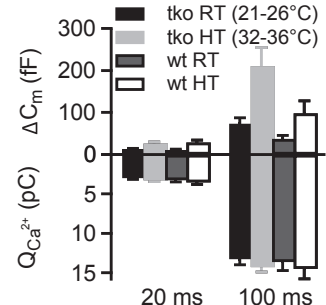

significantly larger in $\mathrm{Pv}^{-/-} \mathrm{Cb}^{-/-} \mathrm{Cr}^{-/-} \mathrm{IHCs}$ as a result of stronger $\mathrm{Ca}^{2+}$-current inactivation (Fig. 3C, Lower). Sustained exocytosis tended to be enhanced for $\mathrm{Pv}^{-/-} \mathrm{Cb}^{-/-} \mathrm{Cr}^{-/-}$IHCs also in near physiological conditions $\left(>32^{\circ} \mathrm{C}, 2 \mathrm{mM}\right.$ extracellular $\left.\left[\mathrm{Ca}^{2+}\right]\right)$, indicating that this likely also occurs in vivo (Fig. $3 G$ and $H$ ). We conclude that the mobile $\mathrm{Ca}^{2+}$ buffers $\mathrm{PV} \alpha, \mathrm{CB}$, and $\mathrm{CR}$ contribute to the regulation of presynaptic $\mathrm{Ca}^{2+}$ influx and exocytosis.

To approximate the concentration and binding kinetics of the mobile $\mathrm{Ca}^{2+}$ buffers of IHCs, we studied the effects of substituting endogenous $\mathrm{Ca}^{2+}$ buffers by BAPTA ("fast binding": association rate constant $k_{\text {on }}=4 \times 10^{8} \mathrm{M}^{-1} \mathrm{~s}^{-1}$; see ref. 20) or EGTA ("slow binding": $k_{\text {on }}=4.5 \times 10^{6}$ or $10^{7} \mathrm{M}^{-1} \mathrm{~s}^{-1}$; see refs. 20, 21) on RRP exocytosis (probed by 20-ms depolarizations) (22) and sustained exocytosis (approximated by the difference between the responses to $100-$ and 20 -ms depolarizations). Synthetic buffers were loaded into $\mathrm{Pv}^{-1-} \mathrm{Cb}^{-1-} \mathrm{Cr}^{-/-}$IHCs to restore the exocytic responses of $\mathrm{Pv}^{+/+} \mathrm{Cb}^{+++} \mathrm{Cr}^{+/+}$IHCs in their native buffering conditions (Fig. 4). We found that $1 \mathrm{mM}$ EGTA or BAPTA reduced sustained exocytosis in $\mathrm{Pv}^{-/-} \mathrm{Cb}^{-1-} \mathrm{Cr}^{-/-}$IHCs to levels comparable to $\mathrm{Pv}^{+++} \mathrm{Cb}^{+/+} \mathrm{Cr}^{+/+}$IHCs recorded in perforated-patch recordings (Fig. $4 B)$. BAPTA $(1 \mathrm{mM})$ best matched to the exocytic responses of $\mathrm{Pv}^{+/+} \mathrm{Cb}^{+++} \mathrm{Cr}^{+/+}$IHC for prolonged depolarizations, but significantly diminished RRP exocytosis (Fig. $4 A$ ). Lower concentrations of fast or slow $\mathrm{Ca}^{2+}$ buffer, on the contrary, left RRP exocytosis unchanged, but could not reinstate the sustained exocytic response as seen in $\mathrm{Pv}^{+/+} \mathrm{Cb}^{+/+} \mathrm{Cr}^{+/+}$IHCs. From these experiments, we conclude that the endogenous concentration of mobile buffers in IHCs is equivalent to $\sim 1 \mathrm{mM}$ synthetic $\mathrm{Ca}^{2+}$-binding sites, half of them with kinetics as fast as BAPTA. Of note, none of the tested concentrations of EGTA or BAPTA was able to accurately restore the exocytic responses as observed in $\mathrm{Pv}^{++} \mathrm{Cb}^{+++} \mathrm{Cr}^{++/} \mathrm{IHCs}$.

The Absence of PV $\alpha, C B$, and CR Has Little Impact on Hearing. We first tested cochlear amplification by measuring distortion product otoacoustic emissions (DPOAEs), which were found to be intact in $\mathrm{Pv}^{-/-} \mathrm{Cb}^{-/-} \mathrm{Cr}^{-/}$mice (Fig. $5 \mathrm{~A}$ and $\mathrm{B}$ ). We then investigated the synchronized neuronal signaling along the auditory pathway by measuring ABRs (Fig. $5 C$ and $D$ ). Over the time course of the project, we encountered variable ABR phenotypes of $\mathrm{Pv}^{-/} \mathrm{Cb}^{-/-}$

Fig. 3. Enhanced sustained exocytosis in the absence of $P V_{\alpha}, C B$, and $C R$. $(A$ and $B)$ Representative $\mathrm{Ca}^{2+}$ currents $(T o p)$ and $C_{m}\left(\Delta C_{m}\right)$ responses (Bottom) of TKO (gray) and WT (black) IHCs to $(A) 20$-ms and $(B)$ 100-ms depolarization to peak $\mathrm{Ca}^{2+}$-current potential, recorded in the perforated-patch configuration. (C) Exocytosis ( $\Delta C_{m i}$ Top) and the corresponding $\mathrm{Ca}^{2+}$-current integrals $\left(Q_{\mathrm{Ca}} ;\right.$ Bottom) as a function of duration of depolarizations to peak $\mathrm{Ca}^{2+}$-current potential. The $C_{m}$ increase in response to 100 -ms depolarization $\left(\Delta C_{m}, 100 m s\right)$ was significantly higher in TKO (gray, $\left.n=24\right)$ compared with WT IHCs [black, $n=23$; ${ }^{*} P=0.0003$, Wilcoxon rank-sum test; ${ }^{\S} P=0.06$, Wilcoxon rank-sum test $(C)$ and Student $t$ test $(D)]$. Note larger $Q_{C a}$ in TKO IHCS at short but not long depolarization $\left({ }^{*} P=0.009\right.$ for $5 \mathrm{~ms}$ and $P=0.02$ for 10 - and 20 ms depolarization, Student $t$ test). All responses are given as grand averages (calculated from the means of the individual cells) $\pm \mathrm{SEM}$. (D) $\Delta C_{m}$ (Top) and $Q_{C a}(B o t t o m)$ in response to short depolarizations evoking the exocytosis of the RRP displayed on an expanded time scale. $(E) \mathrm{Ca}^{2+}$-current amplitudes $\left(I_{C_{a}}\right)$ were significantly increased in the TKO IHCs $(* P<0.0004$, Student $t$ test). $(F)$ The $\mathrm{Ca}^{2+}$ efficiency of exocytosis was higher in the TKO IHCs for long depolarizations $\left({ }^{*} P=1.5 \times 10^{-10}\right.$ for $100 \mathrm{~ms}$ and $P=0.007$ for $200 \mathrm{~ms}$ depolarization, Wilcoxon rank-sum test). The extracellular $\left[\mathrm{Ca}^{2+}\right]$ was $5 \mathrm{mM}$. (G) Representative $\mathrm{Ca}^{2+}$ currents (Top) and $\Delta C_{m}$ (Bottom) of a TKO IHCs recorded at room (black) or close to physiological temperature (gray) with $2 \mathrm{mM}$ extracellular $\left[\mathrm{Ca}^{2+}\right] .(H)$ Average $\Delta C_{\mathrm{m}}$ and $Q_{\mathrm{Ca}}$ in response to 20 - and $100-\mathrm{ms}$ depolarizations recorded at room temperature (RT; black and dark gray) and high temperature (HT; close to physiological temperature, light gray and white) in WT $(n=4)$ and TKO $(n=5)$ IHCs. Note a two- to threefold increase in $\Delta C_{m}$ responses upon increase in temperature in the IHCs of both genotypes.

$\mathrm{Ca}^{2+}$-current integrals (Fig. $3 F$ ). Despite increased initial $\mathrm{Ca}^{2+}$ current, the $\mathrm{Ca}^{2+}$ charge transfer for long depolarizations was not
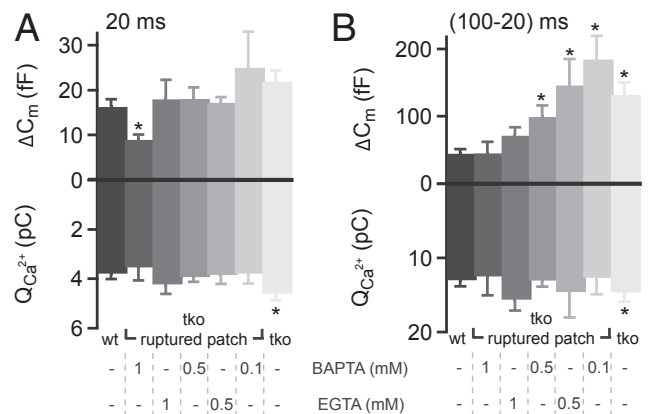

Fig. 4. Functional estimation of the concentration and kinetics of endogenous $\mathrm{Ca}^{2+}$-binding sites in IHCs. $(A) \Delta C_{m}$ and $Q_{C a}$ in response to depolarization for $20 \mathrm{~ms}$ (probing the RRP) in WT and TKO IHCs using perforated-patch (black and light gray) and in $\mathrm{KO} \mathrm{IHCs}$ using ruptured-patch configuration with different concentrations of the exogenously added synthetic $\mathrm{Ca}^{2+}$ chelators BAPTA and EGTA (shades of gray). ( $B$ ) Difference in the $\Delta C_{\mathrm{m}}$ and $Q_{\mathrm{Ca}}$ in response to 100 and $20 \mathrm{~ms}$ (probing the sustained exocytosis). When testing the sustained component of exocytosis $0.5 \mathrm{mM}$ of either buffer was insufficient in $\mathrm{Ca}^{2+}$ buffering. On the contrary, $1 \mathrm{mM}$ BAPTA (but not EGTA) significantly reduced the amount of RRP exocytosis $(A)$. Asterisks denote significant difference vs. WT controls $(P<0.01$, Student $t$ test or Wilcoxon rank-sum test). From left to right, $n=18, n=6, n=5, n=12, n=10$, $n=7$, and $n=12 \mathrm{IHCs}$ were analyzed. 


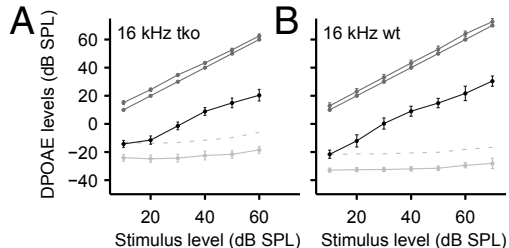

C
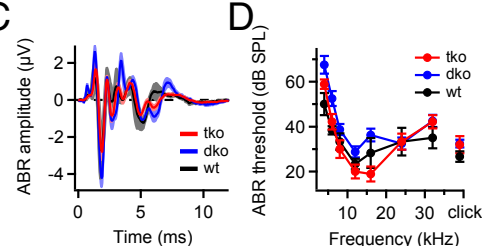

Fig. 5. Cochlear function is preserved in mice lacking the three most prominent EF-hand $\mathrm{Ca}^{2+}$ buffers. ( $A$ and $B$ ) Input/output functions of otoacoustic emission (i.e., DPOAEs; black lines) of the TKO $(A)$ and WT $(B)$ mice at $16 \mathrm{kHz} \mathrm{f}_{2}$ tone. Solid light gray lines indicate the noise floor; dotted light gray lines indicate 2 SDs above the noise floor. The dark gray lines represent both primary tones. All data represent grand averages over several animals. No significant difference was observed between the WT and TKO mice. (C) Average ABR in response to $80 \mathrm{~dB}$ clicks in WT (black), $\mathrm{Pv}^{-1-} \mathrm{Cb}^{-1-}$ (DKO; blue), and TKO (red) mice. (D) Analysis of ABR thresholds revealed no significant difference among the three genotypes. All data are given as mean \pm SEM.

$\mathrm{Cr}^{-/-}$mice. In the majority of animals, there was no noticeable increase in the $\mathrm{ABR}$ thresholds for tone bursts at different frequencies or clicks in comparison with age-matched $\mathrm{Pv}^{+/+} \mathrm{Cb}^{+/+} \mathrm{Cr}^{+/+}$ mice (Fig. 5D). We additionally tested hearing in mice deficient only in $\mathrm{PV} \alpha$ and $\mathrm{CB}\left(\mathrm{Pv}^{-/-} \mathrm{Cb}^{-/-}\right)$. They did not show a significant hearing deficit as assessed by ABR (Fig. $5 C$ and $D$ ), which is consistent with the majority of our $\mathrm{Pv}^{-/-} \mathrm{Cb}^{-/-} \mathrm{Cr}^{-/-}$data.

Next, we studied sound encoding at the single SGN level. Consistent with their near normal population responses (Fig. 5), the single fiber thresholds were comparable between $\mathrm{Pv}^{-1-} \mathrm{Cb}^{-1-} \mathrm{Cr}^{-1-}$ and $\mathrm{Pv}^{+/+} \mathrm{Cb}^{+/+} \mathrm{Cr}^{+/+}$SGNs (Fig. $6 A$ ). The rate of spontaneous SGN firing was increased in $\mathrm{Pv}^{-/-} \mathrm{Cb}^{-/-} \mathrm{Cr}^{-1-}$ mice $(\mathrm{P}=0.04$, KolmogorovSmirnov test; Fig. $6 B)$, which likely reflects enhanced transmitter release from buffer-deficient IHCs in the absence of sound.

We then stimulated each SGN with $50-\mathrm{ms}$ tone bursts at $30 \mathrm{~dB}$ above threshold at the characteristic frequency (CF) to study sound-driven spike rates at saturating sound pressure levels (Fig. $6 C)$. Like in previously published data (23-25), all SGNs showed a high onset firing rate followed by adaptation to reach a lower, but still sizable, steady-state rate. We found no significant differences in the peak rates in SGNs of $\mathrm{Pv}^{-1-} \mathrm{Cb}^{-1-} \mathrm{Cr}^{-/-}$mice (Fig. 6D). Steadystate spike rates were comparable (SI Appendix, Fig. S2B) or even slightly lower in $\mathrm{Pv}^{-1-} \mathrm{Cb}^{-1-} \mathrm{Cr}^{-1-}$ mice (Fig. $6 \mathrm{C}$ and $\mathrm{D}$ ), in apparent contrast to the enhanced sustained exocytosis in IHCs (Fig. 3), an unexpected observation that we investigated in more detail by mathematical modeling (as detailed later). $\mathrm{Pv}^{-1-} \mathrm{Cb}^{-1-} \mathrm{Cr}^{-/-}$SGNs also showed very good temporal precision of sound-onset coding (Fig. $6 E$ ). Forward masking experiments $(26,27)$ revealed a normal extent of depletion of the RRP and unaltered replenishment kinetics (Fig. $6 F$ ). In summary, the single SGN data agree with the notion of a largely unimpaired peripheral auditory function, including a normal temporal precision of sound encoding for transient and sustained stimuli (SI Appendix, Fig. S3).

No Obvious Up-Regulation of Perisynaptic Mitochondria or Plasma Membrane $\mathrm{Ca}^{2+}$ Pumps in IHCs Lacking PVo and CB. The weak effects of $\mathrm{Ca}^{2+}$-buffer deficiency on synaptic sound coding prompted us to test for a potential compensation by enhanced perisynaptic mitochondrial $\mathrm{Ca}^{2+}$ uptake $(11,28)$. To test whether mitochondria are present in increased number or are perhaps enlarged around the synapses in the $\mathrm{Ca}^{2+}$-buffer KOs (28), we performed semiquantitative immunohistochemistry in isolated organs of Corti of $\mathrm{Pv}^{-/-} \mathrm{Cb}^{-/-}$and C57BL/6 mice. Apical coils of the organs of Corti were stained for the mitochondria by using the marker PNPase (29) and ribbons (CtBP2; Fig. $7 A$ and $B$ ). Samples of both genotypes were processed identically and in parallel during immunohistochemistry and confocal imaging (Fig. $7 C$ ). Cumulative PNPase immunofluorescence was analyzed in a total of 1,440 C57BL/6 and 1,260 $\mathrm{Pv}^{-/-} \mathrm{Cb}^{-/-}$synapses from four different experiments on cochleae of eight C57BL/6 and $\mathrm{Pv}^{-/} \mathrm{Cb}^{--}$ mice. In none of the experiments did we observe a significantly increased presynaptic PNPase immunofluorescence in IHCs of $P v^{-/-}$ $\mathrm{Cb}^{-/}$mice, indicating a normal mitochondrial abundance despite the absence of PV $\alpha$ and CB. Similarly, we did not detect an upregulation of the plasma membrane $\mathrm{Ca}^{2+}$-pumps (PMCAs; SI Appendix, Fig. S4).
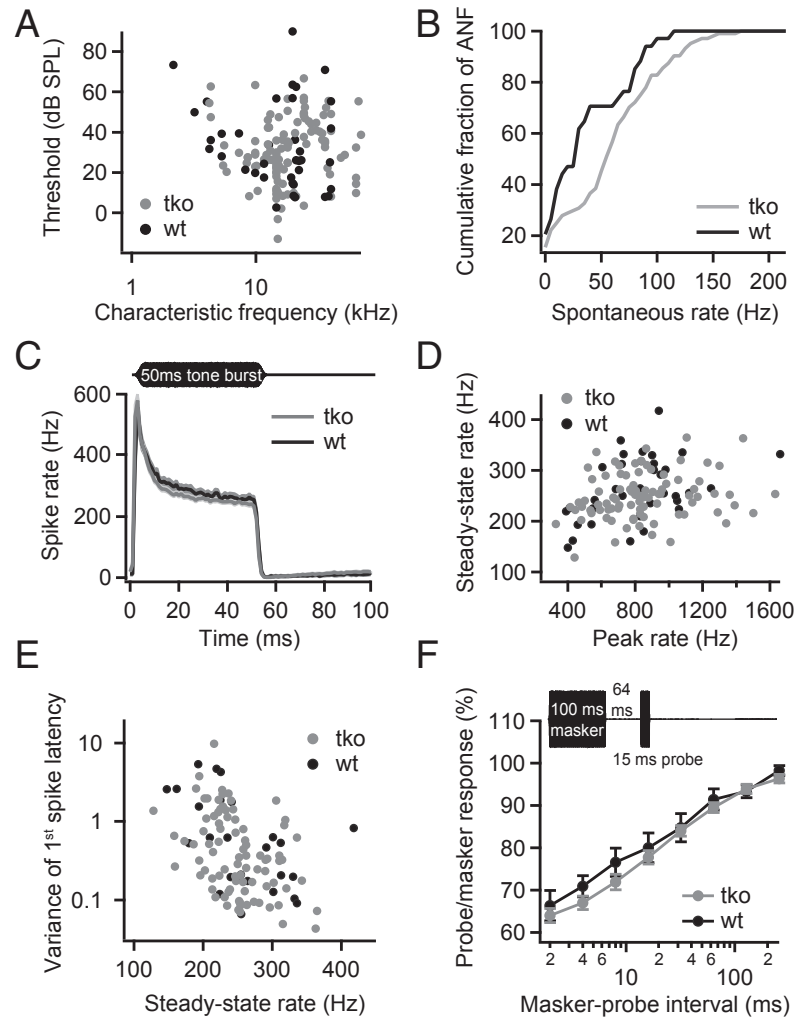

Fig. 6. Disruption of $P V_{\alpha}, C B$, and $C R$ has little impact on sound encoding by single SGNs. (A) Thresholds measured at the CF of each auditory nerve fiber. The thresholds of the TKO fibers (gray, $n=111$ ) were comparable to WT controls (black, $n=37$ ). (B) Spontaneous rate distributions in TKO mice (gray, $n=104$ ) and control groups (black, $n=34$ ). Higher spontaneous rates were observed in SGNs of the TKO animals. (C) Mean peristimulus time histograms $( \pm$ SEM) plotting the instantaneous spiking rate in 1-ms time bins over the time course of stimulation with tone bursts at the CF of each SGN, $30 \mathrm{~dB}$ above threshold. The rates and the time course of adaptation were similar in TKO (gray, $n=87$ ) and WT SGNs (black, $n=35$ ). (D) The analysis of the peak rates (rate in largest 1-ms bin) vs. the steady-state spike rates (averaged over $10 \mathrm{~ms}$ from $35 \mathrm{~ms}$ after stimulus onset) shows comparable rates between TKO SGNs (gray) and WT controls (black). (E) The variance of the first spike latency was similar between fibers from TKO mice and WT controls. $(F)$ Recovery from adaptation probed by a forward-masking paradigm (Inset): maximal masking and the time course of recovery were similar for TKO (gray, $n=35$ ) and WT controls (black, $n=8$ ). Stimuli were applied at CF, $30 \mathrm{~dB}$ above threshold. 

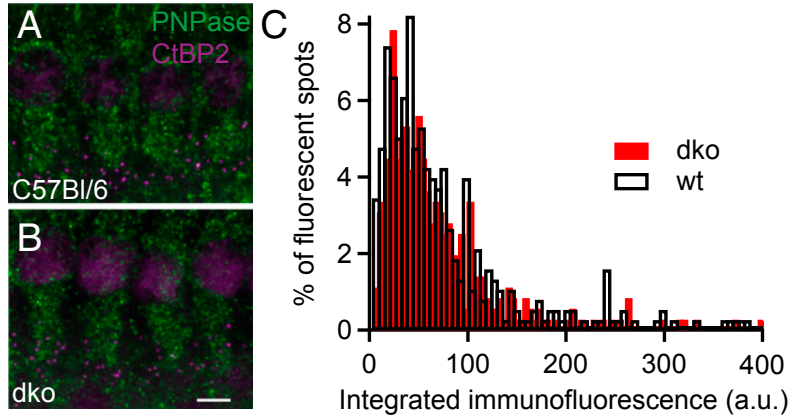

Fig. 7. Immunofluorescence analysis of perisynaptic abundance of mitochondria. ( $A$ and $B$ ) The whole mount of the C57BL/6 (WT) and DKO organs of Corti double stained for the mitochondrial marker PNPase (green) and the presynaptic marker CtBP2 (magenta) reveals similar abundance of mitochondria in IHCs of both genotypes. (Scale bar: $5 \mu \mathrm{m}$.) (C) Perisynaptic PNPase immunofluorescence intensity (integrated within a $0.5-\mu \mathrm{m}$ radius around the center of mass of CtBP2 fluorescence in single confocal sections) in WT and DKO IHCs. A total of 377 synapses from four C57BL/6 (WT) organs and 357 synapses from four DKO organs were analyzed, and the distributions were statistically indistinguishable $(P=0.94$, Wilcoxon rank-sum test).

Computational Analysis of IHC Exocytosis and SGN Spiking Indicates Enhanced Extrasynaptic Release in $\mathrm{Pv}^{-1-} \mathrm{Cb}^{-1-} \mathrm{Cr}^{-1-}$ IHCs. SGN activity is governed by transmitter release at the IHC ribbon synapse. To understand how increased IHC exocytosis of the $\mathrm{Pv}^{-1-} \mathrm{Cb}^{-/-} \mathrm{Cr}^{-1-}$ animals influences SGN spiking rate, we used mathematical modeling (24) (Fig. $8 A$ and Materials and Methods). Specifically, we addressed the question whether the excess sustained exocytosis reflects an acceleration of synaptic vesicle replenishment by $\mathrm{Ca}^{2+}(30,31)$ or extrasynaptic release caused by the wider spread of the synaptic $\mathrm{Ca}^{2+}$ signal. The model converts release events in IHCs into SGN spiking by taking into account neuronal refractoriness $(32,33)$. Refractory periods in WT and TKO SGNs were similar $(1.11 \pm 0.04 \mathrm{~ms}$ and $1.02 \pm 0.03 \mathrm{~ms}$, respectively; SI Appendix, Fig. S5C). By using these parameters and assuming that all sustained IHC exocytosis occurs synaptically, i.e., drives spiking in the postsynaptic SGNs, the spike rates predicted for $\mathrm{Pv}^{-/-} \mathrm{Cb}^{-/-} \mathrm{Cr}^{-/-}$SGNs substantially exceeded the experimentally observed ones (Fig. $8 B$ ). The discrepancy persisted even when assuming unrealistically long refractory periods (SI Appendix, Fig. S5D). This indicates that a large fraction of the increased sustained exocytosis in the $\mathrm{Pv}^{-/-} \mathrm{Cb}^{-/-} \mathrm{Cr}^{-/-}$IHCs occurs at extrasynaptic (i.e., ectopic) locations, and is ineffective in driving SGNs. Indeed, membrane-proximal vesicles outside the AZ are observed in hair cells $(34,35)$ (Fig. $8 C$ ). We then estimated what fraction of the excess sustained exocytosis in $\mathrm{Pv}^{-/}$ $\mathrm{Cb}^{-/-} \mathrm{Cr}^{-/-}$IHCs could be synaptic (Fig. 8D). Only when this fraction was set at less than $10 \%$ did the difference between the computed and experimentally observed spike rates fall within the uncertainty range of the experimental data. Therefore, we conclude that only a minor part of the excess sustained exocytosis, if any, reflects enhanced vesicle replenishment caused by stronger $\mathrm{Ca}^{2+}$ signaling in the absence of mobile $\mathrm{Ca}^{2+}$ buffers.

Tight Coupling Between Presynaptic $\mathrm{Ca}^{2+}$ Channels and $\mathrm{Ca}^{2+}$ Sensors of Exocytosis Limits the Interference of Mobile $\mathrm{Ca}^{2+}$ Buffers with IHC Synaptic Exocytosis. Our experimental observations suggest that endogenous EF-hand $\mathrm{Ca}^{2+}$-binding proteins possess only modest ability to influence synaptic transmitter release in IHCs. This might reflect a very small distance between the $\mathrm{Ca}^{2+}$ source (i.e. $\mathrm{Ca}^{2+}$ channels) and vesicles undergoing fusion at the AZ ("coupling distance"). To determine this coupling distance in IHCs, we constructed a model that predicts the relative amount of exocytosis triggered when changing the distance between the $\mathrm{Ca}^{2+}$ source and the $\mathrm{Ca}^{2+}$ sensor of release (Fig. $9 A$ and Materials and Methods). We used this model to determine the effective coupling distance $R_{\mathrm{c}}$ (weighted average distance for all channels contributing to the $\left[\mathrm{Ca}^{2+}\right]$ at the $\mathrm{Ca}^{2+}$ sensor; Materials and Methods and SI Appendix, section 8) that best matched exocytosis as observed experimentally under various $\mathrm{Ca}^{2+}$-buffering conditions at room temperature. The model was based on the spatiotemporal profile of intracellular $\left[\mathrm{Ca}^{2+}\right]$ resulting from $\mathrm{Ca}^{2+}$ influx through a single 1-nm radius hemispherical source. The integrated exocytosis was assumed to follow $\left[\mathrm{Ca}^{2+}\right]$ according to a power law relation: $\Delta C_{\mathrm{m}} \sim$ $\left(\left[\mathrm{Ca}^{2+}\right]\right)^{m \cdot q}$. The exponent $q$ accounts for the supralinear dependence of $\left[\mathrm{Ca}^{2+}\right]$ on $Q_{\mathrm{Ca}}:\left[\mathrm{Ca}^{2+}\right] \sim Q_{\mathrm{Ca}}{ }^{1 / q}$ that may result from partial buffer depletion at high $\mathrm{Ca}^{2+}$ influx (SI Appendix, section 7.4). The apparent $\mathrm{Ca}^{2+}$ cooperativity of exocytosis $m$ was derived from prior experiments (36) and the value of 1.7 was obtained (SI Appendix, Fig. S6). We note that our previous studies aimed at determining the maximal apparent $\mathrm{Ca}^{2+}$ cooperativity of the RRP exocytosis, which is close to the intrinsic $\mathrm{Ca}^{2+}$ cooperativity, thus yielding an $m$ in the range of 3-4 in 2-wk-old animals (36, 37 ). The model relationship of the present study needs to use an effective $m$ value matched to the experimental conditions [i.e., in the presence of partial pool depletion/sensor saturation observed at high $\left.Q_{\mathrm{Ca}}(36)\right]$.

Fig. $9 C$ displays the discrepancy between modeled and experimentally observed exocytosis for assumed coupling distances between 1 and $100 \mathrm{~nm}$ and for three different values of $m$. When $m$ is equal to 1.7 , the minimum discrepancy is $\sim 11 \%$, which is smaller than the experimental error $[\sim 14 \%$ for the average $\operatorname{SEM}\left(\Delta C_{\mathrm{m}}\right) /$ mean $\left(\Delta C_{\mathrm{m}}\right)$ under different $\mathrm{Ca}^{2+}$ buffering conditions; Fig. $9 C$, dash-dotted line]. For small $\mathrm{Ca}^{2+}$ influx, $i_{\mathrm{Ca}}$ buffer depletion was negligible and the discrepancy between the model and the measurements insensitive to the absolute value of $i_{\mathrm{Ca}}$. For instance, a $\mathrm{Ca}^{2+}$ influx of $0.3 \mathrm{pA}$ (approximating the

A

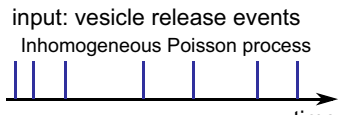
output: spikes $\quad$ time

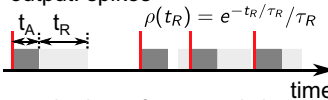
$t_{A}$ - absolute refractory period $t$ $T_{R}$ - mean relative refractory period C
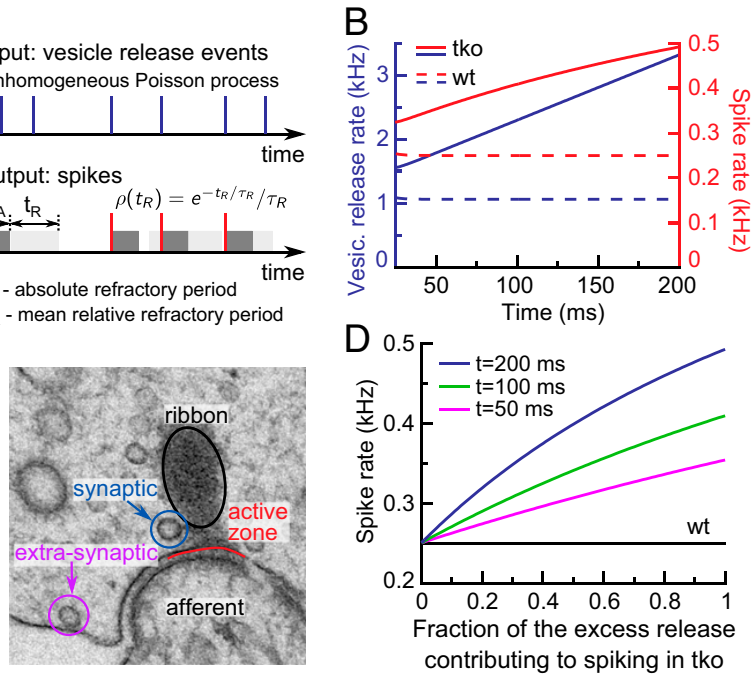

Fig. 8. Mathematical modeling of spike rates in WT and TKO SGNs. (A) Schematic representation of the model for release event to spike conversion (Materials and Methods). (B) Time dependence of vesicle release rate (blue) and spike rate (red) during sustained exocytosis ( $t>25 \mathrm{~ms}$ ) in WT (dashed lines) and TKO (solid lines). (C) An example of a transmission EM micrograph of an $\mathrm{IHC}$ ribbon synapse illustrating the concept of the synaptic vs. ectopic/extrasynaptic vesicle release. Image courtesy of C. Wichmann, University of Göttingen, Göttingen, Germany. $(D)$ Modeled spike rate dependence on a putative fraction of the excess release contributing to the spike generation in SGNs in TKO compared with WT at average overall refractory period $t_{\mathrm{A}}+\tau_{\mathrm{R}}=1.1 \mathrm{~ms}$. 
A

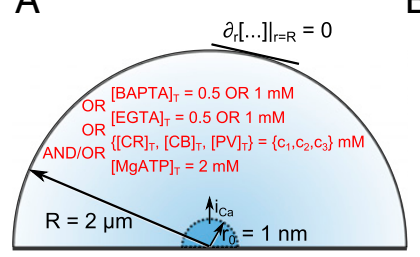

B

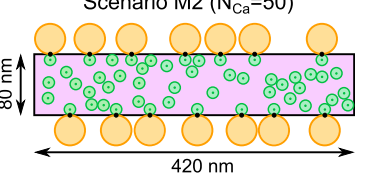

Scenario $\mathrm{M} 3\left(\mathrm{~N}_{\mathrm{Ca}}=14\right)$

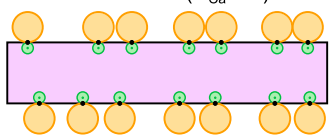

C

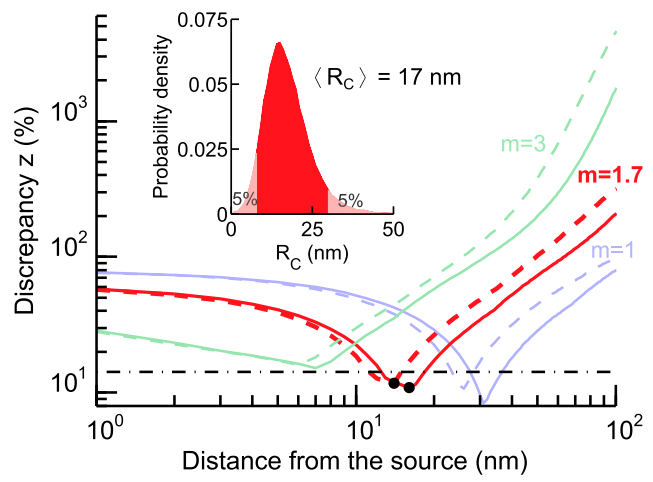

D

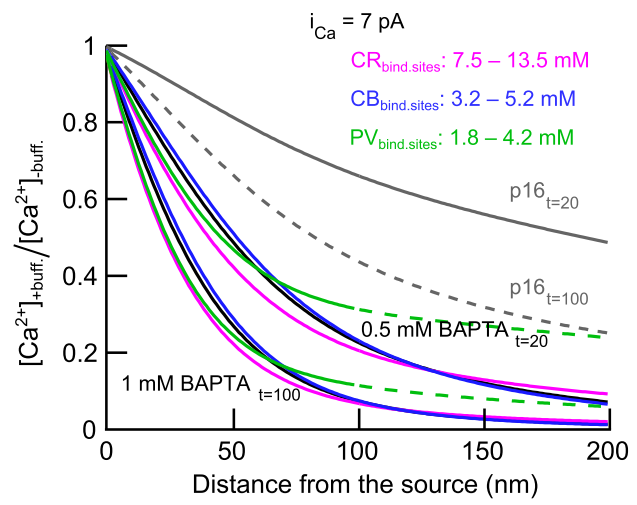

Fig. 9. Estimation of endogenous buffer concentrations and effective coupling distance between presynaptic $\mathrm{Ca}^{2+}$ channels and $\mathrm{Ca}^{2+}$ sensors of exocytosis. (A) Schematic representation of the hemispherical source model. (B) AZ scenarios M2 and M3 from Wong et al. (36) corresponding to the nanodomain-coupling regime. Orange discs represent RRP vesicles, green discs represent $\mathrm{Ca}^{2+}$ channels, black spots represent $\mathrm{Ca}^{2+}$ sensors of exocytosis, and violet area represents the presynaptic density. (C) Dependence of the discrepancy measure $z$ on the effective coupling distance $R_{\mathrm{c}}$. Different colors stand for different values of the apparent $\mathrm{Ca}^{2+}$ cooperativity of exocytosis, $m$. Dashed lines correspond to $i_{\mathrm{Ca}}$ of $0.3 \mathrm{pA}$; solid lines correspond to $i_{\mathrm{ca}}$ of $7 \mathrm{pA}$. Black points denote the local minima of the discrepancy measure $z$ for $m$ of 1.7. Black dash-dotted line corresponds to the average of experimental SEM $\left[\Delta C_{m}\right] /$ mean $\left[\Delta C_{m}\right]$ at different $\mathrm{Ca}^{2+}$ buffering conditions. (Inset) Bootstrap distribution of $R_{\mathrm{c}}$ estimate for $m$ of 1.7 and $i_{\mathrm{Ca}}$ of $7 \mathrm{pA}$, generated as described in Materials and Methods. (D) Spatial profiles of $\left[\mathrm{Ca}^{2+}\right]$ reduction, $\left[\mathrm{Ca}^{2+}\right]_{+ \text {buff. }} /\left[\mathrm{Ca}^{2+}\right]_{- \text {buff., }}$ caused by particular intracellular buffers during $\mathrm{Ca}^{2+}$ influx $\left(i_{\mathrm{Ca}}=7 \mathrm{pA}\right.$ ). Black lines show ratios of the $\mathrm{Ca}^{2+}$ profiles in the presence and absence of $0.5 \mathrm{mM}$ and $1 \mathrm{mM}$ BAPTA, gray lines in the presence and absence of endogenous buffers with concentrations as reported previously (8) in P16 rat IHCs (solid and dashed lines correspond to $\left[\mathrm{Ca}^{2+}\right]$ spatial profiles at $t=20 \mathrm{~ms}$ and $t=100 \mathrm{~ms}$, respectively). Colored lines are the best fits of $\left[\mathrm{Ca}^{2+}\right]_{+ \text {buff. }} /\left[\mathrm{Ca}^{2+}\right]_{- \text {buff. }}$ with CR (magenta), CB (blue), and PV $\alpha$ (green) corresponding to $0.5 \mathrm{mM}$ and $1 \mathrm{mM}$ BAPTA.

single channel current at $-17 \mathrm{mV}$ ) (38) drove negligible buffer depletion and led to estimates of the effective coupling distance of
$15 \mathrm{~nm}$, for $m$ of 1.7 (Fig. $9 C$, dashed red line). As buffer depletion effects might be more prominent for larger $\mathrm{Ca}^{2+}$ influx, we also determined $R_{\mathrm{c}}$ assuming conditions of strong $\mathrm{Ca}^{2+}$ influx. To this end, we calculated the extent of buffer depletion near the AZ by using a hemispherical source equivalent to the entire presynaptic density in a mature synapse (surface area $\sim 420 \times 80 \mathrm{~nm}^{2}$ ) (36) and the maximal depolarization-evoked $\mathrm{Ca}^{2+}$ current per synapse in $\mathrm{PV}^{+/+} \mathrm{Cb}^{+/+} \mathrm{Cr}^{+/+}$IHCs (15 pA). Near a 1-nm hemispherical source, a similar level of buffer depletion was obtained for an $i_{\mathrm{Ca}}$ of $7 \mathrm{pA}(S I$ Appendix, section 7.3). Even for this extreme scenario, partial buffer depletion resulted in only a slight rightward shift of the discrepancy curves (Fig. 9C) with an $R_{\mathrm{c}}$ of $17 \mathrm{~nm}$ for an $m$ of 1.7. We used bootstrapping to estimate the confidence range of the $R_{\mathrm{c}}$ estimate for $i_{\mathrm{Ca}}$ of $7 \mathrm{pA}$, which is affected by the estimation errors of $m$ and $\Delta C_{\mathrm{m}}$. The 5-95 percentile range spanned from 8 to $30 \mathrm{~nm}$ (Fig. 9C, Inset).

In our previous work, we introduced some possible IHC presynaptic $\mathrm{AZ}$ arrangements (36). In spatially resolved simulations with two of these AZ topographies that correspond to the "nanodomain-coupling" regimen (Fig. $9 B$ ), $R_{\mathrm{c}}$ ranged between 15 and $27 \mathrm{~nm}$ (SI Appendix, section 8.4). They might thus be a good representation of the true IHC AZs. At the average $R_{\mathrm{c}}$ of $17 \mathrm{~nm}$, the influence of the $\mathrm{Ca}^{2+}$ buffers on $\left[\mathrm{Ca}^{2+}\right]$ at the $\mathrm{Ca}^{2+}$ sensor is small (0.5 mM BAPTA; Fig. $9 D$, black line). Together, these results indicate that endogenous $\mathrm{Ca}^{2+}$ buffers do not appreciably interfere with exocytosis at the $\mathrm{AZ}$ as a result of tight coupling between $\mathrm{Ca}^{2+}$ channels and $\mathrm{Ca}^{2+}$ sensors of exocytosis in IHCs of 2-3-wk-old mice. However, they effectively reduce extrasynaptic release at sites located more distant from the $\mathrm{Ca}^{2+}$ source.

Predicted Concentrations of Endogenous Buffers in Mature Mouse IHCs. Finally, we used the model to establish lower and upper bounds on the concentrations of endogenous $\mathrm{Ca}^{2+}$ buffers in IHCs. Exocytosis of a given readily releasable vesicle is governed by $\left[\mathrm{Ca}^{2+}\right]$ at the $\mathrm{Ca}^{2+}$ sensor. According to $\Delta C_{\mathrm{m}}$ recordings (Fig. 4), $\left[\mathrm{Ca}^{2+}\right]$ at the $\mathrm{Ca}^{2+}$ sensor of exocytosis in $\mathrm{Pv}^{+/+} \mathrm{Cb}^{+/+} \mathrm{Cr}^{+/+}$should be matched in $\mathrm{Pv}^{-/-} \mathrm{Cb}^{-/-} \mathrm{Cr}^{-/-}$IHCs when they are loaded with $0.5 \mathrm{mM}$ BAPTA. On the contrary, $1 \mathrm{mM}$ BAPTA was required for adjusting sustained exocytosis of $\mathrm{Pv}^{-/-} \mathrm{Cb}^{-/-} \mathrm{Cr}^{--}$IHCs to WT levels, likely by limiting $\left[\mathrm{Ca}^{2+}\right]$ further away from the $\mathrm{Ca}^{2+}$ channel. The spatial $\left[\mathrm{Ca}^{2+}\right]$ profiles in the presence of these two BAPTA concentrations thus define the range of plausible $\left[\mathrm{Ca}^{2+}\right]$ levels in the presence of endogenous buffers.

Fig. $9 D$ shows the predicted ratios of the spatial $\left[\mathrm{Ca}^{2+}\right]$ profiles with 0.5 and $1 \mathrm{mM}$ BAPTA and the best approximation of these $\left[\mathrm{Ca}^{2+}\right]$ profiles in the presence of the endogenous $\mathrm{Ca}^{2+}$ buffers $\mathrm{CB}$, $\mathrm{CR}$, and $\mathrm{PV} \alpha$. The spatial $\left[\mathrm{Ca}^{2+}\right]$ profiles with 0.5 and $1 \mathrm{mM}$ BAPTA were fitted 20 and $100 \mathrm{~ms}$ after stimulus onset, respectively. Solving the model with concentrations of endogenous mobile $\mathrm{Ca}^{2+}$ buffers $\left(0.5 \mathrm{mM} \mathrm{Ca}^{2+}\right.$-binding sites in total $)$ as estimated in rat IHCs by using quantitative immunogold EM (8) showed that these concentrations would be considerably less efficient than 0.5 mM BAPTA. According to our model, the concentration of $\mathrm{Ca}^{2+}$ -binding sites equivalent to $0.5-1 \mathrm{mM}$ BAPTA is between 3.2 and $5.2 \mathrm{mM}$ for $\mathrm{CB}$, between 7.5 and $13.5 \mathrm{mM}$ for CR, and between 1.8 and $4.2 \mathrm{mM}$ for $\mathrm{PV} \alpha$ when each buffer is considered separately (Fig. $9 D$ ). We note that, to convert these concentrations of $\mathrm{Ca}^{2+}$-binding sites into equivalent protein concentrations, the values have to be divided by 4,5 , and 2 for $\mathrm{CB}, \mathrm{CR}$, and $\mathrm{PV}$, respectively; thus, $0.5 \mathrm{mM}$ BAPTA would correspond to $0.8,1.5$, and $0.9 \mathrm{mM}$ of the three buffers. These values are consistent with previous estimates of EF-hand buffer concentrations in neurons (39-41) and hair cells (7). PV $\alpha$ alone could not fully reproduce the spatial $\left[\mathrm{Ca}^{2+}\right]$ profiles predicted for 0.5 or $1 \mathrm{mM}$ BAPTA over distances longer than $100 \mathrm{~nm}$. However, it is likely that, at low concentrations, it acts together with higher amounts of $\mathrm{CB}$ and CR to shape the $\left[\mathrm{Ca}^{2+}\right]$ signal at the IHC synapses.

As shown in Fig. 9D, the upper bounds on endogenous buffer $\mathrm{Ca}^{2+}$-binding site concentrations were estimated by probing 
$\left[\mathrm{Ca}^{2+}\right]$ at a $t=100 \mathrm{~ms}$ after stimulus application. Negligible at first, the effect of accumulated $\mathrm{Ca}^{2+}$ on $\left[\mathrm{Ca}^{2+}\right]$ during stimulation grows with time (SI Appendix, Fig. S7 $A$ and $B$ ). This might affect the estimated upper bounds on endogenous $\mathrm{Ca}^{2+}$-binding site concentrations. We thus reestimated the upper bounds on the $\mathrm{Ca}^{2+}$-binding site concentrations by using spatial profiles of $\left[\mathrm{Ca}^{2+}\right]$ calculated for times between $20 \mathrm{~ms}$ and $100 \mathrm{~ms}$ after stimulus application. As shown in SI Appendix, Fig. S10 (solid lines), the upper bound estimates were not considerably different at intermediate times for $\mathrm{CB}$ and PV (by $\sim 20 \%$ higher at $t=20 \mathrm{~ms}$ than at $t=100 \mathrm{~ms}$ ). For $\mathrm{CR}$, the difference in the estimated $\mathrm{Ca}^{2+}$-binding site concentrations was significant, being $50 \%$ higher at $t=20 \mathrm{~ms}$ than at $t=$ $100 \mathrm{~ms}$. In summary, our results suggest that the concentrations of endogenous buffers in IHCs are (approximately one order) higher than those obtained from immunogold counts (8) or the $k_{\text {on }}$ rates of the buffers in situ (i.e., inside cells) are considerably higher than currently assumed (SI Appendix, section 7.1).

\section{Discussion}

In the present study we addressed the role of $\mathrm{Ca}^{2+}$ buffering EFhand $\mathrm{Ca}^{2+}$-binding proteins in the presynaptic function of IHCs. By using $\mathrm{KO}$ mice lacking $\mathrm{PV} \alpha, \mathrm{CB}$, and $\mathrm{CR}$, we indicate that mobile $\mathrm{Ca}^{2+}$ buffers shape IHC synaptic $\mathrm{Ca}^{2+}$ current by decreasing its amplitude and attenuating inactivation. Independent of their regulation of $\mathrm{Ca}^{2+}$ channels, they constrain transmitter release to AZs, ensuring efficient presynaptic function. By using exocytosis as readout together with mathematical modeling, we provide quantitative functional estimates for the concentration of the endogenous $\mathrm{Ca}^{2+}$ buffers in IHCs. Furthermore, we estimated the effective coupling distance between the $\mathrm{Ca}^{2+}$ channels and sensors for exocytosis in IHCs to be very short (mean value of $R_{\mathrm{c}}=$ $17 \mathrm{~nm}$ ). Surprisingly, sound encoding and hearing were largely intact upon disruption of the three $\mathrm{Ca}^{2+}$ buffers. This can be explained by " $\mathrm{Ca}^{2+}$ nanodomain control" of exocytosis, minimizing the impact of buffers on stimulus-secretion coupling at AZs.

$\mathrm{Ca}^{2+}$ Buffering in Mammalian IHCs. Previous work on hair cells had provided estimates of the concentration of mobile $\mathrm{Ca}^{2+}$-binding sites $(5-9,42-44)$, demonstrated the significance of mobile and immobile $\mathrm{Ca}^{2+}$ buffers for shaping the spatiotemporal properties of synaptic $\mathrm{Ca}^{2+}$ microdomains $(2,44-46)$, and investigated the sensitivity of exocytosis to $\mathrm{Ca}^{2+}$ buffering $(9,22,37,47-49)$. The $\mathrm{Pv}^{-/-} \mathrm{Cb}^{-/-} \mathrm{Cr}^{-1-}$ mice now enabled us to refine the estimates of the concentration of mobile $\mathrm{Ca}^{2+}$-binding sites in hair cells and to study the role of the three endogenous $\mathrm{Ca}^{2+}$ buffers in hair cell presynaptic function and afferent auditory signaling.

We found that the length constants of $\mathrm{Ca}^{2+}$ buffering, defined by $\mathrm{Ca}^{2+}$-binding kinetics, diffusion coefficients, and concentrations of the three $\mathrm{Ca}^{2+}$ buffers, are large relative to the effective $\mathrm{Ca}^{2+}$ channel- $\mathrm{Ca}^{2+}$ sensor coupling distance. Consequently, exocytosis of the RRP, triggered by short depolarization pulses $(<20 \mathrm{~ms})$, was not noticeably affected by the three $\mathrm{Ca}^{2+}$ buffers. During longer depolarizations $(\geq 20 \mathrm{~ms})$, they reduced exocytosis in IHCs primarily by limiting $\mathrm{Ca}^{2+}$ spread from the $\mathrm{AZ}$ to ectopic release sites (Fig. $3 C$ ). In the substitution experiments, $1 \mathrm{mM}$ BAPTA or EGTA reduced sustained exocytosis in $\mathrm{Pv}^{-/-} \mathrm{Cb}^{-/-} \mathrm{Cr}^{-/-}$IHCs to levels observed in $\mathrm{Pv}^{+/+} \mathrm{Cb}^{+/+} \mathrm{Cr}^{+/+}$mice, whereas lower concentrations $(\leq 0.5 \mathrm{mM})$ were insufficient. This is in good agreement with previous results in mice, in which imaging of synaptic $\mathrm{Ca}^{2+}$ microdomains yielded values equivalent to $0.5-2 \mathrm{mM} \mathrm{Ca}^{2+}$-binding sites of synthetic $\mathrm{Ca}^{2+}$ buffers (44). It also largely agrees with functional estimates on hair cells from other species $(5-7,9,42)$. However, when estimating the concentrations of endogenous $\mathrm{Ca}^{2+}$ binding sites, a significant difference in the diffusion coefficients and the $\mathrm{Ca}^{2+}$ (un)binding rates of synthetic and endogenous buffers has to be taken into account. Relying on published $\mathrm{Ca}^{2+}-$ binding rates, our model suggested that the concentration of $\mathrm{Ca}^{2+}$ binding sites is approximately one order of magnitude higher than reported in rat hair cells by using quantitative immunogold labeling ( $\sim 0.54 \mathrm{mM}$ endogenous $\mathrm{Ca}^{2+}$-binding sites) (8). Alternatively, faster $\mathrm{Ca}^{2+}$-binding rates of the endogenous buffers (an order of magnitude higher than published) could account for the observations, or it could be a combination of both effects.

Modulation of $\mathrm{Ca}^{2+}$ Influx by $\mathrm{Ca}^{2+}$ Buffering EF-Hand $\mathrm{Ca}^{2+}$-Binding Proteins. A somewhat unexpected finding was the enhanced initial $\mathrm{Ca}^{2+}$ influx in $\mathrm{Pv}^{-/-} \mathrm{Cb}^{-/-} \mathrm{Cr}^{-/-}$IHCs. We did not observe more synapses in $\mathrm{Pv}^{-/-} \mathrm{Cb}^{-/-} \mathrm{Cr}^{-/-}$IHCs, which could have been an obvious cause for a greater number of $\mathrm{Ca}^{2+}$ channels. Instead, potential mechanisms for the increased $\mathrm{Ca}^{2+}$ influx include more $\mathrm{Ca}^{2+}$ channels per synapse and an enhanced open probability. In addition, we found that $\mathrm{Ca}^{2+}$-current inactivation was enhanced in $\mathrm{Pv}^{-/-} \mathrm{Cb}^{-/-} \mathrm{Cr}^{-/-}$IHCs. Reduced $\mathrm{Ca}^{2+}$ buffering could increase $\mathrm{Ca}^{2+}$-calmodulin-dependent inactivation (CDI). However, the enhanced inactivation was not reversed upon addition of synthetic $\mathrm{Ca}^{2+}$ chelators, which generally show little potency to antagonize CDI in mature IHCs $(50,51)$. Alternatively, EF-hand $\mathrm{Ca}^{2+}$-binding proteins might directly interact with the channel, which is established for calmodulin and CaBPs (52-55). Indeed, $\mathrm{CR}$ suppresses $\mathrm{CDI}$ in $\mathrm{Ca}_{\mathrm{V}} 2.1$ channels (but not $\mathrm{Ca}_{\mathrm{V}} 1.2$ channels) in HEK 293-T cells (56).

$\mathrm{Ca}^{2+}$ Nanodomain Control of Fusion at the IHC Ribbon Synapse. Our previous work suggested that vesicle fusion at IHC ribbon synapses is induced by $\mathrm{Ca}^{2+}$ provided by one or few proximal $\mathrm{Ca}^{2+}$ channels $(36,37)$, nicknamed $\mathrm{Ca}^{2+}$ nanodomain control. In accordance, it has been demonstrated that not even $10 \mathrm{mM}$ BAPTA completely blocks exocytosis in auditory hair cells (57). The present study took advantage of the disruption of endogenous $\mathrm{Ca}^{2+}$ buffers and substitution with synthetic $\mathrm{Ca}^{2+}$ buffers to further test this hypothesis. Based on experiments and modeling, we estimated the effective coupling distance $R_{\mathrm{c}}$ between $\mathrm{Ca}^{2+}$ channels and vesicular $\mathrm{Ca}^{2+}$ sensors at IHC AZs to be $\sim 17 \mathrm{~nm}$ with a 5-95 percentile range of 8-30 nm. Our previously proposed AZ scenarios of nanodomain control (36) (Fig. 9B) give $R_{\mathrm{c}}$ values that fit well into the proposed range and might thus represent a good approximation of the true IHC AZ arrangement. Such tight coupling increases efficacy and speed of synaptic transmission.

Mobile IHC Ca ${ }^{2+}$ Buffering and Hearing. At first sight, finding a near-normal function of the cochlea in the $\mathrm{Pv}^{-/-} \mathrm{Cb}^{-/-} \mathrm{Cr}^{-/-}$ mice is surprising. In SGNs, only spontaneous spiking rates were increased, which might be related to the increased $\mathrm{Ca}^{2+}$ influx. In fact, a regulation of spontaneous firing rates by modulating $\mathrm{Ca}^{2+}$ channel open probability has been shown (58). A minor increase (e.g., $17 \%$ ) in the number or open probability of $\mathrm{Ca}^{2+}$ channels could explain the significantly enhanced spontaneous rate in SGNs of $\mathrm{Pv}^{-/-} \mathrm{Cb}^{-/-} \mathrm{Cr}^{---}$mice, but may not lead to an obvious enhancement of the evoked firing rates when driven by strong supratheshold stimulation (Fig. 6; see also ref. 59).

Sound encoding during continued stimulation was not significantly altered despite increased sustained IHC exocytosis. Mathematical modeling indicated that this discrepancy reflects extrasynaptic exocytosis of vesicles that contributes less efficiently to sound coding, if at all. Alternative explanations such as refractoriness (Fig. $8 B$ and SI Appendix, Fig. S5D) and increased AMPA desensitization (49) seem unlikely. A supralinear rise of exocytosis during prolonged stimulation was reported for turtle hair cells and interpreted as $\mathrm{Ca}^{2+}$-dependent vesicle replenishment (60), but the relation to sound encoding remained to be elucidated, and a contribution of extrasynaptic exocytosis could not be ruled out.

Release away from the ribbon-type $\mathrm{AZ}$ has previously been observed in retinal bipolar cells $(61,62)$, where it is physiologically relevant for communication of bipolar cells to AII amacrine cells (62). The results of our study suggest that such extrasynaptic exocytosis of IHCs has little influence on the postsynaptic SGN 
spiking. Together with previous studies demonstrating a good correlation between the presynaptic release rate and postsynaptic spiking in WT and bassoon mutant animals (24), it further suggests little or no ectopic release in the presence of endogenous $\mathrm{Ca}^{2+}$ buffers. We conclude that the properties of the endogenous buffer $\mathrm{Ca}^{2+}$ binding and their diffusion kinetics allow for a metabolically efficient control of the $\mathrm{Ca}^{2+}$-dependent sound encoding.

\section{Materials and Methods}

Animals. The TKO line $\mathrm{Pv}^{-1-} \mathrm{Cb}^{-1-} \mathrm{Cr}^{-1-}$ was generated by breeding the two double KO (DKO) lines, $\mathrm{Pv}^{-1-} \mathrm{Cb}^{-1-}$ (63) (genetic background: C57BL/6 $\times 129 /$ OlaHsd $\times 129 / \mathrm{SvJ}$ ) and $\mathrm{Pv}^{-1-} \mathrm{Cr}^{-1-}$ (64) (genetic background: C57BL/6 $\times 129 /$ OlaHsd). The initial double-heterozygous litters $\left(\mathrm{Pv}^{-1-} \mathrm{Cb}^{+-} \mathrm{Cr}^{+-}\right)$were used to generate the TKO line. The genotype was determined by PCR for the mutated loci in the three genes, Pvalb, Calb1, and Calb2, as reported before (65-67). The line was then maintained as an inbred line with a mixed C57BL/6 $\times 129 /(\mathrm{OlaHsd} \times$ SvJ) background. We studied the auditory phenotype of DKO $\left(\mathrm{Pv}^{-1-} \mathrm{Cb}^{--}\right)$and TKO $\left(\mathrm{PV}^{-l-} \mathrm{Cb}^{-1-} \mathrm{Cr}^{--}\right)$mice. Control experiments were performed on WT animals $\left(\mathrm{PV}^{+/+} \mathrm{Cb}^{+/+} \mathrm{Cr}^{+/+}\right)$with the most similar background to TKO mice (mixed background of C57BL/6 and 129/OlaHsd). These animals originated from a previous heterozygote $\left(\mathrm{Pv}^{+-} \mathrm{Cr}^{+-}\right)$breeding. All experiments were done in compliance with the national animal care guidelines and were approved by the board for animal welfare of the University Medical Center Goettingen, the animal welfare office of the state of Lower Saxony, and the institutional animal care and use committee of the Massachusetts Eye and Ear Infirmary.

Immunohistochemistry and Confocal Microscopy. Immunohistochemistry was performed as described previously (19). To analyze the abundance of mitochondria, we fixed organs of Corti in methanol at $-20{ }^{\circ} \mathrm{C}$ for $20 \mathrm{~min}$ and double-stained hair cells for PNPase (mitochondria marker) (29) and CtBP2 (19) (to identify ribbons). In all other cases, organs were fixed with $4 \%$ (wt/vol) formaldehyde for 10-60 min on ice. The following antibodies were used: mouse IgG1 anti-CtBP2 (1:200; BD Biosciences), rabbit anti-GluR2/3 (1:200; Chemicon), mouse anti-calbindin-D28k, mouse anti-calretinin, mouse antiparvalbumin- $\alpha$, rabbit anti-oncomodulin (anti-parvalbumin- $\beta$; all 1:500; Swant) rabbit anti-PNPase (1:500; Proteintech), rabbit VGlut3 (1:500; Abcam), mouse 5F10 (pan-PMCA marker, 1:200; Abcam) and secondary Alexa Fluor 488- and Alexa Fluor 568-labeled antibodies (1:200; Molecular Probes). In some instances, nuclei were stained with Hoechst 34580 (1:1,000; Molecular Probes). Confocal images were acquired by using a laser-scanning confocal microscope (TCS SP2 Leica Microsystems) with 488-nm ( $\mathrm{Ar})$ and $561-\mathrm{nm}$ (He-Ne) lasers and a $63 \times$ oil immersion objective. $z$-axis stacks of $2 \mathrm{D}$ images were taken with a step size of $0.5 \mu \mathrm{m}$. $Z$-projections were done in ImageJ (National Institutes of Health). The ribeye/ CtBP2 and GluR2/3 immunofluorescence spots were counted in the $z$-stacks and divided by the number of IHCs. Juxtaposed spots of pre- and postsynaptic immunofluorescence were taken to identify intact IHC ribbon synapses. To assess the perisynaptic abundance of mitochondria, we measured the cumulative PNPase immunofluorescence intensity within a circle with a radius of $0.5 \mu \mathrm{m}$ around the center of mass of the ribbon (CtBP2 immunofluorescence) in confocal sections of IHCs by using a custom-written MATLAB routine.

Patch-Clamp Recordings. KO $\left(\mathrm{Pv}^{-1-} \mathrm{Cb}^{-1-} \mathrm{Cr}^{-/-}\right)$and WT mice $\left(\mathrm{PV}^{+/+} \mathrm{Cb}^{+/+} \mathrm{Cr}^{+/+}\right)$ with postnatal ages of 14-23 d were killed by decapitation. The apical cochlear turns were dissected in Hepes Hanks solution containing (in $\mathrm{mM}$ ): 5.36 $\mathrm{KCl}, 141.7 \mathrm{NaCl}, 1 \mathrm{MgCl}_{2}, 0.5 \mathrm{MgSO}_{4}, 10$ Hepes, 11.1 D-glucose, and 3.42 L-glutamine, $\mathrm{pH}$ 7.2. IHCs were patch-clamped in the perforated-patch configuration as previously described (17). For estimation of endogenous buffe equivalents, $\mathrm{Pv}^{-1-} \mathrm{Cb}^{-1-} \mathrm{Cr}^{-1-} \mathrm{IHCs}$ were patch-clamped in the ruptured-patch configuration. The pipette solution contained (in mM): $130 \mathrm{Cs}$-gluconate 10 TEA-Cl, 10 4-AP (4-aminopyridine; Merck), 1 MgCl 2,10 Hepes, $300 \mu \mathrm{g} / \mathrm{mL}$ amphotericin B (for perforated patch-clamp experiments; Calbiochem) $2 \mathrm{MgATP}$, and $0.3 \mathrm{NaGTP}$ (both for ruptured-patch experiments), pH 7.2 with osmolarity of approximately $290 \mathrm{mOsm}$. In ruptured-patch experiments, pipette solution additionally contained different concentrations of exogenous buffers: $0.1,0.5$, or $1 \mathrm{mM}$ BAPTA or 0.5 or $1 \mathrm{mM}$ EGTA. The extracellular solution contained (in $\mathrm{mM}$ ): $106 \mathrm{NaCl}(113 \mathrm{mM}$ in Fig. $3 \mathrm{G}$ and $H$ ), $35 \mathrm{TEA}-\mathrm{Cl}, 2.8 \mathrm{KCl}, 5 \mathrm{CaCl}_{2}\left(2 \mathrm{mM}\right.$ in Fig. $3 \mathrm{G}$ and $H$ ), $1 \mathrm{MgCl}_{2}, 10$ Hepes, $1 \mathrm{CsCl}, 11.1 \mathrm{D}$-glucose, pH 7.2, with osmolarity of approximately $300 \mathrm{mOsm}$. All chemicals were obtained from Sigma-Aldrich unless stated otherwise.

We used an EPC-9 amplifier (HEKA Electronics) controlled by Pulse software (HEKA Electronics). $C_{m}$ increments $\left(\Delta C_{m}\right)$ were measured as pre viously described (22), averaging $400 \mathrm{~ms}$ before and after (skipping the first $40 \mathrm{~ms}$ ) depolarization. To measure $\Delta C_{\mathrm{m}}$ IHCs were stimulated by depolarizations of different durations to peak $\mathrm{Ca}^{2+}$ current potential at intervals of 30-60 s. For $\mathrm{Ca}^{2+}$ current inactivation experiments, IHCs were depolarized for $100 \mathrm{~ms}$. All voltages were corrected for liquid junction potential $(-17 \mathrm{mV})$. In ruptured-patch experiments, measurements were initiated an average of $2.5 \mathrm{~min}$ after patch rupture to allow for the diffusion of exogenous buffer into the cytosol. The waiting time depended on the series resistance $R_{\mathrm{s}}$, which relates to the kinetics of diffusional exchange as previously described (68). Unless stated otherwise, all recordings were performed at room temperature.

ABRs, Otoacoustic Emissions, and Recordings from Single SGNs. Recordings of ABRs and DPOAEs $(69)$ as well as extracellular recordings from SGNs $(23,24)$ were performed as described previously. Briefly, 12-16-wk-old mice were anesthetized by i.p. injection of xylazine $(2.5 \mathrm{mg} / \mathrm{kg})$ and ketamine $(125 \mathrm{mg} / \mathrm{kg}, A B R$ and DPOAE) or xylazine $(5 \mathrm{mg} / \mathrm{kg})$ and urethane $(1.32 \mathrm{mg} / \mathrm{kg}$, for single SGN recordings). The occipital bone and cerebellum were partly removed, and SGNs near the auditory nerve's entry zone into the cochlear nucleus were approached by a glass microelectrode filled with $2 \mathrm{M} \mathrm{KCl}$ and $4 \%$ (wt/vol) methylene blue during noise burst stimulation. Sound-responsive neurons were first characterized by obtaining their spontaneous rate, tuning curves, and responses to $50-\mathrm{ms}$ tone bursts presented at the CF of the SGN, $30 \mathrm{~dB}$ above threshold. SGNs were distinguished from primary cochlear nucleus units based on their primary-like response characteristics and irregular firing pattern, as well as by the electrode position, which was confirmed after the end of the experiment. Spike detection was performed online based on a manually set amplitude criterion.

Data Analysis. Data analysis was performed in Igor Pro (Wavemetrics) and MATLAB (Mathworks) software, confocal images were assembled for display by using ImageJ and Photoshop (Adobe Systems), and final figures were composed in Illustrator (Adobe Systems). To calculate activation time constants of $\mathrm{Ca}^{2+}$ currents, data were fitted with the following function: $I(t)=I_{0}+I_{\max } \times\left(1-\mathrm{e}^{-t / \tau}\right)^{p}$. In most cases, data could be well fitted with the parameter power $(p)$ fixed to 2 , or else $p$ was set as a free parameter. Mean $\Delta C_{m}$ and $\mathrm{Ca}^{2+}$ current estimates present grand averages calculated from the mean estimates of individual IHCs. All results are expressed as mean \pm SEM. Data were tested for randomness, normality (Jarque-Bera test), and equality of variances ( $F$-test) and compared for statistical significance by using a Student $t$ test (in case of normal distribution and equal variance of both samples) or Wilcoxon rank-sum test.

Mathematical Modeling. Mathematical modeling was done by using MATLAB R2012b (Mathworks).

Conversion of neurotransmitter release to spikes. The release rate was inferred from exocytic $\Delta C_{m}$ estimates of IHCS (at room temperature). In the model, the SGN generates a spike in response to each release event unless it is in the refractory state, which lasts for a period of $t_{\mathrm{A}}+t_{\mathrm{R}}\left(t_{\mathrm{A}}\right.$ is the absolute refractory period, $t_{R}$ the relative refractory period; Fig. 9A). Although $t_{A}$ was fixed for a particular $\mathrm{SGN}, t_{\mathrm{R}}$ was a random number drawn from a monoexponential distribution with a mean value $\tau_{\mathrm{R}}$. The stationary spike rate $\left(R_{\mathrm{sp}}^{\infty}\right)$ is related to the release rate $\left(R_{\mathrm{r}}^{\infty}\right)$ and the refractory period in the following way (32): $R_{\mathrm{sp}}^{\infty}=$ $R_{\mathrm{r}}^{\infty} /\left[1+R_{\mathrm{r}}^{\infty} \cdot\left(t_{\mathrm{A}}+\tau_{\mathrm{R}}\right)\right]$. Given that changes in the sustained release rate (between 50 and $200 \mathrm{~ms}$; Fig. $9 B$ ) are slow in comparison with the duration of the refractory period, we applied the adiabatic approximation: $R_{\mathrm{sp}}(t) \sim R_{\mathrm{r}}(t) /[1+$ $\left.R_{\mathrm{r}}(t) \cdot\left(t_{\mathrm{A}}+\tau_{\mathrm{R}}\right)\right]$. To estimate the release rate $R_{\mathrm{r}}(t)$, the experimental $\Delta C_{\mathrm{m}}(t)$ values were first converted to the number of released synaptic vesicles, $N(t)$ : $N(t)=s c \cdot \Delta C_{m}(t) /\left(C_{s v} \cdot N_{\text {syn }}\right)$. Here, a $C_{\text {sv }}$ of 44.5 aF was taken as average $\Delta C_{m}$ upon fusion of single synaptic vesicle (70), the $N_{\text {syn }}$ of 12 is the number of synapses per IHC as obtained from immunohistochemistry (Fig. 2). $s c \leq 1$ is a scaling coefficient to account for differences between in vitro (patch-clamp) and in vivo (single unit recordings) conditions [such as the amplitude of stimulusevoked IHC depolarization, temperature, ion homeostasis (e.g., $\left[\mathrm{Ca}^{2+}\right]_{\mathrm{e}}$ ), and/or the case that a single release event may correspond to release of a few synaptic vesicles] $(70,71)$. Next, $N(t)$ was fitted by $A \cdot t+B \cdot\left(1-e^{-t / \tau}\right)^{n}$ for the $\mathrm{Pv}^{+/+} \mathrm{Cb}^{+/+} \mathrm{Cr}^{+/+}$ IHCs and $A \cdot t+B \cdot\left(1-\mathrm{e}^{-t / \tau}\right)^{n}+t \cdot(a \cdot t+b)$ for the $P v^{-1-} C b^{-1-} C^{-1-}$ IHCS (SI Appendix, Fig. S2A). Finally, the release rate $R_{\mathrm{r}}(t)$ was obtained by calculating the time derivative of $N(t)$.

Refractory periods. We collected experimental cumulative interspike intervals corresponding to the sustained part of PSTH (30-50-ms interval; Fig. 6C). The parameter $t_{\mathrm{A}}$ was estimated as the interspike interval smaller than $99.5 \%$ of the remaining interspike intervals in the sample from the SGN under consideration. To estimate $\tau_{R}$, the cumulative interspike interval distribution excluding the $0.5 \%$ smallest interspike intervals was fitted for each SGN with the expression (which applies for the model formulated earlier): 


$$
P^{M}(t)=\left\{\begin{array}{c}
1-\frac{\left(\tau_{S}-\tau_{R}-t_{A}\right)^{-1} \cdot e^{-\frac{\left(t-t_{A}\right)}{\tau_{R}}}-\tau_{R}^{-1} \cdot e^{-\frac{\left(t-\tau_{A}\right)}{\tau_{s}-\tau_{R}-\tau_{A}}}}{\left(\tau_{S}-\tau_{R}-t_{A}\right)^{-1}-\tau_{R}^{-1}}, \text { if } t>t_{A} \\
0, \text { if } t \leq t_{A}
\end{array}\right.
$$

where $\tau_{s}$ is experimental estimate of the mean interspike interval. The fitting was done by minimizing the relative discrepancy between the model and the experimental cumulative distributions $\sum_{\mathrm{i}} \mid P_{\mathrm{i}}^{\mathrm{M}}-P_{\mathrm{i}}^{\mathrm{E}} / / P_{\mathrm{i}}^{\mathrm{E}}$ in the range of $P^{\mathrm{E}}$ values between 0 and 0.9 (SI Appendix, Fig. S5 $A$ and $B$ ).

Concentrations of the endogenous $\mathrm{Ca}^{2+}$ buffers and effective coupling distance between presynaptic $\mathrm{Ca}^{2+}$ channels and $\mathrm{Ca}^{2+}$ sensors of exocytosis. Buffer concentrations and the effective $\mathrm{Ca}^{2+}$ channel-exocytosis coupling distance (i.e. $R_{c}$ ) were estimated by using a hemispherical $\mathrm{Ca}^{2+}$ source model (Fig. 9A). A hemispherical $\mathrm{Ca}^{2+}$ source with 1 -nm radius was embedded in a hemispherical volume with $2-\mu \mathrm{m}$ radius, which corresponds to the mean distance between neighboring synapses in IHCs (72). The simulation volume was filled with $2 \mathrm{mM}$ MgATP and the chosen concentrations of BAPTA, EGTA, or endogenous buffers. Initially, the system was in a steady state with uniform distribution of $\mathrm{Ca}^{2+}, \mathrm{Mg}^{2+}$, and buffers. The boundary of the simulation volume was assumed to be reflective for all considered particle species. This effectively mimics the effect of $\mathrm{Ca}^{2+}$ coming from the neighboring synapses and also fixes the amount of buffers available per synapse. On the contrary, pure reflection of $\mathrm{Ca}^{2+}$ from the boundary ignores $\mathrm{Ca}^{2+}$ removal by the pumps and through exchange with the remaining volume of the IHC, which is considerably larger $(2.2 \mathrm{pL})(73)$ than the volume of 12 hemispheres of $2 \mu \mathrm{m}$ each $(\sim 0.02 \mathrm{pL})$. It is shown in SI Appendix, section 7.6, however, that these effects do not considerably influence our results. $\mathrm{Ca}^{2+}$ concentration at time $t$ and distance $r$ from the source, $\left[\mathrm{Ca}^{2+}\right](t, r)$, is governed by a system of reactiondiffusion equations as described in SI Appendix, section 7.1 .

We assumed that the amount of release in a 20-ms time window was proportional to $\left(\left[\mathrm{Ca}^{2+}\right](r)\right)^{m \cdot q(r)}$. $\left[\mathrm{Ca}^{2+}\right](r)$ was estimated $20 \mathrm{~ms}$ after the onset of stimulus. During this time window, $\left[\mathrm{Ca}^{2+}\right](r)$ in simulations was essentially stationary (apart from the initial equilibration of the profile, which, however, is very fast; SI Appendix, section 7.2).

To find $R_{\mathrm{c}}$ based on the experimental data, we minimized the mean relative deviation of the ratios of modeled and experimentally observed release during the first $20 \mathrm{~ms}$ in different $\mathrm{Ca}^{2+}$ buffering conditions:

$$
z(r)=\sum_{j=1}^{4} w_{j} \cdot \frac{\left|a_{j}-b_{j}(r)\right|}{\min \left(a_{j}, b_{j}(r)\right)}
$$

Here, $a_{\mathrm{j}}=\Delta C_{\mathrm{m}, 0} / \Delta C_{\mathrm{m}, \mathrm{j}}$ is the ratio of $\Delta C_{\mathrm{m}}$ values estimated in TKO IHCs (at room temperature) with $1 \mathrm{mM}$ BAPTA $\left(\Delta C_{\mathrm{m}, 0}\right)$ and $1 \mathrm{mM}$ EGTA $(j=1)$, $0.5 \mathrm{mM}$ BAPTA $(j=2), 0.5 \mathrm{mM} \operatorname{EGTA}(j=3)$, or no exogenous buffer $(j=4) . b_{\mathrm{j}}=$

1. Lenzi D, Roberts WM (1994) Calcium signalling in hair cells: Multiple roles in a compact cell. Curr Opin Neurobiol 4(4):496-502.

2. Roberts WM (1994) Localization of calcium signals by a mobile calcium buffer in frog saccular hair cells. J Neurosci 14(5 pt 2):3246-3262.

3. Schwaller B, Meyer M, Schiffmann S (2002) 'New' functions for 'old' proteins: The role of the calcium-binding proteins calbindin D-28k, calretinin and parvalbumin, in cerebellar physiology. Studies with knockout mice. Cerebellum 1(4):241-258.

4. Schneggenburger R, Neher E (2005) Presynaptic calcium and control of vesicle fusion Curr Opin Neurobiol 15(3):266-274.

5. Heller S, Bell AM, Denis CS, Choe Y, Hudspeth AJ (2002) Parvalbumin 3 is an abundant Ca2+ buffer in hair cells. J Assoc Res Otolaryngo/ 3(4):488-498.

6. Roberts WM (1993) Spatial calcium buffering in saccular hair cells. Nature 363(6424): 74-76.

7. Edmonds B, Reyes R, Schwaller B, Roberts WM (2000) Calretinin modifies presynaptic calcium signaling in frog saccular hair cells. Nat Neurosci 3(8):786-790.

8. Hackney CM, Mahendrasingam S, Penn A, Fettiplace R (2005) The concentrations of calcium buffering proteins in mammalian cochlear hair cells. J Neurosci 25(34):7867-7875.

9. Johnson SL, Forge A, Knipper M, Münkner S, Marcotti W (2008) Tonotopic variation in the calcium dependence of neurotransmitter release and vesicle pool replenishment at mammalian auditory ribbon synapses. J Neurosci 28(30):7670-7678.

10. Servais $L$, et al. (2005) Mono- and dual-frequency fast cerebellar oscillation in mice lacking parvalbumin and/or calbindin D-28k. Eur J Neurosci 22(4):861-870.

11. Racay P, Gregory P, Schwaller B (2006) Parvalbumin deficiency in fast-twitch muscles leads to increased 'slow-twitch type' mitochondria, but does not affect the expression of fiber specific proteins. FEBS $J$ 273(1):96-108.

12. Farré-Castany MA, et al. (2007) Differences in locomotor behavior revealed in mice deficient for the calcium-binding proteins parvalbumin, calbindin D-28k or both Behav Brain Res 178(2):250-261.

13. Kreiner L, Christel CJ, Benveniste M, Schwaller B, Lee A (2010) Compensatory regulation of $\mathrm{Cav} 2.1 \mathrm{Ca} 2+$ channels in cerebellar Purkinje neurons lacking parvalbumin and calbindin D-28k. J Neurophysiol 103(1):371-381.
$\left(\left[\mathrm{Ca}^{2+}\right]_{0}(r) /\left(\left[\mathrm{Ca}^{2+}\right]_{j}(r)\right)^{m \cdot q(r)},\left[\mathrm{Ca}^{2+}\right]_{0}(r)\right.$-the model's estimate of $\left[\mathrm{Ca}^{2+}\right]$ at distance $r$ from the source in TKO IHCs loaded with 1 mM BAPTA, $\left[\mathrm{Ca}^{2+}\right]_{j}(r)$ the model's estimates of $\left[\mathrm{Ca}^{2+}\right]$ at distance $r$ from the source in the other four buffering conditions as mentioned earlier. $w_{\mathrm{j}}$ is relative weight of the ith term in the sum, which depends on the ratio of the mean and SEM values of experimental $\Delta C_{m}$ estimates: $w_{j}=\left[\Delta C_{m j} / \operatorname{SEM}\left(\Delta C_{m_{j}}\right)\right] / \sum_{i=1}^{4}\left[\Delta C_{m_{j}} / \operatorname{SEM}\left(\Delta C_{m_{i}}\right)\right]$ Note that $\Delta C_{m}$ estimated in different buffering conditions, correspond to slightly different $Q_{\mathrm{Ca}}$ values which reflect different influx of $\mathrm{Ca}^{2+}$. To take this into account, we rescaled the actual $i_{\mathrm{Ca}}$ values for estimating each $\left[\mathrm{Ca}^{2+}\right.$. This was done so that the average $Q_{\mathrm{Ca}}=3.78 \mathrm{pC}$ measured for $20 \mathrm{~ms}$ depolarization in $\mathrm{PV}^{+/+} \mathrm{Cb}^{+/+} \mathrm{Cr}^{+/+} \mathrm{IHCS}$ corresponded to the nominal $i_{\mathrm{Ca}}$ values. Bootstrapping was used to estimate the confidence interval of $R_{\mathrm{c}}$ (given as a 5-95 percentile range; Fig. $9 C$ ) because of uncertainty in experimental estimates of $m$ and $\Delta C$ for different buffering conditions. A total of $10^{5}$ artificial data sets were generated by drawing numbers from normal distributions with their mean and SD values set to experimentally estimated mean and SEM values.

As shown in $S I$ Appendix, section 8, the $R_{\mathrm{c}}$ of a particular AZ is not simply the average distance between $\mathrm{Ca}^{2+}$ sensors of exocytosis and $\mathrm{Ca}^{2+}$ channels. Rather, it is a nonlinear average of those distances weighted by the contributions of the corresponding channels to the $\left[\mathrm{Ca}^{2+}\right]$ driving exocytosis at the AZ. This aspect is relevant for the functional interpretation of $R_{\mathrm{c}}$. Qualitatively, the more a particular $\mathrm{Ca}^{2+}$ channel contributes to $\left[\mathrm{Ca}^{2+}\right]$ at a particular release site and the more that release site contributes to the overall neurotransmitter release at the $A Z$, the more the distance between the channel and the corresponding $\mathrm{Ca}^{2+}$ sensor of exocytosis weighs in determining the $R_{\mathrm{c}}$.

The fitting procedure for endogenous buffer concentration estimation was based on minimization of a relative discrepancy measure of the form $\sum_{\mathrm{i}} \mid c_{1}\left(r_{\mathrm{i}}\right)-c_{2}\left(r_{\mathrm{i}}\right) / /\left[c_{1}\left(r_{\mathrm{i}}\right)+c_{2}\left(r_{\mathrm{i}}\right)\right]$ for $r \in[0 ; 200] \mathrm{nm}$.

ACKNOWLEDGMENTS. We thank M. Charles Liberman for providing guidance and the facilities for the single auditory nerve fiber recordings, Gerhard Hoch for providing image analysis MATLAB routines, Carolin Wichmann for providing the EM micrograph, Erwin Neher, Aaron B. Wong, and members of the InnerEarLab for discussion and comments on the manuscript and Margitta Köppler, Sonja Blume, Christian Rüdiger, Sandra Gerke, and Christiane Senger-Freitag (Institute for Auditory Neuroscience, University Medical Center Göttingen, ) and Simone Eichenberger and Valérie Salicio (Unit of Anatomy, University of Fribourg) for excellent technical assistance. (Unit of Anatomy, University of Fribourg) for excellent technical assistance. Deutsche Forschungsgemeinschaft (DFG) through a fellowship (to N.S.) and the Collaborative Research Center 889 Projects A2, A6, and C6 (to T.M. N.S., and F.W.), and German Federal Ministry for Education and Research Grants 01GQ0811 (to F.W.) and 01GQ1005A (to T.M.) through the Bernstein Center for Computational Neuroscience.

14. Schwaller $B$ (2012) The use of transgenic mouse models to reveal the functions of Ca2+ buffer proteins in excitable cells. Biochim Biophys Acta 1820(8):1294-1303.

15. Hackney CM, Mahendrasingam S, Jones EM, Fettiplace R (2003) The distribution of calcium buffering proteins in the turtle cochlea. J Neurosci 23(11):4577-4589.

16. Platzer J, et al. (2000) Congenital deafness and sinoatrial node dysfunction in mice lacking class D L-type Ca2+ channels. Cell 102(1):89-97.

17. Brandt A, Striessnig J, Moser T (2003) CaV1.3 channels are essential for development and presynaptic activity of cochlear inner hair cells. J Neurosci 23(34):10832-10840.

18. Dou $\mathrm{H}$, et al. (2004) Null mutation of alpha1D Ca2+ channel gene results in deafness but no vestibular defect in mice. J Assoc Res Otolaryngol 5(2):215-226.

19. Khimich D, et al. (2005) Hair cell synaptic ribbons are essential for synchronous auditory signalling. Nature 434(7035):889-894.

20. Naraghi M, Neher E (1997) Linearized buffered Ca2+ diffusion in microdomains and its implications for calculation of $[\mathrm{Ca} 2+]$ at the mouth of a calcium channel. J Neurosci 17(18):6961-6973.

21. Nägerl UV, Novo D, Mody I, Vergara JL (2000) Binding kinetics of calbindin-D(28k) determined by flash photolysis of caged $\mathrm{Ca}(2+)$. Biophys $J$ 79(6):3009-3018.

22. Moser T, Beutner D (2000) Kinetics of exocytosis and endocytosis at the cochlear inner hair cell afferent synapse of the mouse. Proc Natl Acad Sci USA 97(2):883-888.

23. Taberner AM, Liberman MC (2005) Response properties of single auditory nerve fibers in the mouse. J Neurophysio/ 93(1):557-569.

24. Buran BN, et al. (2010) Onset coding is degraded in auditory nerve fibers from mutant mice lacking synaptic ribbons. J Neurosci 30(22):7587-7597.

25. Jing Z, et al. (2013) Disruption of the presynaptic cytomatrix protein bassoon degrades ribbon anchorage, multiquantal release, and sound encoding at the hair cell afferent synapse. J Neurosci 33(10):4456-4467.

26. Dallos P, Harris D (1978) Properties of auditory nerve responses in absence of outer hair cells. J Neurophysio/ 41(2):365-383.

27. Frank T, et al. (2010) Bassoon and the synaptic ribbon organize $\mathrm{Ca}^{2}+$ channels and vesicles to add release sites and promote refilling. Neuron 68(4):724-738.

28. Chen G, et al. (2006) Deficiency in parvalbumin, but not in calbindin D-28k upregulates mitochondrial volume and decreases smooth endoplasmic reticulum surface 
selectively in a peripheral, subplasmalemmal region in the soma of Purkinje cells. Neuroscience 142(1):97-105.

29. von Ameln S, et al. (2012) A mutation in PNPT1, encoding mitochondrial-RNA-import protein PNPase, causes hereditary hearing loss. Am J Hum Genet 91(5):919-927.

30. Pangršič $\mathrm{T}$, et al. (2010) Hearing requires otoferlin-dependent efficient replenishment of synaptic vesicles in hair cells. Nat Neurosci 13(7):869-876.

31. Cho S, Li G-L, von Gersdorff H (2011) Recovery from short-term depression and facilitation is ultrafast and $\mathrm{Ca} 2+$ dependent at auditory hair cell synapses. $J$ Neurosc 31(15):5682-5692.

32. Young ED, Barta PE (1986) Rate responses of auditory nerve fibers to tones in noise near masked threshold. J Acoust Soc Am 79(2):426-442.

33. Li J, Young ED (1993) Discharge-rate dependence of refractory behavior of cat auditory-nerve fibers. Hear Res 69(1-2):151-162.

34. Lenzi D, Crum J, Ellisman MH, Roberts WM (2002) Depolarization redistributes synaptic membrane and creates a gradient of vesicles on the synaptic body at a ribbon synapse. Neuron 36(4):649-659.

35. Kantardzhieva A, Liberman MC, Sewell WF (2013) Quantitative analysis of ribbons, vesicles, and cisterns at the cat inner hair cell synapse: correlations with spontaneous rate. J Comp Neurol 521(14):3260-3271.

36. Wong $A B$, et al. (2014) Developmental refinement of hair cell synapses tightens the coupling of $\mathrm{Ca} 2+$ influx to exocytosis. EMBO J 33(3):247-264.

37. Brandt A, Khimich D, Moser T (2005) Few CaV1.3 channels regulate the exocytosis of a synaptic vesicle at the hair cell ribbon synapse. I Neurosci $25(50): 11577-11585$.

38. Zampini V, et al. (2013) Burst activity and ultrafast activation kinetics of $\mathrm{CaV} 1.3 \mathrm{Ca}^{2}$ channels support presynaptic activity in adult gerbil hair cell ribbon synapses. J Physio 591(Pt 16):3811-3820.

39. Saftenku EE (2012) Effects of calretinin on Ca2+ signals in cerebellar granule cells: implications of cooperative $\mathrm{Ca} 2+$ binding. Cerebellum 11(1):102-120.

40. Eggermann E, Jonas $\mathrm{P}(2012)$ How the 'slow' $\mathrm{Ca}(2+)$ buffer parvalbumin affects transmitter release in nanodomain-coupling regimes. Nat Neurosci 15(1):20-22.

41. Maeda H, Ellis-Davies GCR, Ito K, Miyashita Y, Kasai H (1999) Supralinear Ca2+ signaling by cooperative and mobile $\mathrm{Ca} 2+$ buffering in Purkinje neurons. Neuron 24(4): 989-1002.

42. Tucker TR, Fettiplace R (1996) Monitoring calcium in turtle hair cells with a calcium activated potassium channel. J Physiol 494(Pt 3):613-626.

43. Ricci AJ, Wu YC, Fettiplace R (1998) The endogenous calcium buffer and the time course of transducer adaptation in auditory hair cells. J Neurosci 18(20):8261-8277.

44. Frank T, Khimich D, Neef A, Moser T (2009) Mechanisms contributing to synaptic Ca2 signals and their heterogeneity in hair cells. Proc Natl Acad Sci USA 106(11): 4483-4488.

45. Tucker T, Fettiplace R (1995) Confocal imaging of calcium microdomains and calcium extrusion in turtle hair cells. Neuron 15(6):1323-1335.

46. Issa NP, Hudspeth AJ (1996) The entry and clearance of Ca2+ at individual presynaptic active zones of hair cells from the bullfrog's sacculus. Proc Natl Acad Sci USA 93(18) 9527-9532.

47. Beutner D, Moser T (2001) The presynaptic function of mouse cochlear inner hair cells during development of hearing. J Neurosci 21(13):4593-4599.

48. Spassova MA, et al. (2004) Evidence that rapid vesicle replenishment of the synaptic ribbon mediates recovery from short-term adaptation at the hair cell afferent synapse. J Assoc Res Otolaryngol 5(4):376-390.

49. Goutman JD, Glowatzki E (2007) Time course and calcium dependence of transmitter release at a single ribbon synapse. Proc Natl Acad Sci USA 104(41):16341-16346.

50. Johnson SL, Marcotti W (2008) Biophysical properties of CaV1.3 calcium channels in gerbil inner hair cells. J Physiol 586(4):1029-1042.

51. Grant L, Fuchs P (2008) Calcium- and calmodulin-dependent inactivation of calcium channels in inner hair cells of the rat cochlea. J Neurophysiol 99(5):2183-2193.
52. Peterson BZ, DeMaria CD, Adelman JP, Yue DT (1999) Calmodulin is the Ca2+ sensor for Ca2+-dependent inactivation of L-type calcium channels. Neuron 22(3):549-558.

53. Lee $\mathrm{A}$, et al. (1999) $\mathrm{Ca}+/ \mathrm{calmodulin}$ binds to and modulates P/Q-type calcium channels. Nature 399(6732):155-159.

54. Lee $\mathrm{A}$, et al. (2002) Differential modulation of $\mathrm{Ca}(\mathrm{v}) 2.1$ channels by calmodulin and Ca2+-binding protein 1. Nat Neurosci 5(3):210-217.

55. Haeseleer $F$, et al. (2000) Five members of a novel $\mathrm{Ca}(2+)$-binding protein (CABP) subfamily with similarity to calmodulin. J Biol Chem 275(2):1247-1260.

56. Christel CJ, et al. (2012) Calretinin regulates Ca2+-dependent inactivation and facilitation of $\mathrm{Ca}(\mathrm{v}) 2.1 \mathrm{Ca} 2+$ channels through a direct interaction with the $\alpha 12.1$ subunit. J Biol Chem 287(47):39766-39775.

57. Graydon CW, Cho S, Li G-L, Kachar B, von Gersdorff H (2011) Sharp Ca ${ }^{2+}$ nanodomains beneath the ribbon promote highly synchronous multivesicular release at hair cell synapses. I Neurosci 31(46): 16637-16650.

58. Robertson D, Paki B (2002) Role of L-type Ca2+ channels in transmitter release from mammalian inner hair cells. II. Single-neuron activity. I Neurophysiol 87(6): 2734-2740

59. Wong AB, et al. (2013) Concurrent maturation of inner hair cell synaptic $\mathrm{Ca} 2+$ influx and auditory nerve spontaneous activity around hearing onset in mice. $J$ Neurosci 33(26):10661-10666.

60. Schnee ME, Santos-Sacchi J, Castellano-Muñoz M, Kong J-H, Ricci AJ (2011) Calciumdependent synaptic vesicle trafficking underlies indefatigable release at the hair cell afferent fiber synapse. Neuron 70(2):326-338.

61. Midorikawa M, Tsukamoto Y, Berglund K, Ishii M, Tachibana M (2007) Different roles of ribbon-associated and ribbon-free active zones in retinal bipolar cells. Nat Neurosci 10(10):1268-1276.

62. Mehta B, et al. (2014) Global Ca2+ signaling drives ribbon-independent synaptic transmission at rod bipolar cell synapses. J Neurosci 34(18):6233-6244.

63. Vecellio M, Schwaller B, Meyer M, Hunziker W, Celio MR (2000) Alterations in Purkinje cell spines of calbindin D-28 k and parvalbumin knock-out mice. Eur $J$ Neurosci 12(3): 945-954.

64. Bouilleret V, Schwaller B, Schurmans S, Celio MR, Fritschy JM (2000) Neurodegenerative and morphogenic changes in a mouse model of temporal lobe epilepsy do not depend on the expression of the calcium-binding proteins parvalbumin, calbindin, or calretinin. Neuroscience 97(1):47-58.

65. Airaksinen MS, et al. (1997) Ataxia and altered dendritic calcium signaling in mice carrying a targeted null mutation of the calbindin D28k gene. Proc Natl Acad Sci USA 94(4): 1488-1493.

66. Schwaller B, et al. (1999) Prolonged contraction-relaxation cycle of fast-twitch muscles in parvalbumin knockout mice. Am J Physiol 276(2 pt 1):C395-C403.

67. Schiffmann SN, et al. (1999) Impaired motor coordination and Purkinje cell excitability in mice lacking calretinin. Proc Natl Acad Sci USA 96(9):5257-5262.

68. Pusch M, Neher E (1988) Rates of diffusional exchange between small cells and a measuring patch pipette. Pflugers Arch 411(2):204-211.

69. Pauli-Magnus D, et al. (2007) Detection and differentiation of sensorineural hearing loss in mice using auditory steady-state responses and transient auditory brainstem responses. Neuroscience 149(3):673-684.

70. Neef A, et al. (2007) Probing the mechanism of exocytosis at the hair cell ribbon synapse. J Neurosci 27(47):12933-12944.

71. Glowatzki E, Fuchs PA (2002) Transmitter release at the hair cell ribbon synapse. Nat Neurosci 5(2):147-154.

72. Meyer AC, et al. (2009) Tuning of synapse number, structure and function in the cochlea. Nat Neurosci 12(4):444-453.

73. Lenzi D, Runyeon JW, Crum J, Ellisman MH, Roberts WM (1999) Synaptic vesicle populations in saccular hair cells reconstructed by electron tomography. J Neurosci 19(1):119-132. 


\title{
Supplementary Material for
}

\section{EF-hand Protein $\mathrm{Ca}^{2+}$ Buffers Regulate $\mathrm{Ca}^{2+}$ Influx and Exocytosis in Sensory Hair Cells}

\author{
by \\ Tina Pangršič, Mantas Gabrielaitis, Susann Michanski, Beat Schwaller, Fred Wolf, Nicola Strenzke, \\ Tobias Moser
}

\section{Contents}

1 Supplementary Figure $1 \quad 2$

2 Supplementary Figure $2 \quad 3$

3 Supplementary Figure $3 \quad 4$

4 Supplementary Figure $4 \quad 5$

5 Supplementary Figure $5 \quad 6$

6 Supplementary Figure $6 \quad 7$

7 Modeling of $\mathrm{Ca}^{2+}$ Dynamics (Including Supplementary Figures 7 - 11) 8

7.1 Reaction-Diffusion equations for $\left[\mathrm{Ca}^{2+}\right]$ dynamics . . . . . . . . . . . . . . . 8

7.2 Temporal evolution of $\left[\mathrm{Ca}^{2+}\right] \ldots \ldots \ldots \ldots \ldots \ldots$

7.3 Extent of $\mathrm{Ca}^{2+}$ buffer depletion due to $\mathrm{Ca}^{2+}$ influx . . . . . . . . . . . . . . . . . 12

7.4 Influence of the partial buffer depletion on the $\left[\mathrm{Ca}^{2+}\right]$ vs. $\mathrm{i}_{\mathrm{Ca}}$ relation . . . . . . . . . . . . . 12

7.5 Effect of accumulated bulk $\mathrm{Ca}^{2+}$ during prolonged stimulation on the model predictions of concentrations of the endogenous $\mathrm{Ca}^{2+}$ buffers . . . . . . . . . . . . . . . . . . . . . 14

7.6 Influence of the type of boundary conditions on the estimates of the endogenous buffer concentrations and the coupling distance $R_{c} \ldots \ldots \ldots \ldots \ldots \ldots$

8 Defining and Evaluating the Effective Coupling Distance $\mathbf{R}_{\mathbf{c}}$ (Including Supplementary Figure 12)

8.1 Active zones with 1 vesicular release site and $\mathrm{N} \mathrm{Ca}^{2+}$ channels, no $\mathrm{Ca}^{2+}$ buffers . . . . . . . 17

8.2 Active zones with 1 vesicular release site and $N \mathrm{Ca}^{2+}$ channels, one set of $\mathrm{Ca}^{2+}$ buffers $\quad$. . . . 19

8.3 Active zones with 1 vesicular release site and $N \mathrm{Ca}^{2+}$ channels, two sets of $\mathrm{Ca}^{2+}$ buffers $\quad \ldots \quad$. 21

8.4 Active zones with $M$ vesicular release sites and $N \mathrm{Ca}^{2+}$ channels . . . . . . . . . . . . . . . . 23

8.5 Influence of the $\mathrm{Ca}^{2+}$ channel opening-closing dynamics on $\mathrm{R}_{\mathrm{c}}$ estimate $\ldots . . . . . . . .26$

\begin{tabular}{ll} 
References & 28 \\
\hline
\end{tabular} 


\section{Supplementary Figure 1}

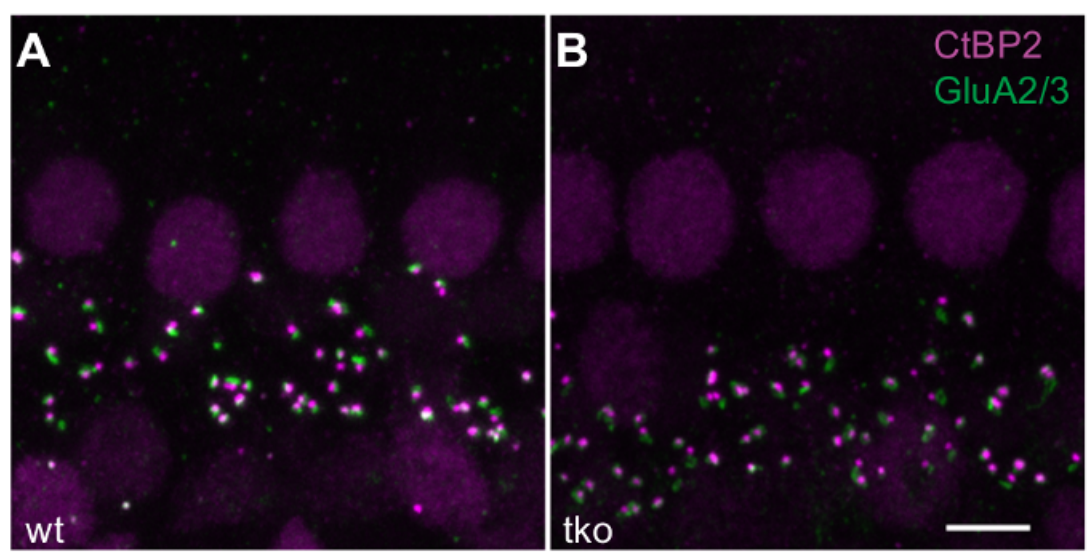

Figure S1: Comparable number of ribbon synapses in the IHCs of the triple buffer knockout and wild-type mice. (A - B) Confocal images of whole-mounts of organs of Corti double stained for the presynaptic marker CtBP2/Ribeye (magenta) and postsynaptic marker GluA 2/3 (green). The number of synapses in tko IHCs (B) was comparable to that of wt IHCs (A). Intact synapses are defined by the juxtaposition of pairs of pre- and postsynaptic fluorescent spots. Scale bar: $5 \mu \mathrm{m}$. 


\section{Supplementary Figure 2}

A

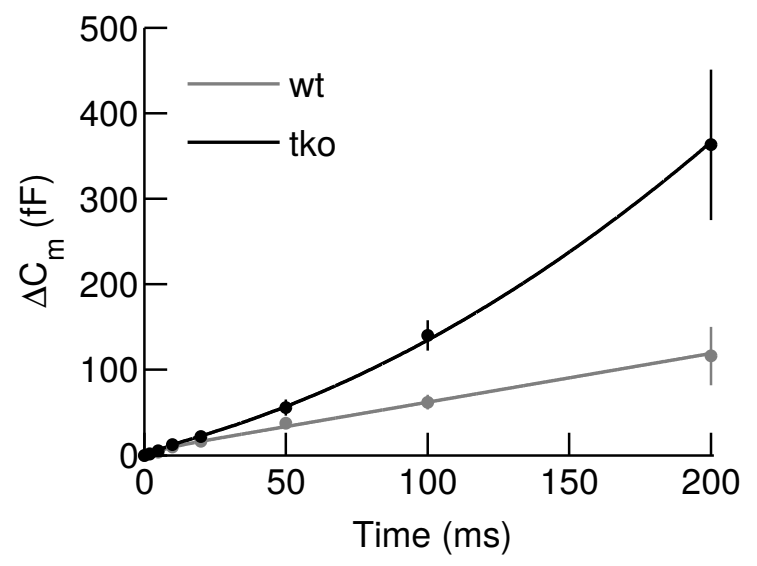

B

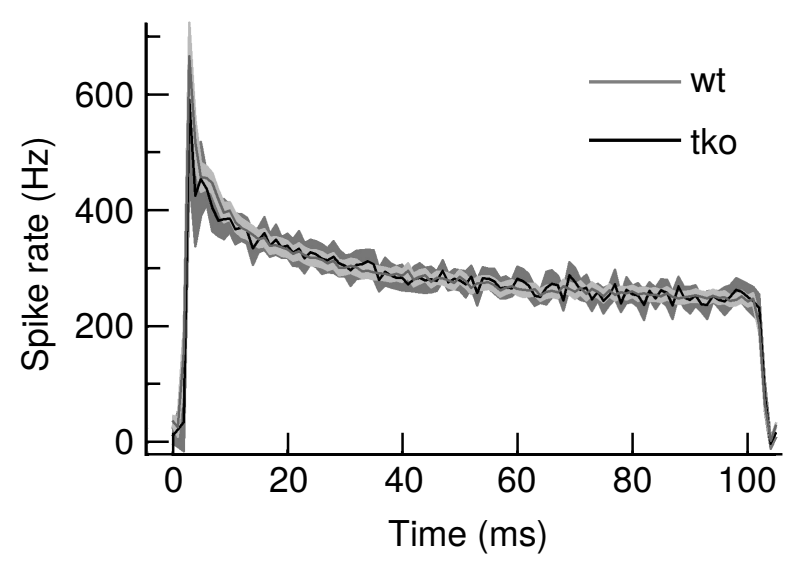

Figure S2: Cumulative exocytosis and SGN spike rates in response to prolonged stimuli in wt and tko mice. (A) Phenomenological fits to the experimental $\Delta \mathrm{C}_{\mathrm{m}}(\mathrm{t})$ data. The wt data were fitted by $\Delta C_{m}(t)=A^{\prime} \cdot t+B^{\prime} \cdot\left(1-e^{-t / \tau}\right)^{n}$ with $\left(A^{\prime}, B^{\prime}, \tau, n\right)$ treated as fitting parameters. The tko data were fitted by $\Delta C_{m}(t)=A^{\prime} \cdot t+B^{\prime} \cdot\left(1-e^{-t / \tau}\right)^{n}+t \cdot\left(a^{\prime} \cdot t+b^{\prime}\right)$ with parameters $\left(A^{\prime}, B^{\prime}, \tau, n\right)$ set to the same values as for the wt data. Parameters $a^{\prime}$ and $b^{\prime}$ were found by fitting the difference between the experimental estimates of $\Delta \mathrm{C}_{\mathrm{m}}(\mathrm{t})$ from tko and wt IHCs by $t \cdot\left(a^{\prime} \cdot t+b^{\prime}\right)$. Note that parameters $(A, B, a, b)$ considered in Methods section "Conversion of neurotransmitter release events to spikes" correspond to the parameters $\left(A^{\prime}, B^{\prime}, a^{\prime}, b^{\prime}\right)$ multiplied by $s c /\left(C_{s v} \cdot N_{s y n}\right)$. (B) Mean peristimulus time histogram ( \pm s.e.m.), plotting the instantaneous spiking rate in 1-ms time bins over the time course of stimulation with $100 \mathrm{~ms}$ long tone bursts at the characteristic frequency of each SGN, $30 \mathrm{~dB}$ above threshold. The rates and the time course of adaptation were similar in tko (grey, $\mathrm{n}=35$ ) and wt SGNs (black, $\mathrm{n}=8$ ). 


\section{Supplementary Figure 3}

A

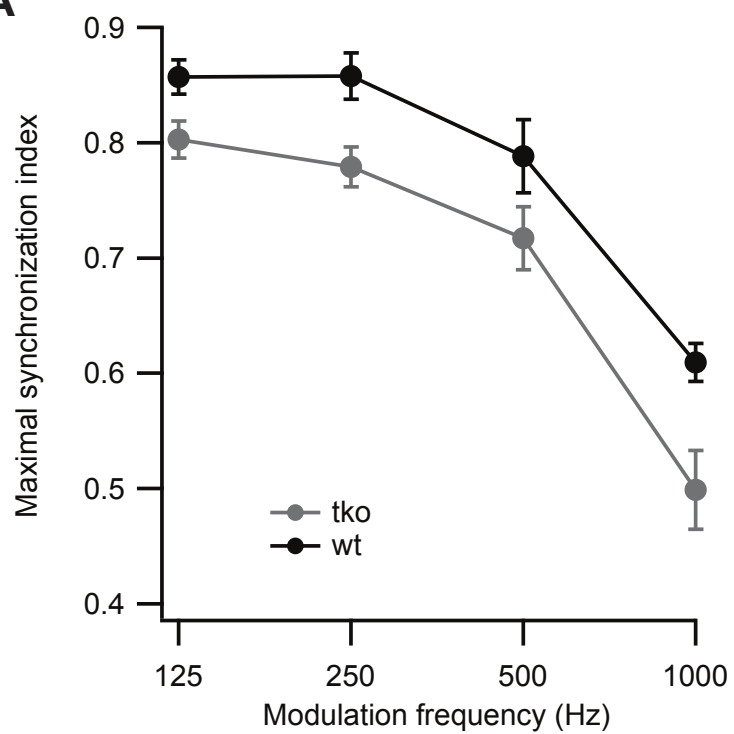

C

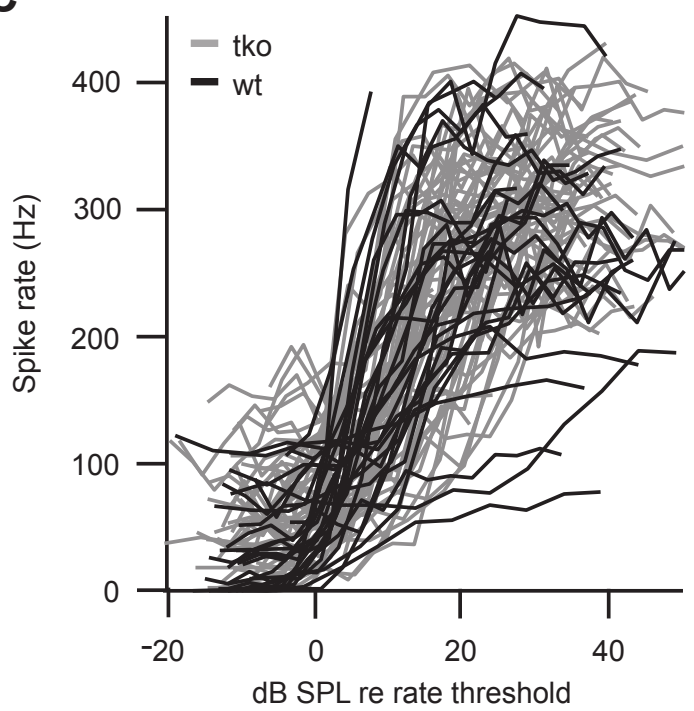

B
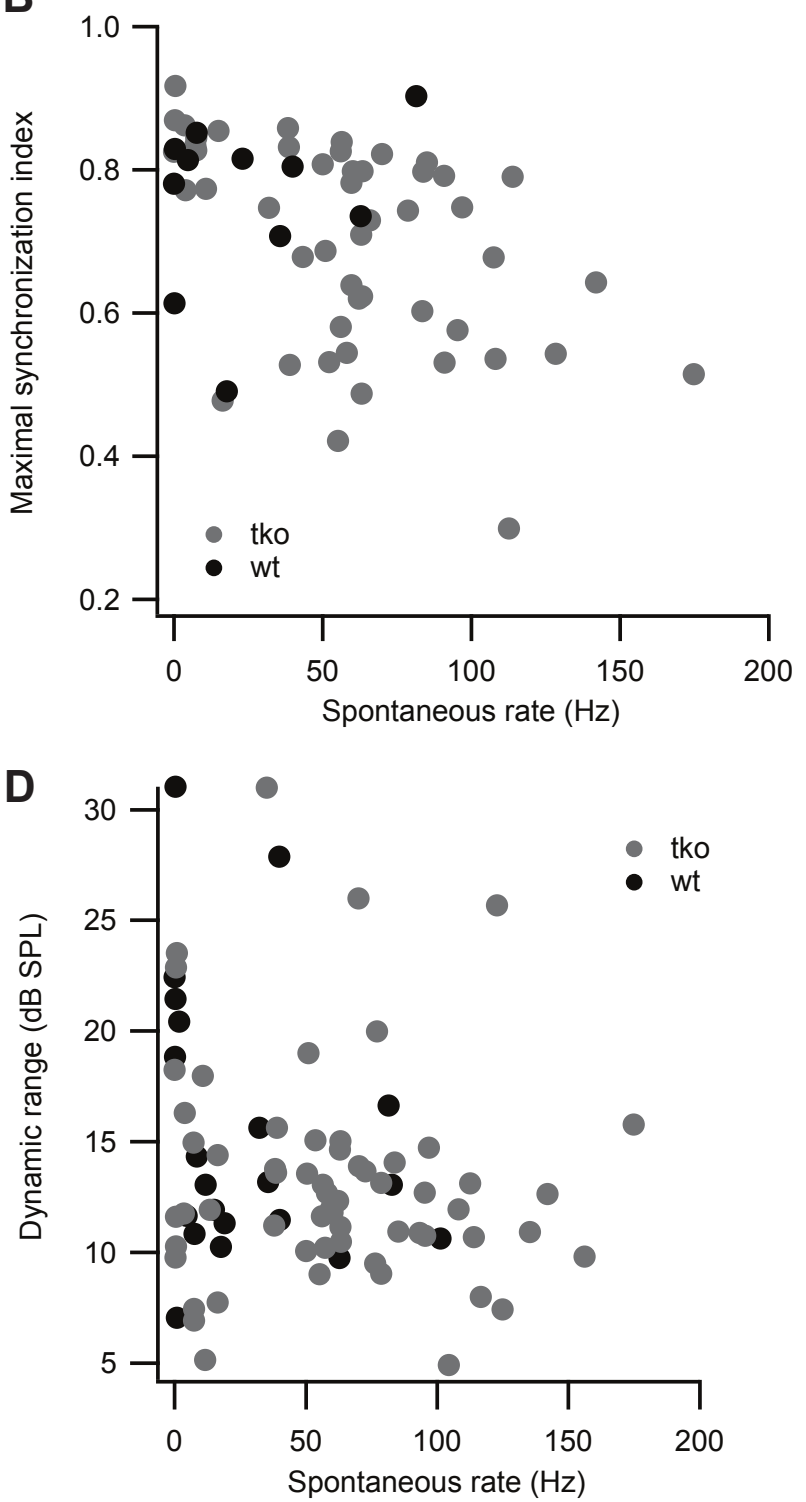

Figure S3: Precision of ongoing sound encoding is not affected by the absence of the three $\mathbf{C a}^{2+}$ buffers. (A) Synchronization of SGN spiking to amplitude modulated transposed tones (formed by modulating a continuous sine wave at CF by a half-wave rectified sinusoid) was assessed at several stimulus intensities and modulation frequencies to determine the maximal synchronization index. (B) Maximal synchronization at $500 \mathrm{~Hz}$ was comparable between tko (grey, n = 49) and wt SGNs (black, $\mathrm{n}=11$ ) in spite of the tendency towards higher spontaneous rates in tko. (C) The steepness of spike rate increase with tone burst intensities was unchanged between tko (grey, $\mathrm{n}=64$ ) and wt (black, $\mathrm{n}=22$ ) SGNs. (D) The dynamic range of sound encoding (range of intensities over which the spike rate increases), which co-varies with spontaneous spiking rate, was similar among genotypes. 


\section{Supplementary Figure 4}
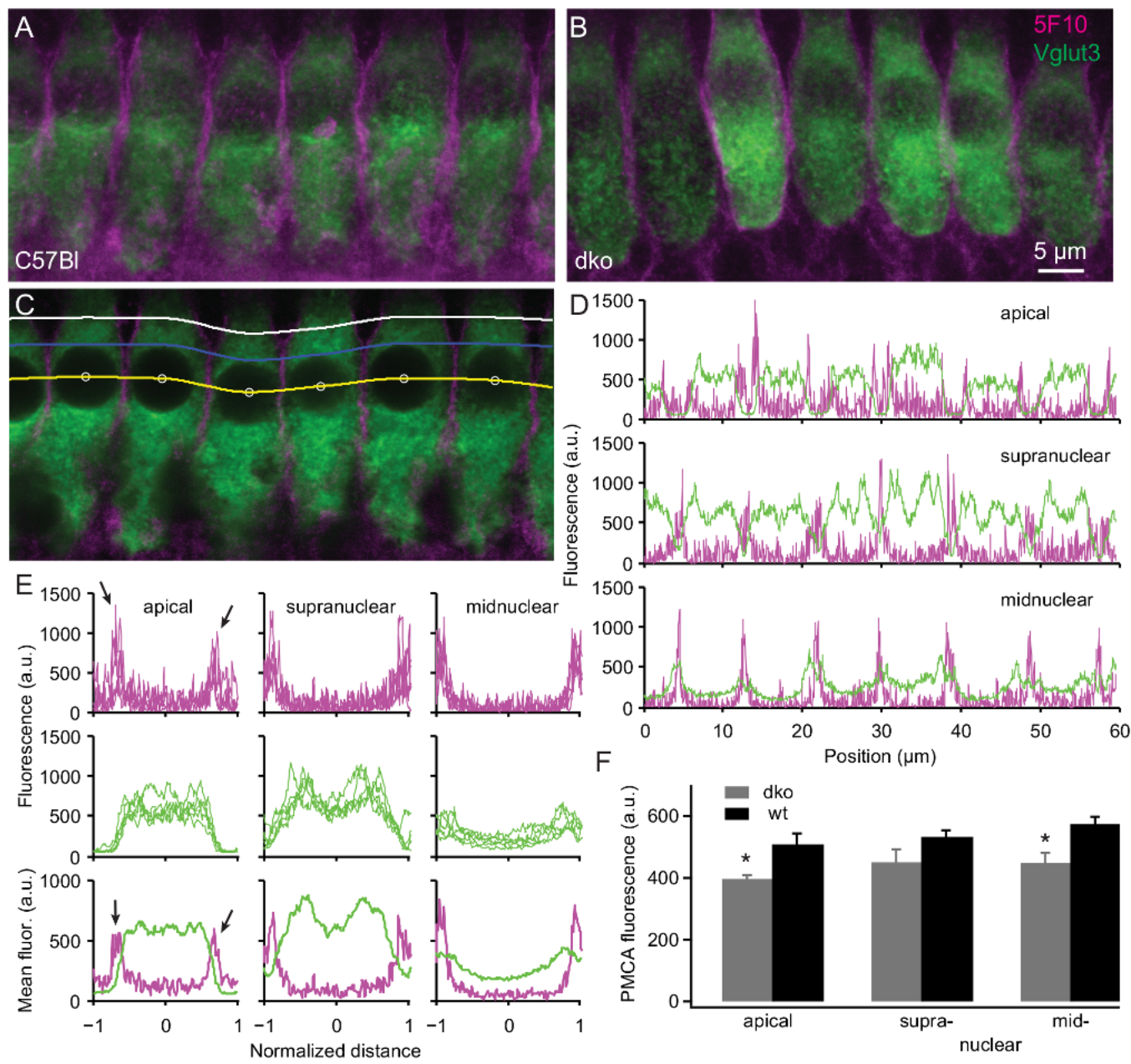

$\mathrm{F}$

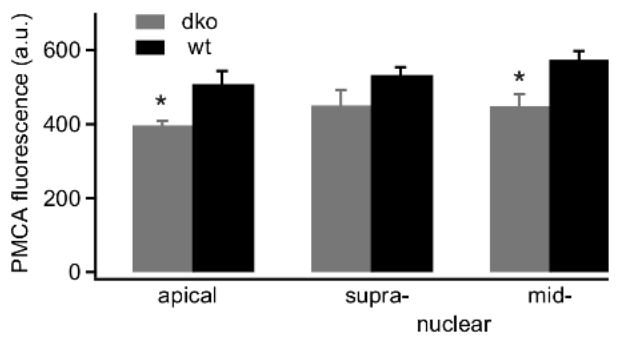

Figure S4: No compensatory upregulation of $\mathrm{Ca}^{2+}$ ATPases (PMCAs) in the plasma membrane of the double buffer knockout IHCs. (A-B) Maximal projections of confocal image stacks of a wt (A) and a dko (B) organ of Corti, stained for panPMCA (5F10, magenta) and an IHC marker Vglut3 (green). (C-E) An example demonstrating how the analysis of the PMCA fluorescence intensity was performed. (C) For each z-stack the immunofluorescence intensity was analyzed in the image plane with the best discernible nuclei (white circles denoting their centers). (D) Line profiles of Vglut3 (green) and PMCA (magenta) fluorescence intensity in the apical, supranuclear and midnuclear IHC region (white, blue and yellow line in panel C, respectively). Please note that we restricted our analysis to these cellular regions because the PMCA immunofluerescence was less well discernible in the basal parts of IHCs of either genotype (due to strong fluorescence in the surrounding tissue, e.g., the nerve fibers). (E) Fluorescence line profiles (from D) of separate cells aligned and superimposed (upper and middle panels). Lower panels show grand averages. Note a visible increase in the PMCA immunofluorescence at the cell borders (arrows). The average fluorescence intensity of these peaks was taken as the IHC PMCA fluorescence. (F) Histogram of average PMCA immunofluorescence in the three cellular regions. Note a significant decrease in the PMCA fluorescence in the plasma membranes of dko IHCs as compared to wt controls (Wilcoxon rank sum test, asterisks, $p<0.05)$. 


\section{$5 \quad$ Supplementary Figure 5}
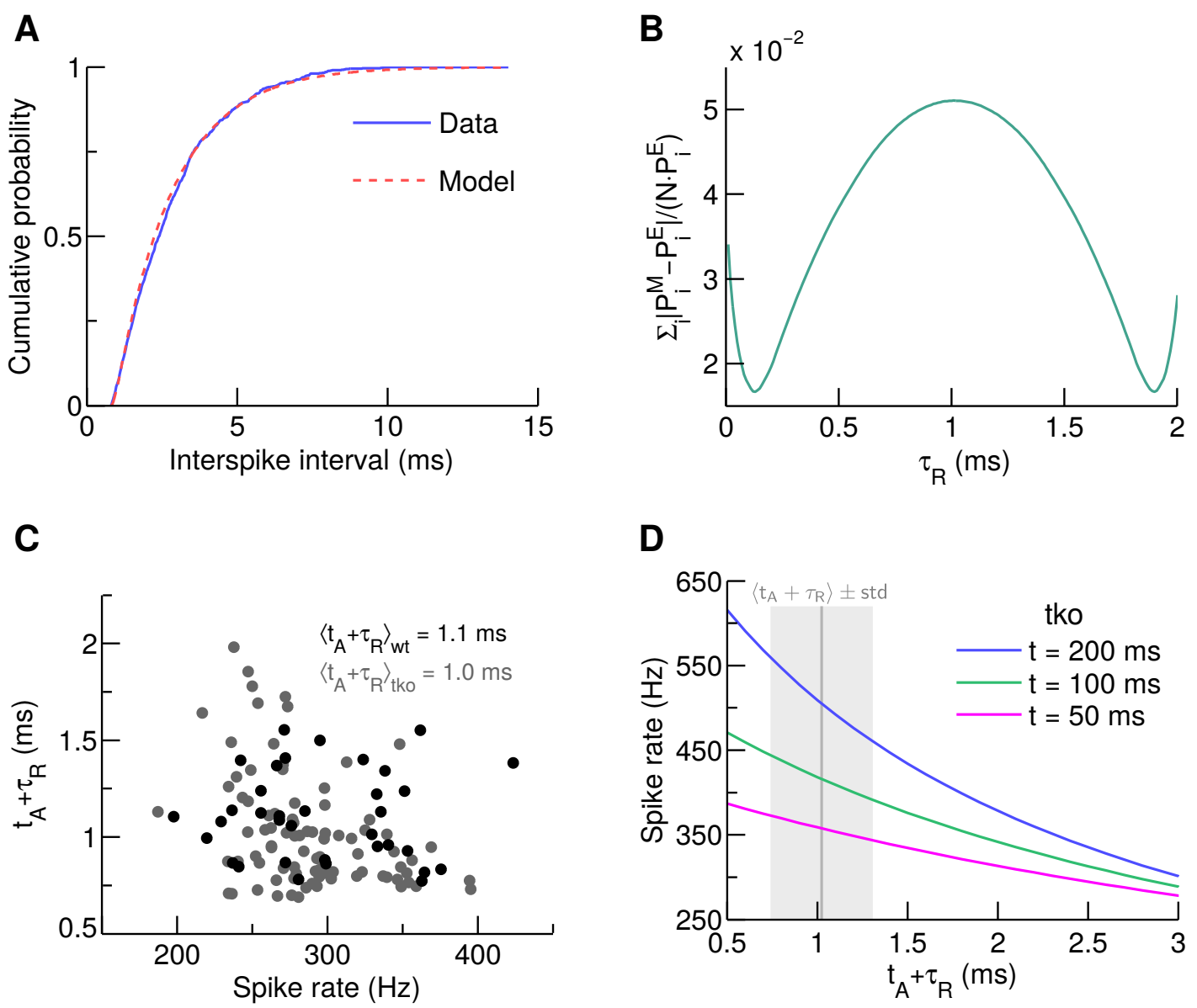

Figure S5: Estimation of the refractory periods and their impact on the discrepancy between the modeled spiking rates in wt and tko SGNs. (A) An exemplary cumulative distribution function of inter-spike intervals of SGNs. Blue solid line - experimental data (wt), red dashed line - the best model fit. $\tau_{\mathrm{R}}$ estimated by this model is likely a minor overestimate because this model ignores the finding that spiking slightly deviates from Poissonian statistics for short inter-spike intervals (1). (B) Dependence of the discrepancy between the experimental data and the model from (A) on the fitting parameter $\tau_{\mathrm{R}}$. As it follows from the expression of $P^{M}(t)$ given in Methods, the discrepancy measure has two equivalent minimum points. We found, however, that, in most of the cases considered, the minimum point corresponding to the larger of the two values of $\tau_{\mathrm{R}}$ resulted in release rate estimates considerably higher than those based on the capacitance increment recordings from wt IHCs. We thus selected the smaller of the two $\tau_{\mathrm{R}}$ estimates, which always gave reasonable release rate estimates. In a few cases, when both estimates of $\tau_{\mathrm{R}}$ resulted in reasonable release rates, the difference between them was small. (C) Scatter plot of the overall refractory period estimate $t_{A}+\tau_{R}$ vs. steady-state spike rate from different SGNs (wt $\mathrm{n}=35$, tko $\mathrm{n}=87$ ). (D) Spike rate dependence on $\mathrm{t}_{\mathrm{A}}+\tau_{\mathrm{R}}$ of modeled tko SGNs. Different colors represent spike rate estimates at different time points after stimulus onset. The scaling coefficient $s c$ was chosen in such a way that the modeled spike rate of wt SGNs, derived from the $\Delta \mathrm{C}_{\mathrm{m}}$ data from wt IHCs, equaled the experimental observation $-250 \mathrm{~Hz}$ - at a given $\mathrm{t}_{\mathrm{A}}+\tau_{\mathrm{R}}, 50 \mathrm{~ms}$ after stimulus onset. The sc values increased from 0.23 at $\mathrm{t}_{\mathrm{A}}+\tau_{\mathrm{R}}=0$ to 0.94 at $\mathrm{t}_{\mathrm{A}}+\tau_{\mathrm{R}}=3 \mathrm{~ms}$. The same $s c$ values were then used for calculating spike rates of modelled tho SGNs based on $\Delta \mathrm{C}_{\mathrm{m}}$ data from tko IHCs. 


\section{Supplementary Figure 6}

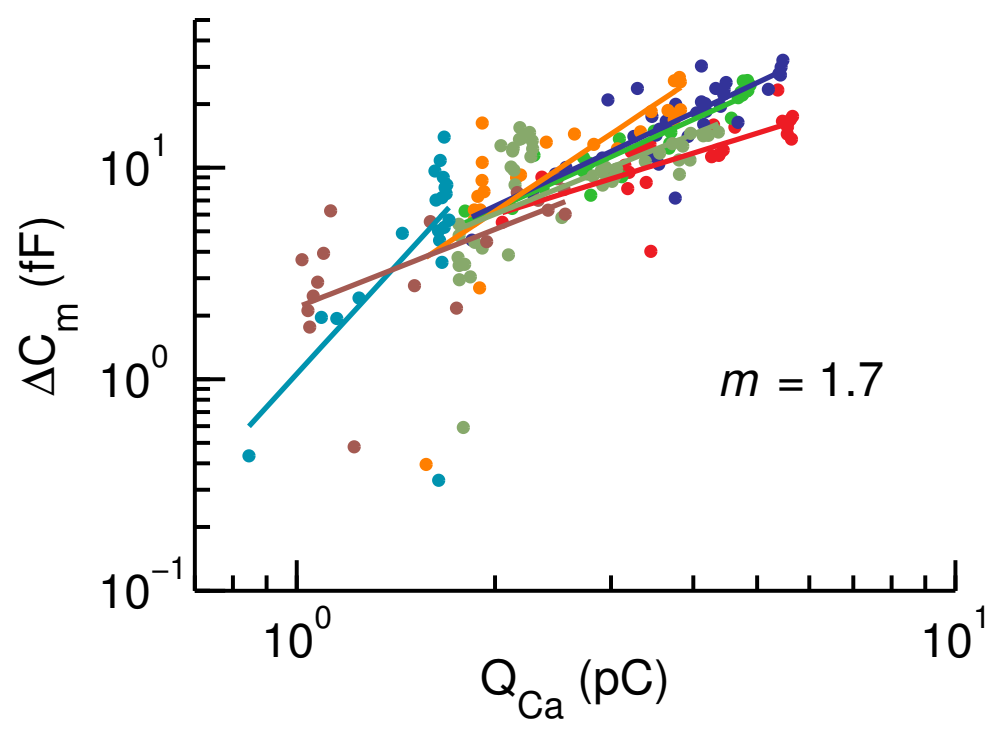

Figure S6: Apparent $\mathrm{Ca}^{2+}$ cooperativity of exocytosis in the operating range of $\mathrm{Ca}^{2+}$ influx at IHC synapses used for estimating the effective coupling distance $\mathbf{R}_{\mathbf{c}}$. Apparent $\mathrm{Ca}^{2+}$ cooperativities were estimated as the slope factors of linear fits (solid lines) of $\Delta C_{m}$ vs $Q_{C a}$ relations based on external $\left[\mathrm{Ca}^{2+}\right]$ manipulation from seven mature IHCs (points) in log-log scale. Each color in the figure represents a particular cell. The data were taken from (2). $Q_{C a}$ interval used for the data fitting shown here was $\left[\max \left[Q_{C a}\right] / 2.5, \max \left[Q_{C a}\right]\right]$ for each cell individually. The resulting $\mathrm{Ca}^{2+}$ cooperativity averaged over all cells, which we denote by $m$, was equal to 1.7. This value of $m$ was used for estimating the effective coupling distance $R_{c}$, as described in Methods. To make sure that the estimate of $m$ was not sensitive to small changes in the choice of the left boundary of the $Q_{C a}$ interval, we also considered values of the left boundary from $\max \left[Q_{C a}\right] / 1.1$ to $\max \left[Q_{C a}\right] / 3$. The resulting $m$ values varied between 1.5 and 1.9 . 


\section{$7 \quad$ Modeling of $\mathrm{Ca}^{2+}$ Dynamics (Including Supplementary Fig- ures 7 - 11)}

In this section, we provide additional information on modeling $\mathrm{Ca}^{2+}$ dynamics in the framework of a single $\mathrm{Ca}^{2+}$ source model formulated in Methods.

\subsection{Reaction-Diffusion equations for $\left[\mathrm{Ca}^{2+}\right]$ dynamics}

In the presence of one of the mobile exogenous buffers, BAPTA or EGTA, the dynamics of $\left[\mathrm{Ca}^{2+}\right]$ and concentrations of the mobile $\mathrm{Ca}^{2+}$ buffers following the onset of $\mathrm{Ca}^{2+}$ influx at $\mathrm{t}=0$ were modeled by the following closed system of reaction-diffusion equations:

$$
\left\{\begin{array}{l}
\partial_{\mathrm{t}}\left[\mathrm{MgB}_{0}\right]=\mathrm{k}_{\mathrm{on}, 0}^{\prime} \cdot\left[\mathrm{Mg}^{2+}\right] \cdot\left(\left[\mathrm{B}_{0}\right]_{\mathrm{T}}-\left[\mathrm{MgB}_{0}\right]-\left[\mathrm{CaB}_{0}\right]\right)-\left(\mathrm{k}_{\mathrm{off}, 0}^{\prime}-\mathrm{D}_{0} \cdot \Delta_{\mathrm{r}}\right) \cdot\left[\mathrm{MgB}_{0}\right] \\
\partial_{\mathrm{t}}\left[\mathrm{Mg}^{2+}\right]=\left(\mathrm{D}_{0} \cdot \Delta_{\mathrm{r}}\left[\mathrm{MgB}_{0}\right]-\partial_{\mathrm{t}}\left[\mathrm{MgB}_{0}\right]\right)+\mathrm{D}^{\prime} \cdot \Delta_{\mathrm{r}}\left[\mathrm{Mg}^{2+}\right] \\
\partial_{\mathrm{t}}\left[\mathrm{CaB}_{0}\right]=\mathrm{k}_{\mathrm{on}, 0} \cdot\left[\mathrm{Ca}^{2+}\right] \cdot\left(\left[\mathrm{B}_{0}\right]_{\mathrm{T}}-\left[\mathrm{MgB}_{0}\right]-\left[\mathrm{CaB}_{0}\right]\right)-\left(\mathrm{k}_{\mathrm{off}, 0}-\mathrm{D}_{0} \cdot \Delta_{\mathrm{r}}\right) \cdot\left[\mathrm{CaB}_{0}\right] \\
\partial_{\mathrm{t}}\left[\mathrm{CaB}_{1}\right]=\mathrm{k}_{\mathrm{on}, 1} \cdot\left[\mathrm{Ca}^{2+}\right] \cdot\left(\left[\mathrm{B}_{1}\right]_{\mathrm{T}}-\left[\mathrm{CaB}_{1}\right]\right)-\left(\mathrm{k}_{\mathrm{off}, 1}-\mathrm{D}_{1} \cdot \Delta_{\mathrm{r}}\right) \cdot\left[\mathrm{CaB}_{1}\right] \\
\partial_{\mathrm{t}}\left[\mathrm{Ca}^{2+}\right]=\sum_{\mathrm{i}=0}^{1}\left(\mathrm{D}_{\mathrm{i}} \cdot \Delta_{\mathrm{r}}\left[\mathrm{CaB}_{\mathrm{i}}\right]-\partial_{\mathrm{t}}\left[\mathrm{CaB}_{\mathrm{i}}\right]\right)+\mathrm{D} \cdot \Delta_{\mathrm{r}}\left[\mathrm{Ca}^{2+}\right]
\end{array} .\right.
$$

Here, $\mathrm{B}_{0}$ stands for ATP, and $\mathrm{B}_{1}$ stands either for BAPTA or EGTA. $\mathrm{k}_{\mathrm{on}}$ and $\mathrm{k}_{\mathrm{off}}$ are $\mathrm{Ca}^{2+}$ binding and dissociation rates of a particular buffer. $\mathrm{k}_{\mathrm{on}}^{\prime}$ and $\mathrm{k}_{\mathrm{off}}^{\prime}$ are $\mathrm{Mg}^{2+}$ binding and dissociation rates of a particular buffer. $\mathrm{D}_{0}$ and $\mathrm{D}_{1}$ are the diffusion coefficients of $\mathrm{B}_{0}$ and $\mathrm{B}_{1}$ respectively. We assumed that the diffusion coefficients of $\mathrm{Ca}^{2+}$ - or $\mathrm{Mg}^{2+}$-bound buffer molecules are equal to the the diffusion coefficients of the corresponding free buffer molecules. D and $\mathrm{D}^{\prime}$ stand for the diffusion coefficients of $\mathrm{Ca}^{2+}$ and $\mathrm{Mg}^{2+}$ respectively. $\Delta_{\mathrm{r}} \equiv \frac{1}{r^{2}} \frac{\partial}{\partial r}\left(r^{2} \frac{\partial}{\partial r} \ldots\right)$ is the radial component of the Laplace operator. Note that concentrations of free buffer molecules at any point of space and time were determined by the difference between the total and $\mathrm{Mg}^{2+}$-bound and/or $\mathrm{Ca}^{2+}$-bound buffer concentrations. The total buffer concentrations were constant in space and time. This applies whenever $\mathrm{Ca}^{2+}$-bound and $\mathrm{Mg}^{2+}$-bound molecules have the same diffusion coefficients as the corresponding free buffer molecules, the total buffer concentrations are distributed uniformly within the simulation volume at $\mathrm{t}=0$ (as it was assumed in our model), and boundary conditions formulated in the next paragraph are used (see (3)).

In the case of the original model, the boundary conditions read as

$$
\left.\frac{\partial\left[\mathrm{Ca}^{2+}\right]}{\partial r}\right|_{r=r_{0}}=-\frac{i_{C a}}{4 \cdot \pi \cdot D \cdot F \cdot r_{0}^{2}} \cdot H(t),\left.\quad \frac{\partial[\mathrm{X}]}{\partial r}\right|_{r=r_{0}}=0,\left.\quad \frac{\partial\left[\mathrm{Ca}^{2+}\right.}{\partial r}\right|_{r=R}=0,\left.\quad \frac{\partial[\mathrm{X}]}{\partial r}\right|_{r=R}=0,
$$

here, $\mathrm{X}-\mathrm{Mg}^{2+}$ or one of the $\mathrm{Ca}^{2+}$ or $\mathrm{Mg}^{2+}$ bound buffer molecules, $F$ - Faraday constant, $H(t)-$ Heaviside step function. In the case of the modified version of the original model considered in section 7.6 , the boundary conditions read as

$$
\left.\frac{\partial\left[\mathrm{Ca}^{2+}\right]}{\partial r}\right|_{r=r_{0}}=-\frac{i_{C a}}{4 \cdot \pi \cdot D \cdot F \cdot r_{0}^{2}} \cdot H(t),\left.\quad \frac{\partial[\mathrm{X}]}{\partial r}\right|_{r=r_{0}}=0,\left.\quad\left[\mathrm{Ca}^{2+}\right]\right|_{r=R}=\left[\mathrm{Ca}^{2+}\right]_{0},\left.\quad[\mathrm{X}]\right|_{r=R}=[\mathrm{X}]_{0} .
$$

Here, $\left[\mathrm{Ca}^{2+}\right]_{0}$ and $[\mathrm{X}]_{0}$ are concentrations of $\mathrm{Ca}^{2+}$ and other ions and molecules in the simulation volume before onset of $\mathrm{Ca}^{2+}$ influx. Noteworthy, due to the symmetry, a hemispherical source model with 
reflecting boundary at the base of the hemispherical simulation volume is equivalent to a spherical source model, with identical $\mathrm{Ca}^{2+}$ flux density, embedded in a spherical simulation volume.

In the presence of mobile endogenous $\mathrm{Ca}^{2+}$ buffers, $\left[\mathrm{Ca}^{2+}\right]$ was determined by the following set of equations (or a particular subset of this set, when not all of the physiological endogenous buffers were considered together):

$$
\left\{\begin{array}{l}
\partial_{\mathrm{t}}\left[\mathrm{MgB}_{0}\right]=\mathrm{k}_{\mathrm{on}, 0}^{\prime} \cdot\left[\mathrm{Mg}^{2+}\right] \cdot\left(\left[\mathrm{B}_{0}\right]_{\mathrm{T}}-\left[\mathrm{MgB}_{0}\right]-\left[\mathrm{CaB}_{0}\right]\right)-\left(\mathrm{k}_{\mathrm{off}, 0}^{\prime}-\mathrm{D}_{0} \cdot \Delta_{\mathrm{r}}\right) \cdot\left[\mathrm{MgB}_{0}\right] \\
\partial_{\mathrm{t}}\left[\mathrm{MgB}_{1}\right]=\mathrm{k}_{\mathrm{on}, 1}^{\prime} \cdot\left[\mathrm{Mg}^{2+}\right] \cdot\left(2 \cdot\left[\mathrm{B}_{1}\right]_{\mathrm{T}}-\left[\mathrm{MgB}_{1}\right]-\left[\mathrm{CaB}_{1}\right]\right)-\left(\mathrm{k}_{\mathrm{off}, 1}^{\prime}-\mathrm{D}_{1} \cdot \Delta_{\mathrm{r}}\right) \cdot\left[\mathrm{MgB}_{1}\right] \\
\partial_{\mathrm{t}}\left[\mathrm{Mg}^{2+}\right]=\sum_{\mathrm{i}=0}^{1}\left(\mathrm{D}_{\mathrm{i}} \cdot \Delta_{\mathrm{r}}\left[\mathrm{MgB}_{\mathrm{i}}\right]-\partial_{\mathrm{t}}\left[\mathrm{MgB}_{\mathrm{i}}\right]\right)+\mathrm{D}^{\prime} \cdot \Delta_{\mathrm{r}}\left[\mathrm{Mg}^{2+}\right] \\
\partial_{\mathrm{t}}\left[\mathrm{CaB}_{0}\right]=\mathrm{k}_{\mathrm{on}, 0} \cdot\left[\mathrm{Ca}^{2+}\right] \cdot\left(\left[\mathrm{B}_{0}\right]_{\mathrm{T}}-\left[\mathrm{MgB}_{0}\right]-\left[\mathrm{CaB}_{0}\right]\right)-\left(\mathrm{k}_{\mathrm{off}, 0}-\mathrm{D}_{0} \cdot \Delta_{\mathrm{r}}\right) \cdot\left[\mathrm{CaB}_{0}\right] \\
\partial_{\mathrm{t}}\left[\mathrm{CaB}_{1}\right]=\mathrm{k}_{\mathrm{on}, 1} \cdot\left[\mathrm{Ca}^{2+}\right] \cdot\left(2 \cdot\left[\mathrm{B}_{1}\right]_{\mathrm{T}}-\left[\mathrm{MgB}_{1}\right]-\left[\mathrm{CaB}_{1}\right]\right)-\left(\mathrm{k}_{\mathrm{off}, 1}-\mathrm{D}_{1} \cdot \Delta_{\mathrm{r}}\right) \cdot\left[\mathrm{CaB}_{1}\right] \\
\partial_{\mathrm{t}}\left[\mathrm{CaB}_{2}\right]=\mathrm{k}_{\mathrm{on}, 2} \cdot\left[\mathrm{Ca}^{2+}\right] \cdot\left(4 \cdot\left[\mathrm{B}_{2}\right]_{\mathrm{T}}-\left[\mathrm{CaB}_{2}\right]\right)-\left(\mathrm{k}_{\mathrm{off}, 2}-\mathrm{D}_{2} \cdot \Delta_{\mathrm{r}}\right) \cdot\left[\mathrm{CaB}_{2}\right] \\
\partial_{\mathrm{t}}\left[\mathrm{CaB}_{3}\right]=\mathrm{k}_{\mathrm{on}, 3} \cdot\left[\mathrm{Ca}^{2+}\right] \cdot\left(\left[\mathrm{B}_{3}\right]_{\mathrm{T}}-\left[\mathrm{CaB}_{3}\right]\right)-\left(\mathrm{k}_{\mathrm{off}, 3}-\mathrm{D}_{3} \cdot \Delta_{\mathrm{r}}\right) \cdot\left[\mathrm{CaB}_{3}\right] \\
\partial_{\mathrm{t}}\left[\mathrm{CaB}_{4}\right]=\mathrm{k}_{\mathrm{on}, 4}^{\mathrm{T}} \cdot\left[\mathrm{Ca}^{2+}\right] \cdot\left(2 \cdot\left[\mathrm{B}_{4}\right]_{\mathrm{T}}-\left[\mathrm{CaB}_{4}\right]-\left[\mathrm{Ca}_{2} \mathrm{~B}_{4}\right]\right)-\left(\mathrm{k}_{\mathrm{off}, 4}^{\mathrm{T}}-\mathrm{D}_{4} \cdot \Delta_{\mathrm{r}}\right) \cdot\left[\mathrm{CaB}_{4}\right] \\
\partial_{\mathrm{t}}\left[\mathrm{Ca}_{2} \mathrm{~B}_{4}\right]=\mathrm{k}_{\mathrm{on}, 4}^{\mathrm{R}} \cdot\left[\mathrm{Ca}^{2+}\right] \cdot\left[\mathrm{CaB}_{4}\right]-\left(\mathrm{k}_{\mathrm{off}, 4}^{\mathrm{R}}-\mathrm{D}_{4} \cdot \Delta_{\mathrm{r}}\right) \cdot\left[\mathrm{Ca}_{2} \mathrm{~B}_{4}\right] \\
\partial_{\mathrm{t}}\left[\mathrm{Ca}^{2+}\right]=\sum_{\mathrm{i}=0}^{3}\left(\mathrm{D}_{\mathrm{i}} \cdot \Delta_{\mathrm{r}}\left[\mathrm{CaB}_{\mathrm{i}}\right]-\partial_{\mathrm{t}}\left[\mathrm{CaB}_{\mathrm{i}}\right]\right)+\mathrm{D}_{\mathrm{i}} \cdot \Delta_{\mathrm{r}}\left[\mathrm{Ca}_{2} \mathrm{~B}_{4}\right]-\partial_{\mathrm{t}}\left[\mathrm{Ca}_{2} \mathrm{~B}_{4}\right]+\mathrm{D} \cdot \Delta_{\mathrm{r}}\left[\mathrm{Ca}^{2+}\right]
\end{array}\right.
$$

Here, $\mathrm{B}_{0}$ stands for ATP, $\mathrm{B}_{1}$ - parvalbumin- $\alpha$ (PV), $\mathrm{B}_{2}$ - calbindin-D28k (CB), $\mathrm{B}_{3}$ - non-cooperative binding site of calretinin $\left(\mathrm{CR}_{\text {non.coop. }}\right), \mathrm{B}_{4}$ - cooperative binding site of calretinin with no $\mathrm{Ca}^{2+}$-bound $\left(\mathrm{CR}_{\text {coop. }}\right), \mathrm{CaB}_{4}-$ cooperative binding site of calretinin with one $\mathrm{Ca}^{2+}$-bound $\left(\mathrm{CaCR}_{\text {coop. }}\right), \mathrm{Ca}_{2} \mathrm{~B}_{4}-$ cooperative binding site of calretinin with two $\mathrm{Ca}^{2+}$ bound. The following $\mathrm{Ca}^{2+}$ and $\mathrm{Mg}^{2+}$ binding and unbinding rates were used for simulations:

$$
\begin{aligned}
& \text { BAPTA }-\mathrm{k}_{\mathrm{on}}=400 \mathrm{mM}^{-1} \cdot \mathrm{ms}^{-1}, \mathrm{k}_{\mathrm{off}}=0.088 \mathrm{~ms}^{-1},(3) \\
& \cdot \mathrm{EGTA}-\mathrm{k}_{\mathrm{on}}=10 \mathrm{mM}^{-1} \cdot \mathrm{ms}^{-1}, \mathrm{k}_{\mathrm{off}}=0.0007 \mathrm{~ms}^{-1},(4) \\
& \cdot \mathrm{ATP}-\mathrm{k}_{\mathrm{on}}=1000 \mathrm{mM}^{-1} \cdot \mathrm{ms}^{-1}, \mathrm{k}_{\mathrm{off}}=90 \mathrm{~ms}^{-1},(5) \\
& \cdot \mathrm{ATP}\left(\mathrm{Mg}^{2+}\right)-\mathrm{k}_{\mathrm{on}}=10 \mathrm{mM}^{-1} \cdot \mathrm{ms}^{-1}, \mathrm{k}_{\mathrm{off}}=0.45 \mathrm{~ms}^{-1},(5) \\
& \cdot \mathrm{PV}-\mathrm{k}_{\mathrm{on}}=103 \mathrm{mM}^{-1} \cdot \mathrm{ms}^{-1}, \mathrm{k}_{\mathrm{off}}=0.00095 \mathrm{~ms}^{-1},(6) \\
& \cdot \mathrm{PV}\left(\mathrm{Mg}^{2+}\right)-\mathrm{k}_{\mathrm{on}}=0.8 \mathrm{mM}^{-1} \cdot \mathrm{ms}^{-1}, \mathrm{k}_{\mathrm{off}}=0.025 \mathrm{~ms}^{-1},(6) \\
& \cdot \mathrm{CB}-\mathrm{k}_{\mathrm{on}}=75 \mathrm{mM}^{-1} \cdot \mathrm{ms}^{-1}, \mathrm{k}_{\mathrm{off}}=0.0295 \mathrm{~ms}^{-1},(7) \\
& \cdot \mathrm{CR}_{\text {non.coop. }}-\mathrm{k}_{\mathrm{on}}=7.3 \mathrm{mM}^{-1} \cdot \mathrm{ms}^{-1}, \mathrm{k}_{\mathrm{off}}=0.252 \mathrm{~ms}^{-1},(8) \\
& \mathrm{CR}_{\text {coop. }}-\mathrm{k}_{\mathrm{on}}=1.8 \mathrm{mM}^{-1} \cdot \mathrm{ms}^{-1}, \mathrm{k}_{\mathrm{off}}=0.053 \mathrm{~ms}^{-1},(8) \\
& \cdot \mathrm{CaCR}_{\text {coop. }}-\mathrm{k}_{\mathrm{on}}=310 \mathrm{mM}^{-1} \cdot \mathrm{ms}^{-1}, \mathrm{k}_{\mathrm{off}}=0.02 \mathrm{~ms}^{-1},(8)
\end{aligned}
$$

The diffusion coefficients were set to $0.22 \mu \mathrm{m}^{2} \cdot \mathrm{ms}^{-1}$ for $\mathrm{Ca}^{2+}, \mathrm{Mg}^{2+}$, ATP, BAPTA, and EGTA (3), to $0.02 \mu \mathrm{m}^{2} \cdot \mathrm{ms}^{-1}$ for PV and CR $(8,9)$, and to $0.043 \mu \mathrm{m}^{2} \cdot \mathrm{ms}^{-1}$ for CB (10). The resting $\mathrm{Ca}^{2+}$ concentration, $\left[\mathrm{Ca}^{2+}\right](\mathrm{r}, \mathrm{t}=0)$, was set to $50 \mathrm{nM}(11)$.

The above systems of partial differential equations were solved numerically in MATLAB using built-in PDE solver pdepe. 


\subsection{Temporal evolution of $\left[\mathrm{Ca}^{2+}\right]$}

Fig. S7A-B show time evolution of normalized $\left[\mathrm{Ca}^{2+}\right]$ after onset of stimulus ${ }^{1}$ at distances up to $100 \mathrm{~nm}$ from the edge of a $\mathrm{r}_{0}=1 \mathrm{~nm}$ hemispherical source $\left(\mathrm{i}_{\mathrm{Ca}}=15 \mathrm{pA}\right)$ embedded in a hemispherical simulation volume of $2 \mu \mathrm{m}$ radius with reflecting boundary conditions. In all plots shown, $\left[\mathrm{Ca}^{2+}\right](r, t)$ was normalized to $\left[\mathrm{Ca}^{2+}\right](r, t=20 \mathrm{~ms})$. Panels on the left in Fig. S7 were obtained for the simulation volume filled with $2 \mathrm{mM} \mathrm{MgATP}$, while panels on the right correspond to the simulation volume filled with $2 \mathrm{mM}$ $\mathrm{MgATP}+0.5 \mathrm{mM}$ BAPTA. It follows from the panels (A) and (B) that the temporal profiles of $\left[\mathrm{Ca}^{2+}\right]$ were approximately characterized by two time scales. The initial time scale corresponded to the quasiequilibration of $\mathrm{Ca}^{2+}$ diffusion and reaction with the buffer molecules. The later, much slower time scale corresponded to accumulation of $\mathrm{Ca}^{2+}$ and the accompanying increase in the fraction of $\mathrm{Ca}^{2+}$-bound buffer molecules due to the prolonged $\mathrm{Ca}^{2+}$ influx. As can be seen in the plots, $\left[\mathrm{Ca}^{2+}\right]$ at distances up to $100 \mathrm{~nm}$ away from the source stayed rather constant during the first $20 \mathrm{~ms}$ after the initial build-up. This was especially true for points located closer to the source. At $\mathrm{t}=100 \mathrm{~ms}$, however, the accumulation of $\mathrm{Ca}^{2+}$ was considerable at the greater distances, in relative terms. This is seen better in Fig. S7C-D, where normalized spatial $\left[\mathrm{Ca}^{2+}\right]$ profiles are shown for selected time points ${ }^{2}$.

In the case of a real presynaptic active zone, a $15 \mathrm{pA}$ influx of $\mathrm{Ca}^{2+}$ ions (a finding of our present work) is spread over an area of the cellular membrane which is considerably larger than the surface area of a $\mathrm{r}_{0}=1 \mathrm{~nm}$ hemispherical source. Thus, the absolute levels of $\left[\mathrm{Ca}^{2+}\right]$ at small distances from the source were overestimated in our model ${ }^{3}$. This overestimation could result in a decreased effect of the accumulated bulk $\mathrm{Ca}^{2+}$ on $\left[\mathrm{Ca}^{2+}\right]$ in the proximity of the source during prolonged depolarizations. To examine this, we repeated the calculations with a $\mathrm{r}_{0}=70 \mathrm{~nm}$ radius hemispherical source. The surface area of this larger hemisphere approximately matched the area of the presynaptic density at IHC synapses $\left(420 \times 80 \mathrm{~nm}^{2},(2)\right)$. We found that, although the influence of the accumulated $\mathrm{Ca}^{2+}$ on $\left[\mathrm{Ca}^{2+}\right]$ was slightly stronger in this case, the conclusions drawn from the model with $\mathrm{r}_{0}=1 \mathrm{~nm}$ radius hemispherical source did not change - compare plots in Fig. S7E-F with the corresponding plots in Fig. S7A-B. Note that, when a point of interest is close to the edge of the $\mathrm{Ca}^{2+}$ source, the average distance between the point of interest and effective $\mathrm{Ca}^{2+}$ point sources which are spread on the surface of the hemisphere is considerably higher in the case of the $\mathrm{r}_{0}=70 \mathrm{~nm}$ radius hemisphere than the $\mathrm{r}_{0}=1 \mathrm{~nm}$ radius hemisphere. This is one of the reasons why the influence of the accumulated $\mathrm{Ca}^{2+}$ on $\left[\mathrm{Ca}^{2+}\right]$ was slightly stronger for the $\mathrm{r}_{0}=70 \mathrm{~nm}$ source than for the $\mathrm{r}_{0}=1 \mathrm{~nm}$ source. The same fact explains why initial phases of the $\left[\mathrm{Ca}^{2+}\right]$ temporal profiles were associated with a slightly slower time scale in the case of the larger, $\mathrm{r}_{0}=70 \mathrm{~nm}$, hemisphere.

\footnotetext{
1 "Onset of stimulus" and "onset of $\mathrm{Ca}^{2+}$ influx" are used as synonyms in sections $6.1-6.6$.

${ }^{2}$ Similar effects were also found when $2 \mathrm{mM} \mathrm{MgATP}+1 \mathrm{mM}$ BAPTA or $2 \mathrm{mM} \mathrm{MgATP}+(0.5-1) \mathrm{mM}$ EGTA were used as $\mathrm{Ca}^{2+}$ buffers. Naturally, the effect of $\mathrm{Ca}^{2+}$ accumulation on $\left[\mathrm{Ca}^{2+}\right]$ is less pronounced for higher concentrations of exogenous $\mathrm{Ca}^{2+}$ buffers and/or smaller $\mathrm{i}_{\mathrm{Ca}}$ levels.

${ }^{3}$ Note that, in this work, estimations of concentrations of the endogenous buffers or the coupling distance between presynaptic $\mathrm{Ca}^{2+}$ channels and $\mathrm{Ca}^{2+}$ sensors of exocytosis were based on the ratios of $\left[\mathrm{Ca}^{2+}\right]$ estimated in different $\mathrm{Ca}^{2+}$ buffering conditions, not the absolute levels of $\left[\mathrm{Ca}^{2+}\right]$.
} 


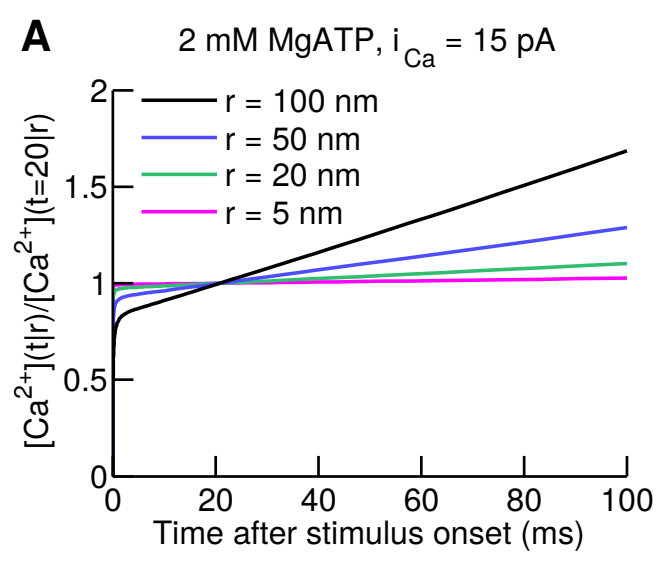

B $0.5 \mathrm{mM} \mathrm{BAPTA}+2 \mathrm{mM}$ MgATP, $\mathrm{i}_{\mathrm{Ca}}=15 \mathrm{pA}$
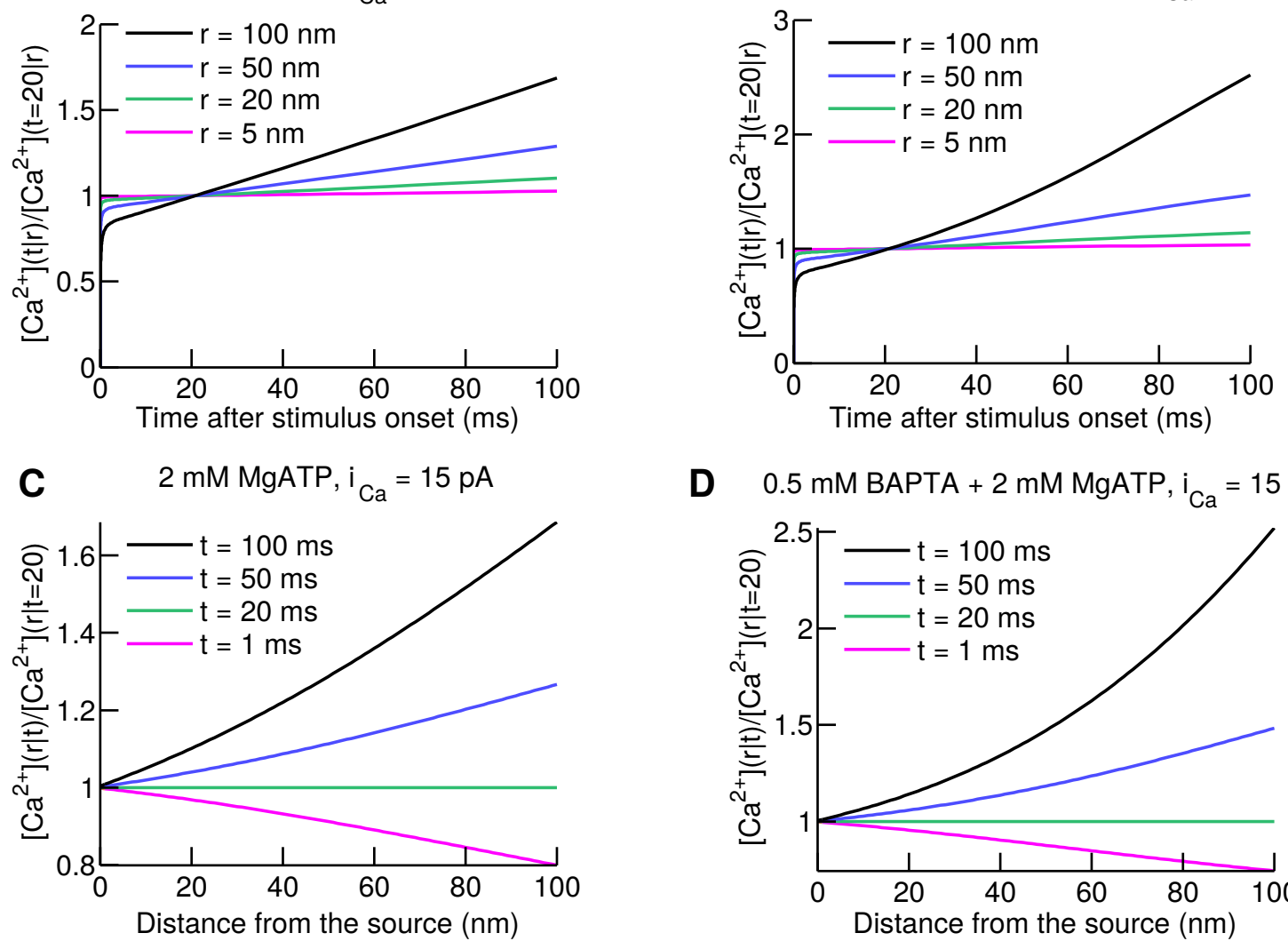

D $\quad 0.5 \mathrm{mM} \mathrm{BAPTA}+2 \mathrm{mM} M g A T P, \mathrm{i}_{\mathrm{Ca}}=15 \mathrm{pA}$
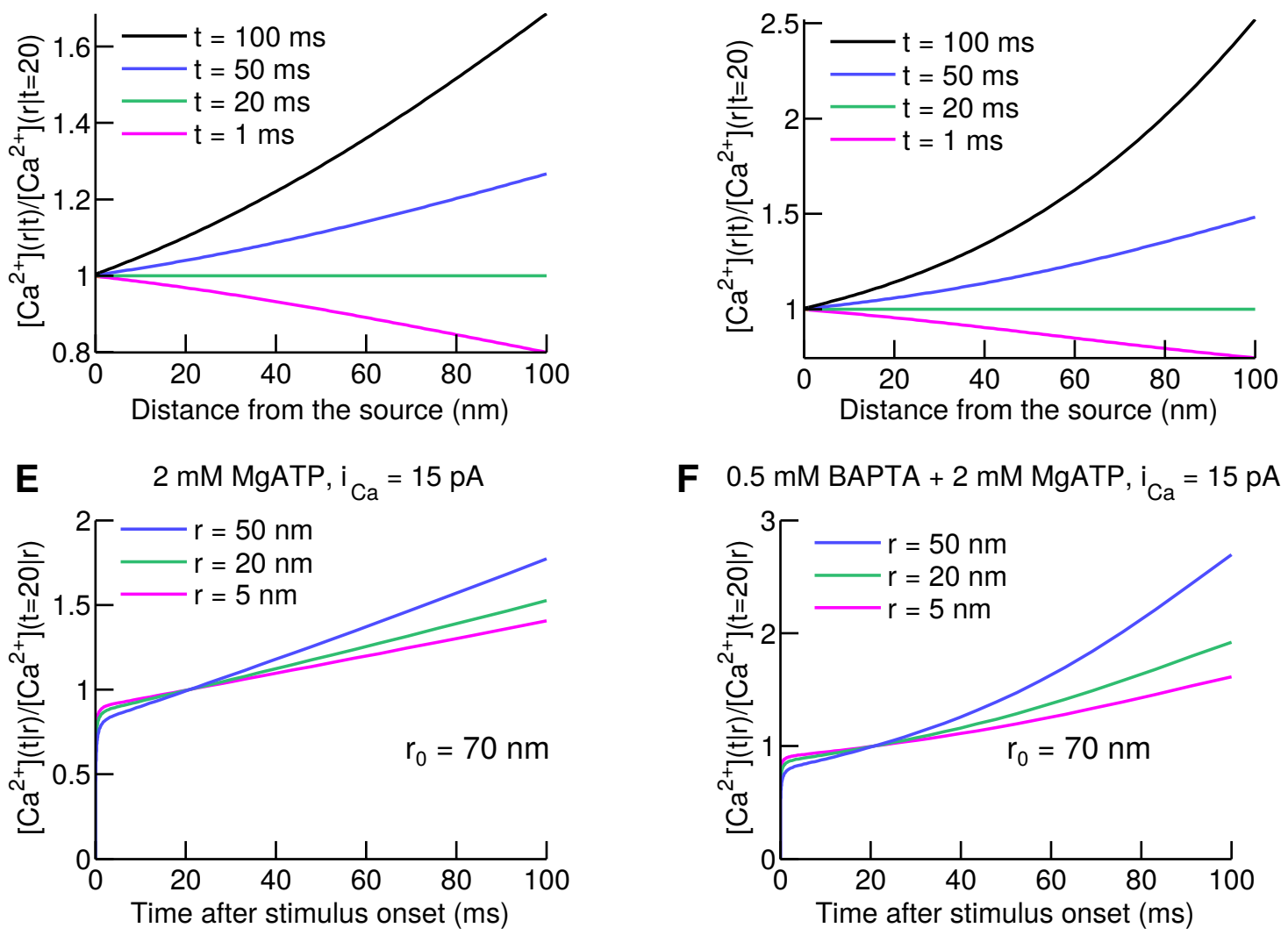

Figure S7: Temporal evolution of $\left[\mathrm{Ca}^{2+}\right]$. (A) Ratios of temporal $\left[\mathrm{Ca}^{2+}\right]$ profiles at certain distances from the source, $\left[\mathrm{Ca}^{2+}\right](\mathrm{t} \mid \mathrm{r}) /\left[\mathrm{Ca}^{2+}\right](\mathrm{t}=20 \mid \mathrm{r})$, based on $\mathrm{i}_{\mathrm{Ca}}=15 \mathrm{pA} \mathrm{Ca}^{2+}$ influx through a $\mathrm{r}_{0}=1 \mathrm{~nm}$ hemispherical source with the simulation volume filled with $2 \mathrm{mM} \mathrm{MgATP}$. (B) The same as (A), but with the simulation volume filled with $0.5 \mathrm{mM}$ BAPTA and $2 \mathrm{mM} \mathrm{MgATP}$. (C) Ratios of spatial $\mathrm{Ca}^{2+}$ profiles at fixed times, $\left[\mathrm{Ca}^{2+}\right](\mathrm{r} \mid \mathrm{t}) /\left[\mathrm{Ca}^{2+}\right](\mathrm{r} \mid \mathrm{t}=20)$, corresponding to the temporal profiles shown in $(\mathrm{A})$. (D) The same as (C), but with the simulation volume filled with $0.5 \mathrm{mM}$ BAPTA and $2 \mathrm{mM} \mathrm{MgATP}$. (E) Temporal profiles of $\left[\mathrm{Ca}^{2+}\right]$ based on $\mathrm{i}_{\mathrm{Ca}}=15 \mathrm{pA} \mathrm{Ca}{ }^{2+}$ influx through a $\mathrm{r}_{0}=70 \mathrm{~nm}$ hemispherical source with simulation volume filled with $2 \mathrm{mM} \operatorname{MgATP}$. (F) The same as (E), but with the simulation volume filled with $0.5 \mathrm{mM}$ BAPTA and $2 \mathrm{mM} \mathrm{MgATP}$. 


\subsection{Extent of $\mathrm{Ca}^{2+}$ buffer depletion due to $\mathrm{Ca}^{2+}$ influx}

To find the value of $i_{\mathrm{Ca}}$ which results in a similar extent of $\mathrm{Ca}^{2+}$ buffer depletion for a $\mathrm{r}_{0}=1 \mathrm{~nm}$ hemispherical source as at the presynaptic active zone, we varied levels of $i_{\mathrm{Ca}}$ and compared the resulting $\mathrm{Ca}^{2+}$ buffer concentrations with the ones corresponding to $\mathrm{r}_{0}=70 \mathrm{~nm}$ source $^{4}$ with $\mathrm{i}_{\mathrm{Ca}}=15 \mathrm{pA}$. Fig. S8 shows the ratios of increments of the $\mathrm{Ca}^{2+}$-bound buffer concentrations due to onset of $\mathrm{Ca}^{2+}$ influx through the $\mathrm{r}_{0}=1 \mathrm{~nm}$ and $\mathrm{r}_{0}=70 \mathrm{~nm}$ hemispherical sources as functions of the distance from the edge of the sources. In the case shown here, $\mathrm{Ca}^{2+}$ influx was set to $15 \mathrm{pA}$ for the $\mathrm{r}_{0}=70 \mathrm{~nm}$ source and $7 \mathrm{pA}$ for the $\mathrm{r}_{0}=1 \mathrm{~nm}$ source. The concentrations were estimated $20 \mathrm{~ms}$ after onset of $\mathrm{Ca}^{2+}$ influx. The simulation volume was a hemisphere of $2 \mu \mathrm{m}$ radius with reflecting boundary conditions. The total concentrations of exogenous buffers BAPTA and EGTA, each of them considered separately, were set to either $0.5 \mathrm{mM}$ or $1 \mathrm{mM}$. Moreover, in all considered cases, a total of $2 \mathrm{mM}$ ATP was included. As can be seen from the plots, the ratios of the amounts of depleted buffers, BAPTA + ATP or EGTA + ATP, were around 1 or higher for the distances considered. This indicates that the $\mathrm{r}_{0}=1 \mathrm{~nm}$ source with $\mathrm{i}_{\mathrm{Ca}}=7 \mathrm{pA}$ resulted in a similar extent of $\mathrm{Ca}^{2+}$ buffer depletion as the $\mathrm{r}_{0}=70 \mathrm{~nm}$ source with $\mathrm{i}_{\mathrm{Ca}}=15 \mathrm{pA}$ over distances 0 to $50 \mathrm{~nm}$ from the $\mathrm{Ca}^{2+}$ source.

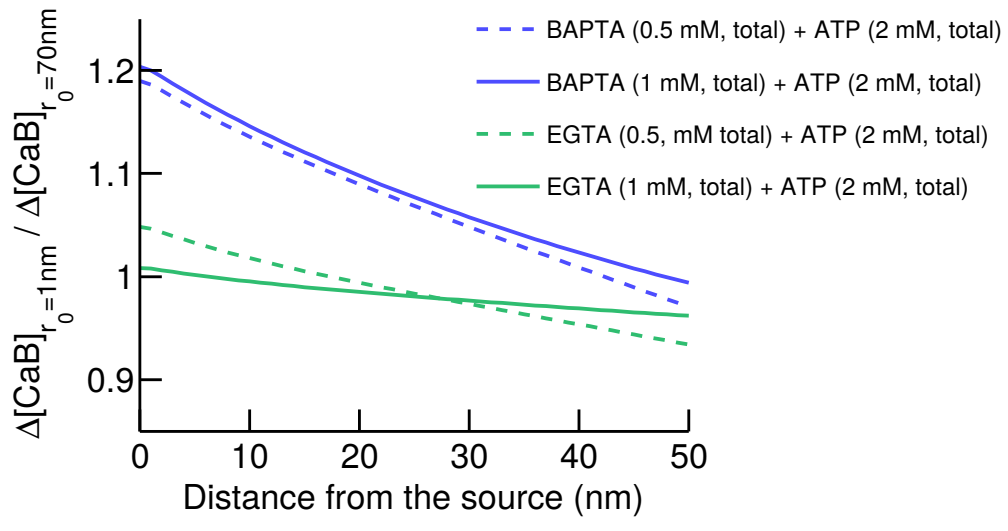

Figure S8: Ratios between increased $\mathrm{Ca}^{2+}$-bound buffer concentrations due to $\mathrm{Ca}^{2+}$ influx for hemispherical $\mathrm{Ca}^{2+}$ sources with $\mathrm{r}_{0}=1 \mathrm{~nm}, \mathrm{i}_{\mathrm{Ca}}=7 \mathrm{pA}$, and $\mathrm{r}_{0}=70 \mathrm{~nm}, \mathrm{i}_{\mathrm{Ca}}=15 \mathrm{pA}$ as functions of the distance from the edge of the sources.

\subsection{Influence of the partial buffer depletion on the $\left[\mathrm{Ca}^{2+}\right]$ vs. $i_{C a}$ relation}

It was assumed in the model considered in Results that integrated exocytosis, $\Delta C_{m}$ follows $\left[\mathrm{Ca}^{2+}\right]$ at the sensor of exocytosis according to a power law relation. However, $\left[\mathrm{Ca}^{2+}\right]$ at the sensor of exocytosis is not an experimentally accessible quantity currently. Thus, in order to determine the exponent parameter of the relation between $\Delta C_{m}$ and $\left[\mathrm{Ca}^{2+}\right]$ at the sensor of exocytosis, we have to employ the experimentally accessible relation between the integrated release and integrated $\mathrm{Ca}^{2+}$ influx, $Q_{C a}: \Delta C_{m} \sim\left(Q_{C a}\right)^{m}$ (see section 6). We show below that $Q_{C a} \sim\left[\mathrm{Ca}^{2+}\right]^{q}$. Thus, $\Delta C_{m} \sim\left[\mathrm{Ca}^{2+}\right]^{m \cdot q}$.

Due to nonlinearities inherent to the dynamics of $\mathrm{Ca}^{2+}$ and buffer concentrations, the dependence of $\left[\mathrm{Ca}^{2+}\right](\mathrm{t}, \mathrm{r})$ on the $\mathrm{Ca}^{2+}$ influx level $\mathrm{i}_{\mathrm{Ca}}$ is nonlinear in general. We simulated the dependence of

\footnotetext{
${ }^{4}$ Which has the surface area similar in extent to that of a real IHC presynaptic density as explained in section 7.2.
} 
$\left[\mathrm{Ca}^{2+}\right](\mathrm{t}, \mathrm{r})$ on $\mathrm{i}_{\mathrm{Ca}}$ to quantitatively check how much this relation deviates from the linear approximation. It turned out that, for all the considered buffering conditions, the relation was well approximated by $\left[\mathrm{Ca}^{2+}\right](r) \sim i_{\mathrm{Ca}}^{1 / q(r)}$ at distances of 0 to $100 \mathrm{~nm}$ from the source. Fig. S9B-D show the $\left[\mathrm{Ca}^{2+}\right]$ vs. $\mathrm{i}_{\mathrm{Ca}}$ relations at distances of $0,10,25,50,100 \mathrm{~nm}$ away from the source, at $\mathrm{t}=20 \mathrm{~ms}$ for three different $\mathrm{Ca}^{2+}$ buffer sets: $2 \mathrm{mM}$ MgATP, $0.5 \mathrm{mM}$ BAPTA $+2 \mathrm{mM} \mathrm{MgATP}, 0.5 \mathrm{mM}$ EGTA $+2 \mathrm{mM}$ MgATP. Black solid lines represent the simulated data, while magenta dashed lines represent the best fits of the form $\left[\mathrm{Ca}^{2+}\right](r)=k(r) \cdot i_{C a}^{1 / q(r)}$. $\mathrm{i}_{\mathrm{Ca}}$ values ranging from $0.3 \mathrm{pA}$ to $7 \mathrm{pA}$ were considered. The radius of the hemispherical source was set to $1 \mathrm{~nm}$. The radius of the simulation volume hemisphere was $2 \mu \mathrm{m}$. The boundary was reflective for all ions and molecules considered. Fig. S9A shows the dependence of the exponent $q$ on the distance from the source, $r$, for the three aforementioned $\mathrm{Ca}^{2+}$ buffering conditions. $q$ values were only slightly smaller than 1 , thus the $\left[\mathrm{Ca}^{2+}\right]$ vs. $\mathrm{i}_{\mathrm{Ca}}$ relation was only slightly supralinear. When concentrations of BAPTA and EGTA were increased from $0.5 \mathrm{mM}$ to $1 \mathrm{mM}, q(r)$ shifted slightly towards 1.

A

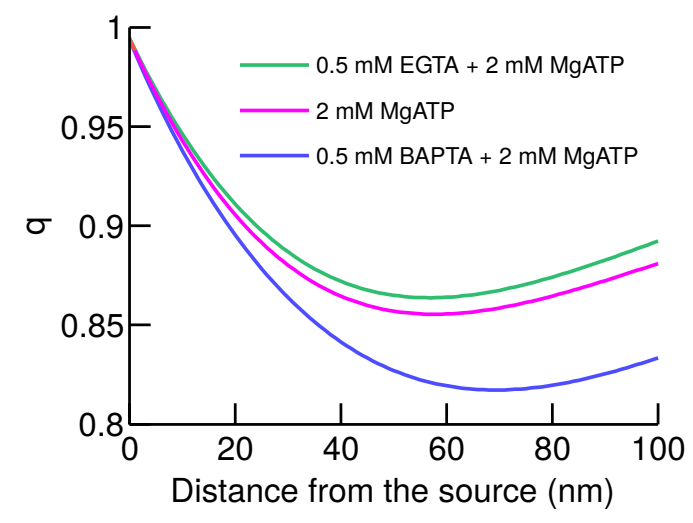

C

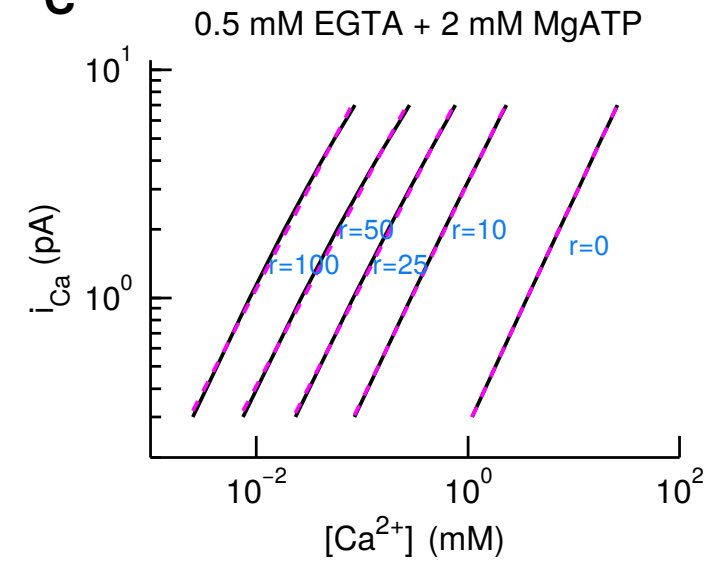

B

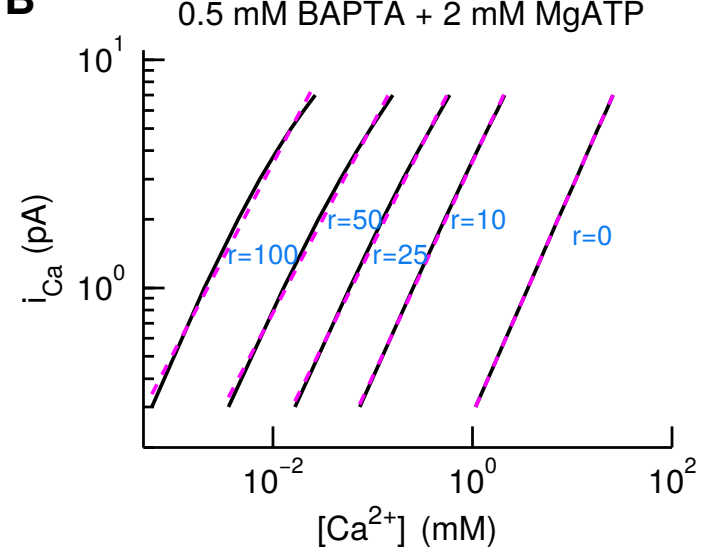

D

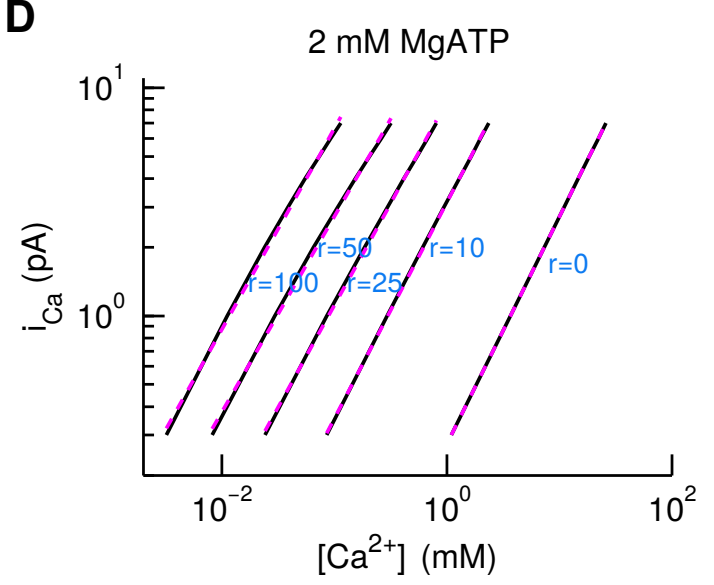

Figure S9: Influence of the partial buffer depletion on $\left[\mathrm{Ca}^{2+}\right]$ vs. $\mathrm{i}_{\mathrm{Ca}}$ relation. (A) Dependence of the exponent $q$ corresponding to the best fits of simulated $\mathrm{i}_{\mathrm{Ca}} \mathrm{vs}$. $\left[\mathrm{Ca}^{2+}\right]$ relations by $i_{C a}=k \cdot\left[\mathrm{Ca}^{2+}\right]^{q}$ on the distance from a $\mathrm{r}_{0}=1 \mathrm{~nm}$ hemispherical $\mathrm{Ca}^{2+}$ source with $\mathrm{i}_{\mathrm{Ca}}=7 \mathrm{pA}$. (B-D) Simulated $\mathrm{i}_{\mathrm{Ca}} \mathrm{vs}$. $\left[\mathrm{Ca}^{2+}\right]$ relations (black solid lines) and the optimal fits of the form $i_{C a}=k \cdot\left[\mathrm{Ca}^{2+}\right]^{q}$ (dashed magenta lines) with different contents of $\mathrm{Ca}^{2+}$ buffers. 
Note that $Q_{C a}=t \cdot i_{C a}$ for constant $i_{C a}$, where $t$ is the time passed since stimulus onset. Thus, $\left[\mathrm{Ca}^{2+}\right](r)=\left(k(r) / t^{1 / q(r)}\right) \cdot Q_{C a}^{1 / q(r)}$. To take into account the effect of partial buffer depletion on the estimate of the effective coupling distance between presynaptic $\mathrm{Ca}^{2+}$ channels and $\mathrm{Ca}^{2+}$ sensors of exocytosis for $\mathrm{i}_{\mathrm{Ca}}=7 \mathrm{pA}$, we used $q(r)$ profiles corresponding to $0.5 \mathrm{mM} \mathrm{BAPTA}+2 \mathrm{mM}$ MgATP buffering conditions, which roughly approximates the natural $\mathrm{Ca}^{2+}$ buffering conditions in IHC as shown in our present work. We used $q(r) \equiv 1$ for estimating the coupling distance for weak $\left[\mathrm{Ca}^{2+}\right]$ influx $\left(\mathrm{i}_{\mathrm{Ca}}=0.3 \mathrm{pA}\right)$.

\subsection{Effect of accumulated bulk $\mathrm{Ca}^{2+}$ during prolonged stimulation on the model predictions of concentrations of the endogenous $\mathrm{Ca}^{2+}$ buffers}

The upper bounds for concentrations of the endogenous $\mathrm{Ca}^{2+}$ buffers shown in Fig. 9C were estimated by probing $\left[\mathrm{Ca}^{2+}\right]$ at $\mathrm{t}=100 \mathrm{~ms}$ after stimulus onset. It was shown in section 7.2 (see Fig. S7A-B) that the effect of the accumulated $\mathrm{Ca}^{2+}$ on $\left[\mathrm{Ca}^{2+}\right]$ increased with time after stimulus onset from negligible at $\mathrm{t}=20 \mathrm{~ms}$ to significant at $\mathrm{t}=100 \mathrm{~ms}$. This could affect the estimates of the upper bounds for the concentrations of the endogenous $\mathrm{Ca}^{2+}$ buffers, which were determined by matching the spatial $\left[\mathrm{Ca}^{2+}\right]$ profiles at $\mathrm{t}=100 \mathrm{~ms}$ (see Results and Fig. 9C). To test this, we re-estimated the upper bounds for the concentrations of the endogenous $\mathrm{Ca}^{2+}$ buffers by probing spatial profiles of $\left[\mathrm{Ca}^{2+}\right]$ at times between $20 \mathrm{~ms}$ and $100 \mathrm{~ms}$ after stimulus onset. Solid lines in Fig. S10 show dependencies of the estimates of $\mathrm{Ca}^{2+}$-binding site concentrations of the endogenous buffers on the time when $\left[\mathrm{Ca}^{2+}\right]$ was probed. The estimates of $\mathrm{Ca}^{2+}$-binding site concentrations for calbindin-D28k and parvalbumin- $\alpha$ decreased only slightly when comparing values obtained at $\mathrm{t}=20 \mathrm{~ms}$ to $\mathrm{t}=100 \mathrm{~ms}$ : from $6.4 \mathrm{mM}$ to $5.2 \mathrm{mM}$ for calbindin-D28k and from $5.0 \mathrm{mM}$ to $4.2 \mathrm{mM}$ for parvalbumin- $\alpha$. The difference was more significant for calretinin - the estimate of the $\mathrm{Ca}^{2+}$-binding site concentration decreased from $21.0 \mathrm{mM}$ at $\mathrm{t}=20 \mathrm{~ms}$ to $13.8 \mathrm{mM} \mathrm{t}=100 \mathrm{~ms}$. The reason why calretinin was so sensitive to the accumulated $\mathrm{Ca}^{2+}$ is that it has highly cooperative $\mathrm{Ca}^{2+}$ binding sites, with the first binding step being much slower than the second one.

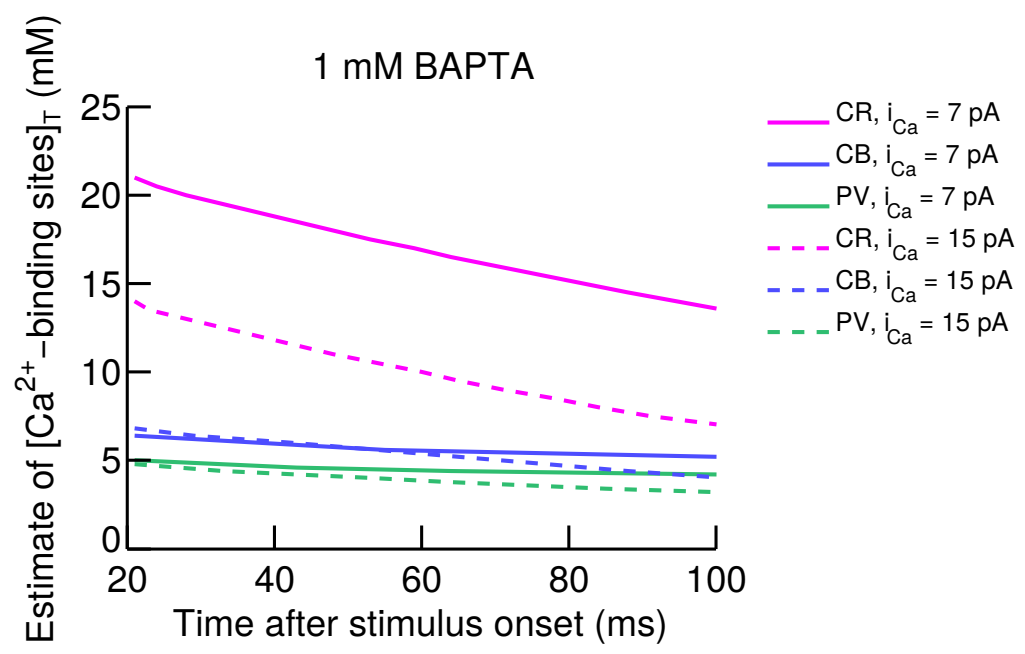

Figure S10: Estimation of concentrations of the endogenous buffer $\mathrm{Ca}^{2+}$-binding sites equivalent to $1 \mathrm{mM}$ BAPTA based on $\left[\mathrm{Ca}^{2+}\right]$ probed at different moments after stimulus onset. 
Dashed lines in Fig. S10 show dependencies of the estimates of the $\mathrm{Ca}^{2+}$-binding site concentrations on the moment when $\left[\mathrm{Ca}^{2+}\right]$ was probed after onset of $\mathrm{i}_{\mathrm{Ca}}$ of $15 \mathrm{pA}$. The results were essentially the same as with $\mathrm{i}_{\mathrm{Ca}}=7 \mathrm{pA}$ for calbindin-D28k and parvalbumin- $\alpha$. However, the concentration estimates were decreased by $\sim 7 \mathrm{mM}$ for calretinin.

\subsection{Influence of the type of boundary conditions on the estimates of the endogenous buffer concentrations and the coupling distance $\boldsymbol{R}_{c}$}

The original model of $\left[\mathrm{Ca}^{2+}\right]$ dynamics presented in this work assumed a hemispherical simulation volume with $2 \mu \mathrm{m}$ radius. The boundaries of the simulation volume were set to be reflective to all molecules and ions considered. The choice of the radius of the simulation volume was based on the finding that the presynaptic active zones of inner hair cells are separated by $2 \mu \mathrm{m}$ on average (12). The reflecting boundary condition set at the hemispherical boundary of the simulation volume was meant to effectively take into account accumulation of $\mathrm{Ca}^{2+}$ due to $\mathrm{Ca}^{2+}$ channels from the synapse under consideration as well as $\mathrm{Ca}^{2+}$ channels from neighboring synapses. On the other hand, such a boundary condition could lead to an overestimation of the $\left[\mathrm{Ca}^{2+}\right]$ because it does not take into account removal of $\mathrm{Ca}^{2+}$ ions and $\mathrm{Ca}^{2+}$-bound buffer molecules from the neighborhood of the presynaptic site due to exchange of the molecules with the large non-synaptic part of the IHC volume. Indeed, 12 hemispheres with $2 \mu \mathrm{m}$ radius correspond to only one percent of hair cell volume $\left(2.2 \cdot 10^{-15} \mathrm{~m}^{-3},(13)\right)$ on average. The purely reflective boundary at the base of the simulation volume ignores $\mathrm{Ca}^{2+}$ removal by $\mathrm{Ca}^{2+}$ pumps. To check how the choice of the boundary conditions affected our estimates of the endogenous buffer concentrations and the coupling distance between presynaptic $\mathrm{Ca}^{2+}$ channels and $\mathrm{Ca}^{2+}$ sensors of exocytosis, we performed equivalent simulations with different boundary conditions. Specifically, we fixed concentrations of all considered molecules and ions at the hemispherical boundary of the simulation volume to their resting levels, i.e., levels before the onset of $\mathrm{Ca}^{2+}$ influx. The base of the hemisphere was chosen to be reflecting as in the original model. The radius of the hemispherical simulation volume, $\mathrm{R}$, was set to either $2 \mu \mathrm{m}$ or $4 \mu \mathrm{m}$. Such boundary conditions can be treated as overestimating the intensity of $\mathrm{Ca}^{2+}$ removal from the neighborhood of the presynaptic active zones.

Fig. S11A-B show plots of ratios $\left[\mathrm{Ca}^{2+}\right](\mathrm{r} \mid \mathrm{t}) /\left[\mathrm{Ca}^{2+}\right]^{*}(\mathrm{r} \mid+\infty)$ at selected times. Here, $\left[\mathrm{Ca}^{2+}\right](\mathrm{r} \mid \mathrm{t})$ stands for $\left[\mathrm{Ca}^{2+}\right]$ at the distance $r$ from the source at moment $t$ after stimulus onset, estimated by using the original model with reflecting boundary conditions. $\left[\mathrm{Ca}^{2+}\right]^{*}(\mathrm{r} \mid+\infty)$ stands for the steady state $\left[\mathrm{Ca}^{2+}\right]$ at the distance $r$ from the source, estimated by using the "resting-level" boundary conditions introduced in the previous paragraph (see also section 7.1). Solid (dashed) lines correspond to $\left[\mathrm{Ca}^{2+}\right]^{*}$ estimated using a $\mathrm{R}=2 \mu \mathrm{m}(\mathrm{R}=4 \mu \mathrm{m})$ simulation volume. Fig. S11A corresponds to $2 \mathrm{mM} \mathrm{MgATP}$, Fig. S11B corresponds to $0.5 \mathrm{mM}$ BAPTA $+2 \mathrm{mM} \mathrm{MgATP}$. These profiles are quantitatively similar to the spatial profiles shown in Fig. S7C-D, where $\left[\mathrm{Ca}^{2+}\right](\mathrm{r} \mid \mathrm{t}=20)$ was used for scaling. The differences between the results corresponding to $\mathrm{R}=2 \mu \mathrm{m}$ and $\mathrm{R}=4 \mu \mathrm{m}$ simulation volumes were negligible (compare solid and dashed lines in Fig. S11A-B). This similarity can be explained by the fact that, in the first $20 \mathrm{~ms}$ after onset of $\mathrm{Ca}^{2+}$ influx, the processes of $\mathrm{Ca}^{2+}$ diffusion and binding to the buffers were confined to a small volume, which was far away from the hemispherical surface of the simulation volume. These results suggest that the estimate of $R_{c}$ is rather insensitive to the precise choice of boundary conditions, given 
that the radius of the simulation volume, $R$, is $\geq 2 \mu \mathrm{m}$. This idea is corroborated by the observation that dependencies of the discrepancy measure $z$ on the effective distance between the $\mathrm{Ca}^{2+}$ source and $\mathrm{Ca}^{2+}$ sensors of exocytosis shown in Fig. S11C were strikingly similar to the equivalent plots shown in Fig. 9B. Estimates of the effective concentrations of the endogenous buffer $\mathrm{Ca}^{2+}$ binding sites did not strongly depend on which of the boundary conditions were used either (compare Fig. S11D with Fig. 9C), except the upper bound estimate for calretinin. The latter was larger by $\sim 50 \%$ in the case of the resting-level boundary conditions because of the $\mathrm{Ca}^{2+}$ binding cooperativity of calretinin.
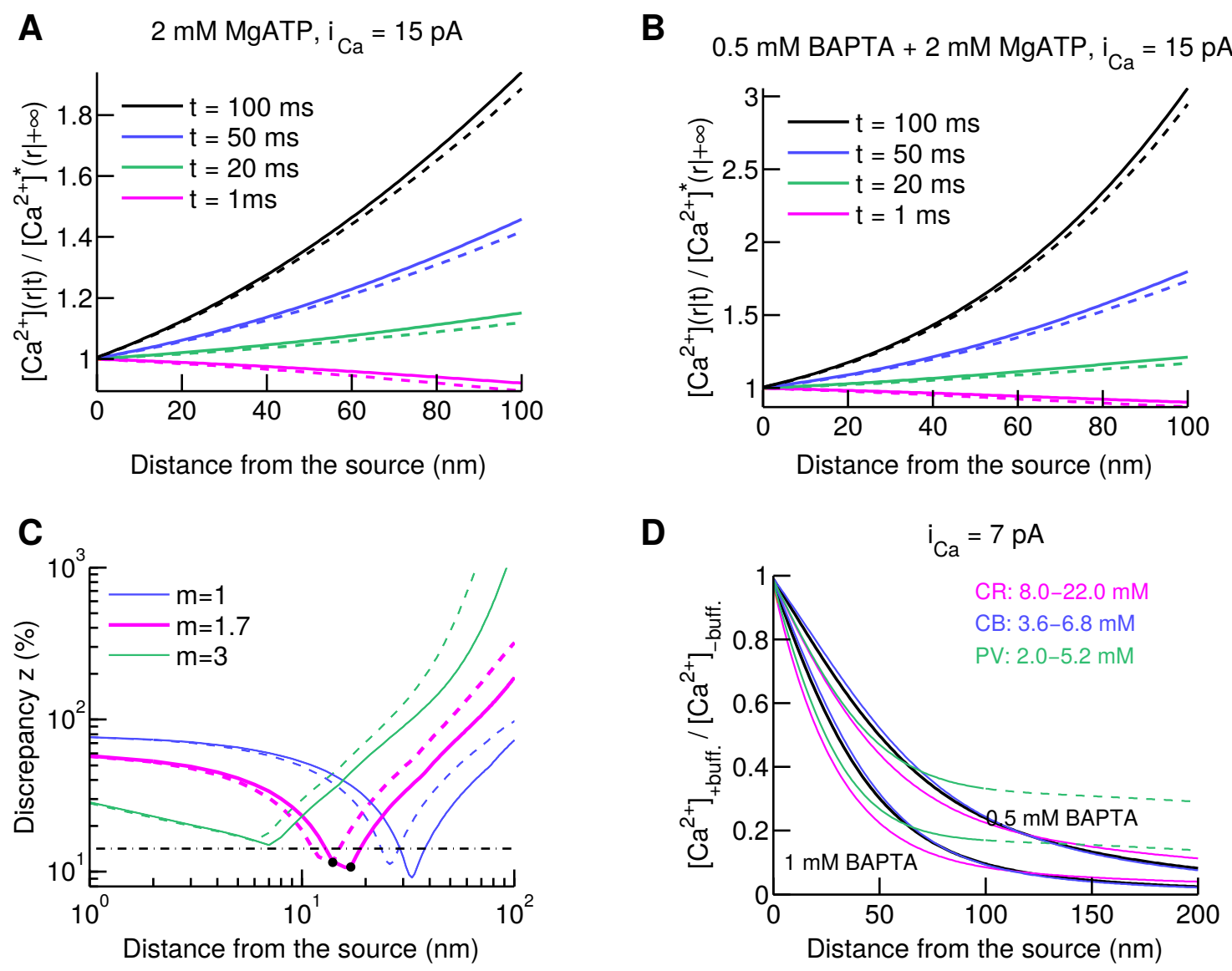

Figure S11: Results based on the simulations with boundary conditions that fix concentrations of all molecular species to the resting levels at the hemispherical surface of the simulation volume. (A-B) Spatial profiles of ratios $\left[\mathrm{Ca}^{2+}\right](\mathrm{r} \mid \mathrm{t}) /\left[\mathrm{Ca}^{2+}\right]^{*}(\mathrm{r} \mid+\infty)$ at selected times. Solid (dashed) lines correspond to $\left[\mathrm{Ca}^{2+}\right]^{*}(\mathrm{r} \mid+\infty)$ estimated with the radius of the simulation volume equal to $\mathrm{R}=2 \mu \mathrm{m}(\mathrm{R}=4 \mu \mathrm{m})$. (C) Dependence of the discrepancy measure $z$ on the effective coupling distance between presynaptic $\mathrm{Ca}^{2+}$ channels and sensors of exocytosis. Dashed lines correspond to $\mathrm{i}_{\mathrm{Ca}}=0.3 \mathrm{pA}$, solid lines correspond to $\mathrm{i}_{\mathrm{Ca}}=7 \mathrm{pA}$. The black dash-dotted line corresponds to the average of the experimental s.e.m. $\left[\Delta \mathrm{C}_{\mathrm{m}}\right] /\left\langle\Delta \mathrm{C}_{\mathrm{m}}\right\rangle$ over different $\mathrm{Ca}^{2+}$ buffering conditions (R=2 $\left.\mu \mathrm{m}\right)$. (D) Ratios of $\left[\mathrm{Ca}^{2+}\right]$ in the presence, denoted by $\left[\mathrm{Ca}^{2+}\right]_{+ \text {buff., }}$, and the absence, denoted by $\left[\mathrm{Ca}^{2+}\right]_{- \text {buff., }}$ of particular buffers as functions of the distance from the $1 \mathrm{~nm}$ radius hemispherical source. Color lines are the best

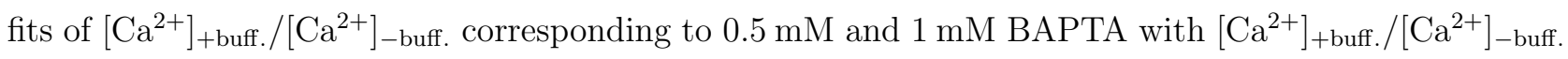
corresponding to either calretinin (magenta), calbindin-D28k (blue) or parvalbumin- $\alpha$ (green). 


\section{Defining and Evaluating the Effective Coupling Distance $\mathbf{R}_{\mathbf{c}}$ (Including Supplementary Figure 12)}

Several versions of the model of a single effective vesicular release site driven by a single effective $\mathrm{Ca}^{2+}$ channel have been applied to evaluate the proximity between presynaptic $\mathrm{Ca}^{2+}$ channels and $\mathrm{Ca}^{2+}$ sensors of exocytosis based on the differential effect of mobile exogenous $\mathrm{Ca}^{2+}$ buffers with different $\mathrm{Ca}^{2+}$ binding kinetics and concentrations on exocytosis (see, for example, $(14-17)$ ). Intuitively, the coupling distance, which we denote by $R_{c}$, estimated in the framework of that model reflects the proximity between the $\mathrm{Ca}^{2+}$ channels and the vesicular release sites within the active zone. However, how $R_{c}$ is quantitatively related to the parameters which define the spatial arrangement of presynaptic $\mathrm{Ca}^{2+}$ channels and vesicular release sites, as well as the kinetic parameters which define the $\mathrm{Ca}^{2+}$ dynamics, has not been analyzed before.

In this section we provide a mathematical analysis for understanding the physical meaning of $R_{c}$. This analysis unravels that $R_{c}$, in general, is a complicated, nonlinear average of the physical distances between the presynaptic $\mathrm{Ca}^{2+}$ channels and $\mathrm{Ca}^{2+}$ sensors of exocytosis. The main advantage of the single $\mathrm{Ca}^{2+}$ source model compared to spatially resolved models is that it produces a single output, $R_{c}$, which embodies the essence of all possible active zone topographies which are compatible with the experimental data. However, as we explain later in this section, the model also has a disadvantage that the exact value of $R_{c}$ depends not only on the structural but also on the kinetic parameters of the system, such as $\mathrm{Ca}^{2+}$ cooperativity of exocytosis or $\mathrm{Ca}^{2+}$ binding kinetics of intracellular $\mathrm{Ca}^{2+}$ buffers. Thus, to avoid misinterpretations of $R_{c}$, care has to be taken when designing the experiments.

In this section, we also provide results of spatially resolved models of IHC presynaptic active zones introduced previously (see (2)). We show that active zone topographies suggested as corresponding to the " $\mathrm{Ca}^{2+}$ nanodomain coupling" regime in (2) are compatible with the $R_{c}$ estimate determined in our present work.

\subsection{Active zones with 1 vesicular release site and $N \mathrm{Ca}^{2+}$ channels, no $\mathrm{Ca}^{2+}$ buffers}

First of all, let us consider a presynaptic active zone which contains $\mathrm{N} \mathrm{Ca}^{2+}$ channels and one vesicular release site (see Fig. S12A). We assume that no $\mathrm{Ca}^{2+}$ buffers are present in the cytoplasm and that $\mathrm{Ca}^{2+}$ diffuses freely above the reflective cellular membrane. In this case, a particular channel $i$ results in an increment of $\left[\mathrm{Ca}^{2+}\right]$, which, averaged over time in steady state at fixed membrane potential, is inversely proportional to the distance from the mouth of that channel ${ }^{5}(3)$ :

$$
\delta\left\langle\left[\mathrm{Ca}^{2+}\right]\right\rangle_{i}=\frac{i_{C a} \cdot P_{o}}{4 \cdot \pi \cdot F \cdot D \cdot R_{i}},
$$

\footnotetext{
${ }^{5}$ For the sake of mathematical tractability, here and further on in sections $8.1-8.5$, we treat $\mathrm{Ca}^{2+}$ channels as point sources and assume open boundary conditions above the plane of the plasma membrane (see (3)), unless stated otherwise. It follows from the results of section 7.6 that such an approximation would have negligible effect on the estimates of the effective coupling distance $R_{c}$.
} 
where $i_{C a}$ is single $\mathrm{Ca}^{2+}$ channel current, $R_{i}$ - distance from the mouth of $i$-th channel to the point of interest, $F$ - Faraday constant, $D$ - diffusion coefficient of $\mathrm{Ca}^{2+}, P_{o}$ - open probability of the channels in steady state at a given membrane potential. Let us now consider a response of such a model synapse to a depolarizing pulse of certain length. We assume that exocytosis at the synapse is fully determined by the time-averaged $\mathrm{Ca}^{2+}$ concentration at the vesicular release $\operatorname{site}^{6},\left\langle\left[\mathrm{Ca}^{2+}\right]\right\rangle=\sum_{i=1}^{N} \delta\left\langle\left[\mathrm{Ca}^{2+}\right]\right\rangle_{i}{ }^{7}$, and is not affected by momentary $\mathrm{Ca}^{2+}$ concentration fluctuations due to the channel opening and closing (the validity of this assumption is considered in section 8.5). We also assume that functional relation between $\left\langle\left[\mathrm{Ca}^{2+}\right]\right\rangle$ and the amount of vesicles released in a chosen time period $T$, which we denote by $\Delta_{\text {rel. }}$, is bijective: $\Delta_{r e l .}=f\left(\left\langle\left[\mathrm{Ca}^{2+}\right]\right\rangle\right)$. Given this, we can apply a single $\mathrm{Ca}^{2+}$ source model to determine the effective coupling distance $R_{c}$ from a measured $\Delta_{r e l .}: R_{c}$ is the distance from a single $\mathrm{Ca}^{2+}$ source, with total $\mathrm{Ca}^{2+}$ influx $I_{C a}=N \cdot i_{C a}$, to the point of interest at which $\mathrm{Ca}^{2+}$ concentration is equal to $\left\langle\left[\mathrm{Ca}^{2+}\right]\right\rangle=f^{-1}\left(\Delta_{\text {rel. }}\right)$. Taking into account equation [1], we can express $R_{c}$ in the following way:

$$
\begin{aligned}
R_{c} & =\frac{I_{C a} \cdot P_{o}}{4 \cdot \pi \cdot F \cdot D \cdot\left\langle\left[\mathrm{Ca}^{2+}\right]\right\rangle}=\frac{\sum_{i=1}^{N}\left(i_{C a} \cdot P_{o}\right) /(4 \cdot \pi \cdot F \cdot D)}{\left\langle\left[\mathrm{Ca}^{2+}\right]\right\rangle}=\frac{\sum_{i=1}^{N} R_{i} \cdot \delta\left\langle\left[\mathrm{Ca}^{2+}\right]\right\rangle_{\mathrm{i}}}{\left\langle\left[\mathrm{Ca}^{2+}\right]\right\rangle}= \\
& =\sum_{i=1}^{N} R_{i} \cdot\left(\frac{\delta\left\langle\left[\mathrm{Ca}^{2+}\right]\right\rangle_{\mathrm{i}}}{\sum_{j=1}^{N} \delta\left\langle\left[\mathrm{Ca}^{2+}\right]\right\rangle_{j}}\right) \equiv\langle R\rangle_{C a}^{\mathcal{L}}
\end{aligned}
$$

Thus, $R_{c}$ is a weighted average of distances from all the channels to the $\mathrm{Ca}^{2+}$ sensor of exocytosis, which we denote by $\langle R\rangle_{C a}^{\mathcal{L}}$. Here, superscript $\mathcal{L}$ stands for "linear" and subscript $C a$ indicates that the averaging weights depend on how $\delta\left\langle\left[\mathrm{Ca}^{2+}\right]\right\rangle_{i}$ depends on $R_{i}{ }^{8}$. The weight for each channel is the relative contribution of that channel to $\left\langle\left[\mathrm{Ca}^{2+}\right]\right\rangle$ at the vesicular release site. The higher is the contribution of a channel to $\left\langle\left[\mathrm{Ca}^{2+}\right]\right\rangle$ and, thus, exocytosis, the closer is $\langle R\rangle_{C a}^{\mathcal{L}}$ to the distance from that channel to the sensor. Note that the choice $I_{C a}=N \cdot i_{C a}$ is derived from the requirement that $R_{c}=R_{i}$ when $R_{i}=R_{j}$ for any $i$ and $j$, i.e., when all the channels are equidistant to the $\mathrm{Ca}^{2+}$ sensor.

\footnotetext{
${ }^{6}$ Here and further on, we assume that the exocytosis rate depends on $\left[\mathrm{Ca}^{2+}\right]$ at a particular point associated with the vesicular release site which we will call " $\mathrm{Ca}^{2+}$ sensor". " $\mathrm{Ca}^{2+}$ concentration at a vesicular release site" and " $\mathrm{Ca}^{2+}$ concentration at a $\mathrm{Ca}^{2+}$ sensor of exocytosis" are used as synonyms in sections $8.1-8.5$.

${ }^{7}$ Here and further on, we assume that the contribution of the background $\left[\mathrm{Ca}^{2+}\right]$, i.e., $\left[\mathrm{Ca}^{2+}\right]$ in the absence of stimulus, is negligible.

${ }^{8} \mathrm{It}$ is shown in section 8.4 that, in general, the averaging weights depend not only on the quantitative expression of $\delta\left\langle\left[\mathrm{Ca}^{2+}\right]\right\rangle_{i}$ vs. $R_{i}$ but also on the properties of release rate dependence on $\left\langle\left[\mathrm{Ca}^{2+}\right]\right\rangle$. Moreover, as it is shown in 8.2 , the averaging is nonlinear if $\mathrm{Ca}^{2+}$ buffers are present in the presynaptic solution.
} 
A

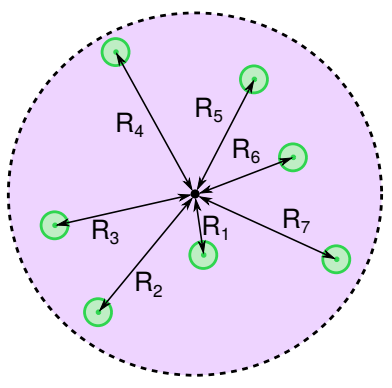

C

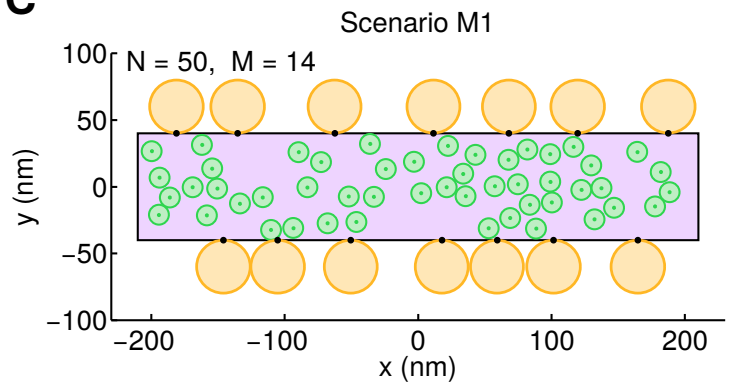

B

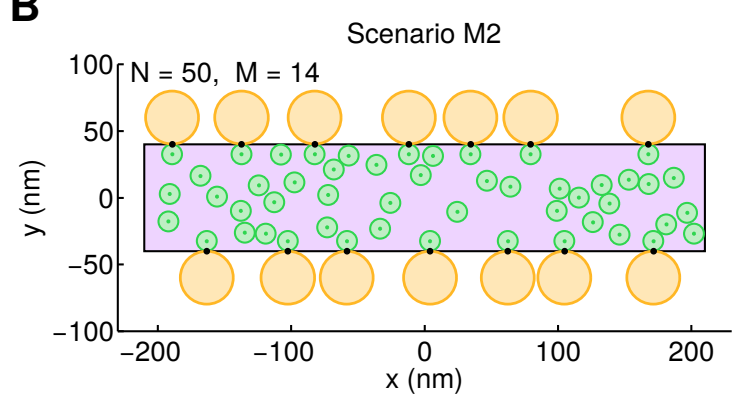

D

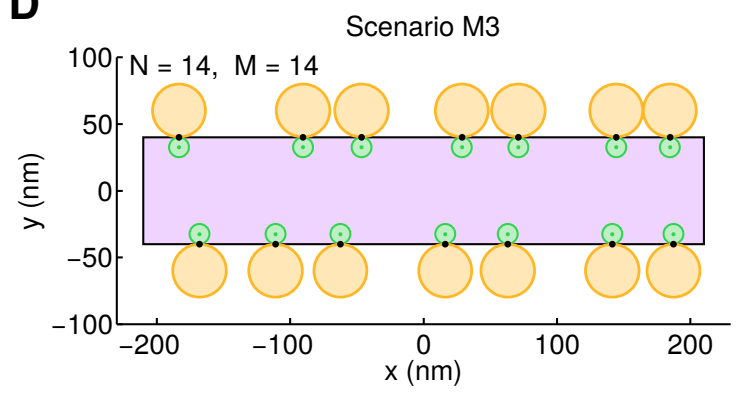

Figure S12: Examples of the active zone topographies considered in section 8. Orange discs $-\mathrm{RRP}$ vesicles, green discs $-\mathrm{Ca}^{2+}$ channels, black spots $-\mathrm{Ca}^{2+}$ sensors of exocytosis, violet area presynaptic density.

\subsection{Active zones with 1 vesicular release site and $\mathrm{N} \mathrm{Ca}^{2+}$ channels, one set of $\mathrm{Ca}^{2+}$ buffers}

Let us next consider a situation equivalent to that introduced in the previous section, except that we now assume the presence of one presynaptic mobile $\mathrm{Ca}^{2+}$ buffer. As it was shown in section 7.4 , the linearized approximation of $\mathrm{Ca}^{2+}$ dynamics works well for the synapse considered in this work ${ }^{9}$. Then, the time-averaged stationary spatial $\mathrm{Ca}^{2+}$ concentration profile resulting from a single $\mathrm{Ca}^{2+}$ channel in the presence of a $\mathrm{Ca}^{2+}$ buffer with a single binding site reads as (3):

$$
\delta\left\langle\left[\mathrm{Ca}^{2+}\right]\right\rangle_{\mathrm{i}}=\frac{i_{C a} \cdot P_{o}}{4 \cdot \pi \cdot F \cdot\left(\kappa \cdot D_{B}+D\right) \cdot R_{i}} \cdot\left(1+\frac{\kappa \cdot D_{B}}{D} \cdot e^{-R_{i} / \lambda}\right)=\frac{i_{C a} \cdot P_{o}}{R_{i}} \cdot\left(A+B \cdot e^{-R_{i} / \lambda}\right),
$$

where $D_{B}$ is diffusion coefficient of the buffer molecules, $\kappa$ and $\lambda$ are parameters dependent on the resting $\mathrm{Ca}^{2+}$ concentration, $\mathrm{Ca}^{2+}$ binding and unbinding rates of the buffer as well as $D$ and $D_{B}$ (see (3) for quantitative definition of $\kappa$ and $\lambda$ ). Let us now, as in the previous section, assume that we estimate $\left\langle\left[\mathrm{Ca}^{2+}\right]\right\rangle$ at the vesicular release site based on an experimentally measured amount of exocytosis: $\left\langle\left[\mathrm{Ca}^{2+}\right]\right\rangle=f^{-1}\left(\Delta_{\text {rel. }}\right)$. Then, we can apply the single $\mathrm{Ca}^{2+}$ source model and estimate the effective coupling distance $R_{c}$ by solving the following equation:

$$
\left\langle\left[\mathrm{Ca}^{2+}\right]\right\rangle=\frac{I_{C a} \cdot P_{o}}{R_{c}} \cdot\left(A+B \cdot e^{-R_{c} / \lambda}\right) \quad \rightarrow \quad R_{c} .
$$

\footnotetext{
${ }^{9}$ This assumption is applied to all the situations considered in sections 8.2-8.5 whenever the presence of $\mathrm{Ca}^{2+}$ buffers is assumed.
} 
If we denote $\delta\left\langle\left[\mathrm{Ca}^{2+}\right]\right\rangle_{\mathrm{i}}=g\left(R_{i}\right)$, equation [4] can be written in the following way:

$$
R_{c}=g^{-1}\left(\frac{\left\langle\left[\mathrm{Ca}^{2+}\right]\right\rangle}{N}\right)=g^{-1}\left(\frac{1}{N} \sum_{i=1}^{N} g\left(R_{i}\right)\right) \equiv\langle R\rangle_{C a}^{\mathcal{N}},
$$

Thus, in this case, $R_{c}$ can be interpreted as a nonlinear average, which we denote by $\langle R\rangle_{C a}^{\mathcal{N}}$, of $R_{i} \mathrm{~s}$ implicitly weighted by contributions of the corresponding channels to $\left\langle\left[\mathrm{Ca}^{2+}\right]\right\rangle$. Here, superscript $\mathcal{N}$ stands for "nonlinear". The higher the contribution of a channel to $\left\langle\left[\mathrm{Ca}^{2+}\right]\right\rangle$ and, thus, exocytosis, the closer $\langle R\rangle_{\mathrm{Ca}}^{\mathcal{N}}$ is to the distance from that channel to the $\mathrm{Ca}^{2+}$ sensor of exocytosis. Equation [5] is valid for any arbitrary well defined dependence of $\delta\left\langle\left[\mathrm{Ca}^{2+}\right]\right\rangle_{i}$ on $R_{i}$, for example, the one in the presence of multiple mobile $\mathrm{Ca}^{2+}$ buffers in the presynaptic solution.

It follows from equation [5] that $\langle R\rangle_{\mathrm{Ca}}^{\mathcal{N}}=\langle R\rangle_{\mathrm{Ca}}^{\mathcal{L}}=R_{i}$ if all the channels are equidistant to the $\mathrm{Ca}^{2+}$ sensor, i.e., $R_{i}=R_{j}$ for any $i$ and $j$. If the channels are not equidistant, $\langle R\rangle_{\mathrm{Ca}}^{\mathcal{N}} \neq\langle R\rangle_{\mathrm{Ca}}^{\mathcal{L}}$. However, note that at sufficiently small distances, i.e., when $R_{i} \ll \lambda$, and at sufficiently large distances, i.e., when $R_{i} \gg \lambda, \delta\left\langle\left[\mathrm{Ca}^{2+}\right]\right\rangle_{i}$ is approximately inversely proportional to $R_{i}$ even in the presence of $\mathrm{Ca}^{2+}$ buffers. This statement follows from equation [3], see also (3). Thus, if a fraction of the channels is sufficiently close to the $\mathrm{Ca}^{2+}$ sensor, while the remaining channels are sufficiently far away from the $\mathrm{Ca}^{2+}$ sensor, $\langle R\rangle_{C a}^{\mathcal{N}} \approx\langle R\rangle_{C a}^{\mathcal{L}}$.

To estimate how big the difference between $\langle R\rangle_{\mathrm{Ca}}^{\mathcal{N}}$ and $\langle R\rangle_{\mathrm{Ca}}^{\mathcal{L}}$ might be in $\mathrm{Ca}^{2+}$ buffering conditions used in this work, we considered a $\mathrm{Ca}^{2+}$ channel cluster consisting of 2 to 5 channels, which were distributed in a 2D area, like in Fig. S12A. Each of the channels was located from the $\mathrm{Ca}^{2+}$ sensor of exocytosis not closer than $1 \mathrm{~nm}$ and not further than $100 \mathrm{~nm} .0 .5 \mathrm{mM}$ and $1 \mathrm{mM}$ of either BAPTA or EGTA were considered as presynaptic $\mathrm{Ca}^{2+}$ buffers. To estimate the maximum and the minimum values of the difference $\langle R\rangle_{C a}^{\mathcal{N}}-\langle R\rangle_{C a}^{\mathcal{L}}$, we used the optimization based on a genetic algorithm (MATLAB function $g a$, Generations $=20 \cdot N$, StallGenLimit $=15 \cdot N$, PopulationSize $=N \cdot 50$, EliteCount $=N \cdot 5$, CrossoverFraction $=0.6$, TolFun $=10^{-12}$ ) followed by Nelder-Mead simplex method (MATLAB function fminsearch) repeated with 100 different initial conditions. In all these cases considered, the numerical optimization suggested that $\langle R\rangle_{\mathrm{Ca}}^{\mathcal{N}} \geq\langle R\rangle_{\mathrm{Ca}}^{\mathcal{L}}$ with the equality between the two present only when all the $\mathrm{Ca}^{2+}$ channels were equidistant to the sensor. $\max \left[\langle R\rangle_{C a}^{\mathcal{N}}-\langle R\rangle_{C a}^{\mathcal{L}}\right]$ was larger for buffers with smaller parameter $\lambda$, and for larger $N$, as summarized in Table S1.

\begin{tabular}{|c||c|c|c|c|}
\hline \multicolumn{1}{|c||}{} & \multicolumn{4}{c|}{$\max \left[\langle R\rangle_{\mathrm{Ca}}^{\mathcal{N}}-\langle R\rangle_{\mathrm{Ca}}^{\mathcal{L}}\right], \mathrm{nm}$} \\
\cline { 2 - 5 } & 0.5 mM EGTA & 1 mM EGTA & $0.5 \mathrm{mM}$ BAPTA & 1 mM BAPTA \\
\hline \hline$N=2$ & 3 & 4 & 9 & 9 \\
\hline$N=3$ & 4 & 6 & 14 & 14 \\
\hline$N=4$ & 5 & 7 & 17 & 17 \\
\hline$N=5$ & 6 & 7 & 19 & 19 \\
\hline
\end{tabular}

Table S1 


\subsection{Active zones with 1 vesicular release site and $N \mathrm{Ca}^{2+}$ channels, two sets of $\mathrm{Ca}^{2+}$ buffers}

As it is formulated in Methods, the single $\mathrm{Ca}^{2+}$ source model was designed to estimate the effective coupling distance $R_{c}$ from experimental data in such a way that knowledge of the absolute values of $\left\langle\left[\mathrm{Ca}^{2+}\right]\right\rangle$ at the vesicular release sites is not required ${ }^{10}$. Indeed, if we measure amounts of released vesicles in two different $\mathrm{Ca}^{2+}$ buffering conditions, then the ratio of these two estimates, $\Delta_{\text {rel.,1 }} / \Delta_{\text {rel.,2}}=$ $f\left(\left\langle\left[\mathrm{Ca}^{2+}\right]_{1}\right\rangle\right) / f\left(\left\langle\left[\mathrm{Ca}^{2+}\right]_{2}\right\rangle\right)=\left(\left\langle\left[\mathrm{Ca}^{2+}\right]_{1}\right\rangle /\left\langle\left[\mathrm{Ca}^{2+}\right]_{2}\right\rangle\right)^{m}$ can be used to extract the $R_{c}$ :

$$
\frac{\Delta_{r e l ., 1}}{\Delta_{r e l, 2}}=\left(\frac{A_{1}+B_{1} \cdot e^{-R_{c} / \lambda_{1}}}{A_{2}+B_{2} \cdot e^{-R_{c} / \lambda_{2}}}\right)^{m} \rightarrow R_{c}
$$

Here, A's and B's depend only on the physical parameters of the buffer molecules, their concentrations, diffusion coefficient of $\mathrm{Ca}^{2+}$ and the resting $\left[\mathrm{Ca}^{2+}\right]$ at the presynaptic site. $m$ is the apparent cooperativity of exocytosis ${ }^{11}$. If we denote $\delta\left\langle\left[\mathrm{Ca}^{2+}\right]_{1}\right\rangle_{\mathrm{i}}=g_{1}\left(R_{i}\right), \delta\left\langle\left[\mathrm{Ca}^{2+}\right]_{2}\right\rangle_{\mathrm{i}}=g_{2}\left(R_{i}\right)$, and $g_{1}(x) / g_{2}(x)=g_{12}(x)$, equation [6] can be rewritten in the following way:

$$
R_{c}=g^{-1}\left(\frac{\left\langle\left[\mathrm{Ca}^{2+}\right]_{1}\right\rangle}{\left\langle\left[\mathrm{Ca}^{2+}\right]_{2}\right\rangle}\right)=g_{12}^{-1}\left(\frac{\sum_{i=1}^{N} g_{1}\left(R_{i}\right)}{\sum_{i=1}^{N} g_{2}\left(R_{i}\right)}\right) \equiv\langle R\rangle_{\text {Ca,12 }}^{\mathcal{N} *},
$$

Here, subscript 12 stands for the two buffering conditions "1" and "2" used to estimate $\langle R\rangle_{\text {Ca,12 }}^{\mathcal{N} *}$. The superscript $*$ is used to emphasize that the effective coupling distance is calculated by estimating exocytosis in two different presynaptic $\mathrm{Ca}^{2+}$ buffering conditions "1" and "2". Like $\langle R\rangle_{\mathrm{Ca}}^{\mathcal{N}},\langle R\rangle_{\mathrm{Ca}, 12}^{\mathcal{N} *}$ can be interpreted as a nonlinear average, of $R_{i} \mathrm{~s}$ implicitly weighted by the contributions of the corresponding channels to $\left\langle\left[\mathrm{Ca}^{2+}\right]\right\rangle$. However, in general, $\langle R\rangle_{\mathrm{Ca}, 12}^{\mathcal{N} *} \neq\langle R\rangle_{\mathrm{Ca}, 1}^{\mathcal{N}}$ and $\langle R\rangle_{\mathrm{Ca}, 12}^{\mathcal{N} *} \neq\langle R\rangle_{\mathrm{Ca}, 2}^{\mathcal{N}}$, unless all the $\mathrm{Ca}^{2+}$ channels are equidistant to the $\mathrm{Ca}^{2+}$ sensor of exocytosis, in which case $\langle R\rangle_{\mathrm{Ca}, 12}^{\mathcal{N} *}=\langle R\rangle_{\mathrm{Ca}, 1}^{\mathcal{N}}=\langle R\rangle_{\mathrm{Ca}, 2}^{\mathcal{N}}=R_{i}$. Clearly, equation [7] is valid for any arbitrary well defined dependence of $\delta\left\langle\left[\mathrm{Ca}^{2+}\right]\right\rangle_{i}$ on $R_{i}$, for example, the one in the presence of multiple mobile $\mathrm{Ca}^{2+}$ buffers in the presynaptic solution.

That we do not need to know the absolute values of $\left\langle\left[\mathrm{Ca}^{2+}\right]\right\rangle$ at the $\mathrm{Ca}^{2+}$ sensor of exocytosis or $\mathrm{Ca}^{2+}$ currents that give rise to $\left\langle\left[\mathrm{Ca}^{2+}\right]\right\rangle$ in order to determine $R_{c}$ as defined by equation [7] is a very important advantage of the mentioned approach over that considered in section 8.2. Indeed, $\left\langle\left[\mathrm{Ca}^{2+}\right]\right\rangle$ at $\mathrm{Ca}^{2+}$ sensors of exocytosis is not a measurable quantity with currently available experimental techniques. On the other hand, this approach has one disadvantage, which always has to be kept in mind when interpreting estimates of $R_{c}$ based on the experimental data. The disadvantage is that it is necessary to measure the release in at least two different $\mathrm{Ca}^{2+}$ buffering conditions, only one of which can be the natural one. Thus, an estimate of $R_{c}$ achieved by using this approach depends not only on the intrinsic properties of the system being studied, but also on how we choose to study the system. It follows from equation [3] that, when the buffering length constant $\lambda$ is decreased (by increasing concentration of the buffer, for example), the contribution of a particular channel to $\left\langle\left[\mathrm{Ca}^{2+}\right]\right\rangle$ at a $\mathrm{Ca}^{2+}$ sensor of exocytosis is decreased the more the further that channel is away from the sensor. Thus, if mobile $\mathrm{Ca}^{2+}$ buffers used for estimating $R_{c}$ are much stronger than the endogenous buffers of the synapse, the coupling distance may be

\footnotetext{
${ }^{10}$ Assuming the linearity between $i_{C a}$ and $\left\langle\left[\mathrm{Ca}^{2+}\right]\right\rangle$ at the vesicular release sites.

${ }^{11}$ Here and further on, we assume that the apparent $\mathrm{Ca}^{2+}$ cooperativity is the same in both $\mathrm{Ca}^{2+}$ buffering conditions used for determining $R_{c}$.
} 
considerably underestimated. Vice versa, if mobile $\mathrm{Ca}^{2+}$ buffers used for estimating $R_{c}$ are much weaker than the endogenous buffers of the synapse, the coupling distance may be overestimated. Nevertheless, as it is shown next, $\langle R\rangle_{\mathrm{Ca}, 12}^{\mathcal{N} *}$ is closely related to quantities uniquely determined by the configuration of the natural system, for example, $\langle R\rangle_{\mathrm{C} a, 1}^{\mathcal{L}}$ or $\langle R\rangle_{\mathrm{C} a, 2}^{\mathcal{L}}$, and carries valuable information about the system if the experiment is designed appropriately. Noteworthy, if spatially resolved simulations are performed to check the hypothesis of particular scenarios of the active zone organization, the experimental estimate of $R_{c}$ can be directly compared to its modeled counterpart independently of the buffering conditions ${ }^{12}$.

To better understand the physical meaning of $\langle R\rangle_{\mathrm{Ca}, 12}^{\mathcal{N} *}$, we compared it with linear weighted average distances $\langle R\rangle_{\mathrm{C} a, 1}^{\mathcal{L}}$ and $\langle R\rangle_{\mathrm{C} a, 2}^{\mathcal{L}}$, estimated separately in the two $\mathrm{Ca}^{2+}$ buffering conditions used for estimating $\langle R\rangle_{\mathrm{Ca}, 12}^{\mathcal{N} *}{ }^{13}$. To this end, we first considered the previously introduced active zone model consisting of one vesicular release site and two to five $\mathrm{Ca}^{2+}$ channels (see section 8.2). Pairs constructed from four $\mathrm{Ca}^{2+}$ buffers introduced above were considered, namely: $1 \mathrm{mM}$ BAPTA \& $0.5 \mathrm{mM}$ BAPTA, $1 \mathrm{mM}$ BAPTA \& $1 \mathrm{mM}$ EGTA, $1 \mathrm{mM}$ BAPTA \& $0.5 \mathrm{mM}$ EGTA, $0.5 \mathrm{mM}$ BAPTA \& $1 \mathrm{mM}$ EGTA, $1 \mathrm{mM}$ EGTA \& $0.5 \mathrm{mM}$ EGTA and $0.5 \mathrm{mM}$ BAPTA \& $0.5 \mathrm{mM}$ EGTA. Numerical global optimization (performed in the same way as introduced in section 8.2) resulted in $\left.\left.\langle R\rangle_{C a, 2}^{\mathcal{L}}\right\rangle\langle R\rangle_{C a, 12}^{\mathcal{N} *}\right\rangle\langle R\rangle_{C a, 1}^{\mathcal{L}}$ for buffers "1" and "2" with $\lambda_{2}>\lambda_{1}$.

Next, we got estimates of $\langle R\rangle_{\text {Ca,1 }}^{\mathcal{L}},\langle R\rangle_{\text {Ca,2 }}^{\mathcal{L}}$, and $\langle R\rangle_{\text {Ca,12 }}^{\mathcal{N} *}$ for examples of physiologically realistic active zone topographies. To this end, we studied three IHC active zone topography scenarios - M1, M2, and M3 - considered in (2), see Fig. S12B-D. In those three scenarios, 14 readily releasable pool vesicles (orange disks, $R=20 \mathrm{~nm}$ ) were randomly distributed at the longer sides of the presynaptic density (violet rectangle). All the $\mathrm{Ca}^{2+}$ sensors of exocytosis (black dots) were located at the level of the plasma membrane, at the longer sides of the presynaptic density. In scenario M1, $50 \mathrm{Ca}^{2+}$ channels (green disks, $R=7.5 \mathrm{~nm}$ ) were distributed randomly within the presynaptic density. In scenario $\mathrm{M} 2,36$ out of $50 \mathrm{Ca}^{2+}$ channels were distributed randomly within the presynaptic density, while the remaining 14 $\mathrm{Ca}^{2+}$ channels were placed in contact with the $\mathrm{Ca}^{2+}$ sensors of exocytosis. In scenario M3, there were only $14 \mathrm{Ca}^{2+}$ channels in contact with the $\mathrm{Ca}^{2+}$ sensors. We considered 100 active zone realizations for each scenario. Four pairs of mobile $\mathrm{Ca}^{2+}$ buffer sets, the same as those used in our experiments, were assumed: $1 \mathrm{mM}$ BAPTA $+2 \mathrm{mM}$ ATP $\& 0.5 \mathrm{mM}$ BAPTA $+2 \mathrm{mM}$ ATP, $1 \mathrm{mM}$ BAPTA $+2 \mathrm{mM}$ ATP $\& 1 \mathrm{mM}$ EGTA $+2 \mathrm{mM}$ ATP, $1 \mathrm{mM}$ BAPTA $+2 \mathrm{mM}$ ATP $\& 0.5 \mathrm{mM}$ EGTA $+2 \mathrm{mM}$ ATP, and $1 \mathrm{mM}$ BAPTA $+2 \mathrm{mM}$ ATP \& $2 \mathrm{mM}$ ATP. Mouth of each $\mathrm{Ca}^{2+}$ channel was treated as a hemispherical source with $1 \mathrm{~nm}$ radius and $i_{C a}=0.3 \mathrm{pA} .\langle R\rangle_{\mathrm{C} a, 1}^{\mathcal{L}},\langle R\rangle_{\mathrm{C} a, 2}^{\mathcal{L}}$, and $\langle R\rangle_{\mathrm{C} a, 12}^{\mathcal{N} *}$ were estimated for each active zone scenario as averages over separate vesicular release sites within a particular realization of the active zone and over 100 realizations of that active zone scenario. The results are summarized in Table S2.

We found that, as in the case of the simplified active zone model considered before, inequality $\left.\left.\langle R\rangle_{\mathrm{C} a, 2}^{\mathcal{L}}\right\rangle\langle R\rangle_{\mathrm{Ca}, 12}^{\mathcal{N} *}\right\rangle\langle R\rangle_{\mathrm{C} a, 1}^{\mathcal{L}}$ holds for active zone scenarios M1, M2, and M3. $\langle R\rangle_{\text {Ca,12 }}^{\mathcal{N} *}$ was shifted more towards $\langle R\rangle_{C a, 1}^{\mathcal{L}}$ than to $\langle R\rangle_{C a, 2}^{\mathcal{L}}$. As expected, values of $\langle R\rangle_{C a, 1}^{\mathcal{L}},\langle R\rangle_{C a, 2}^{\mathcal{L}}$, and $\langle R\rangle_{C a, 12}^{\mathcal{N} *}$ were the

\footnotetext{
${ }^{12}$ Of course, in this case, the exact value of $R_{c}$ depends on the properties of the $\mathrm{Ca}^{2+}$ buffers used. However, now we need to use only one set of $\mathrm{Ca}^{2+}$ buffers. This set of buffers may be chosen, at least in principle, to be that which is present in a real synapse.

${ }^{13}$ The main reason why we chose $\langle R\rangle_{C a, 1}^{\mathcal{L}}$ and $\langle R\rangle_{C a, 2}^{\mathcal{L}}$, not $\langle R\rangle_{C a, 1}^{\mathcal{N}}$ and $\langle R\rangle_{C a, 2}^{\mathcal{N}}$, to compare to $\langle R\rangle_{C a, 12}^{\mathcal{N} *}$ is that $\langle R\rangle_{C a}^{\mathcal{L}}$ have a clearer physical meaning than $\langle R\rangle_{\mathrm{Ca}}^{\mathcal{N}}$ (compare equations [2] and [5]).
} 


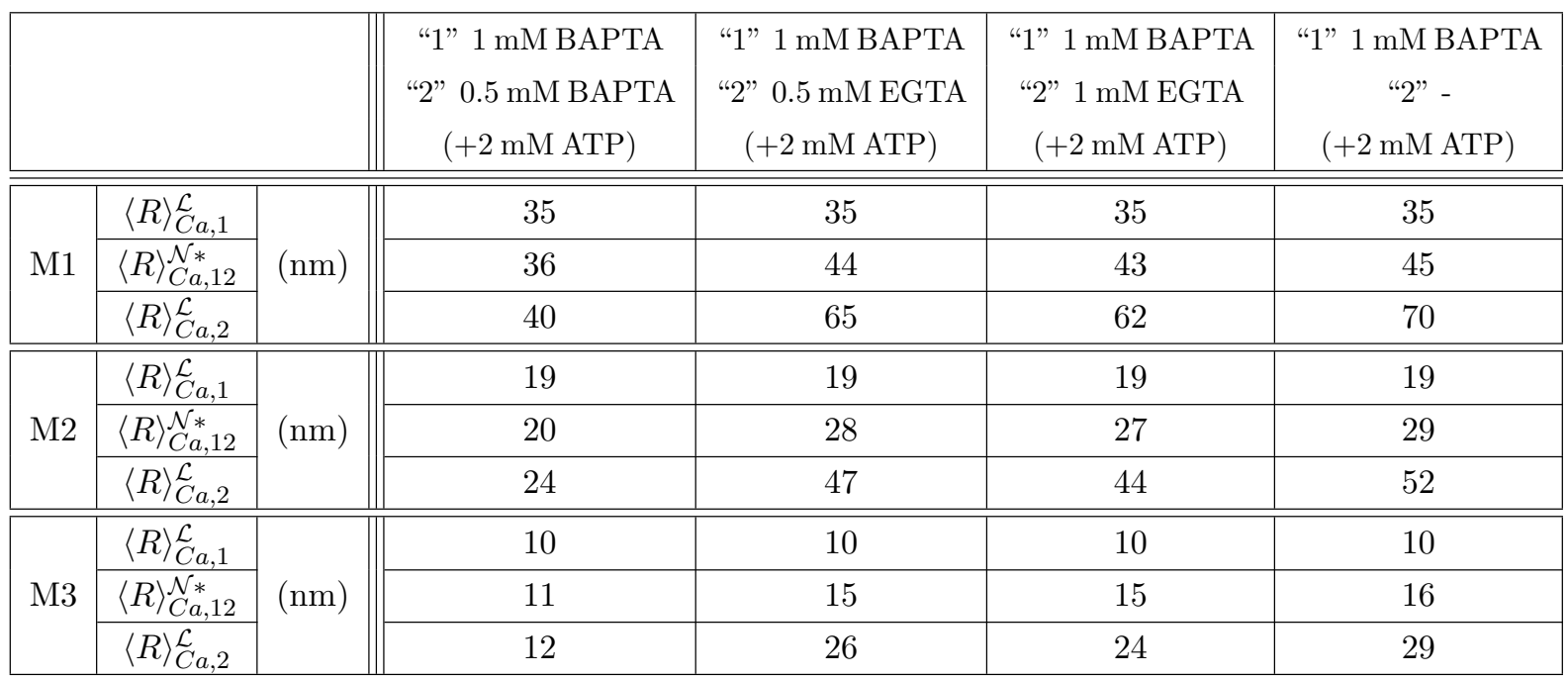

Table S2

smallest for scenario M3 (the most tight coupling), and the largest for scenario M1 (the least tight coupling) at any buffering conditions considered. The largest difference between $\langle R\rangle_{\text {Ca,2 }}^{\mathcal{L}}$ and $\langle R\rangle_{\mathrm{Ca}, 1}^{\mathcal{L}}$, which reflects the sensitivity of $R_{c}$ estimate to the $\mathrm{Ca}^{2+}$ buffering conditions, was found for scenario M1 and the smallest - for scenario M3. This is partially explained by the fact that a tighter coupling with fewer channels contributing $\mathrm{Ca}^{2+}$ to particular vesicular release sites results in a smaller variability of the distances between the channels and the vesicular release site about the average one. Another reason is that the considered mobile $\mathrm{Ca}^{2+}$ buffers have a relatively small effect on $\delta\left\langle\left[\mathrm{Ca}^{2+}\right]\right\rangle_{i}$ when the coupling is so tight as in scenario M3, as mentioned in section 8.2. The estimates of $\langle R\rangle_{\mathrm{Ca}, 1}^{\mathcal{L}},\langle R\rangle_{\mathrm{C} a, 2}^{\mathcal{L}}$, and $\langle R\rangle_{\mathrm{Ca}, 12}^{\mathcal{N} *}$ were smaller with stronger $\mathrm{Ca}^{2+}$ buffering conditions. For example, in the case of scenario $\mathrm{M} 1,\langle R\rangle_{\mathrm{Ca}, 12}^{\mathcal{N} *}$ was equal to $36 \mathrm{~nm}$ for $1 \mathrm{mM}$ BAPTA $+2 \mathrm{mM}$ ATP \& $0.5 \mathrm{mM}$ BAPTA $+2 \mathrm{mM}$ ATP, compared to $45 \mathrm{~nm}$ for $1 \mathrm{mM}$ BAPTA $+2 \mathrm{mM}$ ATP \& $2 \mathrm{mM}$ ATP. This trend is explained by the fact that the relative contributions of more distant $\mathrm{Ca}^{2+}$ channels are reduced when the strength of the $\mathrm{Ca}^{2+}$ buffers is increased.

In conclusion, the results discussed in this section suggest that, at least for the $\mathrm{Ca}^{2+}$ buffering conditions considered in this work, $\langle R\rangle_{\mathrm{Ca}, 12}^{\mathcal{N} *}$ can be treated as an upper or lower bound for $\langle R\rangle_{\mathrm{Ca}}^{\mathcal{L}}$ estimated in the natural $\mathrm{Ca}^{2+}$ buffering conditions. $\langle R\rangle_{\mathrm{Ca}, 12}^{\mathcal{N} *}$ is the upper bound for $\langle R\rangle_{\mathrm{Ca}}^{\mathcal{L}}$ when buffer "2" is weaker, i.e., with higher lambda, than the natural $\mathrm{Ca}^{2+}$ buffer in the synapse while buffer " 1 " is not stronger than the endogenous buffer. $\langle R\rangle_{\mathrm{Ca}, 12}^{\mathcal{N} *}$ is the lower bound for $\langle R\rangle_{\mathrm{Ca}}^{\mathcal{L}}$ when buffer "2" is not stronger than the endogenous $\mathrm{Ca}^{2+}$ buffer in the synapse while buffer "1" is stronger, i.e., with lower lambda, than the natural buffer.

\subsection{Active zones with $M$ vesicular release sites and $N \mathrm{Ca}^{2+}$ channels}

Real presynaptic active zones contain considerably more than one vesicular release site. If the dependence of vesicle release rate on $\left[\mathrm{Ca}^{2+}\right]$ is non-linear, the interpretation of the effective coupling distance $R_{c}$ for an active zone with $M>1$ site is more complicated compared to the situations with $M=1$ considered 
so $\operatorname{far}^{14}$. To see this, let us consider an active zone with $M>1$ vesicular release sites driven by $N$ $\mathrm{Ca}^{2+}$ channels. As before, we assume that the contribution to the time-averaged $\mathrm{Ca}^{2+}$ concentration at a particular vesicular release site $j$ by a particular channel $i$ is a bijective function of the distance between the channel and the corresponding $\mathrm{Ca}^{2+}$ sensor of exocytosis: $\delta\left\langle\left[\mathrm{Ca}^{2+}\right]\right\rangle_{\mathrm{j}, \mathrm{i}}=g\left(R_{j, i}\right)$. We also assume that, for each vesicular release site, the amount of vesicles released in particular time window $T$ depends only on time-averaged $\mathrm{Ca}^{2+}$ concentration at that vesicular release site: $\Delta_{\text {rel., }, j}=f\left(\left\langle\left[\mathrm{Ca}^{2+}\right]\right\rangle_{\mathrm{j}}\right)=$ $f\left(\sum_{i=1}^{N} \delta\left\langle\left[\mathrm{Ca}^{2+}\right]\right\rangle_{\mathrm{j}, \mathrm{i}}\right)$. Then, $R_{c}$ estimated by recording the amount of release at the active zone, $\Delta_{r e l, \Sigma}=$ $\sum_{j=1}^{M} \Delta_{r e l, j}$, in the presence of a particular set of $\mathrm{Ca}^{2+}$ buffers can be expressed in the following way:

$$
R_{c}=g^{-1}\left(\frac{1}{N} f^{-1}\left(\frac{1}{M} \sum_{j=1}^{M} f\left(\sum_{i=1}^{N} g\left(R_{j, i}\right)\right)\right)\right) .
$$

Equation [8] is a generalization of equation [5] for active zones with an arbitrary number of vesicular release sites. $R_{c}$ defined by equation [8] can be interpreted as a nonlinear, implicitly weighted average of distances between a particular channel and a particular $\mathrm{Ca}^{2+}$ sensor of exocytosis over all possible channel-sensor pairs. Differently from the situation with a single vesicular release site, the nonlinear averaging of distances between one of the $\mathrm{Ca}^{2+}$ channels and one of the $\mathrm{Ca}^{2+}$ sensors of exocytosis now depends not only on how $\delta\left\langle\left[\mathrm{Ca}^{2+}\right]\right\rangle_{\mathrm{j}, \mathrm{i}}$ depends on $R_{j, i}$ but also on how $\Delta_{\text {rel., }}$ depends on $\left\langle\left[\mathrm{Ca}^{2+}\right]\right\rangle_{\mathrm{j}}$. Qualitatively, the larger the relative contribution of a particular $\mathrm{Ca}^{2+}$ channel to $\left\langle\left[\mathrm{Ca}^{2+}\right]\right\rangle_{\mathrm{j}}$ at a particular vesicular release site is and the larger the relative contribution of that vesicular release site to the overall release at the active zone $\Delta_{r e l, \Sigma \Sigma}$ is, the closer is $R_{c}$ to the distance between that particular $\mathrm{Ca}^{2+}$ channel and $\mathrm{Ca}^{2+}$ sensor of exocytosis of that particular vesicular release site.

If all vesicular release sites are equivalent in the sense of how the $\mathrm{Ca}^{2+}$ channels are positioned in regards of them, i.e., if $R_{j, i}=R_{j^{\prime}, i} \equiv R_{i}$ for any $j, j^{\prime}$, and $i$, the effective coupling distance for the whole active zone is equal to the effective coupling distance for one of the vesicular release sites. Indeed, if that condition is met, equation [8] reduces to equation [5]. Equation [8] reduces to $R_{c}=R_{i, j}$ when $R_{i, j}=R_{i^{\prime}, j^{\prime}}$ for arbitrary $i, i^{\prime}, j$, and $j^{\prime}$. i.e., when all the $\mathrm{Ca}^{2+}$ channels are at the same distance from all the $\mathrm{Ca}^{2+}$ sensors. In this case, $R_{c}$ is equal to the physical distance between any of those $\mathrm{Ca}^{2+}$ channels and any of those $\mathrm{Ca}^{2+}$ sensors of exocytosis at the active zone. If $\Delta_{r e l, j}$ depends on $\left\langle\left[\mathrm{Ca}^{2+}\right]\right\rangle_{j}$ linearly, i.e., if $f(x)=a \cdot x+b$, the effective coupling distance $R_{c}$ is equal to the effective coupling distance of the "average vesicular release site" within the active zone:

$$
R_{c}=g^{-1}\left(\frac{1}{M} \sum_{j=1}^{M}\left(\frac{1}{N} \sum_{i=1}^{N} g\left(R_{j, i}\right)\right)\right) .
$$

When dependence of $\Delta_{r e l ., j}$ on $\left\langle\left[\mathrm{Ca}^{2+}\right]\right\rangle_{j}$ follows a power law, i.e., when $f(x)=k \cdot x^{m}$, and no $\mathrm{Ca}^{2+}$ buffers are present in the presynaptic solution, i.e., when $\delta\left\langle\left[\mathrm{Ca}^{2+}\right]\right\rangle_{j, i}$ is described by [1], [8] has the

\footnotetext{
${ }^{14}$ This is true independently of how many vesicles are actually released during the observation window.
} 
following form:

$$
\begin{aligned}
R_{c} & =g^{-1}\left(\frac{1}{N}\left(\frac{1}{M} \sum_{j=1}^{M}\left(\sum_{i=1}^{N} g\left(R_{j, i}\right)\right)^{m}\right)^{1 / m}\right)=\frac{M \cdot N \cdot i_{C a} \cdot P_{o} /(4 \cdot \pi \cdot F \cdot D)}{M \cdot\left(\frac{1}{M} \sum_{j=1}^{M}\left(\sum_{i=1}^{N} g\left(R_{j, i}\right)\right)^{m}\right)^{1 / m}}= \\
& =\frac{\sum_{j=1}^{M} \sum_{i=1}^{N} R_{j, i} \cdot g\left(R_{j, i}\right)}{M \cdot\left(\frac{1}{M} \sum_{j=1}^{M}\left(\sum_{i=1}^{N} g\left(R_{j, i}\right)\right)^{m}\right)^{1 / m}}= \\
& =\sum_{j=1}^{M} \sum_{i=1}^{N} R_{j, i} \cdot\left(\frac{\delta\left\langle\left[\mathrm{Ca}^{2+}\right]_{\mathrm{j}, \mathrm{i}}\right\rangle}{M \cdot\left(\frac{1}{M} \sum_{j^{\prime}=1}^{M}\left(\sum_{i^{\prime}=1}^{N} \delta\left\langle\left[\mathrm{Ca}^{2+}\right]_{\mathrm{j}^{\prime}, \mathrm{i}^{\prime}}\right\rangle\right)^{m}\right)^{1 / m}}\right) \equiv\langle\langle R\rangle\rangle_{C a}^{\mathcal{L}, m} .
\end{aligned}
$$

Here, $\left(\frac{1}{M} \sum_{j^{\prime}=1}^{M}\left(\sum_{i^{\prime}=1}^{N} \delta\left\langle\left[\mathrm{Ca}^{2+}\right]_{j^{\prime}, i^{\prime}}\right\rangle\right)^{m}\right)^{1 / m}$ is the power $m$ mean (Hölder mean) of $\left\langle\left[\mathrm{Ca}^{2+}\right]\right\rangle_{j^{\prime}}$ over all $M$ vesicular release sites at the active zone. Thus, the effective coupling distance $R_{c}$, in this case, is a weighted linear average of distances from all the $\mathrm{Ca}^{2+}$ channels to all the $\mathrm{Ca}^{2+}$ sensors in the active zone, which we denote by $\langle\langle R\rangle\rangle_{\mathrm{Ca}}^{\mathcal{L}, m}$. Here, the second angular brackets stand for averaging over vesicular release sites (in addition to the averaging over $\mathrm{Ca}^{2+}$ channels). The superscript $m$ expresses the idea that the effective coupling distance depends on the apparent $\mathrm{Ca}^{2+}$ cooperativity of exocytosis. The weight for distance $R_{j, i}$ between channel $i$ and vesicular release site $j$ is equal to the ratio between the increment of $\mathrm{Ca}^{2+}$ concentration at the vesicular release site $j$ contributed by the channel $i$ and the power $m$ mean of $\mathrm{Ca}^{2+}$ concentrations over all vesicular release sites at the active zone multiplied by the number of the vesicular release sites. It follows from the properties of the Hölder mean that $\left(\frac{1}{M} \sum_{j^{\prime}=1}^{M}\left(\sum_{i^{\prime}=1}^{N} \delta\left\langle\left[\mathrm{Ca}^{2+}\right]_{\mathrm{j}^{\prime}, i^{\prime}}\right\rangle\right)^{m_{2}}\right)^{1 / m_{2}}>\left(\frac{1}{M} \sum_{j^{\prime}=1}^{M}\left(\sum_{i^{\prime}=1}^{N} \delta\left\langle\left[\mathrm{Ca}^{2+}\right]_{\mathrm{j}^{\prime}, i^{\prime}}\right\rangle\right)^{m_{1}}\right)^{1 / m_{1}}$ for $m_{2}>m_{1}$. Thus, the higher is $\mathrm{Ca}^{2+}$ cooperativity of exocytosis, the smaller is $\langle\langle R\rangle\rangle_{\mathrm{Ca}}^{\mathcal{L}, m}$.

When $R_{c}$ is estimated by using the measurements of $\Delta_{r e l, \Sigma \Sigma}$ in two different $\mathrm{Ca}^{2+}$ buffering conditions ${ }^{15}$ and $f(x)=k \cdot x^{m}$, the equivalent of equation [8] reads as

$$
R_{c}=g_{12}^{-1}\left(\left(\frac{\sum_{j=1}^{M}\left(\sum_{i=1}^{N} g_{1}\left(R_{j, i}\right)\right)^{m}}{\sum_{j=1}^{M}\left(\sum_{i=1}^{N} g_{2}\left(R_{j, i}\right)\right)^{m}}\right)^{1 / m}\right) \equiv\langle\langle R\rangle\rangle_{C a, 12}^{\mathcal{N} * m},
$$

where $g_{1}\left(R_{j, i}\right)$ and $g_{2}\left(R_{j, i}\right)$ are dependencies of $\left\langle\left[\mathrm{Ca}^{2+}\right]\right\rangle_{j, i}$ on $R_{j, i}$ in $\mathrm{Ca}^{2+}$ buffering conditions "1" and "2" respectively, $g_{12}(x)=g_{1}(x) / g_{2}(x)$. Taking into account the results from section 8.3, it is tempting to hypothesize that $\langle\langle R\rangle\rangle_{C a, 12}^{\mathcal{N} *, m}$ could be an upper and a lower bound for $\langle\langle R\rangle\rangle_{C a, 1}^{\mathcal{L}, m}$ and $\langle\langle R\rangle\rangle_{C a, 2}^{\mathcal{L}, m}$, respectively, and could be rather well approximated by the average of the later two. We checked this idea with the realistic active zone topographies considered in section 8.3 and shown in Fig. S12B-D. The results of our simulations for $m=1.7$, the experimentally predicted value of the apparent $\mathrm{Ca}^{2+}$ cooperativity of exocytosis (see Fig. S6), are summarized in Table S3. The results are strikingly similar to those for $\langle R\rangle_{C a, 1}^{\mathcal{L}},\langle R\rangle_{C a, 12}^{\mathcal{N} *}$, and $\langle R\rangle_{C a, 2}^{\mathcal{L}}$, shown in Table $\mathbf{S 2}^{16}$. Thus, all the conclusions and comments made at the end of section 8.3 in the context of an active zone with a single vesicular release site, are valid for $R_{c}$ estimated for scenarios M1, M2, and M3 with all vesicular release sites within an active zone considered collectively.

\footnotetext{
${ }^{15} \mathrm{As}$ it was done in our study.

${ }^{16}$ We also considered higher $m$ values. As mentioned before, $R_{c}$ estimates are smaller for larger $m$. However, for active zone topographies scenarios M2 and M3, $m=5$ resulted in estimates of $\langle\langle R\rangle\rangle_{\mathrm{Ca}, 12}^{\mathcal{N} *, m}$ only $<1 \mathrm{~nm}$ smaller than those for
} 


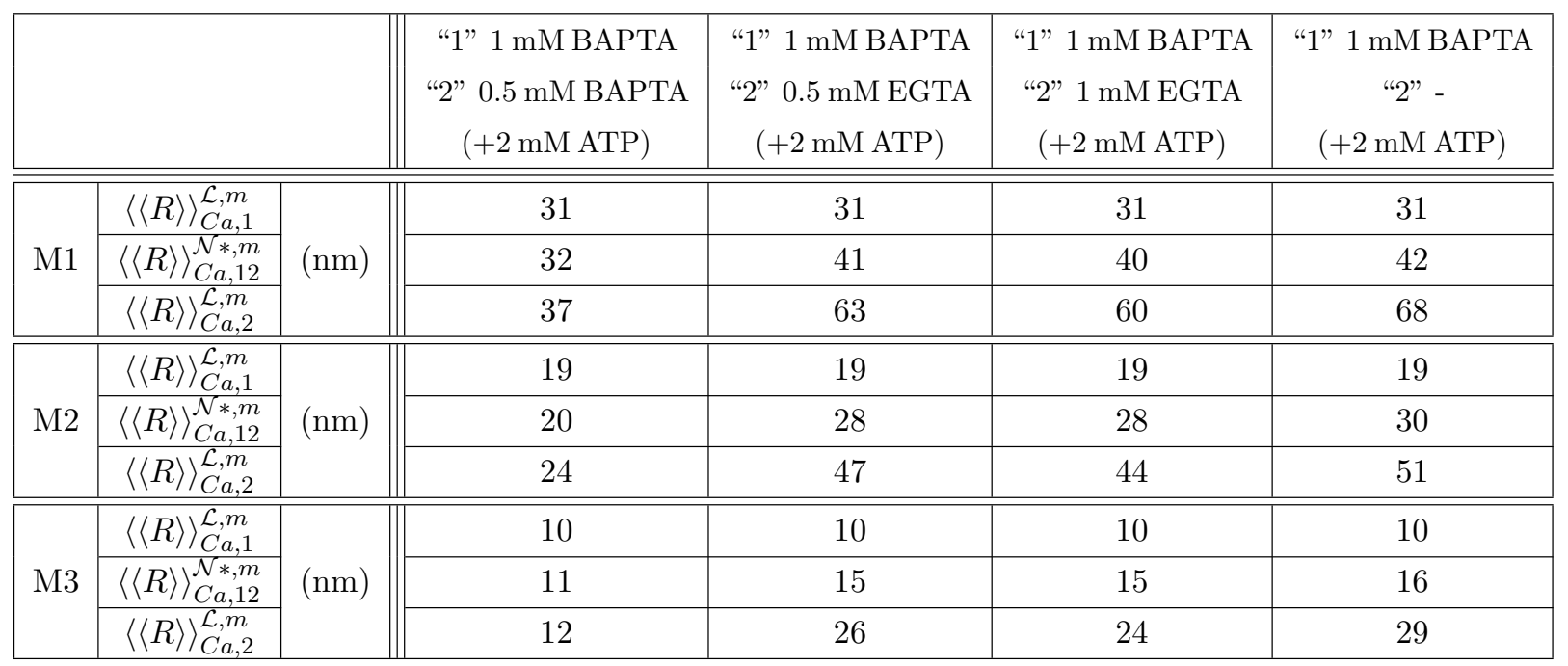

Table S3

Finally, we have to note that we used estimates of capacitance increments in four different pairs of $\mathrm{Ca}^{2+}$ buffering conditions collectively to estimate the $R_{c}$ (see Methods). This was done with the purpose to reduce the influence of measurement noise in the experimental data. Thus, in order to interpret the experimental estimate of $R_{c}$ in the context of spatially resolved active zone models, we have to estimate the same quantity for those modeled active zones. The corresponding model estimates of $R_{c}$ calculated in the mentioned way were $41 \mathrm{~nm}, 28 \mathrm{~nm}$, and $15 \mathrm{~nm}$ for scenarios M1, M2, and M3, respectively. Thus, our experimental estimate $R_{c}=17 \mathrm{~nm}$ with $5-95$ percentile range of $8-30 \mathrm{~nm}$ (see Results) for the single $\mathrm{Ca}^{2+}$ source model is compatible with the active zone topography scenarios M2 and M3 but not scenario M1.

\subsection{Influence of the $\mathrm{Ca}^{2+}$ channel opening-closing dynamics on $\mathrm{R}_{\mathrm{c}}$ estimate}

All considerations related to $R_{c}$ in sections 8.1-8.4 were based on the assumption that exocytosis rate at any vesicular release site is a well defined function of the time-averaged stationary $\mathrm{Ca}^{2+}$ concentration at that vesicular release site. In our experiments, cumulative release was measured $20 \mathrm{~ms}$ after the stimulus onset. $20 \mathrm{~ms}$ is much longer than the characteristic equilibration time of the channel opening, which is $0.5 \mathrm{~ms}$ at the membrane potential corresponding to the peak $\mathrm{Ca}^{2+}$ current in IHCs (18). Thus, the assumption of stationarity is justified. However, "averaging out" the fluctuations of $\mathrm{Ca}^{2+}$ concentration due to opening and closing of the presynaptic $\mathrm{Ca}^{2+}$ channels may still substantially influence the results, if channel gating is not sufficiently fast ${ }^{17}$. To check this averaging assumption we performed additional simulations where the build-up and decay of $\left[\mathrm{Ca}^{2+}\right]$ due to the channel gating was modeled explicitly as

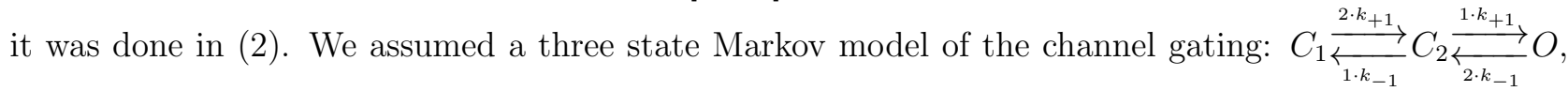
$k_{1}=1.78 \mathrm{~ms}^{-1}, k_{-1}=1.37 \mathrm{~ms}^{-1}$. Vesicle fusion followed the kinetic model proposed by (19). All the $m=1.7$. For scenario M1, the differences were not larger than $4 \mathrm{~nm}$, which is still small compared to the absolute values of $\langle\langle R\rangle\rangle_{\mathrm{Ca}, 12}^{\mathcal{N} *, m}$. Thus, at least for the considered active zone topographies, $m$ does not affect value of $R_{c}$ considerably.

${ }^{17}$ By speed of channel gating we mean the closing and opening rates of the channel. 
kinetic parameters of vesicle fusion were set to the original values except that $k_{\text {on }}$ was reduced four times in order to reproduce reasonable $m$ and $\Delta C_{m}$ values. Vesicle replenishment was treated as a single step process with a fixed rate $k_{r e p}=0.13 \mathrm{~ms}^{-1}$. Other relevant details of the simulation procedure are reported in (2).

Using the aforementioned model to calculate $\Delta_{r e l, \Sigma}$ and its dependence on $\left\langle\left[\mathrm{Ca}^{2+}\right]\right\rangle$, we estimated the coupling distance $R_{c}$ in the same way which was discussed in the last paragraph of section 8.4. The corresponding values of $R_{c}$ for scenarios M1, M2, and M3 were $37 \mathrm{~nm}, 27 \mathrm{~nm}$, and $14 \mathrm{~nm}$, respectively. This is in a very good agreement with the estimates of $R_{c}$ that were achieved by ignoring the fluctuations of $\left[\mathrm{Ca}^{2+}\right]$ due to the $\left[\mathrm{Ca}^{2+}\right]$ channels closing and opening. This result lets us conclude that, for active zone topographies and the kinetic parameters considered in this work, the channel gating noise does not considerably affect the estimation of $R_{c}$. 


\section{References}

1. P. Heil, H. Neubauer, D.R.F. Irvine, and M. Brown. Spontaneous activity of auditory-nerve fibers: insights into stochastic processes at ribbon synapses. J. Neurosci. 2007, 27: $8457-8474$.

2. A.B. Wong, M.A. Rutherford, M. Gabrielaitis, T. Pangršič, F. Göttfert, T. Frank, S. Michanski, S. Hell, F. Wolf, C. Wichmann, and T. Moser. Developmental refinement of hair cell synapses tightens the coupling of $\mathrm{Ca}^{2+}$ influx to exocytosis. $E M B O J$. 2014, 33: $247-264$.

3. M. Naraghi and E. Neher. Linearized buffered $\mathbf{C a}^{2+}$ diffusion in microdomains and its implications for calculation of $\left[\mathbf{C a}^{2+}\right]$ at the mouth of a calcium channel. J. Neurosci. 1997, 17: $6961-6973$.

4. U.V. Nägerl, D. Novo, I. Mody, and J.L. Vergara. Binding kinetics of calbindin-D28k determined by flash photolysis of caged $\mathbf{C a}^{2+}$. Biophys. J. 2000, 79: $3009-3018$.

5. J.H. Bollmann and B. Sakmann. Control of synaptic strength and timing by the release-site Ca ${ }^{2+}$ signal. Nat. Neurosci. 2005, 8: $426-434$

6. S.H. Lee, B. Schwaller, and E. Neher. Kinetics of $\mathbf{C a}^{2+}$ binding to parvalbumin in bovine chromaffin cells: implications for $\left[\mathbf{C a}^{2+}\right]$ transients of neuronal dendrites. J Physiol. 2000, 525: $419-432$.

7. G.C. Faas, S. Raghavachari, J.E. Lisman, and I. Mody. Calmodulin as a direct detector of Ca ${ }^{2+}$ signals. Nat. Neurosci. 2011, 14: $301-304$.

8. G.C. Faas, B. Schwaller, J.L. Vergara, and I. Mody. Resolving the fast kinetics of cooperative binding: Ca ${ }^{2+}$ buffering by calretinin. Plos Biol. 2007, 5: e311.

9. H. Schmidt, E.B. Brown, B. Schwaller, and J. Eilers. Diffusional mobility of parvalbumin in spiny dendrites of cerebellar Purkinje beurons quantified by fluorescence recovery after photobleaching. Biophys. J. 2003, 84: 2599 - 2608.

10. H. Schmidt, B. Schwaller, and J. Eilers. Calbindin D28k targets myo-inositol monophosphatase in spines and dendrites of cerebellar Purkinje neurons PNAS 2005, 102: 5850 - 5855.

11. D. Beutner, T. Moser. The presynaptic function of mouse cochlear inner hair cells during development of hearing. J. Neurosci. 2001, 21: $4593-4599$.

12. A.C. Meyer, T. Frank, D. Khimich, G. Hoch, D. Riedel, N.M. Chapochnikov, Y.M. Yarin, B. Harke, S.W. Hell, A. Egner, and T. Moser. Tuning of synapse number, structure and function in the cochlea. Nat. Neurosci. 2009, 12: $444-453$.

13. D. Lenzi, J.W. Runyeon, J. Crum, M.H. Ellisman, and W.M. Roberts. Synaptic vesicle populations in saccular hair cells reconstructed by electron tomography. J. Neurosci. 1999, 19: $119-132$.

14. E. Neher. Usefulness and limitations of linear approximations to the understanding of Ca ${ }^{2+}$ signals. Cell Calcium 1998 , 24: $345-357$.

15. J.D. Goutman and E. Glowatzki. Time course and calcium dependence of transmitter release at a single ribbon synapse. PNAS 2007, 104: $16341-16346$.

16. I. Bucurenciu, A. Kulik, B. Schwaller, M. Frotscher, and P. Jonas. Nanodomain coupling between $\mathbf{C a}^{2+}$ channels and Ca $^{2+}$ sensors promotes fast and efficient transmitter release at a cortical GABAergic synapse. Neuron 2008, 57: $536-545$.

17. N.P. Vyleta and P. Jonas. Loose coupling btween $\mathbf{C a}^{2+}$ channels and release sensors at a plastic hippocampal synapse. Science 2014, 343: $665-670$.

18. J. Neef, A. Gehrt, A.V. Bulankina, A.C. Meyer, D. Riedel, R.G. Gregg, N. Strenzke, and T. Moser. The Ca ${ }^{2+}$ channel subunit $\boldsymbol{\beta 2}$ regulates $\mathbf{C a}^{2+}$ channel abundance and function in inner hair cells and is required for hearing. J. Neurosci. 2009, 29: $10730-10740$.

19. D. Beutner, T. Voets, E. Neher, and T. Moser. Calcium dependence of exocytosis and endocytosis at the cochlear inner hair cell afferent synapse. Neuron 2001, 29: $681-690$. 
Chapter 4

Presynaptic Mechanisms of

Heterogeneous Information Transfer to the Auditory Nerve 


\section{List of Abbreviations}

$\bar{p}_{o} \quad$ maximum open probability of presynaptic $\mathrm{Ca}^{2+}$ channels

$\Delta_{S P L}\left[R_{s}\right]$ dynamic range of a rate-level function

$\gamma \quad$ irreversible vesicle fusion rate

$\langle t\rangle_{I E I}$ average inter-event time interval

$\langle t\rangle_{I S I}$ average inter-spike time interval

$\max _{S P L}\left[R_{s}\right]$ maximum spike rate of a rate-level function

$\min _{S P L}^{*}\left[R_{s}\right]$ normalized minimum spike rate of a rate-level function

$\min _{S P L}\left[R_{s}\right]$ minimum spike rate of a rate-level function

$\rho_{I E I}(t)$ probability density function of inter-event time intervals

$\rho_{I S I}(t)$ probability density function of inter-spike time intervals

$\rho_{\text {reff }}(t)$ probability density function of the refractory times

$\tau_{R} \quad$ average relative refractory time of spiral ganglion neurons

$\Theta_{S P L}\left[R_{s}\right]$ spiking threshold of a rate-level function

$\tilde{k}_{\text {rep }} \quad$ amplitude of the $V_{m}$ dependence of vesicle replenishment rate

$\tilde{V}_{0.5} \quad$ half-activation voltage of the $V_{m}$ dependent rate of vesicle replenishment

$b \quad$ cooperativity factor of calcium unbinding rate from the sensor of exocytosis

$C_{I E I}$ serial correlation function of inter-event time intervals

$C_{I S I}$ serial correlation function of inter-spike time intervals

$C V_{I E I}$ coefficient of variation of inter-event time intervals

$C V_{I S I}$ coefficient of variation of inter-spike time intervals 
$k_{V} \quad$ voltage sensitivity of the open probability of presynaptic $\mathrm{Ca}^{2+}$ channels

$k_{V}^{\prime} \quad$ voltage sensitivity of the dependence of postsynaptic spiking rate on the presynaptic membrane potential

$k_{+1} \quad$ opening rate of presynaptic $\mathrm{Ca}^{2+}$ channels

$k_{-1} \quad$ closing rate of presynaptic $\mathrm{Ca}^{2+}$ channels

$k_{\text {off }} \quad$ rate of calcium unbinding from the sensor of exocytosis

$k_{o n} \quad$ rate of calcium binding to the sensor of exocytosis

$k_{r e l}$ rate of calcium binding to the sensor of exocytosis multiplied by the concentration of calcium at the sensor

$k_{r e p} \quad$ rate of vesicle replenishment

$m$ number of $\mathrm{Ca}^{2+}$-binding sites of the $\mathrm{Ca}^{2+}$ sensor of exocytosis

$N_{C} \quad$ number of $\mathrm{Ca}^{2+}$ channels per presynaptic vesicular release site

$n_{C} \quad$ number of states of a presynaptic $\mathrm{Ca}^{2+}$ channel

$N_{V} \quad$ number of presynaptic vesicular release sites

$n_{V} \quad$ number of states of a presynaptic vesicular release site

$P \quad$ sound pressure (amplitude)

$p_{o} \quad$ open probability of presynaptic $\mathrm{Ca}^{2+}$ channels

$r \quad$ coefficient of correlation

$R_{r} \quad$ presynaptic vesicle release rate

$R_{s} \quad$ postsynaptic (SGN) spike rate

$S_{r} \quad$ shift of the $R_{r}$ vs $V_{m}$ relation with respect to the $p_{o}$ vs $V_{m}$ dependence along the $V_{m}$ axis

$S_{s} \quad$ shift of the $R_{s}$ vs $V_{m}$ relation with respect to the $R_{r}$ vs $V_{m}$ dependence along the $V_{m}$ axis

$S P L$ sound pressure level

$t_{A} \quad$ absolute refractory time of spiral ganglion neurons

$V_{m} \quad$ presynaptic membrane potential

$V_{0.5}$ half-activation voltage of the open probability of presynaptic $\mathrm{Ca}^{2+}$ channels 
$V_{d c} \quad$ DC component of the presynaptic receptor potential of inner hair cells

$V_{\text {rest }} \quad$ presynaptic resting membrane potential

$\left[\mathrm{Ca}^{2+}\right]$ concentration of presynaptic $\mathrm{Ca}^{2+}$

AZ presynaptic active zone

$\mathrm{CF}$ characteristic frequency

IEI inter-event time interval of presynaptic vesicle release in a steady state

IHC inner hair cell

ISI inter-event time interval of postsynaptic spikes in a steady state

RLF rate-level function

SGN spiral ganglion neuron

SR spontaneous spike rate 


\subsection{Introduction}

Inner hair cell ribbon synapses are the first synapses in the mammalian auditory pathway. They are formed between inner hair cells (IHCs), which transduce the mechanical stimulus into graded voltage changes across their plasma membranes, and spiral ganglion neurons (SGNs), that project to the CNS. Thus, the quality of sound encoding at IHC ribbon synapses sets an upper bound on the information about auditory stimuli entering the CNS. A remarkable feature of the mammalian auditory system is its ability to accurately encode sound levels over a range of 6 orders of magnitude (Riesz, 1928; Viemeister, 1988). The presence of such performance was associated with the apparent pronounced heterogeneity of responses of SGNs to sound stimuli (Viemeister, 1983; Delgutte, 1987; Winslow \& Sachs, 1988; Viemeister, 1988; Yates et al., 1990; Winter \& Palmer, 1991; Colburn et al., 2003). Indeed, the range of sound levels in which each SGN responds with varying spiking is rather limited. However, SGNs emanating from the same cochlear tonotopic position have different spiking thresholds as well as different maximum and spontaneous spiking rates (SRs), adaptation and recovery times, and different spike statistics (Sachs \& Abbas, 1974; Liberman, 1978; Borg et al., 1988; Relkin \& Doucet, 1991; Müller \& Robertson, 1991; Winter et al., 1990; Ohlemiller et al., 1991; Taberner et al., 2005; Prijs et al., 1993; Li \& Young 1993). Fifty years after the first reports (Katsuki et al., 1962; Kiang et al., 1965), biophysical mechanisms of such a high response variability between different SGNs are still not well understood.

The nonlinearity of the basilar membrane response as a key mechanism of widening the dynamic ranges of high characteristic frequency (CF) SGNs with higher thresholds was suggested (Sachs \& Abbas, 1974; Sachs et al., 1989; Yates et al., 1990; Müller \& Robertson, 1991; Robertson \& Winslow, 1991; Cooper \& Yates, 1994). One of the candidate mechanisms contributing to all the remaining important aspects of the apparent response heterogeneity could be the differences in the intrinsic properties of SGNs. However, no direct evidence to support this idea has been reported to date. Though, it was shown that cultured SGNs have a certain level of variability in their resting membrane potentials and spike thresholds (Davis \& Liu, 2011; Liu et al., 2014). Also, it was noticed that the dendrites of high-SR SGNs are thicker, richer in mitochondria (Liberman, 1980; Liberman, 1982), and have larger postsynaptic AMPA receptor clusters than low-SR SGNs (Liberman et al., 2011). Nevertheless, these features could well be a consequence of an upstream presynaptic heterogeneity (Liberman, 1980; Liberman, 1982). Low-SR SGNs were found to form $2-3$ times more synaptic contacts with efferent axons of lateral olivocochlear cells than high-SR SGNs (Liberman, 1990a). Disruption of the whole olivocochlear innervation of the cochlea, however, demonstrated that this system is not necessary for the response heterogeneity of SGNs (Liberman, 1990b).

More evidence exists for the presynaptic mechanisms of the heterogeneity of SGN responses to sound stimuli. First, it was found that the same IHC is contacted by populations of SGNs with thick (high-SR) and thin (low-SR) dendrites (Liberman, 1980; Liberman, 1982). Presy- 
naptic sites corresponding to the high-SR SGNs were shown to have smaller active zones (AZ) with fewer vesicles than the low-SR SGNs (Merchan-Perez \& Liberman, 1996; Kantardzhieva et al., 2013). The presynaptic ribbons of the synapses corresponding to low- and high-SR SGNs were shown to have different morphologies (Liberman et al., 1990; Liberman et al., 2011; Kantardzhieva et al., 2013). Second, a considerable increase in SR was induced by Bay K8644, a presynaptic $\mathrm{Ca}^{2+}$ channel agonist (Robertson \& Paki, 2002; Sueta et al., 2004). In accordance, a considerable decrease in SR and considerable increase in the thresholds of single SGN spiking and of the compound action potential were induced by the presynaptic $\mathrm{Ca}^{2+}$ channel antagonist nimodipine (Zhang et al., 1999; Robertson \& Paki, 2002; Sueta et al., 2004). Third, it was found that the maximum intensities of $\mathrm{Ca}^{2+}$ fluorescence signals at different synapses of the same IHC in response to depolarizing stimuli in vitro, on average, differed by four times between the strongest and the weakest synapse, and that the activation curves of the $\mathrm{Ca}^{2+}$ signal may be shifted along the voltage axis by as much as $15 \mathrm{mV}$ (Frank et al., 2009; Ohn et al., in preparation). These findings are intriguing but do not yet demonstrate to what extent heterogeneity of the presynaptic function accounts for the overall heterogeneity of SGN responses in vivo.

In this work, we extended the understanding of the contribution of the presynaptic heterogeneity of IHC ribbon synapses to sound encoding by mathematical modeling. To this end, we constructed a biophysical model of a high CF IHC ribbon synapse, which includes the key presynaptic mechanisms determining the vesicle fusion dynamics at the presynaptic AZs. We demonstrated that this model, while characterized by multiple parameters, is largely analytically tractable. The analytical tractability allowed us to thoroughly dissect the relation between the structure of the presynaptic AZ and characteristics of the adapted response of the synapse. We then applied the model to describe the in vivo data of SGN responses. All currently documented key aspects of the apparent heterogeneity of the responses of high CF SGNs were reproduced by the model in a detailed and quantitative manner. Moreover, the data analysis provided compelling evidence that the origin of the response heterogeneity is essentially on the presynaptic, not the postsynaptic, side. These findings unraveled certain principles of AZ organization underlying the experimentally observed response heterogeneity. However, we found that different sets of presynaptic parameters were able to reproduce the same aspects of the SGN responses. The results of this work set directions for further experiments to find which of the potential molecular mechanisms of presynaptic heterogeneity prevail.

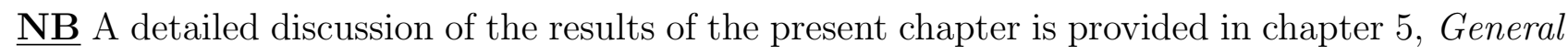
Discussion.

\subsection{Formulation of the model}

The model used in this work encompasses processes from sound application at the outer ear to spike generation by SGNs. For an intuitive understanding, it is convenient to divide the 
model into three stages (see Fig. 4.1A): (1) conversion of sound pressure to depolarization of the IHC, (2) conversion of the depolarization of the IHC to $\mathrm{Ca}^{2+}$ influx through presynaptic $\mathrm{Ca}^{2+}$ channels and the following vesicle release at the presynaptic active zone, (3) conversion of the presynaptic release events to spike generation by postsynaptic SGNs. Stage (2), i.e., the processes taking place at the presynaptic site, was in the focus. Our primary goal in this work was to understand the contribution of the heterogeneity of the presynaptic active zone organization to the apparent heterogeneity of SGN responses to sound stimuli. Therefore, we modeled conditions in which cochlear micromechanics and transduction add the least complexity to sound encoding. First, we considered only responses to high frequency pure tone bursts, such that the AC component of the IHC receptor potential would be negligible compared to the DC component $\left(V_{d c}\right)$. This is due to the low pass filter characteristics of the IHC, and works well for sound frequencies above $2-4 \mathrm{kHz}$ (Johnson, 1980; Palmer \& Russell, 1986). Second, we studied IHC and SGN responses to sound stimuli that are at least 0.5 octaves below the CF. In this regime, the amplitude of the basilar membrane oscillations responds linearly to sound pressure amplitude applied at the ear (Robles \& Ruggero, 2001). Third, we considered only stationary, i.e., adapted, responses of IHCs and SGNs which have been explored experimentally the best. In particular, we considered distributions and serial correlation functions of the inter-spike intervals (ISIs) and inter release event intervals (IEIs) as well as spike and vesicle release rate dependencies on the stimulus intensity (Fig. 4.1B).

\subsubsection{Generation of the IHC receptor potential}

To describe how deflections of IHC hair bundles induced by an applied sound are converted to the receptor potential, we used a biophysical, Hodgkin-Huxley type model proposed by (LopezPoveda \& Eustaquio-Martin, 2006). In this model, depolarization of IHC is shaped by the mechanotransducer channels as well as two voltage dependent basolateral $\mathrm{K}^{+}$currents. All relevant equations and parameters describing the dynamics of IHC membrane potential $\left(V_{m}\right)$ are given in (Lopez-Poveda \& Eustaquio-Martin, 2006). We were interested only in steady state responses to sounds in the limit of high frequencies, when only the DC component of the receptor potential is preserved. Thus, only the steady state solutions of the dynamic equations in response to a certain hair bundle deflection had to be calculated (see Methods). The gain factor $(\kappa)$ between the amplitudes of the sound pressure $(P)$ applied at animal's ear and the deflection of the hair bundle was not known in our case. Thus we treated it as a free parameter.

Despite that the model by (Lopez-Poveda \& Eustaquio-Martin, 2006) can reproduce the $V_{d c}$ vs $P$ relation measured in vivo fairly well, one discrepancy between the two is obvious. $V_{d c}$ increases quadratically with $P$ at small intensities in the model but linearly in the experiment (see Patuzzi \& Sellick, 1983; Fig. 10 in Lopez-Poveda \& Eustaquio-Martin, 2006). Accordingly, an increase of $V_{d c}$ at large $P$ is more compressive in the model than the experiment. This discrepancy can be taken into account by a transformation $P \rightarrow P^{\alpha}$ in the model, with $\alpha \sim 0.5$. This is what we further assumed, with a possibility to vary $\alpha$ slightly around 0.5 when fitting 
A

Stage 1 Conversion of sound pressure

to graded depolarization of the IHC

MU $\quad \begin{aligned} & \text { Hair bundle with } \\ & \text { mechanotransduce } \\ & \text { channels }\end{aligned}$

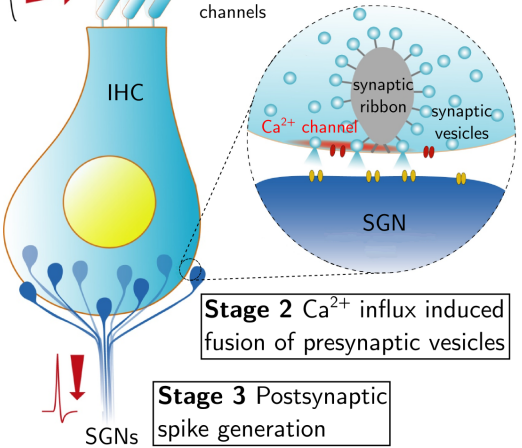

C

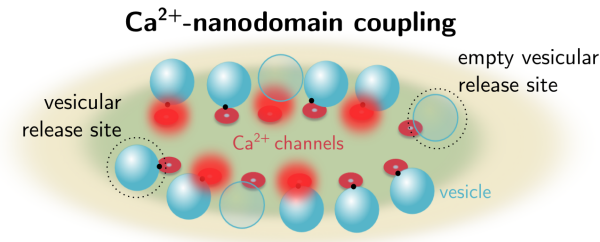

Intermediate $\mathrm{Ca}^{2+}$-domain coupling

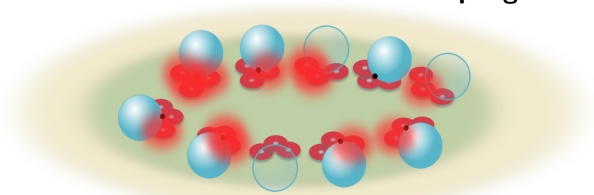

B
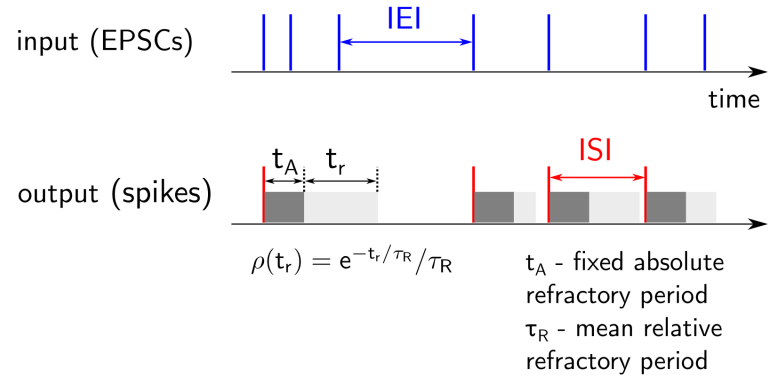

$\mathrm{Ca}^{2+}$-microdomain coupling

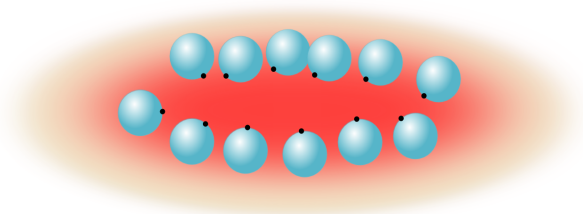

D

$$
\begin{aligned}
& \mathrm{C} \underset{\mathrm{k}_{-1}\left(\mathrm{v}_{\mathrm{m}}\right)}{\stackrel{\mathrm{k}_{+1}\left(\mathrm{v}_{\mathrm{m}}\right)}{\rightleftarrows}} \mathrm{O} \\
& \mathrm{C}_{1} \underset{\mathrm{k}_{1}\left(\mathrm{~V}_{\mathrm{m}}\right)}{\stackrel{\mathrm{k}_{\mathrm{t}}\left(\mathrm{V}_{\mathrm{m}}\right)}{\rightleftarrows}} \stackrel{\odot}{\mathrm{C}_{2}} \cdots \mathrm{C}_{\mathrm{n}} \underset{\mathrm{k}_{\mathrm{n}}\left(\mathrm{V}_{\mathrm{m}}\right)}{\stackrel{\mathrm{k}_{\mathrm{n}}\left(\mathrm{V}_{\mathrm{m}}\right)}{\rightleftarrows}} \mathrm{O}
\end{aligned}
$$

Shared $\mathrm{Ca}^{2+}$-domain coupling

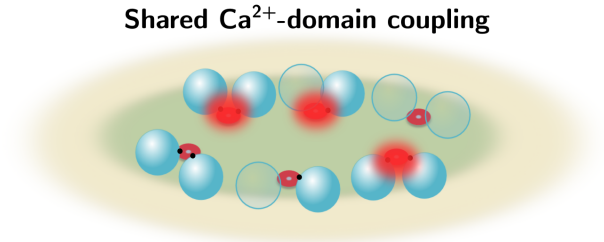

$E$

Figure 4.1: The key components of the model considered in this work. (A) Three main stages of the signal transmission in the IHC-SGN system. (B) The refractory counter model of SGNs. (C) Four characteristic scenarios of the AZ topography. Blue balls: synaptic vesicles of the readily releasable pool, red disks: presynaptic $\mathrm{Ca}^{2+}$ channels, diffused red: clouds of $\mathrm{Ca}^{2+}$ ions, khaki area: presynaptic active zone. (D) Kinetic schemes of presynaptic $\mathrm{Ca}^{2+}$ channel gating. (E) Kinetic schemes of the vesicular release site states underlying the vesicle exocytosis-replenishment processes. 
the data. An example of the $V_{d c}$ vs $P$ relation based on this model is shown in Fig. 4.9C. Note the logarithmic transformation of $P$ into sound pressure level $(S P L)$ there. The $V_{d c}$ vs $P$ relation was relevant in our work only for modeling the spike rate dependencies on $S P L$, the so-called Rate-level functions (RLFs), but not for other aspects of SGN responses.

\subsubsection{Organization of the presynaptic site}

\section{Topography of the presynaptic active zone}

In the most general version of our model, the presynaptic $\mathrm{AZ}$ consisted of $N_{C}$ independent $\mathrm{Ca}^{2+}$ channels and $N_{V}$ independent vesicular release sites of the readily releasable pool (RRP), all positioned arbitrarily. We, however, concentrated on the analysis of several characteristic scenarios of the AZ topography. Data from electron and STED immunofluorescence microscopy jointly showed that presynaptic $\mathrm{Ca}^{2+}$ channels are concentrated within the plasma membrane underneath the presynaptic density at mature IHC AZs (Wong et al., 2014, i.e., the original article of chapter 2 in the present dissertation). A typical presynaptic density area was approximated by a $80 \mathrm{~nm} \times 400 \mathrm{~nm}$ stripe. Our previous experimental and modeling work suggested a particular $\mathrm{Ca}^{2+}$ channel distribution within the presynaptic density. Importantly, the average effective coupling distance $R_{c}$ between the $\mathrm{Ca}^{2+}$ channels and $\mathrm{Ca}^{2+}$ sensors of exocytosis was estimated to be $\sim 20 \mathrm{~nm}$ (Pangršič et al., 2015, i.e., the original article of chapter 3 in the present dissertation). The number of the effective channels contributing to $\left[\mathrm{Ca}^{2+}\right]$ at the vesicle fusion sensors was $\sim 2$ per each vesicle on average (Wong et al., 2014). These findings are compatible with the so-called $\mathrm{Ca}^{2+}$-nanodomain coupling regime of $\mathrm{Ca}^{2+}$ influx to vesicle fusion (Eggermann et al., 2012; Tarr et all., 2013). It is important to note, however, that the numbers mentioned above represent an average synapse, with no information about the heterogeneity across different synapses. Thus, in this work, we also considered the opposite, so-called, $\mathrm{Ca}^{2+}$-microdomain coupling regime between the channels and vesicular release sites. The defining property of this scenario is a large number of $\mathrm{Ca}^{2+}$-channels contributing $\mathrm{Ca}^{2+}$ at any vesicular release site (Eggermann et al., 2012).

To represent the $\mathrm{Ca}^{2+}$-nanodomain coupling regime, we assumed an idealized limiting case when each vesicle release site is associated with one particular $\mathrm{Ca}^{2+}$ channel, which contributes to $\left[\mathrm{Ca}^{2+}\right]$ only at that "coupled" vesicle (Fig. 4.1C). To represent the $\mathrm{Ca}^{2+}$-microdomain coupling scenario, an idealized limiting case when $\left[\mathrm{Ca}^{2+}\right]$ at each particular release site is due to population averaged contribution of $\mathrm{Ca}^{2+}$ channels at the whole presynaptic site was assumed (Fig. 4.1C). Indeed, when the effective number of contributing channels to $\left[\mathrm{Ca}^{2+}\right]$ at a particular location is large, the fluctuations of $\left[\mathrm{Ca}^{2+}\right]$ due to the stochastic opening and closing of the channels are averaged out. In addition to the idealized $\mathrm{Ca}^{2+}$-nanodomain and $\mathrm{Ca}^{2+}$ microdomain coupling regimes, we also studied two more scenarios (but only when analyzing experimental ISI distributions). For one of them, it was assumed that each vesicular release site is associated to a finite number of private $\mathrm{Ca}^{2+}$ channels (Fig. 4.1C). We called it as interme- 
diate $\mathrm{Ca}^{2+}$-domain coupling scenario, because it represents an intermediate case between the limiting scenarios of $\mathrm{Ca}^{2+}$-nanodomain $\left(N_{C}=1\right)$ and $\mathrm{Ca}^{2+}$-microdomain $\left(N_{C}=\infty\right)$ coupling. In another additional scenario, one or a few channels were coupled to several vesicles at the same time (Fig. 4.1C), thus we named it as shared $\mathrm{Ca}^{2+}$-domain coupling scenario.

\section{Dynamics of presynaptic $\mathrm{Ca}^{2+}$ channel gating}

The stochastic process of opening and closing of presynaptic voltage-gated $\mathrm{Ca}^{2+}$ channels was described by a continuous time Markov chain in our model. In the basic version of the model, we considered a two state scheme of the channel gating (Fig. 4.1D), which is characterized by one opening $\left(k_{+1}\right)$ and one closing $\left(k_{-1}\right)$ rates. In agreement to experimental findings (see, e.g., Neef et al., 2009; Zampini et al., 2013), $V_{m}$ dependence of the channel open probability $\left(p_{o}\right)$ was described by a Boltzmann function,

$$
p_{o}\left(V_{m}\right)=\frac{\bar{p}_{o}}{1+e^{-\left(V_{m}-V_{0.5}\right) / k_{V}}} .
$$

Here, $\bar{p}_{o}$ is the maximal open probability, $V_{0.5}$ is the half activation voltage, and $k_{V}$ is the voltage sensitivity. In vitro estimates of $V_{0.5}$ and $k_{V}$ averaged over many IHC synapses are respectively $\sim-30 \mathrm{mV}$ and $\sim 7 \mathrm{mV}$ (see, e.g., Johnson et al., 2005; Neef et al., 2009; Frank et al., 2010). The value of $\bar{p}_{o}$ is more debatable. Whole cell recordings indicated $\bar{p}_{o} \sim 0.4$, while single channel recordings pointed to $\bar{p}_{o} \sim 0.2$ (Zampini, et al., 2013) or even $\bar{p}_{o}<0.1$ (Zampini, et al., 2014). It follows from the whole cell and single channel recordings that a variation of $V_{m}$ in the physiological range mainly affects the channel opening, not the closing rates (see Supplementary Material 1.1). Thus, we assumed $k_{-1}\left(V_{m}\right) \equiv$ const. This, together with Eq. (4.1), constrained the voltage dependence of $k_{+1}$ :

$$
k_{+1}\left(V_{m}\right)=\frac{k_{-1} \cdot \bar{p}_{o}}{1-\bar{p}_{o}+e^{-\left(V_{m}-V_{0.5}\right) / k_{V}}} .
$$

$k_{-1}, V_{0.5}, k_{V}$, and $\bar{p}_{o}$ were considered as free parameters in the model.

To better understand the influence of the details of the channel gating dynamics on the presynaptic vesicle release, we also explored kinetic schemes of channel gating with several intermediate closing states (Fig.4.1D). Currently available single channel recordings are too cursory to properly constrain the kinetic model of $\mathrm{Ca}^{2+}$ channel gating at IHC synapses. However, the dwell time statistics indicate the presence of channels in two different modes (see Zampini, et al., 2011, 2013, 2014). At least one of those modes is apparently described by three, not two gating states (two intermediate closed states followed by one open state). Inactivation of presynaptic $\mathrm{Ca}^{2+}$ influx in IHCs is weak (see, e.g., Johnson et al., 2005, Cui, et al., 2007, Neef et al., 2009) and was not taken into account in our work.

\section{Dynamics of presynaptic $\left[\mathrm{Ca}^{2+}\right]$}

The dynamics of the $\left[\mathrm{Ca}^{2+}\right]$ at presynaptic AZs (denoted by $\left[\mathrm{Ca}^{2+}\right]$ for later use) which arises from influx of $\mathrm{Ca}^{2+}$ through the presynaptic $\mathrm{Ca}^{2+}$ channels is determined by various factors. 
Among them is the geometry of the intracellular space, the kinetics of presynaptic $\mathrm{Ca}^{2+}$ channel gating, as well as the diffusion and binding reactions of $\mathrm{Ca}^{2+}$ to intracellular $\mathrm{Ca}^{2+}$ buffers. However, we found that a few important approximations can be applied to describe the dynamics of $\left[\mathrm{Ca}^{2+}\right]$ in the case of IHC ribbon synapses.

First, we noted that the build-up and collapse of $\left[\mathrm{Ca}^{2+}\right]$ domains within the active zone tightly follows opening and closing of the presynaptic $\mathrm{Ca}^{2+}$ channels. Fig. 4.2A shows examples of $\left[\mathrm{Ca}^{2+}\right]$ dynamics due to $\mathrm{Ca}^{2+}$ influx through a single channel, $20 \mathrm{~nm}$ and $100 \mathrm{~nm}$ from its mouth (see Methods for the details of the model). In the presence of $0.5 \mathrm{mM} \mathrm{BAPTA}+2 \mathrm{mM}$ MgATP, a conservative estimate of the intrinsic $\mathrm{Ca}^{2+}$ buffering strength of IHC (Pangršič, et al., 2015, i.e., the original article of chapter 3 in the present dissertation), the correspondence between the normalized increment of $\left[\mathrm{Ca}^{2+}\right]$ (blue line) and the channel state (grey line) is nearly ideal. Quantitatively, the equilibration times of the $\left[\mathrm{Ca}^{2+}\right]$ increment are much shorter than the opening or closing times of the channel. If the distance from the channel is further increased, the dynamics of $\left[\mathrm{Ca}^{2+}\right]$ increasingly deviates from that of the $\mathrm{Ca}^{2+}$ channel gating. Indeed, the equilibration time of $\left[\mathrm{Ca}^{2+}\right]$ increases with the distance from the channel. This is illustrated in Fig. 4.2B where times necessary to reach $95 \%$ of the steady state level a plotted as functions of the distance from the channel for various $\mathrm{Ca}^{2+}$ buffering conditions. However, the contribution of the channel to $\left[\mathrm{Ca}^{2+}\right]$ decreases exponentially fast with the distance in the presence of $\mathrm{Ca}^{2+}$ buffers (Naraghi \& Neher 1997) and becomes irrelevant at large distances. For example, the steady state level of $\left[\mathrm{Ca}^{2+}\right]$ during continued $\mathrm{Ca}^{2+}$ influx decreases from $25.1 \mu \mathrm{M}$ to $0.6 \mu \mathrm{M}$ when the distance is increased from $20 \mathrm{~nm}$ to $100 \mathrm{~nm}$ in the presence of $0.5 \mathrm{mM}$ BAPTA $+2 \mathrm{mM}$ MgATP.

To show how well the mentioned argument applies to $\left[\mathrm{Ca}^{2+}\right]$ at realistic AZs, we tested the dynamics of the presynaptic $\left[\mathrm{Ca}^{2+}\right]$ at the sensors of exocytosis for two opposing IHC AZ scenarios considered in (Wong et al., 2014). In the so-called scenario M1, the presynaptic $\mathrm{Ca}^{2+}$ channels were distributed randomly in the area of the presynaptic density. In the socalled scenario M3, the presynaptic $\mathrm{Ca}^{2+}$ channels were placed in the physical contact with the RRP vesicles (one channel per vesicle). Further details of the model are given in Methods. The upper sub-panels in Fig. 4.2C, D show the time-averaged $\left[\mathrm{Ca}^{2+}\right]$ profiles at the level of the plasma membrane at $V_{m}=-20 \mathrm{mV}$ in a steady state for particular realizations of scenarios M1 and M3. We considered a random snapshot of the states of the presynaptic $\mathrm{Ca}^{2+}$ channel population in a steady state and estimated the time required for $\left[\mathrm{Ca}^{2+}\right]$ at the sensors of exocytosis to reach $95 \%$ of the steady state level after stimulus onset. Depolarizations to $-40 \mathrm{mV},-30 \mathrm{mV}$, and $-20 \mathrm{mV}$ from an initial state with all $\mathrm{Ca}^{2+}$ channels closed were considered. This time, $t_{0.95}$, was then averaged over all 14 vesicular release sites within the AZ, 50 different realizations of the channel population states, and 10 different realizations of the AZ scenarios. Fig. 4.2E illustrates how $t_{0.95}$ (mean \pm s.d.) depends on $V_{m}$. Note that $V_{m}=-20 \mathrm{mV}$ elicits the near maximal $\mathrm{Ca}^{2+}$ influx level (Wong et al., 2014). Consistent with our above considerations of the single channel system, $\left[\mathrm{Ca}^{2+}\right]$ equilibration was fast $\left(t_{0.95}\right.$ only tens of $\mu$ s even for scenario 
A
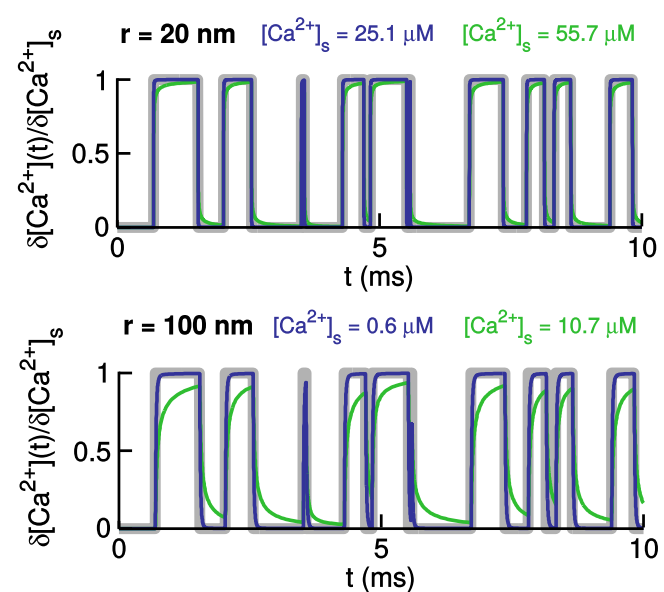

C
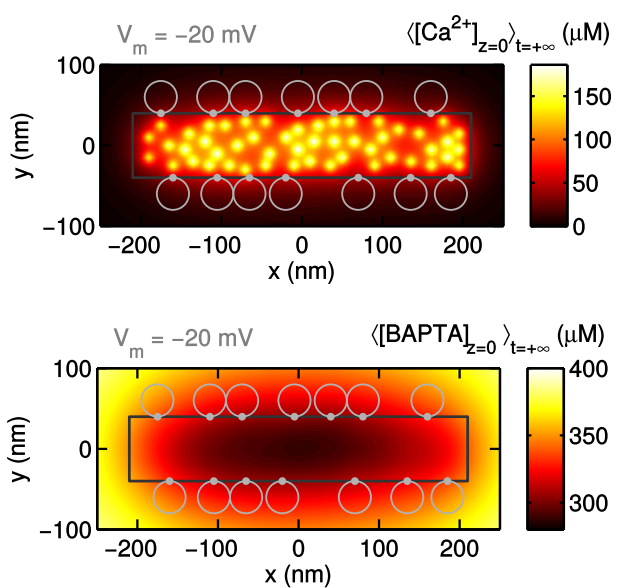

$\mathbf{E}$

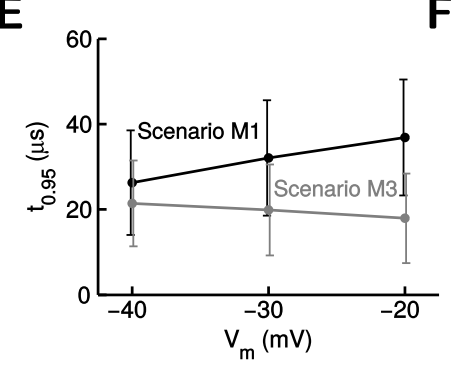

$\mathbf{F}$

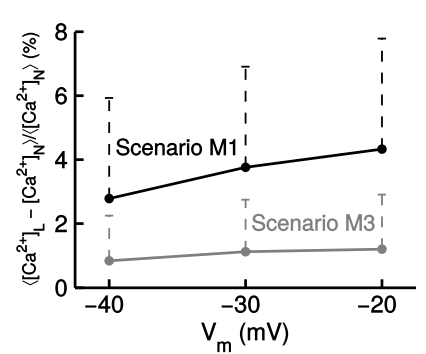

B

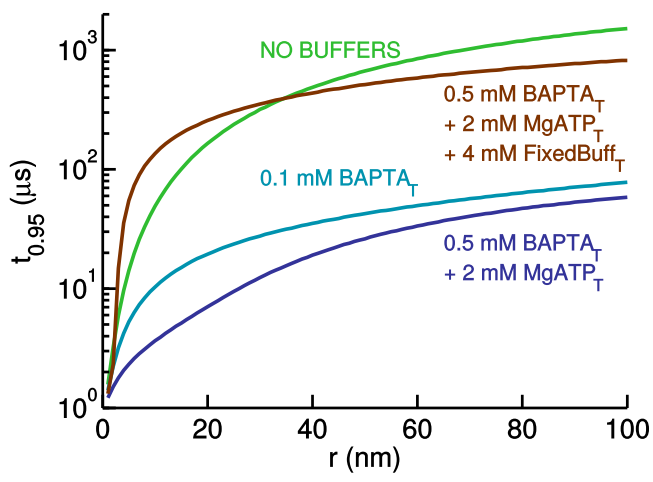

D
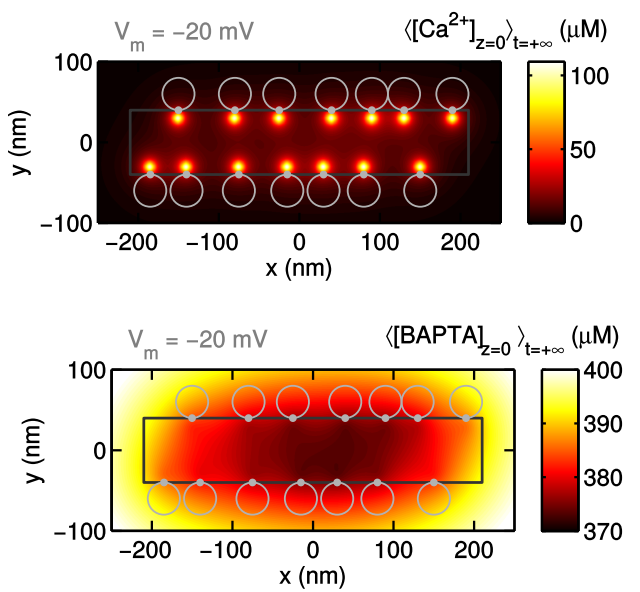

G

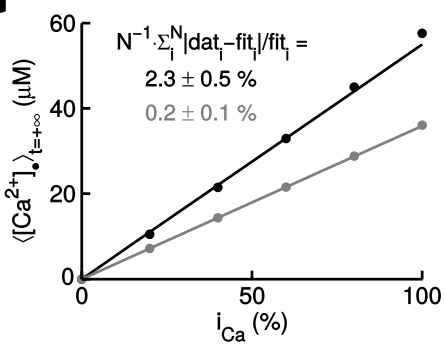

Figure 4.2: Properties of $\left[\mathrm{Ca}^{2+}\right]$ dynamics at IHC active zones. (A) Exemplary trajectories of $\left[\mathrm{Ca}^{2+}\right]$ evolution across time due to opening and closing of a single channel in the presence of $0.5 \mathrm{mM}$ BAPTA $+2 \mathrm{mM} \mathrm{MgATP}$ (blue) and in the absence of $\left[\mathrm{Ca}^{2+}\right]$ buffers (green). The upper and the lower subpanels show $\left[\mathrm{Ca}^{2+}\right] 20 \mathrm{~nm}$ and $100 \mathrm{~nm}$ away from the channel, respectively. (B) The time it takes to reach $95 \%$ of the steady state level of $\left[\mathrm{Ca}^{2+}\right]$ after opening of a $\mathrm{Ca}^{2+}$ channel at different distances from the channel and in different $\mathrm{Ca}^{2+}$ buffering conditions. (C) Time-averaged steady state $\left[\mathrm{Ca}^{2+}\right]$ (upper panel) and free [BAPTA] (lower panel) at the level of the plasma membrane at $V_{m}=-20 \mathrm{mV}$ for AZ scenario M1. (D) The same as (C) but for AZ scenario M3. (E) Time it takes to reach $95 \%$ of the steady state level of $\left[\mathrm{Ca}^{2+}\right]$ at the $\mathrm{Ca}^{2+}$ sensors of exocytosis for AZ scenarios M1 and M3 at different $V_{m}$. (F) Relative deviations between $\left[\mathrm{Ca}^{2+}\right]$ at the $\mathrm{Ca}^{2+}$ sensors of exocytosis obtained by solving generic and linearized reaction diffusion equations. (G) Linear fits of the dependencies of time-averaged steady state $\left[\mathrm{Ca}^{2+}\right]$ at the $\mathrm{Ca}^{2+}$ sensors of exocytosis on the single channel current $(100 \% \rightarrow 0.3 \mathrm{pA})$ at $V_{m}=-20 \mathrm{mV}$ for $\mathrm{AZ}$ scenarios M1 and M3. 
M1). As follows from Fig. 4.2A, B such short times allow to treat the build-up and collapse of increments of $\left[\mathrm{Ca}^{2+}\right]$ as instantaneous upon the channel closing and opening.

The speed of $\left[\mathrm{Ca}^{2+}\right]$ equilibration depends on the concentrations and kinetic properties of the intracellular mobile $\mathrm{Ca}^{2+}$ buffers. In the absence of mobile buffers, the equilibration is slowed (Fig. 4.2B, C, green line). However, even the presence of a small amount of a fast mobile $\mathrm{Ca}^{2+}$ buffer strongly speeds the equilibration. The light blue curve in Fig. 4.2B shows the dependence of $t_{0.95}$ on the distance from a single channel in the presence of $0.1 \mathrm{mM}$ BAPTA. Another important factor shaping the equilibration times of $\left[\mathrm{Ca}^{2+}\right]$ after the channel opening or closing are the immobile $\mathrm{Ca}^{2+}$ buffers. These buffers do not influence the steady state levels of $\left[\mathrm{Ca}^{2+}\right]$, but slow down the equilibration (Naraghi \& Neher 1997). Concentrations and $\left[\mathrm{Ca}^{2+}\right]$ binding properties of immobile $\left[\mathrm{Ca}^{2+}\right]$ buffers in IHCs are not known. However, even assuming the strongest immobile buffering described so far $-4 \mathrm{mM}, k_{o n}=10^{8} \mathrm{M}^{-1} \mathrm{~s}^{-1}$, and $K_{D}=10^{-4} \mathrm{M}$ (Xu et al. 1997, Matthews \& Dietrich 2015) - the equilibration times would still be rather small (Fig. 4.2B, brown line) for the $\mathrm{Ca}^{2+}$-nanodomain and intermediate $\mathrm{Ca}^{2+}$-domain coupling scenarios (Fig. $1 \mathrm{C}$ ). Please note that $\left[\mathrm{Ca}^{2+}\right]$ is constant in a steady state for AZs in the idealized $\mathrm{Ca}^{2+}$-microdomain coupling regime by definition. Thus, immobile $\mathrm{Ca}^{2+}$ buffers have no effect then.

The second important feature of $\left[\mathrm{Ca}^{2+}\right]$ dynamics at the presynaptic AZs we found is the near-linear superposition of $\mathrm{Ca}^{2+}$ domains arising from $\mathrm{Ca}^{2+}$ influx through separate presynaptic channels. To show this, we first calculated the steady state spatial profiles of $\left[\mathrm{Ca}^{2+}\right]$ for the AZ scenarios M1 and M3 by solving the generic reaction diffusion equations (see Methods). Then, we calculated the steady state profiles of $\left[\mathrm{Ca}^{2+}\right]$ by considering each open presynaptic $\mathrm{Ca}^{2+}$ channel in isolation and summing up the spatial $\left[\mathrm{Ca}^{2+}\right]$ profiles corresponding to each open channel. Fig. 4.2F shows the average relative difference between $\left[\mathrm{Ca}^{2+}\right]$ at the $\mathrm{Ca}^{2+}$ sensors of exocytosis calculated by using the superposition $\left(\left[\mathrm{Ca}^{2+}\right]_{\mathrm{L}}\right)$ and by solving the generic reaction-diffusion equations $\left(\left[\mathrm{Ca}^{2+}\right]_{\mathrm{N}}\right)$. The top points of the vertical bars shown in Fig. 4.2F mark the upper limits of the 0 - 95 percentile range of the discrepancy measure. The averaging was performed in the same way as when calculating $t_{0.95}$ described above. The discrepancy between the two estimates is small even for the near-maximal $\mathrm{Ca}^{2+}$ influx at $V_{m}=-20 \mathrm{mV}$. Mathematically, these findings are equivalent to the fact that the reaction-diffusion equations describing the dynamics of $\left[\mathrm{Ca}^{2+}\right]$ can be linearized to a very good approximation. The results shown in Fig. 4.2 were obtained by assuming RRP vesicles and synaptic ribbons being permeable to $\mathrm{Ca}^{2+}$ ions and buffer molecules. However, the same conclusion remained valid when these organelles were treated as diffusion barriers (as described in "Additional results" of chapter 2 in the present dissertation). The average relative discrepancy, equivalent to that shown in Fig. 4.2, did not exceed $10 \%$ then (data not shown).

An important condition for the validity of the linear approximation is the fact that the concentrations of the mobile $\mathrm{Ca}^{2+}$-unbound buffer molecules are rather uniform at the $\mathrm{AZ}$ compared to $\left[\mathrm{Ca}^{2+}\right]_{\mathrm{i}}$ (Fig. 4.2C, D). This renders the concentrations of the $\mathrm{Ca}^{2+}$-unbound buffer 
molecules quite insensitive to the particular combinations of open channels within the AZ. Thus, the reaction-diffusion dynamics can be linearized around the average concentration of the $\mathrm{Ca}^{2+}$ unbound buffer within the AZ for a given overall $\mathrm{Ca}^{2+}$ influx. This would remain reasonable even if the buffer was considerably depleted due to incoming $\mathrm{Ca}^{2+}$. However, then the equations linearized for one particular overall $\mathrm{Ca}^{2+}$ influx could not be generalized to different $\mathrm{Ca}^{2+}$ influx levels. On the other hand, if buffer depletion is not too strong, it is possible to linearize the dynamics around one chosen $\mathrm{Ca}^{2+}$ influx level such that the linearization works well within the whole range of the stimulus intensities of our interest. This turns out to be a very realistic situation in the case of the IHC AZs. Fig. 4.2G shows how average value of $\left[\mathrm{Ca}^{2+}\right]$ at the $\mathrm{Ca}^{2+}$ sensor of exocytosis depends on the single channel current (filled circles) for scenarios M1 and M3. Here, $i_{C a}=100 \%$ corresponds to $V_{m}=-20 \mathrm{mV}$ at which the overall $\mathrm{Ca}^{2+}$ influx is maximal. For both scenarios, the dependence nearly perfectly follows the linear relation $y=k \cdot x$ shown by solid lines in Fig. 4.2G. This means that linearization of the reaction diffusion equations at one particular $i_{C a}$ value works well within the whole range of stimulus intensities of our interest.

Guided by the above results, we assumed in this work that: 1) the dynamics of $\left[\mathrm{Ca}^{2+}\right]$ is instantaneous upon opening and closing of the presynaptic $\mathrm{Ca}^{2+}$ channels, 2) the overall $\left[\mathrm{Ca}^{2+}\right]$ at the presynaptic site is equal to the sum of the contributions of each presynaptic $\mathrm{Ca}^{2+}$ channel when considered separately from each other. If these assumptions are taken into account, then $\left[\mathrm{Ca}^{2+}\right]$ at the $\mathrm{Ca}^{2+}$ sensors of exocytosis are fully described by the conductance states of the presynaptic $\mathrm{Ca}^{2+}$ channels at a given moment and steady state levels of $\left[\mathrm{Ca}^{2+}\right]$ due to each channel in isolation. We denoted the contributions to $\left[\mathrm{Ca}^{2+}\right]$ at the vesicular release sites by each presynaptic channel as $\Delta\left[\mathrm{Ca}^{2+}\right]_{\mathrm{i}, \mathrm{j}}^{\mathrm{s}}$, where, in the most general case, $i=\overline{1, N_{C}}$ and $j=\overline{1, N_{V}}$. In this work, we treated $\Delta\left[\mathrm{Ca}^{2+}\right]_{\mathrm{i}, \mathrm{j}}^{\mathrm{s}}$ as free parameters which determine the dynamics of fusion of presynaptic vesicles. When calculating vesicle release rate dependence on $V_{m}, \Delta\left[\mathrm{Ca}^{2+}\right]_{\mathrm{i}, \mathrm{j}}^{\mathrm{s}}$ were assumed to be independent of $V_{m}$. This is justified by the large difference between the resting membrane potential of IHCs $(-45 \mathrm{mV}$, Cody \& Russell, 1987) and the reversal potential of $\mathrm{Ca}^{2+}$ current $(\sim+40 \mathrm{mV}$, see, e.g., Johnson et al. 2005; Frank et al., 2010) with respect to the range of $V_{m}$ values where the release rate of a synapse varies considerably $(\sim 10 \mathrm{mV}$ see section 4.4.4).

\section{Dynamics of presynaptic vesicle release and replenishment}

Based on presynaptic membrane capacitance measurements combined with photolysis of caged $\mathrm{Ca}^{2+}$, it was suggested that fusion of the RRP vesicles at IHC synapses happens upon binding of $4-5 \mathrm{Ca}^{2+}$ ions to the $\mathrm{Ca}^{2+}$-sensor of exocytosis (Beutner et al., 2001). In our work, the original Markov chain scheme of $\mathrm{Ca}^{2+}$ dependent vesicle fusion proposed by (Beutner et al., 2001) was used as the base model for vesicle fusion (Fig. 4.1E). This scheme is characterized by four parameters: the intrinsic $\mathrm{Ca}^{2+}$ binding rate $\left(k_{o n}\right)$, the $\mathrm{Ca}^{2+}$ unbinding rate $\left(k_{o f f}\right)$, the cooperativity factor of $\mathrm{Ca}^{2+}$ unbinding $(b)$, and the rate of the last irreversible vesicle fusion step 
$(\gamma)$. In vitro estimates of these parameters, based on presynaptic exocytic responses of many IHCs, each containing roughly a dozen synapses, were respectively $0.028 \mu \mathrm{M}^{-1} \mathrm{~ms}^{-1}, 2.2 \mathrm{~ms}^{-1}$, 0.4 , and $1.7 \mathrm{~ms}^{-1}$. Note that the apparent $\mathrm{Ca}^{2+}$-binding rate, which we denoted by $k_{r e l}$, is the product of $k_{o n}$ and $\left[\mathrm{Ca}^{2+}\right]$ at the sensor of exocytosis. In our model, we varied all these parameters, including the number of the $\mathrm{Ca}^{2+}$-binding sites.

The kinetics and $\mathrm{Ca}^{2+}$ dependence of the replenishment of RRP vesicles at IHC synapses, as well as other ribbon synapses, are currently poorly understood. In vitro patch clamp recordings indicated strong positive dependence of the RRP recovery rate on intracellular $\left[\mathrm{Ca}^{2+}\right]$ at auditory hair cell synapses (Moser \& Beutner, 2000; Spassova et al., 2004). In fact, the RRP recovery was abolished by application of $2.5-5 \mathrm{mM} \mathrm{Ca}^{2+}$ buffer EGTA in the paired pulse stimulation paradigm. These findings suggested that the RRP recovery depends on distant $\left[\mathrm{Ca}^{2+}\right]$, several hundred nanometers away from the presynaptic $\mathrm{Ca}^{2+}$ channels (Moser \& Beutner, 2000; Spassova et al., 2004). Nevertheless, no quantitative relations between intracellular $\left[\mathrm{Ca}^{2+}\right]$ and RRP recovery have been reported so far. Vesicle replenishment to a partially depleted RRP during ongoing stimulation likely deviates from that after cessation of a stimulus. Given these uncertainties, vesicle replenishment was treated as a one step process characterized by one rate parameter $k_{r e p}$ in our model. At fixed $V_{m},\left[\mathrm{Ca}^{2+}\right]$ is constant in time at locations distant from the presynaptic channels because the fluctuations due to opening/closing of individual $\mathrm{Ca}^{2+}$ channels are averaged out. Thus, $k_{r e p}$ is a well defined function of $V_{m}$ in a steady state. In the basic version of the model we simply assumed $k_{r e p}\left(V_{m}\right) \equiv k_{r e p}=$ const. More generally, the $k_{r e p}$ vs $V_{m}$ relation was described by a Boltzmann function,

$$
k_{r e p}\left(V_{m}\right)=\frac{\tilde{k}_{r e p}}{1+e^{-\left(V_{m}-\tilde{V}_{0.5}\right) / k_{V}}},
$$

where $\tilde{k}_{r e p}$ is the maximum replenishment rate, $\tilde{V}_{0.5}$ the half activation voltage, $k_{V}$ is the voltage sensitivity (the same as for $p_{o}\left(V_{m}\right)$ ).

In the most basic version of the model considered, vesicle fusion was directly induced upon irreversible binding of one $\mathrm{Ca}^{2+}$ ion (Fig. 4.1E). Such a vesicular release site was characterized by only two states, empty (fused) or filled with a vesicle, and was called as a 2-state site. We used a term $n_{V}$-state vesicular release site to refer to those sites defined by $n_{V} \geq 3$ states. Note that, for these sites, $n_{V}=m+2$, where $m$ is the number of $\mathrm{Ca}^{2+}$-binding sites per $\mathrm{Ca}^{2+}$ sensor of exocytosis.

\subsubsection{Spike generation at the postsynaptic neuron}

To describe spike generation at the postsynaptic site, we used a refractory counter model of SGN with fixed absolute and exponentially distributed relative refractory periods (see Fig. 4.1B) (Schroeder \& Hall, 1974; Young \& Barta, 1986; Li \& Young 1993). In this model, EPSCs and spikes are treated as point events which are characterized only by their occurrence times. Other features, like shape of EPSCs are not explicitly taken into account. Two different states 
of the SGN are distinguished: the refractory state and the nonrefractory state. If in the nonrefractory state, the SGN generates a spike to every incoming EPSC. Immediately after each spike generation, the SGN returns to the refractory state. The SGN switches back to the nonrefractory state after refractory period $T$ which is a sum of two components, $T=t_{A}+t_{R}$. $t_{A}$ stands for the absolute refractory period, which is a fixed number. $t_{R}$ is the so-called relative refractory period, which is a random number with an exponential distribution and average $\tau_{R}$. Despite its simplicity, this model reflects the experimentally determined biophysical peculiarities of spike generation at SGNs well (see Supplementary Material 1.2).

\subsection{Analytical treatment of the model}

\subsubsection{Point process of vesicle release at $\mathrm{AZs}$ in a steady state}

Assuming that presynaptic $\left[\mathrm{Ca}^{2+}\right]$ follows the gating of $\mathrm{Ca}^{2+}$ channels instantaneously (section 4.2.2), the state of a presynaptic AZ in our model is fully characterized by the states of all the presynaptic channels and vesicular release sites. In the following, we used vectors $\vec{c}$ and $\vec{v}$ to denote, respectively, the set of states of all channels and the set of states of all vesicular release sites at an AZ. Particular elements of vectors $\vec{c}$ and $\vec{v}$, denoted by $c_{i}$ and $v_{j}$, correspond to a particular channel $i$ and a particular vesicular release site $j$ at the AZ. To denote the states of all channels but the channel $i$ and states of all vesicular release sites but the site $j, \vec{c}_{-i}$ and $\vec{v}_{-j}$ were used, respectively. Values assigned to the elements $c_{i}$ and $v_{j}$ define the states of the corresponding channel or vesicular release site. We employed a notation in which these values vary from 1 to the number of states each presynaptic channel and vesicular release site has. The state of each vesicular release site, from which the fusion takes place, was assigned the maximum possible value. The moment of the irreversible transition from that state to the state $v_{i}=1$ was treated as a vesicle fusion event. The open state of each channel was assigned the maximum possible value, i.e., the total number of states the channel was described by.

It follows from the formulation of the model (section 4.2), that the dynamics of the presynaptic site is described by a Markovian jump process. This process underlies the events of vesicle fusion and postsynaptic spike generation, which are treated as points in time. As we demonstrated below, quantitative characterization of the stochastic point processes of vesicle fusion and spike generation is based on the solutions of the following two sets of differential equations. The first of them, the so-called master equation, describes the dynamics of the probability $\bar{P}\left(t, \vec{c}, \vec{v} \mid \vec{c}_{0}, \vec{v}_{0}\right)$ of finding the presynaptic site in a particular state $(\vec{c}, \vec{v})$ at moment 
$t>0$ if it was in a particular state $\left(\vec{c}_{0}, \vec{v}_{0}\right)$ at $t=0$ :

$$
\begin{aligned}
& \partial_{t} \bar{P}\left(t, \vec{c}, \vec{v} \mid \vec{c}_{0}, \vec{v}_{0}\right)=\sum_{i=1}^{N_{C}} \sum_{c_{i}^{\prime}=1}^{n_{C}(i)} k_{c_{i}^{\prime} \rightarrow c_{i}}^{C i} \cdot \bar{P}\left(t, c_{i}^{\prime}, \vec{c}_{-i}, \vec{v} \mid \vec{c}_{0}, \vec{v}_{0}\right)+ \\
& +\sum_{j=1}^{N_{V}} \sum_{v_{j}^{\prime}=1}^{n_{V}(j)} k_{v_{j}^{\prime} \rightarrow v_{j}}^{V j} \cdot f_{\vec{c}, v_{j}^{\prime} \rightarrow v_{j}}^{j} \cdot \bar{P}\left(t, \vec{c}, v_{j}^{\prime}, \vec{v}_{-j} \mid \vec{c}_{0}, \vec{v}_{0}\right),
\end{aligned}
$$

where

$$
\begin{aligned}
k_{c_{i} \rightarrow c_{i}}^{C i} & =-\sum_{c_{i}^{\prime}=1 \neq c_{i}}^{n_{C}(i)} k_{c_{i} \rightarrow c_{i}^{\prime}}^{C i}, \quad k_{v_{j} \rightarrow v_{j}}^{V j}=-\sum_{v_{j}^{\prime}=1 \neq v_{j}}^{n_{V}(j)} k_{v_{j} \rightarrow v_{j}^{\prime}}^{V j} \cdot f_{\vec{c}, v_{j} \rightarrow v_{j}^{\prime}}^{j}, \\
f_{\vec{c}, v_{j}^{\prime} \rightarrow v_{j}}^{j} & =1+\left(\sum_{i=1}^{N_{C}} \Delta\left[\mathrm{Ca}^{2+}\right]_{i j}^{s} \cdot \delta_{c_{i}, n_{C}(i)}-1\right) \cdot \delta_{v_{j}^{\prime} \rightarrow v_{j}, \mathrm{Ca}^{2+}}^{j} \cdot\left(1-\delta_{v_{j}^{\prime}, v_{j}}\right) .
\end{aligned}
$$

The second set of equations describes the dynamics of the probability $P\left(t, \vec{c}, \vec{v} \mid \vec{c}_{0}, \vec{v}_{0}\right)$ that no vesicle fusion happened before moment $t$ and that the presynaptic AZ is in a particular state $(\vec{c}, \vec{v})$ at that moment:

$$
\begin{aligned}
& \partial_{t} P\left(t, \vec{c}, \vec{v} \mid \vec{c}_{0}, \vec{v}_{0}\right)=\sum_{i=1}^{N_{C}} \sum_{c_{i}^{\prime}=1}^{n_{C}(i)} k_{c_{i}^{\prime} \rightarrow c_{i}}^{C i} \cdot P\left(t, c_{i}^{\prime}, \vec{c}_{-i}, \vec{v} \mid \vec{c}_{0}, \vec{v}_{0}\right)+ \\
& \quad+\sum_{j=1}^{N_{V}} \sum_{v_{j}^{\prime}=1}^{n_{V}(j)} k_{v_{j}^{\prime} \rightarrow v_{j}}^{V j} \cdot f_{\vec{c}, v_{j}^{\prime} \rightarrow v_{j}}^{j} \cdot\left(1-\delta_{v_{j}, 1} \cdot \delta_{v_{j}^{\prime}, n_{V}(j)}\right) \cdot P\left(t, \vec{c}, v_{j}^{\prime}, \vec{v}_{-j} \mid \vec{c}_{0}, \vec{v}_{0}\right) .
\end{aligned}
$$

In Eqs. $(4.4)-(4.6), N_{C}$ - the number of presynaptic $\mathrm{Ca}^{2+}$ channels, $k_{c_{i}^{\prime} \rightarrow c_{i}}^{C i}$ - the transition rate from state $c_{i}^{\prime}$ to state $c_{i}$ of the $i$-th channel, $n_{C}(i)$ - the number of states of the $i$-th channel, $N_{V}$ - the number of presynaptic vesicular release sites, $k_{v_{j}^{\prime} \rightarrow v_{j}}^{V j}$ - the transition rate from state $v_{j}^{\prime}$ to state $v_{j}$ of the $j$-th release site, $n_{V}(j)$ - the number of states of the $j$-th vesicular release site. $\Delta\left[\mathrm{Ca}^{2+}\right]_{i j}^{s}-\mathrm{Ca}^{2+}$ concentration at the $j$-th site due to the $i$-th channel in the open state. Note that the summation of $\Delta\left[\mathrm{Ca}^{2+}\right]_{i j}^{s}$ over different channels exploits the assumption of the superposition, introduced in section 4.2.2. Variable $\delta_{v_{j}^{\prime} \rightarrow v_{j}, \mathrm{Ca}^{2+}}^{j}$ is equal to 1 if the transition from state $v_{j}^{\prime}$ to state $v_{j}$ of vesicular release site $j$ is $\mathrm{Ca}^{2+}$ dependent, and is equal to 0 otherwise. In all other cases here, the symbol $\delta$ stands for the Kronecker delta. If no transition between two particular states of a channel or vesicular release site exists, the corresponding transition rates $\left(k_{c_{i}^{\prime} \rightarrow c_{i}}^{C i}\right.$ or $\left.k_{v_{j}^{\prime} \rightarrow v_{j}}^{V}\right)$ in Eq. (4.5) are equal to zero. In our model we considered a steady state situation where the receptor potential is constant in time (section 4.2.1). Thus, the channel gating rates in Eqs. (4.5) - (4.9) were constant in time.

The probability distribution of IEIs in a steady state can be constructed by using the solutions of Eq. (4.6). Indeed, let us use $P_{0}^{s}\left(\vec{c}_{0}, \vec{v}_{0}\right)$ to denote the probability that, during a particular vesicle fusion event in a steady state, the system enters state $\left(\vec{c}_{0}, \vec{v}_{0}\right)$. Then, the probability that no vesicle fusion happened in a time window after the previous fusion event in 
a steady state is given by

$$
P_{I E I}(t)=\sum_{\vec{c} \in \mathcal{C}} \sum_{\vec{v} \in \mathcal{V}} \sum_{\vec{c}_{0} \in \mathcal{C}} \sum_{\vec{v}_{0} \in \mathcal{V}} P\left(t, \vec{c}, \vec{v} \mid \vec{c}_{0}, \vec{v}_{0}\right) \cdot P_{0}^{s}\left(\vec{c}_{0}, \vec{v}_{0}\right)
$$

Here, $\mathcal{C}$ and $\mathcal{V}$ stand for the sets of possible realizations of vectors $\vec{c}$ and $\vec{v}$. Accordingly, the probability density function of IEIs can be expressed in the following way:

$$
\begin{aligned}
\rho_{I E I}(t) & =-\frac{d}{d t} P_{I E I}(t)= \\
& =\sum_{\vec{c} \in \mathcal{C}} \sum_{\vec{v} \in \mathcal{V}} F_{\vec{v}} \cdot \sum_{\vec{c}_{0} \in \mathcal{C}} \sum_{\vec{v}_{0} \in \mathcal{V}} P\left(t, \vec{c}, \vec{v} \mid \vec{c}_{0}, \vec{v}_{0}\right) \cdot P_{0}^{s}\left(\vec{c}_{0}, \vec{v}_{0}\right),
\end{aligned}
$$

where $F_{\vec{v}}=\sum_{j=1}^{N_{V}} k_{v_{j} \rightarrow 1}^{V j} \cdot f_{\vec{c}, v_{j} \rightarrow 1}^{j} \cdot \delta_{v_{j}, n_{V}(j)}$. The second equality in Eq. (4.8) follows from Eq. (4.6), after summing both sides of it with respect to $\vec{c}$ and $\vec{v}$ over $\mathcal{C}$ and $\mathcal{V}$. The set of initial probabilities, $P_{0}^{s}(\vec{c}, \vec{v})$, can be found by using the requirement that, in a steady state, the probability of entering the same state after two subsequent vesicle fusion events is equal. Mathematically, this requirement is equivalent to the following system of linear equations:

$$
P_{0}^{s}(\vec{c}, \vec{v})=\sum_{\vec{c}_{*} \in \mathcal{C}} \sum_{\vec{v}_{*} \in \mathcal{V}} T\left(\vec{c}, \vec{v} \mid \vec{c}_{*}, \vec{v}_{*}\right) \cdot P_{0}^{s}\left(\vec{c}_{*}, \vec{v}_{*}\right)
$$

where $T\left(\vec{c}, \vec{v} \mid \vec{c}_{*}, \vec{v}_{*}\right)$ is the probability that a vesicle fusion event occurs when the system transits to state $(\vec{c}, \vec{v})$, if during the previous fusion event the system transited to state $\left(\vec{c}_{*}, \vec{v}_{*}\right)$. $T\left(\vec{c}, \vec{v} \mid \vec{c}_{*}, \vec{v}_{*}\right)$ can be expressed in the following way:

$$
T\left(\vec{c}, \vec{v} \mid \vec{c}_{*}, \vec{v}_{*}\right)=\sum_{j=1}^{N_{V}} \sum_{\vec{v}^{\prime} \in \mathcal{V}} W_{v_{j}^{\prime}}\left(\vec{c}, \vec{v}_{-j}^{\prime}\right) \cdot \tilde{T}\left(\vec{c}, \vec{v}^{\prime} \mid \vec{c}_{*}, \vec{v}_{*}\right) \cdot \delta_{\vec{v}_{-j}^{\prime}, \vec{v}_{-j}} \cdot \delta_{v_{j}, 1},
$$

where

$$
\begin{gathered}
W_{v_{j}^{\prime}}\left(\vec{c}, \vec{v}_{-j}^{\prime}\right)=\frac{k_{v_{j}^{\prime} \rightarrow 1}^{V j} \cdot f_{\vec{c}, v_{j}^{\prime} \rightarrow 1}^{j} \cdot \delta_{v_{j}^{\prime}, n_{V}(j)}}{\sum_{l=1}^{N_{V}} k_{v_{l}^{\prime} \rightarrow 1}^{V l} \cdot f_{\vec{c}, v_{l}^{\prime} \rightarrow 1}^{l} \cdot \delta_{v_{j}^{\prime}, n_{V}(j)}}, \\
\tilde{T}\left(\vec{c}, \vec{v}^{\prime} \mid \vec{c}_{*}, \vec{v}_{*}\right)=\int_{0}^{+\infty}\left(P\left(t, \vec{c}, \vec{v}^{\prime} \mid \vec{c}_{*}, \vec{v}_{*}\right) \cdot \sum_{j=1}^{N_{V}} k_{v_{j}^{\prime} \rightarrow 1}^{V j} \cdot f_{\vec{c}, v_{j}^{\prime} \rightarrow 1}^{j} \cdot \delta_{v_{j}^{\prime}, n_{V}(j)}\right) d t .
\end{gathered}
$$

$\tilde{T}\left(\vec{c}, \vec{v}^{\prime} \mid \vec{c}_{*}, \vec{v}_{*}\right)$ is the probability that a vesicle fusion happens from state $\left(\vec{c}, \vec{v}^{\prime}\right)$ if initially the system started in state $\left(\vec{c}_{*}, \vec{v}_{*}\right) . W\left(j, \vec{c}, \vec{v}^{\prime}\right)$ is the conditional probability of transition $v_{j}^{\prime} \rightarrow n_{V}(j)$ in a steady state given that the system leaves state $\left(\vec{c}, \vec{v}^{\prime}\right)$. Eq. (4.9) has a unique solution which is normalized to one (see Supplementary Material 2.1).

In this work, we were quite often interested only in the first two moments of $\rho_{I E I}(t)$. Calculation of these moments can be reduced to solving systems of linear algebraic equations. This idea can be expressed most clearly in the matrix notation. Thus, let us first rewrite Eq. (4.6) accordingly,

$$
\partial_{t} \mathbf{P}=\mathbf{A} \cdot \mathbf{P}
$$


where elements of the probability vector $\mathbf{P}$ and the transition matrix $\mathbf{A}$ are indexed by a pair $(\vec{c}, \vec{v})$. Then, as shown in Supplementary Material 2.2, the $m$-th moment of $\rho_{I E I}(t)$ is given by

$$
\left\langle t^{m}\right\rangle_{I E I}=m ! \cdot \mathbf{P}_{\mathbf{0}}^{\mathbf{s}} \cdot\left(\left(-\mathbf{A}^{\mathbf{T}}\right)^{-\mathbf{m}} \cdot \mathbf{1}\right)
$$

where vector $\mathbf{P}_{\mathbf{0}}^{\mathbf{s}}$ corresponds to $P_{0}^{s}(\vec{c}, \vec{v})$ introduced above. $\mathbf{1}$ is a vector of the same dimension as $\mathbf{P}_{\mathbf{0}}^{\mathbf{s}}$ and with all its elements equal to 1 . If $\mathbf{P}_{\mathbf{0}}^{\mathbf{s}}$ is known, Eq. (4.14) can be evaluated analytically, at least in principle, even when $\rho_{I E I}(t)$ cannot. In particular, the first moment can always be calculated analytically:

$$
\langle t\rangle_{I E I}=\left(\sum_{\vec{c}_{*} \in \mathcal{C}} \sum_{\vec{v}_{*} \in \mathcal{V}} \sum_{j=1}^{N_{V}} k_{v_{j} \rightarrow 1}^{V j} \cdot f_{\vec{c}, v_{j} \rightarrow 1}^{j} \cdot \delta_{v_{j}, n_{V}(j)} \cdot \bar{P}^{s}(\vec{c}, \vec{v})\right)^{-1},
$$

where $\bar{P}^{s}(\vec{c}, \vec{v})=\bar{P}(t=+\infty, \vec{c}, \vec{v})$ is the stationary solution of Eq. (4.4).

Time intervals between subsequent vesicle fusion events in a steady state are not uncorrelated in general. Indeed, in the most general case, the state through which the system transits to during a particular fusion event correlates with the waiting time for that fusion event, as well as with the waiting time for the next and all the following fusion events. The serial correlation function of IEIs in a steady state, denoted by $C_{I E I}(l)$, can be expressed as

$$
C_{I E I}(l)=\frac{\left\langle t_{i} t_{i+l}\right\rangle-\left\langle t_{i}\right\rangle\left\langle t_{i+l}\right\rangle}{\sqrt{\left\langle t_{i}^{2}\right\rangle-\left\langle t_{i}\right\rangle^{2}} \cdot \sqrt{\left\langle t_{i+l}^{2}\right\rangle-\left\langle t_{i+l}\right\rangle^{2}}}=\frac{\left\langle t_{i} t_{i+l}\right\rangle_{I E I}-\langle t\rangle_{I E I}^{2}}{\left\langle t^{2}\right\rangle_{I E I}-\langle t\rangle_{I E I}^{2}}
$$

where $t_{i}$ and $t_{i+l}$ are the $i$-th and the $(i+l)$-th IEIs of vesicle fusion. Writing (4.16) we used the fact that, in a steady state, the statistical properties of the system are invariant to a shift in time, thus, $\left\langle t_{i}^{m}\right\rangle=\left\langle t_{i+l}^{m}\right\rangle=\left\langle t^{m}\right\rangle_{I E I}$. In a steady state, $\left\langle t_{i} t_{i+l}\right\rangle_{I E I}$ can be expressed through the solutions of Eq. (4.6) and their derivatives in the following way:

$$
\left\langle t_{i} t_{i+l}\right\rangle_{I E I}=\mathbf{1}^{\mathbf{T}} \cdot\left(\mathbf{J} \cdot \mathbf{T}^{\mathbf{l}-\mathbf{1}} \cdot \mathbf{G} \cdot \mathbf{P}_{\mathbf{0}}^{\mathbf{s}}\right),
$$

where elements of matrices $\mathbf{G}, \mathbf{J}$, and $\mathbf{T}$ are

$$
\begin{aligned}
G_{(\vec{c}, \vec{v})\left(\vec{c}_{*}, \vec{v}_{*}\right)} & =\int_{0}^{+\infty}\left[t \cdot \sum_{\vec{v}^{\prime} \in \mathcal{V}}\left(\sum_{i=1}^{N_{V}} W_{v_{i}^{\prime}}\left(\vec{c}, \vec{v}_{-i}^{\prime}\right) \cdot \delta_{\vec{v}_{-i}^{\prime}, \vec{v}_{-i}} \cdot \delta_{v_{i}, 1}\right)\right. \\
& \left.\cdot\left(\sum_{j=1}^{N_{V}} k_{v_{j}^{\prime} \rightarrow 1}^{V j} \cdot f_{\vec{c}, v_{j}^{\prime} \rightarrow 1}^{j} \cdot \delta_{v_{j}^{\prime}, n_{V}(j)}\right) \cdot P\left(t, \vec{c}, \vec{v}^{\prime} \mid \vec{c}_{*}, \vec{v}_{*}\right)\right] d t \\
J_{(\vec{c}, \vec{v})\left(\vec{c}_{*}, \vec{v}_{*}\right)} & =\delta_{(\vec{c}, \vec{v}),\left(\vec{c}_{*}, \vec{v}_{*}\right)} \cdot \int_{0}^{+\infty}\left[\sum_{\vec{c}_{\diamond} \in \mathcal{C}} \sum_{\vec{v}_{\diamond} \in \mathcal{V}} P\left(t, \vec{c}_{\diamond}, \vec{v}_{\diamond} \mid \vec{c}_{*}, \vec{v}_{*}\right)\right] d t \\
T_{(\vec{c}, \vec{v})\left(\vec{c}_{*}, \vec{v}_{*}\right)} & \equiv T\left(\vec{c}, \vec{v} \mid \vec{c}_{*}, \vec{v}_{*}\right) .
\end{aligned}
$$

The physical meanings of the elements of matrices $\mathbf{G}, \mathbf{J}$, and $\mathbf{T}$ are the following. $G_{(\vec{c}, \vec{v})\left(\vec{c}_{*}, \vec{v}_{*}\right)}$ is the mean time to the next vesicle fusion event, given that the system is initially in state $\left(\vec{c}_{*}, \vec{v}_{*}\right)$ 
and during the next fusion event transits into state $(\vec{c}, \vec{v}) . T_{(\vec{c}, \vec{v})\left(\vec{c}, \vec{v}_{*}\right)}^{(n-1)}$ is the probability that the system transits into state $(\vec{c}, \vec{v})$ just after $(n-1)$ subsequent vesicle fusion events, given that it started in state $\left(\vec{c}_{*}, \vec{v}_{*}\right) . J_{(\vec{c}, \vec{v})\left(\vec{c}_{*}, \vec{v}_{*}\right)}$ is the mean time to the next vesicle fusion event, given that the system is initially in state $(\vec{c}, \vec{v})=\left(\vec{c}_{*}, \vec{v}_{*}\right)$. Writing Eq. (4.17) we used the fact that, once the initial conditions for the $(i+n)$-th fusion event are fixed, waiting times for the $i$-th and $(i+n)$-th events are statistically independent. This follows from the fact that the dynamics of the system underlying the vesicle fusion process is Markovian.

Eqs. (4.4) and (4.6) are systems of homogeneous linear differential equations with constant coefficients. In general, the solutions to these equations can be expressed as linear combinations of functions from a set $\left\{t^{\alpha_{i}} \cdot e^{\lambda_{i} \cdot t}, t^{\alpha_{i}} \cdot \cos \left(\lambda_{i} \cdot t\right), t^{\alpha_{i}} \cdot \sin \left(\lambda_{i} \cdot t\right)\right\}$. Solving these equations then reduces to diagonalization of the corresponding transition matrices. This can be done analytically in a number of relevant cases, as is demonstrated throughout this work.

In the case of the $\mathrm{Ca}^{2+}$-nanodomain, $\mathrm{Ca}^{2+}$-microdomain, and intermediate $\mathrm{Ca}^{2+}$-domain coupling scenarios (Fig. 4.1C), vesicular release sites within the AZ are statistically independent. Then, solutions of Eqs. (4.4) and (4.6) can be factorized:

$$
\begin{aligned}
& \bar{P}\left(t, \vec{c}, \vec{v} \mid \vec{c}_{0}, \vec{v}_{0}\right)=\prod_{i=1}^{N_{V}} \bar{P}_{i}\left(t, \vec{c}^{i}, v_{i} \mid \vec{c}_{0}^{i}, v_{0 i}\right), \\
& P\left(t, \vec{c}, \vec{v} \mid \vec{c}_{0}, \vec{v}_{0}\right)=\prod_{i=1}^{N_{V}} P_{i}\left(t, \vec{c}^{i}, v_{i} \mid \vec{c}_{0}^{i}, v_{0 i}\right) .
\end{aligned}
$$

Here, $\bar{P}_{i}\left(t, \vec{c}^{i}, \vec{v}^{i} \mid \vec{c}_{0}^{i}, v_{0 i}\right)$ and $P_{i}\left(t, \vec{c}^{i}, \vec{v}^{i} \mid \vec{c}_{0}^{i}, v_{0 i}\right)$ are solutions of Eqs. (4.4) and (4.6), respectively, for an AZ with a single vesicular release site $i . \vec{c}^{i}$ denotes the state of the $\mathrm{Ca}^{2+}$ channels corresponding to the site $i$ while $v_{i}$ denotes the state of the vesicular release site $i$. The initial condition vector $P_{0}(\vec{c}, \vec{v})$ for calculating $\rho_{I E I}(t)$ can also be factorized for AZs with independent vesicular release sites:

$$
P_{0}^{s}(\vec{c}, \vec{v})=\sum_{i=1}^{N_{V}}\left(P_{i}^{r} \cdot P_{0, i}^{s}\left(\vec{c}^{i}, v_{i}\right) \cdot \prod_{j=1 \neq i}^{N_{V}} \bar{P}_{j}^{s}\left(\vec{c}^{j}, v_{j}\right)\right) .
$$

Here, $\bar{P}_{i}^{s}\left(\vec{c}^{i}, v_{i}\right)=\bar{P}_{i}\left(t=+\infty, \vec{c}^{i}, v_{i}\right)$ is the stationary probability to find vesicular release site $i$ in a state $\left(\vec{c}^{i}, v_{i}\right) . P_{0, i}^{s}\left(\vec{c}^{i}, v_{i}\right)$ is the probability that site $i$ enters state $\left(c_{i}, v_{i}\right)$ during a vesicle fusion event at that site in a steady state. $P_{0, i}^{s}\left(\vec{c}^{i}, v_{i}\right)$ is the solution of Eq. (4.9) formulated for a single vesicular release site. $P_{i}^{r}$ is the probability that a vesicle fusion at the AZ in a steady state is due to site $i . P_{i}^{r}$ is equal to the fraction of the overall release rate at the AZ contributed by site $i$,

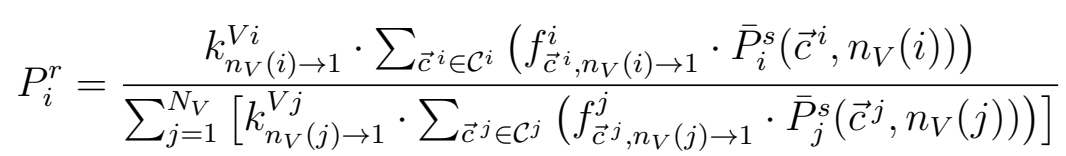

Plugging (4.19) - (4.21) into Eqs. (4.8), (4.14) - (4.15), and (4.16) - (4.17) results in the expressions of $\rho_{I E I}(t),\left\langle t^{m}\right\rangle_{I E I}$, and $C_{I E I}(n)$ for an $\mathrm{AZ}$ with independent vesicular release sites. 
In particular, expressions of $\rho_{I E I}(t)$ and $\langle t\rangle_{I E I}$ are now considerably simpler compared to the original Eqs. (4.8) and (4.15):

$$
\begin{aligned}
& \rho_{I E I}(t)=-\frac{d}{d t} \sum_{i=1}^{N_{V}}\left[P_{i}^{r} \cdot \sum_{\vec{c}_{0}^{i} \in \mathcal{C}^{i}}\left(P_{i}\left(t \mid \vec{c}_{0}^{i}, 1\right) \cdot P_{0, i}^{s}\left(\vec{c}_{0}^{i}, 1\right)\right) \cdot\right. \\
& \left.\cdot \prod_{j=1 \neq i}^{N_{V}} \sum_{\vec{c}_{0}^{j} \in \mathcal{C}^{j}} \sum_{v_{0 j}=1}^{n_{V}(j)}\left(P_{j}\left(t \mid \vec{c}_{0}^{j}, v_{0 j}\right) \cdot \bar{P}_{j}^{s}\left(\vec{c}_{0}^{j}, v_{0 j}\right)\right)\right], \\
& \langle t\rangle=\left(\sum_{j=1}^{N_{V}}\left[k_{n_{V}(j) \rightarrow 1}^{V j} \cdot \sum_{\vec{c}^{j} \in \mathcal{C}^{j}}\left(f_{\vec{c}^{j}, n_{V}(j) \rightarrow 1}^{j} \cdot \bar{P}_{j}^{s}\left(\vec{c}^{j}, n_{V}(j)\right)\right)\right]\right)^{-1},
\end{aligned}
$$

where

$$
P_{i}\left(t \mid \vec{c}_{0}^{i}, v_{0 i}\right)=\sum_{\vec{c}^{i} \in \mathcal{C}^{i}} \sum_{v_{i}=1}^{n_{V}(i)} P_{i}\left(t, \vec{c}^{i}, v_{i} \mid \vec{c}_{0}^{i}, v_{0 i}\right) .
$$

Writing Eq. (4.23) we used the fact that a vesicular release site which results in a vesicle fusion transitions to a state with $v_{i}=1$ during the fusion event. The term in the square brackets in Eq. (4.24) is the vesicle fusion rate due to one of the vesicular release sites at the AZ. In other words, the vesicle fusion rate at the $\mathrm{AZ}$ is a sum of the vesicle fusion rates at separate vesicular release sites.

Expressions (4.19) - (4.24) can be further simplified when considering the $\mathrm{Ca}^{2+}$-nanodomain, $\mathrm{Ca}^{2+}$-microdomain or intermediate $\mathrm{Ca}^{2+}$-domain scenarios individually. For the shared $\mathrm{Ca}^{2+}{ }_{-}$ domain scenario, equivalent expressions to (4.19) - (4.24) apply for the groups of vesicular release sites sharing the same channels (see Supplementary Material 2.3). The fact that solutions of the original Eqs. (4.4) and (4.6) can be factorized is, in practice, crucial to making the analytical approach applicable to systems with a realistic number of vesicular release sites. Indeed, the number of possible states of the system increases exponentially with the number of vesicular release sites. For example, let us assume an AZ with fourteen $\mathrm{Ca}^{2+}$-nanodomain driven release sites and that each unit, consisting of a channel and a vesicular release site, is described by $2 \times 5=10$ states. Then, the number of possible states of the system is equal to $10^{14}$. However, when the factorization is exploited, we have to instead deal with 14 independent systems each characterized by only 10 states.

\subsubsection{Point process of SGN spiking in a steady state}

In our model, the postsynaptic site is described by two states (Fig. 4.1B): the refractory state and the non-refractory state. We introduce a variable $s$ to denote the state of the postsynaptic site: $s=0$ - the refractory state, $s=1$ - the non-refractory state. The state of the joint system of the presynaptic and postsynaptic sites is then uniquely determined by a set $(\vec{c}, \vec{v}, s)$. Let us 
assume that an observation is started at $t=0$, immediately after a spike was generated. The system is initially located in state $\left(\vec{c}_{0}, \vec{v}_{0}, 0\right)$. Then, the probability that the system is in state $(\vec{c}, \vec{v}, 0)$ at moment $t$ reads as

$$
P^{\star}\left(t, \vec{c}, \vec{v}, 0 \mid \vec{c}_{0}, \vec{v}_{0}, 0\right)=\bar{P}\left(t, \vec{c}, \vec{v} \mid \vec{c}_{0}, \vec{v}_{0}\right) \cdot\left(1-\int_{0}^{t} \rho_{r e f}\left(t^{\prime}\right) d t^{\prime}\right)
$$

where $\bar{P}\left(t^{\prime}, \vec{c}, \vec{v} \mid \vec{c}_{0}, \vec{v}_{0}\right)$ is the solution of Eq. (4.4). $\rho_{\text {ref }}(t)$ is the probability density function of the overall refractory period. As follows from the formulation, $\rho_{\text {ref }}(t)=H\left(t-t_{A}\right) \cdot \tau_{R}^{-1} \cdot e^{-\left(t-t_{A}\right) / \tau_{R}}$ in the case of our model (see section $4.2 .3, H(\ldots)$ is the Heaviside step function). The probability that the system is in state $(\vec{c}, \vec{v}, 1)$ at moment $t$, and no spike has been generated by that time, can be expressed as the following convolution integral:

$$
\begin{aligned}
& P^{\star}\left(t, \vec{c}, \vec{v}, 1 \mid \vec{c}_{0}, \vec{v}_{0}, 0\right)= \\
& \quad=\int_{0}^{t} \rho_{r e f}\left(t^{\prime}\right) \cdot \sum_{\vec{c}_{*} \in \mathcal{C}} \sum_{\vec{v}_{*} \in \mathcal{V}} P\left(t-t^{\prime}, \vec{c}, \vec{v} \mid \vec{c}_{*}, \vec{v}_{*}\right) \cdot \bar{P}\left(t^{\prime}, \vec{c}_{*}, \vec{v}_{*} \mid \vec{c}_{0}, \vec{v}_{0}\right) d t^{\prime},
\end{aligned}
$$

where $P\left(t-t^{\prime}, \vec{c}, \vec{v} \mid \vec{c}_{*}, \vec{v}_{*}\right)$ is the solution of Eq. (4.6). $P^{\star}\left(t, \vec{c}, \vec{v}, s \mid \vec{c}_{0}, \vec{v}_{0}, 0\right)$, defined by Eqs. (4.25) - (4.26), can be used to calculate the probability density or serial correlation functions of inter spike intervals, denoted by $\rho_{I S I}(t)$ and $C_{I S I}(t)$. This is achieved by replacing $P\left(t, \vec{c}, \vec{v} \mid \vec{c}_{0}, \vec{v}_{0}\right)$ with $P^{\star}\left(t, \vec{c}, \vec{v}, s \mid \vec{c}_{0}, \vec{v}_{0}, 0\right)$ and including the summation over $s \in\{0,1\}$, in addition to the summation over $c$ and $v$, in the expressions $(4.7)-(4.12)$ and (4.16) - (4.18). In particular, $\rho_{I S I}(t)$ can be expressed as

$$
\begin{gathered}
\rho_{I S I}=-\int_{0}^{t}\left[\rho _ { \text { ref } } ( t ^ { \prime } ) \cdot \sum _ { \vec { c } _ { * } \in \mathcal { C } } \sum _ { \vec { v } _ { * } \in \mathcal { V } } \left(\sum_{\vec{c} \in \mathcal{C}} \sum_{\vec{v} \in \mathcal{V}} \frac{d}{d t} P\left(t-t^{\prime}, \vec{c}, \vec{v} \mid \vec{c}_{*}, \vec{v}_{*}\right) .\right.\right. \\
\left.\left.\cdot \sum_{\vec{c}_{0} \in \mathcal{C}} \sum_{\vec{v}_{0} \in \mathcal{V}} \bar{P}\left(t^{\prime}, \vec{c}_{*}, \vec{v}_{*} \mid \vec{c}_{0}, \vec{v}_{0}\right) \cdot P_{0}^{s}\left(\vec{c}_{0}, \vec{v}_{0}\right)\right)\right] d t^{\prime} .
\end{gathered}
$$

In the case of AZs with the $\mathrm{Ca}^{2+}$-nanodomain, $\mathrm{Ca}^{2+}$-microdomain, and intermediate $\mathrm{Ca}^{2+}$ domain couplings, Eq. (4.27) reduces to the following expression:

$$
\begin{array}{r}
\rho_{I S I}=-\int_{0}^{t}\left[\rho _ { \text { ref } } ( t ^ { \prime } ) \cdot \frac { d } { d t } \sum _ { i = 1 } ^ { N _ { V } } \left[P_{i}^{r} \cdot \sum_{\vec{c}_{*}^{i} \in \mathcal{C}^{i}}\left(P_{i}\left(t-t^{\prime} \mid \vec{c}_{*}^{i}, 1\right) \cdot \bar{P}_{i}\left(t^{\prime}, \vec{c}_{*}^{i}, 1\right)\right) .\right.\right. \\
\left.\left.\cdot \prod_{j=1 \neq i}^{N_{V}} \sum_{\vec{c}_{*}^{j} \in \mathcal{C}^{j}} \sum_{v_{* j}=1}^{n_{V}(j)}\left(P_{j}\left(t-t^{\prime} \mid \vec{c}_{*}^{j}, v_{* j}\right) \cdot \bar{P}_{j}^{s}\left(\vec{c}_{*}^{j}, v_{* j}\right)\right)\right]\right] d t^{\prime},
\end{array}
$$

where

$$
\begin{aligned}
P_{i}\left(t-t^{\prime} \mid \vec{c}_{*}^{i}, v_{* i}\right) & =\sum_{\vec{c}^{i} \in \mathcal{C}^{i}} \sum_{v_{i}=1}^{n_{V}(i)} P_{i}\left(t-t^{\prime}, \vec{c}^{i}, v_{i} \mid \vec{c}_{*}^{i}, v_{* i}\right) \\
\bar{P}_{i}\left(t^{\prime}, \vec{c}_{*}^{i}, v_{* i}\right) & =\sum_{\vec{c}_{0}^{i} \in \mathcal{C}^{i}} \sum_{v_{0 i}=1}^{n_{V}(i)} \bar{P}_{i}\left(t^{\prime}, \vec{c}_{*}^{i}, v_{* i} \mid \vec{c}_{0}^{i}, v_{0 i}\right) \cdot P_{0, i}^{s}\left(\vec{c}_{0}^{i}, v_{0 i}\right) .
\end{aligned}
$$


The statistical moments of ISI, denoted by $\left\langle t^{m}\right\rangle_{I S I}$ have to be calculated directly:

$$
\left\langle t^{m}\right\rangle_{I S I}=\int_{0}^{+\infty}\left(t^{m} \cdot \rho_{I S I}(t)\right) d t=m \cdot \int_{0}^{+\infty}\left(t^{m-1} \cdot P_{I S I}(t)\right) d t .
$$

The fact that $\rho_{\text {ref }}(t)=H\left(t-t_{A}\right) \cdot \tau_{R}^{-1} \cdot e^{-\left(t-t_{A}\right) / \tau_{R}}$ guarantees that the convolution integrals in Eqs. (4.26) - (4.27) can be calculated analytically, whenever the expressions for $P\left(t, \vec{c}, \vec{v} \mid \vec{c}_{0}, \vec{v}_{0}\right)$ and $\bar{P}\left(t, \vec{c}, \vec{v} \mid \vec{c}_{0}, \vec{v}_{0}\right)$ are known. This follows from the fact that $P\left(t, \vec{c}, \vec{v} \mid \vec{c}_{0}, \vec{v}_{0}\right)$ and $\bar{P}\left(t, \vec{c}, \vec{v} \mid \vec{c}_{0}, \vec{v}_{0}\right)$ can be expressed as linear combinations of functions $t^{\alpha_{i}} \cdot e^{\lambda_{i} \cdot t}, t^{\alpha_{i}} \cdot \cos \left(\lambda_{i} \cdot t\right)$, or $t^{\alpha_{i}} \cdot \sin \left(\lambda_{i} \cdot t\right)$.

\subsubsection{The limit of fast $\mathrm{Ca}^{2+}$ channel gating}

Eqs. (4.4) and (4.6) can be considerably reduced if the gating rates of presynaptic $\mathrm{Ca}^{2+}$ channels are much faster than the $\mathrm{Ca}^{2+}$-binding/unbinding rates to the $\mathrm{Ca}^{2+}$ sensor of exocytosis. To show this, we first note that

$$
\bar{P}\left(t, \vec{c}, \vec{v} \mid \vec{c}_{0}, \vec{v}_{0}\right)=\bar{P}\left(t, \vec{c} \mid \vec{v}, \vec{c}_{0}, \vec{v}_{0}\right) \cdot \bar{P}\left(t, \vec{v} \mid \vec{c}_{0}, \vec{v}_{0}\right)
$$

which applies in general. Next, let us assume that the change of $\bar{P}\left(t, \vec{c}, \vec{v} \mid \vec{c}_{0}, \vec{v}_{0}\right)$ due to transitions between any substates $\vec{v}$ and $\vec{v}^{\prime}$ is much smaller than due to transitions between any substates $\vec{c}$ and $\vec{c}$ at any moment $t$. If it is so, then the presynaptic channel subsystem reaches and stays in a steady state before any considerable changes in $\bar{P}\left(t, \vec{c}, \vec{v} \mid \vec{c}_{0}, \vec{v}_{0}\right)$ happen due to the $\vec{v} \rightarrow \vec{v}^{\prime}$ transitions. Taking this into account, and the fact that the presynaptic channels are independent of any other channel or vesicular release site, we can state that

$$
\bar{P}\left(t, \vec{c} \mid \vec{v}, \vec{c}_{0}, \vec{v}_{0}\right) \approx \prod_{i=1}^{N_{C}} \bar{P}_{i}^{s}\left(c_{i}\right), \quad \text { for } t \gg \tau_{c} .
$$

Here, $\bar{P}_{i}^{s}\left(c_{i}\right)$ is the stationary probability to find the $i$-th channel in state $c_{i} . \tau_{c}$ is the characteristic equilibration time of the presynaptic channel subsystem. $\bar{P}_{i}^{s}\left(c_{i}\right)$ is the solution of the following stationary master equation:

$$
0=\sum_{c_{i}^{\prime}=1}^{n_{C}(i)} k_{c_{i}^{\prime} \rightarrow c_{i}}^{C i} \cdot \bar{P}_{i}^{s}, \quad i=\overline{1, N_{C}}
$$

It follows then from Eqs. (4.30) and (4.31) that

$$
\bar{P}\left(t, \vec{c}, \vec{v} \mid \vec{c}_{0}, \vec{v}_{0}\right)=\bar{P}\left(t, \vec{v} \mid \vec{v}_{0}\right) \cdot \prod_{i=1}^{N_{C}} \bar{P}_{i}^{s}\left(c_{i}\right), \quad \text { for } t \gg \tau_{c} .
$$

The term $\bar{P}\left(t, \vec{v} \mid \vec{v}_{0}\right)$ in Eq. (4.33) is not known. However, a closed set of equations can be derived for it. This is achieved by inserting (4.33) into (4.4) and summing both sides of the equation with respect to $\vec{c}$ over $\mathcal{C}$, which gives

$$
\partial_{t} \bar{P}\left(t, \vec{v} \mid \vec{v}_{0}\right)=\sum_{j=1}^{N_{V}} \sum_{v_{j}^{\prime}=1}^{n_{V}(j)} k_{v_{j}^{\prime} \rightarrow v_{j}}^{V j} \cdot \bar{f}_{v_{j}^{\prime} \rightarrow v_{j}}^{j} \cdot \bar{P}\left(t, v_{j}^{\prime}, \vec{v}_{-j} \mid \vec{v}_{0}\right)
$$


where $\bar{f}_{v_{j}^{\prime} \rightarrow v_{j}}^{j}=\sum_{\vec{c} \in \mathcal{C}} f_{\vec{c}, v_{j}^{\prime} \rightarrow v_{j}}^{j} \cdot \prod_{i=1}^{N_{C}} \bar{P}_{i}^{s}\left(c_{i}\right)$. Once the solutions of Eqs. (4.32) and (4.34) are found, the solution of Eq. (4.4) for $t \gg \tau_{c}$ is determined by Eq. (4.33). Likewise, we can reduce Eq. (4.6) to

$$
\partial_{t} P\left(t, \vec{v} \mid \vec{v}_{0}\right)=\sum_{j=1}^{N_{V}} \sum_{v_{j}^{\prime}=1}^{n_{V}(j)} k_{v_{j}^{\prime} \rightarrow v_{j}}^{V j} \cdot \hat{f}_{v_{j}^{\prime} \rightarrow v_{j}}^{j} \cdot P\left(t, v_{j}^{\prime}, \vec{v}_{-j} \mid \vec{v}_{0}\right),
$$

with $\hat{f}_{v_{j}^{\prime} \rightarrow v_{j}}^{j}=\bar{f}_{v_{j}^{\prime} \rightarrow v_{j}}^{j} \cdot\left(1-\delta_{v_{j}, 1} \cdot \delta_{v_{j}^{\prime}, n_{V}(j)}\right)$. Then, accordingly,

$$
P\left(t, \vec{c}, \vec{v} \mid \vec{c}_{0}, \vec{v}_{0}\right)=P\left(t, \vec{v} \mid \vec{v}_{0}\right) \cdot \prod_{i=1}^{N_{C}} \bar{P}_{i}^{s}\left(c_{i}\right), \quad \text { for } t \gg \tau_{c} .
$$

Solutions of Eqs. (4.34) and (4.35) contain all information necessary to fully characterize the point process of vesicle fusion at the AZ. Taking into account that the vesicular release sites are independent, the solutions of (4.34) and (4.35) can be factorized: $\bar{P}\left(t, \vec{v} \mid \vec{v}_{0}\right)=\prod_{i=1}^{N_{V}} \bar{P}_{i}\left(t, v_{i} \mid v_{i 0}\right)$, $P\left(t, \vec{v} \mid \vec{v}_{0}\right)=\prod_{i=1}^{N_{V}} P_{i}\left(t, v_{i} \mid v_{i 0}\right)$.

It follows from the definitions of $f_{\vec{c}, v_{j}^{\prime} \rightarrow v_{j}}^{j}$ and $\bar{P}\left(c_{i}\right)$ that $\bar{f}_{v_{j}^{\prime} \rightarrow v_{j}}^{j}$ is equal to the average [Ca ${ }^{2+}$ ] at vesicular site $j$ for transitions $v_{j}^{\prime} \rightarrow v_{j}$ which depend on $\mathrm{Ca}^{2+} \cdot \bar{f}_{v_{j}^{\prime} \rightarrow v_{j}}^{j}=1$ for transitions $v_{j}^{\prime} \rightarrow v_{j}$ which do not depend on $\mathrm{Ca}^{2+}$. If so, then Eqs. (4.34) and (4.35) are identical to Eqs. (4.4) and (4.6) written for the $\mathrm{Ca}^{2+}$-microdomain coupling scenario! In other words, if the channel gating is fast enough, the vesicle fusion dynamics is independent of the AZ topography, as long as the average $\left[\mathrm{Ca}^{2+}\right]$ at the $\mathrm{Ca}^{2+}$ sensors of exocytosis is the same. One of the consequences of this finding is that vesicle fusion dynamics at $\mathrm{AZs}$ with $\mathrm{Ca}^{2+}$-microdomain coupling forms a subset of the repertoire of the dynamics of other AZ scenarios.

Quantitatively, the requirements for the fast channel gating limit are the following. First, for any $\mathrm{Ca}^{2+}$-dependent transition $v_{j}^{\prime} \rightarrow v_{j}$,

$$
\tau_{c}^{-1} \gg k_{v_{j}^{\prime} \rightarrow v_{j}}^{V j} \cdot \sum_{i=1}^{N_{C}} \Delta\left[\mathrm{Ca}^{2+}\right]_{i j}^{\mathrm{s}} .
$$

Second, if the rate of the transition $v_{j}^{\prime} \rightarrow v_{j}$ is $\mathrm{Ca}^{2+}$-dependent, the backward rate has to be much smaller than $\tau_{c}^{-1}$, independent of its $\mathrm{Ca}^{2+}$-dependence,

$$
\tau_{c}^{-1} \gg k_{v_{j} \rightarrow v_{j}^{\prime}}^{V j} \cdot\left(1+\left(\sum_{i=1}^{N_{C}} \Delta\left[\mathrm{Ca}^{2+}\right]_{i j}^{\mathrm{s}}-1\right) \cdot \delta_{v_{j} \rightarrow v_{j}^{\prime}, \mathrm{Ca}^{2+}}\right) .
$$

Third, if both the backward and the forward transition rates are independent of $\mathrm{Ca}^{2+}$, then the magnitudes of these transition rates do not affect the validity of the fast channel gating approximation. The exact expressions constraining parameters $k_{c_{i}^{\prime} \rightarrow c_{i}}^{C i}$ and $k_{v_{j}^{\prime} \rightarrow v_{j}}^{V j}$ in the limit of fast channel gating depend on the kinetic model of the channel gating. For example, for a two-state $\mathrm{Ca}^{2+}$ channel, $\tau_{c}^{-1}=k_{+1}+k_{-1}$. Thus, if either of $k_{+1}$ or $k_{-1}$ is much higher than the right hand side terms of Eqs. (4.37) and (4.38), the fast channel gating approximation is valid. Finally, it has to be noted that this approximation works even if the aforementioned requirements are not satisfied, but $p_{o}$ is sufficiently close to 1 . Indeed, the channels stay open nearly all the time and $\left[\mathrm{Ca}^{2+}\right]$ is fixed then. 


\subsection{Results}

Results are organized as follows. Sections 4.4.1 and 4.4.2 are dedicated for analyzing how the organization of presynaptic AZs influences the vesicle release process. $\rho_{I E I}(t)$ and $C_{I E I}(l)$ functions of presynaptic vesicle release responses at fixed $V_{m}$ as well as vesicle release rate dependencies on $V_{m}$ are examined there. Section 4.4.1 deals with simplified synapses consisting of a single vesicular release site and lays the foundation for section $\mathbf{4 . 4 . 2}$, where presynaptic sites with multiple vesicular release sites are studied. We first analyze responses of 2-state vesicular release sites and then continue with $n_{V}$-state sites. Despite the fact that the 2-state sites are not biophysically realistic, they are relatively simple to understand and facilitate the evaluation of the properties of more complex features of the $n_{V}$-state sites. As required by the nature of the problem (heterogeneity), we scan a large parameter space instead of concentrating on certain fixed parameter values in order to properly understand the relation between the structure of presynaptic AZs and their responses. Section 4.4.3 considers how the vesicle release process at the presynaptic site is translated into spiking at the postsynaptic site. Finally, in section 4.4.4, previously gained understanding is applied to describe experimental data and to evaluate the hypothesis of the presynaptic origin of SGN response heterogeneity. There, we focus on the rate-level functions as well as ISI distributions and correlations.

NB The equation and figure labels which start with "S" in the text below refer to the material which is provided in the supplementary sections. The numbers which follow symbol " $\mathrm{S}$ " in the labels refer to the numbers of the supplementary sections. Also, please note the list of abbreviations and parameter definitions included at the very beginning of this chapter.

\subsubsection{Response properties of single vesicular release sites}

\section{Inter-event interval distributions}

$\rho_{I E I}(t)$ functions were calculated by evaluating Eq. (4.23) for single vesicular release sites and specified AZ topographies. In order to facilitate understanding of the results, we used graphical schemes which define the underlying kinetic equations. In these schemes, as shown in Figs. 4.3A and $4.4 \mathrm{C}$, open and filled circles represent the states of the system. The initial states of the system, i.e., the states possible just after a particular vesicle release event, are shown as filled circles. Curly lines mark transitions associated with vesicle release events, while regular lines corresponded to the remaining transitions. Taking into account the magnitudes of the transition rates, the kinetic schemes allow for a quick semiquantitative estimation of the properties of time patterns of vesicle release events.

2-state vesicular release site, $\mathbf{C a}^{2+}$-microdomain coupling A 2-state vesicular release site in $\mathrm{Ca}^{2+}$-microdomain coupling with $\mathrm{Ca}^{2+}$ channels is described by only two states: the release site is filled with a vesicle which is ready to release $(v=1)$, or the release site is empty 
$(v=0)\left(\right.$ case $\left[1^{*}\right]$ in Fig. 4.3A). The resulting $\rho_{I E I}(t)$ function and $C V_{I E I}$ are given by

$$
\rho_{I E I}(t)=\frac{k_{r e l} \cdot p_{o} \cdot k_{r e p}}{k_{r e l} \cdot p_{o}-k_{r e p}} \cdot\left(e^{-k_{r e p} \cdot t}-e^{-k_{r e l} \cdot p_{o} \cdot t}\right), \quad C V_{I E I}=\frac{\sqrt{\left(k_{r e l} \cdot p_{o}\right)^{2}+k_{r e p}^{2}}}{k_{r e l} \cdot p_{o}+k_{r e p}} .
$$

It follows from the above expressions that the most regular release patterns generated by such a system are observed when the vesicle fusion and replenishment rates are equal: $k_{r e p}=$ $k_{r e l} \cdot p_{o}$. Then, $C V_{I E I} \equiv \min \left[C V_{I E I}\right]=\sqrt{2}^{-1}$, and the $\rho_{I E I}(t)$ function corresponds to a gamma distribution with shape factor 2 (Fig. 4.4A, light blue line). In the limit when one of the rates, $k_{r e p}$ or $k_{r e l} \cdot p_{o}$, is much larger than the other, the release converges to a homogeneous Poisson point process. The IEIs are distributed exponentially (Fig. 4.4A, grey line) and the release rate is given by the lower of the two rates (see Eq. S3.1). These limiting cases correspond to the most irregular event patterns produced by a 2 -state vesicular release site in $\mathrm{Ca}^{2+}$-microdomain coupling with presynaptic $\mathrm{Ca}^{2+}$ channels. $C V_{I E I} \equiv \max \left[C V_{I E I}\right]=1$ then.

2-state vesicular release site, $\mathbf{C a}^{2+}$-nanodomain coupling A 2-state vesicular release site driven by a single $\mathrm{Ca}^{2+}$ channel has a richer repertoire of vesicle release patterns than its counterpart discussed above (case [1] in Fig. 4.3A). The general expressions of $\rho_{I E I}(t)$ and $C V_{I E I}$ of this system are given by Eqs. (S2.74) and (S2.81). To analyze all possible response scenarios, we scanned the space of the four parameters which define the model, i.e., $k_{-1}, k_{-1}$, $k_{r e p}$, and $k_{r e l}$. In particular, we considered multiple limits of the underlying kinetic equations and their solutions according to six ratios: $k_{1} / k_{r e l}, k_{-1} / k_{r e l}, k_{r e p} / k_{r e l}, k_{1} / k_{-1}, k_{1} / k_{r e p}, k_{1} / k_{r e p}$. All possible cases when any of these ratios goes to either 0,1 , or $+\infty$ and are consistent to each other were taken into account. The expressions of $\rho_{I E I}(t),\left\langle t_{I E I}\right\rangle$, and $C V_{I E I}$ in those limiting cases are provided in Supplementary Material 3.2. The corresponding kinetic schemes and representative examples of the vesicle release and $\mathrm{Ca}^{2+}$ channel gating patterns are shown in Fig. 4.3.

All the limiting cases of the $\rho_{I E I}(t)$ functions can be divided into four groups marked by different colors in Fig. 4.3A. We assigned to the first group all those cases when the vesicular release sites work in the limit of fast channel gating (blue panels in Fig. 4.3A). This requirement is fulfilled when at least one of $k_{-1}$ and $k_{+1}$ is much larger than $k_{r e l}$, or $k_{+1} \gg k_{-1}$, i.e., $p_{o} \sim 1$. As it was shown in section 4.3.3, the vesicle release point process is then identical to that at an equivalent vesicular release site which features $\mathrm{Ca}^{2+}$-microdomain coupling with presynaptic channels. When $k_{-1} \gg k_{r e l}$, the channel closes and opens many times before a docked vesicle fuses, independent of $k_{+1}$ (Fig. 4.3B). Thus the $\mathrm{Ca}^{2+}$ sensor of exocytosis "senses" time averaged $\left[\mathrm{Ca}^{2+}\right]$. On the other hand, if $p_{o} \sim 1$, the channel stays open nearly all the time, thus $\left[\mathrm{Ca}^{2+}\right]$ is fixed.

The second group of the limiting cases is covered by a requirement that $\left(k_{+1}, k_{r e p}\right) \ll k_{-1}$, and $k_{-1} \lesssim k_{\text {rel }}$ (green panels [2-6] in Fig. 4.3A). We called the vesicle release process in these cases as "channel opening limited". In the limiting case [2] $\left(k_{+1} \ll k_{r e p}\right.$ and $\left.k_{-1} \ll k_{r e l}\right)$, each 
A

A
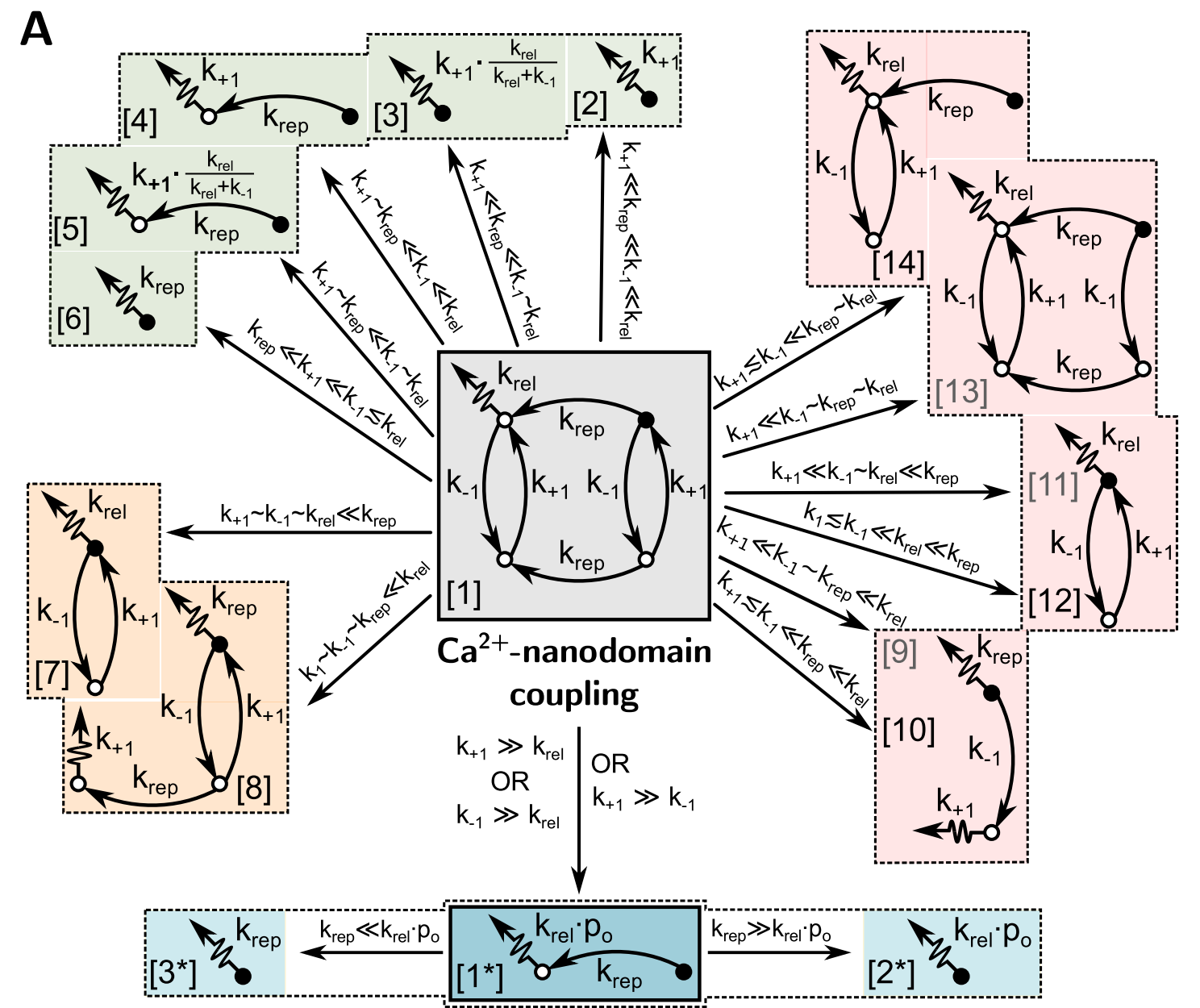

\section{$\mathrm{Ca}^{2+}$-microdomain coupling}

\section{B}

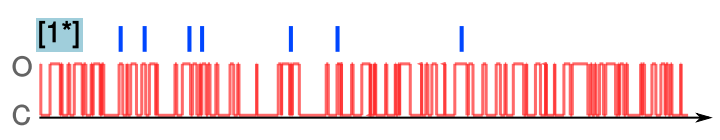

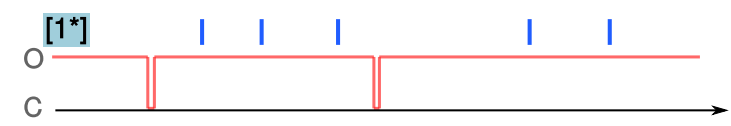
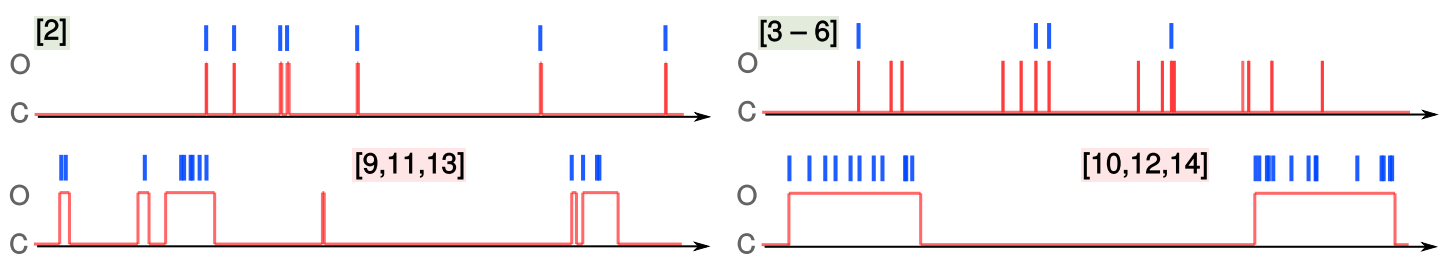

Figure 4.3: Properties of presynaptic vesicle fusion dynamics at single 2-state vesicular release sites. (A) Schemes of the kinetic equations determining the dynamics of a single vesicular release site. The grey and the darker blue panels correspond to the generic schemes of $\mathrm{Ca}^{2+}$-nanodomain and $\mathrm{Ca}^{2+}$-microdomain coupling scenarios, respectively. Limiting cases thereof are shown by panels of other colors. (B) Representative examples of $\mathrm{Ca}^{2+}$ channel gating (red line) and presynaptic vesicle release event (blue vertical bars) time patterns in different limiting cases of a 2-state site shown in (A). Labels "C" and "O" mark, respectively, closed and open conductance states of the channel. 
channel opening induces a single vesicle release event (Fig. 4.3B). When $k_{-1} \sim k_{\text {rel }}$ instead of $k_{-1} \ll k_{\text {rel }}$ (case [3]), only a fraction $k_{-1} /\left(k_{-1}+k_{\text {rel }}\right)$ of channel openings leads to single vesicle release events (Fig. 4.3B). If $k_{+1} \sim k_{\text {rep }}$ (cases [4] and [5]), vesicle release rate is limited not only by $k_{+1}$ but also by $k_{r e p}$. Then, each vesicle release event corresponds to an individual channel opening as in case [3], but even fewer openings are successful. Finally, if $k_{+1} \gg k_{r e p}$ (case [6]), most of the channel openings lead to no vesicle release because of the absence of a vesicle at the release site. The vesicle release rate is limited purely by $k_{r e p}$ then. Limiting cases [2], [3], and [6] are described by a homogeneous Poisson process (Eq. S3.2). Whereas limiting cases [4] and [5] have identical statistical properties as the release process in the limit of fast channel gating (Eq. S3.3). In fact, the limiting cases [4 -5] together with [1*] correspond to the minimum possible $C V_{I E I}=\sqrt{2}^{-1}$ in the case of a 2-state vesicular release site driven by a 2-state $\mathrm{Ca}^{2+}$ channel.

We identified the third group of the limiting cases (red panels [9-14] in Fig. 4.3A) on the basis of bursty vesicle release patterns (see Fig. 4.3B). Then, differently from the two previously considered groups, $C V_{I E I}>1$ (see Supplementary Material 3.2). The bursty character of vesicle release is established by very fast $k_{r e p}$ and $k_{r e l}$ compared to $k_{-1}$ and $k_{+1}$. Cases [9], [11], and [13] lead to what we called "weak bursts", while [10], [12], and [14] give rise to "strong bursts". To understand these response scenarios better, let us consider cases [9] and [10] as two representatives in a more detail. The $\rho_{I E I}(t)$ function and $C V_{I E I}$ are then given by

$$
\rho_{I E I}(t)=\frac{k_{1} \cdot k_{-1}}{k_{-1}+k_{r e p}} \cdot e^{-k_{+1} \cdot t}+k_{r e p} \cdot e^{-\left(k_{-1}+k_{r e p}\right) \cdot t}, \quad C V_{I E I}=\frac{\sqrt{k_{-1} \cdot\left(k_{-1}+2 \cdot k_{r e p}\right)}}{k_{+1}+k_{-1}} .
$$

The first exponential term has a vanishing contribution to $\rho_{I E I}(t)$ in the above expression. However, this term contributes appreciably either to the integral of $\rho_{I E I}(t)$ (case [9]) or, at least, to the first and the second moments (both, [9] and [10]). To see this, let us write the integral and the first two moments of $\rho_{I E I}(t)$ explicitly

$$
\begin{aligned}
& \left\langle t^{0}\right\rangle_{I E I}=\underbrace{\frac{k_{-1}}{k_{-1}+k_{r e p}}+\underbrace{\frac{k_{r e p}}{k_{-1}+k_{r e p}},}_{T_{0,2}}, \frac{T_{0,1}}{T_{0,2}}=\frac{k_{-1}}{k_{r e p}},}_{T_{0,1}} \\
& \left\langle t^{1}\right\rangle_{I E I}=\underbrace{\frac{k_{-1}}{k_{1} \cdot\left(k_{-1}+k_{r e p}\right)}+\underbrace{\frac{k_{r e p}}{\left(k_{-1}+k_{r e p}\right)^{2}}}_{T_{1,2}}, \quad \frac{T_{1,1}}{T_{1,2}}=\frac{k_{-1} \cdot\left(k_{-1}+k_{r e p}\right)}{k_{1} \cdot k_{r e p}},}_{T_{1,1}} \\
& \left\langle t^{2}\right\rangle_{I E I}=\underbrace{\frac{2 \cdot k_{-1}}{k_{1}^{2} \cdot\left(k_{-1}+k_{r e p}\right)}}_{T_{2,1}}+\underbrace{\frac{2 \cdot k_{r e p}}{\left(k_{-1}+k_{r e p}\right)^{3}}}_{T_{2,2}}, \quad \frac{T_{2,1}}{T_{2,2}}=\frac{k_{-1} \cdot\left(k_{-1}+k_{r e p}\right)^{2}}{k_{1}^{2} \cdot k_{r e p}} .
\end{aligned}
$$

It follows from the above expressions that terms $T_{0,1}$ and $T_{0,2}$ are of the same order of magnitude in case [9]. Moreover, the slower exponential term dominates in $\left\langle t^{1}\right\rangle_{I E I}$ and $\left\langle t^{2}\right\rangle_{I E I}$ then: $T_{1,1} \gg T_{1,2}$ and $T_{2,1} \gg T_{2,2}$. Note that $T_{1,2} \sim T_{1,1}^{2}$, thus, $C V$ is finite. In particular, when $k_{-1}=k_{\text {rep }}, C V_{I E I}=\sqrt{3} \approx 1.7$. The release event pattern consists of randomly mixed short and long, exponentially distributed, IEIs in case [9] (see Fig. 4.3B). The averages of those short 
and long IEIs are $k_{\text {rep }}^{-1}$ and $k_{+1}^{-1}$, respectively. The ratio of the numbers of the short and long IEIs is $k_{r e p} / k_{-1} \sim 1$. The only difference between cases [9] and [10] is that $k_{r e p} / k_{-1} \gg 1$ in the later one. Thus the number of the short IEIs is much larger than the long ones and the bursts are "longer" then. Moreover, in case [10], $T_{1,1} \gtrsim T_{1,2}, T_{2,1} \gg T_{2,2}$, and $T_{2,1} \gg T_{1,1}^{2}$, which means that $C V \gg 1$. If $k_{r e p} / k_{-1} \rightarrow+\infty$, so does $T_{2,1} /\left(T_{1,1}\right)^{2}$ and, in turn, $C V_{I E I} \rightarrow+\infty$ ! This result means that $C V_{I E I}$ can be made arbitrarily large in the limiting case [10]. Limiting cases $[11-12]$ and $[13-14]$ are essentially equivalent to $[9-10]$. The only difference is that the short intra-burst IEIs are determined by $k_{r e l}$ in cases $[11-12]$ and by both, $k_{r e p}$ and $k_{r e l}$, in cases $[13-14]$. The corresponding expressions of $\rho_{I E I}(t), C V_{I E I}$, and $\langle t\rangle_{I E I}$ are given in Supplementary Material 3.2. $C V_{I E I}$ is finite in cases [11] and [13], and unbound from above in cases [12] and [14].

Vesicle release patterns in limiting cases [7] and [8], which belong to the last, fourth, identified group, are intermediate between the first two groups (cases $\left[1^{*}-3^{*}\right]$ and $[2-6]$ ) and the third group (cases $[9-14])$. Dependent on the exact values of the parameters, $C V_{I E I}$ can be either slightly $<1$ or $>1$ then (see Eqs. (S.3.6) and (S.3.9)).

$\boldsymbol{n}_{\boldsymbol{V}}$-state vesicular release site, $\mathbf{C a}^{2+}$-microdomain coupling The kinetic scheme $\left[1_{m}^{*}\right]$ in Fig. $4.4 \mathrm{C}$ corresponds to a generic $n_{V}$-state vesicular release site in $\mathrm{Ca}^{2+}$-microdomain coupling with presynaptic ion channels. To understand how the additional $\mathrm{Ca}^{2+}$-binding steps compared to the 2-state model change the time patterns of vesicle release events, we first minimized $C V_{I E I}$. The numerical global optimization showed that a necessary condition for $\min \left[C V_{I E I}\right]$ is a much faster $\mathrm{Ca}^{2+}{ }^{2}$-binding than unbinding rate at any step (limiting case $\left[2_{m}^{*}\right]$ ). As demonstrated in Supplementary Material 3.3, the $\rho_{I E I}(t)$ function and $C V_{I E I}$ are then given by

$$
\rho_{I E I}(t)=\sum_{j=1}^{m+2} \frac{k_{j}^{-m}}{\prod_{i=1 \neq j}^{m+2}\left(k_{j}^{-1}-k_{i}^{-1}\right)} \cdot e^{-t \cdot k_{j}}, \quad C V_{I E I}=\frac{\sqrt{r_{1}^{-2} \cdot \sum_{i=1}^{m} i^{-2}+r_{m+1}^{-2}+r_{0}^{-2}}}{r_{1}^{-1} \cdot \sum_{i=1}^{m} i^{-1}+r_{m+1}^{-1}+r_{0}^{-1}},
$$

where $r_{1}=k_{r e p}, r_{j}=(j-1) \cdot k_{r e l}$ with $1<j<m+2$, and $r_{m+2}=\gamma . C V_{I E I}$ in the above expression is minimized at a chosen $m$ when $k_{r e p}=\gamma=k_{r e l} \cdot \sum_{i=1}^{m} i^{-1} / \sum_{i=1}^{m} i^{-2}$ (see Supplementary Material 3.3). The $\min \left[C V_{I E I}\right]$ is a monotonically decreasing function of $m$ (Fig. 4.4B). Thus, $\mathrm{Ca}^{2+}$ sensors of exocytosis with more binding sites allow for more regular release. The higher regularity of IEIs is reflected by more localized $\rho_{I E I}$ functions (see case $\left[2_{m}^{*}\right]$ with $m=5$ in Fig. $4.4 \mathrm{~A})$.

The fact that $\min \left[C V_{I E I}\right]$ decreases with $m$ explains why the lowest values of $C V_{I E I}$ are attained at negligible $k_{o f f} \cdot b^{i}$. This is well illustrated by limiting case [ $\left.3_{m}^{*}\right]$ (Fig. 4.4C). Then, rates of the first $j \mathrm{Ca}^{2+}$-binding steps are much slower than the corresponding unbinding rates, whereas the opposite is true for all the subsequent $\mathrm{Ca}^{2+}$-binding steps. From the perspective of state $S_{j+3}$, all states from $S_{2}$ to $S_{j+2}$ are in a quasi-equilibrium. Thus, the transition between $S_{2}$ and $S_{j+3}$ is reduced to a single jump with an effective rate $(m-j) \cdot k_{r e l} \cdot \bar{P}_{j+2}$. Here, $\bar{P}_{j+2}$ is a 
A

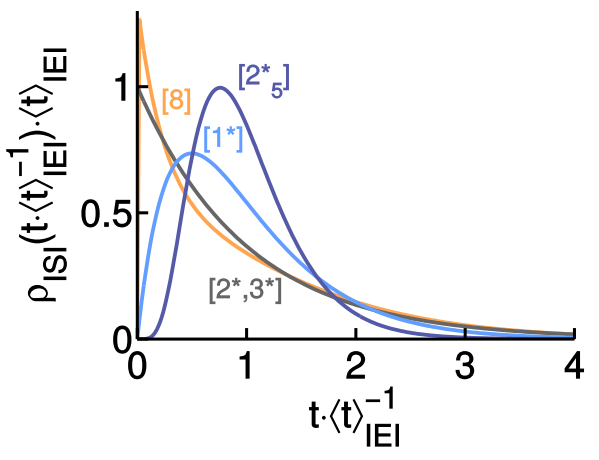

B

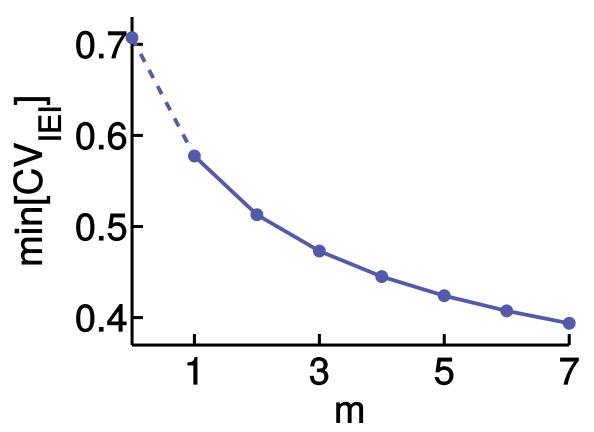

C

\section{$\mathrm{Ca}^{2+}$-microdomain coupling}

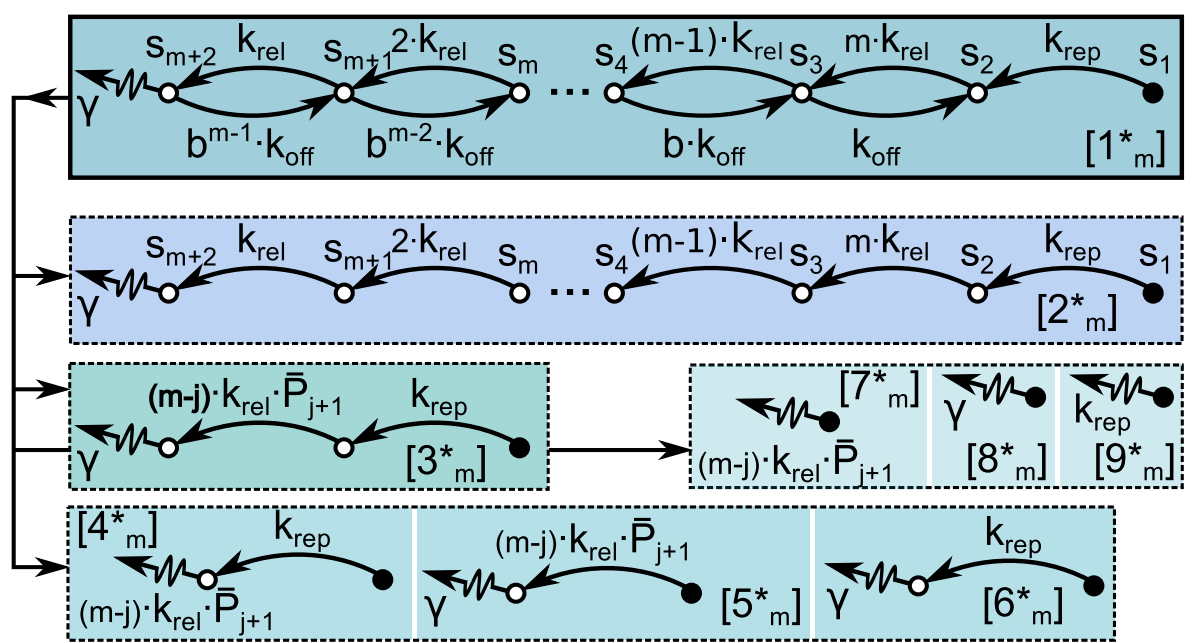

Figure 4.4: Properties of $\rho_{I E I}(t)$ functions and vesicle release dynamics at single 2-state and $\boldsymbol{n}_{\boldsymbol{V}}$-state vesicular release sites. (A) $\rho_{I E I}(t)$ functions of several limiting cases of 2 -state and $n_{V}$-state vesicular release sites. (B) $\min \left[C V_{I E I}\right]$ as a function of the number of $\mathrm{Ca}^{2+}$-binding sites at the $\mathrm{Ca}^{2+}$-sensor of exocytosis. The point at $m=0$ corresponds to the 2 -state vesicular release site. (C) Schemes of the kinetic equations which describe the dynamics of a single vesicular release site. Diagram $\left[1_{m}^{*}\right]$ represents the general case. The remaining diagrams are particular limiting cases of $\left[1_{m}^{*}\right]$. 
steady state probability of state $S_{j+2}$ for a putative system consisting only of states $S_{2}-S_{j+2}$. It can be shown by a simple calculation that

$$
\bar{P}_{j+2}=\frac{\prod_{i=0}^{j-1} \frac{(n-i) \cdot k_{r e l}}{b^{i} \cdot k_{o f f}}}{\sum_{l=1}^{j+1} \prod_{i=0}^{l-2} \frac{(n-i) \cdot k_{r e l}}{b^{i} \cdot k_{o f f}}} .
$$

$\bar{P}_{j+2} \ll 1$, because $\mathrm{Ca}^{2+}$-binding is much slower than unbinding within the subsystem $S_{2}-S_{j+2}$. Thus, the largely irreversible $\mathrm{Ca}^{2+}$-binding rates from state $(j+3)$ onwards are much faster than the effective transition rate between states $S_{2}$ and $S_{j+3}:(m-j-1) \cdot k_{r e l} \gg(m-j) \cdot k_{r e l} \cdot \bar{P}_{j+2}$. This leads to the reduction of $\left[1_{m}^{*}\right]$ to $\left[3_{m}^{*}\right]$. The later has fewer effective forward transitions than $\left[2_{m}^{*}\right]$, which means that vesicle release is less regular then. In a less extreme case, the $\mathrm{Ca}^{2+}$ unbinding may not be much faster than binding. Then, equilibration of the system between states $S_{1}$ and $S_{m+1}$ is only partial. But even in this case $C V_{I E I}>\min \left[C V_{I E I}\right]$. For example, assuming the experimental estimates of average values of $m, k_{o n}, k_{o f f}, b$, and $\gamma$ (see section $4.2 .2),\left[\mathrm{Ca}^{2+}\right]=50 \mu \mathrm{M}$, and $k_{r e p}$ two times faster than the average vesicle release rate in a steady state, $C V_{I E I}=0.56$. This is by more than 0.1 larger than the minimum possible value (see Fig. 4.4B, $m=5$ ).

The numerical global optimization showed that $\max \left[C V_{I E I}\right]=1$ for $n_{V}$-state vesicular release sites, the same as for 2 -state sites. $C V_{I E I}=1$ is observed when one of the rates in the generic scheme $\left[1_{m}^{*}\right]$ limits the overall release rate. Limiting cases $\left[7_{m}^{*}\right],\left[8_{m}^{*}\right]$, and $\left[9_{m}^{*}\right]$ shown in Fig. 4.4 are a few of the possible examples. Various other limiting cases are possible which correspond to $\min \left[C V_{I E I}\right]<C V_{I E I}<1$, as, for example, cases $\left[4_{m}^{*}\right]$, $\left[5_{m}^{*}\right]$, and $\left[6_{m}^{*}\right]$. In conclusion, the increase in the number of $\mathrm{Ca}^{2+}$-binding steps decreases the lower bound for $C V_{I E I}$. How much the actual $C V_{I E I}$ is close to this limit, however, depends on the values of the rate parameters.

$n_{V}$-state vesicular release site, $\mathrm{Ca}^{2+}$-nanodomain coupling Properties of the time patterns of presynaptic vesicle release events for this scenario are largely predictable from our previous results. Indeed, in the limit of fast $\mathrm{Ca}^{2+}$ channel gating, or when $p_{o}=1$, the vesicle release dynamics is identical to that at an $n_{V}$-state vesicular release site in $\mathrm{Ca}^{2+}$-microdomain coupling regime. We showed by global numerical optimization that $\min \left[C V_{I E I}\right]$ for the $n_{V}$-state vesicular site driven by one $\mathrm{Ca}^{2+}$ channel is observed in the fast channel gating limit. Thus, it is the same as for the equivalent vesicular release site with $\mathrm{Ca}^{2+}$-microdomain coupling discussed before. In the case of the channel opening limited release regime, response properties of an $n_{V}$-state vesicular release site is essentially the same as of a 2 -state site. The main difference is that more regular release patterns can be produced by the 2-state site because of an additional irreversible transition defined by $\gamma$. This happens when $k_{+1}=k_{r e p}=\gamma$. The bursty release at $n_{V}$-state vesicular sites also has a lot of similarities to the 2 -state vesicular sites. The interburst times are defined by the channel opening rate in both those cases. The main difference is in the statistics of the intra-burst IEIs. Indeed, within the bursts, the release patterns are 
equivalent to the release patterns in the $\mathrm{Ca}^{2+}$-microdomain coupling regimes with equivalent vesicular release sites. Thus, intraburst IEIs can in principle be more regular for $n_{V}$-state than 2-state sites. This reduces overall $C V_{I E I}$ of the corresponding vesicle release pattern. However, this does not change the situation essentially. For both, 2-state and 7-state, vesicular release sites, $C V_{I E I}$ is higher than one. It is finite in the regime of weak bursty release, but is not bounded from above in the regime of strong bursty release.

$\boldsymbol{n}_{\boldsymbol{C}}$-state $\mathrm{Ca}^{2+}$ channels In a steady state, vesicle release at $\mathrm{AZs}$ with $\mathrm{Ca}^{2+}$-microdomain coupling scenario does not depend on the structure of the channel gating kinetic scheme. Indeed, $\left[\mathrm{Ca}^{2+}\right]$ at the $\mathrm{AZ}$ is constant in time and is determined by $p_{o}$ alone then. The same applies to AZs with any topography scenario in the limit of fast $\mathrm{Ca}^{2+}$ channel gating. In fact, we found by numerical global optimization that, for AZs with channels described by more than one closed state (Fig.4.1D), $\min \left[C V_{I E I}\right]$ is observed in the limit of fast channel gating. Interestingly, in the regime of channel opening limited vesicle release, the most regular release patterns are the same independent of the number of intermediate closed states of the channel. More specifically, we found that, in these cases, all the closed states are in equilibrium due to very fast switching between them. Thus, from the perspective of $\mathrm{Ca}^{2+}$-conductance, $n_{C}$-state channels behave identical to the 2-state channels. In the regimes of bursty release, additional closed states of the $\mathrm{Ca}^{2+}$ channels affect only interburst not the intraburst IEI statistics. Different from a 2-state channel, the interburst IEIs can be multiexponential, not only monoexponential, for $n_{C}$-state channels. This can increase the overall $C V_{I E I}$. It is possible that, for $n_{C}>2$, intraburst release is either channel opening limited or happens in the fast channel gating limit. Mathematically, however, these cases are equivalent to particular instances of the usual bursty release when the channel stays open within each burst.

\section{Inter-event interval correlations}

For 2-state and $n_{V}$-state vesicular release sites which feature $\mathrm{Ca}^{2+}$-microdomain coupling with presynaptic channels, the system returns to one and the same state after each vesicle fusion event (see Fig. 4.3A and Fig. 4.4C). Thus, taking into account that the underlying dynamics is Markovian, IEIs are uncorrelated, i.e., $C_{I E I}(l) \equiv 0$. Then, the vesicle release process is an ordinary renewal process and is fully characterized by the $\rho_{I E I}(t)$ function. The same applies to 2 -state, but not $n_{V}$-state, vesicular release sites in $\mathrm{Ca}^{2+}$-nanodomain coupling with presynaptic channels. Due to $\mathrm{Ca}^{2+}$-independent irreversible vesicle fusion step, the system may transit to $n_{C}$ different states in the later scenario. However, even then, $C_{I E I}(l) \neq 0$ if and only if $l=1$, as shown in Supplementary Material 4.1. That is, only the nearest neighbor IEIs are correlated. The minimum and maximum values of $C_{I E I}(1)$ which we found by numerical global optimization were 0 and $\approx 0.092$, respectively. $C_{I E I}(l)=0$ is achieved, for example, in the limit of fast channel gating. The maximum value of $C_{I E I}(l)$ is observed when $\left(k_{o n}, k_{\text {rep }}\right) \gg\left(k_{1} \sim k_{-1} \sim \gamma\right) \gg k_{\text {off }}$ and is the same for all $n_{C}=\overline{2,4}$ and $n_{V}=\overline{1,5}$, which we 
considered here (see Supplementary Material 4.1 for more details).

\section{Release rate dependence on the membrane potential}

2-state vesicular release site, $\mathrm{Ca}^{2+}$-microdomain coupling First, we assumed that $k_{r e p}$ is stimulus-independent. In this case, the release rate dependence on $V_{m}$, denoted by $R_{r}\left(V_{m}\right)$, is described by a Boltzmann function. Indeed, substituting Eq. (4.1) into Eq. (S2.55) and rearranging terms yields

$$
R_{r}\left(V_{m}\right)=\frac{A_{r}}{1+e^{-\left(V_{m}-V_{0.5}-S_{r}\right) / k_{V}}}
$$

where

$$
A_{r}=\frac{k_{r e l} \cdot \bar{p}_{o} \cdot k_{r e p}}{k_{r e l} \cdot \bar{p}_{o}+k_{r e p}} \leq \min \left[k_{r e l} \cdot \bar{p}_{o}, k_{r e p}\right], \quad S_{r}=k_{V} \cdot \ln \left(\frac{k_{r e p}}{k_{r e p}+k_{r e l} \cdot \bar{p}_{o}}\right) \leq 0 .
$$

Thus, for a 2-state vesicular release site with $\mathrm{Ca}^{2+}$-microdomain coupling regime, $R_{r}\left(V_{m}\right)$ is a scaled and left shifted version of the $p_{o}\left(V_{m}\right)$ function (Fig. $\left.4.5 \mathrm{~A}\right)$. The maximum release rate is set by $A_{r}$, meanwhile the shift is defined by $S_{r}$. The shift can formally be from 0 to $-\infty$ and it is the more negative the smaller is the ratio $k_{r e p} /\left(k_{r e l} \cdot \bar{p}_{o}\right)$. Interestingly, the dynamic ranges of $p_{o}\left(V_{m}\right)$ and $R_{r}\left(V_{m}\right)$ functions, denoted respectively by $\Delta_{V}\left[p_{o}\right]$ and $\Delta_{V}\left[R_{r}\right]$, are the same. For further use, we defined these quantities as the differences between $V_{m}$ values corresponding to $90 \%$ and $10 \%$ of the maximal $p_{o}$ and $R_{r}$, respectively. In general, when $k_{r e p} /\left(k_{r e l} \cdot \bar{p}_{o}\right)$ is decreased, the $R_{r}\left(V_{m}\right)$ is not only shifted left but also scaled down or up (dependent on how the absolute values of $k_{r e p}$ and $k_{r e l} \cdot p_{o}$ are altered, see blue vs green curves in Fig. 4.5A). Nevertheless, it is possible to change both, $k_{r e p}$ and $k_{r e l} \cdot \bar{p}_{o}$, in such a way that $R_{r}\left(V_{m}\right)$ is shifted but the amplitude stays the same (blue vs orange curves in Fig. 4.5A). Indeed, $A_{r}$ can be made arbitrary at any $S_{r}$ by scaling all the rate parameters by the same amount (see also Supplementary Material 5.1).

The possibility of an unlimited leftward shift of $R_{r}\left(V_{m}\right)$ functions upon reduction of $k_{r e p} /\left(k_{r e l}\right.$. $\left.\bar{p}_{o}\right)$ is closely related to the assumed independence of $k_{r e p}$ on $V_{m}$. Indeed, when $k_{r e p} /\left(k_{r e l} \cdot \bar{p}_{o}\right)$ is decreased, the replenishment becomes the rate limiting stage, and $R_{r}\left(V_{m}\right)$ saturates at more negative $V_{m}$. When both of $k_{r e l} \cdot \bar{p}$ and $k_{r e p}$ increase with $V_{m}$, the range of the shift of $R_{r}\left(V_{m}\right)$ with respect to $p_{o}\left(V_{m}\right)$ is considerably restricted (see Eq. (S5.6) and (Fig. 4.5C)). In this case, the $R_{r}\left(V_{m}\right)$ function is positioned between $k_{r e l} \cdot \bar{p}_{o}\left(V_{m}\right)$ and $k_{r e p}\left(V_{m}\right)$ on the abscissa axis. If $k_{r e l} \cdot \bar{p}_{o}\left(V_{m}\right)$ and $k_{r e p}\left(V_{m}\right)$ had identical dependencies on $V_{m}$ (independent of the amplitude), position of the $R_{r}\left(V_{m}\right)$ function on the $V_{m}$ axis would be fixed and would not depend on $k_{r e l} \cdot \bar{p}_{o}$ or the amplitude of $k_{r e p}\left(V_{m}\right)$ at all.

2-state vesicular release site, $\mathbf{C a}^{2+}$-nanodomain coupling In this case, the $R_{r}$ vs $V_{m}$ relation is not strictly described by a Boltzmann function (see Eq. (S5.7)). However, we found that it is approximated by a Boltzmann function exceptionally well in the whole parameter 
space, (see Supplementary Material 5.2 and Fig. S5.1 therein). To understand this fact better quantitatively, we reduced the expression of $R_{r}\left(V_{m}\right)$ for the limiting values of the ratios of any two of three parameters $k_{-1}, k_{r e p}$, and $k_{r e l}$. The expressions of $R_{r}\left(V_{m}\right)$ functions in these limiting cases are provided in Supplementary Material 5.2. Here, we concentrated on the discussion of the shift of $R_{r}\left(V_{m}\right)$ function along the $V_{m}$ axis (see Fig. 4.5B). All considered limiting cases can be divided into three main groups based on the vesicle release pattern type at $V_{m} \ll\left(V_{0.5}+S_{r}\right)$ (marked by different colors in Fig. 4.5). In the limit of fast channel gating (blue band in Fig. 4.5B), the expressions of $R_{r}\left(V_{m}\right)$ functions are identical to those for the $\mathrm{Ca}^{2+}$ microdomain coupling regime studied before. Limiting cases corresponding to bursty release (red band in Fig. 4.5B) are characterized by $S_{r}=0$. Limiting cases corresponding to the channel opening limited vesicle release at (green band in Fig. 4.5B), have $S_{r}<0$. In this case, the smaller is the ratio $k_{r e p} / k_{r e l}$ the more negative is $S_{r}$. Note that $\Delta_{V}\left[R_{r}\right]=\Delta_{V}\left[p_{o}\right]$ for all the 13 limiting cases except $k_{r e l} \gtrsim k_{r e p} \sim k_{-1}$, when $\Delta_{V}\left[R_{r}\right] \sim \Delta_{V}\left[p_{o}\right]$ (see below).

To check if the above analytical analysis of the parameter space did not miss any relevant phenomenon we performed numerical global optimization to find the ranges of the values of $S_{r}$ and $\Delta_{V}\left[R_{r}\right]$. We found that $S_{r} \leq 0$ and $\Delta_{V}\left[p_{o}\right] \leq \Delta_{V}\left[R_{r}\right]$ for any set of parameters $k_{-1}$, $k_{r e l}$, and $k_{r e p}$. The smallest dynamic range corresponds to the limiting case $k_{r e l} \gg k_{-1} \sim k_{r e p}$ mentioned above. However, even in this case the minimum $\Delta_{V}\left[R_{r}\right]$ was $84 \%$ of $\Delta_{V}\left[p_{o}\right]$, and was observed for $\bar{p}_{o}=1$. When the maximally possible $\bar{p}_{o}$ was decreased to more physiological 0.5 , the minimum $\Delta_{V}\left[R_{r}\right]$ was only by $3 \%$ smaller than $\Delta_{V}\left[p_{o}\right]$. Altogether, we conclude that, for a 2-state vesicular release site in $\mathrm{Ca}^{2+}$-nanodomain coupling with a 2-state $\mathrm{Ca}^{2+}$ channel, the $R_{r}\left(V_{m}\right)$ function is shifted to the left with respect to $p_{o}\left(V_{m}\right)$, if shifted at all, and has a nearly identical dynamic range.

$\boldsymbol{n}_{\boldsymbol{V}}$-state vesicular release site, $\mathbf{C a}^{2+}$-microdomain coupling $\mathrm{Ca}^{2+}$ sensor of exocytosis of an $n_{V}$-state vesicular release site has $m=n_{V}-2 \mathrm{Ca}^{2+}$ binding sites. In this case, the expression of the vesicle release rate dependence on the membrane potential is

$$
R_{r}\left(V_{m}\right)=\frac{m ! \cdot \gamma \cdot k_{r e p} \cdot\left(k_{r e l} \cdot p_{o}\left(V_{m}\right)\right)^{m}}{\sum_{i=0}^{m} a_{i} \cdot\left(k_{r e l} \cdot p_{o}\left(V_{m}\right)\right)^{i}}
$$

where coefficients $a_{i}$ are polynomial functions of parameters $k_{o f f}, b, k_{r e p}$ and $\gamma$ (see Eqs. (S2.102) - (S2.106)). For $m=1$, i.e., $n_{V}=3$, the $V_{m}$ dependence of $R_{r}$ is very similar to that of 2 -state vesicular release sites considered earlier. The $R_{r}$ vs $V_{m}$ relation is then described by a Boltzmann function with the dynamic range identical to that of $p_{o}\left(V_{m}\right)$ and $S_{r} \leq 0$ (see Supplementary Material 5.3). In the limiting case when $\mathrm{Ca}^{2+}$ binding to the sensor of exocytosis is much faster than unbinding at every stage (case $\left[2_{m}^{*}\right]$ in Fig. $4.4 \mathrm{C}$ ), $R_{r}\left(V_{m}\right)$ is described by a Boltzmann function with $S_{r} \leq 0$ and the dynamic range equal to that of the $p_{o}\left(V_{m}\right)$ independent of $m$ (green line in Fig. 4.5D, see Supplementary Material 5.3 for details).

We found several important consequences of the multiple $\mathrm{Ca}^{2+}$ binding sites of the $\mathrm{Ca}^{2+}$ sensor of exocytosis to the properties of $R_{r}\left(V_{m}\right)$ functions. First, shifts of $R_{r}\left(V_{m}\right)$ functions with 
A

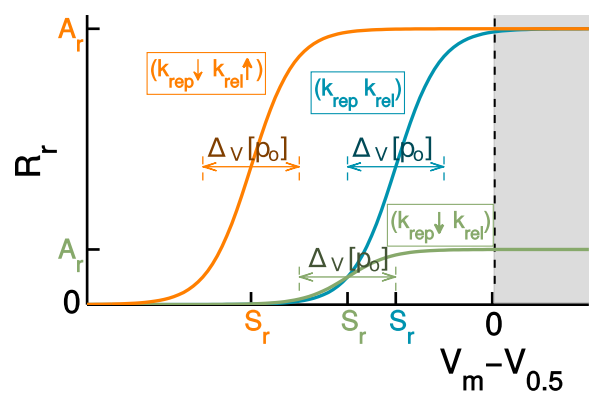

C

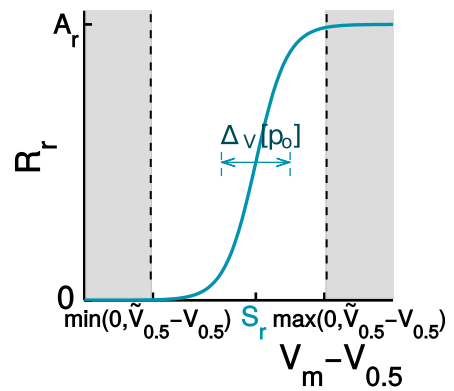

B

$\mathrm{k}_{\text {rel }} \gtrsim \mathrm{k}_{\text {rep }} \gg \mathrm{k}_{-1}=0$
$\mathrm{k}_{\text {rep }} \gg \mathrm{k}_{\text {rel }} \gtrsim \mathrm{k}_{-1}=0$
$\mathrm{k}_{\text {rel }} \gtrsim \mathrm{k}_{\text {rep }} \sim \mathrm{k}_{-1} \approx 0$
$\mathrm{k}_{\text {rel }} \gtrsim \mathrm{k}_{-1} \gg \mathrm{k}_{\text {rep }}=\ln \left(\frac{\mathrm{k}_{\text {rep }} \cdot\left(\mathrm{k}_{-1}+\mathrm{k}_{\text {rel }}\right)}{\mathrm{k}_{-1 \cdot} \cdot \overline{\mathrm{p}}_{\mathrm{o}} \cdot \mathrm{k}_{\text {rel }}+\mathrm{k}_{\text {rep }} \cdot\left(\mathrm{k}_{-1}+\mathrm{k}_{\text {rel }}\right)}\right)<0$
$\mathrm{k}_{\text {rep }} \gtrsim \mathrm{k}_{-1} \gg \mathrm{k}_{\text {rel }}$
$\mathrm{k}_{-1} \gg \mathrm{k}_{\text {rel }} \gtrsim \mathrm{k}_{\text {rep }}=\ln \left(\frac{\mathrm{k}_{\text {rep }}}{\mathrm{k}_{\text {rel }} \cdot \overline{\mathrm{p}}_{\mathrm{o}}+\mathrm{k}_{\text {rep }}}\right) \leq 0$
$\frac{\mathrm{k}_{-1} \gg \mathrm{k}_{\text {rep }} \sim \mathrm{k}_{\text {rel }}}{\frac{\mathrm{S}_{\mathrm{r}} / \mathrm{k}_{\mathrm{V}}}{\text { Limiting case }}}$

E

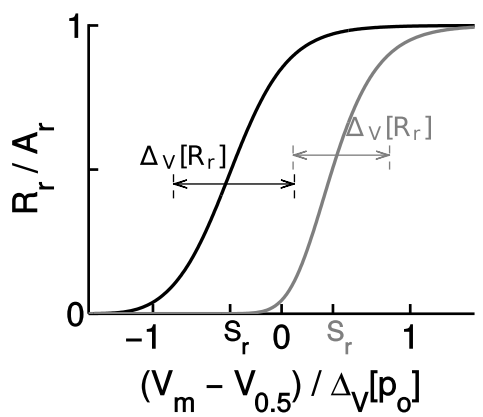

Figure 4.5: Properties of $R_{r}\left(V_{m}\right)$ functions of single vesicular release sites and their dependence on the kinetic parameters of the model. (A) Exemplary $R_{r}\left(V_{m}\right)$ functions of 2state vesicular release sites with $\mathrm{Ca}^{2+}$-microdomain coupling scenario and with $V_{m}$-independent $k_{r e p}$. The downward and upward arrows at $k_{r e p}$ and $k_{r e l}$ indicate the direction in which these parameters have to be changed in order to transform the blue curve to either the green one or orange one. Note that $\Delta_{V}\left[R_{r}\right]=\Delta_{V}\left[p_{o}\right]$ here. (B) Estimates of the shift parameter $S_{r}$ for 2-state vesicular release sites with $\mathrm{Ca}^{2+}$-nanodomain coupling scenario in different limiting cases of the kinetic parameters. (C) The same as (A), but with $V_{m}$-dependent $k_{r e p}$. (D) Exemplary $R_{r}\left(V_{m}\right)$ functions of $n_{V}$-state vesicular release sites with $\mathrm{Ca}^{2+}$-microdomain coupling scenario and $V_{m}$-independent $k_{\text {rep }}$. (E) The same as (D), but with $n_{V}=7$, and the kinetic parameters set to values which maximize the difference between the $S_{r}$ values of the two $R_{r}\left(V_{m}\right)$ functions (see text for more details). 
respect to $p_{o}\left(V_{m}\right)$ along the $V_{m}$ axis can be not only negative but also positive (Fig. 4.5D, blue line). We found by using numerical global optimization that the maximum value of $S_{r}$ is reached in the limit $k_{r e p} /\left(k_{r e l} \cdot \bar{p}_{o}\right) \gg m ! \cdot\left(\gamma+k_{r e p}\right) / \gamma \cdot\left(k_{r e l} \cdot \bar{p}_{o} /\left(b \cdot k_{o f f}\right)\right)^{m}$. In this case, as shown in Supplementary Material 5.3,

$$
R_{r}\left(V_{m}\right)=\frac{m ! \cdot \gamma \cdot k_{r e p} \cdot\left(k_{r e l} \cdot \bar{p}_{o}\right)^{m}}{a_{0} \cdot\left(1+e^{\left.-\left(V_{m}-V_{0.5}\right) / k_{V}\right)^{m}}\right.}
$$

if we assume $k_{r e p}\left(V_{m}\right) \equiv k_{r e p}=$ const. Noteworthy, despite the fact that the above expression is a power $m$ of a Boltzmann function, it can be very well fit by a Boltzmann function with adjusted $k_{V}$ and $V_{0.5}$ (see Fig. S5.3). It follows from Eq. (4.47) that the half maximum vesicle release rate is reached at $V_{m}=V_{0.5}-k_{V} \cdot \ln (\sqrt[m]{2}-1)$. For example, when $m=5, \max \left(S_{r}\right) \approx 1.9 \cdot k_{V}$, which is $\approx 43 \%$ of $\Delta_{V}\left[p_{o}\right]$. The corresponding $\Delta_{V}\left[R_{r}\right]$ is smaller than $\Delta_{V}\left[p_{o}\right]$. The higher the $m$, the smaller the $\Delta_{V}\left[R_{r}\right]$. However, even for $m=5, \Delta_{V}\left[R_{r}\right]$ is $\approx 75 \%$ of $\Delta_{V}\left[p_{o}\right]$. The minimum value of $S_{r}$ for an $n_{V}$-state vesicular release site has no lower bound. This is guaranteed by the fact that the last vesicle fusion step, characterized by rate $\gamma$, is assumed to be $V_{m}$-independent.

Another important feature of $R_{r}\left(V_{m}\right)$ functions brought about by multiple $\mathrm{Ca}^{2+}$-binding sites of the sensor of exocytosis is that $\Delta_{V}\left[R_{r}\right]$ can be considerably smaller than $\Delta_{V}\left[p_{o}\right]$. Indeed, at least for $m$ values from 1 to 5 , the global optimization showed that the minimum of $\Delta_{V}\left[R_{r}\right]$ is $m$ times smaller than the corresponding $\Delta_{V}\left[p_{o}\right]$. This is observed in the limit when $k_{r e p}$ and/or $\gamma$ are much smaller than the average rate of reaching state $S_{n_{V}}$ from state $S_{2}$ and the $\mathrm{Ca}^{2+}$-unbinding rates at every stage are considerably faster than the $\mathrm{Ca}^{2+}$-binding rates (see Supplementary Material 5.3 for details). Low $k_{\text {rep }}$ means that $R_{r}\left(V_{m}\right)$ saturates at very negative $V_{m}$, where $(m-i) \cdot k_{\text {rel }} \cdot p_{o}\left(V_{m}\right)$ may be considerably smaller than $b^{i} \cdot k_{o f f}$, even if $(m-i) \cdot k_{r e l} \cdot \bar{p}_{o} \gtrsim b^{i} \cdot k_{o f f}$. In this case, $R_{r}\left(V_{m}\right)$ is described by a Boltzmann function, but considerably shifted to the left and with $m$ times narrower dynamic range than $p_{o}\left(V_{m}\right)$ (orange line in Fig. 4.5D),

$$
R_{r}\left(V_{m}\right)=\frac{m ! \cdot \gamma \cdot k_{r e p} \cdot\left(k_{r e l} \cdot \bar{p}_{o}\right)^{m}}{a_{m}+a_{0} \cdot e^{-m \cdot\left(V_{m}-V_{0.5}\right) / k_{V}}}
$$

The above expression can be reduced to a canonical form, Eq. (4.44) (see Supplementary Material 5.3). The maximum $\Delta_{V}\left[R_{r}\right]$ for a vesicular release site with $n_{V}$-state $\mathrm{Ca}^{2+}$ sensor of exocytosis and $\mathrm{Ca}^{2+}$-microdomain coupling scenario is equal to $\Delta_{V}\left[p_{o}\right]$. We showed this by using numerical global optimization.

$\boldsymbol{n}_{\boldsymbol{V}}$-state vesicular release site, $\mathbf{C a}^{2+}$-nanodomain coupling In the limit of fast channel gating, the $R_{r}\left(V_{m}\right)$ functions coincide in $\mathrm{Ca}^{2+}$-nanodomain and $\mathrm{Ca}^{2+}$-microdomain coupling regimes. In the limit of bursty vesicle release, $S_{r} \sim 0$ and $\Delta_{V}\left[R_{r}\right]=\Delta_{V}\left[p_{o}\right]$ for $n_{V^{-}}$state sites, as it is for 2 -state sites. Indeed, the release rate is then simply equal to a product of the release rate within a burst and $p_{o}$. The former is not dependent on $V_{m}$, thus, all $V_{m}$ dependence of the $R_{r}$ comes from $p_{o}$ then. In the case of channel opening limited vesicle release, $R_{r}\left(V_{m}\right)$ functions of 
$n_{V}$-state vesicular release sites are almost identical to those of 2 -state sites. The only difference is that the $n_{V}$-state sites have one more $\mathrm{Ca}^{2+}$-independent transition step (defined by rate $\gamma$ ) in addition to vesicle replenishment. Thus, $k_{r e p}$ should be replaced by $\left(k_{r e p} \cdot \gamma\right) /\left(k_{r e p}+\gamma\right)$ in the expressions of $S_{r}$ and $A_{r}$ (see Fig. 4.5B and Eqs. (S5.9 - S5.10)) then.

To study the intermediate cases in the parameter space, we performed global optimization of the parameters $S_{r}$ and $\Delta_{V}\left[R_{r}\right]$. We found that the maximum $S_{r}$ and the minimum $\Delta_{V}\left[R_{r}\right]$ are the same as in the case of $\mathrm{Ca}^{2+}$-microdomain coupling, and are observed in the fast channel gating limit. The maximum $\Delta_{V}\left[R_{r}\right]$ can be slightly larger in $\mathrm{Ca}^{2+}$-nanodomain coupling than $\mathrm{Ca}^{2+}$-microdomain coupling regime. This is observed when $b^{i} \cdot k_{o f f} \gg(m-i) \cdot k_{\text {rel }}$ for $m=\overline{1, m}$ and $\bar{p}_{o}=1$. However, the difference is rather small. For example, the maximum $\Delta_{V}\left[R_{r}\right]$ is larger by only $25 \%$ than $\Delta_{V}\left[p_{o}\right]$ for $m=5$ and is even smaller for $m<5$. Moreover, when $\bar{p}_{o}$ is reduced from one to physiologically more realistic 0.5 or lower, the maximum $\Delta_{V}\left[R_{r}\right]$ is not more than by $1 \%$ larger than $\Delta_{V}\left[p_{o}\right]$. Finally, we found that the $R_{r}$ vs $V_{m}$ relation deviates from a Boltzmann function the most in the limit of fast channel gating when Eq. (4.47) applies. On the other hand, the discrepancy is very small even then, as we showed before (Supplementary Material 5.3). Thus, we can conclude that a Boltzmann function approximates the $R_{r}$ vs $V_{m}$ relations very well not only for 2 -state but also for $n_{V}$-state vesicular release sites.

Extent of changes of $\boldsymbol{S}_{\boldsymbol{r}}$ It follows from the previous results of this section that $R_{r}\left(V_{m}\right)$ functions can be shifted along the $V_{m}$ axis not only by varying $V_{0.5}$ but also by modifying the absolute values of the kinetic parameters of the model. Theoretically, a leftward shift of a $R_{r}\left(V_{m}\right)$ function can be arbitrarily large. However, we found that large changes in the kinetic parameters are necessary in order to produce a considerable difference in $S_{r}$. This is most clearly seen for a 2 -state vesicular release site in $\mathrm{Ca}^{2+}$-microdomain coupling regime. It follows from Eq. (S5.4) that, by allowing to change the magnitudes of $k_{r e p}$ and $k_{r e l} \cdot p_{o}$ up to one order of magnitude, it is possible to change $S_{r}$ by up to $0.52 \cdot \Delta_{V}\left[R_{r}\right]$. To shift $S_{r}$ by $\Delta_{V}\left[R_{r}\right], k_{r e l} \cdot \bar{p}_{o}$ has to be changed 81 times. In the case of $n_{V}$-state vesicular release sites, smaller, but still large, alterations of the kinetic parameters are required. We found by using global numerical optimization that $S_{r}$ could be changed by up to $\sim 0.9 \cdot \Delta_{V}\left[R_{r}\right]$ when all the kinetic parameters of a 7 -state vesicular release site were allowed to differ up to one order of magnitude. Fig. 4.5E shows the pair of $R_{r}\left(V_{m}\right)$ functions obtained by that optimization procedure.

\subsubsection{Response properties of AZs with multiple vesicular release sites}

\section{Release rate dependence on the membrane potential}

Vesicular release sites within an AZ are widely considered to be independent (however, see Glowatzki and Fuchs, 2002; Singer and Diamond, 2004; Chapochnikov et al., 2014). We assumed this in our work too. Therefore, vesicle release rate at an AZ is equal to the sum of 
vesicle release rates at all vesicular release sites within the AZ in our model,

$$
R_{r}\left(V_{m}\right)=\sum_{i=1}^{N_{V}} R_{r, i}\left(V_{m}\right) .
$$

Here, $R_{r, i}\left(V_{m}\right)$ is the vesicle release rate at the $i$-th site, $R_{r}\left(V_{m}\right)$ is the overall vesicle release rate at the $\mathrm{AZ}$. If all the vesicular release sites are identical, the $R_{r}\left(V_{m}\right)$ function is the same as one of the $R_{r, i}\left(V_{m}\right)$ functions scaled up by $N_{V}$ (Fig. 4.6A). If $R_{r, i}\left(V_{m}\right)$ functions differ with respect to their position on the $V_{m}$ axis, the resulting $R_{r}\left(V_{m}\right)$ function of the whole AZ has not only a larger amplitude but also a wider $\Delta_{V}\left[R_{r}\right]$. The larger the shifts are, the higher is the $\Delta_{V}\left[R_{r}\right]$ (Fig. 4.6B). Nevertheless, we showed in the previous section that considerable shifts of $R_{r}\left(V_{m}\right)$ functions are produced only by very large changes in the model parameters. Therefore, we would not expect much of the broadening of $\Delta_{V}\left[R_{r}\right]$ in physiological conditions. This expectation is supported by the results of experimental data analysis in section 4.4.4.

\section{Inter-event interval distributions}

In the case of the $\mathrm{Ca}^{2+}$-nanodomain, $\mathrm{Ca}^{2+}$-microdomain, and intermediate $\mathrm{Ca}^{2+}$-domain coupling scenarios of AZ topographies, vesicle fusion at different vesicular release sites is statistically independent. It is known that the probability distribution of IEIs of a point process resulting from a superposition of $N$ identical independent point processes converges to the exponential distribution when $N \rightarrow+\infty$ (Cox, 1967). Even more, the compound process converges to a Poisson process locally in time then (Cox, 1967; Lindner, 2006). The validity of this statement can be extended to the superposition of non-identical processes if some very general requirements are fulfilled (Khintchin, 1960). This would be true, for example, if the parameters characterizing the vesicular release sites are described by some discrete or continuous normalized distributions of finite width. Physiologically realistic AZ organizations generally fulfill this requirement. Thus, for IEI of vesicle fusion at an $\mathrm{AZ}$ with either $\mathrm{Ca}^{2+}$-nanodomain, $\mathrm{Ca}^{2+}$-microdomain, or shared $\mathrm{Ca}^{2+}$-domain coupling scenario,

$$
\lim _{N \rightarrow \infty} \rho_{I E I}(t)=R_{r} \cdot e^{-R_{r} \cdot t}
$$

The above expression also applies to AZs with shared $\mathrm{Ca}^{2+}$-domain control of exocytosis. However, in that case, $N$ stands for a number of vesicular release site groups driven by shared $\mathrm{Ca}^{2+}$ channels.

Given a physiological number of 10 - 15 vesicular release sites at IHC AZs (Pangršič et al., 2010), how close are $\rho_{I E I}(t)$ functions to an exponential distribution? To understand this, we studied five cases of presynaptic AZs with homogeneous vesicular release sites which correspond to the whole spectrum of vesicle release patterns identified in section 4.4 .1 (see Fig. 4.6C). In cases (1) and (2), respectively 2- and 7-state vesicular release sites with the kinetic parameters set to reproduce IEIs with $\min \left[C V_{I E I}\right]$ were considered. Case (3) described a scenario of vesicle release patterns intermediate between a Poisson process and bursty release with normalized 

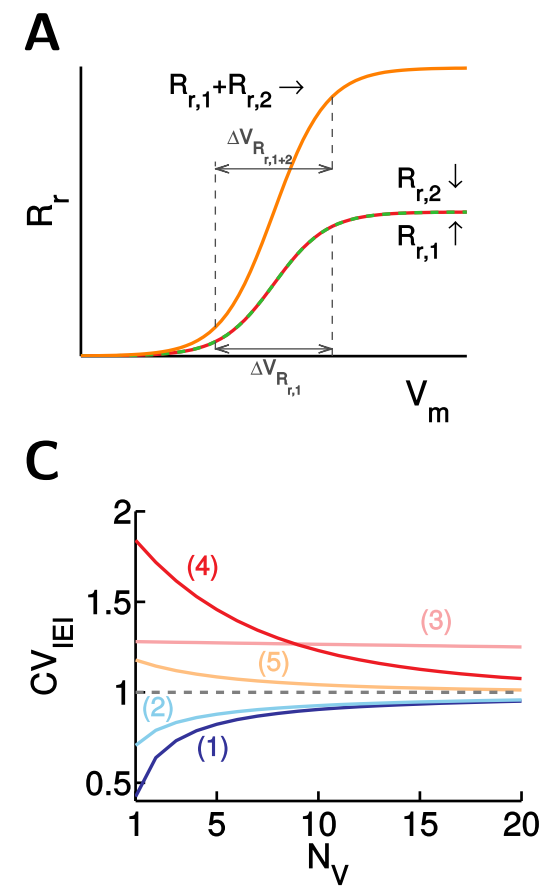

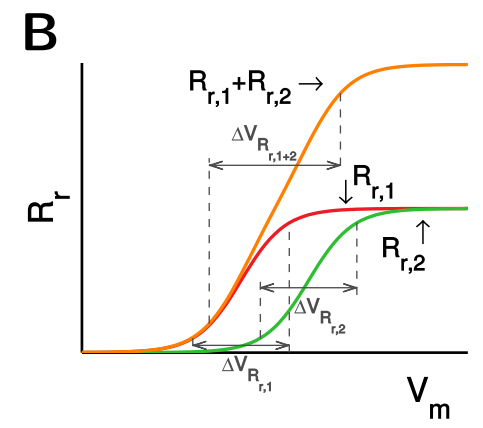

D

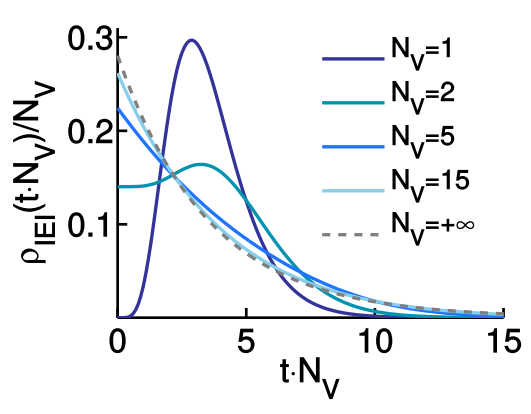

E

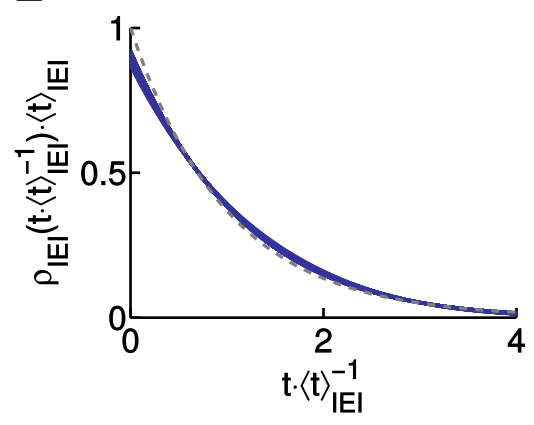

Figure 4.6: Properties of $R_{r}\left(V_{m}\right)$ and $\rho_{I E I}(t)$ functions: from a single vesicular release site to an AZ. (A, B) Two key scenarios of transformation of $R_{r}\left(V_{m}\right)$ functions with increased $N_{V}$. (C) Convergence of $C V_{I E I}$ with increased $N_{V}$ for different presynaptic vesicle release regimes. See text for details. (D) Convergence of the $\rho_{I E I}(t)$ function with increased $N_{V}$. AZ consisting of 7state vesicular release sites with the most regular release patterns possible is considered here. The time variable was scaled by $N_{V}$ to fix $\langle t\rangle_{I E I}$ to the same level for comparison purposes. $(\mathbf{E}) \rho_{I E I}(t)$ functions of an $\mathrm{AZ}$ with fifteen 7 -state vesicular release sites, each with random $k_{\text {rel }}$ values. Overlapping blue lines correspond to $10^{3}$ different realizations of AZs. The dashed grey line plots the exponential distribution. The time variable was scaled by $\langle t\rangle_{I E I}$ for comparative purposes in all cases. See text for more details. 
rate constants $k_{r e l}=1, k_{r e p}=20, k_{+1}=1, k_{-1}=1$. Finally, cases (4) and (5) described AZs with 2-state vesicular release sites in the weak and strong bursty release regimes, respectively. In the former case, we chose $k_{r e l}=1, k_{r e p}=1, k_{+1}=10^{-2}, k_{-1}=1$, in the later case, $k_{\text {rel }}=1$, $k_{r e p}=3, k_{+1}=0.1$, and $k_{+1}=0.3$ were assumed. To quantify the convergence of the $\rho_{I E I}(t)$ functions to the exponential distribution, $C V_{I E I}$ was used for an easier comprehension. Note that $C V_{I E I}=1$ for an exponential distribution. $\rho_{I E I}(t)$ functions at different $N_{V}$ in case (1) are plotted in Fig. 4.6D as a reference for interpreting different $C V_{I E I}$ values.

We found that the convergence of the $\rho_{I E I}(t)$ functions with increased $N_{V}$ was fast in cases $(1-3)$ (Fig. 4.6C, D). $\rho_{I E I}(t) \sim R_{r} \cdot e^{-R_{r} \cdot t}$ for $N_{V} \geq 10$ to a very good approximation with $N_{V}$ as small as 10 in these cases. Moreover, we showed by using numerical global optimization that case (1) corresponds to $\min \left[C V_{I E I}\right]$ not only for $N_{V}=1$ but also for $N_{V}>1$, when all vesicular release sites are identical. Thus, $\rho_{I E I}(t)$ functions are approximated well by $R_{r} \cdot e^{-R_{r} \cdot t}$ at $N_{V} \geq 10$ for identical vesicular release sites working in $\mathrm{Ca}^{2+}$-microdomain coupling regime, in the limit of fast channel gating or regime of channel opening limited vesicle release. The convergence could be slower when vesicular release sites are heterogeneous. To check the extent of this, we considered case $(1)$ with $\log _{10}\left(k_{r e l}\right)$ of different vesicular release sites distributed uniformly in the interval from 0 to 2 , and with $k_{\text {rep }}$ and $\gamma$ adjusted accordingly. This is a safe upper limit of a possible heterogeneity in vivo. $10^{3}$ random realizations of the corresponding $\rho_{I E I}(t)$ functions are shown in Fig. 4.6E. The deviations from $R_{r} \cdot e^{-R_{r} \cdot t}$ were small in the whole sample. Thus, we conclude that the $\rho_{I E I}(t)$ functions are well approximated by $R_{r} \cdot e^{-R_{r} \cdot t}$ for AZs with $N_{V} \geq 10$ in cases $(1-3)$.

In case (4) (weak bursty release), the convergence of $\rho_{I E I}(t)$ functions was very slow (Fig. 4.6C). This is explained by a very low $p_{o}$ then (by definition). Indeed, low $p_{o}$ means that an increase in $N_{V}$ initially results in an increase in the number of bursts with little overlap between them. Thus, $C V_{I E I}$ is little affected then. By choosing $p_{o}$ arbitrarily small we could, in principle, make the convergence arbitrarily slow. In case (5) (strong bursty release), the convergence was relatively fast. However, $C V_{I E I}$ can be made arbitrarily large in this scenario by increasing $k_{r e p} / k_{-1}$. In conclusion, whether $\rho_{I E I}(t)$ functions are close to $R_{r} \cdot e^{-R_{r} \cdot t}$ for bursty vesicular release sites with $N_{V}$ in the range 10 - 15 depends on the exact values of the kinetic parameters.

\section{Inter-event interval correlations}

As we found before in section 4.4.1, vesicle fusion at a single vesicular release site is virtually a renewal process, at least in the case of the $\mathrm{Ca}^{2+}$-nanodomain and $\mathrm{Ca}^{2+}$-microdomain coupling scenarios. A superposition of non-Poisson renewal processes is however not a renewal process in general, even if the superimposed processes are independent (Cox, 1967; Lindner, 2006). Thus, vesicle release at presynaptic AZs is in general a non-renewal process, which is described by finite correlations between IEIs. On the other hand, when $N_{V}$ goes to infinity, the correlations decay to zero and the process converges to a Poisson process locally in time (Cox, 1967; Lindner, 2006; 
Supplementary Material 4.2). Thus, the largest correlations between IEIs should be present in the intermediate range of $N_{V}$ values. Here, we analyzed the magnitudes of these correlations and how they depend on the parameters characterizing the AZs composed of identical vesicular release sites.

2-state vesicular release sites, $\mathbf{C a}^{2+}$-microdomain coupling Fig. 4.7A shows serial correlation functions for an $\mathrm{AZ}$ with a variable number of identical 2-state vesicular release sites and $\mathrm{Ca}^{2+}$-microdomain coupling topography scenarios (see Eqs. (S2.54), (S2.57), (S2.58) - (S2.63) for the analytical expressions). We found by global numerical optimization that, at least for AZs with identical vesicular release sites, $C_{I E I}$ is a non-positive, monotonically increasing function of $l$ with the most negative value at $l=1$. Moreover, for fixed $N_{V}$ and $l, C_{I E I}(l)$ is the most negative when $k_{r e p}=k_{r e l} \cdot p_{o}$. The global minimum values of $C_{I E I}(l)$ vs $l$ relations at different fixed $N_{V}$ are shown by the dark blue line in Fig. 4.7E. The overall global minimum of $C_{I E I} \approx-0.104$ is reached at $N_{V}=3$. The negative correlations between IEIs indicate that shorter than average IEIs are more likely to be followed by longer than average IEIs and vice versa. The negative correlations are due to an interplay between the local vesicle pool depletion and replenishment. If a time interval between two vesicle fusion events is shorter than the average, then the vesicle pool is more depleted after the second fusion event than before the first one. Thus, the IEI between the third and the second fusion events is more likely than not to be longer than the IEI between the second and the first events. The same arguments put in the opposite order explain why longer IEIs tend to be followed by shorter ones. The decrease of $\left|C_{I E I}(l)\right|$ with $l$ is due to the Markovian nature of the processes underlying the vesicle fusion. We found by global numerical optimization that the most positive value of $C_{I E I}(l)$ is zero at any $N_{V}$ (dark blue dashed line in Fig. 4.7E). This is observed in the limit when vesicle fusion at each vesicular release site is described by a Poisson process. Then, $C_{I E I}(l) \equiv 0$.

2-state vesicular release sites, $\mathrm{Ca}^{2+}$-nanodomain coupling $\mathrm{In}$ the case of presynaptic AZs with 2-state vesicular release sites driven by single 2 -state $\mathrm{Ca}^{2+}$ channels, correlations between IEIs of vesicle release can be both negative and positive. The results of global maximization of the positive peak value of $C_{I E I}(l)$ at fixed $N_{V}$ in this $\mathrm{AZ}$ scenario is depicted by the brown line in Fig. 4.7F. As we see, the maximal peak values of the $C_{I E I}(l)$ functions increase from 0.09 at $N_{V}=2$ to 0.18 at $N_{V}=20$. In all these cases, vesicle fusion happens in the bursting mode, with tens of fusion events within each burst. Representative $C_{I E I}(l)$ vs $l$ relations corresponding to the maximal positive peak values are shown in Fig. 4.7B. The positive correlations between IEIs are explained by the overlaps between vesicle fusion bursts at different vesicular release sites within AZs. Indeed, IEIs shorter than the average are more likely to happen when more than the average number of the vesicular release sites are active (i.e., the $\mathrm{Ca}^{2+}$ channels are open). In turn, the next IEI is more likely than not to be shorter than the average. This is because the same vesicular release sites are more likely than not to stay in 
A

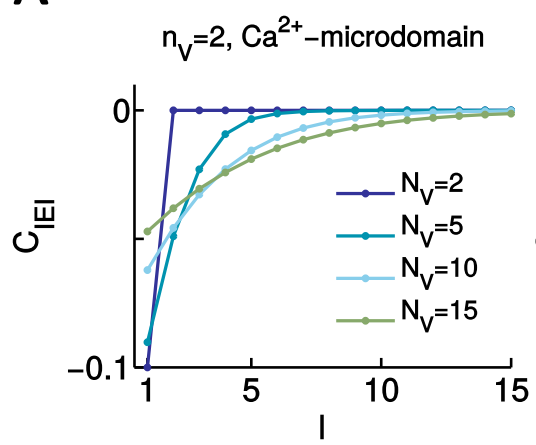

B

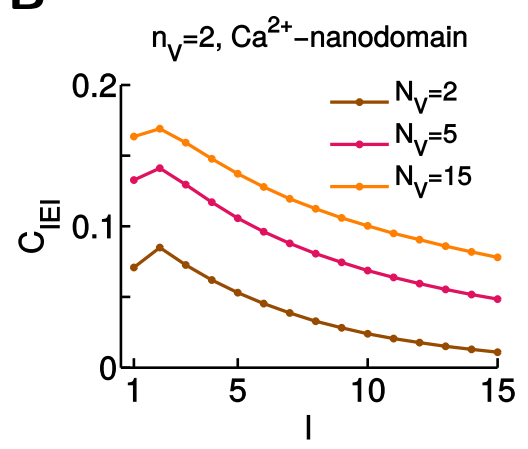

C

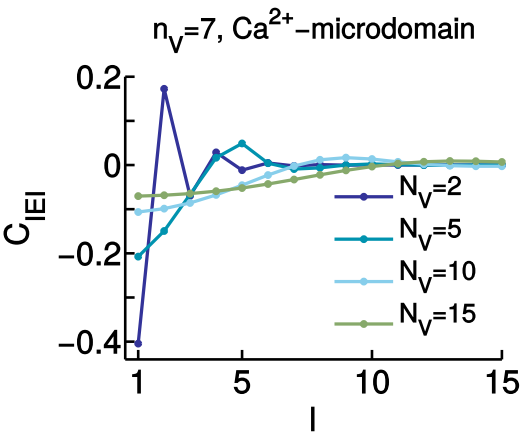

D

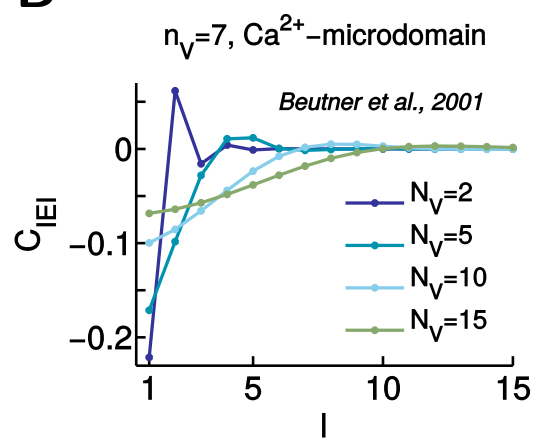

$E$

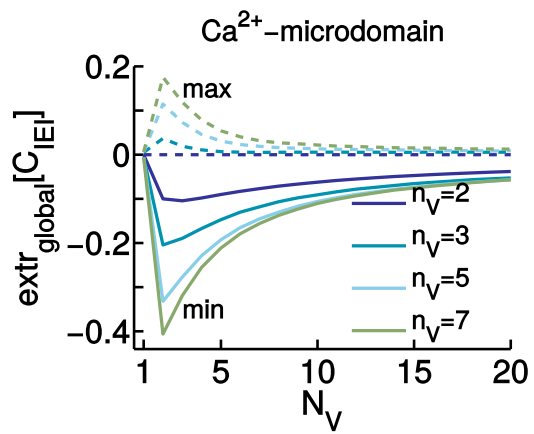

$\mathbf{F}$

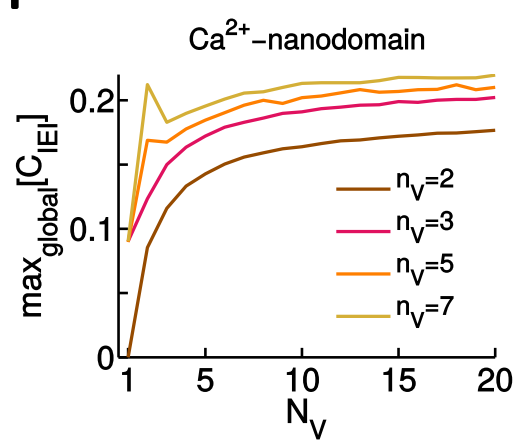

Figure 4.7: Properties of the serial correlation functions of IEIs of presynaptic AZs with multiple vesicular release sites. (A) $C_{I E I}(l)$ functions with the most negative peak values corresponding to AZs built of 2-state vesicular release sites and with $\mathrm{Ca}^{2+}$-microdomain coupling topography. (B) $C_{I E I}(l)$ functions with the most positive peak values corresponding to AZs built of 2state vesicular release sites and with the $\mathrm{Ca}^{2+}$-nanodomain coupling topography. (C) $C_{I E I}(l)$ functions with the most negative and positive peak values corresponding to AZs built of 7 -state vesicular release sites and with $\mathrm{Ca}^{2+}$-microdomain coupling topography. (D) $C_{I E I}(l)$ functions corresponding to AZs built of 7-state vesicular release sites with kinetic parameters taken from (Beutner et al., 2001) and with $\mathrm{Ca}^{2+}$-microdomain coupling topography.

(E) The most positive (dashed lines) and negative (solid lines) values of $C_{I E I}(l)$ functions of AZs built of $n_{V^{-s t a t e}}$ vesicular release sites and with $\mathrm{Ca}^{2+}$ microdomain coupling topography. $(\mathbf{F})$ The most positive values of $C_{I E I}(l)$ functions of AZs built of $n_{V}$-state vesicular release sites and with the $\mathrm{Ca}^{2+}$-nanodomain coupling topography. 
the on mode for more than one IEI. An equivalent line of thinking, but in the opposite direction, applies for longer than the average IEIs. The decrease rate of $C(l)$ with $l$ is slow (compare plots in Fig. 4.7B and Fig. 4.7A). This is because unusually many fusion events happen in a time window required for equilibration of the channel gating, and thus, equilibration of the whole system. We found by global numerical optimization that the smallest values of the $C_{I E I}(l)$ functions are observed in the limit of fast channel gating. Then, the $\mathrm{Ca}^{2+}$-nanodomain and $\mathrm{Ca}^{2+}$-microdomain coupling regimes are equivalent and, thus, properties of the corresponding $C_{I E I}(l)$ functions are the same.

$n_{V^{-}}$-state vesicular release sites, $\mathbf{C a}^{2+}$-microdomain coupling We found that, differently from AZs with 2-state vesicular release sites, values of $C_{I E I}(l)$ functions can be not only negative but also positive for AZs with $n_{V}$-state vesicular release sites (Fig. 4.7C). Moreover, in the later case, $C_{I E I}(l)$ may approach 0 non-monotonically when $l$ is increased. However, these two features of the $C_{I E I}$ vs $l$ relations are pronounced for small $N_{V}$ only (Fig. 4.7C). We showed by numerical global optimization that $C_{I E I}(l=1)$ is always non-positive, the same as in the case of AZs built of 2-state vesicular release sites. However, in the current case, the $C_{I E I}(1)$ values are negative not only due the interplay between vesicle pool depletion and replenishment. The multiple $\mathrm{Ca}^{2+}$-binding sites of the sensor of exocytosis is another contributing factor. Indeed, a shorter than the average time between two fusion events results in a lower than the average occupancy of the sensors of exocytosis by $\mathrm{Ca}^{2+}$ ions after the second event. In turn, the waiting time for the third fusion event is longer than for the second one, which means that the second IEI tends to be longer than the first one. For a larger lag number $l$, though, correlations between the occupancy of the $\mathrm{Ca}^{2+}$ ions-binding sites of the sensors of exocytosis can be positive. This explains the positive values of $C(l)$ at $l>1$ and non-monotonic convergence of $C(l)$ with increased $l$. (see Supplementary Material 4.3 for details).

The higher absolute values of $C_{I E I}(l)$ functions are another feature brought about by the multiple $\mathrm{Ca}^{2+}$-binding sites of the sensor of exocytosis. Fig. 4.7E summarizes our results of global minimization and maximization of $C_{I E I}(l)$ values over the space of the kinetic parameters and different lags $l$ for AZs with different $n_{V}$ and $N_{V}$. As we see, the absolute values of both, the minima and the maxima points of $C_{I E I}(l)$ functions increase with $n_{V}$. This increase is particularly pronounced at small $N_{V}$. We found that the kinetic parameter sets maximizing/minimizing $C_{I E I}(l)$ functions at any tested $n_{V}$ and $N_{V}$ correspond to infinitesimally slow unbinding of $\mathrm{Ca}^{2+}$ ions. Moreover, the most negative and the most positive $C_{I E I}(l)$ values belong to the same serial correlation function (see Fig. 4.7C for examples). As demonstrated in Fig. 4.7D, $C_{I E I}(l)$ functions with qualitatively similar features are found for the kinetic parameter set determined experimentally by (Beutner et al., 2001). Though the magnitudes of the correlations are considerably smaller for small $N_{V}$ in this case, they are rather similar to those shown in Fig. $4.7 \mathrm{C}$ in the physiological range of $N_{V}$ values $(10-15)$. 


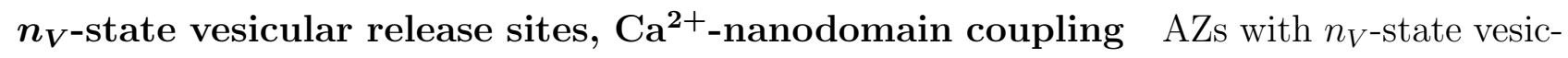
ular release sites and the $\mathrm{Ca}^{2+}$-nanodomain coupling topographies have $C_{I E I}(l)$ vs $l$ relations with positive values in the limit of bursty release. The global maximum values of the positive peaks of $C_{I E I}(l)$ functions at fixed $N_{V}$ are shown in Fig. 4.7F. As we see, the increase in the magnitude of the correlations with growing $n_{V}$ is rather moderate, especially at larger $N_{V}$. The $C_{I E I}(l)$ functions for $n_{V}>2$ are qualitatively similar to those corresponding to $n_{V}=2$ which we studied before. Differently from the maximum positive peak values, the minimum negative peak values of the $C_{I E I}(l)$ vs $l$ relations are the same as for $\mathrm{AZs}$ with $\mathrm{Ca}^{2+}$-microdomain coupling topographies (Fig. 4.7E).

\subsubsection{Conversion of presynaptic vesicle fusion events into spikes}

As we found in section 4.4.2, vesicle fusion at presynaptic AZs is almost always well approximated by a Poisson process if $N_{V}$ is in the physiological range. The only exception is the regime of bursty vesicle release. Thus, in order to understand how refractoriness of SGNs transforms presynaptic fusion into postsynaptic spike trains we needed to concentrate on these two limiting cases only. Of course, when considering the ISI correlations, even relatively small deviations from the Poisson process had to be taken into account explicitly.

\section{Inter-spike interval distributions}

In order to model ISI distributions of high-CF SGNs, the homogeneous Poisson trains convolved with refractoriness have been intensively applied (Teich \& Khanna, 1985; Young \& Barta, 1986; Li \& Young, 1993; Pangršič et al., 2015). As it is shown in Supplementary Material 2.5, the expressions of the ISI interval probability density function $\left(\rho_{I S I}(t)\right)$ and the coefficient of variation $\left(C V_{I S I}\right)$ are then

$$
\begin{aligned}
\rho_{I S I}(t) & =H\left(t-t_{A}\right) \cdot \frac{R_{r}}{1-\tau_{R} \cdot R_{r}} \cdot\left(e^{-R_{r} \cdot\left(t-t_{A}\right)}-e^{-\left(t-t_{A}\right) / \tau_{R}}\right), \\
C V_{I S I}(t) & =\frac{\sqrt{1+\left(R_{r} \cdot \tau_{R}\right)^{2}}}{1+R_{r} \cdot\left(t_{A}+\tau_{R}\right)},
\end{aligned}
$$

where $H(\ldots)$ is the Heaviside step function. Two properties relevant for our further analysis follows from Eq. (4.51). First, the spiking becomes more regular when $t_{A}$ is increased, as higher and higher fraction of each ISI corresponds to the $t_{A}$, which is constant. Second, not taking into account the rightward shift by $t_{A}, \rho_{I S I}(t)$ varies between an exponential distribution and a gamma distribution with shape parameter 2 (Fig. 4.8A). The former is observed when $\tau_{R} \gg\langle t\rangle_{I E I}$ or $\tau_{R} \ll\langle t\rangle_{I E I}$. The latter is observed when $\tau_{R}=\langle t\rangle_{I E I}$. A large fraction of SGNs in vivo indeed demonstrate distributions in the range between the two shown in Fig. 4.8A, as discussed in detail in section 4.4.4.

When presynaptic vesicle fusion is bursty, fusion events falling in the refractory periods of SGNs are preferentially inside the bursts because intraburst IEIs are much shorter than 
interburst IEIs. Thus, the resulting spike bursts produced by SGNs have fewer events than the vesicle fusion bursts, even if the numbers of bursts are similar. In turn, the spike trains are more regular than the patterns of the corresponding presynaptic vesicle release. Of course, when $p_{o}$ and $N_{V}$ are large, bursts from several release sites overlap and the probability of at least one active site at any moment is high. In these cases, the refractoriness preferentially reduces the number of events within those time periods when many of the vesicular release sites are active. The regularizing effect of SGN refractoriness is illustrated in Fig. 4.8B, where the dependencies of $C V_{I S I}$ on the overall refractory period $\left(t_{A}+\tau_{R}\right)$ are plotted. Here, we considered an $\mathrm{AZ}$ with 2-state release sites in $\mathrm{Ca}^{2+}$-nanodomain coupling with 2-state $\mathrm{Ca}^{2+}$ channels. The kinetic parameters were set to reproduce an experimentally observed $\rho_{I S I}(t)$ function shown in Fig. 4.12D with $N_{V}=10$. As we see, $C V_{I E I}$ initially rapidly decreases with $t_{A}+\tau_{R}$. $C V_{I E I}=1$ is reached below $\left(t_{A}+\tau_{R}\right) /\langle t\rangle_{I E I}=0.5$ for all $N_{V}$ considered (up to 15). At very long refractory periods, the spike times are mainly determined by $t_{A}$ and $\tau_{R}$ independent of the statistics of the underlying presynaptic release process. Thus, at large $\left(t_{A}+\tau_{R}\right) /\langle t\rangle_{I E I}, C V_{I S I}$ approaches those of the Poisson process convolved with the refractoriness (dashed grey line in Fig. 4.8B). Black dot in Fig. $4.8 \mathrm{~B}\left(C V_{I S I} \approx 1\right.$ and $\left.\left(t_{A}+\tau_{R}\right) /\langle t\rangle_{I E I} \approx 0.33\right)$ corresponds to the ISI distribution shown in Fig. $4.12 \mathrm{D}$. In this case, in spite of $C V_{I S I} \approx 1$, the spike process is still quite different from the Poisson process convolved with the refractoriness. This is clearly visible from the shape of $\rho_{I S I}$ functions shown in Fig.4.12D. Note that $t_{A}=\tau_{R}$ was assumed in Fig. 4.8B. We obtained very similar results when one of $t_{A}$ and $\tau_{R}$ was set to zero (Fig. S6.3). Only the asymptotic values of $C V_{I S I}$ in the limit of large $t_{A}+\tau_{R}$, determined by Eq. (4.51), are different in all these cases.

The extent of the reduction of $C V_{I E I}$ by the refractoriness depends on the exact statistics of the bursty vesicle release patterns. Given the same average presynaptic fusion rates, patterns with a higher fraction of periods when bursts are on would be more resistant to the refractoriness (compare curves for different $N_{V}$ in in Fig. 4.8B). The example considered in Fig. 4.8B corresponds to one of the most bursty fusion, i.e., with the highest $\mathrm{CV}$, in the literature. Thus, it can be used to judge the upper limit for how much the refractoriness regularizes the vesicle release patterns. In mice, the average value of $t_{A}+\tau_{R}$ was $1.0 \mathrm{~ms}$ (Pangršič et al., 2015). $t_{A}+\tau_{R}=1.2 \mathrm{~ms}$ (median value) was reported in cats by (Heil et al., 2007). We reanalyzed data from (Pangršič et al., 2015) and found that the average value of the ratio $\left(t_{A}+\tau_{R}\right) /\left\langle t_{I E I}\right\rangle$ for the maximally driven responses was 0.46 with the $5-95$ percentile range of $0.25-0.85$. Compared to the modeling results shown in Fig. 4.8B, these estimates suggest that the regularizing effect of the refractory period of the bursty spike trains can be considerable for the maximally driven responses in vivo. For moderate spike rates, the opposite is expected. For example, $\left(t_{A}+\tau_{R}\right) /\left\langle t_{I E I}\right\rangle$ is only 0.05 for $t_{A}+\tau_{R}=1.0 \mathrm{~ms}$ and $R_{s}=50 \mathrm{~s}^{-1}$. 


\section{Spike rate dependence on the presynaptic membrane potential}

As shown in section 4.4.1, the dependence of $R_{r}$ on $V_{m}$ is very well described by a Boltzmann function within the entire space of the kinetic parameters. In addition, we showed that the dependence of the spike rate on $V_{m}$ is also described by a Boltzmann function if the presynaptic vesicle release follows a Poisson process (Supplementary Material 6.2),

$$
R_{s}\left(V_{m}\right)=\frac{A_{s}}{1+e^{-\left(V_{m}-V_{0.5}-S_{r}-S_{s}\right) / k_{V}^{\prime}}},
$$

with

$$
A_{s}=\frac{A_{r}}{1+A_{r} \cdot\left(t_{A}+\tau_{R}\right)} \leq A_{r}, \quad S_{s}=-k_{V}^{\prime} \cdot \ln \left(1+A_{r} \cdot\left(t_{A}+\tau_{R}\right)\right) \leq 0 .
$$

Here, $R_{s}$ is the spike rate, $A_{s}$ is the maximum spike rate, $S_{s}$ is the shift of the $R_{s}$ vs $V_{m}$ relation with respect to the $R_{r}$ vs $V_{m}$ dependence along the $V_{m}$ axis. Thus, $R_{s}\left(V_{m}\right)$ functions are leftshifted and scaled down versions of the $R_{r}\left(V_{m}\right)$ functions, with identical dynamic ranges. This finding shows that, in principal, the variability of the refractory properties could contribute to the heterogeneity of SGN responses to sound stimuli. Recent experimental data, however, suggest that variability of the refractory times in cats and mice is small. Indeed, (Heil et al., 2007 ) reported that spontaneous $R_{s}$ from different SGNs of cats could be well reproduced with fixed $t_{A}=0.59$ and $\tau_{r}=0.65$, by varying only $R_{r}$. In accordance, the average of the total refractory period $t_{A}+\tau_{R}$ was $1.0 \mathrm{~ms}$ with the $5-95$ percentile range of $0.7-1.7 \mathrm{~ms}^{-1}$ for maximally driven SGNs in mice (see Fig. S5 in Pangršič et al., 2015). We performed additional analysis of the data from (Pangršič et al., 2015) by using Eq. (4.53) and found that the average of $S_{s}$ was only $-0.08 \cdot \Delta_{V}\left[R_{s}\right]$, with the $5-95$ percentile range of $-0.14 \cdot \Delta_{V}\left[R_{s}\right]--0.05 \cdot \Delta_{V}\left[R_{s}\right]$. Here, we used the fact that, by definition, $\Delta_{V}\left[R_{s}\right]=k_{V} \cdot \ln (81)$. Accordingly, the measured $R_{s}$ values were tightly correlated with the modeled ones when assuming $t_{A}+\tau_{R}$ fixed to $1.0 \mathrm{~ms}$ (Fig. S6.2A). Differently, the match between the measured and modeled $R_{s}$ was very poor when fixing the $R_{r}$ to the average over the whole population (Fig. S6.2B). Thus, we conclude that the heterogeneity of $R_{s}$ of SGNs is mainly due to the heterogeneity of $R_{r}$, with only a minor contribution by the variability of $t_{A}+\tau_{R}$.

In the case of bursty presynaptic vesicle release, the $R_{r}$ dependence on $V_{m}$ results purely from the modulation of $p_{o}$. Indeed, the release rate within bursts does not depend on $V_{m}$. On the other hand, the postsynaptic refractoriness primarily reduces the number of events within bursts without affecting the burst length at any $V_{m}$ in this release scenario. Thus, we can conclude that the $R_{s}$ vs $V_{m}$ relation is a scaled down version of the corresponding $R_{r}$ vs $V_{m}$ relation, described by a Boltzmann function (section 4.4.1). Of course, when $N_{V}$ is so large that the vesicular release is approximated by a homogeneous Poisson process, a leftward shift of $R_{s}$ vs $V_{m}$ with respect to $R_{r}$ vs $V_{m}$ is expected. However, as shown above, physiological values of the refractory times $t_{A}$ and $\tau_{R}$ are too small to cause any considerable shift. To demonstrate the correctness of these statements we considered six different limiting $R_{r}$ vs $V_{m}$ 
relations with bursty vesicle release patterns identified in section 4.4.1 (Fig.4.3). As shown in Fig. S6.3 (2-state vesicular release sites) and Fig. S6.4 (7-state vesicular release sites), the normalized $R_{r}$ vs $V_{m}$ and $R_{s}$ vs $V_{m}$ relations indeed coincide well even when $\left(t_{A}+\tau_{R}\right) /\langle t\rangle_{I E I}$ is as high as 0.5 . It has to be noted that the amplitude reduction of the $R_{s}$ vs $V_{m}$ relations by the postsynaptic refractoriness in the case of bursty release is larger than in the case of a more regular release (Fig. S6.5). Indeed, intraburst IEIs form the majority of all IEIs and they are considerably shorter than the average IEI. Thus, more of these events fall in the refractory periods of SGNs compared to more regular vesicle release patterns.

\section{Inter-spike interval correlations}

In our model, each ISI is equal to the corresponding IEI or a sum of subsequent IEIs which fall in the time window of the ISI due to the refractoriness. Thus, if the IEIs are uncorrelated so are the ISIs. In other words, the refractoriness itself does not introduce correlations between ISIs. If IEIs are correlated, then correlations between the merged events at particular lag $l$ are smaller in magnitude than the correlations between the original events at the same lag number. This is a natural consequence of the Markovian nature of the presynaptic vesicle release process. Longer refractory periods result in larger numbers of subsequent IEIs merged, and thus, in smaller correlations between the subsequent ISIs. Fig. $4.8 \mathrm{C}$ compares $C_{I E I}$ vs $l$ and the corresponding $C_{I S I}$ vs $l$ relations for an $\mathrm{AZ}$ built of 7 -state vesicular release sites with $\mathrm{Ca}^{2+}$-microdomain topography scenario. There, the kinetic parameters of the model were fixed as reported by (Beutner et al., 2001), $k_{\text {rep }}$ was set so that the replenishment stage took half of the time of the whole vesicle cycle on average. The ratio $\left(t_{A}+\tau_{R}\right) /\langle t\rangle_{I E I}$ was set to 0.46 , the average value in mice SGN for maximally driven responses as shown in section 4.4.3. The reduction of ISI correlations was very pronounced for $N_{V}=2\left(C_{I E I}(1) / C_{I E I}(1) \sim 20\right)$ and considerably milder for $N_{V}=10$. As we showed in section 4.4.2, the IEI correlations decay faster with the lag number $l$ for $\mathrm{AZ}$ with smaller than larger $N_{V}$ for $\mathrm{AZs}$ with $\mathrm{Ca}^{2+}$-microdomain coupling regime or in the limit of fast channel gating. That is why correlations between the subsequent ISIs, which result from combining subsequent IEIs, are reduced more for AZs with smaller $N_{V}$. The situation is different in the regime of bursty vesicle release. Indeed, $C_{I E I}(l)$ decreases slowly with increased $l$ in this case (see section 4.4.2) and, thus, the reduction of the correlations between ISIs is expected to be considerably weaker even at small $N_{V}$. This is exactly what we see in Fig. 4.8D, where AZ with 2-state vesicular release sites in $\mathrm{Ca}^{2+}$-nanodomain coupling with 2-state $\mathrm{Ca}^{2+}$ channels were examined. The kinetic parameters there were set to the values which produce the maximum peaks of the $C_{I E I}$ vs $l$ relations found in section 4.4.2 (Fig. 4.7B). 
A

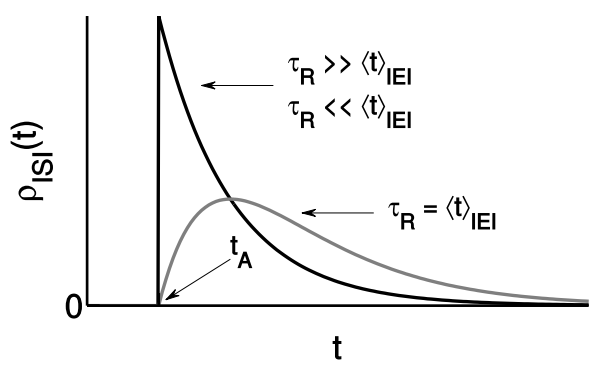

C

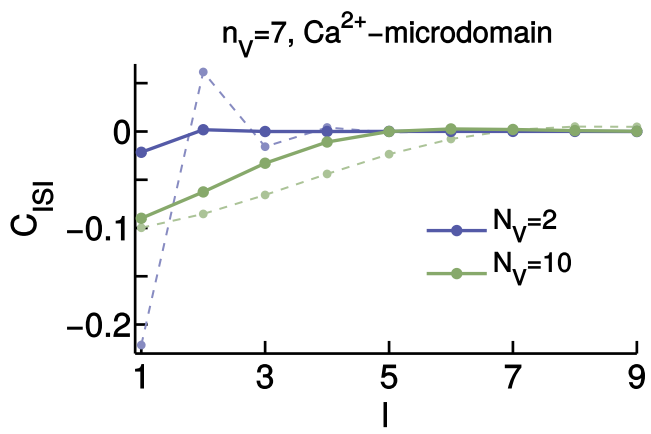

B

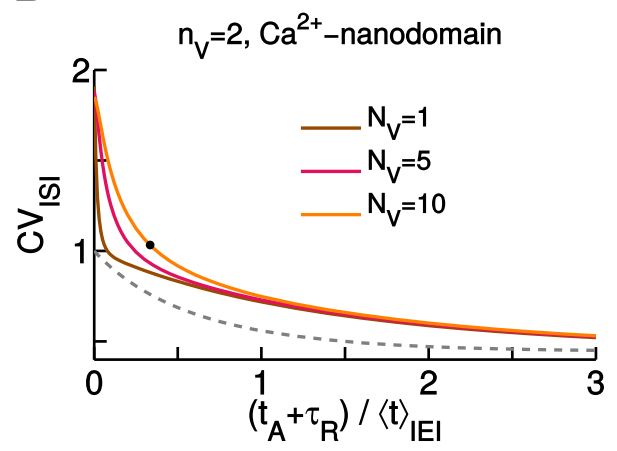

D

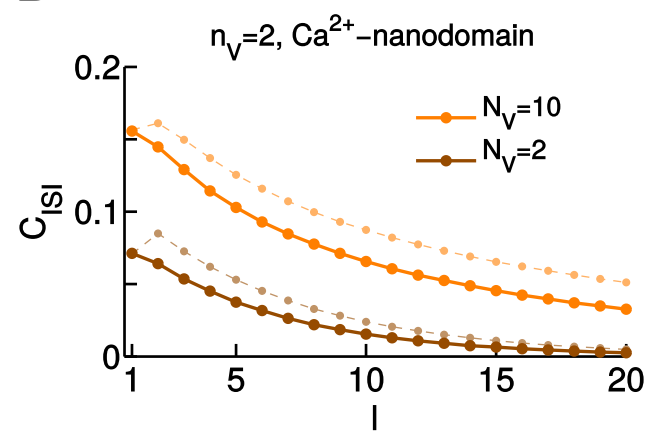

Figure 4.8: Impact of the refractoriness on the statistics of SGN spike trains. (A) Two limiting cases of $\rho_{I S I}(t)$ when the underlying presynaptic vesicle fusion is approximated by a homogeneous Poisson process. (B) The dependence of $C V_{I S I}$ on the overall refractory period in the case of a bursty vesicle release $\left(\mathrm{Ca}^{2+}\right.$-nanodomain coupling). Grey dashed line shows the $C V_{I S I}$ vs $\left(t_{A}+\tau_{R}\right) /\langle t\rangle_{I E I}$ relation for the presynaptic release described by a homogeneous Poisson process. (C) Influence of the refractoriness on the serial correlation functions of ISIs $\left(\mathrm{Ca}^{2+}\right.$-microdomain coupling, kinetic parameters taken from Beutner et al., 2001). Thin dashed lines show the corresponding $C_{I E I}$ vs $l$ relations. (D) Influence of the refractoriness on the serial correlation functions of ISIs $\left(\mathrm{Ca}^{2+}\right.$-nanodomain coupling, bursty release). Thin dashed lines show the corresponding $C_{I E I}$ vs $l$ relations.

\subsubsection{Analysis of experimental data: the origin of SGN response heterogeneity}

\section{Rate-level functions}

Most of the currently available knowledge about the heterogeneity of SGN responses to sound stimuli in vivo comes from the analysis of RLFs. A typical RLF has a sigmoid shape (Fig. 4.11) and is characterized by the minimum spike rate $\left(\min _{S P L}\left[R_{s}\right]\right)$, the maximum spike rate $\left(\max _{S P L}\left[R_{s}\right]\right)$, the dynamic range $\left(\Delta_{S P L}\left[R_{s}\right]\right)$, and the threshold $\left(\Theta_{S P L}\left[R_{s}\right]\right)$. The latter describes a shift of an RLF along the abscissa axis. One of the core results of our theoretical analysis in the previous sections was that the $R_{s}$ vs $V_{m}$ relations are approximated well by Boltzmann functions. Thus, we hypothesized that this dependence underlies the experimentally observed RLFs (after transforming $S P L$ to $V_{m}$ ). To test this hypothesis, we modeled the 
data from (Heil et al., 2011). In total, responses of 72 cat SGNs to sound bursts 0.75 octaves below the $\mathrm{CF}$ were considered (see Methods for more details).

From $\boldsymbol{S P L}$ to $\boldsymbol{V}_{\boldsymbol{m}}$ Before delving into a detailed analysis of the data, we summarize the most important qualitative features of the $S P L$ to $V_{m}$ transformation we found. Fig. 4.9A shows four normalized $R_{s}$ vs $V_{m}$ plots with identical dynamic ranges $\Delta_{V}\left[R_{s}\right]$ but shifted from each other along the $V_{m}$ axis. The corresponding $R_{s}$ vs $S P L$ plots, calculated by using the modified model of (Lopez-Poveda \& Eustaquio-Martin, 2006) (see section 4.2.1) are shown in Fig. 4.9B. In this case, the coefficients $\alpha$ and $\kappa$ were set to 0.5 and $44 \mathrm{~nm} \cdot \mathrm{Pa}^{-1}$, respectively. The maximum of the receptor potential $V_{d c}$ was assumed to be three times larger than $\Delta_{V}\left[R_{s}\right]$. As we see from Fig. 4.9B, the $R_{s}$ vs $S P L$ relations are described by sigmoid functions, however several important differences between them and the $R_{s}$ vs $V_{m}$ dependencies can be identified. First, $\min _{S P L}\left[R_{s}\right]$ is above zero in the case of the $R_{s}$ vs $S P L$ relations. Indeed, in the absence of sound, i.e., $S P L=-\infty, V_{m}=V_{\text {rest }}$ and, thus, $\min _{S P L}\left[R_{s}\right]=R_{s}\left(V_{m}=V_{\text {rest }}\right)>0$. A larger rightward shift of the $R_{s}$ vs $V_{m}$ relation along the $V_{m}$ axis results in a smaller $\min _{S P L}\left[R_{s}\right]$, and vice versa. Accordingly, a larger rightward shift of $R_{s}$ vs $V_{m}$ relation along the $V_{m}$ axis results in a larger $\Theta_{S P L}\left[R_{s}\right]$. That is why RLFs with larger $\Theta_{S P L}\left[R_{s}\right]$ have smaller minimum spike rates.
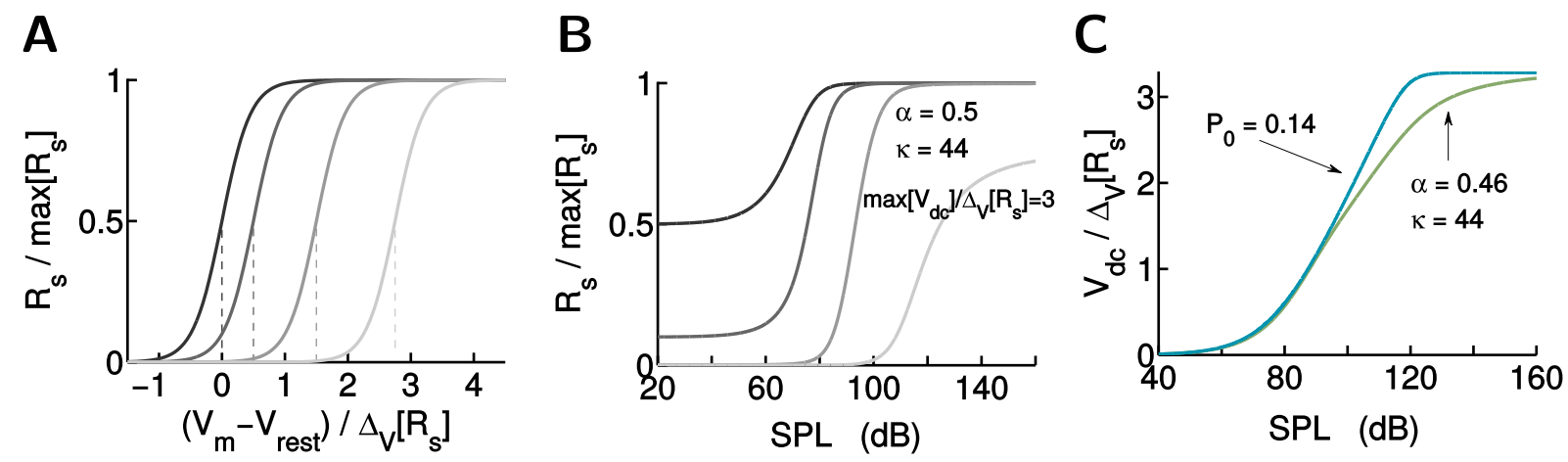

Figure 4.9: Properties of RLFs determined by the $V_{m} \rightarrow S P L$ transformations. (A) Four normalized $R_{s}$ vs $V_{m}$ dependencies shifted along the $V_{m}$ axis but with identical dynamic ranges. (B) $R_{s}$ vs $S P L$ relations corresponding to those shown in (A). The color code is the same in both panels. (C) Normalized $V_{d c}$ vs SPL dependencies. Green lines - the model of (Lopez-Poveda \& Eustaquio-Martin, 2006), blue line - the dependence described by Eq. (4.54).

Another important feature of the $R_{s}$ vs $S P L$ relation which we found was the dependence of $\Delta_{S P L}\left[R_{s}\right]$ on $\Theta_{S P L}\left[R_{s}\right]$. RLFs with different thresholds shown in Fig. 4.9B have different dynamic ranges on the $S P L$ axis, despite the fact that the dynamic ranges are identical on the $V_{m}$ axis. Two competing factors give rise to these differences: (1) the saturating dependence of $V_{m}$ on $P$ and (2) the logarithmic transformation between $P$ and $S P L$. Indeed, $V_{m}$ increases with $P$ at decreasing rate. Thus, the same increment of $V_{m}$ requires larger and larger increments of $P$ when the absolute value of $P$ is increased. On the other hand, the same increment of 
$P$ results in smaller increments of $\log _{10}(P)$, when the absolute value of $P$ is increased. These two factors do not compensate for each other exactly in general, and thus, $\Delta_{S P L}\left[R_{s}\right]$ changes with $\Theta_{S P L}\left[R_{s}\right]$. Finally, it is important to note that $\max _{S P L}\left[R_{s}\right]$ starts decreasing with the threshold in the range of very small $\min _{S P L}^{*}\left[R_{s}\right]$ (Fig. 4.9A) because of the saturation of the $V_{d c}$ vs $P$ relation (Fig. $\left.4.9 \mathrm{C}\right)$.

Dynamic ranges We started data analysis with the scatter plots of variables $\min _{S P L}\left[R_{s}\right] / \max _{S P L}\left[R_{s}\right] \equiv \min _{S P L}^{*}\left[R_{s}\right]$, and $\Delta_{S P L}\left[R_{s}\right]$ of the whole population of SGNs. The relation between $\min _{S P L}^{*}\left[R_{s}\right]$ and $\Delta_{S P L}\left[R_{s}\right]$ (Fig. 4.10A) was described by a smooth one-toone function. Formally, this is an intrinsic property of the empirical function used to fit the experimental data, which was necessary to evaluate $\Delta_{S P L}\left[R_{s}\right]$ (see Supplementary Material $\% .1$ for the expression). However, the fact that this empirical function describes the data very tightly (see Heil et al., 2011) means that the relation shown in Fig. 4.10A is a real phenomenon rather than a consequence of the choice of the fitting expression. The black dashed line in Fig. 4.10A corresponds to the best fit of the data by the model which assumes that each data point results from a shift of an $R_{s}$ vs $V_{m}$ relation described by a Boltzmann function along the $V_{m}$ axis. The underlying $V_{d c}$ vs $P$ relation here follows the modified model of (Lopez-Poveda \& Eustaquio-Martin, 2006), the same as in the plots shown in Fig. 4.9A, B (see section 4.2.1). Due to the uncertainty of the parameters $\alpha$ and $\max \left[V_{d c}\right] / \Delta_{V}\left[R_{s}\right]$, we varied both of them. The optimal values corresponding to the best fit were $\alpha=0.46$ and $\max \left[V_{d c}\right] / \Delta_{V}\left[R_{s}\right]=3.3$. As we see, the model deviated from the experiment noticeably only at $\min _{S P L}^{*}\left[R_{s}\right]<0.01$. This match can be further improved by using the data points shown in Fig. 4.10A themselves. Indeed, as shown in Supplementary Material \%.1, if the $R_{s}$ vs $V_{m}$ dependence is described by a Boltzmann function, then

$$
V_{d c}(P)=k_{V}^{\prime} \cdot \ln \left(\frac{1}{e^{-\max \left[V_{d c}\right] / k_{V}^{\prime}}+\left(1-e^{-\max \left[V_{d c}\right] / k_{V}^{\prime}}\right) \cdot\left(P / P_{0}+1\right)^{-3}}\right) .
$$

Here, $k_{V}^{\prime}$ is the voltage sensitivity of the $R_{s}$ vs $V_{m}$ relation, $P_{0}$ is the parameter which controls the shift of the curve along the $P$ axis and comes originally from the empirical model of RLFs introduced in (Heil et al., 2011). Please note that the meaning of $P_{0}$ is different from the hearing threshold $20 \mu \mathrm{Pa}$, which is often denoted by the same symbol in the literature. The $V_{d c}$ vs $S P L$ dependence calculated by using Eq. (4.54) with $\max \left[V_{d c}\right] / \Delta_{V}\left[R_{s}\right]=3$ is shown in Fig. 4.9C (blue curve). Along with it, the equivalent relation corresponding to the black dashed line in Fig. 4.10A is shown for comparison (green line). As expected, the two differ from each other only at higher $S P L$ values which fall within the dynamic ranges of RLFs with low $\min _{S P L}^{*}\left[R_{s}\right]$. Here, $\kappa$ and $P_{0}$ were chosen such that they reproduce the $\Theta_{S P L}\left[R_{s}\right]$ vs $\min _{S P L}^{*}\left[R_{s}\right]$ relations discussed later. 

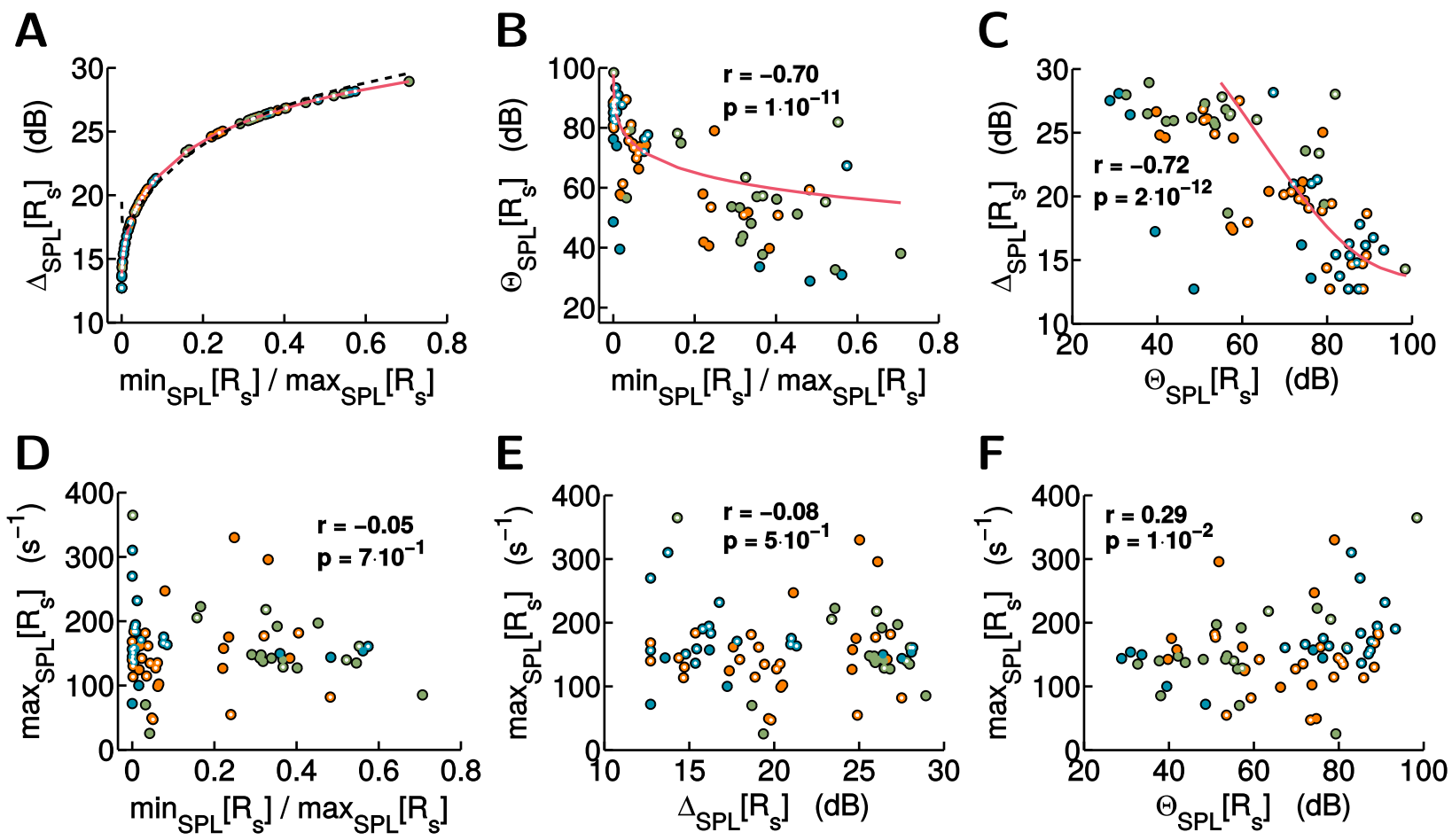

Figure 4.10: Scatter plots of the four parameters characterizing RLFs: $\min \left[R_{s}\right]$, $\max \left[R_{s}\right], \boldsymbol{\Delta}_{S P L}\left[\boldsymbol{R}_{s}\right]$, and $\Theta_{S P L}\left[\boldsymbol{R}_{s}\right]$. In all panels, filled circles represent data from different SGNs. Data points from the same animal are marked by the same color. White points within the filled circles stand for SGNs with CFs in the range $4-30 \mathrm{kHz}$. Solid red lines correspond to the best fits of the data points by the model. Insets: Pearson's correlation coefficients (denoted by r) between the plotted variables, and the corresponding $\mathrm{p}$ values.

How well Eq. (4.54) reproduces the data shown in Fig. 4.10A depends on the value of $\max \left[V_{d c}\right] / k_{V}^{\prime}$, or, equivalently, on the value of $\max \left[V_{d c}\right] / \Delta_{V}\left[R_{s}\right]$. The red line in Fig. 4.10A plots the estimate of $\Delta_{S P L}\left[R_{s}\right]$ vs $\min _{S P L}^{*}\left[R_{s}\right]$ relation assuming $\max \left[V_{d c}\right] / \max _{V}\left[R_{s}\right]=2$. It matches the data nearly perfectly. In order to estimate the lower bound for $\max \left[V_{d c}\right] / \max _{V}\left[R_{s}\right]$ values which could still reproduce the data within the limits of the measurement errors, we performed a bootstrap analysis (see Methods). No essential improvement of the fits was achieved at values higher than 2 (Fig. S7.3A, C). Noteworthy, Eq. (4.54) simplifies to $V_{d c}=3 \cdot k_{V}^{\prime} \cdot \ln \left(P / P_{0}+1\right)$ in the range of relevant $P$ values, if $\max \left[V_{d c}\right] / \max _{V}\left[R_{s}\right] \gtrsim 2$ (see Supplementary Material 7.1 ). In contrast, a decrease of $\max \left[V_{d c}\right] / \max _{V}\left[R_{s}\right]$ below 2 led to a rapid deterioration of the fits. These findings are in line with in vivo intracellular recordings from gerbils and guinea pigs, which showed that the dynamic range of $V_{d c}$ is over $60 \mathrm{~dB}$ SPL when stimulating at $\mathrm{CF}$, at least two times larger than the dynamic ranges of the RLFs (Goodman et al., 1982; Patuzzi \& Sellick, 1983; Cody \& Russell, 1986). Similar findings were published with stimulations below CF (see Figs. 4,6 in Patuzzi \& Sellick, 1983).

The value of $\gtrsim 2$ of $\max \left[V_{d c}\right] / \Delta_{V}\left[R_{s}\right]$ suggests an important physiological role of the multiple $\mathrm{Ca}^{2+}$ binding sites of the $\mathrm{Ca}^{2+}$ sensor of exocytosis. Indeed, the $\max \left[V_{d c}\right]$ was reported to be 
up to $\sim 20 \mathrm{mV}$ (Russell \& Sellick, 1978; Patuzzi \& Sellick; 1983, Cody \& Russell, 1986). It is possible that this value was underestimated due to shunting conductances introduced by penetrating IHCs with the recording electrodes. However, it could hardly be larger than $30 \mathrm{mV}$ as this would result in decreasing RLFs at very high SPLs due to a decreased rate of exocytosis (see Fig. 2B2 in Goutman \& Glowatzki, 2007). Thus, if $\max \left[V_{d c}\right] / \max _{V}\left[R_{s}\right] \sim 2$, then $\Delta_{V}\left[R_{s}\right] \sim 10-15 \mathrm{mV}$. On the other hand, the voltage sensitivity of the $\mathrm{Ca}^{2+}$ channel open probability, $k_{V}$, was found to be $\sim 7 \mathrm{mV}$ in mature IHCs (Johnson et al., 2005; Frank et al., 2010; Ohn et al., in preparation). $k_{V} \sim 7 \mathrm{mV}$ is equivalent to $\Delta_{V}\left[p_{o}\right] \sim 30 \mathrm{mV}$, i.e., at least two times that of the $\Delta_{V}\left[R_{s}\right]$. As we learned before (section 4.4.1), this is possible to achieve only with $\mathrm{Ca}^{2+}$ sensors of exocytosis with multiple $\mathrm{Ca}^{2+}$ binding sites.

The fact that $\Delta_{S P L}\left[R_{s}\right]$ and $\min _{S P L}^{*}\left[R_{s}\right]$ of RLFs are tightly related (Fig. 4.10A) means that $\Delta_{V}\left[R_{s}\right]$ varies very little from one SGN to another. In order to estimate the range of $\Delta_{S P L}\left[R_{s}\right]$ values which could reproduce the experimental estimates of RLFs within the range of measurement errors, we performed a bootstrap analysis. The result was that the $5-95$ percentile range of $\Delta_{S P L}\left[R_{s}\right]$ scaled by the average value of this parameter for each RLF was $0.95-1.06$. It is also important to note here that the relative changes in $\Delta_{S P L}\left[R_{s}\right]$ are considerably smaller than the corresponding relative changes of $\Delta_{V}\left[R_{s}\right]$ (Fig. S7.3D).

Thresholds If a shift of the $R_{s}\left(V_{m}\right)$ function along the $V_{m}$ axis is indeed the mechanism underlying the relation between $\Delta_{S P L}\left[R_{s}\right]$ and $\min _{S P L}^{*}\left[R_{s}\right]$, it should also explain the dependence of $\Theta_{S P L}\left[R_{s}\right]$ on $\min _{S P L}^{*}\left[R_{s}\right]$. Unfortunately, in this case, the comparison between the model and the experiment was not straightforward. Different from $\Delta_{S P L}\left[R_{s}\right], \Theta_{S P L}\left[R_{s}\right]$ showed a strong dependence on CF (Fig. S7.1). In the CF range from 0 to $20 \mathrm{kHz}$, average $\Theta_{S P L}\left[R_{s}\right]$ increased from $\sim 40 \mathrm{~dB}$ (low $\mathrm{CF}$ ) to $\sim 90 \mathrm{~dB}$ (high CF). This dependence of $\Theta_{S P L}\left[R_{s}\right]$ on $\mathrm{CF}$ is most likely explained by the particularities of cochlear micromechanics (Supplementary Material 7.1). Thus, we found, unsurprisingly, a rather large scatter of data points in the plot of $\Theta_{S P L}\left[R_{s}\right]$ vs $\min _{S P L}^{*}\left[R_{s}\right]$ relation (Fig. 4.10B). Nevertheless, there was still a strong negative correlation between the two parameters $\left(r=0.70, p=10^{-11}\right)$. We fitted the data with the model by assuming that the $R_{s}$ vs $V_{m}$ relation is described by a Boltzmann function and the receptor potential $V_{d c}$ follows Eq. (4.54) $\left(\max \left[V_{d c}\right] / \Delta\left[R_{s}\right]=2\right)$. The only free parameter which we varied to minimize the sum of the absolute differences between the model and experimental estimates of $\Theta_{S P L}\left[R_{s}\right]$ was $P_{0}$ (see Eq. (4.54)). Despite the scatter, the model with optimal $P_{0}=0.14 \mathrm{~Pa}$ could reproduce the main trend of the data (red line in Fig. 4.10B). The agreement between the experiment and the model was considerably improved when the $\mathrm{CF}$ range was narrowed to $4-20 \mathrm{kHz}$ (white points in Fig. 4.10B). Fig. 4.10C shows the corresponding relation between $\Delta_{S P L}\left[R_{s}\right]$ and $\Theta_{S P L}\left[R_{s}\right]$. This relation is fully determined by those which are shown in panels A and B of Fig 4.10.

If the scatter of data points in Fig. $4.10 \mathrm{~B}, \mathrm{C}$ is mainly due the $\mathrm{CF}$ dependent cochlear micromechanics, then it should be much smaller when reproducing $\Theta_{S P L}\left[R_{s}\right]$ of SGNs with 
similar CF. To examine this, we selected pairs of SGNs such that the distance between the innervated IHCs was not larger than $5 \%$ of the total length of the organ of Corti (see Methods). Then, we calculated the absolute difference of the thresholds of RLFs within each pair and subtracted it from the model estimate of this difference. In 67 selected pairs, the absolute difference between the model and the experiment was only $6.7 \mathrm{~dB}$, on average. This corresponds to average value of the difference between the model and the experimental estimates of $\Theta_{S P L}\left[R_{s}\right]$ of $6.7 / \sqrt{2} \sim 4.7 \mathrm{~dB}$. Such a small remaining difference can be accounted for by the measurement precision, the microstructure of the spiking thresholds of SGNs (Wilson, 1980; Long, 1984; Mauermann et al., 2004), and the variability of $V_{\text {rest }}$ across different IHCs. Thus, we conclude that the shift of a $R_{s}$ vs $V_{m}$ relation along the $V_{m}$ axis explains the dependence of $\Theta_{S P L}\left[R_{s}\right]$ on $\min _{S P L}^{*}\left[R_{s}\right]$. In accordance, the presence of a tight relation between the $\Theta_{S P L}\left[R_{s}\right]$ and $\min _{S P L}\left[R_{s}\right]$ was also reported for SGNs with similar CFs from guinea pigs, but stimulated at CF (Yates, 1991).

It follows from Fig. 4.10B, C that, after removing the scatter due to the chosen stimulation paradigm, the range of $\Theta_{S P L}\left[R_{s}\right]$ should be $\sim 40 \mathrm{~dB}$. Approximately this range was reported for SGNs from cats (Liberman, 1978, Fig. 12 therein), guinea pigs (Winter et al., 1990; Müller \& Robertson, 1991), gerbils (Ohlemiller et al., 1991), and mice (Taberner \& Liberman, 2005), when stimulating at CF. Note that, at least in guinea pigs, the thresholds of most of those RLFs occurred in the range where the dynamics of the basilar membrane is linear (Fig. 5 in Müller \& Robertson, 1991). This supports the idea that the strong CF dependence of $\Theta_{S P L}\left[R_{s}\right]$ found in our analysis is due to pecularities of the cochlear micromechanics, as discussed in Supplementary Material 7.1.

Maximum spike rates Next, we analyzed the heterogeneity of amplitudes of RLFs. There was no correlation between $\max _{S P L}\left[R_{s}\right]$ and $\min _{S P L}^{*}\left[R_{s}\right]$ in the population of RLFs studied (Fig. 4.10D). Thus, shifts of $R_{s}$ vs $V_{m}$ relations along $V_{m}$ axis, which give rise to different $\min _{S P L}\left[R_{s}\right]$, are independent of changes in $\max _{S P L}\left[R_{s}\right]$. This finding sets constraints on the transformations of the model parameters which underly the shifts and the maximum rates, as we learned before in sections 4.4.1 and 4.4.3. If $V_{d c}$ does not saturate in the relevant range of $P$ values, then the transformation of the model parameters has to preserve $\max _{V}\left[R_{s}\right]$. In agreement with our findings, no considerable variation of $\max _{S P L}\left[R_{s}\right]$ with $\min _{S P L}\left[R_{s}\right]$ was reported in cats (Evans \& Palmer, 1980), gerbils (Ohlemiller et al., 1991), and mice (Taberner $\&$ Liberman, 2005). In guinea pigs, $\mathrm{SGNs}$ with the highest $\min _{S P L}\left[R_{s}\right]$ were reported to have $\max _{S P L}\left[R_{s}\right]$ on average $\approx 100 \mathrm{~s}^{-1}$ lower than SGNs with the lowest $\min _{S P L}\left[R_{s}\right]$ (Yates, 1991; Müller \& Robertson, 1991). However, this finding was simply explained by a slower adaptation of spike rates in SGNs with low $\min _{S P L}\left[R_{s}\right]$ (Müller \& Robertson, 1991). Differently from guinea pigs, the adapting part seems not to contribute considerably to the estimates of $\max _{S P L}\left[R_{s}\right]$ in the other systems mentioned above. In cats (Heil et al., 2011) and mice (Taberner \& Liberman, 2005 ), the initial adaptation was considerably faster than the response time windows (100 and 
$50 \mathrm{~ms}$, respectively). In gerbils (Ohlemiller et al., 1991), spike rates in the adapted part of the responses were estimated.

In the sample of RLFs with $\mathrm{CF}>4 \mathrm{kHz}$ which was considered in this work, $\max _{S P L}\left[R_{s}\right]$ had a median value of $160 \mathrm{~s}^{-1}$ with the $5-95$ percentile range of $80-270 \mathrm{~s}^{-1}$. As we showed and discussed in section 4.4.3, the origin of this heterogeneity is primarily in the varying vesicle release rates, rather than the refractory periods. Assuming the median values of $t_{A}=0.59 \mathrm{~ms}$ and $\tau_{R}=0.65 \mathrm{~ms}$ in cat (Heil et al., 2007), we found that the median and the $5-95$ percentile range of $R_{r}$ were $200 \mathrm{~s}^{-1}$ and $90-400 \mathrm{~s}^{-1}$ respectively. Differences of $R_{s}$ could be due to the variability of the kinetic rates, $\left[\mathrm{Ca}^{2+}\right]$ levels at the sensors of exocytosis, the number of functional vesicular release sites at the presynaptic AZs. Interestingly, in cats, gerbils, and chinchillas, SGNs with low $\min _{S P L}\left[R_{s}\right]$ showed slower recovery kinetics of $R_{s}$ after stimulation (Rhode \& Smith, 1985; Ohlemiller et al., 1991; Relkin \& Doucet, 1991). This is compatible with the slower initial adaptation of $R_{s}$ in SGNs of lower $\min _{S P L}\left[R_{s}\right]$ from guinea pigs in response to pure tone sound bursts (Yates, 1991; Müller \& Robertson, 1991). The only way to have the same rates for synapses with slower kinetics per vesicular release site would be to have more vesicular release sites. Larger presynaptic bodies and $\mathrm{Ca}^{2+}$ channel clusters were indeed documented on the modiolar side of IHC, which is preferentially innervated by low-SR SGNs, in cats and mice (Merchan-Perez \& Liberman 1996; Liberman et al., 2011; Ohn et al., in preparation). One possibility is that the slowed kinetics of the vesicle release is a consequence of the kinetic parameter readjustment for a larger rightward shift of the $R_{s}$ vs $V_{m}$ relations along the $V_{m}$ axis.

We found that $\max _{S P L}\left[R_{s}\right]$ was not correlated with $\Delta_{S P L}\left[R_{s}\right]$ (Fig. 4.10E). This is a direct consequence of the results shown in panels A and D of Fig. 4.10. The correlation between $\max _{S P L}\left[R_{s}\right]$ and $\Theta_{S P L}\left[R_{s}\right]$ was weak $(r=0.29)$ with a rather high $p$ value $(0.01)$ (Fig. 4.10F). However, there was no considerable average increase in $\max _{S P L}\left[R_{s}\right]$ with $\Theta_{S P L}\left[R_{s}\right]$. 

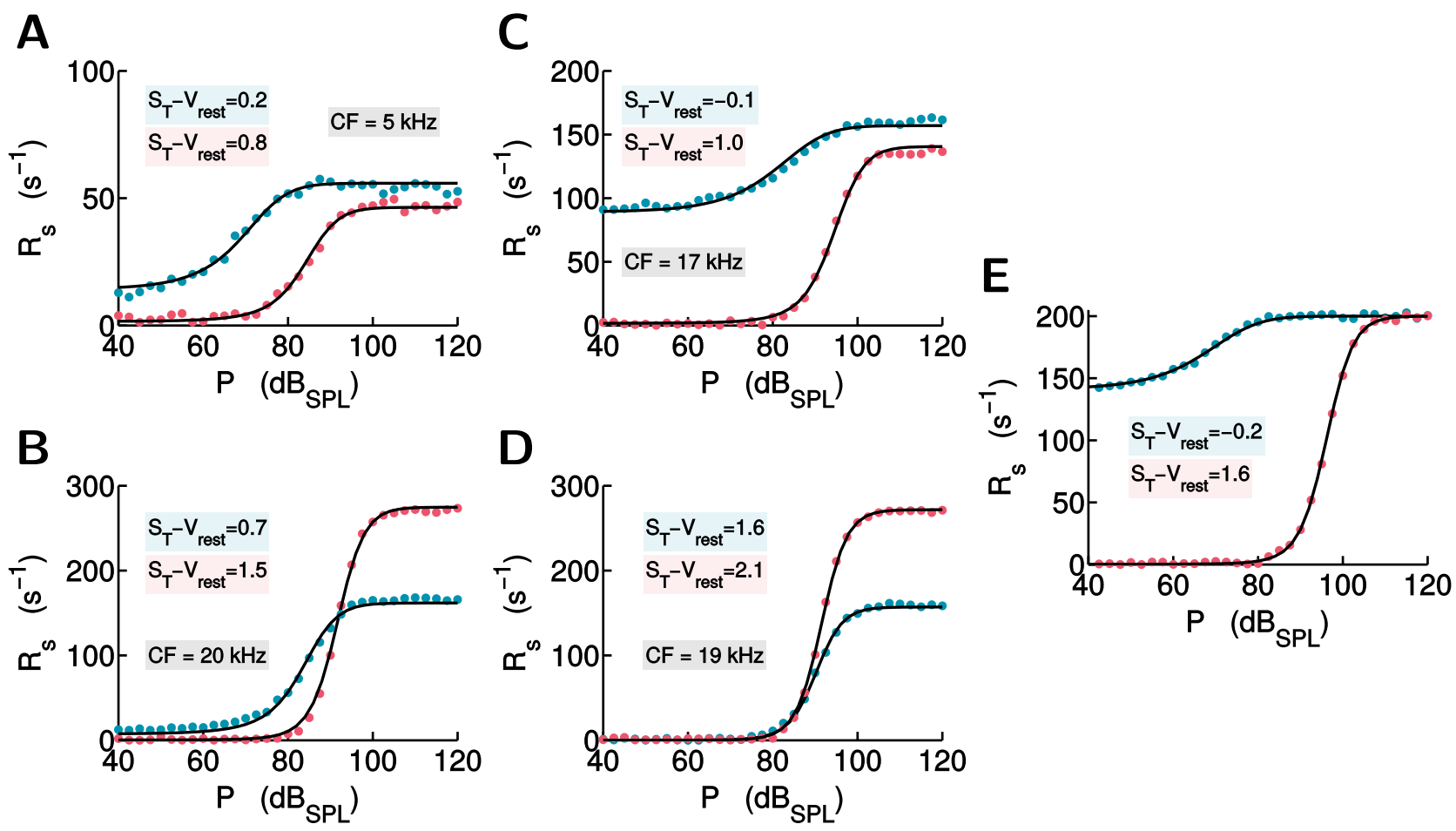

Figure 4.11: Model fits of selected RLFs. (A - D) show RLFs from selected pairs of SGNs with no more than $1 \%$ difference in the tonotopic distance. Pairs of RLFs with maximally different spike thresholds (A), dynamic ranges (B), spontaneous rates (C), and maximum rates (D) were considered. Blue and red points show data corresponding to the RLFs with respectively lower and higher thresholds in each pair. Black solid lines show model fits. (E) A surrogate pair of RLFs with identical $P_{0}$ whose thresholds differ by $40 \mathrm{~dB}$ (see text for more details). The colored insets show the differences between the values of parameters $S_{T}$ and $V_{\text {rest }}$ scaled by $\Delta_{V}\left[R_{s}\right]$ for each RLF. The grey insets show the CFs.

The extent of shifts of the $R_{s}\left(V_{m}\right)$ functions along the $V_{m}$ axis It follows from the above results that the heterogeneity of RLF functions can be fully accounted for by variations of the amplitudes and the $V_{m}$ shifts of the underlying $R_{s}\left(V_{m}\right)$ functions with respect to $V_{\text {rest }}$. The ability of our model to explain the relations between $\min _{S P L}^{*}\left[R_{s}\right], \Delta_{S P L}\left[R_{s}\right], \Theta_{S P L}\left[R_{s}\right]$ in detail guarantees the reproducibility of the whole spectrum of RLFs. To show this explicitly, we selected pairs of SGNs which innervated IHCs with up to $1 \%$ difference in the relative distance along the organ Corti. We then fitted their RLFs by assuming that $R_{s}$ vs $V_{m}$ relations are described by a Boltzmann function, and the $V_{m}$ vs $P$ relation by Eq. (4.54). An absolute difference of $P_{0}$ up to $5 \mathrm{~dB}$ was allowed within each pair of SGNs. Selected examples of the fitting results are plotted in Fig. 4.11. Notably, the RLFs shown in panels A, B, C, and D had maximum differences in $\Theta_{S P L}\left[R_{s}\right], \Delta_{S P L}\left[R_{s}\right], \min _{S P L}^{*}\left[R_{s}\right]$, and $\max _{S P L}\left[R_{s}\right]$, respectively, among all SGN pairs selected. Similar fit quality was obtained for the remaining of the selected SGN pairs (data not shown).

We further used the fitting results shown in Fig.4.11 to evaluate the range of the total shifts $\left(S_{T}=V_{0.5}+S_{r}+S_{s}\right)$ of the $R_{s}\left(V_{m}\right)$ functions with respect to $V_{\text {rest }}$. For RLFs shown in the 
panels A $-\mathrm{D}$, the differences between $S_{T}-V_{\text {rest }}$ in each pair of SGNs were up to $1.1 \cdot \Delta_{V}\left[R_{s}\right]$. Values of $\Theta_{S P L}\left[R_{s}\right]$ in each of these pairs differed by up to $20 \mathrm{~dB}$, which is approximately only half of the available range. To evaluate the difference in $S_{T}-V_{\text {rest }}$ values of SGNs on different sides of the spectrum of possible spiking thresholds, we generated two surrogate RLFs by using the empirical model by (Heil et al., 2011) (see Fig. 4.11E). $P_{0}$ was set to $0.14 \mathrm{~Pa}$ in both cases; $\Theta_{S P L}\left[R_{s}\right]$ differed by $40 \mathrm{~dB}$. After fitting this surrogate data with our model, we found that the underlying difference in $S_{T}-V_{\text {rest }}$ values was $1.8 \cdot \Delta_{V}\left[R_{s}\right]$.

Our results from section 4.4 .3 clearly showed that the variability of the refractory periods of SGNs contribute to the variability of $S_{T}-V_{\text {rest }}$ only marginally. Thus, the origin of the heterogeneity of this parameter should lie predominantly on the presynaptic side. The first possible mechanism is based on the differences in $V_{0.5}$. (Ohn et al., in preparation) found that $V_{0.5}$ varied in a range of $15 \mathrm{mV}$ in a population of 210 synapses from 25 IHCs studied in vitro. This would cover at least one half of the range of the $S_{T}-V_{\text {rest }}$ values if we assume that $\max \left[V_{d c}\right]$ does not exceed $30 \mathrm{mV}$ (see above). The second possible mechanism of the $S_{T}-V_{\text {rest }}$ heterogeneity is based on the differences in the kinetic parameters of the AZs. No experimental estimates of this variability are known to exist. However, our modeling results suggests this as one of the main candidates for explaining the remaining part of the RLF heterogeneity. The third potential mechanism of the $S_{T}-V_{\text {rest }}$ heterogeneity could be the variability of $V_{\text {rest }}$ among different IHCs. This scenario cannot explain the heterogeneity of SGNs innervating the same IHC. However, it could contribute to the range of the response heterogeneity of SGNs innervating different IHCs from nearly the same tonotopic position in the cochlear.

\section{Inter-spike interval distributions}

Fig. 4.12A - D show four different ISI probability density functions from SGNs recorded in vivo. Here, filled circles represent the experimental data, solid lines plot the model fits. The data in Fig. 4.12A, B were reproduced by assuming that presynaptic vesicle fusion is described by a homogeneous Poisson process. In fact, the $\rho_{I S I}(t)$ functions displayed in panels $\mathrm{A}$ and $\mathrm{B}$ are the two limiting cases corresponding to presynaptic vesicle release which follows a homogeneous Poisson process, as discussed in section 4.4.3 (Fig. 4.8A); $\tau_{R} \ll\langle t\rangle_{I E I}$ for $\rho_{I S I}(t)$ shown in Fig. 4.12A, and $\tau_{R} \sim\langle t\rangle_{I E I}$ for $\rho_{I S I}(t)$ shown Fig. 4.12B. $\rho_{I S I}(t)$ functions plotted in panels $\mathrm{A}$ and $\mathrm{B}$, and the functions intermediate between them, are found in the majority of SGNs from different animals (Li \& Young, 1993; Prijs et al., 1993; Heil et al., 2007; Pangršič et al., 2015). Our theoretical results provide a straightforward explanation for such an abundance of this type of $\rho_{I S I}(t)$ functions. Indeed, we showed in section 4.4 .2 that the presynaptic vesicle release converges to a homogeneous Poisson process in the limit of large $N_{V}$ at fixed $V_{m}$. Moreover, in a larger part of the space of kinetic parameters, the Poisson approximation works well already for AZs with a physiological $N_{V}(10-15)$. The only exception to this rule is made by the bursty vesicle release, when the convergence can be very slow (Fig. 4.6C). 

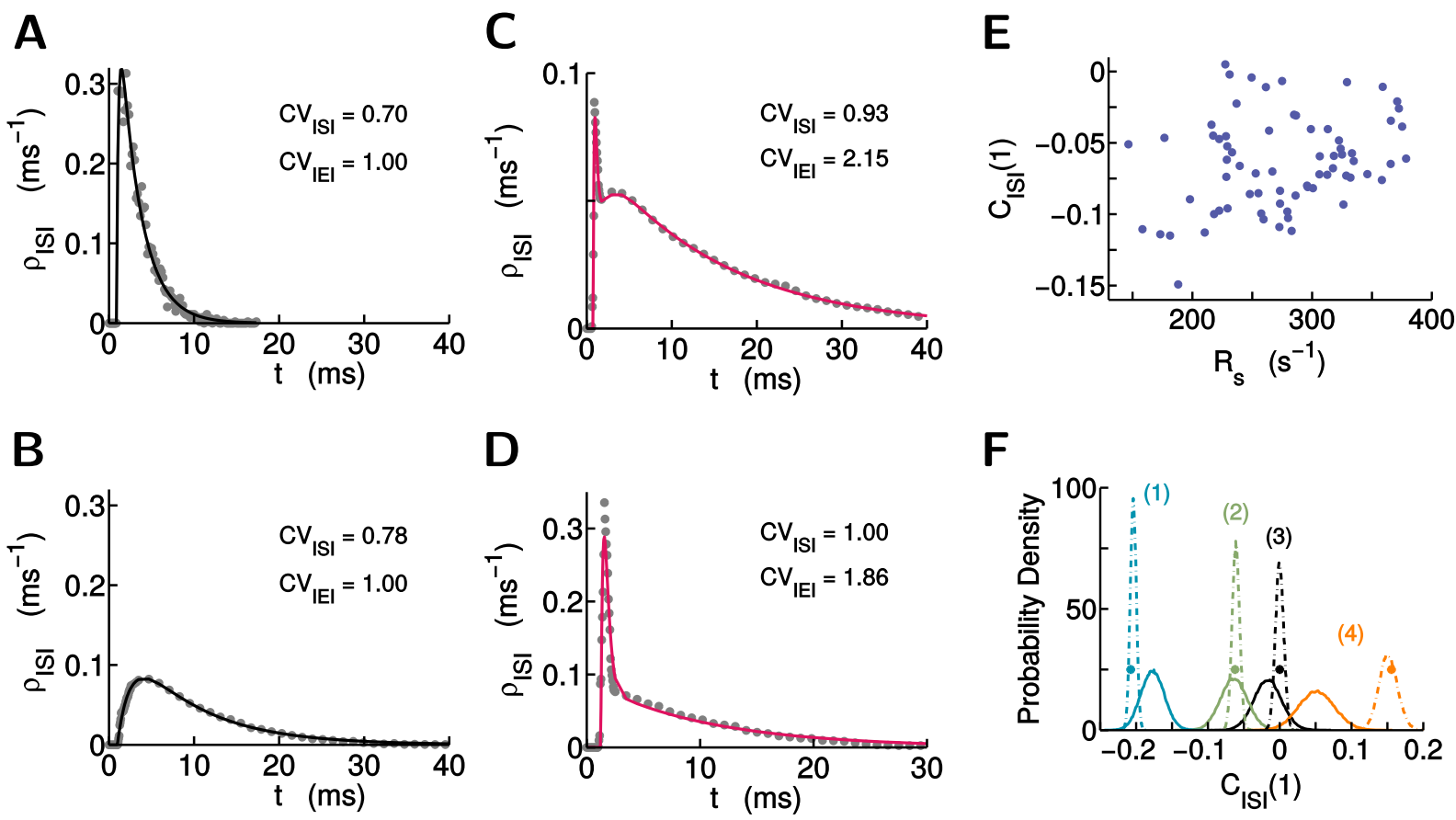

Figure 4.12: Experimental estimates of ISI distributions and correlations. $(\mathrm{A}, \mathrm{B})$ Exemplary $\rho_{I S I}$ vs $t$ relations which are well described by assuming that the presynaptic vesicle release is a homogeneous Poisson process. In (A), $\langle t\rangle_{I E I} \sim \tau_{R}$. In (B), $\langle t\rangle_{I E I} \gg \tau_{R}$. (C, D) Exemplary $\rho_{I S I}$ vs $t$ relations which are described by assuming bursty presynaptic vesicle release (taken from Prijs et al., 1993). In (A - D), the experimental data are shown by filled circles; the solid and dashed lines represent the model fits. The insets report $C V_{I S I}$ and the underlying $C V_{I E I}$ values of the best fits. (E) Scatter plot of the $C_{I S I}(1)$ vs $R_{s}$ relation. (F) Distributions of the estimated $C_{I S I}(1)$ due to finite size samples in four different scenarios of AZ organization, each represented by a different color (see text for details).

To estimate the extent of the deviation of presynaptic vesicle release in vivo from Poisson process, we fitted $72 \rho_{I S I}(t)$ functions of the same type as those shown in Fig. 4.12A, B taken from different SGNs from mice (see Methods). For this purpose, we considered the Poisson model and the biophysical model of AZs with ten 7-state vesicular release sites in $\mathrm{Ca}^{2+}$-nanodomain coupling scenario. We found that the discrepancy between the model and the data was only $11 \%$ smaller on average in the case of the biophysical model. This difference was, however, within the limits of noise due to a finite sample size (3100 ISIs per $\rho_{I S I}(t)$ on average) (see Fig. S7.4A). We found only small relative differences between the $\langle t\rangle_{I E I}$ and $C V_{I S I}$ of the experimental data and the Poisson model, $7 \%$ and $4 \%$ on average, respectively. This provides further support that IEIs underlying the $\rho_{I S I}(t)$ functions from Fig. 4.12A, B are, to a good approximation, distributed exponentially.

(Heil et al., 2007) proposed an empirical model which could fit the experimental $\rho_{I S I}(t)$ functions of the type shown in Fig. 4.12A, B better than the Poisson model. Instead of assuming exponentially distributed IEIs as in the Poisson model, (Heil et al., 2007) assumed a mixture of 
the exponential and gamma distributions. As we showed in the previous paragraph, not much space for an improvement is possible. And indeed, we found that the discrepancy between the mentioned model and the data was only $11 \%$ smaller on average compared to the Poisson model when fitting the $\rho_{I S I}(t)$ functions from mice, introduced above. Despite this, (Heil et al., 2007) found that fitting the experimental data with the Poisson model resulted in unrealistically large estimates of the refractory periods ( $\sim 3 \mathrm{~ms}$ on average). Suspiciously large values of $t_{A}+\tau_{R}$ were also reported in other previous works based on this model (Young \& Barta, 1986; Prijs, et al., 1993; Buran et al., 2010). In contrast, the empirical model of (Heil et al., 2007) resulted in physiological estimates of the refractory times (in the range $1-2 \mathrm{~ms}$ ). Given that the differences in $\rho_{I S I}(t)$ functions reproduced by both models are small, it is unexpected that the estimates of the refractory periods could differ so much. The resolution of this apparent paradox follows from findings by (Pangršič et al., 2015). We pointed out, in this work, that the $\rho_{I S I}(t)$ function of the Poisson model is symmetric to the exchange of $\tau_{R}$ and $R_{r}^{-1}$ (see Eq. (4.51)). Thus, there always exist two different values of $\tau_{R}$ which result in exactly the same $\rho_{I S I}(t)$ function. It was shown in (Pangršič et al., 2015), however, that the larger of the two values of $\tau_{R}$ always required unrealistically high $R_{r}$, which might exceed the experimental estimates up to 10 times. When only the smaller $\tau_{R}$ were assumed, the overall refractory periods fell within a physiological range $(0.75-2 \mathrm{~ms}$ with average of $1 \mathrm{~ms})$. If the larger $\tau_{R}$ had been chosen in that study, the overall refractory period would have instead been $3.3 \mathrm{~ms}$ on average.

Plots in Fig. 4.12C, D represent another group of $\rho_{I S I}(t)$ functions, which are qualitatively different from those shown in Fig. 4.12A, B. The key difference between the two groups is the presence of a sharp peak at small ISI times in the former one (Fig. 4.12C, D). This peak indicates that ISIs much shorter than the average ISI occur with a relatively high probability. And indeed, clusterings of spikes into short bursts of two or three spikes were documented in SGNs described by these $\rho_{I S I}(t)$ functions (Teich \& Khanna, 1985; Li \& Young, 1993; Prijs, et al., 1993). Only a minority of SGNs were reported to have $\rho_{I S I}(t)$ functions corresponding to the bursty spiking in cats: $6 \%$ by (Goumond et al., 1982), 14\% by (Li \& Young, 1993). Whereas $43 \%$ of SGNs from guinea pigs studied by (Prijs, et al., 1993) were of this type. Only 2 of 72 SGNs from mice analyzed by us had an early sharp peak in their $\rho_{I S I}(t)$ functions, which, however, corresponded to a rather small fraction of ISIs (see Fig. S7.4B, C). One of the possible reasons for such a low abundance of bursty spike trains in mice SGNs might be the underlying experimental procedure specific to these animals (see Methods). It was noticed, in the original studies, that such $\rho_{I S I}(t)$ functions cannot be explained by the Poissonian vesicle release convolved with the standard model of the refractoriness, as discussed above. (Miller \& Wang, 1993) introduced a physiologically inspired statistical model which could reproduce bursty spiking, at least semiquantitatively. In this model, the presynaptic vesicle release was described by a homogeneous Poisson process. However, a single EPSC could excite the SGN twice in a row with a certain probability. This model is, however, challenged by data from the intracellular recordings, which showed that SGNs are very phasic and the same EPSCs could 
hardly excite them twice (Rutherford et al., 2012; Siegel, 1992).

The results of our theoretical analysis in sections 4.4.1 and 4.4.2 suggest that a potential reason behind the bursty responses of SGNs could be the bursty presynaptic vesicle release. In order to examine how well this mechanism would explain the data at a quantitative level, we fitted the $\rho_{I S I}(t)$ functions showed in Fig. 4.12C, D. First, we considered the biophysical model of an AZ with 2-state vesicular release sites in $\mathrm{Ca}^{2+}$-nanodomain coupling regime. The match between the model fits and experimental data was very good (Fig. 4.12C, D, $N_{V}=10$ there). Both $\rho_{I S I}(t)$ functions could be fit sufficiently well with any $N_{V}$ in the range $1-10$. However, the fits were better for $N_{V}=10$ or higher (Fig. S7.5A, B). We also fit the same data by assuming AZ topographies of the intermediate $\mathrm{Ca}^{2+}$-domain coupling (Fig. S7.5C, D). The quality of the fit deteriorated with increased $N_{C}$ per vesicular release site, which emphasizes the importance of the $\mathrm{Ca}^{2+}$-nanodomain coupling in reproducing the bursty release.

\section{Inter-spike interval correlations}

Correlations between two subsequent ISIs, i.e., $C_{I S I}(1)$, that are significantly different from zero were reported in spontaneous spike trains from cats (Lowen \& Teich, 1992; Peterson et al., 2014) and guinea pigs (Prijs et al., 1993). Besides these studies, ISI correlations in SGNs have been largely ignored in the literature. However, our theoretical analysis from sections 4.4.2 and 4.4.3 showed that, compared to $\rho_{I S I}(t)$ functions and RLFs, this aspect of SGN responses carries valuable and unique information about the organization of presynaptic AZs. In this work, we studied ISI correlations of steady state spike trains in mouse SGNs of high CF driven to maximum rates by pure tone sound bursts (see Methods).

A scatter plot of the estimated $C_{I S I}(1)$ and spike rate for each neuron is shown in Fig. $4.12 \mathrm{E}$ $(n=72)$. The $C_{I S I}(1)$ values were broadly distributed between -0.149 and 0.005 , with average of -0.064. To understand how much the variability of these estimates could be affected by the finite size of ISI samples, we performed additional numerical modeling. For this purpose, we considered four $\mathrm{AZ}$ scenarios to cover a wide range of theoretically possible values of $C_{I S I}(1)$ : (1) five 7 -state vesicular release sites, $\mathrm{Ca}^{2+}$-microdomain coupling, (2) ten 2-state vesicular release sites, $\mathrm{Ca}^{2+}$-microdomain coupling, (3) vesicle release in the Poisson limit, (4) ten 2state vesicular release sites, $\mathrm{Ca}^{2+}$-nanodomain coupling. The kinetic parameters of the model were adjusted so that the $C_{I S I}(1)$ for the scenarios $(1),(2),(3)$, and (4) were respectively -0.20 , $-0.06,0$, and 0.15 . We then generated spike trains with each of these models, cut them into 260 pieces, each $50 \mathrm{~ms}$ long, and estimated $C_{I S I}(1)$, as in the case of the experimental data. The simulations were based on Gillespie's method (Gillespie, 1977). The event rate was set to $250 \mathrm{~s}^{-1}$, the average of the experimental $R_{s}$ over all SGNs studied. For each of the four scenarios we repeated the same procedure $10^{5}$ times to find the distribution of the estimate of $C_{I S I}(1)$ (Fig. $4.12 \mathrm{~F}$, solid lines). The widths of the distributions were large, comparable in magnitude to the average of our experimental estimate of $C_{I S I}(1)$. Indeed, widths of the $5-95$ percentile ranges for the four scenarios were from 0.051 (scenario (1)) to 0.074 (scenario (4)). 
In addition to the spread of the estimates, they were also shifted with respect to theoretical estimates (shown by filled circles of the respective color in Fig. 4.12F). The bias was positive at very negative values of $C_{I S I}(1)$ and negative at more positive values of $C_{I S I}(1)$. While the spread of the estimates is caused by the finite size of the data sample, the reason behind the bias is the finite number of ISIs in the measurement window (12.5 on average in our case). If the measurement window was increased from $50 \mathrm{~ms}$ to $500 \mathrm{~ms}$, both the bias and the spread would be reduced to a satisfactory level (dashed plots in Fig. 4.12F). The requirement of such a large sample size for estimating ISI correlations reliably is rather unexpected. Indeed, for the four scenarios shown in Fig. $4.12 \mathrm{~F}$, the $5-95$ percentile ranges of estimates of $\langle t\rangle_{I S I}$ and $C V_{I S I}$ would form only $\sim 6 \%$ of their absolute values if the sample size was only 3200 ISIs (the average per SGN in our data sample).

The uncertainties due to the finite size effects prohibited us to study the heterogeneity aspect of the ISI correlations and to study whole $C_{I S I}(l)$ vs $l$ relations in addition to $C_{I S I}(1)$. However, it was still possible to make important statements about the average values. First, we found that the probability of an average $C_{I S I}(1)$ of -0.064 or smaller was zero $\left(10^{-183}\right)$ if ISIs in all neurons studied were uncorrelated (see Methods for the procedure). The average $C_{I S I}(1)$ of the population would be only $\sim 0.006$ if all the neurons were uncorrelated. In fact, the probability of $C_{I S I}(1)$ as low or lower than estimated, if there were no real correlations, was higher than 0.01 only for 20 of 72 SGNs studied. Thus, ISIs were correlated in the majority of the neurons with an average $C_{I S I}(1) \sim 0.06$. A similar range of negative $C_{I S I}(1)$ were reported in spontaneous spike trains in cats (Peterson et al., 2014). As it follows from the results of sections 4.4.2 and 4.4.3, the whole range of $C_{I S I}(1)$ values shown in Fig. $4.12 \mathrm{E}$ can be reproduced assuming the physiological number of $N_{V}$. The fact that the magnitude of the correlations is relatively high (compared to what it maximally can be in the negative range) means that the vesicle release process is not limited by a single step at the maximally driving stimuli in vivo. Indeed, vesicle release which is limited by a single step in the chain of presynaptic processes follows the Poisson process very closely and would result in negligible $C_{I S I}(1)$. For example, if $k_{r e p}$ was decreased from $R_{r} / 2$ to $R_{r} / 10$ in the model considered in Fig. 4.7D $\left(N_{V}=10\right.$, green solid line), the $C_{I E I}$ would decrease from -0.09 to -0.006 .

The fact that $C_{I S I}(1)$ were preferentially negative in the SGNs studied here is in line with the finding that essentially none of these SGNs had signatures of bursty spiking (see section 4.4.4). The negative values of $C_{I S I}(1)$ could formally correspond to either the $\mathrm{AZ}$ working in the fast channel gating limit, and any desirable topography, or the AZ with the $\mathrm{Ca}^{2+}$ microdomain coupling topographies, and arbitrary kinetic parameters. In vitro estimates of the kinetic parameters (averaged over many synapses) are compatible with the fast channel gating scenario, or at least something close to it. Indeed, activation times of $\mathrm{Ca}^{2+}$ channels of several hundred microseconds were reported at $V_{m}$ corresponding to the peak $\mathrm{Ca}^{2+}$ influx (Frank et al., 2010; Zampini et al., 2013). Whereas the kinetic rates associated with the transitions between the states of vesicular release sites are around $1 \mathrm{~ms}^{-1}$ (Beutner et al., 2001). Despite 
the absence of positive correlations in the SGNs studied by us, slightly positive $C_{I S I}(1)$ were reported by (Prijs et al., 1993) in spontaneous spike trains of SGNs from guinea pigs. As predicted by our theoretical analysis, most of those spike trains were bursty. (Peterson et al., 2014) proposed that the positive $C_{I S I}(1)$ estimated in cat SGNs by them could be due to the non-stationarity of the spiking. Though this could indeed be the case for some SGNs, especially those with very positive $C_{I S I}(1)$, slightly positive $C_{I S I}(1)$, like those in (Prijs et al., 1993), could be genuine. To test this reliably, however, considerably larger sample sizes of ISIs would be required than those employed in (Peterson et al., 2014) or this dissertation.

\subsection{Methods}

All analysis of the experimental and modeling data was done with MATLAB (release R2012b, Mathworks).

\subsubsection{Analytical calculations}

All calculations based on the analytical expressions provided throughout the work were performed in MATLAB. In those cases when the transition matrices of the kinetic equations could not be diagonalized analytically, this was done numerically with the built-in function eig. It has to be noticed that, in some cases, the numerical diagonalization had to be done by using high precision arithmetics. Usually, this happened when performing the global optimization with loosely restricted parameter ranges, which could result in a substantial separation of timescales. The Symbolic Math Toolbox was used for this purpose.

\subsubsection{Numerical optimization}

Global numerical optimization was extensively used for analyzing properties of the models and fitting of the experimental data in this work. In all cases, global optimization based on a genetic algorithm (MATLAB function $g a$ ) was followed by Nelder-Mead simplex method (MATLAB function fminsearch). For the first step, the following optimization options were used: Generations $=200$, StallGenLimit $=150$, PopulationSize $=100$, EliteCount $=10$, CrossoverFraction=0.6, TolFun $=10^{-10}$. Ranges of the allowed values of the optimization variables were customized for each optimization problem. The initial choice of the range was based on general understanding of the limiting cases obtained from the analytical treatment of the model. In all cases, it was made sure that the optimal solutions were not limited by the choice of the boundaries of the parameter space. The initial values of the optimization variables were uniformly distributed over the allowed parameter space. The optimal solution from the first optimization step was used as the initial condition for the second optimization step. The output of the second optimization step was taken as the final result. The optimization procedure was repeated with 100 different realizations of initial conditions. In cases when optimization with constrains was 
performed, the second step was skipped; the number of the optimization repetitions was then increased from 100 to 1000. Depending on the problem, high performance computing facilities were used in some cases to reduce the computation time to convenient levels (up to several days, up to several hundred CPU cores).

\subsubsection{Calculation of presynaptic $\left[\mathrm{Ca}^{2+}\right]$}

Simulations of the dynamics of presynaptic $\left[\mathrm{Ca}^{2+}\right]$ determined by $\mathrm{Ca}^{2+}$ influx through a single channel, as shown in Fig. 4.2A, were done by using the same method as described in chapter 3 (the single channel current was set to $0.3 \mathrm{pA}$ ). The only difference was that the timecourse of random gating of the channel was assumed in the present case. A random realization of switching between the closed and open states of the channel was generated using Gillespie's algorithm (Gillespie, 1977) and assuming $k_{-1}=1.4 \mathrm{~ms}^{-1}$ and $k_{+1}=1.8 \mathrm{~ms}^{-1}$ which correspond to $V_{m}=-20 \mathrm{mV}$ (see Supplementary Material 9 in chapter 2). The remaining parameters were the same as provided in section 7.1 of the supplementary material of chapter 3.

Simulations of the dynamics of $\left[\mathrm{Ca}^{2+}\right]$ at the presynaptic AZ determined by $\mathrm{Ca}^{2+}$ influx through an array of channels was done using the same method as described in the last subsection of "Additional results" in chapter 2. The only difference was that we calculated not only time-averaged steady state $\left[\mathrm{Ca}^{2+}\right]$ but also steady state $\left[\mathrm{Ca}^{2+}\right]$ due to a particular set of open channels in a frozen configuration in the present case. We considered depolarizations of the plasma membrane to three levels: $-40 \mathrm{mV},-30 \mathrm{mV}$, and $-20 \mathrm{mV}$. The respective channel open probabilities were 0.08, 0.2, and 0.32 at (based on the data in Wong et al., 2014). Accordingly, the single channel currents were set to $0.46 \mathrm{pA}, 0.36 \mathrm{pA}$, and $0.3 \mathrm{pA}$.

\subsubsection{Calculation of IHC receptor potential}

In order to estimate the receptor potential of IHCs, we solved the original equations of the biophysical model by (Lopez-Poveda \& Eustaquio-Martin, 2006). We were interested only in responses to high frequency stimuli in a steady state. Thus, we only had to find the stationary solutions of those equations, assuming the time-averaged level of the mechanotransducer current at certain hair bundle deflection amplitude. These equations were solved numerically, by using MATLAB's built-in solver of nonlinear algebraic equations fsolve. All the equations and model parameters are provided in (Lopez-Poveda \& Eustaquio-Martin, 2006).

\subsubsection{Analysis of experimental data}

\section{Rate-level functions}

The data related to the experimental estimates of rate-level functions were kindly provided by Peter Heil, and were the same as in (Heil et al., 2011). In total, RLFs from 72 SGNs from 3 cats were considered. Details of the data collection can be found in the original paper. In that 
work, an empirical model was introduced which provides very good fits to the experimental spike rate vs sound level relations. Thus, instead of considering the raw data, we used the best fit parameters of the empirical model in this dissertation. Exemplary RLFs shown in Fig. 4.11 were generated by using the best fit parameters of the corresponding experimentally estimated RLFs and adding the Gaussian noise with zero mean. The standard deviation of the noise was set to the mean square root deviation between the experimentally measured RLFs and their best fits with the empirical model of (Heil et al., 2011).

To select SGNs which innervate IHCs of similar cochlear tonotopic position (section 4.4.4), we had to determine the relative positions of the innervation points along the organ of Corti. This was done by using an empirical expression by (Liberman, 1982)

$$
x=\log _{10}(\mathrm{CF} / 456+0.8) / 2.1,
$$

where $\mathrm{CF}$ is the characteristic frequency in $\mathrm{Hz}$, and $x$ is the proportion of the total normalized length from the apex of the cochlea to the innervation point.

In order to estimate the distribution of optimal values of $\max \left[V_{d c}\right] / \Delta_{V}\left[R_{s}\right]$ due to the noise in the experimental data, we performed bootstrapping analysis (section 4.4.4). Each RLF was generated by using the empirical model of (Heil et al., 2011) with the optimal fit parameters and Gaussian noise of zero mean added on the top. The standard deviation of the noise term was set to the mean square root deviation between the experimentally measured RLFs and their best fits with the empirical model. Then, the RLFs were refitted over 1000 different realizations of the noise terms. This fitting procedure was repeated at different values of $\max \left[V_{d c}\right] / \Delta_{V}\left[R_{s}\right]$, which ranged from 0.1 to 5 by a step of 0.1 . The three fitting parameters were $V_{0.5}-V_{\text {rest }}, A_{s}$, and $P_{0}$. The fitting was performed by using the optimization method described above (section $4.5 .2)$.

\section{ISI distributions}

$\rho_{I S I}(t)$ functions corresponding to the SGNs from guinea pigs (Fig. 4.12B - D) were taken from Fig. 2 in (Prijs et al., 1993). All other $\rho_{I S I}(t)$ functions considered in this work were based on the recordings from mouse SGNs and were estimated by us. One part of the data was taken from (Pangršič et al., 2015), the original article of chapter 3 of this dissertation. Another part of the data, based on the same experimental procedures as in (Pangršič et al., 2015), was kindly provided by Nicola Strenzke and her group, InnerEarLab, University of Goettingen. SGN responses to $100 \mathrm{~ms}$ long pure tone bursts at CF, with the presentation rate of $2 \mathrm{~s}^{-1}$, were recorded. The stimulus intensity was set to $30 \mathrm{~dB}$ above the spiking threshold, which drove SGNs to maximal or nearly maximal spiking rates (Taberner \& Liberman, 2005). The number of stimulus bursts per SGN varied between 200 and 600 with average of 260. Only SGNs with $\mathrm{CF}>4 \mathrm{kHz}$ were selected for analysis to make sure that the AC component of the receptor potentials of IHC is negligible. In fact, CFs of $80 \%$ of the SGNs studied were higher than $10 \mathrm{kHz}$. The SGNs were approached with glass microelectordes through the cochlear nucleus. 
The details of the experimental procedures used to collect the data from mice SGNs can be found in (Taberner \& Liberman, 2005).

ISIs were estimated from the last $50 \mathrm{~ms}$ of responses to the repetitive, $100 \mathrm{~ms}$ long stimuli. The number of the resulting ISIs per each neuron varied from 1200 to 8900 with average of 3200. Only responses with sufficiently stationary spike rates throughout the whole stimulation time were considered. In order to evaluate the stationarity of ISIs of a particular SGN, we calculated a normalized cumulative ISI time as a function of the number of the ISIs: $\bar{c}(l)=$ $\sum_{i=1}^{l} t_{I S I}(i) / \sum_{i=1}^{n_{I S I}} t_{I S I}(i)$. Then, we compared the resulting cumulative function with $\tilde{c}(l)=l$. The later would correspond to ideally stationary sequence ISIs of fixed length. Only SGNs with $\max _{l}|\bar{c}(l)-\tilde{c}(l)|<0.04$ and $1 / l \sum_{l=1}^{n_{I S I}}|\bar{c}(l)-\tilde{c}(l)|<0.02$ were selected for further analysis. For 72 out of 77 SGNs which were selected, the average $\max _{l}|\bar{c}(l)-\tilde{c}(l)|$ and $1 / l \sum_{l}|\bar{c}(l)-\tilde{c}(l)|$ were equal to 0.016 and 0.007 respectively.

The bin sizes, $n_{\text {bin }}$, of the three $\rho_{I S I}(t)$ functions from guinea pigs shown in Fig. $4.12 \mathrm{~B}-$ D were 69,63 , and 63 , respectively. The binning was not uniform, with more points in the regions with faster variations of the functions. For all $\rho_{I S I}(t)$ functions from mice, $n_{b i n}$ was 100 . The experimental $\rho_{I S I}(t)$ functions were fitted with the models by minimizing the following discrepancy function

$$
d=\frac{\frac{1}{n_{b i n}} \cdot \sqrt{\sum_{i=1}^{n_{b i n}}\left(\rho_{I S I}^{e}\left(t_{i}\right)-\rho_{I S I}^{m}\left(t_{i}\right)\right)^{2}}}{\frac{1}{n_{b i n}} \cdot \sum_{i=1}^{n_{b i n}} \rho_{I S I}^{e}\left(t_{i}\right)}
$$

where superscripts $e$ and $m$ denote, respectively, the experimental and the model estimates.

\section{ISI correlations}

ISI correlations were estimated from the same data samples of recordings of mouse SGNs as the $\rho_{\text {ISI }}(t)$ functions described above. The following estimator of the values of the serial correlation function was used:

$$
\bar{C}_{I S I}(l)=\frac{\frac{1}{\sum_{i=1}^{n_{r e p}}\left(n_{I S I, i}-l\right)} \cdot \sum_{i=1}^{n_{\text {rep }}} \sum_{j=1}^{n_{I S I, i}-l}\left(t_{I S I}(i, j)-\langle t\rangle_{I S I}\right) \cdot\left(t_{I S I}(i, j+l)-\langle t\rangle_{I S I}\right)}{\frac{1}{\sum_{i=1}^{n_{r e p}} n_{I S I, i}} \cdot \sum_{i=1}^{n_{\text {rep }}} \sum_{j=1}^{n_{I S I, i}}\left(t_{I S I}(i, j)-\langle t\rangle_{I S I}\right)^{2}},
$$

where $n_{\text {rep }}$ is the number of tone burst applications for each SGN, $n_{I S I, i}$ is the number of ISIs for SGN response to the $i$-th repetition of the tone burst.

To test the hypothesis that estimates of $\bar{C}_{I S I}(1)$ could result from spike trains of uncorrelated events, we had to calculate the distributions of the $\bar{C}_{I S I}(1)$ based on the uncorrelated spike trains. This was done in the following way. First, we fitted the experimental $\rho_{I S I}$ functions with the Poisson model (including the convolution with the refractoriness, see section 4.4.3) for all SGNs studied. As we found in 4.4.3, this model could reproduce the $\rho_{I S I}$ functions of mouse SGNs well. Next, based on the best fitting parameters, we performed stochastic simulations and generated $N 50 \mathrm{~ms}$ long windows of spike trains. The simulations were done by using Gillespie's 
method. $N$ was set to match the number of the tone burst applications used in the experiments for each SGN individually. Then, we used the simulated data to calculate $\bar{C}_{I S I}(1)$ based on Eq. (4.57). The same procedure was repeated $10^{5}$ times, which resulted in the distribution of the estimator $\bar{C}_{I S I}(1)$. 


\section{Supplementary Material 1: Formulation of the model}

\subsection{Dependence of $\mathrm{Ca}^{2+}$ channel gating rates on the membrane po- tential}

To evaluate the $V_{m}$ dependence of the channel gating kinetics, we used data from whole cell recordings provided in (Neef et al., 2009). In that work, $\mathrm{Ca}^{2+}$ current activation kinetics was

described by a three state channel model $C_{1} \underset{1 \cdot k_{-1}}{\stackrel{2 \cdot k_{+1}}{\rightleftarrows}} C_{2} \underset{2 \cdot k_{-1}}{\stackrel{1 \cdot k_{+1}}{\rightleftarrows}} O$. The $V_{m}$ dependence of $\mathrm{Ca}^{2+}$ channel open probability was well described by a Boltzman function, $p_{o}=\bar{p}_{o} /\left(1+e^{-\left(V_{m}-V_{0.5}\right) / k_{V}}\right)$. Fig. S1.1A shows the $p_{o}$ vs $V_{m}$ relation based on the best fit parameters of the experimental data. There, $\bar{p}_{o}$ was set to 0.4 , as it follows from nonstationary fluctuation analysis of $\mathrm{Ca}^{2+}$-tail currents (see Wong et al., 2014 and Supplementary Material 9 therein). Note that $p_{o}=\left(k_{+1} /\left(k_{-1}+k_{+1}\right)\right)^{2}$. The activation time constant $\tau=\left(k_{-1}+k_{+1}\right)^{-1}$ could not be reliably evaluated by fitting the $\mathrm{Ca}^{2+}$-current activation at very negative $V_{m}$ because of the low signalto-noise ratio. Thus, we extrapolated its values based on the fit of the available estimates by $\tau\left(V_{m}\right)=a \cdot e^{b \cdot V_{m}}$ (Fig. S1.1B).

Based on the plots shown in Fig. S1.1A - B, we then reconstructed the average times the channel spends in its closed and open states at fixed $V_{m}$ (Fig. S1.1C). Note that $\tau_{\text {open }}=1 /\left(2 \cdot k_{-1}\right)$ and $\tau_{\text {closed }}=\left(2 \cdot k_{+1}+k_{-1}\right) /\left(2 \cdot k_{+1}^{2}\right)$. The solid lines in Fig. S1.1C show estimates in the range of $V_{m}$ values on the left of $-20 \mathrm{mV}$, which encompass the physiological range of the $V_{m}$ values. Indeed, it is known that the maximum $\mathrm{Ca}^{2+}$-influx is observed at $V_{m} \sim-20 \mathrm{mV}$ (see, e.g., Johnson et al.; 2008, Wong et al., 2014) and that exocytosis rate starts decreasing if IHC is depolarized further (Goutman \& Glowatzki, 2007). On the other hand, spike rates of SGNs are saturating functions of the sound level. This means that $V_{m}$ of IHCs should not exceed $-20 \mathrm{mV}$. Estimates of the resting potential and maximum values of the receptor potential of IHCs in vivo supports the idea that the $V_{m}$ value corresponding to the maximum $\mathrm{Ca}^{2+}$ influx is not exceeded (see Sellick \& Patuzzi, 1983; Cody \& Russell, 1987). As it follows from (Fig. S1.1C), the change in $V_{m}$ in the physiological range causes essentially no change in $\tau_{\text {open }}$, but strongly affects $\tau_{\text {closed }}$. This means that the channel closing rates depend on $V_{m}$ very little and that the majority increase of $p_{o}$ with increased $V_{m}$ is due to the increase in the channel opening frequency, not the lifetime of the open state. Data from single channel recordings by (Zampini, et al., 2011; Zampini, et al., 2013; Zampini, et al., 2014) support this idea.

\subsection{Physiological basis of the refractory counter model of SGNs}

The refractory time counter model of SGN was introduced on a phenomenological basis with a purpose to quantitatively describe experimental data of SGN spiking statistics (see, e.g., Teich et al., 1978; Teich \& Lachs, 1979). Current knowledge based on the intracellular recordings, however, suggests that the model reflects the biophysical peculiarities of spike generation at 

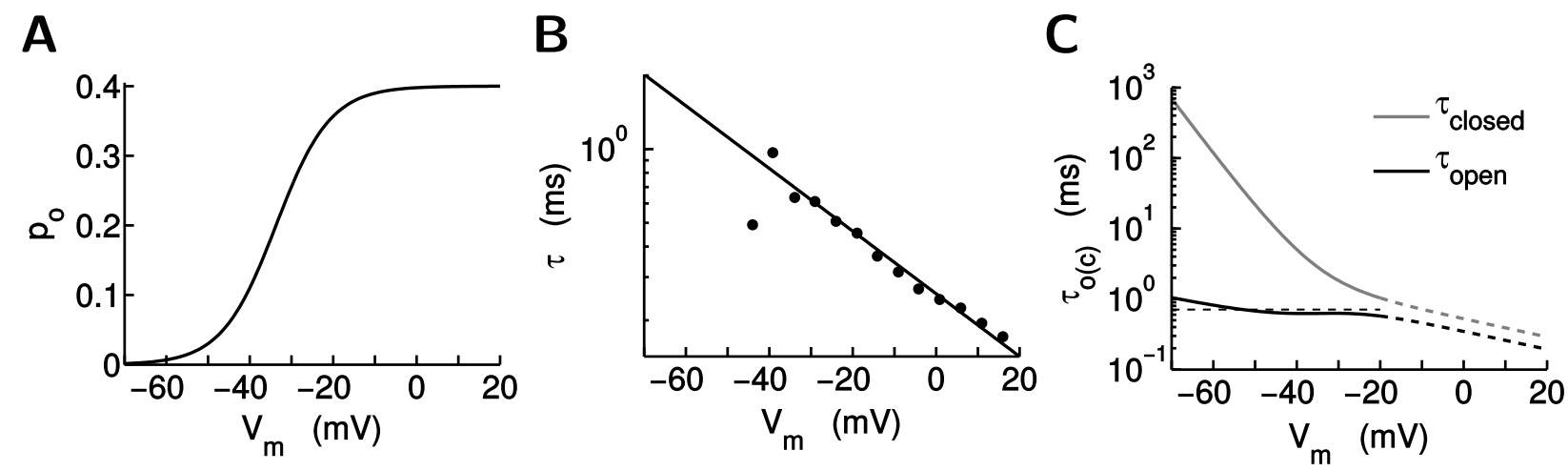

Figure S1.1: (A) $\mathrm{Ca}^{2+}$ channel open probability as a function of $V_{m}$, evaluated by using the best fit parameters from (Neef et al., 2009). (B) $V_{m}$ dependence of the channel activation time constant $\tau$. Filled circles correspond to the experimental data from (Neef et al., 2009). The best fit of the data is plotted by the solid line. (C) Average times the channel spends in its open and closed states at different $V_{m}$ as reconstructed from the data shown in (A) and (B). The horizontal dashed line marks the average value of $\tau_{\text {open }}$ in the range of $V_{m}$ from $-70 \mathrm{mV}$ to $-20 \mathrm{mV}$.

SGNs quite well. Indeed, it was shown that SGNs are phasic neurons which generate not more than one spike in response to EPSCs induced by the presynaptic vesicle release (Siegel, 1992; Rutherford et al., 2012). At small spontaneous spiking rates (3 spike/s), 81\% and 97\% of the EPSCs induced spikes in two mature SGNs studied by (Rutherford et al., 2012). This number was $84 \%$ and $92 \%$ for 57 spike/s and 51 spike/s spontaneous firing fibers studied by (Siegel, 1992). More detailed analysis by (Siegel, 1992) showed that nearly all but a few EPSCs that occurred $20 \mathrm{~ms}$ or later after a previous spike, were capable of inducing another spike. At smaller inter event times, the success rate of spike generation was smaller and had a pronounced dependence on EPSP rise time: slower initial depolarizations meant lower success rate (Siegel, 1992). It was shown later that shapes of EPSCs recorded at SGN boutons are indeed highly variable and with no evident correlation from one event to another (Glowatzki \& Fuchs, 2002; Grant et al., 2010; Rutherford et al., 2012). This would explain why the relative refractory period in the refractory counter model of SGNs is random, not deterministic. The presence of the fixed absolute refractory period in SGNs follows from the properties of voltage gated $\mathrm{Na}^{+}$ channels, as in all other spiking neurons. 


\section{Supplementary Material 2: Analytical Treatment of the Model}

\subsection{Proof of the existence and uniqueness of the solution of equation (4.9) in section 4.3}

Here, we show that Eq. (4.9) in section 4.3 has a unique solution which is normalized to 1. For convenience, let us rewrite the original equation here,

$$
P_{0}^{s}(\vec{c}, \vec{v})=\sum_{\vec{c} * \mathcal{C}} \sum_{\vec{v}_{*} \in \mathcal{V}} T\left(\vec{c}, \vec{v} \mid \vec{c}_{*}, \vec{v}_{*}\right) \cdot P_{0}^{s}\left(\vec{c}_{*}, \vec{v}_{*}\right)
$$

In the above expression, $T$ is a known matrix whose rows and columns are indexed correspondingly by $(\vec{c}, \vec{v})$ and $\left(\vec{c}_{*}, \vec{v}_{*}\right)$. $P_{0}^{s}(\vec{c}, \vec{v})$ is an unknown column vector indexed by $(\vec{c}, \vec{v})$. Sum of the elements of each column of $T$ is equal to 1 by definition. All matrices of that form have a left eigenvector with all its elements being equal and with the corresponding eigenvalue equal to 1 ,

$$
\mathbf{v}_{\mathbf{L}} \cdot \mathbf{T}=\mathbf{v}_{\mathbf{L}}, \quad \mathbf{v}_{\mathbf{L}}=N \cdot \mathbf{1}^{\mathbf{T}}
$$

Here, $N$ is any number, which, for example can be chosen in such a way that the norm of $\mathbf{v}_{\mathbf{L}}$ is equal to one. If such a left eigenvector of $\mathbf{T}$ exists, so does the corresponding right eigenvector $\mathbf{v}_{\mathbf{R}}$, because existence of both of them are based on the same necessary and sufficient condition: $\operatorname{det}(\mathbf{T}-\mathbf{I})=0$. But the right eigenvector of $\mathbf{T}$ corresponding to eigenvalue 1 is exactly the solution of $(\mathrm{S} 2.1)$, where $\mathbf{v}_{\mathbf{R}} \equiv P_{0}^{s}\left(\vec{c}_{*}, \vec{v}_{*}\right)$. Moreover, the eigenvalue equal to 1 is the largest eigenvalue of matrix $\mathbf{T}$ (see, for example, section 5.C.1 in Reichl, 1998 for the proof).

The uniqueness of $\mathbf{v}_{\mathbf{R}}$ can be shown in the following way. First, we note that, during a vesicle fusion event, the system may transit only to a particular subset of all possible states of the system. Moreover, if we start in one of those particular states, then, during the next vesicle fusion event, the system transits to one of the states which belongs to that special subset. Indeed, our system is ergodic by definition. This means that, after reordering of indexes, $\mathbf{T}$ can always be expressed as a matrix with all elements equal to zeros except one square block, with all elements positive, laying on the main diagonal. We denote this square block by $\mathbf{T}^{\prime}$. Then, taking into account what was said above it is obvious that: 1) the sum of the elements of each column of $\mathbf{T}^{\prime}$ are equal to one, 2) $\mathbf{T}^{\prime} \cdot \mathbf{v}_{\mathbf{R}}^{\prime}=\mathbf{v}_{\mathbf{R}}^{\prime}$, and 3) the largest eigenvalue of $\mathbf{T}^{\prime}$ is equal to one. It follows from the Perron-Frobenius theorem that matrices with all elements real and positive have a unique normalized right eigenvector which corresponds to the largest eigenvalue and whose all elements are real and positive (see, e.g., Bellman, 1970). Thus, normalized $\mathbf{v}_{\mathbf{R}}^{\prime}$ is unique. It follows from the fact that all nonzero elements of $\mathbf{T}$ reside in the block $\mathbf{T}^{\prime}$ that $\mathbf{v}_{\mathbf{R}}^{\prime}$ defines all nonzero elements of $\mathbf{v}_{\mathbf{R}}$. Then, if normalized $\mathbf{v}_{\mathbf{R}}^{\prime}$ is uniques so is $\mathbf{v}_{\mathbf{R}}$. This is what we had to show. 


\subsection{Derivation of equation (4.14) in section 4.3}

Let us denote by $\mathbf{P}_{\mathbf{0}}$ a column vector which has all elements equal to zero except the $j$-th element, which is equal to one. Then, the solution of Eq. (4.14) which coincides with $\mathbf{P}_{0 j}$ at $t=0$ can be written as

$$
\mathbf{P}_{j}(t)=e^{\mathbf{A} \cdot t} \cdot \mathbf{P}_{0 j}
$$

Note that subscript $j$ does not denote the components of the vectors here. By definition, the $m$-th moment of the IEI distribution which corresponds to the initial condition described by the vector $\mathbf{P}_{0 j}$ is

$$
\begin{aligned}
\left\langle t^{m}\right\rangle_{I E I, j} & =\int_{0}^{+\infty}\left(t^{m} \cdot \rho_{I E I, j}(t)\right) d t=-\int_{0}^{+\infty}\left(t^{m} \cdot \frac{d}{d t} P_{I E I, j}(t)\right) d t= \\
& =m \cdot \int_{0}^{+\infty}\left(t^{m-1} \cdot P_{I E I, j}(t)\right) d t=m \cdot \int_{0}^{+\infty}\left(t^{m-1} \cdot \mathbf{1}^{\mathbf{T}} \cdot \mathbf{P}_{j}(t)\right) d t .
\end{aligned}
$$

Writing the third equality we integrated by parts and used the fact that $t^{m} \cdot P_{I E I, j}(t)$ vanishes at $t=+\infty$. This is so, because $P_{I E I, j}(t)$ is equal to a superposition of decaying exponential functions. The last equality follows from the definition $P_{I E I}(t)=\sum_{i=1}^{N} P_{i}(t)$. Substituting (S2.3) into (S2.4) and rearranging terms yields

$$
\begin{aligned}
\left\langle t^{m}\right\rangle_{I E I, j} & =\int_{0}^{+\infty}\left(\mathbf{1}^{\mathbf{T}} \cdot e^{\mathbf{A} \cdot t} \cdot \mathbf{P}_{0 j}\right) d t=\int_{0}^{+\infty}\left(\left(e^{\mathbf{A} \cdot t} \cdot \mathbf{P}_{0 j}\right)^{\mathbf{T}} \cdot \mathbf{1}\right) d t= \\
& =\int_{0}^{+\infty}\left(\mathbf{P}^{\mathbf{T}}{ }_{0 j} \cdot e^{\mathbf{A}^{\mathbf{T}} \cdot t} \cdot \mathbf{1}\right) d t=\int_{0}^{+\infty}\left(e^{\mathbf{A}^{\mathbf{T}} \cdot t} \cdot \mathbf{1}\right)_{j} d t
\end{aligned}
$$

where $\left(e^{\mathbf{A}^{\mathbf{T}} \cdot t} \cdot \mathbf{1}\right)_{j}$ denotes the $j$-th component of vector $\left(e^{\mathbf{A}^{\mathbf{T}} \cdot t} \cdot \mathbf{1}\right)$. Writing the last equality of $(\mathrm{S} 2.5)$ we used the fact that only the $j$-th component of $\mathbf{P}_{0 j}$ is equal to one, while the remaining components are equal to zero. Values of $\left\langle t^{m}\right\rangle_{I E I, j}$ corresponding to different $j$ s form a vector, and (S2.5) can be rewritten as

$$
\left\langle\mathbf{t}^{\mathbf{m}}\right\rangle_{\mathbf{I E I}}=\int_{0}^{+\infty}\left(e^{\mathbf{A}^{\mathbf{T}} \cdot t} \cdot \mathbf{1}\right) d t .
$$

Multiplying both sides of (S2.6) by $\left(\mathbf{A}^{\mathbf{T}}\right)^{\mathbf{m}}$, integrating the right hand size by parts $m$ times, and taking into account that $e^{\mathbf{A}^{\mathbf{T}} \cdot t}$ is equal to 1 and 0 at $t=0$ and $t=+\infty$, respectively, results in

$$
\left(\mathbf{A}^{\mathbf{T}}\right)^{\mathbf{m}} \cdot\left\langle\mathbf{t}^{\mathbf{m}}\right\rangle_{\mathbf{I E I}}=m ! \cdot(-1)^{m} \cdot \mathbf{1}
$$

or, equivalently,

$$
\left\langle\mathbf{t}^{\mathbf{m}}\right\rangle_{\mathbf{I E I}}=m ! \cdot\left(\left(-\mathbf{A}^{\mathbf{T}}\right)^{-\mathbf{m}} \cdot \mathbf{1}\right)
$$

The $m$-th moment of $t_{I E I}$ in a steady state is simply a scalar product of $\left\langle\mathbf{t}^{\mathbf{m}}\right\rangle_{\text {IEI }}$ and the special vector of initial conditions $\mathbf{P}_{\mathbf{0}}^{\mathbf{s}}$ introduced in section 4.3. Multiplying both sides of (S2.8) by $\mathbf{P}_{\mathbf{0}}^{\mathbf{s}} \mathbf{T}$ results in Eq. (4.14). 


\subsection{Factorization of the solutions of equations (4.4) and (4.6) in section 4.3}

Here, we consider particular AZ scenarios introduced in section 4.2 (see Fig. 4.1C). In particular, we present more explicit expressions of the factorized solutions of Eqs. (4.4), (4.6) and other relevant quantities which correspond to Eqs. (4.19) - (4.24) in the main text.

\section{Active zones with $\mathrm{Ca}^{2+}$-nanodomain coupling scenario}

In the case of the nanodomain coupling scenario (Fig. 1C), each vesicular release site is driven by a single, "private" $\mathrm{Ca}^{2+}$ channel. Thus, $\vec{c}^{i} \equiv c_{i}$. Eqs. (4.19) - (4.24) are reduced to

$$
\begin{aligned}
& \bar{P}\left(t, \vec{c}, \vec{v} \mid \vec{c}_{*}, \vec{v}_{*}\right)=\prod_{i=1}^{N_{V}} \bar{P}_{i}\left(t, c_{i}, v_{i} \mid c_{* i}, v_{* i}\right), \\
& P\left(t, \vec{c}, \vec{v} \mid \vec{c}_{*}, \vec{v}_{*}\right)=\prod_{i=1}^{N_{V}} P_{i}\left(t, c_{i}, v_{i} \mid c_{* i}, v_{* i}\right), \\
& P_{0}^{s}\left(\vec{c}_{*}, \vec{v}_{*}\right)=\sum_{i=1}^{N_{V}}\left(P_{i}^{r} \cdot P_{0, i}^{s}\left(c_{* i}, v_{* i}\right) \cdot \prod_{j=1 \neq i}^{N_{V}} \bar{P}_{j}^{s}\left(c_{* j}, v_{* j}\right)\right), \\
& P_{i}^{r}=\frac{k_{n_{V}(i) \rightarrow 1}^{V_{i}} \cdot \sum_{c_{i}=1}^{n_{C}(i)}\left(f_{c_{i}, n_{V}(i) \rightarrow 1}^{i} \cdot \bar{P}_{i}^{s}\left(c_{i}, n_{V}(i)\right)\right)}{\sum_{j=1}^{N_{V}}\left[k_{n_{V}(j) \rightarrow 1}^{V_{j}} \cdot \sum_{c_{j}=1}^{n_{C}(j)}\left(f_{c_{j}, n_{V}(j) \rightarrow 1}^{j} \cdot \bar{P}_{j}^{s}\left(c_{j}, n_{V}(j)\right)\right)\right]}, \\
& \rho_{I E I}(t)=-\frac{d}{d t} \sum_{i=1}^{N_{V}}\left[P_{i}^{r} \cdot \sum_{c_{i *}=1}^{n_{C}(i)}\left(P_{i}\left(t \mid c_{i *}, 1\right) \cdot P_{0, i}^{s}\left(c_{i *}, 1\right)\right) .\right. \\
& \left.\cdot \prod_{j=1 \neq i}^{N_{V}} \sum_{c_{j *}=1}^{n_{C}(j)} \sum_{v_{j *}=1}^{n_{V}(j)}\left(P_{j}\left(t \mid c_{j *}, v_{j *}\right) \cdot \bar{P}_{j}^{s}\left(c_{j *}, v_{j *}\right)\right)\right] \text {, } \\
& \langle t\rangle=\left(\sum_{j=1}^{N_{V}}\left[k_{n_{V}(j) \rightarrow 1}^{V_{j}} \cdot \sum_{c_{j}=1}^{n_{C}(j)}\left(f_{c_{j}, n_{V}(j) \rightarrow 1}^{j} \cdot \bar{P}_{j}^{s}\left(c_{j}, n_{V}(j)\right)\right)\right]\right)^{-1}
\end{aligned}
$$

where

$$
P_{i}\left(t \mid c_{i *}, v_{i *}\right)=\sum_{c_{i}=1}^{n_{C}(i)} \sum_{v_{i}=1}^{n_{V}(i)} P_{i}\left(t, c_{i}, v_{i} \mid c_{i *}, v_{i *}\right)
$$

and

$$
f_{c_{i}, n_{V}(i) \rightarrow 1}^{i}=1+\left(\Delta\left[\mathrm{Ca}^{2+}\right]_{i i}^{s} \cdot \delta_{c_{i}, n_{C}(i)}-1\right) \cdot \delta_{n_{V}(i) \rightarrow 1, \mathrm{Ca}^{2+}}^{i}
$$




\section{Active zones with $\mathrm{Ca}^{2+}$-microdomain coupling scenario}

In the case of AZs with $\mathrm{Ca}^{2+}$-microdomain coupling scenarios, the sate of the system is fully characterized by the states of the vesicular release sites. Indeed, in this case $\left[\mathrm{Ca}^{2+}\right]$ is constant at each vesicular release site in a steady state. Thus, $\bar{P}\left(t, \vec{c}, \vec{v} \mid \vec{c}_{*}, \vec{v}_{*}\right) \equiv \bar{P}\left(t, \vec{v} \mid \vec{v}_{*}\right)$ and $P\left(t, \vec{c}, \vec{v} \mid \vec{c}_{*}, \vec{v}_{*}\right) \equiv P\left(t, \vec{v} \mid \vec{v}_{*}\right)$, and Eqs. $(4.19)-(4.24)$ are reduced to

$$
\begin{gathered}
\bar{P}\left(t, \vec{v} \mid \vec{v}_{*}\right)=\prod_{i=1}^{N_{V}} \bar{P}_{i}\left(t, v_{i} \mid v_{* i}\right), \\
P\left(t, \vec{v} \mid \vec{v}_{*}\right)=\prod_{i=1}^{N_{V}} P_{i}\left(t, v_{i} \mid v_{* i}\right), \\
P_{0}^{s}\left(\vec{v}_{*}\right)=\sum_{i=1}^{N_{V}}\left(P_{i}^{r} \cdot P_{0, i}^{s}\left(v_{* i}\right) \cdot \prod_{j=1 \neq i}^{N_{V}} \bar{P}_{j}^{s}\left(v_{* j}\right)\right) \\
P_{i}^{r}=\frac{k_{n_{V}(i) \rightarrow 1}^{V_{i}} \cdot f_{\infty, n_{V}(i) \rightarrow 1}^{i} \cdot \bar{P}_{i}^{s}\left(n_{V}(i)\right)}{\sum_{j=1}^{N_{V}}\left(k_{n_{V}(j) \rightarrow 1}^{V_{j}} \cdot f_{\infty, n_{V}(j) \rightarrow 1}^{j} \cdot \bar{P}_{j}^{s}\left(n_{V}(j)\right)\right)}, \\
\rho_{I E I}(t)=-\frac{d}{d t} \sum_{i=1}^{N_{V}}\left[P_{i}^{r} \cdot P_{i}(t \mid 1) \cdot P_{0, i}^{s}(1) \cdot \prod_{j=1 \neq i}^{N_{V}} \sum_{v_{j *}=1}^{n_{V}(j)}\left(P_{j}\left(t \mid v_{j *}\right) \cdot \bar{P}_{j}^{s}\left(v_{j *}\right)\right)\right] \\
\langle t\rangle=\left(\sum_{j=1}^{N_{V}}\left[k_{n_{V}(j) \rightarrow 1}^{V_{j}} \cdot\left(f_{\infty, n_{V}(j) \rightarrow 1}^{j} \cdot \bar{P}_{j}^{s}\left(n_{V}(j)\right)\right)\right]\right)^{-1},
\end{gathered}
$$

where

$$
P_{i}\left(t \mid v_{i *}\right)=\sum_{v_{i}=1}^{n_{V}(i)} P_{i}\left(t, v_{i} \mid v_{i *}\right),
$$

and

$$
f_{\infty, n_{V}(i) \rightarrow 1}^{i}=1+\left(p_{o} \cdot\left[\mathrm{Ca}^{2+}\right]_{i}^{s}-1\right) \cdot \delta_{n_{V}(i) \rightarrow 1, \mathrm{Ca}^{2+}}^{i}
$$

$p_{o}$ denotes the open probability of the presynaptic $\mathrm{Ca}^{2+}$ channels, while $\left[\mathrm{Ca}^{2+}\right]_{i}^{s}$ denotes $\mathrm{Ca}^{2+}$ concentration at the $i$-th vesicular release site if all the presynaptic $\mathrm{Ca}^{2+}$ channels were open. Thus, $p_{o} \cdot\left[\mathrm{Ca}^{2+}\right]_{i}^{s}$ is equal to the time average $\mathrm{Ca}^{2+}$ concentration at the $i$-th site

\section{Active zones with intermediate $\mathrm{Ca}^{2+}$-domain coupling scenario}

Equations in the main text apply to an $\mathrm{AZ}$ with the most general intermediate case of the $\mathrm{Ca}^{2+}$ domain coupling scenario. In this work, we were mostly interested in the case of the intermediate 
$\mathrm{Ca}^{2+}$-domain coupling scenario with $\mathrm{Ca}^{2+}$ channels equidistant to the corresponding vesicular release site and identical gating kinetics. Equidistant $\mathrm{Ca}^{2+}$ channels influence vesicle fusion at a particular docking site identically. In this case, the number of the degrees of freedom which characterize the system can be considerably reduced. To this end, we have to change the $\vec{c}^{i}$, to $\vec{z}^{i}$, which characterizes the numbers of the channels of the site $i$ in one of the available states $\left\{c_{i}\right\}_{i=1}^{n_{C}(i)} . \vec{z}^{i}$ has $n_{C}(i)$ number of elements $z_{j}^{i}$. Taking into account that the channel number is fixed, $\sum_{j=1}^{n_{C}(i)} z_{j}^{i}=N_{C}^{i}$. This equality constrains the number of different states the subsystem of identical $\mathrm{Ca}^{2+}$ channels of the release site $i$ is characterized by. For example, the number of possible states of a cluster of $N_{C} 2$-state channels is in general equal to $2^{N_{C}}$. However, if the channels are identical and equidistant, this number is reduced to $N_{C}+1$. In general, if the $\mathrm{Ca}^{2+}$ channel is characterized by $n_{C}$ gating states, the number of the states of a set of identical, equidistant $\mathrm{Ca}^{2+}$ channels is equal to $\prod_{j=1}^{n_{C}-1}\left(1+N_{C} / j\right)$.

For a single release site $i$ driven by $N_{C}^{i}$ identical equidistant 2 -state channels, Eqs. (4.4) and (4.6) simplify to

$$
\begin{aligned}
& \partial_{t} \bar{P}_{i}\left(t, \vec{z}^{i}, v_{i} \mid \vec{z}_{0}^{i}, v_{0 i}\right)=\sum_{j=1}^{n_{C}(i)} z_{j}^{i} \cdot k_{j \rightarrow j}^{C_{i}} \cdot \bar{P}_{i}\left(t, \vec{z}^{i}, v_{i} \mid \vec{z}_{0}^{i}, v_{0 i}\right)+ \\
& +\sum_{j=1}^{n_{C}(i)} z_{j}^{i} \cdot \sum_{l=1 \neq j}^{n_{C}(i)} k_{j \rightarrow l}^{C_{i}} \cdot \bar{P}_{i}\left(t, \vec{z}_{-(j, l)}^{i}, z_{j}^{i}+1, z_{l}^{i}-1, v_{i} \mid \vec{z}_{0}^{i}, v_{0 i}\right)+ \\
& +\sum_{v_{i}^{\prime}=1}^{n_{V}(i)} k_{v_{i}^{\prime} \rightarrow v_{i}}^{V_{i}} \cdot f_{z_{n_{C}(i)}^{i}, v_{i}^{\prime} \rightarrow v_{i}}^{i} \cdot \bar{P}_{i}\left(t, \vec{z}^{i}, v_{i}^{\prime} \mid \vec{z}_{0}^{i}, v_{0 i}\right) .
\end{aligned}
$$

and

$$
\begin{aligned}
& \partial_{t} P_{i}\left(t, \vec{z}^{i}, v_{i} \mid \vec{z}_{0}^{i}, v_{0 i}\right)=\sum_{j=1}^{n_{C}(i)} z_{j}^{i} \cdot k_{j \rightarrow j}^{C_{i}} \cdot P_{i}\left(t, \vec{z}^{i}, v_{i} \mid \vec{z}_{0}^{i}, v_{0 i}\right)+ \\
& +\sum_{j=1}^{n_{C}(i)} z_{j}^{i} \cdot \sum_{l=1 \neq j}^{n_{C}(i)} k_{j \rightarrow l}^{C_{i}} \cdot P_{i}\left(t, \vec{z}_{-(j, l)}^{i}, z_{j}^{i}+1, z_{l}^{i}-1, v_{i} \mid \vec{z}_{0}^{i}, v_{0 i}\right)+ \\
& +\sum_{v_{i}^{\prime}=1}^{n_{V}(i)} k_{v_{i}^{\prime} \rightarrow v_{i}}^{V_{i}} \cdot f_{z_{n_{C}(i)}^{i}, v_{i}^{\prime} \rightarrow v_{i}}^{i} \cdot\left(1-\delta_{v_{i}, 1} \cdot \delta_{v_{i}^{\prime}, n_{V}(i)}\right) \cdot P_{i}\left(t, \vec{z}^{i}, v_{i}^{\prime} \mid \vec{z}_{0}^{i}, v_{0 i}\right),
\end{aligned}
$$

where

$$
f_{z_{n_{C}(i)}^{i}, v_{i}^{\prime} \rightarrow v_{i}}^{i}=1+\left(z_{n_{C}(i)}^{i} \cdot \Delta\left[\mathrm{Ca}^{2+}\right]_{i i}-1\right) \cdot \delta_{v_{i}^{\prime} \rightarrow v_{i}, \mathrm{Ca}^{2+}}^{i} \cdot\left(1-\delta_{v_{i}^{\prime}, v_{i}}\right)
$$

When the presynaptic channels are characterized by two states (kinetic scheme $C-O),(\mathrm{S} 2.21)$ 
and (S2.22) are reduced to

$$
\begin{aligned}
& \partial_{t} \bar{P}_{i}\left(t, z_{2}^{i}, v_{i}\right)=-\left(k_{1 \rightarrow 2}^{C_{i}} \cdot\left(N_{C}^{i}-z_{2}^{i}\right)+k_{2 \rightarrow 1}^{C_{i}} \cdot z_{2}^{i}\right) \cdot \bar{P}_{i}\left(t, z_{2}^{i}, v_{i}\right)+ \\
& +k_{1 \rightarrow 2}^{C_{i}} \cdot\left(N_{C}^{i}-z_{2}^{i}+1\right) \cdot \bar{P}_{i}\left(t, z_{2}^{i}-1, v_{i}\right)+k_{2 \rightarrow 1}^{C_{i}} \cdot\left(z_{2}^{i}+1\right) \cdot \bar{P}_{i}\left(t, z_{2}^{i}+1, v_{i}\right)+ \\
& +\sum_{v_{i}^{\prime}=1}^{n_{V}(i)} k_{v_{i}^{\prime} \rightarrow v_{i}}^{V} \cdot f_{z_{2}^{i}, v_{i}^{\prime} \rightarrow v_{i}}^{i} \cdot \bar{P}_{i}\left(t, z_{2}^{i}, v_{i}^{\prime}\right)
\end{aligned}
$$

and

$$
\begin{aligned}
& \partial_{t} P_{i}\left(t, z_{2}, v_{i}\right)=-\left(k_{1 \rightarrow 2}^{C_{i}} \cdot\left(N_{C}^{i}-z_{2}^{i}\right)+k_{2 \rightarrow 1}^{C_{i}} \cdot z_{2}^{i}\right) \cdot P_{i}\left(t, z_{2}^{i}, v_{i}\right)+ \\
& +k_{1 \rightarrow 2}^{C_{i}} \cdot\left(N_{C}^{i}-z_{2}^{i}+1\right) \cdot P_{i}\left(t, z_{2}^{i}-1, v_{i}\right)+k_{2 \rightarrow 1}^{C_{i}} \cdot\left(z_{2}^{i}+1\right) \cdot P_{i}\left(t, z_{2}^{i}+1, v_{i}\right)+ \\
& +\sum_{v_{i}^{\prime}=1}^{n_{V}(i)} k_{v_{i}^{\prime} \rightarrow v_{i}}^{V_{i}} \cdot f_{z_{2}^{i}, v_{i}^{\prime} \rightarrow v_{i}}^{i} \cdot\left(1-\delta_{v_{i}, 1} \cdot \delta_{v_{i}^{\prime}, n_{V}(i)}\right) \cdot P_{i}\left(t, z_{2}^{i}, v_{i}^{\prime}\right) .
\end{aligned}
$$

Here, we used the fact that $z_{1}^{i}+z_{2}^{i}=N_{C}^{i}$, thus, only one of the $z_{1}^{i}$ and $z_{2}^{i}$ is free to vary between 0 and $N_{C}^{i}$. For an equivalent system with 3 -state $\mathrm{Ca}^{2+}$ channels (kinetic scheme $C_{1}-C_{2}-O$ ), equations equivalent to (S2.24) and (S2.25) read as

$$
\begin{aligned}
& \partial_{t} \bar{P}_{i}\left(t, z_{2}^{i}, z_{3}^{i}, v_{i}\right)=-\left(k_{1 \rightarrow 2}^{C_{i}} \cdot\left(N_{C}^{i}-z_{2}^{i}-z_{3}^{i}\right)+\left(k_{2 \rightarrow 1}^{C_{i}}+k_{2 \rightarrow 3}^{C_{i}}\right) \cdot z_{2}^{i}+\right. \\
& \left.+k_{3 \rightarrow 2}^{C_{i}} \cdot z_{3}^{i}\right) \cdot \bar{P}_{i}\left(t, z_{2}^{i}, z_{3}^{i}, v_{i}\right)+k_{2 \rightarrow 1}^{C_{i}} \cdot\left(z_{2}^{i}+1\right) \cdot \bar{P}_{i}\left(t, z_{2}^{i}+1, z_{3}^{i}, v_{i}\right)+ \\
& +k_{1 \rightarrow 2}^{C_{i}} \cdot\left(N_{C}^{i}-z_{2}^{i}-z_{3}^{i}+1\right) \cdot \bar{P}_{i}\left(t, z_{2}^{i}-1, z_{3}^{i}, v_{i}\right)+ \\
& +k_{2 \rightarrow 3}^{C_{i}} \cdot\left(z_{2}^{i}+1\right) \cdot \bar{P}_{i}\left(t, z_{2}^{i}+1, z_{3}^{i}-1, v_{i}\right)+ \\
& +k_{3 \rightarrow 2}^{C_{i}} \cdot\left(z_{3}^{i}+1\right) \cdot \bar{P}_{i}\left(t, z_{2}^{i}-1, z_{3}^{i}+1, v_{i}\right)+ \\
& +\sum_{v_{i}^{\prime}=1}^{n_{V}} k_{v_{i}^{\prime} \rightarrow v_{i}}^{V_{i}} \cdot f_{z_{3}^{i}, v_{i}^{\prime} \rightarrow v_{i}}^{i} \cdot \bar{P}_{i}\left(t, z_{2}^{i}, z_{3}^{i}, v_{i}^{\prime}\right),
\end{aligned}
$$

and

$$
\begin{aligned}
& \partial_{t} P_{i}\left(t, z_{2}^{i}, z_{3}^{i}, v_{i}\right)=-\left(k_{1 \rightarrow 2}^{C_{i}} \cdot\left(N_{C}^{i}-z_{2}^{i}-z_{3}^{i}\right)+\left(k_{2 \rightarrow 1}^{C_{i}}+k_{2 \rightarrow 3}^{C_{i}}\right) \cdot z_{2}^{i}+\right. \\
& \left.+k_{3 \rightarrow 2}^{C_{i}} \cdot z_{3}^{i}\right) \cdot P_{i}\left(t, z_{2}^{i}, z_{3}^{i}, v_{i}\right)+k_{2 \rightarrow 1}^{C_{i}} \cdot\left(z_{2}^{i}+1\right) \cdot P_{i}\left(t, z_{2}^{i}+1, z_{3}^{i}, v_{i}\right)+ \\
& +k_{1 \rightarrow 2}^{C_{i}} \cdot\left(N_{C}^{i}-z_{2}^{i}-z_{3}^{i}+1\right) \cdot P_{i}\left(t, z_{2}^{i}-1, z_{3}^{i}, v_{i}\right)+ \\
& +k_{2 \rightarrow 3}^{C_{i}} \cdot\left(z_{2}^{i}+1\right) \cdot P_{i}\left(t, z_{2}^{i}+1, z_{3}^{i}-1, v_{i}\right)+ \\
& +k_{3 \rightarrow 2}^{C_{i}} \cdot\left(z_{3}^{i}+1\right) \cdot P_{i}\left(t, z_{2}^{i}-1, z_{3}^{i}+1, v_{i}\right)+ \\
& +\sum_{v_{i}^{\prime}=1}^{n_{V}} k_{v_{i}^{\prime} \rightarrow v_{i}}^{V_{i}} \cdot f_{z_{3}^{i}, v_{i}^{\prime} \rightarrow v_{i}}^{i} \cdot\left(1-\delta_{v_{i}, 1} \cdot \delta_{v_{i}^{\prime}, n_{V}(i)}\right) \cdot P_{i}\left(t, z_{2}^{i}, z_{3}^{i}, v_{i}^{\prime}\right) .
\end{aligned}
$$

The initial condition vectors $P_{0, i}^{s}\left(z_{2}^{i}\right)$ and $P_{0, i}^{s}\left(z_{2}^{i}, z_{3}^{i}\right)$ are found by solving the following 
equations, which are particular versions of Eq. (4.9):

$$
\begin{aligned}
& P_{0, i}^{s}\left(z_{2}^{i}\right)=\sum_{z_{* 2}^{i}=0}^{N_{C}^{i}} T_{i}\left(z_{2}^{i} \mid z_{* 2}^{i}\right) \cdot P_{0, i}^{s}\left(z_{* 2}^{i}\right), \\
& P_{0, i}^{s}\left(z_{2}^{i}, z_{3}^{i}\right)=\sum_{z_{* 2}^{i}=0}^{N_{C}^{i}} \sum_{z_{* 3}^{i}=0}^{N_{C}^{i}-z_{2 *}^{i}} T_{i}\left(z_{2}^{i}, z_{3}^{i} \mid z_{* 2}^{i}, z_{* 3}^{i}\right) \cdot P_{0, i}^{s}\left(z_{* 2}^{i}, z_{* 3}^{i}\right),
\end{aligned}
$$

where

$$
\begin{aligned}
T_{i}\left(z_{2}^{i} \mid z_{* 2}^{i}\right)=k_{n_{V}(i) \rightarrow 1}^{V_{i}} & \cdot f_{z_{2}^{i}, n_{V}(i) \rightarrow 1}^{i} \cdot \int_{0}^{+\infty} P_{i}\left(t, z_{2}^{i}, n_{V}(i) \mid z_{* 2}^{i}, 0\right) d t \\
T_{i}\left(z_{2}^{i}, z_{3}^{i} \mid z_{* 2}^{i}, z_{* 3}^{i}\right)= & k_{n_{V}(i) \rightarrow 1}^{V_{i}} \cdot f_{z_{3}^{i}, n_{V}(i) \rightarrow 1}^{i} \cdot \\
& \cdot \int_{0}^{+\infty} P_{i}\left(t, z_{2}^{i}, z_{3}^{i}, n_{V}(i) \mid z_{* 2}^{i}, z_{* 3}^{i}, 0\right) d t .
\end{aligned}
$$

\section{Active zones with shared $\mathrm{Ca}^{2+}$-domain coupling scenario}

In the case of the shared $\mathrm{Ca}^{2+}$-domain scenario, the solutions of Eqs. (4.4) and (4.6) can be factorized. However, differently from $\mathrm{Ca}^{2+}$-nanodomain, $\mathrm{Ca}^{2+}$-microdomain or intermediate $\mathrm{Ca}^{2+}$-domain scenarios, the independent unit now consists of several vesicular release sites which share the same $\mathrm{Ca}^{2+}$ channels. Let us denote states of the vesicular release sites of each independent unit by $\vec{v}^{i}$, and states of the corresponding $\mathrm{Ca}^{2+}$ channels by $\vec{c}^{i}$. Then, Eqs. (4.19) - (4.24) simplify,

$$
\begin{aligned}
& \bar{P}\left(t, \vec{c}, \vec{v} \mid \vec{c}_{0}, \vec{v}_{0}\right)=\prod_{i=1}^{N_{\text {unit }}} \bar{P}_{i}\left(t, \vec{c}^{i}, \vec{v}^{i} \mid \vec{c}_{0}^{i}, \vec{v}_{0}^{i}\right) \\
& P\left(t, \vec{c}, \vec{v} \mid \vec{c}_{0}, \vec{v}_{0}\right)=\prod_{i=1}^{N_{\text {unit }}} P_{i}\left(t, \vec{c}^{i}, \vec{v}^{i} \mid \vec{c}_{0}^{i}, \vec{v}_{0}^{i}\right) \\
& P_{0}^{s}(\vec{c}, \vec{v})=\sum_{i=1}^{N_{\text {unit }}}\left(P_{i}^{r} \cdot P_{0, i}^{s}\left(\vec{c}^{i}, \vec{v}^{i}\right) \cdot \prod_{j=1 \neq i}^{N_{\text {unit }}} \bar{P}_{j}^{s}\left(\vec{c}^{j}, \vec{v}^{j}\right)\right) \\
& \left.\left.P_{i}^{r}=\frac{\sum_{j=1}^{N_{V}^{i}}\left[k_{n_{V}(i, j) \rightarrow 1}^{V_{i j}} \cdot \sum_{\vec{c}^{i} \in \mathcal{C}^{i}}\left(f_{\vec{c}^{i}, n_{V}(i, j) \rightarrow 1}^{i j} \cdot \bar{P}_{i j}^{s}\left(\vec{c}^{i}, n_{V}(i, j)\right)\right)\right]}{\sum_{l=1}^{N_{u n i t}} \sum_{j=1}^{N_{V}^{i}}\left[k _ { n _ { V } ( l , j ) \rightarrow 1 } ^ { V _ { l j } } \cdot \sum _ { \vec { c } ^ { l } \in \mathcal { C } ^ { l } } \left(f_{\vec{c}^{l}, n_{V}}^{l j}(l, j) \rightarrow 1\right.\right.} \cdot \bar{P}_{l j}^{s}\left(\vec{c}^{l}, n_{V}(l, j)\right)\right)\right], \\
& \rho_{I E I}(t)=-\frac{d}{d t} \sum_{i=1}^{N_{\text {unit }}}\left[P_{i}^{r} \cdot \sum_{\vec{c}_{0}^{i} \in \mathcal{C}^{i}} \sum_{\vec{v}_{0}^{i} \in \mathcal{V}^{i}}\left(P_{i}\left(t \mid \vec{c}_{0}^{i}, \vec{v}_{0}^{i}\right) \cdot P_{0, i}^{s}\left(\vec{c}_{0}^{i}, \vec{v}_{0}^{i}\right)\right) .\right. \\
& \left.\cdot \prod_{j=1 \neq i}^{N_{\text {unit }}} \sum_{\vec{c}_{0}^{j} \in \mathcal{C}^{j}} \sum_{\vec{v}_{0}^{j} \in \mathcal{V}^{j}}\left(P_{j}\left(t \mid \vec{c}_{0}^{j}, \vec{v}_{0}^{j}\right) \cdot \bar{P}_{j}^{s}\left(\vec{c}_{0}^{j}, \vec{v}_{0}^{j}\right)\right)\right]
\end{aligned}
$$




$$
\langle t\rangle=\left(\sum_{l=1}^{N_{\text {unit }}} \sum_{j=1}^{N_{V}^{l}}\left[k_{n_{V}(l, j) \rightarrow 1}^{V_{l j}} \cdot \sum_{\vec{c}^{l} \in \mathcal{C}^{l}}\left(f_{\vec{c}^{l}, n_{V}(l, j) \rightarrow 1}^{l j} \cdot \bar{P}_{l j}^{s}\left(\vec{c}^{l}, n_{V}(l, j)\right)\right)\right]\right)^{-1},
$$

where

$$
P_{i}\left(t \mid \vec{c}_{0}^{i}, \vec{v}_{0}^{i}\right)=\sum_{\vec{c}^{i} \in \mathcal{C}^{i}} \sum_{\vec{v}^{i} \in \mathcal{V}^{i}} P_{i}\left(t, \vec{c}^{i}, \vec{v}^{i} \mid \vec{c}_{0}^{i}, \vec{v}_{0}^{i}\right)
$$

and

$$
f_{\vec{c}^{i}, n_{V}(i, j) \rightarrow 1}^{i j}=1+\left(\sum_{l=1}^{N_{c}^{i}} \Delta\left[\mathrm{Ca}^{2+}\right]_{l j}^{s, i} \cdot \delta_{c_{l}^{i}, n_{C}(i, l)}-1\right) \cdot \delta_{n_{V}(i, j) \rightarrow 1, \mathrm{Ca}^{2+}}^{i j} .
$$

In this work, we were in particular interested in the shared $\mathrm{Ca}^{2+}$-domain scenario with identical vesicular release sites and identical, equidistant 2 -state $\mathrm{Ca}^{2+}$ channels. Then, the number of the degrees of freedom which characterize the system can be considerably reduced. To achieve this, we have to replace $\vec{c}^{i}$ and $\vec{v}^{i}$ by two new state variables $\vec{z}^{i}$ and $\vec{w}^{i}$. $\vec{z}^{i}$ characterizes the numbers of the channels of the unit $i$ in one of the available states $\left\{c_{i}\right\}_{i=1}^{n_{C}(i)} . \vec{w}^{i}$ characterizes the numbers of the vesicular release sites of the unit $i$ in one of the available states $\left\{v_{i}\right\}_{i=1}^{n_{V}(i)} . \vec{z}^{i}$ has $n_{C}(i)$ number of elements $z_{j}^{i}, \vec{w}^{i}$ has $n_{V}(i)$ number of elements $w_{j}^{i}$. Taking into account that the channel and vesicular release site numbers are fixed, $\sum_{j=1}^{n_{C}(i)} z_{j}^{i}=N_{C}^{i}$ and $\sum_{j=1}^{n_{V}(i)} w_{j}^{i}=N_{V}^{i}$. These equalities constrain the number of different states the subsystems of identical $\mathrm{Ca}^{2+}$ channels or vesicular release sites of the independent unit $i$ may be characterized by. Moreover, these equalities mean that only $N_{C}^{i}-1$ out of $N_{C}^{i} z_{j}^{i}$ s and $N_{V}^{i}-1$ out of $N_{V}^{i}$ $w_{j}^{i}$ s are independent. The numbers of states of the $\mathrm{Ca}^{2+}$ channel and vesicular release site subsystems are, respectively, $\prod_{j=1}^{n_{C}(i)-1}\left(1+N_{C}^{i} / j\right)$ and $\prod_{j=1}^{n_{V}(i)-1}\left(1+N_{V}^{i} / j\right)$.

For a shared $\mathrm{Ca}^{2+}$-domain scenario with $N_{V}^{i}$ identical vesicular release sites driven by $N_{C}^{i}$ equidistant, identical $\mathrm{Ca}^{2+}$ channels, Eqs. (4.4) and (4.6) reduce to

$$
\begin{aligned}
& \partial_{t} \bar{P}_{i}\left(t, z_{2}^{i}, \vec{w}^{i} \mid z_{0,2}^{i}, \vec{w}_{0}^{i}\right)=\sum_{j=1}^{n_{V}(i)} w_{j}^{i} \cdot k_{j \rightarrow j}^{V_{i}} \cdot \bar{P}_{i}\left(t, z_{2}^{i}, \vec{w}^{i} \mid z_{0,2}^{i}, \vec{w}_{0}^{i}\right)+ \\
& +\sum_{j=1}^{n_{V}(i)} w_{j}^{i} \cdot \sum_{l=1 \neq j}^{n_{V}(i)} k_{j \rightarrow l}^{V_{i}} \cdot f_{z_{2}^{i}, j \rightarrow l}^{i} \cdot \bar{P}_{i}\left(t, z_{2}^{i}, \vec{w}_{-(j, l)}^{i}, w_{j}^{i}+1, w_{l}^{i}-1 \mid z_{0,2}^{i}, \vec{w}_{0}^{i}\right)- \\
& -\left(k_{1 \rightarrow 2}^{C_{i}} \cdot\left(N_{C}^{i}-z_{2}^{i}\right)+k_{2 \rightarrow 1}^{C_{i}} \cdot z_{2}^{i}\right) \cdot \bar{P}_{i}\left(t, z_{2}^{i}, \vec{w}^{i} \mid z_{0,2}^{i}, \vec{w}_{0}^{i}\right)+ \\
& +k_{1 \rightarrow 2}^{C_{i}} \cdot\left(N_{C}^{i}-z_{2}^{i}+1\right) \cdot \bar{P}_{i}\left(t, z_{2}^{i}-1, \vec{w}^{i} \mid z_{0,2}^{i}, \vec{w}_{0}^{i}\right)+ \\
& +k_{2 \rightarrow 1}^{C_{i}} \cdot\left(z_{2}^{i}+1\right) \cdot \bar{P}_{i}\left(t, z_{2}^{i}+1, \vec{w}^{i} \mid z_{0,2}^{i}, \vec{w}_{0}^{i}\right),
\end{aligned}
$$


and

$$
\begin{aligned}
\partial_{t} P_{i}( & \left.t, z_{2}^{i}, \vec{w}^{i} \mid z_{2}^{i}, \vec{w}_{0}^{i}\right)=\sum_{j=1}^{n_{V}(i)} w_{j}^{i} \cdot k_{j \rightarrow j}^{V_{i}} \cdot \bar{P}_{i}\left(t, z_{2}^{i}, \vec{w}^{i} \mid z_{0,2}^{i}, \vec{w}_{0}^{i}\right)+ \\
& +\sum_{j=1}^{n_{V}(i)} w_{j}^{i} \cdot \sum_{l=1 \neq j}^{n_{V}(i)}\left[k_{j \rightarrow l}^{V_{i}} \cdot f_{z_{2}^{i}, j \rightarrow l}^{i} \cdot\left(1-\delta_{l, 1} \cdot \delta_{j, n_{V}(i)}\right) \cdot\right. \\
& \left.\cdot P_{i}\left(t, z_{2}^{i}, \vec{w}_{-(j, l)}^{i}, w_{j}^{i}+1, w_{l}^{i}-1 \mid z_{0,2}^{i}, \vec{w}_{0}^{i}\right)\right]- \\
& -\left(k_{1 \rightarrow 2}^{C_{i}} \cdot\left(N_{C}^{i}-z_{2}^{i}\right)+k_{2 \rightarrow 1}^{C_{i}} \cdot z_{2}^{i}\right) \cdot P_{i}\left(t, z_{2}^{i}, \vec{w}^{i} \mid z_{0,2}^{i}, \vec{w}_{0}^{i}\right)+ \\
& +k_{1 \rightarrow 2}^{C_{i}} \cdot\left(N_{C}^{i}-z_{2}^{i}+1\right) \cdot P_{i}\left(t, z_{2}^{i}-1, \vec{w}^{i} \mid z_{0,2}^{i}, \vec{w}_{0}^{i}\right)+ \\
& +k_{2 \rightarrow 1}^{C_{i}} \cdot\left(z_{2}^{i}+1\right) \cdot P_{i}\left(t, z_{2}^{i}+1, \vec{w}^{i} \mid z_{0,2}^{i}, \vec{w}_{0}^{i}\right) .
\end{aligned}
$$

In this case, equation for the initial condition vector for calculating the $\rho_{I E I}(\mathrm{t})$, the equivalent of Eq. (4.9), can be written as

$$
P_{0, i}^{s}\left(z_{2}^{i}, \vec{w}^{i}\right)=\sum_{z_{*, 2}^{i}=0}^{N_{C}^{i}} \sum_{\vec{w}_{*}^{i} \in \mathcal{W}} T^{i}\left(z_{2}^{i}, \vec{w}^{i} \mid z_{*, 2}^{i}, \vec{w}_{*}^{i}\right) \cdot P_{0, i}^{s}\left(z_{*, 2}^{i}, \vec{w}_{*}^{i}\right),
$$

where,

$$
\begin{aligned}
T^{i}\left(z_{2}^{i}, \vec{w}^{i} \mid z_{*, 2}^{i}, \vec{w}_{*}^{i}\right)=z_{2}^{i} \cdot\left(w_{n_{V}(i)}^{i}+1\right) \cdot k_{n_{V}(i) \rightarrow 1}^{V_{i}} \cdot f_{z_{2}^{i}, n_{V}(i) \rightarrow 1}^{i} \cdot \\
\cdot \int_{0}^{+\infty} P^{i}\left(t, z_{2}^{i}, w_{1}-1, \vec{w}_{-1,-n_{V}(i)}^{i}, w_{n_{V}(i)}+1 \mid z_{2}^{i}, \vec{w}_{*}^{i}\right) d t
\end{aligned}
$$

if $w_{n_{V}(i)} \geq w_{*, n_{V}(i)}-1$, and $T^{i}\left(z_{2}^{i}, \vec{w}^{i} \mid z_{*, 2}^{i}, \vec{w}_{*}^{i}\right)=0$ otherwise.

\subsection{Analytical expressions of $\rho_{I E I},\langle t\rangle_{I E I}, C V_{I E I}$, and $C_{I E I}(l)$}

\section{AZs with 2-state vesicular release sites, $\mathrm{Ca}^{2+}$-microdomain coupling regime}

In this case, Eqs. (4.4), (4.6) for a vesicular release site $i$ in the main text read as

$$
\begin{gathered}
\left\{\begin{array}{l}
\partial_{t} \bar{P}_{i}\left(t, 1 \mid v_{0}\right)=-k_{r e p, i} \cdot \bar{P}_{i}\left(t, 1 \mid v_{0}\right)+k_{r e l, i} \cdot \bar{P}_{i}\left(t, 2 \mid v_{0}\right) \\
\partial_{t} \bar{P}_{i}\left(t, 2 \mid v_{0}\right)=+k_{r e p, i} \cdot \bar{P}_{i}\left(t, 1 \mid v_{0}\right)-k_{r e l, i} \cdot \bar{P}_{i}\left(t, 2 \mid v_{0}\right)
\end{array}\right. \\
\left\{\begin{array}{l}
\partial_{t} P_{i}\left(t, 1 \mid v_{0}\right)=-k_{r e p, i} \cdot P_{i}\left(t, 1 \mid v_{0}\right) \\
\partial_{t} P_{i}\left(t, 2 \mid v_{0}\right)=+k_{r e p, i} \cdot P_{i}\left(t, 1 \mid v_{0}\right)-k_{r e l, i} \cdot P_{i}\left(t, 2 \mid v_{0}\right)
\end{array}\right.
\end{gathered}
$$

Solving Eq. (S2.45) results in

$$
\begin{aligned}
& P_{i}(t \mid 1)=\left\{\begin{array}{l}
\left(1+k_{r e l, i} \cdot t\right) \cdot e^{-k_{r e l, i},} \quad \text { if } k_{r e p, i}=k_{r e l, i} \\
\frac{k_{r e l, i}}{k_{r e l, i}-k_{r e p, i}} \cdot e^{-k_{r e p, i} \cdot t}-\frac{k_{r e p, i}}{k_{r e l, i}-k_{r e p, i}} \cdot e^{-k_{r e l, i} \cdot t}, \quad \text { otherwise }
\end{array}\right. \\
& P_{i}(t \mid 2)=e^{-k_{r e l, i} \cdot t},
\end{aligned}
$$


where $P_{i}\left(t \mid v_{0}\right)=P_{i}\left(t, 1 \mid v_{0}\right)+P_{i}\left(t, 2 \mid v_{0}\right)$. Stationary solutions of $(\mathrm{S} 2.44)$ are

$$
\bar{P}_{i}^{s}(1)=\frac{k_{r e l, i}}{k_{r e p, i}+k_{r e l, i}}, \quad \bar{P}_{i}^{s}(2)=\frac{k_{r e p, i}}{k_{r e p, i}+k_{r e l, i}} .
$$

Then, $P_{I E I}(t)$ and $\rho_{I E I}(t)$ for an $\mathrm{AZ}$ with $N_{V}$ vesicular release sites can be expressed as follows:

$$
\begin{gathered}
P_{I E I}(t)=\sum_{i=1}^{N_{V}}\left[P_{i}^{r} \cdot P_{i}(t \mid 1) \cdot \prod_{j=1 \neq i}^{N_{V}} \sum_{v_{0}=1}^{2} P_{j}\left(t \mid v_{0}\right) \cdot \bar{P}_{j}^{s}\left(v_{0}\right)\right], \\
\rho_{I E I}(t)=-\sum_{i=1}^{N_{V}}\left[P_{i}^{r} \cdot \frac{d}{d t} P_{i}(t \mid 1) \cdot \prod_{j=1 \neq i}^{N_{V}} \sum_{v_{0}=1}^{2} P_{j}\left(t \mid v_{0}\right) \cdot \bar{P}_{j}^{s}\left(v_{0}\right)\right]- \\
-\sum_{i=1}^{N_{V}}\left[P _ { i } ^ { r } \cdot P _ { i } ( t | 1 ) \cdot \sum _ { j = 1 \neq i } ^ { N _ { V } } \left(\sum_{v_{0}=1}^{2}\left(\frac{d}{d t} P_{j}\left(t \mid v_{0}\right) \cdot \bar{P}_{j}^{s}\left(v_{0}\right)\right) .\right.\right. \\
\left.\left.\cdot \prod_{k=1 \neq i, j}^{N_{V}} \sum_{v_{0}=1}^{2} P_{k}\left(t \mid v_{0}\right) \cdot \bar{P}_{k}^{s}\left(v_{0}\right)\right)\right],
\end{gathered}
$$

where

$$
P_{i}^{r}=\frac{k_{r e l, i} \cdot P_{i}^{s}(2)}{\sum_{j=1}^{N_{V}} k_{r e l, j} \cdot P_{j}^{s}(2)} .
$$

In particular, for an $\mathrm{AZ}$ with one vesicular release site $\left(N_{V}=1\right)$, the expressions for $P_{I E I}(t)$ and $\rho_{I E I}(t)$ are as follows:

$$
\begin{aligned}
& P_{I E I}(t)=\left\{\begin{array}{l}
\left(1+k_{r e l} \cdot t\right) \cdot e^{-k_{r e l},} \text { if } k_{r e p}=k_{r e l} \\
\frac{k_{r e l}}{k_{r e l}-k_{r e p}} \cdot e^{-k_{r e p} \cdot t}-\frac{k_{r e p}}{k_{r e l}-k_{r e p}} \cdot e^{-k_{r e l} \cdot t}, \quad \text { otherwise }
\end{array},\right. \\
& \rho_{I E I}=\left\{\begin{array}{l}
k_{r e l}^{2} \cdot t \cdot e^{-k_{r e l} \cdot t}, \quad \text { if } k_{r e p}=k_{r e l} \\
\frac{k_{r e l} \cdot k_{r e p}}{k_{r e l}-k_{r e p}} \cdot\left(e^{-k_{r e p} \cdot t}-e^{-k_{r e l} \cdot t}\right), \quad \text { otherwise }
\end{array}\right.
\end{aligned}
$$

$\langle t\rangle_{I E I}$ for an $\mathrm{AZ}$ with $N_{V}$ vesicular release sites follows from

$$
\langle t\rangle_{I E I}=\left(\sum_{i=1}^{N_{V}} \frac{k_{r e p, i} \cdot k_{r e l, i}}{k_{r e p, i}+k_{r e l, i}}\right)^{-1} .
$$

Here, the term inside the sum is the vesicle release rate, which we denote by $R_{r, i}$ at the $i$-th site. The sum of $R_{r, i}$ over different sites is the overall vesicle release rate at the AZ, which we denote by $R_{r}$,

$$
R_{r}=\sum_{i=1}^{N_{V}} R_{r, i}, \quad R_{r, i}=\frac{k_{r e p, i} \cdot k_{r e l, i}}{k_{r e p, i}+k_{r e l, i}}
$$

CV for an $\mathrm{AZ}$ with $N_{V}$ identical vesicular release sites can be expressed as

$$
C V_{I E I}=\frac{\sqrt{\left\langle t^{2}\right\rangle_{I E I}-\langle t\rangle_{I E I}^{2}}}{\langle t\rangle_{I E I}},
$$


where $\langle t\rangle_{I E I}$ follows from (S2.54), and

$$
\begin{aligned}
& \left\langle t^{2}\right\rangle_{I E I}=\sum_{b=0}^{N_{V}-1}\left[\frac{\left(N_{V}-1\right) !}{b ! \cdot\left(N_{V}-1-b\right) !} \cdot\left(\frac{k_{r e p}}{k_{r e l}+k_{r e p}}\right)^{b} \cdot\left(\frac{k_{r e l}}{k_{r e l}+k_{r e p}}\right)^{N_{V}-b-1} .\right. \\
& \quad \sum_{i=b-1}^{N_{V}-1}\left[\left(\sum_{j=b}^{i+1}\left(k_{r e l} \cdot j+k_{r e p} \cdot\left(N_{V}-j\right)\right)^{-2}+\right.\right. \\
& \left.+\left(\sum_{j=b}^{i+1}\left(k_{r e l} \cdot j+k_{r e p} \cdot\left(N_{V}-j\right)\right)^{-1}\right)^{2}\right) . \\
& \left.\left.\quad \prod_{j=b}^{i}\left(\frac{k_{r e p} \cdot\left(N_{V}-j\right)}{k_{r e l} \cdot j+k_{r e p} \cdot\left(N_{V}-j\right)}\right) \cdot \frac{k_{r e l} \cdot(i+1)}{k_{r e l} \cdot(i+1)+k_{r e p} \cdot\left(N_{V}-i-1\right)}\right]\right]
\end{aligned}
$$

For an $\mathrm{AZ}$ with only one vesicular release site, the expression for $C V_{I E I}$ is

$$
C V_{I E I}=\frac{\sqrt{k_{r e l}^{2}+k_{r e p}^{2}}}{k_{r e l}+k_{r e p}} .
$$

Calculation of $C_{I E I}(l)$ for an $\mathrm{AZ}$ with $N_{V}$ identical vesicular release sites is based on Eqs. (4.16) - (4.17) in the main text:

$$
C_{I E I}(l)=\frac{\left\langle t_{i} t_{i+l}\right\rangle_{I E I}-\langle t\rangle_{I E I}^{2}}{\left\langle t^{2}\right\rangle_{I E I}-\langle t\rangle_{I E I}^{2}}, \quad\left\langle t_{i} t_{i+l}\right\rangle_{I E I}=\mathbf{1}^{\mathbf{T}} \cdot\left(\mathbf{J} \cdot \mathbf{T}^{\mathbf{l}-\mathbf{1}} \cdot \mathbf{G} \cdot \mathbf{P}_{\mathbf{0}}^{\mathbf{s}}\right) .
$$

In this particular case, the expression of $\left\langle t^{2}\right\rangle_{I E I}$ is given by Eq. (S2.57). The expressions of $\mathbf{P}_{\mathbf{0}}^{\mathbf{s}}$, $\mathbf{G}, \mathbf{T}$, and $\mathbf{J}$ are as follows:

$$
\begin{aligned}
& P_{0}^{s}(a)=\frac{\left(N_{V}-1\right) !}{a ! \cdot\left(N_{V}-1-a\right) !} \cdot\left(\frac{k_{k r e p}}{k_{r e l}+k_{r e p}}\right)^{a} \cdot\left(\frac{k_{k r e l}}{k_{r e l}+k_{r e p}}\right)^{N_{v}-1-a}, \\
& T(b \mid a)=\left\{\begin{array}{l}
\frac{k_{r e l} \cdot(b+1)}{k_{r e l} \cdot(b+1)+k_{r e p} \cdot\left(N_{V}-(b+1)\right)} \cdot \\
\cdot \prod_{i=0}^{b-a} \frac{k_{r e p} \cdot\left(N_{V}-a-i\right)}{k_{r e l} \cdot(a+i)+k_{r e p} \cdot\left(N_{V}-a-i\right)}, \quad \text { if } a \leq b+1 \\
0, \quad \text { otherwise }
\end{array}\right. \\
& \left.G(b \mid a)=T(b \mid a) \cdot \sum_{i=0}^{b-a}\left(k_{r e p} \cdot\left(N_{V}-a-i\right)+k_{r e l} \cdot(a+i)\right)\right)^{-1}, \\
& J(b \mid b)=\sum_{i=b}^{N_{V}}\left[\frac{1}{k_{r e l} \cdot i+k_{r e p} \cdot\left(N_{V}-i\right)} \cdot \prod_{j=b}^{i-1} \frac{k_{r e p} \cdot\left(N_{v}-j\right)}{k_{r e l} \cdot j+k_{r e p} \cdot\left(N_{V}-j\right)}\right] .
\end{aligned}
$$


AZs with 2-state vesicular release sites, $\mathrm{Ca}^{2+}$-nanodomain coupling regime, 2-state $\mathrm{Ca}^{2+}$ channels

In this case, Eqs. (4.4) and (4.6) in the main text for a vesicular release site $i$ are simplified to

$$
\begin{aligned}
& \begin{cases}\partial_{t} P_{i}\left(t, 1,1 \mid c_{0}, v_{0}\right) & =-\left(k_{+1, i}+k_{r e p, i}\right) \cdot P_{i}\left(t, 1,1 \mid c_{0}, v_{0}\right)+ \\
& +k_{-1, i} \cdot P_{i}\left(t, 2,1 \mid c_{0}, v_{0}\right) \\
\partial_{t} P_{i}\left(t, 2,1 \mid c_{0}, v_{0}\right) & =+k_{+1, i} \cdot P_{i}\left(t, 1,1 \mid c_{0}, v_{0}\right)- \\
& -\left(k_{-1, i}+k_{r e p, i}\right) \cdot P_{i}\left(t, 2,1 \mid c_{0}, v_{0}\right) \\
\partial_{t} P_{i}\left(t, 1,2 \mid c_{0}, v_{0}\right) & =+k_{r e p, i} \cdot P_{i}\left(t, 1,1 \mid c_{0}, v_{0}\right)- \\
& -k_{+1, i} \cdot P_{i}\left(t, 1,2 \mid c_{0}, v_{0}\right)+k_{-1, i} \cdot P_{i}\left(t, 2,2 \mid c_{0}, v_{0}\right) \\
& =+k_{r e p, i} \cdot P_{i}\left(t, 2,1 \mid c_{0}, v_{0}\right)+ \\
& +k_{+1, i} \cdot P_{i}\left(t, 1,2 \mid c_{0}, v_{0}\right)- \\
& -\left(k_{-1, i}+k_{r e l, i}\right) \cdot P_{i}\left(t, 2,2 \mid c_{0}, v_{0}\right) \\
& =-\left(k_{+1, i}+k_{r e p, i}\right) \cdot \bar{P}_{i}\left(t, 1,1 \mid c_{0}, v_{0}\right)+ \\
& +k_{-1, i} \cdot \bar{P}_{i}\left(t, 2,1 \mid c_{0}, v_{0}\right)+k_{r e l, i} \cdot \bar{P}_{i}\left(t, 2,2 \mid c_{0}, v_{0}\right) \\
& =+k_{+1, i} \cdot \bar{P}_{i}\left(t, 1,1 \mid c_{0}, v_{0}\right)- \\
& -\left(k_{-1, i}+k_{r e p, i}\right) \cdot \bar{P}_{i}\left(t, 2,1 \mid c_{0}, v_{0}\right) \\
\partial_{t} \bar{P}_{i}\left(t, 2,1 \mid c_{0}, v_{0}\right) & \\
\partial_{t} \bar{P}_{i}\left(t, 1,1 \mid c_{0}, v_{0}\right) & +k_{+1, i} \cdot \bar{P}_{i}\left(t, 1,2 \mid c_{0}, v_{0}\right)- \\
\partial_{t} \bar{P}_{i}\left(t, 1,2 \mid c_{0}, v_{0}\right) & \left.+k_{r e l, i}\right) \cdot \bar{P}_{i}\left(t, 2,2 \mid c_{0}, v_{0}\right) \\
& -k_{+1, i} \cdot \bar{P}_{i}\left(t, 1,1 \mid c_{0}, v_{0}\right)- \\
& =+k_{r e p, i} \cdot \bar{P}_{i}\left(t, 2,1 \mid c_{0}, v_{0}\right)+k_{-1, i} \cdot \bar{P}_{i}\left(t, 2,2 \mid c_{0}, v_{0}\right)+\end{cases}
\end{aligned}
$$

Solving Eq. (S2.64) gives us

$$
\begin{aligned}
& P_{i}(t, \mid 1,1)=A_{i} \cdot F_{a, i}+B_{i}^{+} \cdot F_{b, i}-C_{i} \cdot F_{c, i}-B_{i}^{+} \cdot F_{d, i}, \\
& P_{i}(t, \mid 2,1)=A_{i} \cdot F_{a, i}-B_{i}^{-} \cdot F_{b, i}-C_{i} \cdot F_{c, i}+B_{i}^{-} \cdot F_{d, i}, \\
& P_{i}(t, \mid 1,2)=\frac{\lambda_{2, i}}{\lambda_{2, i}-\lambda_{1, i}} \cdot e^{-\lambda_{1, i} \cdot t}-\frac{\lambda_{1, i}}{\lambda_{2, i}-\lambda_{1, i}} \cdot e^{-\lambda_{2, i} \cdot t}, \\
& P_{i}(t, \mid 2,2)=\frac{\left(\lambda_{2, i}-k_{r e l, i}\right)}{\lambda_{2, i}-\lambda_{1, i}} \cdot e^{-\lambda_{1, i} \cdot t}-\frac{\lambda_{1, i}-k_{r e l, i}}{\lambda_{2, i}-\lambda_{1, i}} \cdot e^{-\lambda_{2, i} \cdot t}
\end{aligned}
$$

where

$$
P_{i}\left(t \mid c_{0}, v_{0}\right)=\sum_{c_{i}=1}^{2} \sum_{v_{i}=1}^{2} P_{i}\left(t, c_{i}, v_{i} \mid c_{0}, v_{0}\right)
$$




$$
\begin{aligned}
& \lambda_{i, 1}=\frac{\left(k_{+1, i}+k_{-1, i}+k_{r e l, i}\right) \pm \sqrt{\left(k_{+1, i}+k_{-1, i}+k_{r e l, i}\right)^{2}-4 \cdot k_{+1, i} \cdot k_{r e l, i}}}{2}, \\
& A_{i}=\frac{k_{r e p, i} \cdot\left(\lambda_{2, i} \cdot k_{-1, i}+k_{+1, i}\left(\lambda_{2, i}-k_{r e l, i}\right)\right)}{\left(\lambda_{2, i}-\lambda_{1, i}\right) \cdot\left(k_{+1, i}+k_{-1, i}\right)}, \\
& B_{i}^{+}=\frac{k_{+1, i} \cdot k_{r e l, i} \cdot k_{r e p, i}}{\left(\lambda_{2, i}-\lambda_{1, i}\right) \cdot\left(k_{+1, i}+k_{-1, i}\right)}, \quad B_{i}^{-}=\frac{k_{-1, i} \cdot k_{r e l, i} \cdot k_{r e p, i}}{\left(\lambda_{2, i}-\lambda_{1, i}\right) \cdot\left(k_{+1, i}+k_{-1, i}\right)}, \\
& C_{i}=\frac{k_{r e p, i} \cdot\left(\lambda_{1, i} \cdot k_{-1, i}+k_{+1, i} \cdot\left(\lambda_{1, i}-k_{r e l, i}\right)\right)}{\left(\lambda_{2, i}-\lambda_{1, i}\right) \cdot\left(k_{+1, i}+k_{-1, i}\right)}, \\
& F_{x, i}=\left\{\begin{array}{l}
t \cdot e^{-r_{x 1, i}}, \quad \text { if } r_{x 1, i}=r_{x 2, i} \\
\left(e^{-r_{x 1, i} \cdot t}-e^{-r_{x 2, i} \cdot t}\right) /\left(r_{x 2, i}-r_{x 1, i}\right), \quad \text { otherwise }
\end{array}\right. \\
& r_{x 1, i}=k_{r e p, i}, \text { if } x=a, c, \quad r_{x 1, i}=k_{r e p, i}+k_{+1, i}+k_{-1, i}, \text { if } x=b, d, \\
& r_{x 2, i}=\lambda_{1, i}, \text { if } x=a, b, \quad r_{x 2, i}=\lambda_{2, i}, \text { if } x=c, d .
\end{aligned}
$$

The expressions of the stationary solutions of Eq. (S2.65) are as follows:

$$
\begin{aligned}
& \bar{P}_{i}^{s}(1,1)=\frac{k_{-1, i} \cdot k_{+1} \cdot k_{r e l, i}}{D_{i}}, \quad \bar{P}_{i}^{s}(2,1)=\frac{k_{+1, i} \cdot k_{r e l, i} \cdot\left(k_{+1, i}+k_{r e p, i}\right)}{D_{i}}, \\
& \bar{P}_{i}^{s}(1,2)=\frac{k_{-1, i} \cdot k_{r e p, i} \cdot\left(k_{-1, i}+k_{+1, i}+k_{r e l, i}+k_{r e p, i}\right)}{D_{i}}, \\
& \bar{P}_{i}^{s}(2,2)=\frac{k_{+1, i} \cdot k_{r e p, i} \cdot\left(k_{-1, i}+k_{+1, i}+k_{r e p, i}\right)}{D_{i}}, \\
& D_{i}=\left(k_{+1, i}+k_{-1, i}\right) \cdot\left(k_{-1, i} \cdot k_{r e p, i}+\left(k_{r e p, i}+k_{+1, i}\right) \cdot\left(k_{r e p, i}+k_{r e l, i}\right)\right) .
\end{aligned}
$$

Then, $P_{I E I}(t)$ and $\rho_{I E I}(t)$ for an $\mathrm{AZ}$ with $N_{V}$ vesicular release sites can be expressed as

$$
\begin{aligned}
& P_{I E I}(t)=\sum_{i=1}^{N_{V}}\left[P_{i}^{r} \cdot P_{i}(t \mid 2,1) \cdot \prod_{j=1 \neq i}^{N_{V}} \sum_{c_{0}=1}^{2} \sum_{v_{0}=1}^{2} P_{j}\left(t \mid c_{0}, v_{0}\right) \cdot \bar{P}_{j}^{s}\left(c_{0}, v_{0}\right)\right], \\
& \rho_{I E I}(t)=-\sum_{i=1}^{N_{V}}\left[P_{i}^{r} \cdot \frac{d}{d t} P_{i}(t \mid 2,1) \cdot \prod_{j=1 \neq i}^{N_{V}} \sum_{c_{0}=1}^{2} \sum_{v_{0}=1}^{2} P_{j}\left(t \mid c_{0}, v_{0}\right) \cdot \bar{P}_{j}^{s}\left(c_{0}, v_{0}\right)\right]- \\
& -\sum_{i=1}^{N_{V}}\left[P _ { i } ^ { r } \cdot P _ { i } ( t | 2 , 1 ) \cdot \sum _ { j = 1 \neq i } ^ { N _ { V } } \left(\sum_{c_{0}=1}^{2} \sum_{v_{0}=1}^{2}\left(\frac{d}{d t} P_{j}\left(t \mid c_{0}, v_{0}\right) \cdot \bar{P}_{j}^{s}\left(c_{0}, v_{0}\right)\right) .\right.\right. \\
& \left.\left.\cdot \prod_{k=1 \neq i, j}^{N_{V}} \sum_{c_{0}=1}^{2} \sum_{v_{0}=1}^{2} P_{k}\left(t \mid c_{0}, v_{0}\right) \cdot \bar{P}_{k}^{s}\left(c_{0}, v_{0}\right)\right)\right]
\end{aligned}
$$

where

$$
P_{i}^{r}=\frac{\bar{P}_{i}^{s}(2,2) \cdot k_{r e l, i}}{\sum_{j=1}^{N_{V}} \bar{P}_{j}^{s}(2,2) \cdot k_{r e l, j}} .
$$

In particular, for an $\mathrm{AZ}$ with one vesicular release site $\left(N_{V}=1\right)$, the expressions of $P_{I E I}(t)$ and $\rho_{I E I}(t)$ are

$$
P_{I E I}(t)=P_{1}(t \mid 2,1), \quad \rho_{I E I}(t)=-\frac{d}{d t} P_{1}(t \mid 2,1) .
$$


If $\lambda_{2} \neq k_{r e p}$ and $\lambda_{1} \neq k_{r e p}+k_{-1}+k_{-1}, \rho_{I E I}(t)$ for an $\mathrm{AZ}$ with one vesicular release site can be expressed in the following way:

$$
\begin{aligned}
& \rho_{I E I}(t)=\sum_{i=1}^{4} M_{i} e^{-m_{i} t}, \\
& m_{1}=k_{r e p}, \quad m_{2}=k_{-1}+k_{1}+k_{r e p}, \quad m_{3}=\lambda_{1} \quad m_{4}=\lambda_{2}, \\
& M_{1}=\frac{k_{1} k_{r e l} k_{r e p}\left(k_{-1}+k_{1}-k_{r e p}\right)}{\left(k_{-1}+k_{1}\right)\left(k_{r e p}^{2}-k_{r e p}\left(k_{-1}+k_{1}+k_{r e l}\right)+k_{1} k_{r e l}\right)}, \\
& M_{2}=\frac{k_{-1} k_{r e l} k_{r e p}\left(k_{-1}+k_{1}+k_{r e p}\right)}{\left(k_{-1}+k_{1}\right)\left(-k_{r e p}^{2}+k_{r e p}\left(-k_{-1}-k_{1}+k_{r e l}\right)+k_{-1} k_{r e l}\right)}, \\
& M_{3}=\frac{k_{r e p} k_{r e l}\left(4 k_{-1} k_{1}+\left(k_{1}-k_{-1}-k_{r e l}+\lambda_{2}-\lambda_{1}\right)\left(2 k_{r e p}+k_{1}-k_{-1}-k_{r e l}+\lambda_{2}-\lambda_{1}\right)\right)}{\left(\lambda_{2}-\lambda_{1}\right)\left(2 k_{r e p}-k_{1}-k_{-1}-k_{r e l}+\lambda_{2}-\lambda_{1}\right)\left(2 k_{r e p}+k_{1}+k_{-1}-k_{r e l}+\lambda_{2}-\lambda_{1}\right)}, \\
& M_{4}=\frac{k_{r e p} k_{r e l}\left(4 k_{-1} k_{1}+\left(k_{1}-k_{-1}-k_{r e l}+\lambda_{1}-\lambda_{2}\right)\left(2 k_{r e p}+k_{1}-k_{-1}-k_{r e l}+\lambda_{1}-\lambda_{2}\right)\right)}{\left(\lambda_{1}-\lambda_{2}\right)\left(2 k_{r e p}-k_{1}-k_{-1}-k_{r e l}+\lambda_{1}-\lambda_{2}\right)\left(2 k_{r e p}+k_{1}+k_{-1}-k_{r e l}+\lambda_{1}-\lambda_{2}\right)} .
\end{aligned}
$$

$\langle t\rangle_{I E I}$ for an $\mathrm{AZ}$ with $N_{V}$ vesicular release sites can be expressed as

$$
\begin{aligned}
& \langle t\rangle_{I E I}=\left(R_{r}\right)^{-1}, \quad R_{r}=\sum_{i=1}^{N_{V}} R_{r, i}, \\
& R_{r, i}=\frac{k_{+1, i} \cdot k_{r e l, i} \cdot k_{r e p, i} \cdot\left(k_{r e p, i}+k_{+1, i}+k_{-1, i}\right)}{\left(k_{+1, i}+k_{-1, i}\right) \cdot\left(k_{-1, i} \cdot k_{r e p, i}+\left(k_{r e p, i}+k_{+1, i}\right) \cdot\left(k_{r e p, i}+k_{r e l, i}\right)\right)} .
\end{aligned}
$$

Here, $R_{r, i}$ the vesicle release rate at the $i$-th site. $R_{r}$ stands for the overall vesicle release rate at the AZ.

The expression of $C V_{I E I}$ is

$$
C V_{I E I}=\frac{\sqrt{\left\langle t^{2}\right\rangle_{I E I}-\langle t\rangle_{I E I}^{2}}}{\langle t\rangle_{I E I}}
$$

where $\langle t\rangle_{I E I}$ is given by $(\mathrm{S} 2.75)$ - (S2.76). In order to calculate $\left\langle t^{2}\right\rangle_{I E I}$ for an AZ with $N_{V}$ identical vesicular release sites, we first of all introduced four new variables to characterize the state of the system: $\left(m_{1}, m_{2}, m_{3}, m_{4}\right) \equiv \vec{m}$. The variable $m_{1}$ is equal to the number of the vesicular release sites in the state $(c=2, v=1)$. The variable $m_{2}$ corresponds to the number of vesicular release sites in the state $(c=1, v=1)$, the variable $m_{3}$ - to the number of the vesicular release sites in state $(c=1, v=2)$, the variable $m_{4}$ - to the number of the vesicular release sites in state $(c=2, v=2) . m_{i}=\overline{0, N_{V}}$ with a constrain $\sum_{i=1}^{4} m_{i}=N_{V} \cdot \vec{m}$ fully characterizes the state of the system. The number of different states is equal to $\prod_{i=1}^{3}\left(1+N_{V} / i\right)$. The transition 
matrix A in Eq. 4.13) can be expressed as

$$
\begin{aligned}
A\left(\vec{m} \mid \vec{m}_{*}\right) & =m_{* 1} \cdot k_{-1} \cdot\left[\delta_{m_{* 1}, m_{1}+1} \cdot \delta_{m_{* 2}, m_{2}-1} \cdot \delta_{m_{* 3}, m_{3}} \cdot \delta_{m_{* 4}, m_{4}}\right]+ \\
& +m_{* 1} \cdot k_{r e p} \cdot\left[\delta_{m_{* 1}, m_{1}+1} \cdot \delta_{m_{* 2}, m_{2}} \cdot \delta_{m_{* 3}, m_{3}} \cdot \delta_{m_{* 4}, m_{4}-1}\right]+ \\
& +m_{* 2} \cdot k_{+1} \cdot\left[\delta_{m_{* 1}, m_{1}-1} \cdot \delta_{m_{* 2}, m_{2}+1} \cdot \delta_{m_{* 3}, m_{3}} \cdot \delta_{m_{* 4}, m_{4}}\right]+ \\
& +m_{* 2} \cdot k_{r e p} \cdot\left[\delta_{m_{* 1}, m_{1}} \cdot \delta_{m_{* 2}, m_{2}+1} \cdot \delta_{m_{* 3}, m_{3}-1} \cdot \delta_{m_{* 4}, m_{4}}\right]+ \\
& +m_{* 3} \cdot k_{+1} \cdot\left[\delta_{m_{* 1}, m_{1}} \cdot \delta_{m_{* 2}, m_{2}} \cdot \delta_{m_{* 3}, m_{3}+1} \cdot \delta_{m_{* 4}, m_{4}-1}\right]+ \\
& +m_{* 4} \cdot k_{-1} \cdot\left[\delta_{m_{* 1}, m_{1}} \cdot \delta_{m_{* 2}, m_{2}} \cdot \delta_{m_{* 3}, m_{3}-1} \cdot \delta_{m_{* 4}, m_{4}+1}\right]+ \\
& +m_{* 4} \cdot k_{r e l} \cdot\left[\delta_{m_{* 1}, m_{1}} \cdot \delta_{m_{* 2}, m_{2}} \cdot \delta_{m_{* 3}, m_{3}} \cdot \delta_{m_{* 4}, m_{4}+1}\right]- \\
& -\left(m_{* 1} \cdot\left(k_{-1}+k_{r e p}\right)+m_{* 2} \cdot\left(k_{+1}+k_{r e p}\right)+m_{* 3} \cdot k_{+1}+\right. \\
& \left.+m_{* 4} \cdot\left(k_{-1}+k_{r e l}\right)\right) \cdot\left[\delta_{m_{* 1}, m_{1}} \cdot \delta_{m_{* 2}, m_{2}} \cdot \delta_{m_{* 3}, m_{3}} \cdot \delta_{m_{* 4}, m_{4}}\right] .
\end{aligned}
$$

Elements of the initial condition vector $\mathbf{P}_{\mathbf{0}}^{\mathbf{s}}$ are expressed as follows:

$$
P_{0}^{s}(\vec{m})=\frac{N_{V} !}{m_{1} ! \cdot m_{2} ! \cdot m_{3} ! \cdot m_{4} !} \cdot \prod_{i=1}^{4}\left(\bar{P}^{s}(i)\right)^{m_{i}}
$$

where $\bar{P}^{s}(1) \equiv \bar{P}^{s}(2,1), \bar{P}^{s}(2) \equiv \bar{P}^{s}(1,1), \bar{P}^{s}(3) \equiv \bar{P}^{s}(1,2)$ and $\bar{P}^{s}(4) \equiv \bar{P}^{s}(2,2)$. Then, according to Eq. (4.14), $\left\langle t^{2}\right\rangle_{I E I}$ is expressed as

$$
\left\langle t^{2}\right\rangle_{I E I}=2 \cdot \mathbf{P}_{\mathbf{0}}^{\mathbf{s}} \cdot\left(\left(-\mathbf{A}^{\mathbf{T}}\right)^{-\mathbf{2}} \cdot \mathbf{1}\right) .
$$

The inverse of $\mathbf{A}^{\mathbf{T}}$ can be found analytically if necessary. However, the resulting expressions for $\left\langle t^{2}\right\rangle_{I E I}$ are very complicated. Thus, the analytical calculation of $\left(\mathbf{A}^{\mathbf{T}}\right)^{-\mathbf{1}}$ for $N_{V}>1$ is of little practical use. For an $\mathrm{AZ}$ with only one vesicular release site, i.e., $N_{V}=1$, the expression of $C V_{I E I}$ is

$$
C V_{I E I}=\frac{\sqrt{E_{1}+E_{2}+E_{3}}}{\left(k_{+1}+k_{-1}\right) \cdot\left(k_{-1} \cdot k_{r e p}+\left(k_{+1}+k_{r e p}\right) \cdot\left(k_{r e l}+k_{r e p}\right)\right)},
$$

where

$$
\begin{aligned}
& E_{1}=k_{r e p}^{4} \cdot\left(2 \cdot k_{-1} \cdot k_{r e l}+\left(k_{-1}+k_{+1}\right)^{2}\right)+ \\
& +2 \cdot k_{r e p}^{3} \cdot\left(2 \cdot k_{-1} \cdot k_{r e l}\left(k_{-1}+k_{+1}\right)+k_{-1} \cdot k_{r e l}^{2}+\left(k_{-1}+k_{+1}\right)^{3}\right), \\
& E_{2}=k_{r e p}^{2} \cdot\left(2 \cdot k_{-1} \cdot k_{r e l} \cdot\left(k_{-1}+k_{+1}\right)^{2}+\right. \\
& \left.+k_{r e l}^{2} \cdot\left(k_{-1}^{2}+4 \cdot k_{+1} \cdot k_{-1}+k_{+1}^{2}\right)+\left(k_{-1}+k_{+1}\right)^{4}\right), \\
& E_{3}=k_{r e p} \cdot 2 \cdot k_{+1}^{2} \cdot k_{r e l}^{2} \cdot\left(k_{-1}+k_{+1}\right)+k_{+1}^{2} \cdot k_{r e l}^{2} \cdot\left(k_{-1}+k_{+1}\right)^{2} .
\end{aligned}
$$

Calculation of $C_{I E I}(l)$ is based on Eqs. (4.16) - (4.17), which we rewrite here,

$$
C_{I E I}(l)=\frac{\left\langle t_{i} t_{i+l}\right\rangle_{I E I}-\langle t\rangle_{I E I}^{2}}{\left\langle t^{2}\right\rangle_{I E I}-\langle t\rangle_{I E I}^{2}}, \quad\left\langle t_{i} t_{i+l}\right\rangle_{I E I}=\mathbf{1}^{\mathbf{T}} \cdot\left(\mathbf{J} \cdot \mathbf{T}^{\mathbf{l}-\mathbf{1}} \cdot \mathbf{G} \cdot \mathbf{P}_{\mathbf{0}}^{\mathbf{s}}\right) .
$$


For an $\mathrm{AZ}$ with $N_{V}$ identical vesicular release sites, the expressions of $\langle t\rangle_{I E I},\left\langle t^{2}\right\rangle_{I E I}$, and $\mathbf{P}_{\mathbf{0}}^{\mathbf{s}}$ are given by (S2.77), (S2.80), and (S2.79) respectively. The expressions of the elements of $\mathbf{G}$, $\mathbf{T}$, and $\mathbf{J}$ are as follows:

$$
\begin{gathered}
T\left(\vec{m} \mid \vec{m}_{*}\right)=k_{r e l} \cdot\left(m_{4}+1\right) \cdot \int_{0}^{+\infty} P\left(t, m_{1}-1, m_{2}, m_{3}, m_{4}+1 \mid \vec{m}_{*}\right) d t \\
G\left(\vec{m} \mid \vec{m}_{*}\right)=k_{r e l} \cdot\left(m_{4}+1\right) \cdot \int_{0}^{+\infty} t \cdot P\left(t, m_{1}-1, m_{2}, m_{3}, m_{4}+1 \mid \vec{m}_{*}\right) d t \\
J\left(\vec{m}_{*} \mid \vec{m}_{*}\right)=\int_{0}^{+\infty} \sum_{m_{4}=0}^{N_{V}} \sum_{m_{3}=0}^{N_{m_{3}}} \sum_{m_{2}=0}^{N_{m_{2}}} \sum_{m_{1}=0}^{N_{m_{1}}} P\left(t, \vec{m} \mid \vec{m}_{*}\right) d t
\end{gathered}
$$

Where, $N_{m_{3}}=N_{V}-m_{4}, N_{m_{2}}=N_{V}-m_{4}-m_{3}$, and $N_{m_{1}}=N_{V}-m_{4}-m_{3}-m_{2}$. If all the eigenvalues of the matrix A, defined by Eq. (S2.78), are different, we can write $P(t, \vec{m} \mid \vec{m})$ as

$$
P\left(t, \vec{m} \mid \vec{m}_{*}\right)=\sum_{i=1}^{\prod_{j=1}^{3}\left(1+N_{V} / j\right)} \alpha_{\vec{m}_{*}, i} \cdot \Lambda_{\vec{m}, i} \cdot e^{\lambda_{i} \cdot t},
$$

where $\lambda_{i}<0$ and $\Lambda_{\vec{m}, i}$ are, respectively, the $i$-th eigenvalue and the $i$-th eigenvector of the matrix A, and $\alpha_{\vec{m}_{*}, i}$ is the $i$-th integration constant. Accordingly, $T\left(\vec{m} \mid \vec{m}_{*}\right), G\left(\vec{m} \mid \vec{m}_{*}\right)$, and $J\left(\vec{m}_{*} \mid \vec{m}_{*}\right)$ can be expressed in the following way:

$$
\begin{gathered}
T\left(\vec{m} \mid \vec{m}_{*}\right)=-k_{r e l} \cdot\left(m_{4}+1\right) \cdot \sum_{i=1}^{\prod_{j=1}^{3}\left(1+N_{V} / j\right)} \alpha_{\vec{m}_{*}, i} \cdot \Lambda_{\left(m_{1}-1, m_{2}, m_{3}, m_{4}+1\right), i} \cdot \lambda_{i}^{-1}, \\
G\left(\vec{m} \mid \vec{m}_{*}\right)=k_{r e l} \cdot\left(m_{4}+1\right) \cdot \sum_{i=1}^{\prod_{j=1}^{3}\left(1+N_{V} / j\right)} \alpha_{\vec{m}_{*}, i} \cdot \Lambda_{\left(m_{1}-1, m_{2}, m_{3}, m_{4}+1\right), i} \cdot \lambda_{i}^{-2}, \\
J\left(\vec{m}_{*} \mid \vec{m}_{*}\right)=-\sum_{i=1}^{\prod_{j=1}^{3}\left(1+N_{V} / j\right)} \alpha_{\vec{m}_{*}, i} \cdot \lambda_{i}^{-1} .
\end{gathered}
$$

When writing Eq. (S2.89), we assumed that eigenvectors $\Lambda_{\vec{m}, i}$ are normalized, which can be done without loss of generality. If some of the eigenvalues of the matrix $\mathbf{A}$ are degenerate, (S2.86) - (S2.89) can be modified as taught in standard mathematical textbooks on differential equations.

\section{AZs with $n_{V^{-}}$state vesicular release sites, $\mathrm{Ca}^{2+}$-microdomain coupling regime}

In this case, Eqs. (4.4) and (4.6) in the main text for a vesicular release site $i$ are simplified to

$$
\partial_{t} \bar{P}_{i}\left(t, v \mid v_{0}\right)=\sum_{v^{\prime}=v-1}^{v+1} k_{v^{\prime} \rightarrow v}^{V i} \cdot \bar{f}_{v^{\prime} \rightarrow v}^{i} \cdot \bar{P}_{i}\left(t, v^{\prime} \mid v_{0}\right)
$$




$$
\partial_{t} P_{i}\left(t, v \mid v_{0}\right)=\sum_{v^{\prime}=v-1}^{v+1} k_{v^{\prime} \rightarrow v}^{V i} \cdot \bar{f}_{v^{\prime} \rightarrow v}^{i} \cdot P_{i}\left(t, v^{\prime} \mid v_{0}\right) \cdot\left(1-\delta_{v, 1} \cdot \delta_{v^{\prime}, n_{V}(i)}\right)
$$

where

$$
\begin{aligned}
k_{n_{V}(i) \rightarrow 1}^{V i} \cdot \bar{f}_{n_{V}(i) \rightarrow 1}^{i} & =\gamma_{i}, \quad k_{1 \rightarrow n_{V}(i)}^{V i} \cdot \bar{f}_{1 \rightarrow n_{V}(i)}^{i}=0, \\
k_{1 \rightarrow 2}^{V i} \cdot \bar{f}_{1 \rightarrow 2}^{i} & =k_{r e p, i}, \quad k_{2 \rightarrow 1}^{V i} \cdot \bar{f}_{2 \rightarrow 1}^{i}=0, \\
k_{v \rightarrow v+1}^{V i} \cdot \bar{f}_{v \rightarrow v+1}^{i} & =\left(n_{V}(i)-v\right) \cdot k_{o n, i} \cdot p_{o} \cdot \Delta\left[\mathrm{Ca}^{2+}\right]_{\mathrm{i}} \\
& =\left(n_{V}(i)-v\right) \cdot k_{r e l, i}, \quad \text { for } 2 \leq v<n_{V}(i)-1, \\
k_{v+1 \rightarrow v}^{V i} \cdot \bar{f}_{v+1 \rightarrow v}^{i} & =b^{v-2} \cdot k_{o f f}, \quad \text { for } 2 \leq v<n_{V}(i)-1, \\
k_{n_{V}(i) \rightarrow n_{V}(i)}^{V i} \bar{f}_{n_{V}(i) \rightarrow n_{V}(i)}^{i} & =-\left(b^{n_{V}(i)-3} \cdot k_{o f f, i}+\gamma_{i}\right), \\
k_{1 \rightarrow 1}^{V i} \cdot \bar{f}_{1 \rightarrow 1}^{i} & =-k_{r e p, i}, \\
k_{2 \rightarrow 2}^{V i} \cdot \bar{f}_{2 \rightarrow 2}^{i} & =-\left(n_{V}(i)-2\right) \cdot k_{o n, i} \cdot p_{o} \cdot \Delta\left[\mathrm{Ca}^{2+}\right]_{\mathrm{i}} \\
& =-\left(n_{V}(i)-2\right) \cdot k_{r e l, i}, \\
k_{v \rightarrow v}^{V i} \cdot \bar{f}_{v \rightarrow v}^{i} & =-\left(\left(n_{V}(i)-v\right) \cdot k_{o n, i} \cdot p_{o} \cdot \Delta\left[\mathrm{Ca}^{2+}\right]_{\mathrm{i}}+b^{v-3} \cdot k_{o f f}\right) \\
& =-\left(\left(n_{V}(i)-2\right) \cdot k_{r e l, i}+b^{v-3} \cdot k_{o f f}\right), \\
& \text { for } 2<v \leq n_{V}(i)-1 .
\end{aligned}
$$

In Eqs. (S2.90) - (S2.91), it is assumed that $\left(v^{\prime}=0\right) \equiv\left(v^{\prime}=n_{V}(i)\right)$ and $\left(v^{\prime}=n_{V}(i)+1\right) \equiv$ $\left(v^{\prime}=1\right)$. The solutions of Eqs. (S2.90) - (S2.91) can be written as

$$
\begin{aligned}
& P\left(t, v \mid v_{0}\right)=\sum_{i=1}^{n_{V}(i)} \alpha_{v_{0}, i} \cdot \Lambda_{v, i} \cdot e^{\gamma_{i} \cdot t} \\
& \bar{P}\left(t, v \mid v_{0}\right)=\sum_{i=1}^{n_{V}(i)} \bar{\alpha}_{v_{0}, i} \cdot \bar{\Lambda}_{v, i} \cdot e^{\bar{\gamma}_{i} \cdot t} .
\end{aligned}
$$

Here, $\lambda_{i}<0$ and $\Lambda_{v, i}$ are respectively the $i$-th eigenvalue and the $i$-th eigenvector of the transition matrix corresponding to $(\mathrm{S} 2.90)$, and $\alpha_{v_{0}, i}$ is the $i$-th integration constant. $\bar{\lambda}_{i} \leq 0$ and $\bar{\Lambda}_{v, i}$ stand, respectively, for the $i$-th eigenvalue and the $i$-th eigenvector of the transition matrix of the Eq. (S2.91). $\bar{\alpha}_{v_{0}, i}$ is the corresponding $i$-th integration constant. It follows from Eq. (S2.93) that

$$
P\left(t \mid v_{0}\right)=\sum_{i=1}^{n_{V}(i)} \alpha_{v_{0}, i} \cdot e^{\lambda_{i} \cdot t}
$$

if we use normalized eigenvectors $\Lambda_{v, i}$. In general, the transition matrices corresponding to Eqs. (S2.90) - (S2.91) cannot be diagonalized analytically, thus, relevant numerical methods have to be employed. Stationary solutions of (S2.90) can be found analytically. To do this, we used computational software program Mathematica (Wolfram Research) in this work. Due to the large size and complexity of the expressions, we do not provide them here. They can be obtained readily by the reader by using the aforementioned software. 
$P_{I E I}(t)$ and $\rho_{I E I}(t)$ for an $\mathrm{AZ}$ with $N_{V}$ vesicular release sites can be expressed as

$$
\begin{gathered}
P_{I E I}(t)=\sum_{i=1}^{N_{V}}\left[P_{i}^{r} \cdot P_{i}(t \mid 1) \cdot \prod_{j=1 \neq i}^{N_{V}} \sum_{v_{0}=1}^{n_{V}(i)} P_{j}\left(t \mid v_{0}\right) \cdot \bar{P}_{j}^{s}\left(v_{0}\right)\right], \\
\rho_{I E I}(t)=-\sum_{i=1}^{N_{V}}\left[P_{i}^{r} \cdot \frac{d}{d t} P_{i}(t \mid 1) \cdot \prod_{j=1 \neq i}^{N_{V}} \sum_{v_{0}=1}^{n_{V}(i)} P_{j}\left(t \mid v_{0}\right) \cdot \bar{P}_{j}^{s}\left(v_{0}\right)\right]- \\
-\sum_{i=1}^{N_{V}}\left[P _ { i } ^ { r } \cdot P _ { i } ( t | 1 ) \cdot \sum _ { j = 1 \neq i } ^ { N _ { V } } \left(\sum_{v_{0}=1}^{n_{V}(i)}\left(\frac{d}{d t} P_{j}\left(t \mid v_{0}\right) \cdot \bar{P}_{j}^{s}\left(v_{0}\right)\right) .\right.\right. \\
\left.\left.\cdot \prod_{k=1 \neq i, j}^{N_{V}} \sum_{v_{0}=1}^{n_{V}(i)} P_{k}\left(t \mid v_{0}\right) \cdot \bar{P}_{k}^{s}\left(v_{0}\right)\right)\right],
\end{gathered}
$$

where

$$
P_{i}^{r}=\frac{\gamma_{i} \cdot \bar{P}_{i}^{s}\left(n_{V}(i)\right)}{\sum_{j=1}^{N_{V}} \gamma_{j} \cdot \bar{P}_{j}^{s}\left(n_{V}(j)\right)} .
$$

In particular, for an $\mathrm{AZ}$ with one vesicular release site $\left(N_{V}=1\right)$, the expressions of $P_{I E I}(t)$ and $\rho_{I E I}(t)$ are given by

$$
P_{I E I}(t)=P_{1}(t \mid 1), \quad \rho_{I E I}(t)=-\frac{d}{d t} P_{1}(t \mid 1) .
$$

$\langle t\rangle_{I E I}$ for an $\mathrm{AZ}$ with $N_{V}$ vesicular release sites can be expressed as

$$
\begin{aligned}
\langle t\rangle_{I E I} & =\left(R_{r}\right)^{-1}, \quad R_{r}=\sum_{i=1}^{N_{V}} R_{r, i}, \\
R_{r, i} & =\gamma_{i} \cdot \bar{P}_{i}^{s}\left(n_{V}(i)\right)= \\
& =\frac{\left(n_{V}(i)-2\right) ! \cdot \gamma_{i} \cdot k_{r e p, i} \cdot\left(k_{r e l, i}\right)^{n_{V}(i)-2}}{\sum_{j=0}^{n_{V}(i)-2} B_{i, j} \cdot\left(k_{r e l, i}\right)^{j}} .
\end{aligned}
$$

Here, $R_{r, i}$ the vesicle release rate at the $i$-th site. $R_{r}$ stands for the overall vesicle release rate at the AZ. Below, we provide the expressions of coefficients $B_{i, j}$ for the $\mathrm{Ca}^{2+}$ sensor of exocytosis with one to five $\mathrm{Ca}^{2+}$-binding sites, i.e., with $n_{V}(i)$ from three to seven. When $n_{V}(i)=3$,

$$
B_{i, 0}=\left(\gamma_{i}+k_{o f f, i}\right) \cdot k_{r e p, i}, \quad B_{i, 1}=\gamma_{i}+k_{r e p, i},
$$

When $n_{V}(i)=4$,

$$
\begin{aligned}
B_{i, 0} & =k_{\text {rep }, i} \cdot k_{o f f, i} \cdot\left(\gamma_{i}+b_{i} \cdot k_{o f f, i}\right), \quad B_{i, 1}=k_{r e p, i} \cdot\left(2 \cdot b_{i} \cdot k_{o f f, i}+3 \cdot \gamma_{i}\right), \\
i, 2 & =2 \cdot\left(\gamma_{i}+k_{r e p, i}\right) .
\end{aligned}
$$

When $n_{V}(i)=5$,

$$
\begin{aligned}
& B_{i, 0}=k_{r e p, i} \cdot\left(\left(b_{i} \cdot k_{o f f, i}\right)^{3}+\gamma_{i} \cdot b_{i} \cdot\left(k_{o f f, i}\right)^{2}\right), \\
& B_{i, 1}=k_{r e p, i} \cdot\left(3 \cdot\left(b_{i}\right)^{3} \cdot\left(k_{o f f, i}\right)^{2}+3 \cdot \gamma_{i} \cdot b_{i} \cdot k_{o f f, i}+\gamma_{i} \cdot k_{o f f, i}\right), \\
& B_{i, 2}=k_{r e p, i} \cdot\left(6 \cdot\left(b_{i}\right)^{2} \cdot k_{o f f, i}+11 \cdot \gamma_{i}\right), \quad B_{i, 3}=6 \cdot\left(\gamma_{i}+k_{r e p, i}\right) .
\end{aligned}
$$


When $n_{V}(i)=6$,

$$
\begin{aligned}
& B_{i, 0}=k_{r e p, i} \cdot\left(b_{i} \cdot k_{o f f, i}\right)^{3} \cdot\left(\gamma_{i}+\left(b_{i}\right)^{3} \cdot k_{o f f, i}\right), \\
& B_{i, 1}=k_{r e p, i} \cdot b_{i} \cdot k_{o f f, i}^{2} \cdot\left(\gamma_{i}+4 \cdot\left(b_{i}\right)^{2} \cdot \gamma_{i}+4 \cdot\left(b_{i}\right)^{5} \cdot k_{o f f, i}\right), \\
& B_{i, 2}=2 \cdot k_{r e p, i} \cdot k_{o f f, i} \cdot\left(\gamma_{i}+2 \cdot b_{i} \cdot \gamma_{i}+6 \cdot\left(b_{i}\right)^{2} \cdot \gamma_{i}+6 \cdot\left(b_{i}\right)^{5} \cdot k_{o f f, i}\right), \\
& B_{i, 3}=2 \cdot k_{r e p, i} \cdot\left(25 \cdot \gamma_{i}+12 \cdot\left(b_{i}\right)^{3} \cdot k_{o f f, i}\right), \quad B_{i, 4}=24 \cdot\left(\gamma_{i}+k_{r e p, i}\right) .
\end{aligned}
$$

When $n_{V}(i)=7$,

$$
\begin{aligned}
& B_{i, 0}=k_{r e p, i} \cdot\left(b_{i}\right)^{6} \cdot\left(k_{o f f, i}\right)^{4} \cdot\left(\gamma_{i}+\left(b_{i}\right)^{4} \cdot k_{o f f, i}\right), \\
& B_{i, 1}=k_{r e p, i} \cdot\left(b_{i} \cdot k_{o f f, i}\right)^{3} \cdot\left(\gamma_{i}+5 \cdot\left(b_{i}\right)^{3} \cdot \gamma_{i}+5 \cdot\left(b_{i}\right)^{7} \cdot k_{o f f, i}\right), \\
& B_{i, 2}=k_{r e p, i} \cdot b_{i} \cdot\left(k_{o f f, i}\right)^{2} \cdot\left(\left(2+5 \cdot\left(b_{i}\right)^{2}+20 \cdot\left(b_{i}\right)^{4}\right) \cdot \gamma_{i}+20 \cdot\left(b_{i}\right)^{8} \cdot k_{o f f, i}\right), \\
& B_{i, 3}=2 \cdot k_{r e p, i} \cdot k_{o f f, i} \cdot\left(\left(3+5 \cdot b_{i} \cdot\left(1+2 \cdot b_{i}+6 \cdot\left(b_{i}\right)^{2}\right)\right) \cdot \gamma_{i}+30 \cdot\left(b_{i}\right)^{7} \cdot k_{o f f, i}\right), \\
& B_{i, 4}=2 \cdot k_{r e p, i} \cdot\left(137 \cdot \gamma_{i}+60 \cdot\left(b_{i}\right)^{4} \cdot k_{o f f, i}\right), \quad B_{i, 5}=120 \cdot\left(\gamma_{i}+k_{r e p, i}\right) .
\end{aligned}
$$

The expression of $C V_{I E I}$ for an AZ with arbitrary number of vesicular release sites can be written as

$$
C V_{I E I}=\frac{\sqrt{\left\langle t^{2}\right\rangle_{I E I}-\langle t\rangle_{I E I}^{2}}}{\langle t\rangle_{I E I}},
$$

where $\langle t\rangle_{I E I}$ is given by $(\mathrm{S} 2.100)-(\mathrm{S} 2.101)$. In order to calculate $\left\langle t^{2}\right\rangle_{I E I}$ for an $\mathrm{AZ}$ with $N_{V}$ identical vesicular release sites, we, first of all, introduced $n_{V}$ new variables to characterize the state of the system: $\left(m_{1}, m_{2}, \ldots, m_{N_{V}}\right) \equiv \vec{m}$. Variable $m_{i}$ is equal to the number of vesicular release sites in state $(v=i)$ at the AZ. $m_{i}=\overline{0, N_{V}}$ with a constrain $\sum_{i=1}^{n_{V}} m_{i}=$ $N_{V} \cdot \vec{m}$ fully characterizes the state of the system. The number of different states is equal to $\prod_{i=1}^{n_{V}-1}\left(1+N_{V} / i\right)$. Elements of the initial condition vector $\mathbf{P}_{\mathbf{0}}^{\mathbf{s}}$ are expressed as follows:

$$
P_{0}^{s}(\vec{m})=\frac{N_{V} !}{\prod_{i=1}^{n_{V}} m_{i} !} \cdot \prod_{i=1}^{n_{V}}\left(\bar{P}^{s}(i)\right)^{m_{i}} .
$$

According to Eq. (4.14), $\left\langle t^{2}\right\rangle_{I E I}$ is equal to

$$
\left\langle t^{2}\right\rangle_{I E I}=2 \cdot \mathbf{P}_{\mathbf{0}}^{\mathbf{s}} \cdot\left(\left(-\mathbf{A}^{\mathbf{T}}\right)^{-\mathbf{2}} \cdot \mathbf{1}\right)
$$

The inverse of $\mathbf{A}^{\mathbf{T}}$ can be found analytically if necessary. However, the resulting expressions for $\left\langle t^{2}\right\rangle_{I E I}$ are very complicated. Thus, the analytical calculation of $\left(\mathbf{A}^{\mathbf{T}}\right)^{-\mathbf{1}}$ for $N_{V}>1$ is of little practical use.

Calculation of $C_{I E I}(l)$ is based on Eqs. (4.16) - (4.17), which we rewrite here,

$$
C_{I E I}(l)=\frac{\left\langle t_{i} t_{i+l}\right\rangle_{I E I}-\langle t\rangle_{I E I}^{2}}{\left\langle t^{2}\right\rangle_{I E I}-\langle t\rangle_{I E I}^{2}}, \quad\left\langle t_{i} t_{i+l}\right\rangle_{I E I}=\mathbf{1}^{\mathbf{T}} \cdot\left(\mathbf{J} \cdot \mathbf{T}^{\mathbf{l}-\mathbf{1}} \cdot \mathbf{G} \cdot \mathbf{P}_{\mathbf{0}}^{\mathbf{s}}\right) .
$$


For an $\mathrm{AZ}$ with $N_{V}$ identical vesicular release sites, the expressions of $\langle t\rangle_{I E I},\left\langle t^{2}\right\rangle_{I E I}$, and $\mathbf{P}_{\mathbf{0}}^{\mathbf{s}}$ are given by (S2.100), (S2.109), and (S2.108) respectively. The expressions of the elements of $\mathbf{G}, \mathbf{T}$, and $\mathbf{J}$ are as follows:

$$
\begin{gathered}
T\left(\vec{m} \mid \vec{m}_{*}\right)=\gamma \cdot\left(m_{n_{V}}+1\right) \cdot \int_{0}^{+\infty} P\left(t, m_{1}-1, \vec{m}_{-\left(1, n_{V}+1\right)}, m_{n_{V}}+1 \mid \vec{m}_{*}\right) d t \\
G\left(\vec{m} \mid \vec{m}_{*}\right)=\gamma \cdot\left(m_{n_{V}}+1\right) \cdot \int_{0}^{+\infty} t \cdot P\left(t, m_{1}-1, \vec{m}_{-\left(1, n_{V}+1\right)}, m_{n_{V}}+1 \mid \vec{m}_{*}\right) d t \\
J\left(\vec{m}_{*} \mid \vec{m}_{*}\right)=\int_{0}^{+\infty} \sum_{m_{n_{V}}=0}^{N_{V}} \sum_{m_{n_{V}-1}=0}^{N_{m_{n_{V}}-1}} \ldots \sum_{m_{2}=0}^{N_{m_{2}}} \sum_{m_{1}=0}^{N_{m_{1}}} P\left(t, \vec{m} \mid \vec{m}_{*}\right) d t
\end{gathered}
$$

Where, $N_{m_{i}}=N_{V}-\sum_{j=i+1}^{n_{V}} m_{j}$. If all eigenvalues of the transition matrix $\mathbf{A}$ are different, we can write $P(t, \vec{m} \mid \vec{m})$ as

$$
P\left(t, \vec{m} \mid \vec{m}_{*}\right)=\sum_{i=1}^{M} \alpha_{\vec{m}_{*}, i} \cdot \Lambda_{\vec{m}, i} \cdot e^{\gamma_{i} \cdot t},
$$

where $\lambda_{i}<0$ and $\Lambda_{\vec{m}, i}$ are respectively the $i$-th eigenvalue and the $i$-th eigenvector of the matrix A, and $\alpha_{\vec{m}_{*}, i}$ is the $i$-th integration constant. $M=\prod_{j=1}^{n_{V}-1}\left(1+N_{V} / j\right)$. Accordingly, $T\left(\vec{m} \mid \vec{m}_{*}\right)$, $G\left(\vec{m} \mid \vec{m}_{*}\right)$, and $J\left(\vec{m}_{*} \mid \vec{m}_{*}\right)$ can be expressed in the following way:

$$
\begin{gathered}
T\left(\vec{m} \mid \vec{m}_{*}\right)=-\gamma \cdot\left(m_{n_{V}}+1\right) \cdot \sum_{i=1}^{M} \alpha_{\vec{m}_{*}, i} \cdot \Lambda_{\left(m_{1}-1, \vec{m}_{-(1,4)}, m_{4}+1\right), i} \cdot \lambda_{i}^{-1}, \\
G\left(\vec{m} \mid \vec{m}_{*}\right)=\gamma \cdot\left(m_{n_{V}}+1\right) \cdot \sum_{i=1}^{M} \alpha_{\vec{m}_{*}, i} \cdot \Lambda_{\left(m_{1}-1, \vec{m}_{-(1,4)}, m_{4}+1\right), i} \cdot \lambda_{i}^{-2}, \\
J\left(\vec{m}_{*} \mid \vec{m}_{*}\right)=-\sum_{i=1}^{M} \alpha_{\vec{m}_{*}, i} \cdot \lambda_{i}^{-1} .
\end{gathered}
$$

When writing Eq. (S2.117), we assumed that the eigenvectors $\Lambda_{\vec{m}, i}$ are normalized, which can be done without loss of generality. If some of the eigenvalues of the matrix $\mathbf{A}$ are degenerate, (S2.114) - (S2.117) can be modified, as taught in standard mathematical textbooks on differential equations (e.g., see Korn \& Korn, 1968).

\section{AZs with $\mathbf{n}_{V^{-s t a t e}}$ vesicular release sites, $\mathrm{Ca}^{2+}$-nanodomain coupling regime, $\mathbf{n}_{\mathrm{C}^{-}}$ state $\mathrm{Ca}^{2+}$ channels}

In this case, Eqs. (4.4) and (4.6) in the main text for a vesicular release site $i$ are simplified to

$$
\begin{aligned}
\partial_{t} \bar{P}_{i}\left(t, c, v \mid c_{0}, v_{0}\right) & =\sum_{c^{\prime}=c-1}^{c+1} k_{c^{\prime} \rightarrow c}^{C i} \cdot \bar{P}_{i}\left(t, c^{\prime}, v^{\prime} \mid c_{0}, v_{0}\right)+ \\
& +\sum_{v^{\prime}=v-1}^{v+1} k_{v^{\prime} \rightarrow v}^{V i} \cdot \bar{f}_{v^{\prime} \rightarrow v}^{i} \cdot \bar{P}_{i}\left(t, c^{\prime}, v^{\prime} \mid c_{0}, v_{0}\right)
\end{aligned}
$$




$$
\begin{aligned}
\partial_{t} \bar{P}_{i}\left(t, c, v \mid c_{0}, v_{0}\right)= & \sum_{c^{\prime}=c-1}^{c+1} k_{c^{\prime} \rightarrow c}^{C i} \cdot \bar{P}_{i}\left(t, c^{\prime}, v^{\prime} \mid c_{0}, v_{0}\right)+ \\
& +\sum_{v^{\prime}=v-1}^{v+1} k_{v^{\prime} \rightarrow v}^{V i} \cdot \bar{f}_{v^{\prime} \rightarrow v}^{i} \cdot \bar{P}_{i}\left(t, c^{\prime}, v^{\prime} \mid c_{0}, v_{0}\right) \cdot\left(1-\delta_{v, 1} \cdot \delta_{v^{\prime}, n_{V}(i)}\right),
\end{aligned}
$$

where

$$
\begin{aligned}
k_{n_{C}(i) \rightarrow 1}^{C i} & =0, \quad k_{1 \rightarrow n_{C}(i)}^{C i}=0, \\
k_{c \rightarrow c+1}^{C i} & =k_{+c}, \quad k_{c+1 \rightarrow c}^{C i}=k_{-c}, \quad \text { for } 1 \leq c<n_{C}(i), \\
k_{1 \rightarrow 1}^{C i} & =-k_{+1}, \quad k_{n_{V} \rightarrow n_{V}}^{C i}=-k_{-\left(n_{V}-1\right)}, \\
k_{c \rightarrow c}^{C i} & =-\left(k_{-(c-1)}+k_{+c}\right), \quad \text { for } 2 \leq c<n_{C}(i),
\end{aligned}
$$

and

$$
\begin{aligned}
k_{n_{V}(i) \rightarrow 1}^{V i} \cdot \bar{f}_{n_{V}(i) \rightarrow 1}^{i} & =\gamma_{i}, \quad k_{1 \rightarrow n_{V}(i)}^{V i} \cdot \bar{f}_{1 \rightarrow n_{V}(i)}^{i}=0, \\
k_{1 \rightarrow 2}^{V i} \cdot \bar{f}_{1 \rightarrow 2}^{i} & =k_{r e p, i}, \quad k_{2 \rightarrow 1}^{V i} \cdot \bar{f}_{2 \rightarrow 1}^{i}=0, \\
k_{v \rightarrow v+1}^{V i} \cdot \bar{f}_{v \rightarrow v+1}^{i} & =\left(n_{V}(i)-v\right) \cdot k_{o n, i} \cdot p_{o} \cdot \Delta\left[\mathrm{Ca}^{2+}\right]_{\mathrm{i}} \\
& =\left(n_{V}(i)-v\right) \cdot k_{r e l, i}, \quad \text { for } 2 \leq v<n_{V}(i)-1, \\
k_{v+1 \rightarrow v}^{V i} \cdot \bar{f}_{v+1 \rightarrow v}^{i} & =b^{v-2} \cdot k_{o f f}, \quad \text { for } 2 \leq v<n_{V}(i)-1, \\
k_{n_{V}(i) \rightarrow n_{V}(i)}^{V i} \bar{f}_{n_{V}(i) \rightarrow n_{V}(i)}^{i} & =-\left(b^{n_{V}(i)-3} \cdot k_{o f f, i}+\gamma_{i}\right), \\
k_{1 \rightarrow 1}^{V i} \cdot \bar{f}_{1 \rightarrow 1}^{i} & =-k_{r e p, i}, \\
k_{2 \rightarrow 2}^{V i} \cdot \bar{f}_{2 \rightarrow 2}^{i} & =-\left(n_{V}(i)-2\right) \cdot k_{o n, i} \cdot p_{o} \cdot \Delta\left[\mathrm{Ca}^{2+}\right]_{\mathrm{i}} \\
& =-\left(n_{V}(i)-2\right) \cdot k_{r e l, i}, \\
k_{v \rightarrow v}^{V i} \cdot \bar{f}_{v \rightarrow v}^{i} & =-\left(\left(n_{V}(i)-v\right) \cdot k_{o n, i} \cdot p_{o} \cdot \Delta\left[\mathrm{Ca}^{2+}\right]_{\mathrm{i}}+b^{v-3} \cdot k_{o f f}\right) \\
& =-\left(\left(n_{V}(i)-2\right) \cdot k_{r e l, i}+b^{v-3} \cdot k_{o f f}\right), \\
& \text { for } 2<v \leq n_{V}(i)-1 .
\end{aligned}
$$

In Eqs. (S2.118) - (S2.119), it is assumed that $\left(v^{\prime}=0\right) \equiv\left(v^{\prime}=n_{V}(i)\right),\left(v^{\prime}=n_{V}(i)+1\right) \equiv\left(v^{\prime}=\right.$ $1),\left(c^{\prime}=0\right) \equiv\left(c^{\prime}=n_{C}(i)\right)$ and $\left(c^{\prime}=n_{C}(i)+1\right) \equiv\left(c^{\prime}=1\right)$. The solutions of Eqs. (S2.118) (S2.119) can be written as

$$
\begin{aligned}
& P\left(t, c, v \mid c_{0}, v_{0}\right)=\sum_{i=1}^{n_{C}(i) \cdot n_{V}(i)} \alpha_{c_{0}, v_{0}, i} \cdot \Lambda_{c, v, i} \cdot e^{\lambda_{i} \cdot t}, \\
& \bar{P}\left(t, c, v \mid c_{0}, v_{0}\right)=\sum_{i=1}^{n_{C}(i) \cdot n_{V}(i)} \bar{\alpha}_{c_{0}, v_{0}, i} \cdot \bar{\Lambda}_{c, v, i} \cdot e^{\bar{\lambda}_{i} \cdot t} .
\end{aligned}
$$

Here, $\lambda_{i}<0$ and $\Lambda_{c, v, i}$ are respectively the $i$-th eigenvalue and the $i$-th eigenvector of the transition matrix corresponding to (S2.118), and $\alpha_{c_{0}, v_{0}, i}$ is the $i$-th integration constant. $\overline{\lambda_{i}} \leq 0$ and $\bar{\Lambda}_{c, v, i}$ stand, respectively, for the $i$-th eigenvalue and the $i$-th eigenvector of the transition 
matrix of the Eq. (S2.119). $\quad \bar{\alpha}_{c_{0}, v_{0}, i}$ is the corresponding $i$-th integration constant. It follows from Eq. (S2.122) that

$$
P\left(t \mid c_{0}, v_{0}\right)=\sum_{i=1}^{n_{C}(i) \cdot n_{V}(i)} \alpha_{c_{0}, v_{0}, i} \cdot e^{\lambda_{i} \cdot t}
$$

if we use normalized eigenvectors $\Lambda_{v, i}$. In general, the transition matrices corresponding of Eqs. (S2.118) - (S2.119) cannot be diagonalized analytically, thus, relevant numerical methods have to be employed. Stationary solutions of $(\mathrm{S} 2.119), \bar{P}^{s}(c, v)$, can be found analytically. To do this, we used computational software program Mathematica (Wolfram Research) in this work. Due to their large size, we do not provide the expressions here.

$\rho_{I E I}(t)$ for an $\mathrm{AZ}$ with $N_{V}$ vesicular release sites can be expressed as

$$
\begin{aligned}
\rho_{I E I}(t)=-\frac{d}{d t} \sum_{i=1}^{N_{V}}[ & P_{i}^{r} \cdot \sum_{c_{0}=1}^{n_{C}(i)}\left(P_{i}\left(t \mid c_{0}, 1\right) \cdot P_{0, i}^{s}\left(c_{0}, 1\right)\right) . \\
& \left.\cdot \prod_{j=1 \neq i}^{N_{V}} \sum_{c_{0}=1}^{n_{C}(j)} \sum_{v_{0}=1}^{n_{V}(j)}\left(P_{j}\left(t \mid c_{0}, v_{0}\right) \cdot \bar{P}_{j}^{s}\left(c_{0}, v_{0}\right)\right)\right],
\end{aligned}
$$

where

$$
P_{i}^{r}=\frac{\gamma_{i} \cdot \bar{P}_{i}^{s}\left(n_{V}(i)\right)}{\sum_{j=1}^{N_{V}} \gamma_{j} \cdot \bar{P}_{j}^{s}\left(n_{V}(j)\right)}
$$

In particular, for an $\mathrm{AZ}$ with one vesicular release site $\left(N_{V}=1\right)$, we have

$$
\rho_{I E I}(t)=-\frac{d}{d t} \sum_{c_{0}=1}^{n_{C}(i)}\left(P_{i}\left(t \mid c_{0}, 1\right) \cdot P_{0, i}^{s}\left(c_{0}, 1\right)\right) .
$$

Writing Eqs. (S2.125) and (S2.127) we used the fact that $P_{0, i}^{s}\left(c_{0}, v_{0}\right)=0$ for $v_{0} \neq 1 . P_{0, i}^{s}\left(c_{0}, 1\right)$ is the solution of

$$
P_{0, i}^{s}\left(c_{0}, 1\right)=\sum_{c_{*}=1}^{n_{C}(i)} T_{i}\left(c_{0} \mid c_{*}\right) \cdot P_{0, i}^{s}\left(c_{*}, 1\right),
$$

which is a particular case of Eq. (4.9), and

$$
T_{i}\left(c_{0} \mid c_{*}\right)=\gamma_{i} \cdot \int_{0}^{+\infty} P_{i}\left(t, c_{0}, n_{V}(i) \mid c_{*}, 1\right) d t .
$$

\subsection{Analytical expressions of $\rho_{I S I},\langle t\rangle_{I S I}, C V_{I S I}$}

Once the solutions of Eqs. (4.4) and (4.6) are known, $\rho_{I S I}$ and $\left\langle t^{m}\right\rangle_{I S I}$ follow from Eqs. (4.27) and (4.29) for AZs with any chosen organization. 


\section{The Poisson limit of the vesicle fusion point process}

Let us denote the vesicle fusion rate by $R_{r}$. When the vesicle fusion process is described by a homogeneous Poisson process, the expression for $\rho_{I S I}(t)$, Eq. (4.28), is simplified to

$$
\begin{aligned}
\rho_{I S I}(t) & =\int_{0}^{t} H\left(t^{\prime}-t_{A}\right) \cdot e^{-\left(t^{\prime}-t_{A}\right) / \tau_{R}} \cdot R_{r} \cdot e^{-R_{r} \cdot\left(t^{\prime}-t\right)} \\
& =H\left(t-t_{A}\right) \cdot \frac{R_{r}}{1-\tau_{R} \cdot R_{r}} \cdot\left(e^{-R_{r} \cdot\left(t-t_{A}\right)}-e^{-\left(t-t_{A}\right) / \tau_{R}}\right) .
\end{aligned}
$$

Accordingly,

$$
\begin{gathered}
\langle t\rangle_{I S I}=\frac{1+R_{r} \cdot\left(t_{A}+\tau_{R}\right)}{R_{r}}, \\
\left\langle t^{2}\right\rangle_{I S I}=\frac{\left(R_{r} \cdot\left(\tau_{R}+t_{A}\right)\right)^{2}+2 \cdot R_{r} \cdot\left(\tau_{R}+t_{A}\right)+\left(R_{r} \cdot \tau_{R}\right)^{2}+2}{\left(R_{r}\right)^{2}}, \\
C V_{I S I}(t)=\frac{\sqrt{1+\left(R_{r} \cdot \tau_{R}\right)^{2}}}{1+R_{r} \cdot\left(t_{A}+\tau_{R}\right)} .
\end{gathered}
$$




\section{Supplementary Material 3: Analysis of the inter-release time interval distributions and their moments}

\subsection{Single 2-state vesicular release site, $\mathrm{Ca}^{2+}$-microdomain coupling regime}

In this case, the analytical expressions for $\rho_{I E I}(t),\langle t\rangle_{I E I}$ and $C V_{I E I}$ are given by Eq. (4.39). As shown in Fig. 4.3, there exist only two limiting cases of the vesicle release: $\left[2^{*}\right]$ and $\left[3^{*}\right]$. In both of these limiting cases vesicular release is described by a homogeneous Poisson process,

$$
\rho_{I E I}(t)=r \cdot e^{-r \cdot t}, \quad\langle t\rangle_{I E I}=r^{-1}, \quad C V_{I E I}=1,
$$

where

$$
r=\left\{\begin{array}{ll}
k_{r e l}, & \text { if } k_{r e p} \gg k_{r e l},\left[2^{*}\right] \\
k_{r e p}, & \text { if } k_{r e p} \ll k_{r e l},\left[3^{*}\right]
\end{array} .\right.
$$

\subsection{Single 2-state vesicular release site, $\mathrm{Ca}^{2+}$-nanodomain coupling regime with a 2 -state $\mathrm{Ca}^{2+}$ channel}

In this case, the general expressions for $\rho_{I E I}(t),\langle t\rangle_{I E I}$, and $C V_{I E I}$ are given, respectively, by Eqs. (S2.74 - S2.76), (S2.81). Below, we provide the reduced versions of these expressions which apply in the limiting cases considered in section 4.4.1 and illustrated in Fig. 4.3.

In the limiting cases $[2],[3],[6],\left[2^{*}\right]$, and $\left[3^{*}\right]$, vesicle release is described by a homogeneous Poisson process,

$$
\rho_{I E I}(t)=r \cdot e^{-r \cdot t}, \quad\langle t\rangle_{I E I}=r^{-1}, \quad C V_{I E I}=1,
$$

where

$$
r=\left\{\begin{array}{ll}
k_{+1}, & \text { if } k_{+1} \ll k_{r e p} \ll k_{-1} \ll k_{r e l}, \quad[2] \\
k_{+1} \cdot \frac{k_{r e l}}{k_{r e l}+k_{-1}}, & \text { if } k_{+1} \sim k_{r e p} \ll k_{-1} \sim k_{r e l},[3] \\
k_{r e p}, & \text { if } k_{r e p} \ll k_{+1} \ll k_{-1} \lesssim k_{r e l},[6] \\
k_{r e l} \cdot \frac{k_{+1}}{k_{+1}+k_{-1}}, & \text { if } k_{+1} \gg k_{r e p} \gg k_{r e l} \cdot p_{o},\left[2^{*}\right] \\
k_{r e p}, & \text { if } k_{+1} \gg k_{r e p} \ll k_{r e l} \cdot p_{o},\left[3^{*}\right]
\end{array} .\right.
$$

Limiting cases [4], [5], and [1*] correspond to the vesicle release processes limited by two steps. One of those steps is replenishment, while the other one is either $\mathrm{Ca}^{2+}$ channel opening (cases [4], and [5]) or $\mathrm{Ca}^{2+}$ binding to the sensor of exocytosis (case [1*]),

$$
\rho_{I E I}(t)=\frac{k \cdot k_{\text {rep }}}{k-k_{\text {rep }}} \cdot\left(e^{-k_{\text {rep }} t}-e^{-k t}\right), \quad\langle t\rangle_{I E I}=\frac{k+k_{\text {rep }}}{k \cdot k_{\text {rep }}}, \quad C V_{\text {IEI }}=\frac{\sqrt{\left(k_{\text {rep }} / k\right)^{2}+1}}{k_{\text {rep }} / k+1},
$$


where

$$
k=\left\{\begin{array}{ll}
k_{+1}, & \text { if } k_{+1} \sim k_{r e p} \ll k_{-1} \ll k_{r e l}, \quad[4] \\
k_{+1} \cdot p_{o}, & \text { if } k_{+1} \sim k_{r e p} \ll k_{-1} \sim k_{r e l},[5] \\
k_{r e l} \cdot p_{o}, & \text { if } k_{+1} \gg k_{r e p} \sim k_{r e l} \cdot p_{o},\left[1^{*}\right]
\end{array} .\right.
$$

In the limiting case [7] $\left(k_{+1} \sim k_{-1} \sim k_{r e l} \ll k_{r e p}\right)$,

$$
\begin{aligned}
\rho_{I E I}(t) & =\frac{k_{r e l}}{\lambda_{2}-\lambda_{1}} \cdot\left(\left(k_{+1}-\lambda_{1}\right) \cdot e^{-\lambda_{1} \cdot t}-\left(k_{+1}-\lambda_{2}\right) \cdot e^{-\lambda_{2} \cdot t}\right) \\
\langle t\rangle_{I E I} & =\frac{k_{-1}+k_{+1}}{k_{+1} \cdot k_{r e l}}=\left(k_{r e l} \cdot p_{o}\right)^{-1}, \\
C V_{I E I} & =\frac{\sqrt{2 \cdot k_{-1} \cdot k_{r e l}+\left(k_{-1}+k_{+1}\right)^{2}}}{k_{-1}+k_{+1}}>1,
\end{aligned}
$$

It is easy to check that $C V_{I E I} \sim 1$, if $k_{-1}, k_{+1}$, and $k_{r e l}$ are similar. In particular, if $k_{+1}=$ $k_{-1}=k_{\text {rel }}, C V_{I E I}=\sqrt{3 / 2} \approx 1.2$.

In the limiting case [8] $\left(k_{+1} \sim k_{-1} \sim k_{r e p} \ll k_{r e l}\right)$,

$$
\begin{aligned}
\rho_{I E I}(t)= & -\frac{k_{+1} \cdot k_{-1} \cdot k_{r e p}}{\left(k_{+1}-k_{r e p}\right) \cdot\left(k_{-1}+k_{r e p}\right)} \cdot e^{-k_{+1} \cdot t}+ \\
& +\frac{k_{+1} \cdot k_{r e p} \cdot\left(k_{+1}+k_{-1}-k_{r e p}\right)}{\left(k_{+1}-k_{r e p}\right) \cdot\left(k_{-1}+k_{+1}\right)} \cdot e^{-k_{r e p} \cdot t}+ \\
& +\frac{k_{-1} \cdot k_{r e p} \cdot\left(k_{+1}+k_{-1}+k_{r e p}\right)}{\left(k_{-1}+k_{r e p}\right) \cdot\left(k_{-1}+k_{+1}\right)} e^{-\left(k_{+1}+k_{-1}+k_{r e p}\right) \cdot t}, \\
\langle t\rangle_{I E I}= & \frac{\left(k_{+1}+k_{-1}\right) \cdot\left(k_{+1}+k_{r e p}\right)}{k_{+1} \cdot k_{r e p} \cdot\left(k_{+1}+k_{-1}+k_{r e p}\right)}, \\
C V_{I E I}= & \frac{\sqrt{A}}{\left(k_{+1}+k_{-1}\right)\left(k_{+1}+k_{r e p}\right)} \sim 1,
\end{aligned}
$$

where

$$
\begin{aligned}
A & =k_{+1}^{4}+2 \cdot k_{+1}^{3} \cdot\left(k_{-1}+k_{r e p}\right)+k_{+1}^{2} \cdot\left(k_{-1}+k_{r e p}\right)^{2} \\
& +4 \cdot k_{+1} \cdot k_{-1} \cdot k_{r e p}^{2}+k_{-1} \cdot k_{r e p}^{2} \cdot\left(k_{-1}+2 \cdot k_{r e p}\right) .
\end{aligned}
$$

It is easy to check that $C V_{I E I} \sim 1$ in this case. In particular, if $k_{+1}=k_{-1}=k_{r e p}, C V_{I E I}=1$. Otherwise, $C V_{I E I}$ is either slightly lower or slightly higher than one. 
In the limiting cases [9] $\left(k_{+1} \ll k_{-1} \sim k_{r e p} \ll k_{r e l}\right)$ and [10] $\left(k_{+1} \lesssim k_{-1} \ll k_{r e p} \ll k_{r e l}\right)$,

$$
\begin{aligned}
\rho_{I E I}(t) & =\frac{k_{+1} \cdot k_{-1}}{k_{-1}+k_{r e p}} \cdot e^{-k_{+1} \cdot t}+k_{r e p} \cdot e^{-\left(k_{-1}+k_{r e p}\right) \cdot t} \\
\langle t\rangle_{I E I} & =\frac{k_{+1}+k_{-1}}{k_{+1} \cdot\left(k_{-1}+k_{r e p}\right)}, \quad C V_{I E I}=\frac{\sqrt{k_{-1} \cdot\left(k_{-1}+2 \cdot k_{r e p}\right)}}{k_{+1}+k_{-1}}>1 .
\end{aligned}
$$

Note that the first exponential in the expression (S3.10) has vanishing weight. However, it contributes appreciably either to the integral of $\rho_{I E I}(t)$ (case [9]) or, at least, to the statistical moments (cases [9] and [10]), as explained in section 4.4.1.

In the limiting case $[11]\left(k_{+1} \ll k_{-1} \sim k_{r e l} \ll k_{r e p}\right)$,

$$
\begin{gathered}
\rho_{I E I}(t)=\frac{k_{r e l}}{k_{-1}+k_{r e l}} \cdot\left(\frac{k_{+1} \cdot k_{-1}}{k_{-1}+k_{r e l}} \cdot e^{-\frac{k_{+1} \cdot k_{r e l}}{k_{-1}+k_{r e l}} \cdot t}+\left(k_{-1}+k_{r e l}\right) \cdot e^{-\left(k_{-1}+k_{r e l}\right) \cdot t}\right), \\
\langle t\rangle_{I E I}=\frac{k_{-1}+k_{+1}}{k_{+1} \cdot k_{r e l}}=\left(k_{r e l} \cdot p_{o}\right)^{-1}, \quad C V_{I E I}=\sqrt{1+2 \cdot\left(k_{r e l} / k_{-1}\right)}>1 .
\end{gathered}
$$

As in the limiting case [9], term with the slower exponent, $k_{+1} \cdot k_{r e l} /\left(k_{-1}+k_{r e l}\right)$, has similar probability weight to that of the faster term, and has dominant contributions to the statistical moments:

$$
\begin{aligned}
& \left\langle t^{0}\right\rangle=\underbrace{\frac{k_{-1}}{k_{-1}+k_{r e l}}}_{T_{1}^{0}}+\underbrace{\frac{k_{r e l}}{k_{-1}+k_{r e l}}}_{T_{2}^{0}}, \quad \frac{T_{1}^{0}}{T_{2}^{0}}=\frac{k_{-1}}{k_{r e l}} \sim 1, \\
& \left\langle t^{1}\right\rangle=\underbrace{\frac{k_{-1}}{k_{+1} \cdot k_{r e l}}+\underbrace{\frac{k_{r e l}}{\left(k_{-1}+k_{r e l}\right)^{2}}}_{T_{2}^{1}}, \quad \frac{T_{1}^{1}}{T_{2}^{1}}=\frac{k_{-1} \cdot\left(k_{-1}+k_{r e l}\right)^{2}}{k_{+1} \cdot\left(k_{r e l}\right)^{2}} \gg 1,}_{T_{1}^{1}} \\
& \left\langle t^{2}\right\rangle=\underbrace{\frac{2 \cdot k_{-1} \cdot\left(k_{-1}+k_{r e l}\right)}{\left(k_{+1} \cdot k_{r e l}\right)^{2}}}_{T_{1}^{2}}+\underbrace{\frac{2 \cdot k_{r e l}}{\left(k_{-1}+k_{r e l}\right)^{3}}}_{T_{2}^{2}}, \quad \frac{T_{1}^{2}}{T_{2}^{2}}=\frac{k_{-1} \cdot\left(k_{-1}+k_{r e l}\right)^{4}}{k_{r e l} \cdot\left(k_{+1} \cdot k_{r e l}\right)^{2}} \gg 1 .
\end{aligned}
$$

$T_{1}^{2} /\left(T_{1}^{1}\right)^{2}=2 \cdot\left(k_{-1}+k_{r e l}\right) / k_{-1}$ is bound from above and so is $C V_{I E I}$. In particular, when $k_{-1}=k_{r e l}, C V_{I E I}=\sqrt{3} \approx 1.7$, like in the limiting case [9].

In the limiting case $[12]\left(k_{+1} \lesssim k_{-1} \ll k_{r e l} \ll k_{r e p}\right)$,

$$
\begin{aligned}
\rho_{I E I}(t) & =\frac{k_{+1} \cdot k_{-1}}{k_{r e l}} \cdot e^{-k_{+1} \cdot t}+k_{r e l} \cdot e^{-k_{r e l} \cdot t} \\
\langle t\rangle_{I E I} & =\frac{k_{-1}+k_{+1}}{k_{+1} \cdot k_{r e l}}=\left(k_{r e l} \cdot p_{o}\right)^{-1}, \quad C V_{I E I}=\frac{\sqrt{2 \cdot k_{-1} \cdot k_{r e l}}}{k_{+1}+k_{-1}}>1 .
\end{aligned}
$$


As in the limiting case [10], term with the slower exponent, $k_{+1}$, has vanishing probability weight. However, that term dominates in the statistical moments,

$$
\begin{aligned}
& \left\langle t^{0}\right\rangle=\underbrace{\frac{k_{-1}}{k_{r e l}}+\underbrace{1}_{T_{2}^{0}}, \quad \frac{T_{1}^{0}}{T_{2}^{0}}=\frac{k_{-1}}{k_{r e l}} \ll 1,}_{T_{1}^{0}} \\
& \left\langle t^{1}\right\rangle=\underbrace{\frac{k_{-1}}{k_{+1} \cdot k_{r e l}}}_{T_{1}^{1}}+\underbrace{\frac{1}{k_{r e l}}}_{T_{2}^{1}}, \quad \frac{T_{1}^{1}}{T_{2}^{1}}=\frac{k_{-1}}{k_{+1}} \gtrsim 1, \\
& \left\langle t^{2}\right\rangle=\underbrace{\frac{2 \cdot k_{-1}}{\left(k_{+1}\right)^{2} \cdot k_{r e l}}}_{T_{1}^{2}}+\underbrace{\frac{2}{\left(k_{r e l}\right)^{2}}}_{T_{2}^{2}}, \quad \frac{T_{1}^{2}}{T_{2}^{2}}=\frac{k_{-1} \cdot k_{r e l}}{\left(k_{+1}\right)^{2}} \gg 1 .
\end{aligned}
$$

$T_{1}^{2} /\left(T_{1}^{1}\right)^{2}=2 \cdot k_{r e l} / k_{-1}$ is unbound from above and so is $C V_{I E I}$.

In the limiting case $[13]\left(k_{+1} \ll k_{-1} \sim k_{r e p} \sim k_{r e l}\right)$,

$$
\begin{aligned}
\rho_{I E I}(t)= & \frac{k_{r e l} \cdot k_{r e p}}{k_{r e l}-k_{r e p}} \cdot e^{-\left(k_{-1}+k_{r e p}\right) \cdot t}+\frac{k_{r e p} \cdot k_{r e l}}{\left(k_{r e p}-k_{r e l}\right)} \cdot e^{-\left(k_{-1}+k_{r e l}\right) \cdot t}+ \\
& +\frac{k_{+1} \cdot k_{-1} \cdot k_{r e l} \cdot\left(k_{-1}+k_{r e l}+k_{r e p}\right)}{\left(k_{-1}+k_{r e p}\right) \cdot\left(k_{-1}+k_{r e l}\right)^{2}} \cdot e^{-\frac{k_{+1} \cdot k_{r e l}}{k_{-1}+k_{r e l}} \cdot t}, \\
\langle t\rangle_{I E I}= & \frac{k_{-1} \cdot\left(k_{-1}+k_{r e l}+k_{r e p}\right)}{k_{+1} \cdot k_{r e l} \cdot\left(k_{-1}+k_{r e p}\right)}, \\
C V_{I E I}= & \frac{\sqrt{2 \cdot k_{-1} \cdot k_{r e p}^{2} \cdot\left(2 \cdot k_{r e l}+k_{-1}\right)+k_{-1} \cdot\left(2 \cdot k_{r e p}+k_{-1}\right) \cdot\left(k_{-1}+k_{r e l}\right)^{2}}}{k_{-1} \cdot\left(k_{r e l}+k_{r e p}\right)}>1 .
\end{aligned}
$$

The third exponential in (S3.18) has vanishing weight and its contribution to $\rho_{I E I}(t)$ is negligible. However, that term is associated with a finite probability weight and dominant contribution to the statistical moments. $C V_{I E I}>1$ but is finite. In particular, when $k_{-1}=k_{r e p}=k_{r e l}$, $C V_{I E I}=\sqrt{3} \approx 1.7$, like in the limiting cases [9] and [11].

\subsection{Single $\mathrm{n}_{\mathrm{V}}$-state vesicular release site, $\mathrm{Ca}^{2+}$-microdomain cou- pling regime}

In the limiting case $\left[\mathbf{2}_{m}^{*}\right]$, the vesicle cycle at each release site consists of $n_{V}=m+2$ irreversible steps, which are independent exponentially distributed random variables. Thus, the average of the vesicle release waiting time is a sum of the average waiting times corresponding to each of the stages of the cycle,

$$
\langle t\rangle_{I E I}=\gamma^{-1}+k_{r e p}^{-1}+\sum_{i=1}^{m} \frac{1}{i \cdot k_{r e l}} .
$$


The same applies to the standard deviation,

$$
\left\langle t^{2}\right\rangle_{I E I}-\langle t\rangle_{I E I}^{2}=\gamma^{-2}+k_{r e p}^{-2}+\sum_{i=1}^{m} \frac{1}{\left(i \cdot k_{r e l}\right)^{2}} .
$$

Thus,

$$
C V_{I E I}=\frac{\sqrt{\left\langle t^{2}\right\rangle_{I E I}-\langle t\rangle_{I E I}^{2}}}{\langle t\rangle_{I E I}}=\frac{\sqrt{k_{r e l}^{-2} \cdot \sum_{i=1}^{m} i^{-2}+\gamma^{-2}+k_{r e p}^{-2}}}{k_{r e l}^{-1} \cdot \sum_{i=1}^{m} i^{-1}+\gamma^{-1}+k_{r e p}^{-1}} .
$$

It is straightforward to show by using standard methods of mathematical analysis that the global minimum of $C V_{I E I}$ as a function of $\gamma$ and $k_{\text {rep }}$ is located at

$$
\gamma^{-1}=k_{r e p}^{-1}=k_{r e l}^{-1} \cdot \frac{\sum_{i=1}^{m} i^{-2}}{\sum_{i=1}^{m} i^{-1}}
$$

Substituting (S3.24) into (S3.23) yields

$$
\min \left[C V_{I E I}\right]=\sqrt{\frac{\sum_{i=1}^{m} i^{-2}}{\left(\sum_{i=1}^{m} i^{-1}\right)^{2}+2 \cdot \sum_{i=1}^{m} i^{-2}}} .
$$

As mentioned above, in the limiting case $\left[2_{m}^{*}\right]$, IEI is a sum of $n_{V}$ independent exponentially distributed random variables. Thus, the characteristic function of $\rho_{I E I}(t)$, which we denote by $\tilde{\rho}_{I E I}(\omega)$, is a product of the characteristic functions of exponential distributions in this case,

$$
\tilde{\rho}_{I E I}(\omega)=\prod_{j=1}^{n_{V}} \frac{1}{1-\imath \cdot k_{j}^{-1} \cdot \omega}
$$

where $k_{1}=k_{r e p}, k_{n_{V}}=\gamma, k_{j}=(j-1) \cdot k_{r e l}, j=\overline{2, n_{V}-1} . \rho_{I E I}(t)$ is equal to the inverse Fourier transform of its characteristic function. Thus,

$$
\rho_{I E I}(t)=\frac{1}{2 \pi} \cdot \int_{-\infty}^{+\infty}\left[e^{-\imath \cdot \omega \cdot t} \cdot \prod_{j=1}^{n_{V}} \frac{1}{1-\imath \cdot k_{j}^{-1} \cdot \omega}\right] d \omega .
$$

Integral (S3.27) can be calculated by using the residue theorem and taking into account that the integration contour can be closed by the lower semicircle whose contribution is equal to zero. Doing this leads to

$$
\rho_{I E I}(t)=\sum_{j=1}^{n_{V}} \frac{k_{j}^{-\left(n_{V}-2\right)}}{\prod_{i=1 \neq j}^{n_{V}}\left(k_{j}^{-1}-k_{i}^{-1}\right)} \cdot e^{-k_{j} \cdot t} .
$$




\section{Supplementary Material 4: Analysis of the serial correla- tion functions of inter-release time intervals}

\subsection{AZ with a single $n_{V^{-}}$-state vesicular release site driven by a $n_{C^{-}}$ state channel $\left(\mathrm{Ca}^{2+}\right.$-nanodomain coupling regime)}

First, we show that $C_{I E I}(l) \neq 1$ if and only if $l=1$ for this system. Indeed, independently from which state the system starts just after a vesicle fusion event, the system must go through the state when the channel is open before the first $\mathrm{Ca}^{2+}$ ion binds to the sensor of exocytosis. The dynamics of the system is Markovian. Thus, all further development of the system from the aforementioned state does not depend on the past of the system. In turn, the probability to find the system in a particular state just after a release event does not depend on the state in which the system was just after the previous release events. If it is so, then $I E I_{i+l}$ and $I E I_{i}$ are independent for any $l \geq 2$. $I E I_{i}$ and $I E I_{i+1}$ are correlated because the waiting time for the previous vesicle release event depends on the state to which the the system transits during this event. The same state influences the waiting time for the next vesicular release event as well.

Next, we consider the limit $\left(k_{r e l}, k_{r e p}\right) \gg\left(k_{+1} \sim k_{-1} \sim \gamma\right) \gg k_{\text {off }}$, when $\max \left[C_{I E I}\right]$ is observed for an $n_{V}$-state vesicular release site in $\mathrm{Ca}^{2+}$-nanodomain coupling regime with presynaptic $\mathrm{Ca}^{2+}$-channels. We found by global optimization that $\max \left[C_{I E I}\right]$ does not depend on $n_{C}$. Thus we considered $n_{C}=2$ below. In this case, the kinetic schemes corresponding to the calculation of IEI are shown in Fig. S4.1. Effectively, the system is described by two

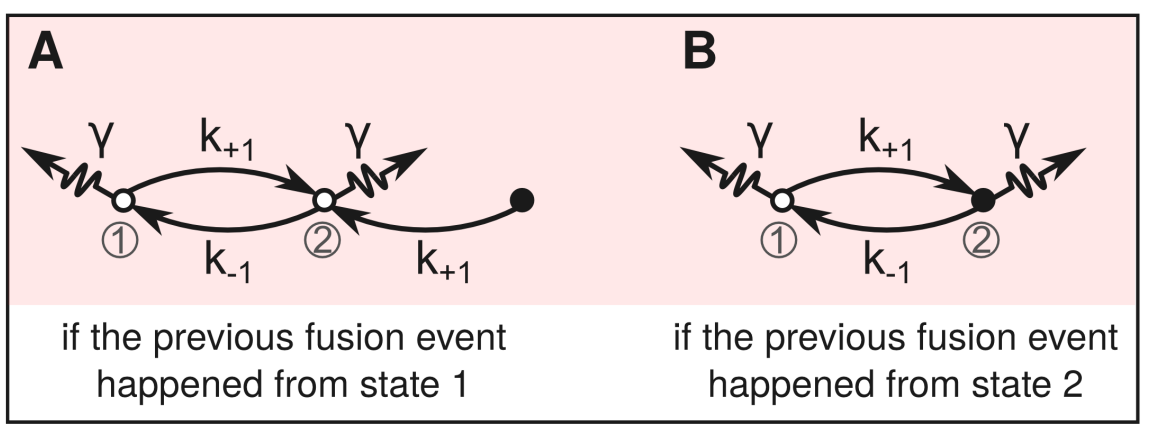

Figure S4.1: Kinetic schemes for calculating IEIs for an $n_{V}$-state vesicular release site in $\mathrm{Ca}^{2+}$ nanodomain coupling regime with a 2 -state $\mathrm{Ca}^{2+}$ channel. The limiting case $\left(k_{r e l}, k_{r e p}\right) \gg\left(k_{+1} \sim\right.$ $\left.k_{-1} \sim \gamma\right) \gg k_{o f f}$ is considered here.

states, which correspond to the channel gating variable $c$. If the previous vesicle fusion event happened from state 1 , the kinetic scheme A applies for calculating $P(t, c \mid 1)$. If the previous vesicle fusion event happened from state 2 , the kinetic scheme $\mathrm{B}$ applies for calculating $P(t, c \mid 2)$. 
Accordingly, the dynamics of $P\left(t, c \mid c_{0}\right)$ is described by the following equations:

$$
\begin{aligned}
& \partial_{t} P(t, 1 \mid 1)=-\left(k_{+1}+\gamma\right) \cdot P(t, 1 \mid 1)+k_{-1} \cdot P(t, 2 \mid 1) \\
& \partial_{t} P(t, 2 \mid 1)=k_{+1} \cdot P(t, 1 \mid 1)-\left(k_{-1}+\gamma\right) \cdot P(t, 2 \mid 1)+k_{+1} \cdot e^{-k_{+1} \cdot t^{\prime}}
\end{aligned}
$$

with $P(t=0,1 \mid 1)=P(t=0,2 \mid 1)=0$, and

$$
\begin{aligned}
& \partial_{t} P(t, 1 \mid 2)=-\left(k_{+1}+\gamma\right) \cdot P(t, 1 \mid 2)+k_{-1} \cdot P(t, 2 \mid 2) \\
& \partial_{t} P(t, 2 \mid 2)=k_{+1} \cdot P(t, 1 \mid 2)-\left(k_{-1}+\gamma\right) \cdot P(t, 2 \mid 2)
\end{aligned},
$$

with $P(t=0,1 \mid 2)=0, P(t=0,2 \mid 2)=1$. Solving (S4.1) and (S4.2) results in

$$
\begin{aligned}
P(t, 1 \mid 1) & =\frac{k_{-1} \cdot k_{+1}}{\left(k_{-1}+k_{+1}\right) \cdot\left(\gamma+k_{-1}\right) \cdot\left(\gamma-k_{+1}\right)} \cdot\left(\left(k_{-1}+k_{+1}\right) \cdot e^{-k_{+1} \cdot t}-\right. \\
& \left.-\left(\gamma+k_{-1}\right) \cdot e^{-\gamma \cdot t}+\left(\gamma-k_{+1}\right) \cdot e^{-\left(k_{-1}+k_{+1}+\gamma\right) \cdot t}\right) \\
P(t, 2 \mid 1) & =\frac{k_{+1}}{\left(k_{-1}+k_{+1}\right) \cdot\left(\gamma+k_{-1}\right) \cdot\left(\gamma-k_{+1}\right)} \cdot\left(\gamma \cdot\left(k_{-1}+k_{+1}\right) \cdot e^{-k_{+1} \cdot t}-\right. \\
& \left.-k_{+1} \cdot\left(\gamma+k_{-1}\right) \cdot e^{-\gamma \cdot t}-k_{-1} \cdot\left(\gamma-k_{+1}\right) \cdot e^{-\left(k_{-1}+k_{+1}+\gamma\right) \cdot t}\right) \\
P(t, 1 \mid 2) & =\frac{k_{-1}}{\left(k_{-1}+k_{+1}\right)} \cdot\left(e^{-\gamma \cdot t}-e^{-\left(k_{-1}+k_{+1}+\gamma\right) \cdot t}\right), \\
P(t, 2 \mid 2) & =\frac{1}{\left(k_{-1}+k_{+1}\right)} \cdot\left(k_{+1} \cdot e^{-\gamma \cdot t}+k_{-1} \cdot e^{-\left(k_{-1}+k_{+1}+\gamma\right) \cdot t}\right) .
\end{aligned}
$$

Then, the expressions of elements of the matrix $\mathbf{T}$ are given by

$$
T(1 \mid 1)=T(1 \mid 2)=\frac{k_{-1}}{k_{-1}+k_{+1}+\gamma}, \quad T(2 \mid 1)=T(2 \mid 2)=\frac{k_{+1}+\gamma}{k_{-1}+k_{+1}+\gamma} .
$$

Here, $T(i \mid j)$ were calculated by using $T(i \mid j)=\int_{0}^{+\infty} P(t, i \mid j) d t$. Elements of the initial condition vector $\mathbf{P}_{\mathbf{0}}^{\mathbf{s}}$, which follows from $\mathbf{T} \cdot \mathbf{P}_{\mathbf{0}}^{\mathbf{s}}=\mathbf{P}_{\mathbf{0}}^{\mathbf{s}}$, are

$$
P_{0}^{s}(1)=\frac{k_{-1}}{k_{-1}+k_{+1}+\gamma}, \quad P_{0}^{s}(2)=\frac{k_{+1}+\gamma}{k_{-1}+k_{+1}+\gamma} .
$$

The expressions of elements of the matrix $\mathbf{G}$ are

$$
\begin{aligned}
& G(1 \mid 1)=\frac{k_{-1} \cdot\left(\left(k_{+1}+\gamma\right) \cdot\left(k_{-1}+k_{+1}+\gamma\right)+k_{+1} \cdot \gamma\right)}{\gamma \cdot k_{+1} \cdot\left(k_{-1}+k_{+1}+\gamma\right)^{2}} \\
& G(1 \mid 2)=\frac{k_{-1} \cdot\left(k_{-1}+k_{+1}+2 \cdot \gamma\right)}{\gamma \cdot\left(k_{-1}+k_{+1}+\gamma\right)^{2}} \\
& G(2 \mid 1)=\frac{\left(k_{+1}+\gamma\right)^{2} \cdot\left(k_{-1}+k_{+1}+\gamma\right)-k_{-1} \cdot k_{+1} \cdot \gamma}{\gamma \cdot k_{+1} \cdot\left(k_{-1}+k_{+1}+\gamma\right)^{2}} \\
& G(2 \mid 2)=\frac{\left(k_{+1}+\gamma\right)^{2}+k_{-1} \cdot k_{+1}}{\gamma \cdot\left(k_{-1}+k_{+1}+\gamma\right)^{2}}
\end{aligned}
$$

Here, $G(i \mid j)$ were calculated by using $G(i \mid j)=\int_{0}^{+\infty}[t \cdot P(t, i \mid j)] d t$. The expressions of the elements of the diagonal matrix $\mathbf{J}$ are as follows:

$$
J(1,1)=\frac{k_{+1}+\gamma}{k_{+1} \cdot \gamma}, \quad J(2 \mid 2)=\frac{1}{\gamma} .
$$


Substituting the above expressions of $\mathbf{J}, \mathbf{T}, \mathbf{G}$ and $\mathbf{P}_{\mathbf{0}}^{\mathbf{s}}$ into Eq. (14) in the section Analytical treatment of the model yields

$$
\left\langle t_{i} \cdot t_{i+1}\right\rangle_{I E I}=\frac{k_{-1}}{k_{+1} \cdot\left(k_{-1}+k_{+1}+\gamma\right)^{2}} .
$$

The first and the second moments of the $\rho_{I E I}(t)$ function in this case are

$$
\begin{aligned}
\langle t\rangle_{I E I} & =\sum_{i=1}^{2}\left(P_{0}^{s}(i) \cdot \int_{0}^{+\infty}(P(t, 1 \mid i)+P(t, 2 \mid i)) d t\right) \\
& =\frac{\left(k_{+1}+\gamma\right) \cdot\left(k_{-1}+k_{+1}\right)}{k_{+1} \cdot \gamma \cdot\left(k_{-1}+k_{+1}+\gamma\right)}, \\
\left\langle t^{2}\right\rangle_{I E I}=2 \cdot \sum_{i=1}^{2}\left(P_{0}^{s}(i) \cdot \int_{0}^{+\infty}(t \cdot P(t, 1 \mid i)+t \cdot P(t, 2 \mid i)) d t\right) & \\
=2 & \frac{\left(k_{+1}+\gamma\right) \cdot\left(\left(k_{+1}\right)^{2}+k_{-1} \cdot \gamma\right)+\left(k_{+1}\right)^{2} \cdot k_{-1}}{\left(k_{+1} \cdot \gamma\right)^{2} \cdot\left(k_{-1}+k_{+1}+\gamma\right)},
\end{aligned}
$$

Finally, we can write the correlation coefficient between two subsequent IEIs as

$$
\begin{aligned}
C_{I E I}(1) & =\frac{\left\langle t_{i} \cdot t_{i+1}\right\rangle_{I E I}-\langle t\rangle_{I E I}^{2}}{\left\langle t^{2}\right\rangle_{I E I}-\langle t\rangle_{I E I}^{2}}= \\
& =\frac{k_{-1} \cdot k_{+1} \cdot \gamma^{2}}{\left(k_{+1} \cdot\left(k_{-1}+k_{+1}+\gamma\right)\right)^{2}+k_{-1} \cdot \gamma^{2} \cdot\left(k_{-1}+2 \cdot\left(k_{+1}+\gamma\right)\right)} .
\end{aligned}
$$

Let us now introduce two new variables, $\tilde{k}_{-1}=k_{-1} / \gamma$ and $\tilde{k}_{+1}=k_{+1} / \gamma$. Then, we can rewrite Eq. (S4.17) as

$$
C_{I E I}(1)=\frac{\tilde{k}_{-1} \cdot \tilde{k}_{+1}}{\left(\tilde{k}_{+1} \cdot\left(\tilde{k}_{-1}+\tilde{k}_{+1}+1\right)\right)^{2}+\tilde{k}_{-1} \cdot\left(\tilde{k}_{-1}+2 \cdot\left(\tilde{k}_{+1}+1\right)\right)}
$$

$\left(\tilde{k}_{-1}, \tilde{k}_{+1}\right)$ corresponding to the maximum value of $C_{I E I}(1)$ can be found by equating the partial derivatives of $C_{I E I}(1)$ with respect to $\tilde{k}_{-1}$ and $\tilde{k}_{+1}$ to zero. This results in the following system of polynomial equations:

$$
\begin{aligned}
& 3 \cdot\left(\tilde{k}_{+1}\right)^{4}+4 \cdot\left(1+\tilde{k}_{-1}\right) \cdot\left(\tilde{k}_{+1}\right)^{3}+\left(1+\tilde{k}_{-1} \cdot\left(2+\tilde{k}_{-1}\right)\right) \cdot\left(\tilde{k}_{+1}\right)^{2}- \\
& -\tilde{k}_{-1} \cdot\left(2+\tilde{k}_{-1}\right)=0 \\
& \left(\tilde{k}_{+1}\right)^{4}+2 \cdot\left(\tilde{k}_{+1}\right)^{3}+\left(1-\left(\tilde{k}_{-1}\right)^{2}\right) \cdot\left(\tilde{k}_{+1}\right)^{2}-\left(\tilde{k}_{-1}\right)^{2}=0
\end{aligned} .
$$

Solving these equations numerically results in $\tilde{k}_{-1} \approx 0.687, \tilde{k}_{+1} \approx 0.511$, and $\max \left[C_{I E I}(1)\right] \approx$ 0.092 . 


\subsection{Convergence of the vesicle fusion process in the limit of infinite number of vesicular release sites}

A superposition of $N$ independent renewal processes in the limit $N \rightarrow \infty$ is not in general a Poisson process, even though the correlations between IEIs decay to zero and the IEI distribution converges to an exponential (Lindner, 2006). It is so because the sum of correlations between a particular IEI and all subsequent IEIs remains larger than zero in the limit $N \rightarrow \infty$, despite the fact that correlations between any chosen IEIs decay to zero then. This is a deviation from a homogeneous Poisson process. However, if the observation window is finite and the rate of the combined point process is fixed for all $N$, the sum of IEI correlations over all lags decays to zero when $N \rightarrow \infty$. The combined process converges to a Poisson process then. This is what is called convergence of the point process locally in time. And this is the convergence relevant to us. Indeed when we compare two presynaptic AZs with different number of vesicular release sites, we assume that the overall release rate at those AZs is the same. Though this requirement is meaningful when considering vesicle release at presynaptic AZs, it may not be so in general (see Lindner, 2006).

\subsection{Explanation of $C_{I E I}(l)>0$ at $l>1$ for AZs with the $\mathrm{Ca}^{2+}$ microdomain coupling topographies}

Here, we provide a qualitative explanation for positive values of $C_{I E I}(l)$ at $l>1$ in the case of AZs with $n_{V}$-state vesicular release sites in $\mathrm{Ca}^{2+}$-microdomain coupling regime with $\mathrm{Ca}^{2+}$ channels. The same arguments provide an intuitive understanding of the presence of nonmonotonic convergence of $C_{I E I}(l)$ to 0 with increased $l$. For simplicity, we considered an AZ with two vesicular release sites. But the same line of thinking applies to AZs with more than two vesicular release sites.

Let us say that two vesicle fusion events happen in a shorter than average time interval. If so, then it is more likely than not that, just before the first fusion event, $\mathrm{Ca}^{2+}$-binding sites of the sensors of exocytosis of both vesicular release sites are fully or nearly fully occupied by $\mathrm{Ca}^{2+}$ ions. At the same time, the occupancy of both sensors of exocytosis by $\mathrm{Ca}^{2+}$ is very low just after the second fusion event. This, more likely than unlikely, results in a longer time gap between the third and the second than between the second and the first fusion events. In turn, $C(1)<0$. Moreover, just before the third fusion even happens, both of the vesicular release sites, more likely than not, have almost all $\mathrm{Ca}^{2+}$-binding sites of their sensors of exocytosis occupied (similar state as just before the first fusion event). In turn, the time gap between the fourth and the third vesicle fusion events is more likely than not to be closer to the time gap between the second and the first fusion events than the average time gap between any two fusion events in a sequence. This means that $C_{I E I}(2)>0$. We could now apply the same line of thinking for the subsequent fusion events and see that $C_{I E I}(3)<0, C_{I E I}(4)>0$, and so on. Thus, $C_{I E I}$ oscillates between the negative and positive values with increased $l$. Of course, with 
increasing $l$, the absolute values of the correlations decrease due to the Markovian nature of the presynaptic processes. The lag between the negative and positive peaks of $C_{I E I}(l)$ increases with an increased number of the vesicular release sites. 


\section{Supplementary Material 5: Analysis of the vesicle release rate dependence on the membrane potential}

\subsection{Single 2-state vesicular release site, $\mathrm{Ca}^{2+}$-microdomain coupling regime}

First, we consider the situation when $k_{r e p}\left(V_{m}\right) \equiv k_{r e p}=$ const and $k_{r e l} \cdot p_{o}\left(V_{m}\right)=k_{r e l} \cdot \bar{p}_{o} /(1+$ $\left.e^{-\left(V_{m}-V_{0.5}\right) k_{V}}\right)$. Then, as given in Results, the expression for the release rate dependence on $V_{m}$ is

$$
R_{r}\left(V_{m}\right)=\frac{A_{r}}{1+e^{-\left(V_{m}-V_{0.5}-S_{r}\right) / k_{V}}}
$$

where

$$
\begin{aligned}
& A_{r}=\frac{k_{r e l} \cdot \bar{p}_{o} \cdot k_{r e p}}{k_{r e l} \cdot \bar{p}_{o}+k_{r e p}} \leq \min \left[k_{r e l} \cdot \bar{p}_{o}, k_{r e p}\right], \\
& S_{r}=k_{V} \cdot \ln \left(\frac{k_{r e p}}{k_{r e p}+k_{r e l} \cdot \bar{p}_{o, \max }}\right) \leq 0 .
\end{aligned}
$$

According to Eq. (S5.2), the requirement for a pure-shift transformation $\left(k_{r e p}, k_{r e l} \cdot \bar{p}\right) \rightarrow$ $\left(k_{r e p}^{\prime}, k_{r e l}^{\prime} \cdot \bar{p}^{\prime}\right)$, such that $S_{r}^{\prime}-S_{r}=\Delta S_{r}$ and $A_{r}=A_{r}^{\prime}$, is

$$
\begin{aligned}
& \frac{k_{r e p} \cdot k_{r e l} \cdot \bar{p}_{o}}{k_{r e p}+k_{r e l} \cdot \bar{p}_{o}}=\frac{k_{r e p}^{\prime} \cdot k_{r e l}^{\prime} \cdot \bar{p}_{o}^{\prime}}{k_{r e p}^{\prime}+k_{r e l}^{\prime} \cdot \bar{p}^{\prime}}, \\
& k_{V} \cdot \ln \left(\frac{k_{r e p}^{\prime}}{k_{r e p}^{\prime}+k_{r e l}^{\prime} \cdot \bar{p}_{o}^{\prime}}\right)-k_{V} \cdot \ln \left(\frac{k_{r e p}}{k_{r e p}+k_{r e l} \cdot \bar{p}_{o}}\right)=\Delta S_{r},
\end{aligned}
$$

By solving Eq. (S5.3) for $k_{r e p}^{\prime}$ and $k_{r e l}^{\prime} \cdot \bar{p}^{\prime}$ we obtain

$$
\begin{aligned}
k_{r e l}^{\prime} \cdot \bar{p}_{o}^{\prime} & =k_{r e l} \cdot \bar{p}_{o} \cdot e^{-\Delta S_{r} / k_{V}}, \\
k_{r e p}^{\prime} & =\frac{k_{r e p} \cdot k_{r e l} \cdot \bar{p}_{o} \cdot e^{-\Delta S_{r} / k_{V}}}{\left(k_{r e p}+k_{r e l} \cdot \bar{p}_{o}\right) \cdot e^{-\Delta S_{r} / k_{V}}-k_{r e p}} .
\end{aligned}
$$

Note that $S_{r}^{\prime} \leq 0$. Otherwise, the denominator of the second expression of Eq. (S5.4) would be negative.

Next, we consider the situation when both, $k_{r e p}$ and $k_{r e l} \cdot p_{o}$ depend on $V_{m}$. As before, we assume that $k_{r e l} \cdot p_{o}\left(V_{m}\right)=k_{r e l} \cdot \bar{p}_{o} /\left(1+e^{-\left(V_{m}-V_{0.5}\right) / k_{V}}\right)$. In addition, we assume that $k_{\text {rep }}=\tilde{k}_{r e p} /\left(1+e^{-\left(V_{m}-\tilde{V}_{0.5}\right) / \tilde{k}_{V}}\right)$. Substituting these expressions into Eq. (S2.54) with $N_{V}=1$ we get

$$
R_{r}\left(V_{m}\right)=\frac{A_{r}}{1+e^{-\left(V_{m}-V_{0.5}-S_{r}\right) / k_{V}}}
$$

where,

$$
\begin{aligned}
& A_{r}=\frac{k_{r e l} \cdot \bar{p}_{o} \cdot \tilde{k}_{r e p}}{k_{r e l} \cdot \bar{p}_{o}+\tilde{k}_{r e p}} \leq \min \left[k_{r e l} \cdot \bar{p}_{o}, \tilde{k}_{r e p}\right] \\
& S_{r}=k_{V} \cdot \ln \left(\frac{\tilde{k}_{r e p}}{\tilde{k}_{r e p}+k_{r e l} \cdot \bar{p}_{o}}+\frac{k_{r e l} \cdot \bar{p}_{o},}{\tilde{k}_{r e p}+k_{r e l} \cdot \bar{p}_{o}} \cdot e^{\left(\tilde{V}_{0.5}-V_{0.5}\right) / k_{V}}\right) .
\end{aligned}
$$


Thus, the release rate dependence on $V_{m}$ is described by a Boltzmann function, as it was before. However, in this case, $R_{r}\left(V_{m}\right)$ is positioned between $k_{r e l} \cdot p_{o}\left(V_{m}\right)$ and $k_{r e p}\left(V_{m}\right)$ on the $V_{m}$ axis, i.e., $\min \left(0, \tilde{V}_{0.5}-V_{0.5}\right) \leq S_{r} \leq \max \left(0, \tilde{V}_{0.5}-V_{0.5}\right)$.

\subsection{Single 2-state vesicular release site, $\mathrm{Ca}^{2+}$-nanodomain coupling regime with a 2-state $\mathrm{Ca}^{2+}$ channel}

We first demonstrate that the $R_{r}\left(V_{m}\right)$ vs $V_{m}$ relations are very well described by Boltzmann functions. Here, we assume $k_{r e p}\left(V_{m}\right) \equiv k_{r e p}=$ const. Substituting Eq. (4.2) into Eq. (S2.76) yields

$$
R_{r}\left(V_{m}\right)=\frac{A+B \cdot e^{-\left(V_{m}-V_{0.5}\right) / k_{V}}}{\left(1+e^{-\left(V_{m}-V_{0.5}\right) / k_{V}}\right) \cdot\left(C+D \cdot e^{\left.-\left(V_{m}-V_{0.5}\right) / k_{V}\right)}\right.},
$$

where

$$
\begin{aligned}
& A=k_{r e p} \cdot k_{r e l} \cdot \bar{p}_{o} \cdot\left(k_{-1}+k_{r e p} \cdot\left(1-\bar{p}_{o}\right)\right), \\
& B=k_{r e p} \cdot k_{r e l} \cdot \bar{p}_{o} \cdot\left(k_{-1}+k_{r e p}\right), \\
& C=k_{-1} \cdot\left(k_{r e p}+k_{r e l} \cdot \bar{p}_{o}\right)+k_{r e p} \cdot\left(k_{r e p}+k_{r e l}\right) \cdot\left(1-\bar{p}_{o}\right), \\
& D=k_{r e p} \cdot\left(k_{-1}+k_{r e l}+k_{r e p}\right) .
\end{aligned}
$$

Red curves in Fig. S5.1 display $R_{r}\left(V_{m}\right)$ functions for different values of parameters $k_{-1}, k_{r e p}$, and $k_{r e l}$. Here, we considered all possible combinations when $k_{r e l}=1$ (we assume dimensionless time) and each of $k_{-1}$ and $k_{r e p}$ is independently equal to one of the following: $10^{-2}, 10^{-1}, 10^{0}$, $10^{1}, 10^{2}$. Each panel in Fig. S5.1 shows normalized $R_{r}$ vs $V_{m}$ plots for fixed $k_{r e l}$, and $k_{-1}$ and different values of $k_{r e p}$. Curves in each plot are shifted only for display purposes. Blue dashed lines in Fig. S5.1 show the best fits of $R_{r}\left(V_{m}\right)$ with $f\left(V_{m}\right)=\left(1+e^{-\left(V_{m}-p_{2}\right) / p_{1}}\right)^{-1}$ with two free parameters $p_{1}$ and $p_{2}$. As we see, the fits are hard to distinguish from the original functions visually.

Next, we consider approximate expressions for the $R_{r}\left(V_{m}\right)$ vs $V_{m}$ relations in different parts of the parameter space. At fixed $V_{0.5}$ and $k_{V}$, the $R_{r}\left(V_{m}\right)$ functions are fully characterized by three parameters: $\left(k_{-1}, k_{r e p}, k_{r e l} \cdot \bar{p}\right)$. To scan the parameter space, we calculated triple limits of $R_{r}\left(V_{m}\right)$ when each of $\left(k_{-1} / k_{r e l}, k_{r e p} / k_{r e l}\right.$, and $\left.k_{-1} / k_{r e p}\right)$ independently goes either to 0,1 , or $+\infty$. There exist 13 different limits of this kind. In all but two of those cases, $R_{r}\left(V_{m}\right)$ converges to a Boltzmann function exactly as shown below. In the remaining two cases, the $R_{r}\left(V_{m}\right)$ vs $V_{m}$ relation is still approximated by a Boltzmann function very well. Below, we provide the expressions of $R_{r}\left(V_{m}\right)$ in each of the mentioned limiting cases. 

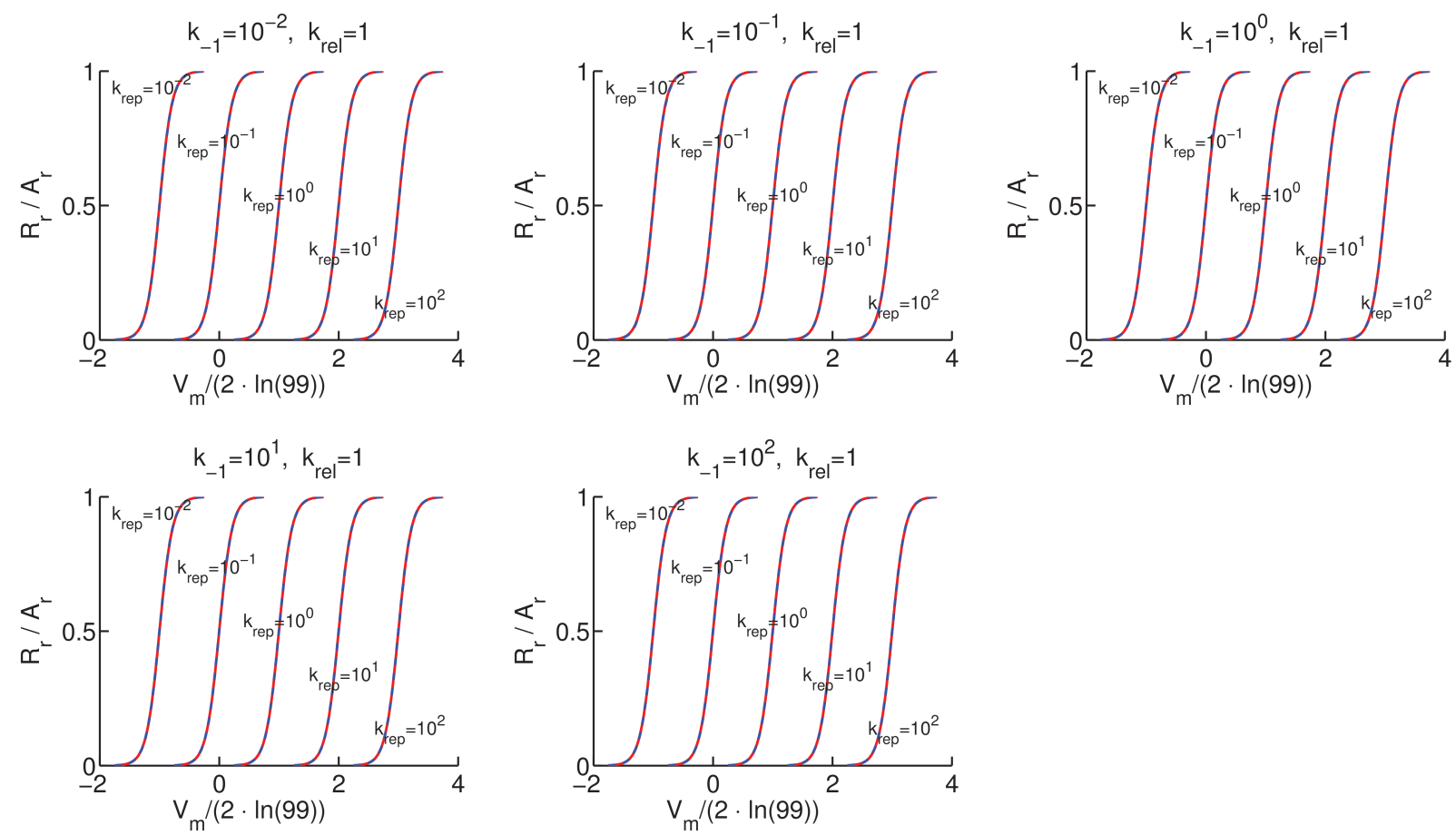

Figure S5.1: Plots of $R_{r}\left(V_{m}\right)$ functions (red lines) for different values of parameters $k_{-1}, k_{r e l}$, and $k_{r e p}$. Dashed blue lines correspond to the best fits of the $R_{r}\left(V_{m}\right)$ functions by Boltzmann functions.

\section{Limiting case $\left(k_{r e l} \gg k_{-1} \gg k_{r e p}\right)$}

$$
\begin{aligned}
R_{r}\left(V_{m}\right) & =\frac{A_{r}}{1+e^{-\left(V_{m}-V_{0.5}-S_{r}\right) / k_{V}}}, \\
A_{r} & =\frac{k_{-1} \cdot \bar{p}_{o} \cdot k_{r e p}}{k_{-1} \cdot \bar{p}_{o}+k_{r e p} \cdot\left(1-\bar{p}_{o}\right)}, \\
S_{r} & =k_{V} \cdot \ln \left(\frac{k_{r e p}}{k_{-1} \cdot \bar{p}_{o}+k_{r e p} \cdot\left(1-\bar{p}_{o}\right)}\right) .
\end{aligned}
$$

\section{Limiting case $\left(k_{r e l} \sim k_{-1} \gg k_{r e p}\right)$}

$$
\begin{aligned}
R_{r}\left(V_{m}\right) & =\frac{A_{r}}{1+e^{-\left(V_{m}-V_{0.5}-S_{r}\right) / k_{V}}}, \\
A_{r} & =\frac{k_{-1} \cdot \bar{p}_{o} \cdot k_{r e p} \cdot k_{r e l}}{k_{-1} \cdot \bar{p}_{o} \cdot k_{r e l}+k_{r e p} \cdot\left(1-\bar{p}_{o}\right) \cdot\left(k_{-1}+k_{r e l}\right)} \\
S_{r} & =k_{V} \cdot \ln \left(\frac{k_{r e p} \cdot\left(k_{-1}+k_{r e l}\right)}{k_{-1} \cdot \bar{p}_{o} \cdot k_{r e l}+k_{r e p} \cdot\left(1-\bar{p}_{o}\right) \cdot\left(k_{-1}+k_{r e l}\right)}\right) .
\end{aligned}
$$

\section{Limiting case $\left(k_{r e l} \gg k_{r e p} \gg k_{-1}\right)$}

$$
R_{r}\left(V_{m}\right)=\frac{A_{r}}{1+e^{-\left(V_{m}-V_{0.5}-S_{r}\right) / k_{V}}}, \quad A_{r}=k_{r e p} \cdot \bar{p}_{o}, \quad S_{r}=0 .
$$


Limiting case $\left(k_{r e l} \sim k_{r e p} \gg k_{-1}\right)$

$$
R_{r}\left(V_{m}\right)=\frac{A_{r}}{1+e^{-\left(V_{m}-V_{0.5}-S_{r}\right) / k_{V}}}, \quad A_{r}=\frac{k_{r e l} \cdot k_{r e p}}{k_{r e l}+k_{r e p}} \cdot \bar{p}_{o}, \quad S_{r}=0 .
$$

Limiting cases $\left(k_{r e p} \gg k_{r e l} \gtrsim k_{-1}\right)$

$$
R_{r}\left(V_{m}\right)=\frac{A_{r}}{1+e^{-\left(V_{m}-V_{0.5}-S_{r}\right) / k_{V}}}, \quad A_{r}=k_{r e l} \cdot \bar{p}_{o}, \quad S_{r}=0 .
$$

Limiting cases $\left(k_{r e p} \gtrsim k_{-1} \gg k_{r e l}\right)$ and $\left(k_{-1} \gg k_{r e p} \gg k_{r e l}\right)$

$$
R_{r}\left(V_{m}\right)=\frac{A_{r}}{1+e^{-\left(V_{m}-V_{0.5}-S_{r}\right) / k_{V}}}, \quad A_{r}=k_{r e l} \cdot \bar{p}_{o}, \quad S_{r}=0 .
$$

Limiting cases $\left(k_{-1} \gg k_{r e l} \gtrsim k_{r e p}\right)$

$$
\begin{aligned}
R_{r}\left(V_{m}\right) & =\frac{A_{r}}{1+e^{-\left(V_{m}-V_{0.5}-S_{r}\right) / k_{V}}}, \\
A_{r} & =\frac{k_{r e l} \cdot \bar{p}_{o} \cdot k_{r e p}}{k_{r e l} \cdot \bar{p}_{o}+k_{r e p}}, \quad S_{r}=k_{V} \cdot \ln \left(\frac{k_{r e p}}{k_{r e l} \cdot \bar{p}_{o}+k_{r e p}}\right) .
\end{aligned}
$$

$$
\begin{aligned}
& \text { Limiting case }\left(\boldsymbol{k}_{\text {rel }} \gg \boldsymbol{k}_{-\mathbf{1}} \sim \boldsymbol{k}_{\boldsymbol{r e p}}\right) \\
& \qquad \begin{aligned}
R_{r}\left(V_{m}\right) & =k_{r e p} \cdot p_{o}\left(V_{m}\right) \cdot \frac{k_{1}\left(V_{m}\right)+k_{-1}+k_{r e p}}{k_{1}\left(V_{m}\right)+k_{r e p}} \\
& =\frac{k_{r e p} \cdot \bar{p}_{o}}{1+e^{-\left(V_{m}-V_{0.5}\right) / k_{V}}} \cdot \frac{k_{-1} \cdot \bar{p}_{o}+\left(k_{-1}+k_{r e p}\right) \cdot\left(1-\bar{p}_{o}+e^{-\left(V_{m}-V_{0.5}\right) / k_{V}}\right)}{k_{-1} \cdot \bar{p}_{o}+k_{r e p} \cdot\left(1-\bar{p}_{o}+e^{-\left(V_{m}-V_{0.5}\right) / k_{V}}\right)}
\end{aligned}
\end{aligned}
$$

As it follows from Fig. S5.1, $R_{r}\left(V_{m}\right)$ is still well approximated by a Boltzmann function in this limiting case. Moreover, $S_{r} \sim 0$ then.

\section{Limiting case $\left(k_{r e l} \sim k_{-1} \sim k_{r e p}\right)$}

In this case, the original expression for $R_{r}\left(V_{m}\right)$, Eq. (S5.7), applies. However, at negative $V_{m}$, where $p_{o}\left(V_{m}\right)$ is low, the vesicle release is of the bursty type and the $R_{r}\left(V_{m}\right)$ function is approximated by (S5.12). As it follows from the last panel of Fig. S5.2, the approximation works relatively well for higher $V_{m}$ values too. $S_{r} \sim 0$ in this case.

A relevant question related to the approximations of the $R_{r}\left(V_{m}\right)$ functions given by Eqs. (S5.9) - (S5.16) is how much the "》" or "«" relations mean from the perspective of the two compared parameters. As demonstrated in Fig. S5.2, the approximations of the generic expression (S5.7) work very well already when the requirement of one parameter being much greater than the other one corresponds to the difference of one order of magnitude. 

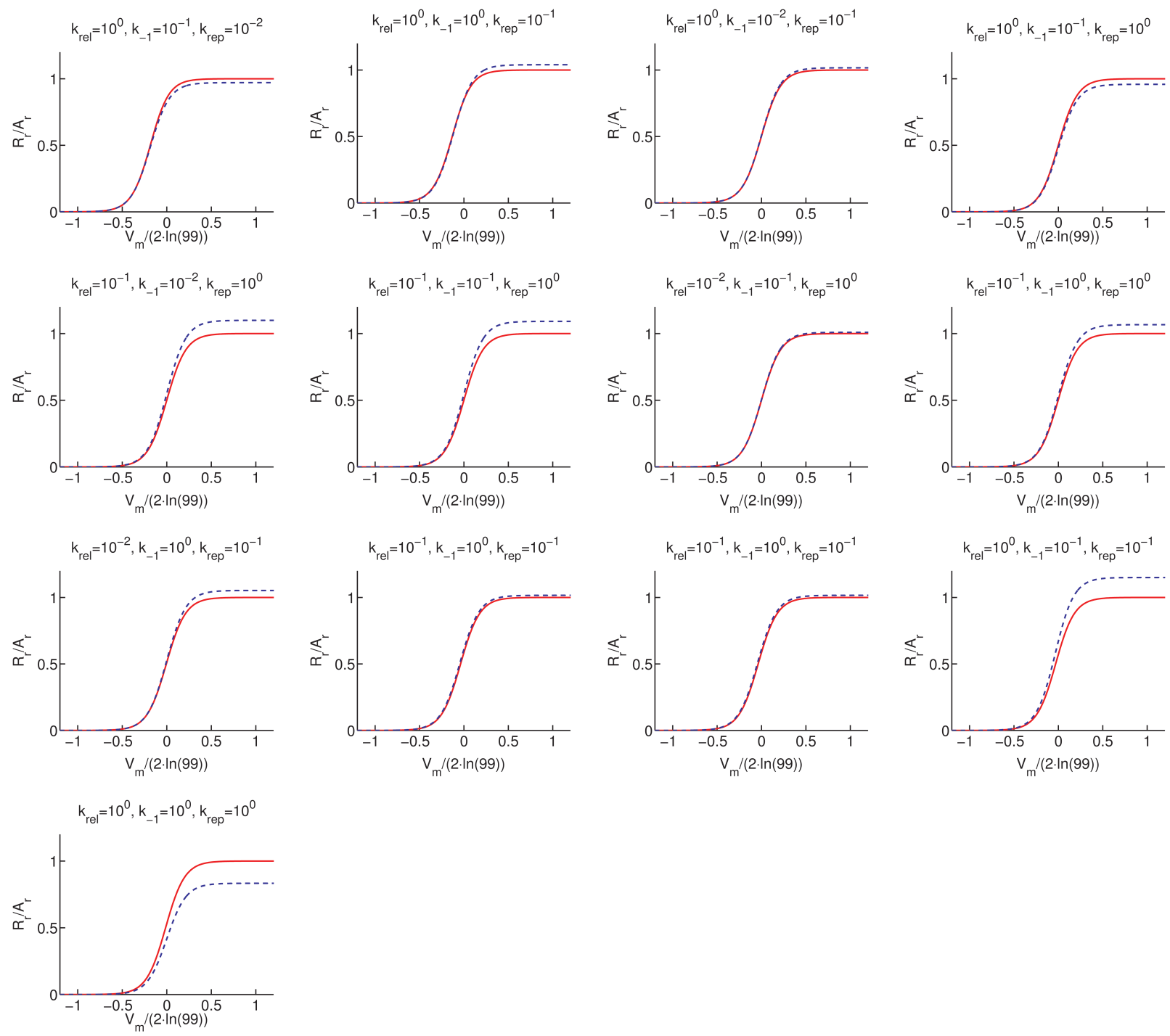

Figure S5.2: Comparisons between the original $R_{r}\left(V_{m}\right)$ function (Eq. (S5.7)) and its approximations (Eqs. (S5.9) - (S5.16)) in different regions of the parameter space.

\subsection{Single $n_{V}$-state vesicular release site, the $\mathrm{Ca}^{2+}$-microdomain coupling regime}

\section{Sensor of exocytosis with one $\mathrm{Ca}^{2+}$-binding site}

First, we consider the $R_{r}\left(V_{m}\right)$ function of a 3-state vesicular release site, which contains a $\mathrm{Ca}^{2+}$ sensor of exocytosis with one binding site $(m=1)$. Assuming that $k_{r e p}\left(V_{m}\right) \equiv k_{\text {rep }}=$ const, it follows from Eqs. (S2.101) - (S2.102) that

$$
R_{r}\left(V_{m}\right)=\frac{\gamma \cdot k_{r e p} \cdot k_{r e l} \cdot p_{o}\left(V_{m}\right)}{\left(\gamma+k_{o f f}\right) \cdot k_{r e p}+\left(\gamma+k_{r e p}\right) \cdot k_{r e l} \cdot p_{o}\left(V_{m}\right)} .
$$

Substituting $p_{o}\left(V_{m}\right)=\frac{\bar{p}_{o}}{1+e^{-\left(V_{m}-V_{0.5}\right) / k_{V}}}$, one can rewrite the above expression as

$$
R_{r}\left(V_{m}\right)=\frac{A_{r}}{1+e^{-\left(V_{m}-V_{0.5}-S_{r}\right) / k_{V}}},
$$


where

$$
\begin{aligned}
& A_{r}=\frac{\gamma \cdot k_{r e p} \cdot k_{r e l} \cdot \bar{p}_{o}}{\left(\gamma+k_{o f f}\right) \cdot k_{r e p}+\left(\gamma+k_{r e p}\right) \cdot k_{r e l} \cdot \bar{p}_{o}}, \\
& S_{r}=k_{V} \cdot \ln \left(\frac{k_{r e p}}{k_{r e p}+\frac{\left(\gamma+k_{r e p}\right)}{\left(\gamma+k_{o f f}\right)} \cdot k_{r e l} \cdot \bar{p}_{o}}\right) \leq 0 .
\end{aligned}
$$

As in the case of a 2-state vesicular release site, the resulting $R_{r}$ vs $V_{m}$ relation is described by a Boltzmann function.

\section{The limit of infinitely slow $\mathrm{Ca}^{2+}$-unbinding from the sensor of exocytosis}

Next, we consider a vesicular release site with arbitrary $m$ in the limit when $\mathrm{Ca}^{2+}$ binding to the sensor of exocytosis is much faster than unbinding at every step. The expression of $\langle t\rangle_{I E I}=R_{r}^{-1}$ is given by Eq. (S3.21) in this case. Substituting $p_{o}\left(V_{m}\right)=1 /\left(1+e^{-\left(V_{m}-V_{0.5}\right) / k_{V}}\right)$ into (S3.21) and rearranging terms yields

$$
R_{r}\left(V_{m}\right)=\frac{A_{r}}{1+e^{-\left(V_{m}-V_{0.5}-S_{r}\right) / k_{V}}}
$$

where

$$
\begin{aligned}
& A_{r}=\frac{\gamma \cdot k_{r e p} \cdot k_{r e l} \cdot \bar{p}_{o}}{\gamma \cdot k_{r e p} \cdot \sum_{i=1}^{m} i^{-1}+\left(\gamma+k_{r e p}\right) \cdot k_{r e l} \cdot \bar{p}_{o}} \\
& S_{r}=k_{V} \cdot \ln \left(\frac{\gamma \cdot k_{r e p} \cdot \sum_{i=1}^{m} i^{-1}}{\gamma \cdot k_{r e p} \cdot \sum_{i=1}^{m} i^{-1}+\left(\gamma+k_{r e p}\right) \cdot k_{r e l} \cdot \bar{p}_{o}}\right) \leq 0
\end{aligned}
$$

Expressions (S5.21) and (S5.19) are equivalent under the transformation $\gamma+k_{o f f}=\gamma \cdot \sum_{i=1}^{m} i^{-1}$. Thus, in the limiting case considered here, the $R_{r}\left(V_{m}\right)$ function has all key properties equivalent to those of a vesicular release site with the linear $\mathrm{Ca}^{2+}$ sensor $(m=1)$. It has to be noted that, unless we fix $k_{o f f}=0$, it is always possible to find such $V_{m}$ at which $k_{r e l} \cdot p_{o}\left(V_{m}\right) \lesssim k_{o f f}$ even if $k_{r e l} \cdot \bar{p}_{o} \gg k_{o f f}$. However, if $k_{r e p} \gg k_{o f f}$ and $\gamma \gg k_{o f f}$, the inequality $k_{r e l} \cdot p_{o}\left(V_{m}\right) \lesssim k_{o f f}$ is valid only in the range of $V_{m}$ values where $R_{r}$ is much smaller than the maximum release rate.

\section{The limit of the maximum $S_{r}$ value}

We found by numerical global optimization that $S_{r}$ is maximized in the limit $k_{r e p} /\left(k_{r e l} \cdot \bar{p}_{o}\right) \gg$ $m ! \cdot\left(\gamma+k_{r e p}\right) / \gamma \cdot\left(k_{r e l} \cdot \bar{p}_{o} /\left(b \cdot k_{o f f}\right)\right)^{m}$. It follows from Eqs. $(\mathrm{S} 2.102)-(\mathrm{S} 2.106)$ that $a_{0} \gg$ $a_{i} \cdot\left(k_{r e l} \cdot p_{o}\left(V_{m}\right)\right)^{i}$ for $i=\overline{1, m}$ in this limit. Taking this into account, the generic Eq. (4.46) is reduced to Eq. (4.47). It is then straightforward to show by using Eq. (4.47) that the halfmaximum value of $R_{r}$ is reached at $V_{m}=k_{V} \cdot \ln (\sqrt[m]{2}-1)$. Fig. S5.3 shows plots of normalized $R_{r}$ vs $V_{m}$ relations corresponding to the $\max \left[S_{r}\right]$ at $m=3$ and $m=5$, and their best fits with Boltzmann functions. As we see, the fits approximate the original expressions in terms of the absolute values of $R_{r} / A_{r}$ very well (Fig. S5.3). At first sight, such result may look unexpected, because, in relative terms, a Boltzmann function deviates from the function defined by Eq. (4.47) 
at sufficiently negative $V_{m}$ considerably. However, then, the absolute values of $R_{r} / A_{R}$ are close to 0 and are of no relevance in the context of our problem. It also has to be noted that, after nondimensionalization of Eq. (4.46), the only free parameter left is $m$. Thus, the approximations shown in Fig. S5.3 work well with arbitrary values of the kinetic parameters, as long as they satisfy the constraints set by the definition of the limit of maximum $S_{r}$.
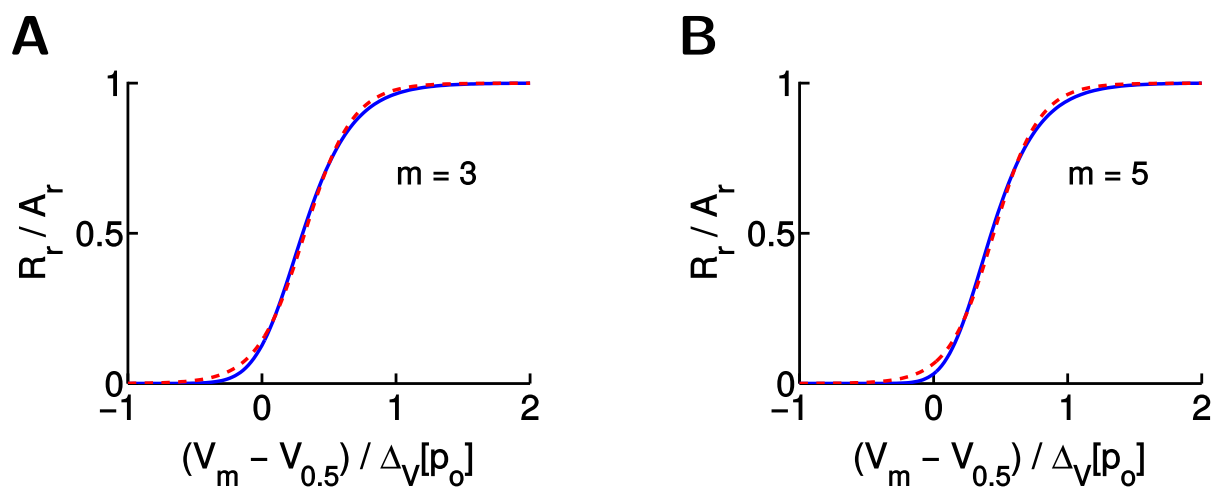

Figure S5.3: Comparison between the $R_{r}\left(V_{m}\right)$ functions of $n_{V}$-state vesicular release sites in the limit of maximum $S_{r}$ value (blue solid lines) and their best fits with Boltzmann functions (red dashed lines). (A) and (B) correspond to $\mathrm{Ca}^{2+}$-sensors of exocytosis with, respectively, three and five binding sites.

\section{The limit of the minimum dynamic range of $R_{r}\left(V_{m}\right)$}

After rearranging terms, Eq. (4.48) in section 4.4.1 can be reduced to

$$
R_{r}\left(V_{m}\right)=\frac{A_{r}}{1+e^{-m \cdot\left(V_{m}-V_{0.5}-S_{r}\right) / k_{V}}}
$$

where

$$
A_{r}=\frac{m ! \cdot \gamma \cdot k_{r e p} \cdot\left(k_{r e l} \cdot \bar{p}_{o}\right)^{m}}{a_{m}}, \quad S_{r}=\frac{k_{V}}{m} \cdot \log \left(a_{0} / a_{m}\right) \leq 0 .
$$




\section{Supplementary Material 6: Conversion of presynaptic vesi- cle fusion events into spikes by SGNs}

\subsection{Influence of the SGN refractoriness on $C V_{I S I}$ in the case of bursty presynaptic release}

A

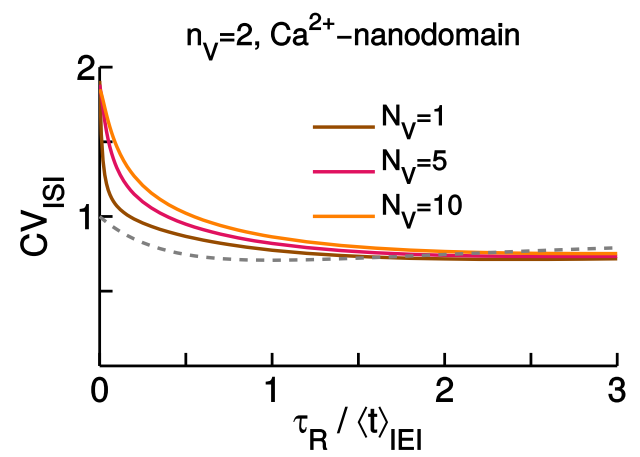

B

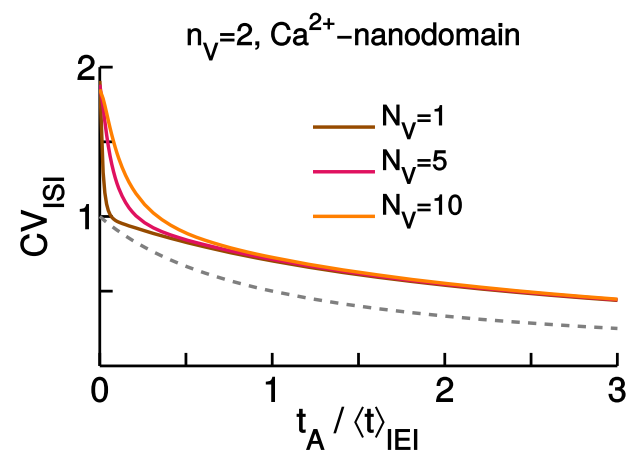

Figure S6.1: Dependence of $C V_{I S I}$ on the refractory times. Here, the same presynaptic site model was considered as in Fig. 4.8 (section 4.4.3). The difference is that here we set either $t_{A}$, panel (A), or $\tau_{R}$, panel (B), to zero.

\subsection{Spike rate dependence on the membrane potential}

The expression of the $R_{s}$ vs $V_{m}$ relation for presynaptic vesicle release described by a homogeneous Poisson process

The expression of the $R_{s}$ vs $V_{m}$ relation for presynaptic vesicle release which is described by a homogeneous Poisson process follows from Eq. (S2.131), which relates $R_{s}$ and $R_{r}$. If we assume that $R_{r}=1 /\left(1+e^{-\left(V_{m}-V_{0.5}-S_{r}\right) / k_{V}^{\prime}}\right)$, then substituting this expression to Eq. (S2.131) and rearranging the terms results in Eq. (4.52). 


\section{Refractory times vs release rates as determinants of the heterogeneity of maximum spike rates in mice SGNs}

A

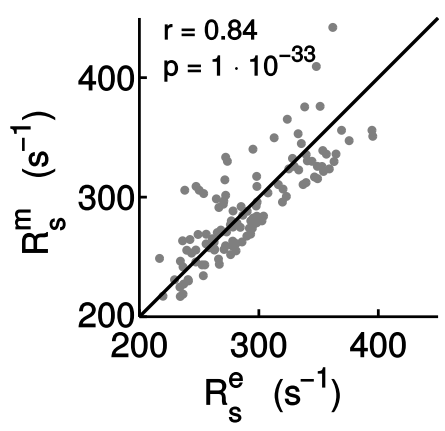

B

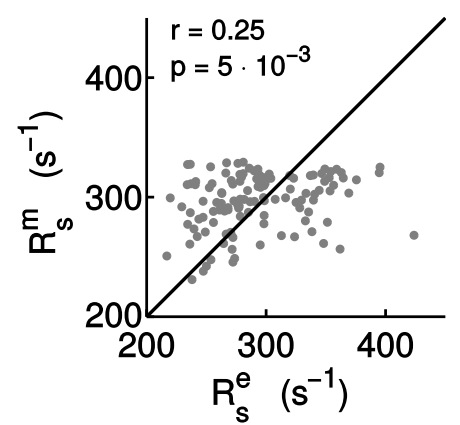

Figure S6.2: Reproduction of $R_{s}$ values in the population of SGNs studied by (Pangršič et al., 2015) (see Fig. S5 therein) under two assumptions. $R_{s}^{e}$ stands for the experimental estimate of $R_{s}$, while $R_{s}^{m}$ marks the model estimate of the $R_{s}$ of the same neuron. In panel $(\mathbf{A}), t_{A}+\tau_{R}$ was fixed to $1.0 \mathrm{~ms}$, the average value in the SGN population. The $R_{s}^{m}$ was then calculated by assuming the $R_{r}$ as determined by the fitting procedure in (Pangršič et al., 2015) for each neuron. In panel (B), $R_{r}$ was fixed to $430 \mathrm{~s}^{-1}$, the average value in the SGN population. The $R_{s}^{m}$ was then calculated by assuming the $t_{A}+\tau_{R}$ as determined by the fitting procedure in (Pangršič et al., 2015) for each neuron. The Pearson's correlation coefficients and the corresponding $\mathrm{p}$ values are provided in the insets of each panel.

\section{$R_{s}$ vs $V_{m}$ relations for bursty presynaptic vesicle fusion}

We concluded in section 4.4.3 that, when the presynaptic vesicle release is bursty, the $R_{s}$ vs $V_{m}$ relation is a scaled down version of the corresponding $R_{r}$ vs $V_{m}$ relation to a very good approximation. To demonstrate the correctness of this statement we considered six different limiting $R_{r}$ vs $V_{m}$ relations which feature the burtsy vesicle release, as identified in section 4.4.1. For this purpose, we considered presynaptic AZs which consist either of 2-state vesicular release sites or 7 -state vesicular release sites with $k_{\text {off }}=0$. As it follows from our results in section 4.4.1, these scenarios represent the two limiting cases of the spectrum of the IEI statistics within the vesicle release bursts. The kinetic parameters used are defined in Tab. S6.1. The results are shown in Fig. S6.3 (2-state vesicular release sites) and Fig. S6.4 (7-state vesicular release sites). Each panel in these figure feature the six normalized $R_{s}$ vs $V_{m}$ dependencies (red lines). Different panel corresponds to different $P_{o, \max }$ and $N_{V}$. Blue dashed lines in the same figures show the corresponding normalized $R_{r}$ vs $V_{m}$ relations. Note that the $R_{s}$ vs $V_{m}$ plots are shifted from each other along the $V_{m}$ axis just for the purpose of visualization. 


\begin{tabular}{|c||c|c|}
\hline & $k_{r e p} / k_{f}$ & $k_{-1} / k_{f}$ \\
\hline \hline$(1)$ & $10^{0}$ & $10^{-1}$ \\
\hline$(2)$ & $10^{-1}$ & $10^{-2}$ \\
\hline$(3)$ & $10^{1}$ & $10^{0}$ \\
\hline$(4)$ & $10^{1}$ & $10^{-1}$ \\
\hline$(5)$ & $10^{0}$ & $10^{0}$ \\
\hline$(6)$ & $10^{-1}$ & $10^{-1}$ \\
\hline
\end{tabular}

Table S6.1: Sets of the kinetic parameters corresponding to bursty presynaptic vesicle release. In the case of AZs with 2 -state vesicular release sites, $k_{f}$ corresponds to $k_{r e l}$. In the case of AZs with 7 -state vesicular release sites, $k_{f}$ corresponds to the effective rate of the forward crossing from the 2 -nd to the 7 -th state of the vesicular release site: $k_{f}=k_{r e l} \cdot \sum_{i=1}^{5} i^{-1}$.
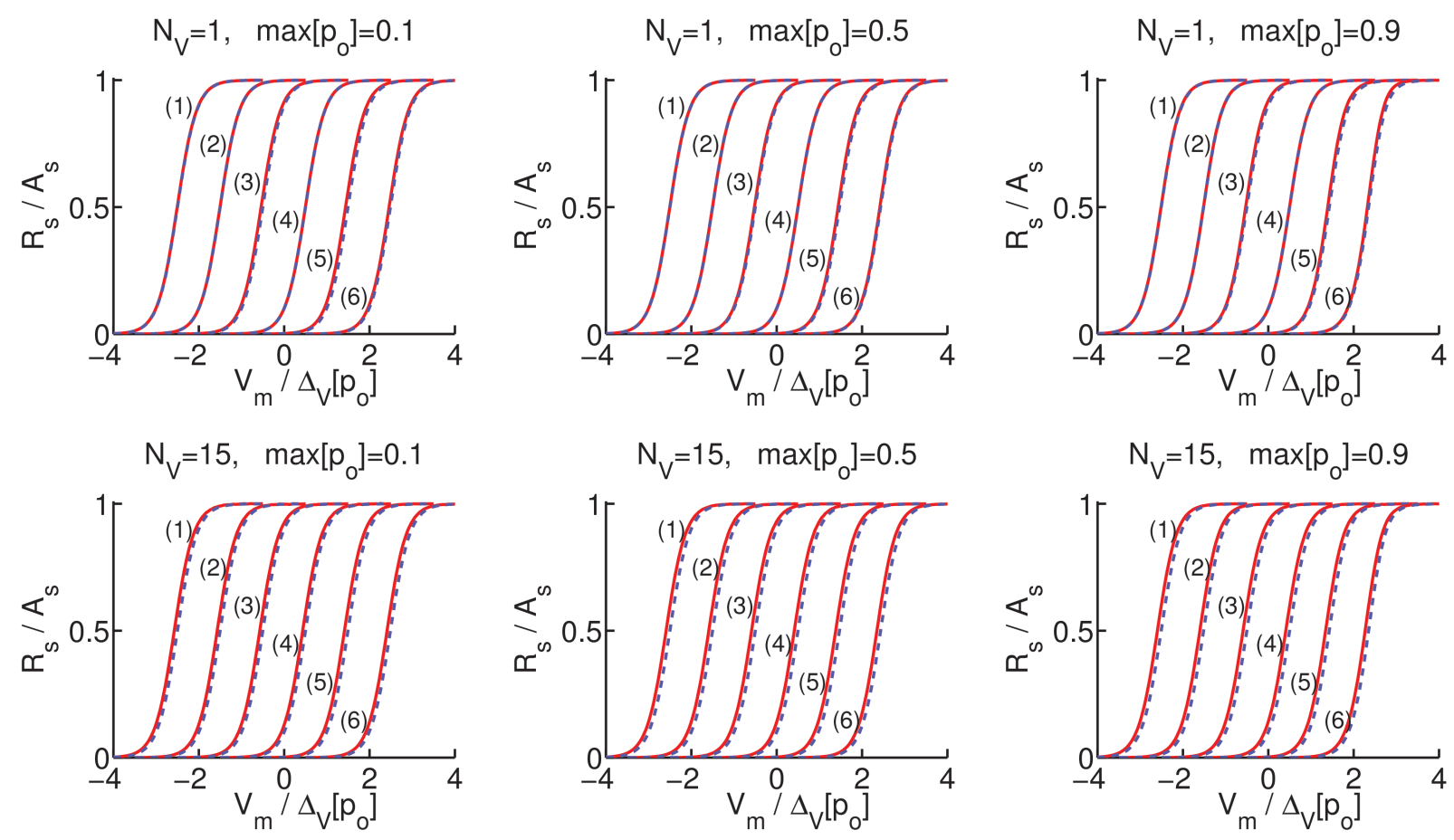

Figure S6.3: Plots of normalized $R_{s}$ vs $V_{m}$ relations (red lines) for six different sets of parameters $k_{-1}, k_{r e l}$, and $k_{r e p}$ defined in Tab.S6.1 (AZs with 2-state vesicular release sites and 2-state $\mathrm{Ca}^{2+}$ channels). Dashed blue lines show the corresponding normalized $R_{r}$ vs $V_{m}$ relations. 

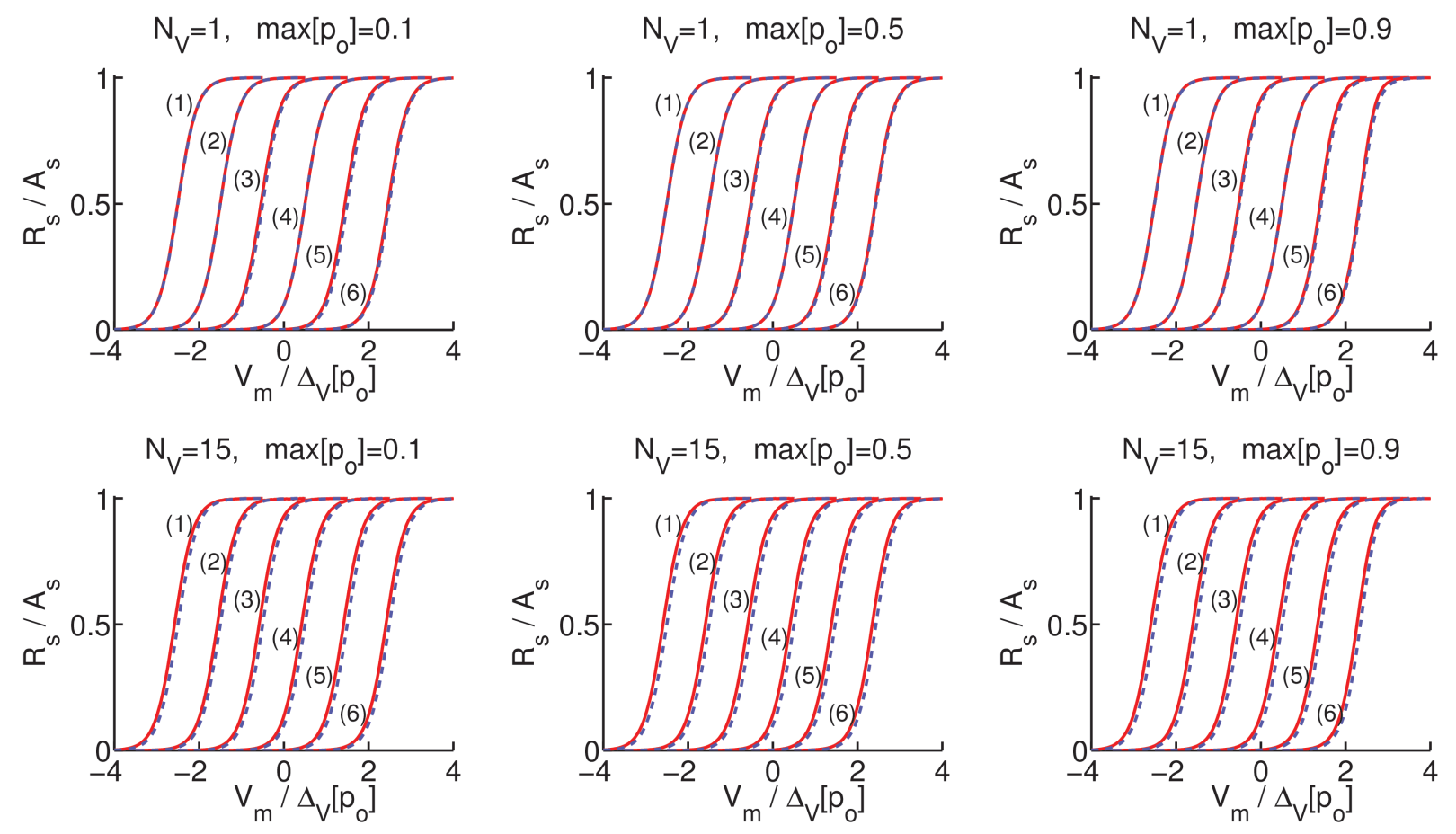

Figure S6.4: Plots of normalized $R_{s}$ vs $V_{m}$ relations (red lines) for six different sets of parameters $k_{-1}, k_{r e l}$, and $k_{r e p}$ defined in table Tab. S6.1 (AZs with 7-state vesicular release sites with $k_{\text {off }}=0$ and 2-state $\mathrm{Ca}^{2+}$ channels). Dashed blue lines show the corresponding normalized $R_{r}$ vs $V_{m}$ relations.

Fig. S6.5 demonstrates that SGN refractoriness reduces the maximum spike rate in the case of the bursty release more than in the case of vesicle release described by a homogeneous Poisson process. There, we plotted the ratio of spike rates resulting from bursty presynaptic vesicle release and release described by a homogeneous Poisson process (both with the same rate). The same model scenarios as in Figs. S6.3 and S6.4 are considered in Fig. S6.5. 

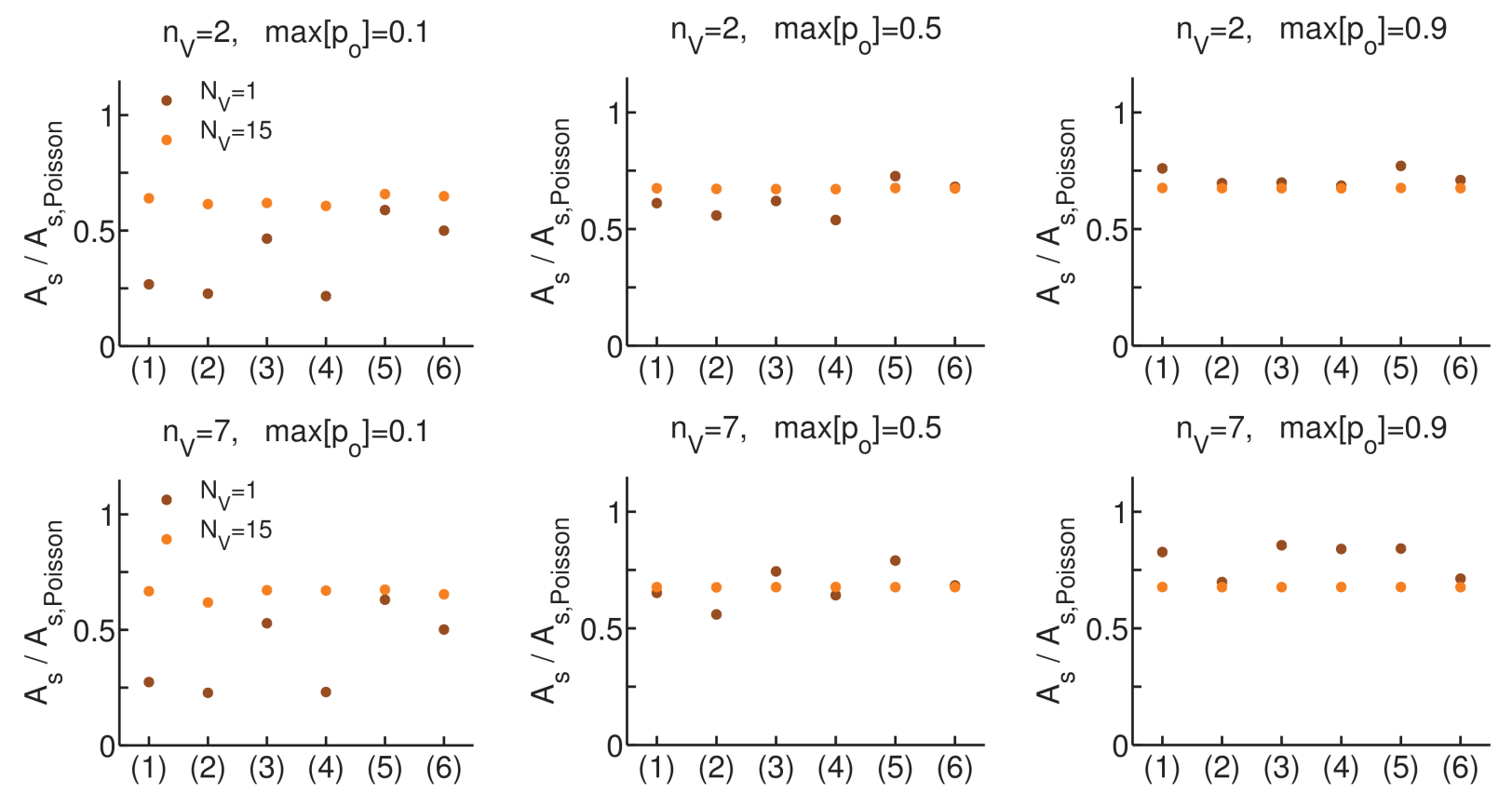

Figure S6.5: Reduction of the maximum spike rate due to refractoriness of SGNs for six different sets of parameters $k_{-1}, k_{r e l}$, and $k_{r e p}$ corresponding to the bursting release. AZs with 2-state vesicular release sites and 2-state $\mathrm{Ca}^{2+}$ channels were assumed in the top row. AZs with 7-state vesicular release sites and 2-state $\mathrm{Ca}^{2+}$ channels were considered in the bottom row. The ratio between the spike rate amplitudes estimated by assuming bursty vesicle fusion and vesicle fusion described by a homogeneous Poisson process is on the ordinate axis. 


\section{Supplementary Material 7: Analysis of experimental data}

\subsection{Rate-level functions}

Variation of the properties of rate-level functions with the characteristic frequency

Fig. S7.1 shows how spiking thresholds, dynamic ranges, spontaneous rates, and maximum rates of SGNs vary with CF. The data points were calculated from the best fits of RLFs of cat SGNs with the empirical model introduced in (Heil et al., 2011).
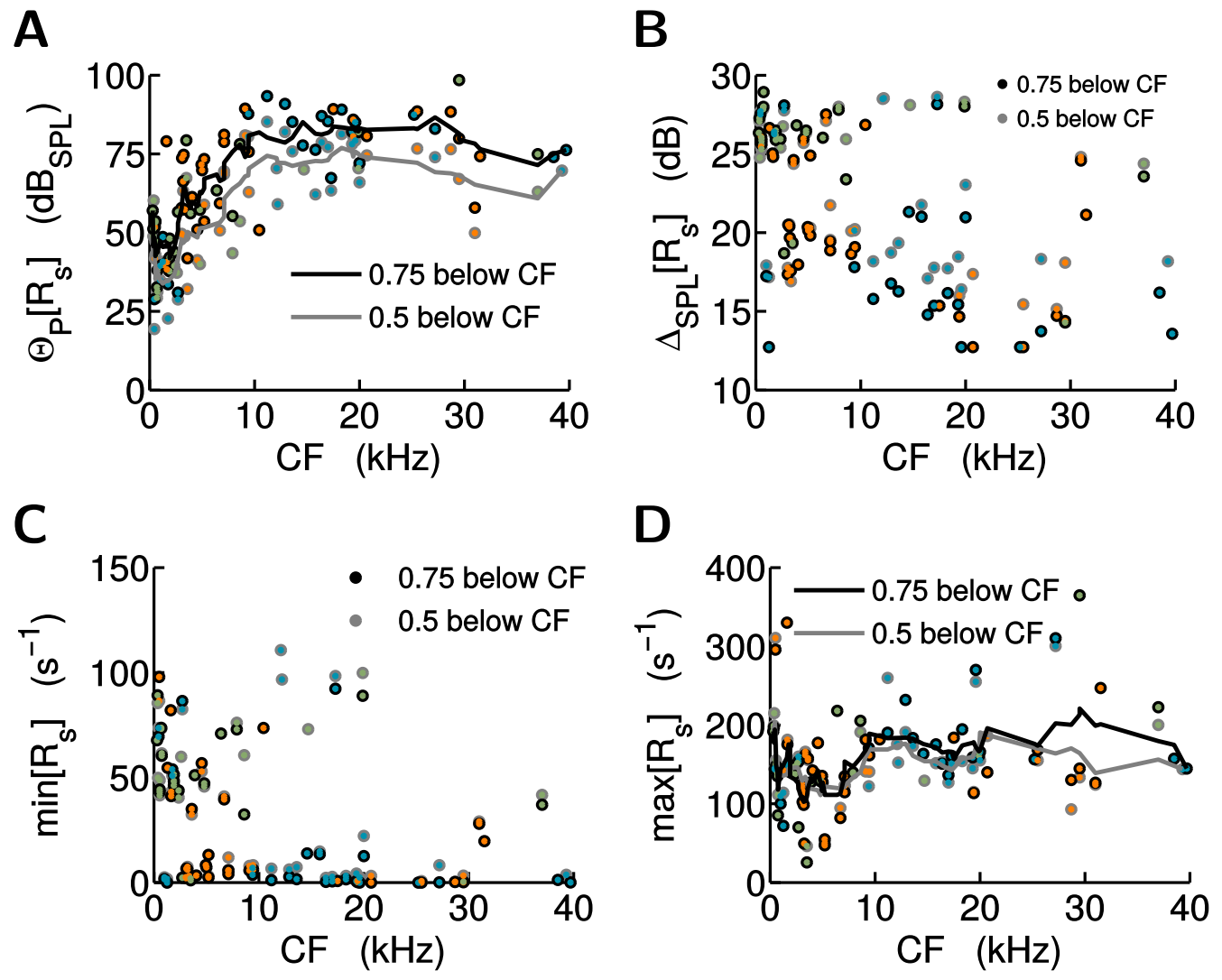

Figure S7.1: Dependence of the RLF properties on CF. (A) Spike threshold. (B) Dynamic range. (C) Spontaneous (minimum) spike rate. (D) Maximum spike rate. In all panels, black color stands for responses to stimuli 0.75 octaves below CF, grey color stands for responses to stimuli 0.5 octaves below CF. Orange, blue, and green colors represent data points from three different animals. In (A) and (D), the solid lines show the running averages with spans of seven points of the corresponding data points.

A likely explanation of the clearly pronounced increase of $\Theta_{S P L}\left[R_{s}\right]$ with CF (Fig. S7.1A) is the following. In the considered experiments, the sound stimuli were applied at fixed relative difference ( 0.5 or 0.75 octaves) below the CF. Thus, the absolute difference between the stimulation frequency and CF was higher for ANFs with higher CFs. On the other hand, it is known that the sharpness of SGN tuning increases with CF (Liberman, 1978; see also Fig. 11 
in Taberner and Liberman, 2005). This means that the gain between the sound intensity at the external ear and the deflection of basilar membrane decreased with CF for the stimulation paradigm considered in the experiments by (Heil et al., 2011). The contribution of middle ear filtering (Pickles, 2008) to the CF dependence of the spiking thresholds is also very likely.

In panels B and C of Fig. S7.1 a clear split of the data points into two groups is present. This is a well known phenomenon of the division of SGNs into subpopulations of low SR, high threshold neurons, and high SR, low threshold neurons (see, e.g., Liberman 1978).

\section{Differences of the rate-level functions between SGNs with similar characteristic frequencies}

Fig. S7.2 shows how differences in spiking thresholds, dynamic ranges, spontaneous rates, and maximum spike rates between two nearest-neighbor SGNs along the CF axis vary with the CF. The same data as in section 7.1 are considered. 

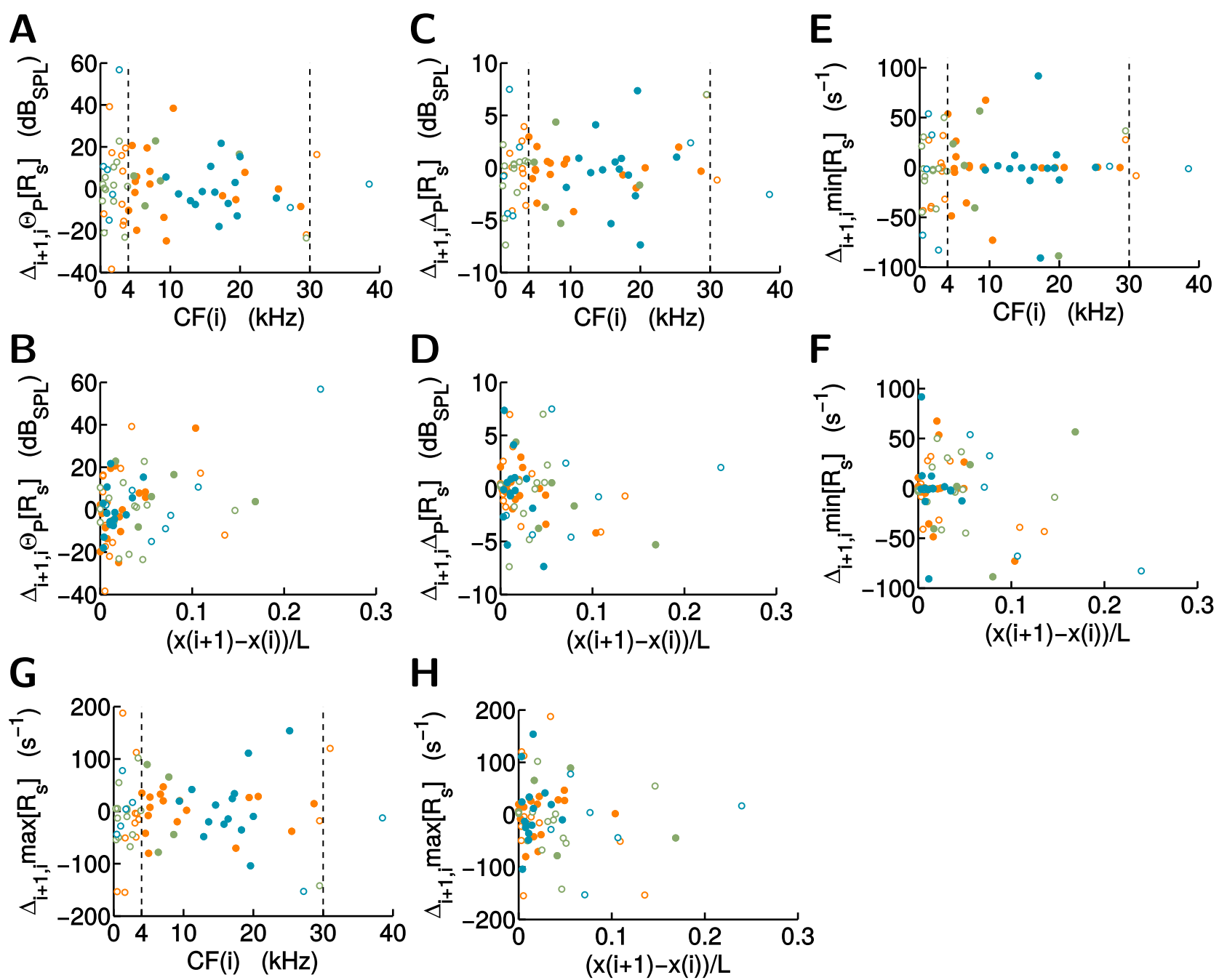

Figure S7.2: Differences in RLF properties between the nearest-neighbor SGNs along the $\mathbf{C F}$ axis. (A, B) Spike threshold. (C, D) Dynamic range. (E, F) Spontaneous (minimum) spike rate. $(\mathbf{G}, \mathbf{H})$ Maximum spike rate. The abscissa of plots in panels A, C, E, and G represents the smaller of the two CFs in each pair of the nearest-neighbor SGNs ordered with increasing $\mathrm{CF}$ value. In panels $\mathrm{B}, \mathrm{D}, \mathrm{F}$, and $\mathrm{H}$, the relative distance between the nearest neighbor SGNs along the organ of Corti are on the abscissa. In all panels, orange, blue, and green stand for data points from three different animals. In all cases, the SGNs were stimulated with sound bursts 0.75 octaves below the CF. Filled circles correspond to the nearest-neighbor pairs with CF of both SGNs between 4 and $30 \mathrm{kHz}$. Open circles correspond to the remaining data points. Black dashed lines mark $4-30 \mathrm{kHz}$ interval on the $\mathrm{CF}$ axis. 
Derivation of the relation between the dynamic range and minimum spike rate in the model of (Heil et al., 2011)

As it was shown in (Heil et al., 2011), the spike rate dependence on sound pressure of SGNs from cats is, to a very good approximation, described by

$$
R_{s}(P)=\frac{\max \left[R_{s}\right] \cdot\left(P+P_{0}\right)^{\beta}}{K_{A A}^{-1}+\left(P+P_{0}\right)^{\beta}}=\frac{\max \left[R_{s}\right]}{1+K_{A A}^{-1} \cdot\left(P+P_{0}\right)^{-\beta}} .
$$

Here, $\max \left[R_{s}\right] \equiv \max _{S P L}\left[R_{S}\right]$ is the maximum spike rate as observed at $P=+\infty . P_{0}, K_{A A}^{-1}$, and $\beta$ are other parameters of the model. In fact, it was found by (Heil et al., 2011) that $\beta=3$ for all SGNs considered in that work. The minimum spike rate, i.e., spike rate at $P=0$, is given by

$$
\min \left[R_{s}\right]=R_{s}(P=0)=\frac{\max \left[R_{s}\right]}{1+K_{A A}^{-1} \cdot P_{0}^{-\beta}} .
$$

By using (S7.2), (S7.1) can be rearranged as

$$
R_{s}(P)=\frac{\min \left[R_{s}\right] \cdot \max \left[R_{s}\right]}{\min \left[R_{s}\right]+\left(\max \left[R_{s}\right]-\min \left[R_{s}\right]\right) \cdot\left(P / P_{0}+1\right)^{-\beta}} .
$$

In our work, the dynamic range is defined by

$$
\Delta_{S P L}\left[R_{s}\right]=20 \cdot \log _{10}\left(P_{0.9} / P_{0.1}\right)
$$

where $P_{0.9}$ and $P_{0.1}$ are sound pressure amplitudes such that

$$
\begin{aligned}
R_{s}\left(P_{f}\right) & =\min \left[R_{s}\right]+f \cdot\left(\max \left[R_{s}\right]-\min \left[R_{s}\right]\right) \\
& =(1-f) \cdot \min \left[R_{s}\right]+f \cdot \max \left[R_{s}\right]
\end{aligned}
$$

Plugging (S7.3) into (S7.5) and rearranging the terms gives

$$
P_{f}=P_{0} \cdot\left(\frac{f+(1-f) \cdot \min \left[R_{s}\right] / \max \left[R_{s}\right]}{(1-f) \cdot \min \left[R_{s}\right] / \max \left[R_{s}\right]}\right)^{1 / \beta}-P_{0} .
$$

Finally, substituting (S7.6) into (S7.4) yields

$$
\begin{aligned}
\Delta_{S P L}\left[R_{s}\right] & =20 \cdot \log _{10}\left(\left(\frac{9+\min \left[R_{s}\right] / \max \left[R_{s}\right]}{\min \left[R_{s}\right] / \max \left[R_{s}\right]}\right)^{1 / \beta}-1\right)- \\
& -20 \cdot \log _{10}\left(\left(\frac{1+9 \cdot \min \left[R_{s}\right] / \max \left[R_{s}\right]}{9 \cdot \min \left[R_{s}\right] / \max \left[R_{s}\right]}\right)^{1 / \beta}-1\right) .
\end{aligned}
$$

It follows from Eq. (S7.7) that the minimum value of $\Delta_{S P L}\left[R_{s}\right]$ is $20 \cdot \log _{10} 9^{2 / \beta}$, and is attained at $\min \left[R_{s}\right] / \max \left[R_{s}\right]=0$. The maximum value of $\Delta_{S P L}\left[R_{s}\right]$ is $20 \cdot \log _{10}\left(\left(10^{1 / \beta}-\right.\right.$ $\left.1) /\left(0.9^{-1 / \beta}-1\right)\right)$ and is attained at $\min \left[R_{s}\right] / \max \left[R_{s}\right]=1$. Taking into account that $\beta=3$ (Heil et al., 2011), the minimum and maximum values of $\Delta_{S P L}\left[R_{s}\right]$ are respectively $\sim 12.7 \mathrm{~dB}$ and $\sim 30.2 \mathrm{~dB}$. 


\section{Derivation of the relation between the receptor potential of IHC and sound pressure}

Here, we derive the relation between the d.c. component of the IHC receptor potential $\left(V_{d c}\right)$ and sound pressure based on the experimental data from (Heil et al., 2011). The following considerations apply to high CF SGNs stimulated well below the CF, where the response of the basilar membrane to sound is linear and the a.c. component of the IHC receptor potential is negligible.

We start with Eq. (S7.3) which relates the spike rate with the sound pressure. As we learned in Results, the spike rate dependence on the membrane potential is, to a very good approximation, described by the Boltzmann function

$$
R_{s}\left(V_{m}\right)=\frac{A_{s}}{1+e^{-\left(V_{m}-S^{\prime}\right) / k_{V}^{\prime}}} .
$$

Accordingly, the membrane potential corresponding to $R_{s}\left(V_{m}\right)$ can be expressed as

$$
V_{m}\left(R_{s}\right)=S^{\prime}+k_{V}^{\prime} \cdot \ln \left(\frac{R_{s}}{A_{s}-R_{s}}\right) .
$$

By definition, the receptor potential $V_{d c}$ corresponding to the sound pressure $P$ is equal to the difference between the membrane potentials corresponding to $R_{s}(P)$ and $\min \left[R_{s}\right]$. By using Eq. (S7.9), this difference can be expressed as

$$
V_{d c}\left(R_{s}\right)=k_{V}^{\prime} \cdot \ln \left(\frac{R_{s}(P)}{A_{s}-R_{s}(P)} \cdot \frac{A_{s}-\min \left[R_{s}\right]}{\min \left[R_{s}\right]}\right)
$$

Substituting (S7.3) into (S7.10) gives

$$
V_{d c}(P)=k_{V}^{\prime} \cdot \ln \left(\frac{\max \left[R_{s}\right] \cdot\left(A_{s}-\min \left[R_{s}\right]\right)}{A_{s} \cdot \min \left[R_{s}\right]+A_{s} \cdot\left(\max \left[R_{s}\right]-\min \left[R_{s}\right]\right) \cdot\left(P / P_{0}+1\right)^{-\beta}-\min \left[R_{s}\right] \cdot \max \left[R_{s}\right]}\right) .
$$

$\max \left[R_{s}\right]$ can be expressed through other parameters of the model. Indeed, the membrane potential corresponding to $\max \left[R_{s}\right]$ is equal to the sum of the membrane potential corresponding to $\min \left[R_{s}\right]$ and the maximum of the receptor potential

$$
V_{m}\left(\max \left[R_{s}\right]\right)=S^{\prime}+k_{V}^{\prime} \cdot \ln \left(\frac{\min \left[R_{s}\right]}{A_{s}-\min \left[R_{s}\right]}\right)+\max \left[V_{d c}\right]
$$

Substituting Eq. (S7.12) back into (S7.8) and rearranging some terms yields

$$
\max \left[R_{s}\right]=\frac{\min \left[R_{s}\right] \cdot A_{s}}{\min \left[R_{s}\right]+\left(A_{s}-\min \left[R_{s}\right]\right) \cdot e^{-\max \left[V_{d c}\right] / k_{V}^{\prime}}} .
$$

Combining Eqs. (S7.13) and (S7.11) gives us the final expression of the $V_{d c}$ vs $P$ relation

$$
V_{d c}(P)=k_{V}^{\prime} \cdot \ln \left(\frac{1}{e^{-\max \left[V_{d c}\right] / k_{V}^{\prime}}+\left(1-e^{-\max \left[V_{d c}\right] / k_{V}^{\prime}}\right) \cdot\left(P / P_{0}+1\right)^{-\beta}}\right) .
$$


This equation has four independent parameters. The exponent $\beta$ was shown to be equal to 3 for all RLFs studied in (Heil et al., 2011). $P_{0}$ can be determined by fitting the experimental data of $\Delta_{S P L}\left[R_{s}\right]$ vs $\Theta_{S P L}\left[R_{s}\right]$ relation as shown in Results. In a logarithmic scale, $P_{0}$ merely determines the shift of the $V_{d c}$ vs $P$ relation along the pressure axis. $k_{V}^{\prime}$ describes the voltage sensitivity of the $R_{s}$ vs $V_{m}$ dependence. $\max \left[V_{d c}\right]$ is the maximum of the d.c. component of the receptor potential.

In the limit $\max \left[V_{d c}\right] / k_{V}^{\prime} \rightarrow+\infty$, Eq. (S7.14) reduces to

$$
V_{d c}(P)=\beta \cdot k_{V}^{\prime} \cdot \ln \left(P / P_{0}+1\right)
$$

When $\max \left[V_{d c}\right] / k_{V}^{\prime}$ is finite but $\gg 1$, Eq. (S7.15) approximates the $V_{d c}$ calculated by Eq. (S7.14) for $P$ values below those where $V_{d c}$ saturates (see Fig. S7.3C). Plugging expression (S7.15) into (S7.8) yields the original expression of the $R_{s}$ dependence on $P$, Eq. (S7.1), with $\max \left[R_{s}\right]=A_{s}$ and $K_{A A}=e^{\left(V_{\text {rest }}-S^{\prime}\right) / k_{V}^{\prime}}$. The reason why the original $R_{s}$ vs $P$ relation is restored only in the limit $\max \left[V_{d c}\right] / k_{V}^{\prime} \rightarrow+\infty$ is that $\max \left[R_{s}\right]$ and $\min \left[R_{s}\right] / \max \left[R_{s}\right]$ are independent quantities in the empirical model by (Heil et al., 2011; see Eq. (S7.2)). This is possible only if the receptor potential is not bound from above. However, as we described in Results, the data can be fitted with essentially the same quality for all $\max \left[V_{d c}\right] / \Delta_{V}\left[R_{s}\right]>2$. Note that $\Delta_{V}\left[R_{s}\right]=k_{V}^{\prime} \cdot \ln (81)$ by definition. 
Influence of $\max \left[V_{d c}\right]$ and $\Delta_{V}\left[R_{s}\right]$ on the properties of RLFs

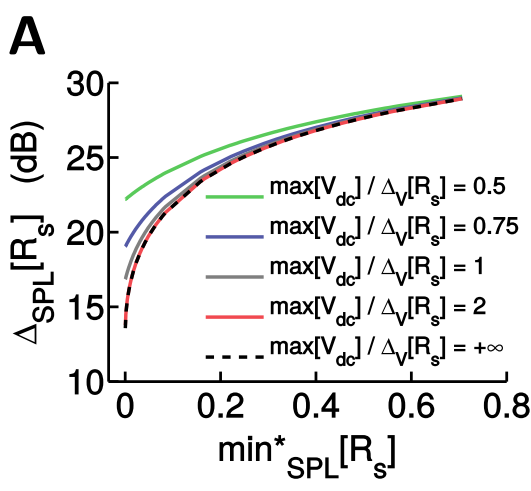

D

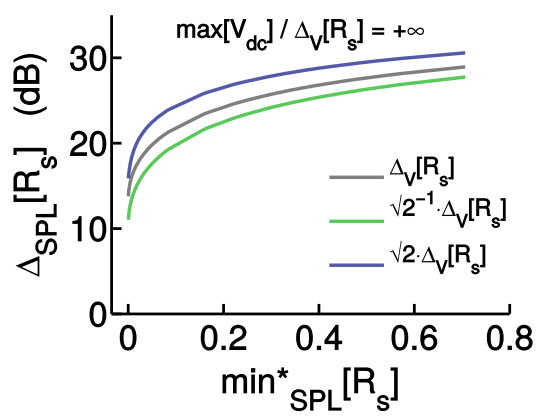

B

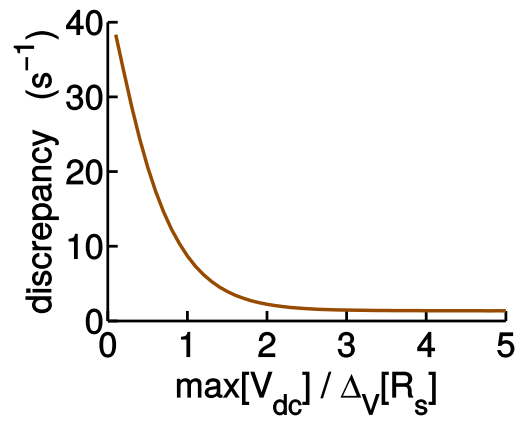

C

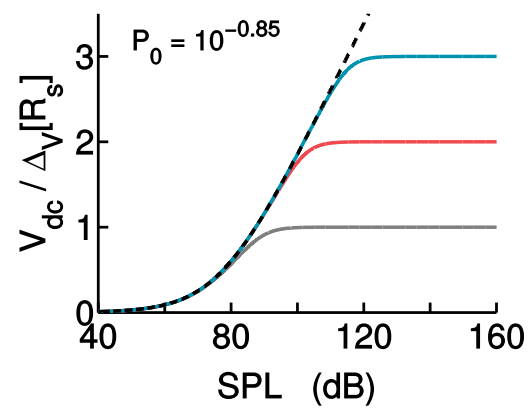

$E$

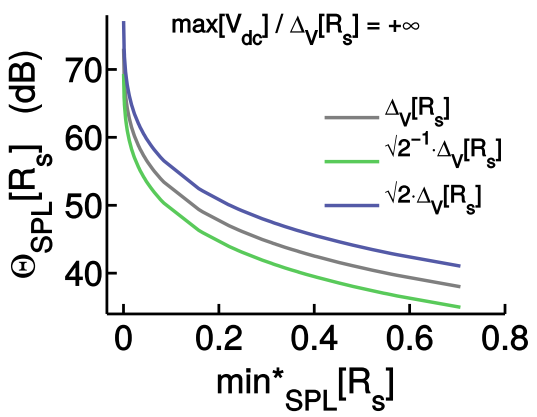

Figure S7.3: (A) Relations between $\Delta_{S P L}\left[R_{s}\right]$ and $\min _{S P L}^{*}\left[R_{s}\right]$ at different $\max \left[V_{d c}\right] / \Delta_{V}\left[R_{s}\right]$ (B) The average discrepancy between the data and the model fits of RLFs in the bootstrap sample at different $\max \left[V_{d c}\right] / \Delta_{V}\left[R_{s}\right]$. The discrepancy for each RLF was evaluated by an absolute difference between the experimental and modeled estimates of $R_{s}$ per each data point. (C) $V_{d c}$ vs $S P L$ plots at different $\max \left[V_{d c}\right] / \Delta_{V}\left[R_{s}\right]$. The dashed black line corresponds to $\max \left[V_{d c}\right] / \Delta_{V}\left[R_{s}\right]=+\infty$. (D) The dependence of $\Delta_{S P L}\left[R_{s}\right]$ on $\min _{S P L}^{*}\left[R_{s}\right]$ at different $\Delta_{V}\left[R_{s}\right]$. The grey line corresponds to the value of $\Delta_{V}\left[R_{s}\right]$ which was used to calculate the $S P L$ dependence on $V_{d c}$. (E) The same as (D) but for $\Theta_{S P L}\left[R_{s}\right]$. 


\subsection{Interspike interval distributions}
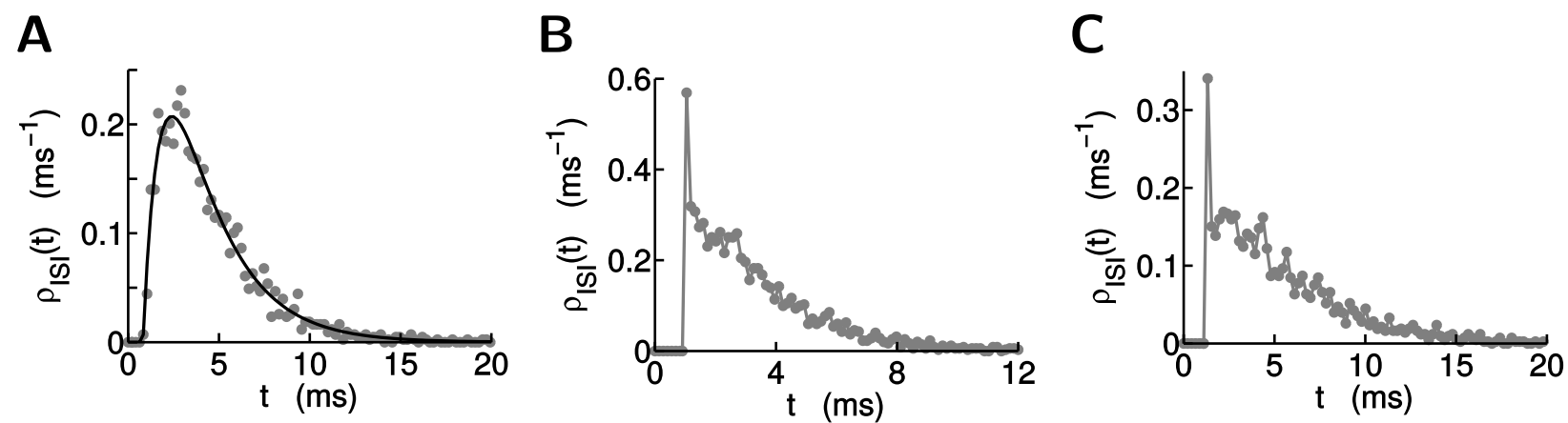

Figure S7.4: Selected examples of $\rho_{I S I}(t)$ functions of mice SGNs studied in this work. (A) An example of the main group of $\rho_{I S I}(t)$ functions which are well reproduced by the Poisson model (including the convolution with the SGN refractoriness). Filled circles mark the experimental data, the solid line corresponds to the best fit with the Poisson model. The discrepancy between the data and the fit of this particular selection corresponds to the average discrepancy in the sample of mice SGNs analyzed in our work. (B, C) Two examples of $\rho_{I S I}(t)$ functions with sharp early peaks from mice SGNs.

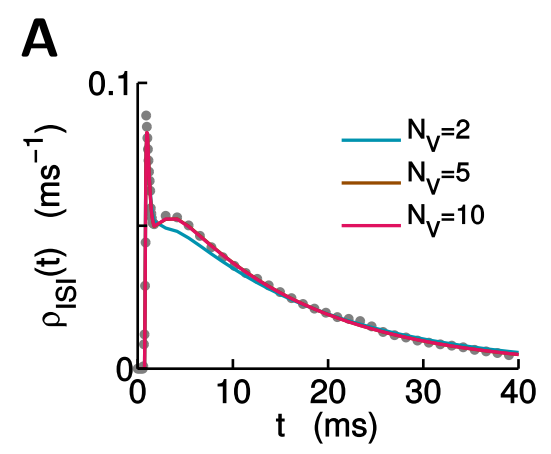

B

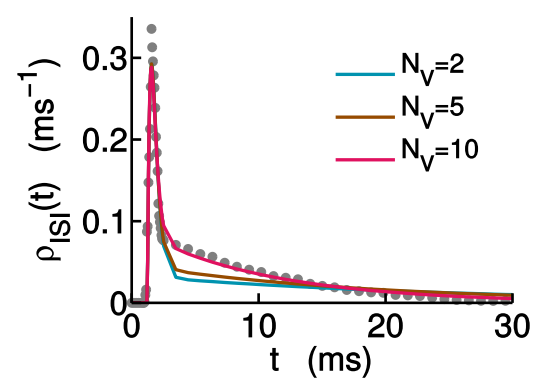

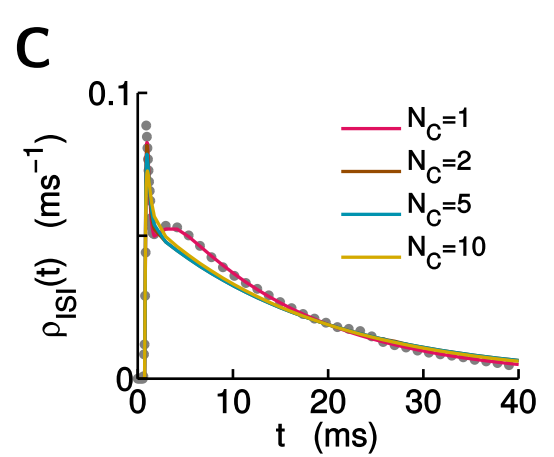

D

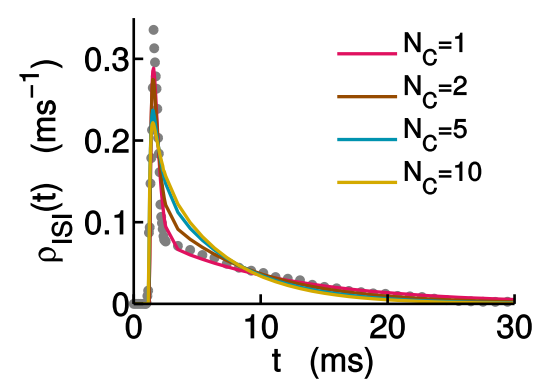

Figure S7.5: Reproduction of experimental $\rho_{I S I}(t)$ functions of bursty spike patterns by $\mathrm{AZ}$ models with different $\boldsymbol{N}_{\boldsymbol{C}}$ and $\boldsymbol{N}_{\boldsymbol{V}}$ numbers. $(\mathrm{A}, \mathrm{B})$ Fits of the same $\rho_{I S I}(t)$ functions as in Fig. 4.12C, D at different $N_{V}$. (C, D) Fits of the same $\rho_{I S I}(t)$ functions as in Fig. 4.12C, D at different $N_{C}$. 


\section{Chapter 5}

\section{General Discussion}

In this chapter, we provide an overview and discussion of the main results of the present doctoral dissertation. We compare our findings with relevant results of the previous works and comment on their importance and implications on auditory information processing at IHC ribbon synapses. Finally, relevant questions remaining to be approached in future studies are addressed.

\subsection{Presynaptic mobile $\mathrm{Ca}^{2+}$ buffers}

\subsubsection{Summary}

EF-hand $\mathrm{Ca}^{2+}$ buffers are important players in regulating presynaptic $\mathrm{Ca}^{2+}$ signals and neurotransmitter release (see, e.g., Roberts, 1993; Neher, 1998; Caillard et al., 2000; Vyleta \& Jonas, 2014); these proteins are prominently expressed in sensory hair cells of the ear (Edmonds et al., 2000; Heller et al., 2002; Hackney et al., 2005). In chapter 3 of this thesis, we studied how parvalbumin- $\alpha$ (PV- $\alpha$ ), calbindin-D28k (CB-D28k), and calretinin (CR), three main proteinaceous $\mathrm{Ca}^{2+}$ buffers found in IHCs, influence exocytosis and sound encoding at the ribbon synapses.

Patch clamp recordings were employed to track the voltage-gated $\mathrm{Ca}^{2+}$ currents and exocytosis under different intracellular $\mathrm{Ca}^{2+}$ buffering conditions. Extracellular recordings from SGNs were used to probe the postsynaptic readout of the presynaptic vesicle fusion in wild-type mice and those lacking PV- $\alpha$, CB-D28k, and $\mathrm{CR}\left(\mathrm{PV}^{-/-} \mathrm{CB}^{-/-} \mathrm{CR} \mathrm{R}^{-/-}\right)$. The auditory function was examined by measuring brainstem responses and otoacoustic emissions. Mathematical modeling was performed to evaluate the concentrations of the three $\mathrm{Ca}^{2+}$ buffers and to better understand their involvement in shaping presynaptic $\left[\mathrm{Ca}^{2+}\right]$ and control of exocytosis.

Our findings suggest that the proteinaceous $\mathrm{Ca}^{2+}$ buffers are present, altogether, at millimolar concentration at IHC ribbon synapses. Due to the tight coupling between the presynaptic channels and sensors of exocytosis, CB-D28k, CR, and PV- $\alpha$, do not interfere with fusion of RRP vesicles of the ribbon synapses considerably. Instead, we conclude that they prevent ex- 
trasynaptic exocytosis by reducing $\left[\mathrm{Ca}^{2+}\right]$ outside the AZs and allow for metabolically more efficient encoding of sound.

\subsubsection{Overview of the main experimental findings}

In $P V^{-/-} C B^{-/-} C R^{-/-}$IHCs, the kinetics of initial exocytosis $(t<20 \mathrm{~ms}$ ) was largely unchanged. However, during prolonged depolarizations $(t \geq 20 \mathrm{~ms})$, exocytosis was significantly enhanced: while the rate of vesicle release rapidly reached the steady-state level in wild-type IHCs, it continuously increased in the $\mathrm{PV}^{-/-} \mathrm{CB}^{-/-} C R^{-/-}$IHCs. The initial $\mathrm{Ca}^{2+}$ current was enhanced by $20 \%$ but, at the same time, showed faster initial inactivation. Thus, differences in the levels of $\mathrm{Ca}^{2+}$ influx became insignificant in both systems quickly after stimulus application. It was next found by loading different exogenous $\mathrm{Ca}^{2+}$ buffers to these cells that exocytosis could be restored to normal levels by applying $0.5 \mathrm{mM}$ of fast $\mathrm{Ca}^{2+}$ chelator BAPTA and $0.5 \mathrm{mM}$ slow $\mathrm{Ca}^{2+}$ chelator EGTA. These estimates are compatible with previously reported estimates of the buffering power of the endogenous buffers in IHCs (Johnson et al., 2008; Frank et al., 2010). Strikingly, despite the differences in the late-phase of exocytosis, sound encoding by spiral ganglion neurons (SGNs) was largely unaffected in animals lacking PV- $\alpha$, CB-D28k, and CR. The onset and sustained rates of spiking, average first spike latency, dynamic ranges, thresholds, as well as time-course of recovery from stimuli in SGNs of both genotypes were not significantly different. Further, mostly unaltered auditory brainstem potentials and otoacoustic emissions were found.

\subsubsection{Overview of the main modeling results}

By modeling the spatial profiles of $\left[\mathrm{Ca}^{2+}\right]$ corresponding to the exogenous buffer concentrations which could match the initial and sustained exocytosis, we found that the overall buffering effect of the proteinaceous buffers of IHCs is equivalent to either $\sim 1.5 \mathrm{mM} \mathrm{PV}-\alpha$, or $\sim 1 \mathrm{mM}$ of CB-D28k, or $\sim 2 \mathrm{mM}$ of CR. The obtained experimental data did not, however, allow us to constrain the abundance ratios of these three proteins.

Next, we modeled the relation between the exocytosis rate of IHCs and the spike rate of associated SGNs in wild-type and $P V^{-/-} C B^{-/-} C R^{-/-}$animals and concluded that little, if any, of the late-phase excess exocytosis observed in the later case contributes to SGN firing. We thus proposed that the excess exocytosis in IHCs lacking PV- $\alpha$, CB-D28k, and CR is mainly extrasynaptic. To reconcile different effects of the buffers on the initial and sustained exocytosis, we further modeled the effects of different exogenous buffers on the presynaptic exocytosis rate and estimated the effective coupling distance between presynaptic $\mathrm{Ca}^{2+}$ channels and $\mathrm{Ca}^{2+}$ sensors of exocytosis of $\sim 17 \mathrm{~nm}$. It turned out that this distance is considerably shorter than the characteristic length-constants of the predetermined endogenous $\mathrm{Ca}^{2+}$ buffers, thus, explaining the vanishing effect of them on the initial-phase exocytosis. This finding further supports the idea that the excess exocytosis observed in $P V^{-/-} C B^{-/-} C R^{-/-}$IHCs occurs 
distantly from the presynaptic AZs.

\subsubsection{Concentrations}

Our model estimates of endogenous EF-hand $\mathrm{Ca}^{2+}$ buffer concentrations are compatible with previous estimates of concentrations of these proteins in saccular hair cells (Edmonds et al., 2000) and some central neurons (Saftenku, 2012; Eggermann \& Jonas, 2012). However, they are around one order of magnitude higher than the estimates based on quantitative EM immunogold labeling in mature IHCs (Hackney et al., 2005). The origin of this discrepancy is not clear. However, it should be taken into account that the efficiency of immunolabeling is usually not properly controllable and may vary among different preparations. Alternatively, faster $\mathrm{Ca}^{2+}$-binding rates of the endogenous buffers than currently assumed (see section 7.1 in the supplementary material of chapter 3) could partially account for this discrepancy. Moreover, it has to be taken into consideration that, while loading the exogenous $\mathrm{Ca}^{2+}$ buffers, other proteins with $\mathrm{Ca}^{2+}$ binding capacity might have been washed out during our experiments. Though the concentrations of potential candidates calmodulin and the so-called $\mathrm{Ca}^{2+}$-binding proteins are low in neurons $(10 \mu \mathrm{M}$ or smaller; Manalan \& Klee, 1984; Yang et al., 2014), they are still to be determined in IHCs.

\subsubsection{Functional role}

Our findings suggest that proteinaceous $\mathrm{Ca}^{2+}$ buffers PV- $\alpha$, CB-D28k, and CR do not affect synaptic neurotransmitter release strongly due to tight coupling between the $\mathrm{Ca}^{2+}$ channels and sensors of exocytosis. However, as it follows from the model calculations, they are expected to strongly attenuate $\left[\mathrm{Ca}^{2+}\right]$ outside the presynaptic AZs. Firstly, this attenuation can be considered to be important for spatial separation of synaptic exocytosis from other $\mathrm{Ca}^{2+}$ driven processes. Secondly, it is predicted to speed up removal of $\mathrm{Ca}^{2+}$ from $\mathrm{AZs}$ and reduce build-up of bulk $\left[\mathrm{Ca}^{2+}\right]$, as was proposed in saccular hair cells (Roberts, 1994; Issa \& Hudspeth, 1996). In this context, the fact that our experiments showed no considerable changes of sound driven responses of SGNs in animals deficient of PV- $\alpha, \mathrm{CB}-\mathrm{D} 28 \mathrm{k}$, and CR, is relatively unexpected. On the other hand, only short stimuli (up to $100 \mathrm{~ms}$ long) were used in our in vivo experiments. It cannot be rejected that $\mathrm{Ca}^{2+}$ accumulates and affects sound encoding significantly only for longer ongoing stimulation times (seconds or minutes long). This remains to be answered in future studies.

In spite of undetected effects of the genetic ablation of PV- $\alpha$, CB-D28k, and CR on synaptic exocytosis, our findings suggest the presence of vesicle release outside the ribbon synapses during sustained stimulations. Ectopic neuronal exocytosis was implied and/or demonstrated in previous works. Synaptic vesicles docked away from presynaptic AZs were documented in large amounts around ribbon synapses of saccular hair cells (Lenzi et al., 1999). Ectopic vesicle release was shown to be physiologically relevant for communication between bipolar cells to AII 
amacrine cells (Mehta et al., 2014) and for broadening the repertoire of neuronal communication at the ciliary ganglion calyx synapses (Coggan et al., 2005). In IHCs, however, the extrasynaptic release does not contribute to sound encoding, as our results show. Moreover, the fact that it is induced by a non-natural perturbation of a physiological system, suggests its involvement in non-optimal functioning of IHCs. This phenomenon may resemble unwanted, premature exocytosis or a compensatory signaling pathway. Whichever is true, vesicle release outside AZs is associated with additional metabolic and energetic load, which makes sound encoding at these fast synapses less efficient. The differential sensitivity of the synaptic and the proposed ectopic release to mobile $\mathrm{Ca}^{2+}$ buffers at IHCs suggests that the later is induced by $\mathrm{Ca}^{2+}$ microdomains. The location of the ectopic release sites and the origin of $\mathrm{Ca}^{2+}$ which triggers exocytosis therein (synaptic channels vs extrasynaptic channels) remains to be determined in future studies.

\subsection{Active zone topography and coupling of $\mathrm{Ca}^{2+}$ influx to exocytosis}

\subsubsection{Summary}

The topographic arrangement of fusion-competent vesicles and voltage-gated $\mathrm{Ca}^{2+}$ channels within presynaptic AZs is one of the key determinants of neurotransmitter release dynamics and has been intensely investigated in the past decades (see, e.g., Zucker \& Fogelson, 1985; Augustine et al., 1991; Stanley 1993; Meinrenken et al., 2002; Shahrezaei et al., 2006; Bucurenciu et al., 2008; Nakamura et al., 2015). In this thesis, we studied the properties of presynaptic AZ topography of mature IHC ribbon synapses (chapter 2 and, partially, chapter 3).

Transmission electron microscopy, immunohistochemistry, STED and confocal microscopy techniques were applied to probe the structure of the presynaptic sites. Patch clamp electrophysiology was used to estimate the relation between $\mathrm{Ca}^{2+}$ influx and exocytosis at these synapses. A mathematical model of AZs of IHC ribbon synapses was then created based on these findings and previously published work. The model was used to further constrain the AZ topography based on the electrophysiological findings, as well as to study basic properties of the spatiotemporal dynamics of presynaptic $\left[\mathrm{Ca}^{2+}\right]$ (partially done in chapter 4). Changes of the presynaptic site organization during maturation were also considered, but to a lesser extent.

Our findings suggest that an equivalent of only two tightly coupled channels controls fusion of the presynaptic vesicles in mature IHC ribbon synapses. Such a tight coupling results in fast and strong $\mathrm{Ca}^{2+}$ signals at the sensors of exocytosis. Interestingly, the arrangement between the channels and vesicles considerably tightens during development around the onset of hearing, leading to a higher efficiency of exocytosis. We conclude that the nanodomain coupling between $\mathrm{Ca}^{2+}$ channels and sensors of exocytosis at IHC ribbon synapses is important for metabolically efficient and robust high precision encoding of sound. 


\subsubsection{Overview of the main experimental findings}

In agreement with previous EM measurements (Frank et al., 2010; Pangršič et al., 2010), a typical presynaptic density of mature IHC ribbon synapses was stripe-shaped, with the approximate dimensions of $400 \mathrm{~nm} \times 100 \mathrm{~nm}$. Two rows of tightly packed membrane-proximal vesicles positioned along the longer edges of the presynaptic density, presumably constituting the RRP, were identified. The EM data also revealed that bassoon is essentially concentrated at the presynaptic density. In addition, STED immunofluerescence measurements showed a tight colocalization of bassoon and $\mathrm{Ca}_{\mathrm{V}} 1.3$ channels, both of which form stripe shaped arrays resembling the area of the presynaptic density. These two findings provide additional evidence that the voltage-gated $\mathrm{Ca}^{2+}$ channels cluster predominantly within the presynaptic density, as previously proposed based on the overlaps of RIBEYE and Cav 1.3 immunofluorescence signals (Brandt et al., 2005; Frank et al., 2010). The average exponent $m$ of the relation between $\mathrm{Ca}^{2+}$ influx and exocytosis based on the change of the number of active $\mathrm{Ca}^{2+}$ channels was 1.4, in agreement with the previous observations (Brandt et al., 2005). Whereas the estimate of the intrinsic $\mathrm{Ca}^{2+}$ cooperativity of exocytosis, evaluated as the exponent $m$ upon change of the single channel current, was $\sim 3$. The effective coupling distance $R_{c}$ assessed by measuring exocytosis in response to short depolarizations in different intracellular $\mathrm{Ca}^{2+}$ buffering conditions was on average $17 \mathrm{~nm}$, with the $5-95$ percentile range of $8-30 \mathrm{~nm}$.

\subsubsection{Overview of the main modeling results}

As no reliable information about the exact positions of $\mathrm{Ca}_{\mathrm{V}} 1.3$ channels within the presynaptic density was available, we first tried to distribute the channels uniformly therein. It turned out that the experimentally obtained exponent $m=1.4$ could be reproduced with $\sim 20$ channels per AZ, amounting to $\sim 2$ effective channels, i.e., $n_{c h}$, per vesicle. However, such a scenario resulted in unrealistically slow release (due to low $\left[\mathrm{Ca}^{2+}\right]$ at the sensors of exocytosis). Moreover, the corresponding value of the effective coupling distance $R_{c}$ was $\sim 50 \mathrm{~nm}, \sim 3$ times higher than the experimental estimate. Increasing the number of randomly distributed channels within the presynaptic density to 75 decreased the $R_{c}$ only to $40 \mathrm{~nm}$ at the same time, resulting in exponent $m$ values considerably higher than 1.4. Altogether, these results strongly suggested that some of the presynaptic $\mathrm{Ca}^{2+}$ channels are systematically positioned close to presynaptic vesicles. Further analysis revealed that, in order to reproduce the experimentally determined values of $m$ and $R_{c}$, it was necessary to place one or two channels in close proximity $(\lesssim 10 \mathrm{~nm})$ to each RRP vesicle. In the case when only one channel was tightly coupled to each RRP vesicle, it was necessary to include additional channels within the presynaptic density, preferentially closer to the edges of the AZ, so that they altogether contributed similar amount of $\mathrm{Ca}^{2+}$ for exocytosis of each vesicle as the tightly coupled channels $\left(n_{c h} \sim 2\right)$. Currently available data do not allow to reliably distinguish between these two possibilities.

In spite of some uncertainty about the exact AZ topography, our findings imply that the 
density of $\mathrm{Ca}^{2+}$ channels should be shifted towards the longer edges of the presynaptic density area, where RRP vesicles are located. To some extent, this arrangement resembles the AZs from frog neuromuscular junction (Heuser, 1979; Harlow et al., 2001; Luo et al., 2011). Further refinement of the $\mathrm{AZ}$ topography in IHC ribbon synapses could be achieved by probing the exponent $m$ in different buffering conditions of intracellular $\mathrm{Ca}^{2+}$, by increasing the signal to noise ratio of the exocytosis measurements (for example, by using simultaneous patch clamp of pre- and postsynaptic sites; Goutman \& Glowatzki, 2007), by using super-resolution localization microscopy (see, e.g., Sauer, 2013), and/or freeze-fracture replica immunogold labeling of Cav1.3 channels (see, e.g., Nakamura et al., 2015; Baur et al., 2015)

\subsubsection{A note on heterogeneity}

Accumulating evidence suggests that presynaptic AZs are different among ribbon synapses within individual IHCs and that this heterogeneity plays an important role in sound encoding (Frank et al., 2009; Meyer et al., 2010; Liberman et al., 2011; Kantardzhieva et al., 2013; chapter 4). Due to the nature of the recording technique, the experimental estimates of the exponent $m$ of the exocytosis vs $\mathrm{Ca}^{2+}$ influx relation and the effective coupling distance $R_{c}$ were obtained based on combined exocytosis of all synapses of single IHCs in our work. Moreover, to increase the signal to noise ratio, we used $m$ values averaged over the whole sample of IHCs studied as a reference for the modeling results. Thus, the AZ topography scenarios identified by our modeling efforts correspond to an average view, which does not reflect possible variations of the presynaptic structure across different synapses. This question remains to be elucidated in future studies, e.g., by using simultaneous patch clamp recordings from IHCs and postsynaptic boutons of SGNs (see e.g., Goutman \& Glowatzki, 2007).

\subsubsection{Properties of $\mathrm{Ca}^{2+}$ dynamics within the active zones}

Modeling of presynaptic $\mathrm{Ca}^{2+}$ dynamics in chapter 4 revealed that build-up and collapse of $\left[\mathrm{Ca}^{2+}\right]$ domains within AZs tightly follows opening and closing of the presynaptic $\mathrm{Ca}^{2+}$ channels. Equilibration of $\left[\mathrm{Ca}^{2+}\right]$ at the $\mathrm{Ca}^{2+}$ sensors of exocytosis of these synapses is nearly complete within the first few tens of $\mu$ s after opening or closing of any of the channels. This is much smaller than the characteristic open and closed times of $\mathrm{Ca}_{\mathrm{V}} 1.3$ channels (hundreds of $\mu \mathrm{s}$ to ms, Neef et al., 2009). The same conclusion is valid even for those scenarios of AZ topographies in which none of the $\mathrm{Ca}^{2+}$ channels located within the presynaptic density is preferentially positioned close to the sensors of exocytosis. We showed that the abundant mobile $\mathrm{Ca}^{2+}$ buffers at these synapses play an important role in making the equilibration of $\left[\mathrm{Ca}^{2+}\right]$ at $\mathrm{AZs}$ as fast as it is. Similar conclusions have been reached for $\left[\mathrm{Ca}^{2+}\right]$ dynamics at saccular hair cell synapses (Roberts, 1994).

Another important feature of $\left[\mathrm{Ca}^{2+}\right]$ at AZs of IHC ribbon synapses, which we found by modeling, is that $\left[\mathrm{Ca}^{2+}\right]$ dynamics can be linearized, to a very good approximation, for different 
AZ topographies tested, even at maximal $\mathrm{Ca}^{2+}$ influx. This means that domains of $\left[\mathrm{Ca}^{2+}\right]$ attributed to opening of separate $\mathrm{Ca}^{2+}$ channels superimpose almost linearly. In our case, the validity of this approximation is partially guaranteed by the relatively high concentrations of the presynaptic $\mathrm{Ca}^{2+}$ buffers, which are not depleted considerably during $\mathrm{Ca}^{2+}$ influx. General properties of the linearized $\left[\mathrm{Ca}^{2+}\right]$ dynamics have been analyzed in detail in previous works (see, e.g., Pape et al., 1995; Naraghi \& Neher, 1997) and are well understood. The linear approximation as well as fast equilibration of $\left[\mathrm{Ca}^{2+}\right]$ upon channel opening and closing allow for substantial simplifications of the underlying calculations of the spatiotemporal dynamics of $\mathrm{Ca}^{2+}$, and were exploited throughout this thesis.

\subsubsection{Developmental changes}

In immature IHC ribbon synapses, we found the presynaptic density to be divided into multiple small patches localized in the contact areas between postsynaptic boutons of SGNs and IHCs. Small roundish ribbons were anchored to some of those patches and presynaptic vesicles tended to cluster around them. The immature IHCs just before the developmental acquisition of hearing function featured two times higher $\mathrm{Ca}^{2+}$ influx, mainly due to the proportionally larger number of channels, which were broadly spread outside the presynaptic densities throughout whole basolateral membrane of IHCs. The exponent $m$ obtained by manipulating the number of open $\mathrm{Ca}^{2+}$ channels was $\sim 2.3 \pm 0.2$, substantially higher than in mature IHCs. Application of mathematical modeling was considerably limited in this case. First, differently from the mature presynaptic AZs, the immature synapses showed more complex, heterogeneous patterns of organization that could not be formalized well at a quantitative level with the data obtained in our experiments. Second, a large amount of extrasynaptic $\mathrm{Ca}^{2+}$ channels could potentially drive extrasynaptic exocytosis, which is included in, but not resolved by, our membrane capacitance measurements. Finally, the estimates of the effective coupling distance $R_{c}$ in the immature synapses were not available to us. Nevertheless, the experimentally determined value of exponent $m=2.3$ could be readily reproduced with exemplary immature presynaptic AZ topographies if none of the RRP vesicles were associated with tightly coupled channels $\left(n_{c h} \sim 4\right)$.

The confinement of presynaptic $\mathrm{Ca}^{2+}$ channels to presynaptic AZs and/or decrease of the coupling distance between them and fusion-competent vesicles during development were also documented in synapses between cerebellar cortical parallel fibers and Purkinje cells (Baur et al., 2015), and the calyx of Held (Fedchyshyn \& Wang, 2005; Wang et al., 2008; Nakamura et al., 2015). This phenomenon is likely in other central synapses too (reviewed in Eggermann et al., 2012). Our findings provide further support to the notion of the developmental tightening of $\mathrm{Ca}^{2+}$ influx to exocytosis coupling as a widespread feature of synaptogenesis (but see Vyleta \& Jonas, 2014). 


\subsubsection{Functional implications}

Different AZ topographies equip synapses with distinct response properties which may be advantageous or disadvantageous depending on the context (reviewed in chapter 1; Eggermann, 2012). What are advantageous effects of the tight coupling between $\mathrm{Ca}^{2+}$ channels and sensors of exocytosis at mature IHC ribbon synapses? First, this regime of exocytosis control results in lower energetic expenses due to lower demand for intracellular $\mathrm{Ca}^{2+}$ extrusion. Second, as it follows from our results obtained in chapter 3, the nanodomain coupling established between $\mathrm{Ca}^{2+}$ channels and sensors of exocytosis at IHC ribbon synapses allows for selective regulation of $\mathrm{Ca}^{2+}$ dependent processes outside the presynaptic site by avoiding any interference with synaptic exocytosis. Third, the nanodomain $\mathrm{Ca}^{2+}$ endows synapses with higher temporal precision, compared to what the microdomain coupling could provide. This is partially achieved by fast equilibration of $\left[\mathrm{Ca}^{2+}\right]$ at the $\mathrm{Ca}^{2+}$ sensors of exocytosis of these synapses, as we mentioned above. Even more importantly, the tight coupling with $\mathrm{Ca}^{2+}$ channels results in high- $\left[\mathrm{Ca}^{2+}\right]$ domains at the sensors of exocytosis, which renders the presynaptic vesicle release more resistant to accumulating global $\mathrm{Ca}^{2+}$ during prolonged stimulations. Given that the dynamics of the bulk $\left[\mathrm{Ca}^{2+}\right]$ can be as slow as hundreds of milliseconds or even seconds (see, e.g., Moser \& Beutner, 2000; Billups \& Forsythe, 2002; Neher \& Taschenberger, 2013), the advantage provided by $\mathrm{Ca}^{2+}$ nanodomain coupling may be crucial for the synaptic release to phase lock to sounds with frequencies as high as $2 \mathrm{kHz}$ over prolonged stimulations (Kiang et al., 1965; Rose et al., 1967; Palmer \& Russell, 1986) or react to changes in the amplitude of an ongoing stimulation with temporal precision of a few ms in the case of sounds of higher frequencies (Kiang et al., 1965).

One of potential disadvantages of the nanodomain coupling between $\mathrm{Ca}^{2+}$ channels and sensors of exocytosis are the expected random fluctuations of $\left[\mathrm{Ca}^{2+}\right]$ at the sensors of exocytosis due to stochastic openings of the presynaptic $\mathrm{Ca}^{2+}$ channels (Eggermann et al., 2012). Our detailed quantitative analysis in chapter 4 revealed that this noise affects the statistics of presynaptic vesicle fusion only if the channel opening/closing is much slower than the $\mathrm{Ca}^{2+}$ binding to the sensors of exocytosis. Current experimental estimates of the channel gating kinetics suggest that the activation constant of the channel opening is about several hundred microseconds (Neef et al., 2009; Frank et al., 2010; Zampini et al., 2013; Zampini et al., 2014). Meanwhile, $\mathrm{Ca}^{2+}$-binding rates to the sensor of exocytosis are expected to be similar or even slower (Beutner et al., 2001). Thus, we expect that the stochastic nature of $\mathrm{Ca}^{2+}$ channel gating does not deteriorate sound encoding at these synapses during ongoing stimulation noticeably. In turn, due to the advantages mentioned above, this makes the nanodomain coupling regime of exocytosis a preferred choice over the microdomain coupling for synapses with high temporal resolution.

The nanodomain coupling between $\mathrm{Ca}^{2+}$ channels and sensors of exocytosis was also proposed in retinal and amphibian auditory ribbon synapses (Jarsky et al., 2011; Graydon et al., 2011). Due to signal processing advantages granted by the $\mathrm{Ca}^{2+}$ nanodomain coupling, it looks 
reasonable that this coupling scenario could be a preferred choice for afferent synapses of many different sensory cells.

\subsection{Analytically tractable biophysical model of IHC rib- bon synapses}

In chapter 4, we developed an analytical framework for calculating steady state properties of the stochastic process of presynaptic vesicle release in response to constant depolarizations of the plasma membrane. In the context of IHC ribbon synapses, such responses are evoked when stimulating the ear by high-frequency pure tones in vivo (Kiang et al., 1965). Moreover, step depolarizations are commonly used during in vitro electrophysiology studies of the function of IHC ribbon synapses (see, e.g., Goutman \& Glowatzki, 2007; Pangršič et al., 2010; Zampini et al., 2013). Our analytical approach is based on two key approximations: 1) $\left[\mathrm{Ca}^{2+}\right]$ follows gating of presynaptic $\mathrm{Ca}^{2+}$ channels instantaneously, and 2) dynamics of presynaptic $\left[\mathrm{Ca}^{2+}\right]$ is linear. We demonstrated the validity of these assumptions using the biophysical model of IHC ribbon synapses introduced in chapter 2, confirming the applicability of our analytical method for treating this model.

The developed method played a crucial role in our analysis of the presynaptic mechanisms of information decomposition in the auditory nerve (chapter 4). One intriguing future application of this approach would be studying IHC ribbon synapses by using tools of information theory. The later has been successfully applied to various problems in neuroscience and provided a framework to evaluate neurons from the perspective of their most important biological function - information processing (see, e.g., Steveninck \& Bialek, 1988; Rieke et al., 1999; Dimitrov et al., 2011). Calculations of information theoretic measures are computationally demanding and, thus, analytical models are of great relevance (Strong et al., 1998; Kang \& Sompolinsky, 2001; Shneidman et al., 2006).

\subsection{Presynaptic mechanisms of information decomposi- tion in the auditory nerve}

\subsubsection{Motivation}

Different spiral ganglion neurons (SGNs) innervating IHCs of the same cochlear position show contrasting responses to sound stimuli (Sachs \& Abbas, 1974; Liberman, 1978; Winter et al., 1990; Relkin \& Doucet, 1991; Li \& Young, 1993). Strong evidence exists that even SGNs contacting the same IHC can have substantially different response properties (Liberman, 1980; Liberman, 1982). This heterogeneity is believed to be crucial for the capacity of the mammalian auditory system to encode a wide range of sound levels with high precision (Viemeister, 1988; 
Colburn, 2003). Nevertheless, the mechanisms underlying this phenomenon are still poorly understood. Three mutually non-exclusive hypotheses have been put forward: (1) variation of the intrinsic biophysical properties of SGNs (Liberman, 1982; Liberman et al., 2011; Liu et al., 2014), (2) variation of the efferent innervation of SGNs by lateral olivocochlear system (Liberman, 1990; Groff \& Liberman, 2003) (3) variation of the presynaptic properties of IHCs (Merchan-Perez \& Liberman, 1996; Robertson \& Paki, 2002; Frank et al., 2009). In chapter 4 of this thesis, we addressed the hypothesis of the presynaptic mechanism of the SGN response heterogeneity by using extensive mathematical modeling and analysis of in vivo SGN responses to pure tones.

\subsubsection{Approach}

A biophysical model of IHC ribbon synapses, which includes the key aspects of the mechanisms determining vesicle fusion dynamics at the presynaptic AZs, was constructed. To minimize the complication arising from the cochlear micromechanics and transduction, we studied steady state responses to high-frequency pure tone bursts, at least 0.5 octaves below the CF. In particular, we considered rate-level functions (RLFs) as well as distributions and serial correlation functions of the inter-spike intervals (ISIs) or inter release event intervals (IEIs). The analytical tractability of the model allowed us to thoroughly dissect the relation between the structure of the presynaptic AZ and characteristics of the adapted response of the synapse. Despite the fact that our work of previous chapters of this dissertation suggested the nanodomain coupling between $\mathrm{Ca}^{2+}$ channels and sensors of exocytosis, we took advantage of our model and analyzed a broader range of hypothetical synapses working in both, $\mathrm{Ca}^{2+}$ nanodomain, and $\mathrm{Ca}^{2+}$ microdomain coupling regimes. This was done with a purpose to understand possible advantages or disadvantages exerted on the coding properties of these synapses by the $\mathrm{Ca}^{2+}$ nanodomain coupling regime. Finally, we applied the model to describe the in vivo data.

Our main findings are summarized and discussed in the subsequent sections.

\subsubsection{Heterogeneity of rate-level functions}

The RLF is one of the main properties characterizing SGNs as information coding devices, and, thus, is among the most well studied features of these neurons. In previous studies, empirical models were introduced to describe experimental RLFs (Sachs \& Abbas, 1974; Yates et al., 1990; Sumner et al., 2002; Heil et al., 2011). These models played an important role in quantifying the differences between RLFs and identifying the contribution of the basilar membrane nonlinearity to the heterogeneity of RLF shapes. However, they, by design, could not reveal the underlying presynaptic and/or postsynaptic mechanisms of the quantified phenomena. In chapter 4 of this thesis, we showed that the variability of the parameters which characterize IHC ribbon synapses is able to account for the heterogeneity of RLFs in a detailed and quantitative manner. Our results provided straightforward mechanistic explanations of well known (but not 
understood) features of RLFs. By using our framework, we were able to quantify the effect of some already measured properties of the presynaptic and postsynaptic site heterogeneities on the RLF variability. Moreover, we were able to make further quantitative predictions that suggest directions for future experiments.

\section{Overview of the main results}

One of the key results of our theoretical analysis is that the SGN spike rate dependence on the presynaptic (!) membrane potential $\left(V_{m}\right)$ is very well approximated by a Boltzmann function in the framework of our biophysical model of IHC ribbon synapse. Thus, despite the fact that the synapse is characterized by a large number of microscopic variables, their effect on the spike rate dependence on $V_{m}$ reduces to three effective parameters: 1) half-activation voltage, 2) dynamic range (voltage sensitivity), and 3) amplitude. This result led us to hypothesize that RLFs follow from (approximately) Boltzmann functions after transforming $V_{m}$ to $S P L$. We confirmed this hypothesis by analyzing RLFs from a sample of 72 cat SGNs and showed that whole spectrum of RLFs arising from the same cochlear tonotopic position could be reproduced with our model in a detailed manner. This required only a scaling of the amplitude of the postsynaptic rate dependence on $V_{m}$ and changing its half-activation voltage with respect to the presynaptic resting membrane potential $\left(V_{\text {rest }}\right)$, without modifying the dynamic range.

As predicted by our model, a variation of the amplitude of the Boltzmann function determined only the maximum spike rate and was not correlated with the remaining aspects of RLFs in the data. In accordance, no considerable variation in the maximum spike rate of adapted responses with the spontaneous spike rate was reported in the previous studies (Evans \& Palmer, 1980; Ohlemiller et al., 1991; Taberner \& Liberman, 2005). The remaining parameters of RLFs were controlled by a change of the half-activation voltage, and covaried closely, as predicted by our model. Due to the properties of the $S P L$ to $V_{m}$ transformation, a difference in the half-activation voltage induces not only a change in the RLF threshold but also the dynamic range and the spontaneous rate. We found that the dynamic range decreases monotonically from $\sim 30 \mathrm{~dB}$ to $\sim 15 \mathrm{~dB}$ when the spontaneous rate decreases from $\sim 1$ to $\sim 0$. Correspondingly, this is accompanied by an increment of the RLF threshold by $\sim 40 \mathrm{~dB}$ (for SGNs from the same cochlear tonotopic position). Approximately this range of thresholds was reported for SGNs from different animals (Winter et al., 1990; Müller \& Robertson, 1991; Ohlemiller et al., 1991; Taberner \& Liberman, 2005), when stimulating at CF. Regarding the dynamic ranges, previous studies concentrated mainly on SGN responses to pure tone stimuli at CF. Then, it was found that the dynamic range increases considerably with threshold due to the saturating nonlinearity of the dependence of the basilar membrane deflection on the sound level (Sachs \& Abbas, 1974; Yates et al., 1990).

We estimated further that, in order for one IHC to host SGNs on different sides of the spectrum of possible RLF thresholds (and, thus, spontaneous rates and dynamic ranges), the maximum level of the IHC receptor potential has to be at least two times higher than the 
dynamic range of the vesicle release rate dependence on $V_{m}$. This is compatible with in vivo intracellular recordings which showed that the IHC receptor potential can vary in a range of $60 \mathrm{~dB}$ SPL without pronounced saturation when stimulating at $\mathrm{CF}$, at least two times larger than the dynamic ranges of the RLFs (Goodman et al., 1982; Patuzzi \& Sellick, 1983; Cody \& Russell, 1986). Similar findings were obtained with stimulations below CF (see Figs. 4,6 in Patuzzi \& Sellick, 1983).

In the framework of our model of IHC ribbon synapses, the half-activation voltage of the spike rate dependence on $V_{m}$ can be changed with respect to $V_{\text {rest }}$ by both, presynaptic and postsynaptic mechanisms. We, thus, aimed to evaluate the contributions of both of them.

\section{Evaluating the postsynaptic mechanisms}

It was shown previously that heterogeneity of spontaneous spike rates of SGNs from cats can be primarily accounted for by the variation of presynaptic vesicle release rates, with the postsynaptic refractory period fixed to the median level (Heil et al., 2007). In chapter 4 of the present dissertation, we evaluated the contribution of the heterogeneity of refractory periods to the variation of the maximum spike rates in response to high-frequency pure tones in mouse SGNs. Similarly to (Heil et al., 2007), we found a rather limited range of the overall refractory periods, the $5-95$ percentile range of $0.7-1.7 \mathrm{~ms}^{-1}$. We also demonstrated that it was mainly the variation of the presynaptic vesicle release rate, not the variation of postsynaptc refractory periods, which accounted for the differences in the spike rates. Next, by using our biophysical model, we estimated that the refractory period influenced the half-activation voltage of the SGN spike rate dependence on $V_{m}$ only by $8 \%$ of its dynamic range on average $(5-95$ percentile range from $5 \%$ to $14 \%$ ). Thus, we concluded that the contribution of the postsynaptic mechanisms to the RLF heterogeneity in response to high-CF pure tone bursts is marginal.

It has to be taken into account that the above reasoning applies not only to the intrinsic properties of SGNs but also to the effect exerted by their efferent innervation by the olivovohlear system. Indeed, taken into account that olivocochlear fibers synapse on the peripheral axons of SGNs (Liberman, 1980), where spikes are likely to be initiated (Hossain et al., 2005), the effect of their excitatory or inhibitory inputs effectively increases or decreases the refractory period of these neurons, as seen from the perspective of synaptic input from the ribbon synapses. Nevertheless, our findings do not reject the possibility that the lateral olivocochlear innervation could play an important role in modulation of SGN responses in more complex and time varying acoustic signal setups (see, e.g., Groff \& Liberman, 2003; Darrow et al., 2006; Darrow et al., 2007). Further work is necessary to better understand the effects of the olivocochlear system on the dynamic range of the peripheral auditory system. 


\section{Evaluating the presynaptic mechanisms}

The finding of a likely small contribution of the postsynaptic site to the heterogeneity of RLFs suggests that the mechanisms of the variation of SGN responses to high-frequency pure tones reside predominantly on the presynaptic side. In the framework of our biophysical model, three mutually non-exclusive scenarios for this exist, as discussed below.

First, IHCs may have different resting potentials. This mechanism cannot explain the fact that the same IHC can be contacted by low and high spontaneous rate SGNs, as strongly supported by experimental findings (Liberman, 1980; Liberman, 1982), and, thus, most likely, does not have an exclusive role in sound encoding. However, it could still widen the range of the response heterogeneity of SGNs innervating different IHCs from nearly the same tonotopic cochlear position. Due to the complexity of in vivo recordings from IHCs, no reliable estimates of the variation of their $V_{\text {rest }}$ are currently available. In different studies based on the sharp electrode technique, IHCs with $V_{\text {rest }}$ different by as much as $20 \mathrm{mV}$ were documented (see, e.g., Dallos, 1985; Russell et al., 1986). However, most of this variation, very likely, can be attributed to the damage to some of IHCs done by penetrating them with electrodes, as suggested by the strong variation of the maximum levels of the receptor potentials and input resistances in these recordings. Further studies and technical advances are needed to quantify the relative impact of $V_{\text {rest }}$ variation to the RFL heterogeneity and sound encoding.

Second, as it follows from in vitro $\mathrm{Ca}^{2+}$ imaging experiments by (Frank et al., 2009) and (Ohn et al., in preparation), presynaptic $\mathrm{Ca}^{2+}$ channels at different $\mathrm{AZs}$ of the same IHC can have different half-activation voltages of their open probabilities. Indeed, (Ohn et al., in preparation) found that the half-activation voltage of the open probabilities of the presynaptic $\mathrm{Ca}^{2+}$ channel clusters varied over a range of $15 \mathrm{mV}$ in a population of $210 \mathrm{AZs}$ from 25 IHCs studied in vitro. According to our modeling results, this spans around one half of the range of the SGN spike rate dependencies on $V_{m}$ which is necessary to reproduce the whole spectrum of RLFs at the same cochlear tonotopic position. Thus, the variation in the half-activation voltage seems to be a substantial component in the presynaptic heterogeneity scheme. On its own, however, it cannot quantitatively account for the entire heterogeneity.

Third, as it follows from our general model analysis, any factors which change the intrinsic $\mathrm{Ca}^{2+}$ binding rates of the sensor of exocytosis, the absolute values of the $\mathrm{Ca}^{2+}$ channel gating rates, vesicle replenishment rates, and the $\mathrm{Ca}^{2+}$ concentration at the presynaptic AZs, can affect the half-activation voltages of the presynaptic vesicle release rates. Differences in the presynaptic $\mathrm{Ca}^{2+}$ concentration are in particular relevant. As we learned throughout this thesis, even small changes in the positions of presynaptic $\mathrm{Ca}^{2+}$ channels can have a large effect on $\left[\mathrm{Ca}^{2+}\right]$ at the sensors of exocytosis. So far, the obtained experimental data does not allow to quantify the significance of the aforementioned differences in presynaptic AZs for the diverse coding properties of SGNs. However, at qualitative level, the evidence is substantial. Firstly, presynaptic sites corresponding to high SR SGNs were shown to have smaller AZs with fewer vesicles than the low SR SGNs (Merchan-Perez \& Liberman, 1996; Kantardzhieva et 
al., 2013). In addition, different morphology and size of presynaptic ribbons of the synapses corresponding to low and high SR SGNs have been described (Liberman et al., 1990; MerchanPerez \& Liberman, 1996; Liberman et al., 2011). Secondly, it was found that the maximum intensities of $\mathrm{Ca}^{2+}$ fluorescence signals at synapses of the same IHC in response to depolarizing stimuli in vitro differed by four times between the strongest and the weakest synapse on average (Frank et al., 2009; Ohn et al., in preparation). Stronger $\mathrm{Ca}^{2+}$ signals were more frequently found on the modiolar side of IHCs (Ohn et al., in preparation), which is preferentially contacted by low spontaneous rate SGNs (Liberman, 1980; Liberman, 1982). Analogous results have been obtained for $\mathrm{Ca}_{\mathrm{V}} 1.3$ immunofluorescence signals, suggesting that higher $\left[\mathrm{Ca}^{2+}\right]$ around the AZs results from a higher number of $\mathrm{Ca}^{2+}$ channels. Moreover, the $\mathrm{Ca}^{2+}$ signal amplitudes were shown to positively correlate with the ribbon fluorescence intensity (Frank et al., 2009).

Findings introduced in the previous paragraph support the concept of a substantial contribution of the variation of presynaptic AZ organization to the heterogeneity of SGN responses. So far, however, the key observations appeared as rather puzzling to researchers in the field (Liberman, 1990; Merchan-Perez \& Liberman, 1996; Liberman et al., 2011); it is at first sight counterintuitive that SGNs with lower spontaneous rates receive synaptic input from larger presynaptic AZs. In the light of our modeling results, these observations appear as a useful design. It has been repeatedly shown that SGNs with lower spontaneous rates have slower recovery (Rhode \& Smith, 1985; Ohlemiller et al., 1991; Relkin \& Doucet, 1991), and adaptation (Yates, 1991; Müller \& Robertson, 1991) kinetics for driven spike rates than high spontaneous rate SGNs. However, the adapted maximum spike rates of both types of SGNs are not significantly different (Evans \& Palmer, 1980; Ohlemiller et al., 1991; Taberner \& Liberman, 2005). The only way to obtain the same rates for synapses with slower kinetics per vesicular release site is to have more vesicular release sites, i.e., larger presynaptic AZs. While this resolves the paradox, it does not explain why it is so. One possibility is that the slowed kinetics of the release is a direct consequence of the kinetic parameter readjustment for larger rightward shifts of the half-activation voltages of vesicle release rates. This scenario remains to be addressed in future studies.

\section{A role of high $\mathrm{Ca}^{2+}$ cooperativity of exocytosis in sound encoding}

Many different synaptic systems feature high intrinsic $\mathrm{Ca}^{2+}$ cooperativity of exocytosis. The physiological function of this property is possibly multifaceted and is not well understood (reviewed in Schneggenburger \& Neher, 2005). Our theoretical work and analysis of SGN responses to pure tone bursts in vivo suggested that multiple $\mathrm{Ca}^{2+}$-binding sites of the sensor of exocytosis at IHC ribbon synapses is one of the prerequisites for encoding a wide range of sound amplitudes by the auditory system. Indeed, as we indicated in chapter 4, the dynamic range of the voltage activation of presynaptic $\mathrm{Ca}^{2+}$ channels is as wide as the range of IHC receptor potential values. Thus, the only way to have SGNs with contrasting RLF thresholds arising from the same IHC is to make the dynamic ranges of the dependencies of the presynaptic vesicle 
release rate on $V_{m}$ severalfold smaller than the voltage activation ranges of the $\mathrm{Ca}^{2+}$ channels. In the framework of our biophysical model of IHC ribbon synapses, the above requirement can be met only if the vesicle fusion is triggered by binding of multiple $\mathrm{Ca}^{2+}$ ions to the sensor of exocytosis. These findings motivate further studies of the molecular identity and $\mathrm{Ca}^{2+}$ binding properties of these proteins in IHCs.

\section{Nanodomain versus microdomain coupling}

We found that, given the same sets of the kinetic parameters and average $\left[\mathrm{Ca}^{2+}\right]$ at all the sensors of exocytosis at an AZ, RLFs produced by the nanodomain and microdomain coupling regimes do not coincide in general. However, it turned out that the sets of all possible RLFs corresponding to synapses with either of $\mathrm{Ca}^{2+}$ nanodomain or $\mathrm{Ca}^{2+}$ microdomain control of exocytosis are overlapping, to a good approximation. Importantly, this shows that there is no absolute need to use a superposition of many $\mathrm{Ca}^{2+}$ sources to sufficiently average out temporal single channel fluctuations. Thus, assuming no additional constraints, none of the two coupling regimes would have an advantage over the other, and the $\mathrm{Ca}^{2+}$-nanodomain coupling does not have an intrinsic disadvantage. In a real biological system, achieving some of the parameter values is likely to be metabolically more expensive or technically impossible compared to the others. Hence, a presynaptic site which results in an RLF with desirable properties might be more economical to implement in one of the coupling regimes than the other one. An obvious candidate to favor $\mathrm{Ca}^{2+}$ nanodomain coupling would be the metabolic cost, as a lower number of $\mathrm{Ca}^{2+}$ channels implies a reduced effort for $\mathrm{Ca}^{2+}$-extrusion and homeostasis.

\subsubsection{Heterogeneity of spike train statistics}

How well information is encoded in spike trains of neurons depends not only on their tuning curves but also on the spike statistics (Rieke et al., 1999). Thus, besides RLFs, we also studied interspike interval (ISI) distributions and correlations of SGNs in chapter 4 of this dissertation.

\section{Interspike interval distributions}

Two types of ISI distributions were identified in experimental studies of SGN responses to high-frequency pure tone bursts or spontaneous activity. For the majority of SGNs, the ISI distributions can be reproduced by convolving a presumed Poisson like vesicle release with SGN refractoriness (Li \& Young, 1993; Prijs et al., 1993; Heil et al., 2007; Pangršič et al., 2015). However, a small fraction of SGNs exhibit spike trains with a relatively high fraction of ISIs that are substantially smaller than the average ISI. Qualitatively, this manifests by an increased tendency of spikes to form small clusters as compared to the first group of ISI distributions (Gaumond et al., 1982; Teich \& Khanna, 1985; Li \& Young, 1993; Prijs et al., 1993). The mechanism underlying this phenomenon is not known. 
Our theoretical analysis of the biophysical model of presynaptic AZs provided a straightforward explanation for a high abundance of SGNs with the ISI distributions accounted for by the Poisson model. We showed that the presynaptic vesicle release converges to a homogeneous Poisson process with increased number of vesicular release sites fast. In the larger part of the kinetic parameter space, the Poisson approximation works well already for AZs with as few as ten vesicular release sites, even if exocytosis at individual release sites deviates from a Poisson process substantially. Indeed, we demonstrated that no considerable improvement in fitting ISI distributions of spike trains of maximally driven mice SGNs could be achieved with our biophysical model compared to the Poisson model.

The capacity of the Poisson model to describe the ISI distributions has been questioned in previous works (Heil et al., 2007; Peterson et al., 2014). The authors of the first study noted that fitting ISI distributions of spontaneous spiking from cat SGNs by the Poisson model resulted in unrealistically large estimates of the refractory periods ( $\sim 3 \mathrm{~ms}$ on average). Suspiciously large values of the refractory periods were also reported by other studies based on this model (Young \& Barta, 1986; Prijs et al., 1993; Buran et al., 2010). We could resolve this discrepancy in chapter 3 of this thesis by noting that, due to a particular symmetry of the Poisson model, there always exist two different values of the refractory period which result in exactly the same ISI distribution. It turned out that the larger of the two values of the refractory period always required unrealistically high presynaptic vesicle release rates, which might exceed the experimental estimates up to 10 -fold, in mice. When only the smaller fitting refractory period value was used, estimates fell within a physiologically plausible range $(0.75-2 \mathrm{~ms}$, with average of $1 \mathrm{~ms}$ ). If we had chosen the larger value of the refractory period, the overall refractory period would instead have been $3.3 \mathrm{~ms}$ on average.

It has to be noted that, despite the fact that the Poisson model can account for ISI distributions for a certain population of SGNs, it ignores the presence of ISI correlations by definition. As we discuss in the next section, ISI correlations are present, but are weak in biological situations and the Poisson model describes the spike process, to a first approximation, well. Nevertheless, ISI correlations, in spite of being small, contain valuable information about the structure of the presynaptic sites and are worth studying on their own, as detailed in the next section.

Our modeling results also suggested a potential explanation for the second class of ISI distributions of SGNs, which show an increased fraction of very short ISIs. We could reproduce representative experimental ISI probability density functions when fitting was performed assuming the nanodomain coupling between $\mathrm{Ca}^{2+}$ channels and sensors and exocytosis. An alternative explanation provided in a previous modeling study (Miller \& Wang, 1993) associated this type of SGN spiking to the intrinsic properties of SGNs. Hopefully, these two orthogonal scenarios will be tested quantitatively in future experimental studies. 


\section{Interspike interval correlations}

Correlations between two subsequent ISIs that are significantly different from zero were reported in spontaneous spike trains of SGNs from cats (Lowen \& Teich, 1992; Peterson et al., 2014) and guinea pigs (Prijs et al., 1993) SGNs. Besides these studies, the ISI correlations have been largely ignored in the literature, likely due to their relatively small size and the success of the Poisson model, which by definition assumes no correlations, to describe the ISI distributions of the majority of SGNs. In chapter 4, we showed theoretically that, compared to RLFs and probability density functions of ISIs, the correlations carry valuable and unique information about the organization of presynaptic AZs. This fact alone makes ISI correlations a feature worth studying, even if, due their small size, they do not significantly shape coding properties of SGNs.

We found that, in the framework of our biophysical model, ISI correlations can be negative and positive, as previously shown experimentally. Interestingly, the positive values are possible only in the regime of $\mathrm{Ca}^{2+}$ nanodomain coupling between $\mathrm{Ca}^{2+}$ channels and sensors of exocytosis, and only if the presynaptic vesicle release is bursty. We predicted computationally that, for the bursty spike trains observed in previous works, the correlation between two subsequent ISIs should be positive, but very weak $(\leq 0.01)$. It was proposed by (Peterson et al., 2014) that the positive correlations which they found in cat SGNs could be predominantly due to the non-stationarity of the spiking. Though this is a likely explanation for SGNs with the correlation coefficients of $>0.05$, our results demonstrate that weak positive ISI correlations are biophysically possible even in a completely stationary situation.

Negative correlations between subsequent ISIs were documented in the majority of SGNs in previous studies, with values between -0.1 and 0 (Prijs et al., 1993; Peterson et al., 2014). Our biophysical model could well reproduce these estimates. We found that AZs which feature either $\mathrm{Ca}^{2+}$ nanodomain or $\mathrm{Ca}^{2+}$ microdomain coupling regimes can generate negative correlations of ISIs. Differently from the positive ISI correlations, the negative ones are associated with presynaptic release patterns more regular than a Poisson process $(C V<1)$. In the case of $\mathrm{Ca}^{2+}$ nanodomain coupling, negative correlations are compatible with the working regime of the synapse when channel gating is either much faster or, at least, not slower than $\mathrm{Ca}^{2+}$ binding to the sensors of exocytosis. As discussed in the previous sections, any possible disadvantages of the signal processing due to the channel gating noise are bypassed in this regime. Interestingly, we found that the decay of ISI serial correlation functions becomes slower once the number of vesicular release sites per AZ is increased, despite the decreased magnitude of the correlations. Moreover, if ISIs are negative, AZs with only a few vesicular sites have ISI correlation functions with a pronounced oscillatory decay. These features make the serial correlation functions more informative of the number of the vesicular release sites at the presynaptic AZs compared to ISI distributions.

In chapter 4, we studied ISI correlations in spike trains of mouse SGNs of high CF that were driven to maximum rates by pure tone sound bursts. We confirmed that, like in the case 
of spontaneous activity (reported in previous studies), ISIs of driven responses are correlated too. In the sample of 72 SGNs studied by us, correlation coefficients between two subsequent ISIs were predominantly negative, in the range from -0.15 to 0 , with an average value of 0.06. Nevertheless, we found that the estimators of the correlation coefficients of ISIs are considerably more sensitive to the length of the measurement window and the overall number of ISIs, compared to other measures based on the second order moments, like the coefficient of variation. It turned out that a conclusive interpretation of the heterogeneity of these values in our data sample was impossible, though we showed that the correlations are statistically significant. The same problem prohibited us from studying serial ISI correlation functions instead of estimating only correlations between subsequent spikes. Our estimates were based on SGN responses to 200 repetitive, short $(100 \mathrm{~ms})$ tone bursts. This protocol is adapted for estimating spike rates and is standard in studies of SGN responses in vivo. We estimated that, in order to reduce the bias and uncertainty of the estimates of ISI correlations to a negligible level, such that the information about the heterogeneity of the responses and full ISI serial correlation functions of ISIs can be extracted, the stimulation window should be increased to $500 \mathrm{~ms}$. More generally, this requirement can be stated as $\sim 10^{2}$ ISIs per repetition $\sim 2 \cdot 10^{4}$ ISIs in total.

In view of their unexplored features and the distinct information carried about the IHC ribbon synapses, ISI serial correlation functions are one of the response characteristics of SGNs that should be addressed in more detail in future works. 


\section{Bibliography}

[1] E. Adler, G. Augustine, S. Duffy, and M. Charlton. Alien intracellular calcium chelators attenuate neurotransmitter release at the squid giant synapse. The Journal of Neuroscience, 11(6):1496 -1507 , June 1991.

[2] J. A. Assad, G. M. G. Shepherd, and D. P. Corey. Tip-link integrity and mechanical transduction in vertebrate hair cells. Neuron, 7(6):985-994, Dec. 1991.

[3] G. J. Augustine, E. M. Adler, and M. P. Charlton. The calcium signal for transmitter secretion from presynaptic nerve terminals. Annals of the New York Academy of Sciences, 635(1):365-381, Oct. 1991.

[4] G. J. Augustine, F. Santamaria, and K. Tanaka. Local calcium signaling in neurons. Neuron, 40(2):331-346, Oct. 2003.

[5] M. Avissar, A. C. Furman, J. C. Saunders, and T. D. Parsons. Adaptation reduces spikecount reliability, but not spike-timing precision, of auditory nerve responses. The Journal of Neuroscience, 27(24):6461-6472, June 2007.

[6] D. Baur, G. Bornschein, D. Althof, M. Watanabe, A. Kulik, J. Eilers, and H. Schmidt. Developmental tightening of cerebellar cortical synaptic influx-release coupling. The Journal of Neuroscience, 35(5):1858-1871, Feb. 2015.

[7] G. Bekesy. Experiments in hearing. McGraw-Hill, New York, 1960.

[8] R. Bellman. Introduction to matrix analysis. McGraw-Hill, London, 1960.

[9] M. J. Berridge, M. D. Bootman, and H. L. Roderick. Calcium signalling: dynamics, homeostasis and remodelling. Nature Reviews Molecular Cell Biology, 4(7):517-529, July 2003.

[10] M. Beurg, M. G. Evans, C. M. Hackney, and R. Fettiplace. A large-conductance calcium-selective mechanotransducer channel in mammalian cochlear hair cells. The Journal of Neuroscience, 26(43):10992-11000, Oct. 2006.

[11] M. Beurg, R. Fettiplace, J.-H. Nam, and A. J. Ricci. Localization of inner hair cell mechanotransducer channels using high-speed calcium imaging. Nature Neuroscience, 12(5):553-558, May 2009.

[12] D. Beutner, T. Voets, E. Neher, and T. Moser. Calcium dependence of exocytosis and endocytosis at the cochlear inner hair cell afferent synapse. Neuron, 29(3):681-690, Mar. 2001. 
[13] B. Billups and I. D. Forsythe. Presynaptic mitochondrial calcium sequestration influences transmission at mammalian central synapses. The Journal of Neuroscience, 22(14):5840-5847, July 2002 .

[14] E. Borg, B. Engström, G. Linde, and K. Marklund. Eighth nerve fiber firing features in normalhearing rabbits. Hearing Research, 36(23):191-201, Nov. 1988.

[15] M. Bortolozzi, A. Lelli, and F. Mammano. Calcium microdomains at presynaptic active zones of vertebrate hair cells unmasked by stochastic deconvolution. Cell Calcium, 44(2):158-168, Aug. 2008.

[16] A. Brandt, D. Khimich, and T. Moser. Few Cav1.3 channels regulate the exocytosis of a synaptic vesicle at the hair cell ribbon synapse. The Journal of Neuroscience, 25(50):11577-11585, Dec. 2005 .

[17] A. Brandt, J. Striessnig, and T. Moser. Cav1.3 channels are essential for development and presynaptic activity of cochlear inner hair cells. The Journal of Neuroscience, 23(34):1083210840, Nov. 2003.

[18] I. Bucurenciu, A. Kulik, B. Schwaller, M. Frotscher, and P. Jonas. Nanodomain coupling between $\mathrm{Ca}^{2+}$ channels and $\mathrm{Ca}^{2+}$ sensors promotes fast and efficient transmitter release at a cortical GABAergic synapse. Neuron, 57(4):536-545, Feb. 2008.

[19] A. H. Bunt. Enzymatic digestion of synaptic ribbons in amphibian retinal photoreceptors. Brain Research, 25(3):571-577, Feb. 1971.

[20] O. Caillard, H. Moreno, B. Schwaller, I. Llano, M. R. Celio, and A. Marty. Role of the calciumbinding protein parvalbumin in short-term synaptic plasticity. Proceedings of the National Academy of Sciences, 97(24):13372-13377, Nov. 2000.

[21] N. M. Chapochnikov, H. Takago, C.-H. Huang, T. Pangršič, D. Khimich, J. Neef, E. Auge, F. Göttfert, S. W. Hell, C. Wichmann, F. Wolf, and T. Moser. Uniquantal release through a dynamic fusion pore is a candidate mechanism of hair cell exocytosis. Neuron, 83(6):1389-1403, Sept. 2014.

[22] A. R. Cody and I. J. Russell. The response of hair cells in the basal turn of the guinea-pig cochlea to tones. The Journal of Physiology, 383(1):551-569, Feb. 1987.

[23] J. S. Coggan, T. M. Bartol, E. Esquenazi, J. R. Stiles, S. Lamont, M. E. Martone, D. K. Berg, M. H. Ellisman, and T. J. Sejnowski. Evidence for ectopic neurotransmission at a neuronal synapse. Science, 309(5733):446-451, July 2005.

[24] P. R. S. Cohen. Cell Biology of the Synapse. In D. W. Pfaff, editor, Neuroscience in the 21st Century, pages 309-349. Springer New York, 2013.

[25] S. H. Colburn, L. H. Carney, and M. G. Heinz. Quantifying the information in auditorynerve responses for level discrimination. JARO: Journal of the Association for Research in Otolaryngology, 4(3):294-311, Sept. 2003. 
[26] N. P. Cooper and G. K. Yates. Nonlinear input-output functions derived from the responses of guinea-pig cochlear nerve fibres: Variations with characteristic frequency. Hearing Research, 78(2):221-234, Aug. 1994.

[27] D. P. Corey and A. J. Hudspeth. Response latency of vertebrate hair cells. Biophysical Journal, 26(3):499-506, June 1979.

[28] D. Cox. Renewal theory. Science Paperbacks, Great Britain, 1962.

[29] G. Cui, A. C. Meyer, I. Calin-Jageman, J. Neef, F. Haeseleer, T. Moser, and A. Lee. Ca ${ }^{2+}$ binding proteins tune $\mathrm{Ca}^{2+}$-feedback to Cav1.3 channels in mouse auditory hair cells. The Journal of Physiology, 585(3):791-803, Dec. 2007.

[30] P. Dallos. Response characteristics of mammalian cochlear hair cells. The Journal of Neuroscience, 5(6):1591-1608, June 1985.

[31] K. N. Darrow, S. F. Maison, and M. C. Liberman. Cochlear efferent feedback balances interaural sensitivity. Nature Neuroscience, 9(12):1474-1476, Dec. 2006.

[32] K. N. Darrow, S. F. Maison, and M. C. Liberman. Selective removal of lateral olivocochlear efferents increases vulnerability to acute acoustic injury. Journal of Neurophysiology, 97(2):17751785, Feb. 2007.

[33] R. L. Davis and Q. Liu. Complex primary afferents: What the distribution of electrophysiologically-relevant phenotypes within the spiral ganglion tells us about peripheral neural coding. Hearing Research, 276(12):34-43, June 2011.

[34] B. Delgutte. Peripheral auditory processing of speech information: implications from a physiological study of intensity discrimination. In M. E. H. Schouten, editor, The psychophysics of speech perception, number 39 in NATO ASI Series, pages 333-353. Springer Netherlands, 1987.

[35] W. Denk, J. R. Holt, G. M. G. Shepherd, and D. P. Corey. Calcium imaging of single stereocilia in hair cells: Localization of transduction channels at both ends of tip links. Neuron, 15(6):13111321, Dec. 1995.

[36] A. G. Dimitrov, A. A. Lazar, and J. D. Victor. Information theory in neuroscience. Journal of Computational Neuroscience, 30(1):1-5, Jan. 2011.

[37] S. V. Duncker, C. Franz, S. Kuhn, U. Schulte, D. Campanelli, N. Brandt, B. Hirt, B. Fakler, N. Blin, P. Ruth, J. Engel, W. Marcotti, U. Zimmermann, and M. Knipper. Otoferlin couples to clathrin-mediated endocytosis in mature cochlear inner hair cells. The Journal of Neuroscience, 33(22):9508-9519, May 2013.

[38] B. Edmonds, R. Reyes, B. Schwaller, and W. M. Roberts. Calretinin modifies presynaptic calcium signaling in frog saccular hair cells. Nature Neuroscience, 3(8):786-790, Aug. 2000.

[39] E. Eggermann, I. Bucurenciu, S. P. Goswami, and P. Jonas. Nanodomain coupling between $\mathrm{Ca}^{2+}$ channels and sensors of exocytosis at fast mammalian synapses. Nat Rev Neurosci, 13(1):7-21, Jan. 2012. 
[40] E. Eggermann and P. Jonas. How the "slow" $\mathrm{Ca}^{2+}$ buffer parvalbumin affects transmitter release in nanodomain-coupling regimes. Nature Neuroscience, 15(1):20-22, Jan. 2012.

[41] S. Elis F. Single calcium channels and acetylcholine release at a presynaptic nerve terminal. Neuron, 11(6):1007-1011, Dec. 1993.

[42] E. F. Evans and A. R. Palmer. Relationship between the dynamic range of cochlear nerve fibres and their spontaneous activity. Experimental Brain Research, 40(1):115-118, Aug. 1980.

[43] M. Eybalin. Neurotransmitters and neuromodulators of the mammalian cochlea. Physiological Reviews, 73(2):309-373, Apr. 1993.

[44] R. R. Fay, A. N. Popper, R. R. Fay, and A. N. Popper, editors. Comparative hearing: mammals, volume 4 of Springer handbook of auditory research. Springer New York, New York, NY, 1994.

[45] M. J. Fedchyshyn and L.-Y. Wang. Developmental transformation of the release modality at the calyx of Held synapse. The Journal of Neuroscience, 25(16):4131-4140, Apr. 2005.

[46] T. Frank, D. Khimich, A. Neef, and T. Moser. Mechanisms contributing to synaptic $\mathrm{Ca}^{2+}$ signals and their heterogeneity in hair cells. Proceedings of the National Academy of Sciences of the United States of America, 106(11):4483-4488, Mar. 2009.

[47] T. Frank, M. A. Rutherford, N. Strenzke, A. Neef, T. Pangršič, D. Khimich, A. Fejtova, E. D. Gundelfinger, M. C. Liberman, B. Harke, K. E. Bryan, A. Lee, A. Egner, D. Riedel, and T. Moser. Bassoon and the synaptic ribbon organize $\mathrm{Ca}^{2+}$ channels and vesicles to add release sites and promote refilling. Neuron, 68(4):724-738, Nov. 2010.

[48] T. Furukawa, Y. Hayashida, and S. Matsuura. Quantal analysis of the size of excitatory postsynaptic potentials at synapses between hair cells and afferent nerve fibres in goldfish. The Journal of Physiology, 276(1):211-226, Mar. 1978.

[49] T. J. Garfinkle and J. C. Saunders. Morphology of inner hair cell stereocilia in C57bl/6j mice as studied by scanning electron microscopy. Otolaryngology - Head and Neck Surgery, 91(4):421426, Aug. 1983.

[50] R. P. Gaumond, C. E. Molnar, and D. O. Kim. Stimulus and recovery dependence of cat cochlear nerve fiber spike discharge probability. Journal of neurophysiology, 48(3):856-873, Sept. 1982.

[51] D. T. Gillespie. Exact stochastic simulation of coupled chemical reactions. The Journal of Physical Chemistry, 81(25):2340-2361, Dec. 1977.

[52] E. Glowatzki and P. A. Fuchs. Transmitter release at the hair cell ribbon synapse. Nature Neuroscience, 5(2):147-154, Jan. 2002.

[53] D. Goodman, R. Smith, and S. Chamberlain. Intracellular and extracellular responses in the organ of Corti of the gerbil. Hearing Research, 7(2):161-179, July 1982. 
[54] J. D. Goutman and E. Glowatzki. Time course and calcium dependence of transmitter release at a single ribbon synapse. Proceedings of the National Academy of Sciences of the United States of America, 104(41):16341-16346, Oct. 2007.

[55] L. Grant, E. Yi, and E. Glowatzki. Two modes of release shape the postsynaptic response at the inner hair cell ribbon synapse. The Journal of Neuroscience, 30(12):4210-4220, Mar. 2010.

[56] C. W. Graydon, S. Cho, G.-L. Li, B. Kachar, and H. Von Gersdorff. Sharp $\mathrm{Ca}^{2+}$ nanodomains beneath the ribbon promote highly synchronous multivesicular release at hair cell synapses. The Journal of Neuroscience, 31(46):16637-16650, Nov. 2011.

[57] C. W. Graydon, J. Zhang, N. W. Oesch, A. A. Sousa, R. D. Leapman, and J. S. Diamond. Passive diffusion as a mechanism underlying ribbon synapse vesicle release and resupply. The Journal of Neuroscience, 34(27):8948-8962, July 2014.

[58] J. A. Groff and M. C. Liberman. Modulation of cochlear afferent response by the lateral olivocochlear system: activation via electrical stimulation of the inferior colliculus. Journal of Neurophysiology, 90(5):3178-3200, Nov. 2003.

[59] C. M. Hackney, S. Mahendrasingam, A. Penn, and R. Fettiplace. The concentrations of calcium buffering proteins in mammalian cochlear hair cells. The Journal of Neuroscience, 25(34):78677875, Aug. 2005.

[60] M. L. Harlow, D. Ress, A. Stoschek, R. M. Marshall, and U. J. McMahan. The architecture of active zone material at the frog's neuromuscular junction. Nature, 409(6819):479-484, Jan. 2001.

[61] R. S. Heffner and H. E. Heffner. Hearing range of the domestic cat. Hearing Research, 19(1):8588, Jan. 1985.

[62] R. S. Heffner, G. Koay, and H. E. Heffner. Audiograms of five species of rodents: implications for the evolution of hearing and the perception of pitch. Hearing Research, 157(12):138-152, July 2001.

[63] P. Heil, H. Neubauer, and D. R. F. Irvine. An improved model for the rate-level functions of auditory-nerve fibers. The Journal of Neuroscience, 31(43):15424-15437, Oct. 2011.

[64] P. Heil, H. Neubauer, D. R. F. Irvine, and M. Brown. Spontaneous activity of auditory-nerve fibers: insights into stochastic processes at ribbon synapses. The Journal of Neuroscience, $27(31): 8457-8474,2007$.

[65] S. Heller, A. M. Bell, C. S. Denis, Y. Choe, and A. J. Hudspeth. Parvalbumin 3 is an abundant Ca2+ buffer in hair cells. Journal of the Association for Research in Otolaryngology, 3(4):488498, Dec. 2002.

[66] J. E. Heuser, T. S. Reese, M. J. Dennis, Y. Jan, L. Jan, and L. Evans. Synaptic vesicle exocytosis captured by quick freezing and correlated with quantal transmitter release. The Journal of Cell Biology, 81(2):275 -300, May 1979. 
[67] W. A. Hossain, S. D. Antic, Y. Yang, M. N. Rasband, and D. K. Morest. Where is the spike generator of the cochlear nerve? Voltage-gated sodium channels in the mouse cochlea. The Journal of Neuroscience, 25(29):6857-6868, July 2005.

[68] A. J. Hudspeth and D. P. Corey. Sensitivity, polarity, and conductance change in the response of vertebrate hair cells to controlled mechanical stimuli. Proceedings of the National Academy of Sciences, 74(6):2407-2411, June 1977.

[69] N. P. Issa and A. J. Hudspeth. The entry and clearance of $\mathrm{Ca}^{2+}$ at individual presynaptic active zones of hair cells from the bullfrog's sacculus. Proceedings of the National Academy of Sciences, 93(18):9527-9532, Sept. 1996.

[70] P. R. Jahn and D. J. Boyken. Molecular regulation of synaptic release. In D. W. Pfaff, editor, Neuroscience in the 21st century, pages 351-401. Springer New York, 2013.

[71] R. Jahn and D. Fasshauer. Molecular machines governing exocytosis of synaptic vesicles. Nature, 490(7419):201-207, Oct. 2012.

[72] T. Jarsky, M. Tian, and J. H. Singer. Nanodomain control of exocytosis is responsible for the signaling capability of a retinal ribbon synapse. Journal of Neuroscience, 30(36):11885-11895, Sept. 2010.

[73] Z. Jing, M. A. Rutherford, H. Takago, T. Frank, A. Fejtova, D. Khimich, T. Moser, and N. Strenzke. Disruption of the presynaptic cytomatrix protein bassoon degrades ribbon anchorage, multiquantal release, and sound encoding at the hair cell afferent synapse. The Journal of Neuroscience, 33(10):4456-4467, Mar. 2013.

[74] D. H. Johnson. The relationship between spike rate and synchrony in responses of auditory-nerve fibers to single tones. The Journal of the Acoustical Society of America, 68(4):1115, 1980.

[75] S. L. Johnson, A. Forge, M. Knipper, S. Münkner, and W. Marcotti. Tonotopic variation in the calcium dependence of neurotransmitter release and vesicle pool replenishment at mammalian auditory ribbon synapses. The Journal of Neuroscience, 28(30):7670-7678, July 2008.

[76] S. L. Johnson and W. Marcotti. Biophysical properties of Cav1.3 calcium channels in gerbil inner hair cells. The Journal of Physiology, 586(4):1029-1042, Feb. 2008.

[77] S. L. Johnson, W. Marcotti, and C. J. Kros. Increase in efficiency and reduction in $\mathrm{Ca}^{2+}$ dependence of exocytosis during development of mouse inner hair cells. The Journal of Physiology, 563(1):177-191, Feb. 2005.

[78] S. Jung, T. Maritzen, C. Wichmann, Z. Jing, A. Neef, N. H. Revelo, H. Al-Moyed, S. Meese, S. M. Wojcik, I. Panou, H. Bulut, P. Schu, R. Ficner, E. Reisinger, S. O. Rizzoli, J. Neef, N. Strenzke, V. Haucke, and T. Moser. Disruption of adaptor protein 2 (AP-2) in cochlear hair cells impairs vesicle reloading of synaptic release sites and hearing. The EMBO Journal, Oct. 2015 . 
[79] S. Jung, T. Oshima-Takago, R. Chakrabarti, A. B. Wong, Z. Jing, G. Yamanbaeva, M. M. Picher, S. M. Wojcik, F. Göttfert, F. Predoehl, K. Michel, S. W. Hell, S. Schoch, N. Strenzke, C. Wichmann, and T. Moser. Rab3-interacting molecules $2 \alpha$ and $2 \beta$ promote the abundance of voltage-gated $\mathrm{Ca}_{\mathrm{V}} 1.3 \mathrm{Ca}^{2+}$ channels at hair cell active zones. Proceedings of the National Academy of Sciences, page 201417207, June 2015.

[80] D. Kamin, N. H. Revelo, and S. O. Rizzoli. FM dye photo-oxidation as a tool for monitoring membrane recycling in inner hair cells. PLoS ONE, 9(2):e88353, Feb. 2014.

[81] K. Kang and H. Sompolinsky. Mutual information of population codes and distance measures in probability space. Physical Review Letters, 86(21):4958-4961, May 2001.

[82] A. Kantardzhieva, M. C. Liberman, and W. F. Sewell. Quantitative analysis of ribbons, vesicles, and cisterns at the cat inner hair cell synapse: correlations with spontaneous rate. Journal of Comparative Neurology, 521(14):3260-3271, 2013.

[83] Y. Katsuki, N. Suga, and Y. Kanno. Neural mechanism of the peripheral and central auditory system in monkeys. The Journal of the Acoustical Society of America, 34(9B):1396-1410, Sept. 1962.

[84] P. Kazmierczak and U. Müller. Sensing sound: molecules that orchestrate mechanotransduction by hair cells. Trends in Neurosciences, 35(4):220-229, Apr. 2012.

[85] D. Khimich, R. Nouvian, R. Pujol, S. tom Dieck, A. Egner, E. D. Gundelfinger, and T. Moser. Hair cell synaptic ribbons are essential for synchronous auditory signalling. Nature, 434(7035):889-894, Apr. 2005.

[86] A. J. Khintchine. Mathematical methods in the theory of queueing. Griffin, London, 1960.

[87] N. Kiang, T. Watanabe, E. Thomas, and T. Clark. Discharge patterns of single fibers in the cat's auditory nerve. Cambridge, MA: MIT, 1965.

[88] N. Y. Kiang, J. M. Rho, C. C. Northrop, M. C. Liberman, and D. K. Ryugo. Hair-cell innervation by spiral ganglion cells in adult cats. Science, 217(4555):175-177, July 1982.

[89] M.-H. Kim, N. Korogod, R. Schneggenburger, W.-K. Ho, and S.-H. Lee. Interplay between $\mathrm{Na}^{+} / \mathrm{Ca}^{2+}$ exchangers and mitochondria in $\mathrm{Ca}^{2+}$ clearance at the calyx of Held. The Journal of Neuroscience, 25(26):6057-6065, June 2005.

[90] G. A. Korn and T. M. Korn. Mathematical handbook for scientists and engineers. McGraw-Hill, New York, 1968.

[91] A. S. Kozlov, T. Risler, and A. J. Hudspeth. Coherent motion of stereocilia assures the concerted gating of hair-cell transduction channels. Nature Neuroscience, 10(1):87-92, Jan. 2007.

[92] C. J. Kros and A. C. Crawford. Potassium currents in inner hair cells isolated from the guinea-pig cochlea. The Journal of Physiology, 421(1):263-291, Feb. 1990. 
[93] C. J. Kros, J. P. Ruppersberg, and A. Rüsch. Expression of a potassium current in inner hair cells during development of hearing in mice. Nature, 394(6690):281-284, July 1998.

[94] D. Lee, K.-H. Lee, W.-K. Ho, and S.-H. Lee. Target cell-specific involvement of presynaptic mitochondria in post-tetanic potentiation at hippocampal mossy fiber synapses. The Journal of Neuroscience, 27(50):13603-13613, Dec. 2007.

[95] S.-H. Lee, M.-H. Kim, J.-Y. Lee, S. H. Lee, D. Lee, K. H. Park, and W.-K. Ho. Na ${ }^{+} / \mathrm{Ca}^{2+}$ exchange and $\mathrm{Ca}^{2+}$ homeostasis in axon terminals of mammalian central neurons. Annals of the New York Academy of Sciences, 1099(1):396-412, Mar. 2007.

[96] A. Lelli, P. Perin, M. Martini, C. D. Ciubotaru, I. Prigioni, P. Valli, M. L. Rossi, and F. Mammano. Presynaptic calcium stores modulate afferent release in vestibular hair cells. The Journal of Neuroscience, 23(17):6894-6903, July 2003.

[97] D. Lenzi, J. Crum, M. H. Ellisman, and W. M. Roberts. Depolarization redistributes synaptic membrane and creates a gradient of vesicles on the synaptic body at a ribbon synapse. Neuron, 36(4):649-659, Nov. 2002.

[98] D. Lenzi, J. W. Runyeon, J. Crum, M. H. Ellisman, and W. M. Roberts. Synaptic vesicle populations in saccular hair cells reconstructed by electron tomography. The Journal of Neuroscience, 19(1):119-132, Jan. 1999.

[99] D. Lenzi and H. von Gersdorff. Structure suggests function: the case for synaptic ribbons as exocytotic nanomachines. BioEssays, 23(9):831-840, Sept. 2001.

[100] J. Li and E. D. Young. Discharge-rate dependence of refractory behavior of cat auditory-nerve fibers. Hearing Research, 69(12):151-162, Sept. 1993.

[101] L. D. Liberman, H. Wang, and M. C. Liberman. Opposing gradients of ribbon size and AMPA receptor expression underlie sensitivity differences among cochlear-nerve/hair-cell synapses. The Journal of Neuroscience, 31(3):801-808, Jan. 2011.

[102] M. Liberman. Morphological differences among radial afferent fibers in the cat cochlea: An electron-microscopic study of serial sections. Hearing Research, 3(1):45-63, July 1980.

[103] M. C. Liberman. Auditory-nerve response from cats raised in a low-noise chamber. The Journal of the Acoustical Society of America, 63(2):442-455, 1978.

[104] M. C. Liberman. The cochlear frequency map for the cat: Labeling auditorynerve fibers of known characteristic frequency. The Journal of the Acoustical Society of America, 72(5):14411449, Nov. 1982.

[105] M. C. Liberman. Single-neuron labeling in the cat auditory nerve. Science, 216(4551):1239-1241, June 1982.

[106] M. C. Liberman. Effects of chronic cochlear de-efferentation on auditory-nerve response. Hearing Research, 49(1-3):209-223, Nov. 1990. 
[107] M. C. Liberman, L. W. Dodds, and S. Pierce. Afferent and efferent innervation of the cat cochlea: Quantitative analysis with light and electron microscopy. The Journal of Comparative Neurology, 301(3):443-460, 1990.

[108] D. J. Lim. Functional structure of the organ of Corti: a review. Hearing Research, 22(1):117-146, Jan. 1986.

[109] B. Lindner. Superposition of many independent spike trains is generally not a Poisson process. Physical Review E, 73(2):022901, Feb. 2006.

[110] Q. Liu, E. Lee, and R. L. Davis. Heterogeneous intrinsic excitability of murine spiral ganglion neurons is determined by Kv1 and HCN channels. Neuroscience, 257:96-110, Jan. 2014.

[111] G. R. Long. The microstructure of quiet and masked thresholds. Hearing Research, 15(1):73-87, July 1984.

[112] E. A. Lopez-Poveda and A. Eustaquio-Martn. A biophysical model of the inner hair cell: the contribution of potassium currents to peripheral auditory compression. JARO: Journal of the Association for Research in Otolaryngology, 7(3):218-235, Sept. 2006.

[113] S. B. Lowen and M. C. Teich. Auditory-nerve action potentials form a nonrenewal point process over short as well as long time scales. The Journal of the Acoustical Society of America, 92(2):803-806, Aug. 1992.

[114] E. A. Lumpkin, R. E. Marquis, and A. J. Hudspeth. The selectivity of the hair cells mechanoelectrical-transduction channel promotes $\mathrm{Ca}^{2+}$ flux at low $\mathrm{Ca}^{2+}$ concentrations. Proceedings of the National Academy of Sciences, 94(20):10997-11002, Sept. 1997.

[115] F. Luo, M. Dittrich, J. R. Stiles, and S. D. Meriney. Single-pixel optical fluctuation analysis of calcium channel function in active zones of motor nerve terminals. The Journal of Neuroscience, 31(31):11268-11281, Aug. 2011.

[116] V. G. Magupalli, K. Schwarz, K. Alpadi, S. Natarajan, G. M. Seigel, and F. Schmitz. Multiple RIBEYE-RIBEYE interactions create a dynamic scaffold for the formation of synaptic ribbons. The Journal of Neuroscience, 28(32):7954-7967, Aug. 2008.

[117] A. Manalan and C. Klee. Calmodulin. Advances in Cyclic Nucleotide and Protein Phosphorylation Research, 18:227-278, 1984.

[118] D. C. Marcus, T. Wu, P. Wangemann, and P. Kofuji. KCNJ10 (Kir4.1) potassium channel knockout abolishes endocochlear potential. American Journal of Physiology - Cell Physiology, 282(2):C403-C407, Feb. 2002.

[119] E. A. Matthews and D. Dietrich. Buffer mobility and the regulation of neuronal calcium domains. Frontiers in Cellular Neuroscience, 9:48, 2015.

[120] G. Matthews and P. Fuchs. The diverse roles of ribbon synapses in sensory neurotransmission. Nat Rev Neurosci, 11(12):812-822, Dec. 2010. 
[121] V. Matveev, A. Sherman, and R. S. Zucker. New and corrected simulations of synaptic facilitation. Biophysical Journal, 83(3):1368-1373, Sept. 2002.

[122] M. Mauermann, G. R. Long, and B. Kollmeier. Fine structure of hearing threshold and loudness perception. The Journal of the Acoustical Society of America, 116(2):1066-1080, Aug. 2004.

[123] B. Mehta, J.-B. Ke, L. Zhang, A. D. Baden, A. L. Markowitz, S. Nayak, K. L. Briggman, D. Zenisek, and J. H. Singer. Global $\mathrm{Ca}^{2+}$ signaling drives ribbon-independent synaptic transmission at rod bipolar cell synapses. The Journal of Neuroscience, 34(18):6233-6244, Apr. 2014.

[124] C. J. Meinrenken, J. G. G. Borst, and B. Sakmann. Calcium secretion coupling at calyx of Held governed by nonuniform channel-vesicle topography. The Journal of Neuroscience, 22(5):16481667, Mar. 2002.

[125] A. Merchan-Perez and M. C. Liberman. Ultrastructural differences among afferent synapses on cochlear hair cells: Correlations with spontaneous discharge rate. The Journal of Comparative Neurology, 371(2):208-221, 1996.

[126] S. D. Meriney and M. Dittrich. Organization and function of transmitter release sites at the neuromuscular junction. The Journal of Physiology, 591(13):3159-3165, July 2013.

[127] A. C. Meyer, T. Frank, D. Khimich, G. Hoch, D. Riedel, N. M. Chapochnikov, Y. M. Yarin, B. Harke, S. W. Hell, A. Egner, and T. Moser. Tuning of synapse number, structure and function in the cochlea. Nature Neuroscience, 12(4):444-453, Apr. 2009.

[128] A. C. Meyer and T. Moser. Structure and function of cochlear afferent innervation. Current Opinion in Otolaryngology \& Head and Neck Surgery, 18(5):441-446, Oct. 2010.

[129] M. I. Miller and J. Wang. A new stochastic model for auditory-nerve discharge. The Journal of the Acoustical Society of America, 94(4):2093-2107, Oct. 1993.

[130] A. G. Moller. Hearing: its physiology and pathophysiology. Academic Press Inc, 2000.

[131] T. Moser and D. Beutner. Kinetics of exocytosis and endocytosis at the cochlear inner hair cell afferent synapse of the mouse. Proceedings of the National Academy of Sciences, 97(2):883 -888, Jan. 2000.

[132] M. Müller and D. Robertson. Relationship between tone burst discharge pattern and spontaneous firing rate of auditory nerve fibres in the guinea pig. Hearing Research, 57(1):63-70, Dec. 1991.

[133] M. Müller and D. Robertson. Shapes of rate-versus-level functions of primary auditory nerve fibres: Test of the basilar membrane mechanical hypothesis. Hearing Research, 57(1):71-78, Dec. 1991.

[134] U. Müller. Cadherins and mechanotransduction by hair cells. Current Opinion in Cell Biology, 20(5):557-566, Oct. 2008. 
[135] Y. Nakamura, H. Harada, N. Kamasawa, K. Matsui, J. S. Rothman, R. Shigemoto, R. A. Silver, D. A. DiGregorio, and T. Takahashi. Nanoscale distribution of presynaptic $\mathrm{Ca}^{2+}$ channels and its impact on vesicular release during development. Neuron, 85(1):145-158, Jan. 2015.

[136] S. Nakayama, H. Kawasaki, and R. Kretsinger. Evolution of EF-hand proteins. In Topics in Biological Inorganic Chemistry. Calcium homeostasis, volume 3, pages 29-58. 2000.

[137] M. Naraghi and E. Neher. Linearized buffered $\mathrm{Ca}^{2+}$ diffusion in microdomains and its implications for calculation of $\left[\mathrm{Ca}^{2+}\right]$ at the mouth of a calcium channel. The Journal of Neuroscience, 17(18):6961 -6973, 1997.

[138] A. Neef, D. Khimich, P. Pirih, D. Riedel, F. Wolf, and T. Moser. Probing the mechanism of exocytosis at the hair cell ribbon synapse. The Journal of Neuroscience, 27(47):12933-12944, Nov. 2007.

[139] J. Neef, A. Gehrt, A. V. Bulankina, A. C. Meyer, D. Riedel, R. G. Gregg, N. Strenzke, and T. Moser. The $\mathrm{Ca}^{2+}$ channel subunit $\beta 2$ regulates $\mathrm{Ca}^{2+}$ channel abundance and function in inner hair cells and is required for hearing. The Journal of Neuroscience, 29(34):10730-10740, Aug. 2009.

[140] J. Neef, S. Jung, A. B. Wong, K. Reuter, T. Pangršič, R. Chakrabarti, S. Kügler, C. Lenz, R. Nouvian, R. M. Boumil, W. N. Frankel, C. Wichmann, and T. Moser. Modes and regulation of endocytic membrane retrieval in mouse auditory hair cells. The Journal of Neuroscience, 34(3):705-716, Jan. 2014.

[141] E. Neher. Usefulness and limitations of linear approximations to the understanding of $\mathrm{Ca}^{2+}$ signals. Cell Calcium, 24(5-6):345-357, 1998.

[142] E. Neher and H. Taschenberger. Transients in global $\mathrm{Ca}^{2+}$ concentration induced by electrical activity in a giant nerve terminal. The Journal of Physiology, 591(13):3189-3195, July 2013.

[143] L. Nizami. Estimating auditory neuronal dynamic range using a fitted function. Hearing Research, 167(12):13-27, May 2002.

[144] R. Nobili, F. Mammano, and J. Ashmore. How well do we understand the cochlea? Trends in Neurosciences, 21(4):159-167, Apr. 1998.

[145] R. Nouvian, J. Neef, A. V. Bulankina, E. Reisinger, T. Pangršič, T. Frank, S. Sikorra, N. Brose, T. Binz, and T. Moser. Exocytosis at the hair cell ribbon synapse apparently operates without neuronal SNARE proteins. Nature Neuroscience, 14(4):411-413, Mar. 2011.

[146] K. K. Ohlemiller, S. M. Echteler, and J. H. Siegel. Factors that influence rate-versus-intensity relations in single cochlear nerve fibers of the gerbil. The Journal of the Acoustical Society of America, 90(1):274-287, 1991.

[147] T. L. Ohn, M. A. Rutherford, C. D. Alfonso, A. Scharinger, G. Hoch, and T. Moser. Positionspecific regulation of presynaptic function in cochlear inner hair cells. In preparation. 
[148] D. Oliver, M. Knipper, C. Derst, and B. Fakler. Resting potential and submembrane calcium concentration of inner hair cells in the isolated mouse cochlea are set by KCNQ-type potassium channels. The Journal of Neuroscience, 23(6):2141-2149, Mar. 2003.

[149] A. R. Palmer and I. J. Russell. Phase-locking in the cochlear nerve of the guinea-pig and its relation to the receptor potential of inner hair-cells. Hearing Research, 24(1):1-15, Jan. 1986.

[150] B. Pan, G. S. Gloc, Y. Asai, G. C. Horwitz, K. Kurima, K. Ishikawa, Y. Kawashima, A. J. Griffith, and J. R. Holt. TMC1 and TMC2 are components of the mechanotransduction channel in hair cells of the mammalian inner ear. Neuron, 79(3):504-515, Aug. 2013.

[151] Z. P. Pang and T. C. Südhof. Cell biology of $\mathrm{Ca}^{2+}$-triggered exocytosis. Current Opinion in Cell Biology, 22(4):496-505, Aug. 2010.

[152] T. Pangršič, M. Gabrielaitis, S. Michanski, B. Schwaller, F. Wolf, N. Strenzke, and T. Moser. EFhand protein $\mathrm{Ca}^{2+}$ buffers regulate $\mathrm{Ca}^{2+}$ influx and exocytosis in sensory hair cells. Proceedings of the National Academy of Sciences, 112(9):E1028-E1037, Mar. 2015.

[153] T. Pangršič, L. Lasarow, K. Reuter, H. Takago, M. Schwander, D. Riedel, T. Frank, L. M. Tarantino, J. S. Bailey, N. Strenzke, N. Brose, U. Müller, E. Reisinger, and T. Moser. Hearing requires otoferlin-dependent efficient replenishment of synaptic vesicles in hair cells. Nature Neuroscience, 13(7):869-876, June 2010.

[154] T. Pangršič, E. Reisinger, and T. Moser. Otoferlin: a multi-C2 domain protein essential for hearing. Trends in Neurosciences, 35(11):671-680, Nov. 2012.

[155] P. C. Pape, D. S. Jong, and W. K. Chandler. Calcium release and its voltage dependence in frog cut muscle fibers equilibrated with $20 \mathrm{mM}$ EGTA. The Journal of General Physiology, 106(2):259-336, Aug. 1995.

[156] R. Patuzzi and P. M. Sellick. A comparison between basilar membrane and inner hair cell receptor potential input-output functions in the guinea pig cochlea. The Journal of the Acoustical Society of America, 74(6):1734-1741, 1983.

[157] A. W. Peng, F. T. Salles, B. Pan, and A. J. Ricci. Integrating the biophysical and molecular mechanisms of auditory hair cell mechanotransduction. Nat Commun, 2:523, Nov. 2011.

[158] A. J. Peterson, D. R. F. Irvine, and P. Heil. A model of synaptic vesicle-pool depletion and replenishment can account for the interspike interval distributions and nonrenewal properties of spontaneous spike trains of auditory-nerve fibers. The Journal of Neuroscience, 34(45):1509715109, Nov. 2014.

[159] J. O. Pickles. An introduction to the physiology of hearing. Emerald Group Publishing Ltd., Bingley, 3rd edition, 2008.

[160] J. O. Pickles, S. D. Comis, and M. P. Osborne. Cross-links between stereocilia in the guinea pig organ of Corti, and their possible relation to sensory transduction. Hearing Research, 15(2):103112, Aug. 1984. 
[161] J. Platzer, J. Engel, A. Schrott-Fischer, K. Stephan, S. Bova, H. Chen, H. Zheng, and J. Striessnig. Congenital deafness and sinoatrial node dysfunction in mice lacking class D L-type $\mathrm{Ca}^{2+}$ channels. Cell, 102(1):89-97, July 2000.

[162] A. N. Popper, R. R. Fay, R. R. Fay, and A. N. Popper, editors. Hearing by bats, volume 5 of Springer handbook of auditory research. Springer New York, New York, NY, 1995.

[163] V. F. Prijs, J. Keijzer, H. Versnel, and R. Schoonhoven. Recovery characteristics of auditory nerve fibres in the normal and noise-damaged guinea pig cochlea. Hearing Research, 71(12):190201, Dec. 1993.

[164] D. Purves, G. J. Augustine, D. Fitzpatrick, W. C. Hall, A. LaMantia, J. O. McNamara, and M. S. Williams, editors. Neuroscience. Sinauer Associates, 3rd edition, 2004.

[165] N. P. Raybould, D. J. Jagger, and G. D. Housley. Positional analysis of guinea pig inner hair cell membrane conductances: implications for regulation of the membrane filter. JARO: Journal of the Association for Research in Otolaryngology, 2(4):362-376, Dec. 2001.

[166] H. Regus-Leidig, M. Fuchs, M. Löhner, S. R. Leist, S. Leal-Ortiz, V. A. Chiodo, W. W. Hauswirth, C. C. Garner, and J. H. Brandstätter. In vivo knockdown of Piccolino disrupts presynaptic ribbon morphology in mouse photoreceptor synapses. Frontiers in Cellular Neuroscience, 8, Sept. 2014.

[167] L. E. Reichl. A modern course in statistical physics. John Wiley and Sons, 2nd edition, 1998.

[168] E. M. Relkin and J. R. Doucet. Recovery from prior stimulation. I: Relationship to spontaneous firing rates of primary auditory neurons. Hearing Research, 55(2):215-222, Oct. 1991.

[169] N. H. Revelo, D. Kamin, S. Truckenbrodt, A. B. Wong, K. Reuter-Jessen, E. Reisinger, T. Moser, and S. O. Rizzoli. A new probe for super-resolution imaging of membranes elucidates trafficking pathways. The Journal of Cell Biology, 205(4):591-606, May 2014.

[170] W. S. Rhode and P. H. Smith. Characteristics of tone-pip response patterns in relationship to spontaneous rate in cat auditory nerve fibers. Hearing Research, 18(2):159-168, May 1985.

[171] A. Ricci, A. Crawford, and R. Fettiplace. Tonotopic variation in the conductance of the hair cell mechanotransducer channel. Neuron, 40(5):983-990, Dec. 2003.

[172] A. Ricci, H. Kennedy, A. Crawford, and R. Fettiplace. The transduction channel filter in auditory hair cells. Journal of Neuroscience, 25(34):7831-7839, Aug. 2005.

[173] F. Rieke, D. Warland, R. de Ruyter van Steveninck, and W. Bialek. Spikes: exploring the neural code. A Bradford Book, 1999.

[174] R. R. Riesz. Differential intensity sensitivity of the ear for pure tones. Physical Review, 31(5):867875, May 1928.

[175] W. M. Roberts. Spatial calcium buffering in saccular hair cells. Nature, 363(6424):74-76, May 1993. 
[176] W. M. Roberts. Localization of calcium signals by a mobile calcium buffer in frog saccular hair cells. The Journal of Neuroscience, 14(5):3246-3262, May 1994.

[177] D. Robertson. Centrifugal control in mammalian hearing. Clinical and Experimental Pharmacology and Physiology, 36(7):603-611, July 2009.

[178] D. Robertson and B. Paki. Role of L-type $\mathrm{Ca}^{2+}$ channels in transmitter release from mammalian inner hair cells. II. Single-neuron activity. Journal of Neurophysiology, 87(6):2734-2740, June 2002 .

[179] D. Robertson and S. A. Wilson. Changes in cochlear sensitivity do not alter relative thresholds of different spontaneous rate categories of primary auditory nerve fibres. Hearing Research, 51(1):29-32, Jan. 1991.

[180] L. Robles and M. A. Ruggero. Mechanics of the mammalian cochlea. Physiological Reviews, 81(3):1305-1352, July 2001.

[181] J. E. Rose, J. F. Brugge, D. J. Anderson, and J. E. Hind. Phase-locked response to lowfrequency tones in single auditory nerve fibers of the squirrel monkey. Journal of Neurophysiology, 30(4):769-793, July 1967.

[182] I. Roux, S. Safieddine, R. Nouvian, M. Grati, M.-C. Simmler, A. Bahloul, I. Perfettini, M. Le Gall, P. Rostaing, G. Hamard, A. Triller, P. Avan, T. Moser, and C. Petit. Otoferlin, defective in a human deafness form, is essential for exocytosis at the auditory ribbon synapse. Cell, 127(2):277-289, Oct. 2006.

[183] J. Ruel, R. Nouvian, C. G. D'Aldin, R. Pujol, M. Eybalin, and J.-L. Puel. Dopamine inhibition of auditory nerve activity in the adult mammalian cochlea. European Journal of Neuroscience, 14(6):977-986, Sept. 2001.

[184] I. Russell, A. Cody, and G. Richardson. The responses of inner and outer hair cells in the basal turn of the guinea-pig cochlea and in the mouse cochlea grown in vitro. Hearing Research, 22(13):199-216, 1986.

[185] I. J. Russell and P. M. Sellick. Intracellular studies of hair cells in the mammalian cochlea. The Journal of Physiology, 284(1):261-290, Nov. 1978.

[186] M. A. Rutherford, N. M. Chapochnikov, and T. Moser. Spike encoding of neurotransmitter release timing by spiral ganglion neurons of the cochlea. The Journal of Neuroscience, 32(14):4773-4789, Apr. 2012.

[187] M. A. Rutherford and T. Pangršič. Molecular anatomy and physiology of exocytosis in sensory hair cells. Cell Calcium, 52(34):327-337, Sept. 2012.

[188] M. B. Sachs and P. J. Abbas. Rate versus level functions for auditory-nerve fibers in cats: tone-burst stimuli. The Journal of the Acoustical Society of America, 56(6):1835-1847, 1974.

[189] M. B. Sachs, R. L. Winslow, and B. H. Sokolowski. A computational model for rate-level functions from cat auditory-nerve fibers. Hearing Research, 41(1):61-69, Aug. 1989. 
[190] E. Saftenku. Effects of calretinin on $\mathrm{Ca}^{2+}$ signals in cerebellar granule cells: implications of cooperative $\mathrm{Ca}^{2+}$ binding. The Cerebellum, 11(1):102-120, Mar. 2011.

[191] M. Sauer. Localization microscopy coming of age: from concepts to biological impact. Journal of Cell Science, 126(16):3505-3513, Aug. 2013.

[192] H. Schmidt, S. Brachtendorf, O. Arendt, S. Hallermann, S. Ishiyama, G. Bornschein, D. Gall, S. N. Schiffmann, M. Heckmann, and J. Eilers. Nanodomain coupling at an excitatory cortical synapse. Current Biology, 23(3):244-249, Feb. 2013.

[193] F. Schmitz. The making of synaptic ribbons: how they are built and what they do. The Neuroscientist, Aug. 2009.

[194] F. Schmitz, A. Königstorfer, and T. C. Südhof. RIBEYE, a component of synaptic ribbons: a protein's journey through evolution provides insight into synaptic ribbon function. Neuron, 28(3):857-872, Dec. 2000.

[195] R. Schneggenburger and E. Neher. Presynaptic calcium and control of vesicle fusion. Current Opinion in Neurobiology, 15(3):266-274, June 2005.

[196] E. Schneidman, M. J. Berry, R. Segev, and W. Bialek. Weak pairwise correlations imply strongly correlated network states in a neural population. Nature, 440(7087):1007-1012, Apr. 2006.

[197] M. R. Schroeder and J. L. Hall. Model for mechanical to neural transduction in the auditory receptor. The Journal of the Acoustical Society of America, 55(5):1055-1060, 1974.

[198] B. Schwaller. Cytosolic $\mathrm{Ca}^{2+}$ buffers. Cold Spring Harbor Perspectives in Biology, 2(11), Nov. 2010.

[199] B. Schwaller. The regulation of a cell's $\mathrm{Ca}^{2+}$ signaling toolkit: the $\mathrm{Ca}^{2+}$ homeostasome. In M. S. Islam, editor, Calcium signaling, pages 1-25. Springer Netherlands, 2012.

[200] C. S. Scullin and L. D. Partridge. Contributions of SERCA pump and ryanodine-sensitive stores to presynaptic residual $\mathrm{Ca}^{2+}$. Cell Calcium, 47(4):326-338, Apr. 2010.

[201] P. Sellick and I. Russell. The responses of inner hair cells to basilar membrane velocity during low frequency auditory stimulation in the guinea pig cochlea. Hearing Research, 2(34):439-445, June 1980.

[202] V. Shahrezaei, A. Cao, and K. R. Delaney. $\mathrm{Ca}^{2+}$ from one or two channels controls fusion of a single vesicle at the frog neuromuscular junction. The Journal of Neuroscience, 26(51):1324013249, Dec. 2006.

[203] V. Shahrezaei and K. R. Delaney. Consequences of molecular-level $\mathrm{Ca}^{2+}$ channel and synaptic vesicle colocalization for the $\mathrm{Ca}^{2+}$ microdomain and neurotransmitter exocytosis: a Monte Carlo study. Biophysical Journal, 87(4):2352-2364, Oct. 2004. 
[204] V. Shahrezaei and K. R. Delaney. Brevity of the $\mathrm{Ca}^{2+}$ microdomain and active zone geometry prevent $\mathrm{Ca}^{2+}$-sensor saturation for neurotransmitter release. Journal of Neurophysiology, 94(3):1912-1919, Sept. 2005.

[205] J. Siegel. Spontaneous synaptic potentials from afferent terminals in the guinea pig cochlea. Hearing Research, 59(1):85-92, Apr. 1992.

[206] J. H. Siegel and E. M. Relkin. Antagonistic effects of perilymphatic calcium and magnesium on the activity of single cochlear afferent neurons. Hearing Research, 28(23):131-147, 1987.

[207] J. H. Singer, L. Lassov, N. Vardi, and J. S. Diamond. Coordinated multivesicular release at a mammalian ribbon synapse. Nature Neuroscience, 7(8):826-833, Aug. 2004.

[208] J. Snellman, B. Mehta, N. Babai, T. M. Bartoletti, W. Akmentin, A. Francis, G. Matthews, W. Thoreson, and D. Zenisek. Acute destruction of the synaptic ribbon reveals a role for the ribbon in vesicle priming. Nature Neuroscience, 14(9):1135-1141, Sept. 2011.

[209] M. A. Spassova, M. Avissar, A. C. Furman, M. A. Crumling, J. C. Saunders, and T. D. Parsons. Evidence that rapid vesicle replenishment of the synaptic ribbon mediates recovery from shortterm adaptation at the hair cell afferent synapse. JARO: Journal of the Association for Research in Otolaryngology, 5(4):376-390, Dec. 2004.

[210] R. D. R. V. Steveninck and W. Bialek. Real-time performance of a movement-sensitive neuron in the blowfly visual system: coding and information transfer in short spike sequences. Proceedings of the Royal Society of London B: Biological Sciences, 234(1277):379-414, Sept. 1988.

[211] N. Strenzke, S. Chanda, C. Kopp-Scheinpflug, D. Khimich, K. Reim, A. V. Bulankina, A. Neef, F. Wolf, N. Brose, M. A. Xu-Friedman, and T. Moser. Complexin-I is required for highfidelity transmission at the endbulb of Held auditory synapse. The Journal of Neuroscience, 29(25):7991-8004, June 2009.

[212] S. P. Strong, R. Koberle, R. R. de Ruyter van Steveninck, and W. Bialek. Entropy and information in neural spike trains. Physical Review Letters, 80(1):197-200, Jan. 1998.

[213] T. C. Südhof. The presynaptic active zone. Neuron, 75(1):11-25, Dec. 2012.

[214] T. Sueta, S. Y. Zhang, P. M. Sellick, R. Patuzzi, and D. Robertson. Effects of a calcium channel blocker on spontaneous neural noise and gross action potential waveforms in the guinea pig cochlea. Hearing Research, 188(12):117-125, Feb. 2004.

[215] C. J. Sumner, E. A. Lopez-Poveda, L. P. O'Mard, and R. Meddis. A revised model of the inner-hair cell and auditory-nerve complex. The Journal of the Acoustical Society of America, 111(5):2178, 2002.

[216] A. M. Taberner and M. C. Liberman. Response properties of single auditory nerve fibers in the mouse. Journal of Neurophysiology, 93(1):557-569, Jan. 2005.

[217] T. B. Tarr, M. Dittrich, and S. D. Meriney. Are unreliable release mechanisms conserved from NMJ to CNS? Trends in Neurosciences, 36(1):14-22, Jan. 2013. 
[218] M. C. Teich and S. M. Khanna. Pulse-number distribution for the neural spike train in the cat's auditory nerve. The Journal of the Acoustical Society of America, 77(3):1110-1128, 1985.

[219] M. C. Teich and G. Lachs. A neural-counting model incorporating refractoriness and spread of excitation. I. Application to intensity discrimination. The Journal of the Acoustical Society of America, 66(6):1738-1749, Dec. 1979.

[220] M. C. Teich, L. Matin, and B. I. Cantor. Refractoriness in the maintained discharge of the cat's retinal ganglion cell. Journal of the Optical Society of America, 68(3):386-402, Mar. 1978.

[221] R. C. Uthaiah and A. J. Hudspeth. Molecular anatomy of the hair cell's ribbon synapse. The Journal of Neuroscience, 30(37):12387-12399, Sept. 2010.

[222] N. F. Viemeister. Intensity coding and the dynamic range problem. Hearing Research, 34(3):267274, Aug. 1988.

[223] C. Vogl, B. H. Cooper, J. Neef, S. M. Wojcik, K. Reim, E. Reisinger, N. Brose, J.-S. Rhee, T. Moser, and C. Wichmann. Unconventional molecular regulation of synaptic vesicle replenishment in cochlear inner hair cells. Journal of Cell Science, 128(4):638-644, Feb. 2015.

[224] N. P. Vyleta and P. Jonas. Loose coupling between $\mathrm{Ca}^{2+}$ channels and release sensors at a plastic hippocampal synapse. Science, 343(6171):665-670, Feb. 2014.

[225] L.-Y. Wang, M. J. Fedchyshyn, and Y.-M. Yang. Action potential evoked transmitter release in central synapses: insights from the developing calyx of Held. Molecular Brain, 2(1):36, Nov. 2009 .

[226] L.-Y. Wang, E. Neher, and H. Taschenberger. Synaptic vesicles in mature calyx of Held synapses sense higher nanodomain calcium concentrations during action potential-evoked glutamate release. The Journal of Neuroscience, 28(53):14450-14458, Dec. 2008.

[227] P. Wangemann. Supporting sensory transduction: cochlear fluid homeostasis and the endocochlear potential. The Journal of Physiology, 576(Pt 1):11-21, Oct. 2006.

[228] S. Weiler, S. Krinner, A. B. Wong, T. Moser, and T. Pangršič. ATP hydrolysis is critically required for function of Cav1.3 channels in cochlear inner hair cells via fueling $\mathrm{Ca}^{2+}$ clearance. The Journal of Neuroscience, 34(20):6843-6848, May 2014.

[229] C. Weisz, E. Glowatzki, and P. Fuchs. The postsynaptic function of type II cochlear afferents. Nature, 461(7267):1126-1129, Oct. 2009.

[230] W. L. Whitlow. Hearing in whales and dolphins: an overview. In W. L. Whitlow, R. R. Fay, and A. N. Popper, editors, Hearing by whales and dolphins, number 12 in Springer handbook of auditory research, pages 1-42. Springer New York, 2000.

[231] C. Wichmann and T. Moser. Relating structure and function of inner hair cell ribbon synapses. Cell and Tissue Research, pages 1-20, Jan. 2015. 
[232] J. P. Wilson. Evidence for a cochlear origin for acoustic re-emissions, threshold fine-structure and tonal tinnitus. Hearing Research, 2(34):233-252, June 1980.

[233] R. L. Winslow and M. B. Sachs. Single-tone intensity discrimination based on auditory-nerve rate responses in backgrounds of quiet, noise, and with stimulation of the crossed olivocochlear bundle. Hearing Research, 35(23):165-189, Sept. 1988.

[234] I. M. Winter and A. R. Palmer. Intensity coding in low-frequency auditory-nerve fibers of the guinea pig. The Journal of the Acoustical Society of America, 90(4):1958-1967, 1991.

[235] I. M. Winter, D. Robertson, and G. K. Yates. Diversity of characteristic frequency rate-intensity functions in guinea pig auditory nerve fibres. Hearing Research, 45(3):191-202, May 1990.

[236] A. B. Wong, M. A. Rutherford, M. Gabrielaitis, T. Pangršič, F. Göttfert, T. Frank, S. Michanski, S. Hell, F. Wolf, C. Wichmann, and T. Moser. Developmental refinement of hair cell synapses tightens the coupling of $\mathrm{Ca}^{2+}$ influx to exocytosis. The EMBO Journal, 33(3):247-264, Feb. 2014 .

[237] T. Xu, M. Naraghi, H. Kang, and E. Neher. Kinetic studies of $\mathrm{Ca}^{2+}$ binding and $\mathrm{Ca}^{2+}$ clearance in the cytosol of adrenal chromaffin cells. Biophysical Journal, 73(1):532-545, July 1997.

[238] P. S. Yang, M. B. Johny, and D. T. Yue. Allostery in $\mathrm{Ca}^{2+}$ channel modulation by calciumbinding proteins. Nature Chemical Biology, 10(3):231-238, Mar. 2014.

[239] S. Yasunaga, M. Grati, M. Cohen-Salmon, A. El-Amraoui, M. Mustapha, N. Salem, E. El-Zir, J. Loiselet, and C. Petit. A mutation in OTOF, encoding otoferlin, a FER-1-like protein, causes DFNB9, a nonsyndromic form of deafness. Nature Genetics, 21(4):363-369, Apr. 1999.

[240] G. K. Yates. Auditory-nerve spontaneous rates vary predictably with threshold. Hearing Research, 57(1):57-62, Dec. 1991.

[241] G. K. Yates, I. M. Winter, and D. Robertson. Basilar membrane nonlinearity determines auditory nerve rate-intensity functions and cochlear dynamic range. Hearing Research, 45(3):203219, May 1990.

[242] E. D. Young and P. E. Barta. Rate responses of auditory nerve fibers to tones in noise near masked threshold. The Journal of the Acoustical Society of America, 79(2):426-442, 1986.

[243] V. Zampini, S. L. Johnson, C. Franz, M. Knipper, M. C. Holley, J. Magistretti, S. Masetto, and W. Marcotti. Burst activity and ultrafast activation kinetics of Cav1.3 $\mathrm{Ca}^{2+}$ channels support presynaptic activity in adult gerbil hair cell ribbon synapses. The Journal of Physiology, 591(16):3811-3820, Aug. 2013.

[244] V. Zampini, S. L. Johnson, C. Franz, M. Knipper, M. C. Holley, J. Magistretti, G. Russo, W. Marcotti, and S. Masetto. Fine tuning of Cav1.3 $\mathrm{Ca}^{2+}$ channel properties in adult inner hair cells positioned in the most sensitive region of the gerbil cochlea. PLoS ONE, 9(11):e113750, Nov. 2014. 
[245] V. Zampini, S. L. Johnson, C. Franz, N. D. Lawrence, S. Muenkner, J. Engel, M. Knipper, J. Magistretti, S. Masetto, and W. Marcotti. Elementary properties of Cav $1.3 \mathrm{Ca}^{2+}$ channels expressed in mouse cochlear inner hair cells. Journal of Physiology-London, 588(1):187-199, Jan. 2010.

[246] S. Y. Zhang, D. Robertson, G. Yates, and A. Everett. Role of L-type Ca ${ }^{2+}$ channels in transmitter release from mammalian inner hair cells I. Gross sound-evoked potentials. Journal of Neurophysiology, 82(6):3307-3315, Dec. 1999.

[247] R. S. Zucker and A. L. Fogelson. Relationship between transmitter release and presynaptic calcium influx when calcium enters through discrete channels. Proceedings of the National Academy of Sciences, 83(9):3032 -3036, May 1986. 